\title{
The Advanced Chemistry Basins Project Final Report
}

Covering the period of June 1999-November, 2003

William Goddard, Principle Investigator

California Institute of Technology

1200 E. California Blvd, M/C 135-79

Pasadena, CA 91125

Issued April 5, 2004

Submitted in fulfillment of DOE Contract DE-AC26-99BC15217

Sections prepared by

Peter Meulbroek

Yongchun Tang (co-PI)

Lawrence Cathles III (co-PI)

NOTE THAT: the Semi-annual technical progress report covering the period from June, 2003 to the end of the project is contained within this document. 


\section{Disclaimer}

This report was prepared as an account of work sponsored by an agency of the United States Government. Neither the United States Government nor any agency thereof, nor any of their employees, makes any warranty, express or implied, or assumes any legal liability or responsibility for the accuracy, completeness, or usefulness of any information, apparatus, product, or process disclosed, or represents that its use would not infringe privately owned rights. Reference herein to any specific commercial product, process, or service by trade name, trademark, manufacturer, or otherwise does not necessarily constitute or imply its endorsement, recommendation, or favoring by the United States Government or any agency thereof. The views and opinions of authors expressed herein do not necessarily state or reflect those of the United States Government or any agency thereof. 


\section{Abstract}

In the next decades, oil exploration by majors and independents will increasingly be in remote, inaccessible areas, or in areas where there has been extensive shallow exploration but deeper exploration potential may remain; areas where the collection of data is expensive, difficult, or even impossible, and where the most efficient use of existing data can drive the economics of the target. The ability to read hydrocarbon chemistry in terms of subsurface migration processes by relating it to the evolution of the basin and fluid migration is perhaps the single technological capability that could most improve our ability to explore effectively because it would allow us to use a vast store of existing or easily collected chemical data to determine the major migration pathways in a basin and to determine if there is deep exploration potential. To this end a the DOE funded a joint effort between California Institute of Technology, Cornell University, and GeoGroup Inc. to assemble a representative set of maturity and maturation kinetic models and develop an advanced basin model able to predict the chemistry of hydrocarbons in a basin from this input data. The four year project is now completed and has produced set of public domain maturity indicator and maturation kinetic data set, an oil chemistry and flash calculation tool operable under Excel, and a user friendly, graphically intuitive basin model that uses this data and flash tool, operates on a PC, and simulates hydrocarbon generation and migration and the chemical changes that can occur during migration (such as phase separation and gas washing).

The DOE Advanced Chemistry Basin Model includes a number of new methods that represent advances over current technology. The model is built around the concept of handling arbitrarily detailed chemical composition of fluids in a robust finite-element 2-D grid. There are three themes on which the model focuses: chemical kinetic and equilibrium reaction parameters, chemical phase equilibrium, and physical flow through porous media. The chemical kinetic scheme includes thermal indicators including vitrinite, sterane ratios, hopane ratios, and diamonoids; and a user-modifyable reaction network for primary and secondary maturation. Also provided is a database of typespecific kerogen maturation schemes. The phase equilibrium scheme includes modules for primary and secondary migration, multi-phase equilibrium (flash) calculations, and viscosity predictions. 


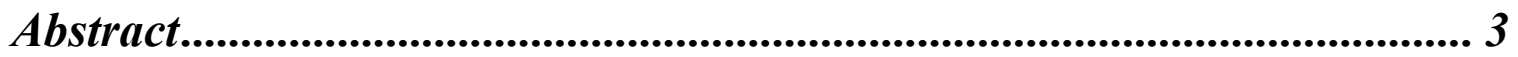

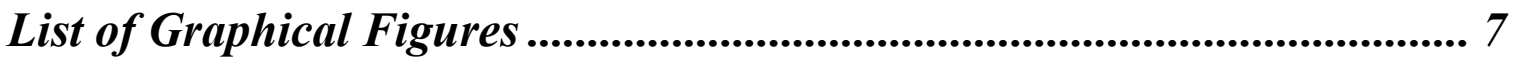

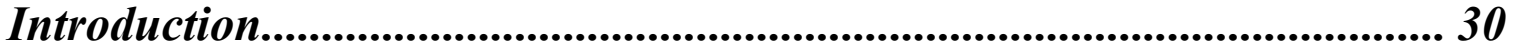

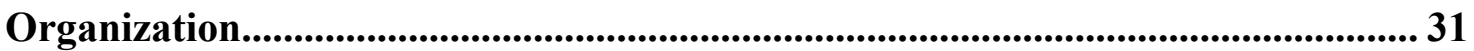

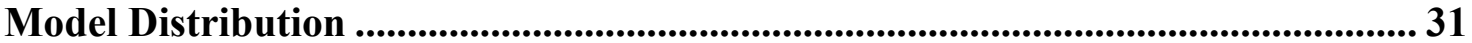

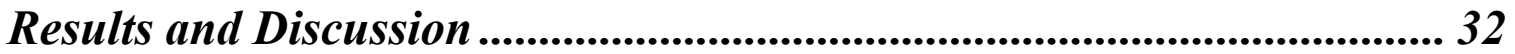

Paleo-Heat Flow Calibration Using Chemical Maturity indicators.......... 32

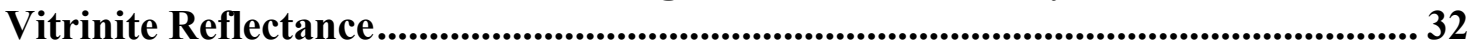

MethylPhenanthrene Index (MPI) ...................................................................33

MethylDibenzothiophene Index (MDI) ............................................................................. 33

Hopane and Sterane ratios............................................................................................... 33

20S/20R diastereomer ratios............................................................................................. 35

Apatite Fission-Track ................................................................................................... 36

Smectite/Illite .......................................................................................................................... 36

Compositional Modeling of Hydrocarbon Generation and Destruction................. 42

Technical Approach ............................................................................................................ 43

Compositional Modeling of Hydrocarbon Generation and Destruction... 43

Methods and Materials ..................................................................................... 45

Kerogen Sources and Types............................................................................................... 45

Computational Methods....................................................................................................... 50

References ....................................................................................................... 54

Appendix 1: Data Tables ....................................................................................... 63

Appendix 2: Experimental and theoretical data plots..............................8 87

Modeling Phase Behavior................................................................................. 110

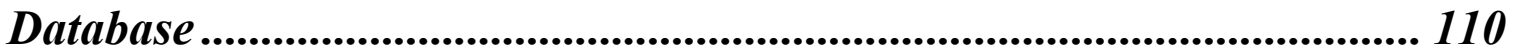

Tables............................................................................................................................... 111

Perl Scripts and Modules............................................................................ 114

1 PT-PhaseDiagram.pl ............................................................................................... 115

2 PX-PhaseDiagram.pl .................................................................................................. 117

3 getIDs.pl..................................................................................................................... 118

4 creatComponent.pl ........................................................................................................ 119

5 EOSInterface................................................................................................................... 120

6 Phase::Database::Access................................................................................ 123

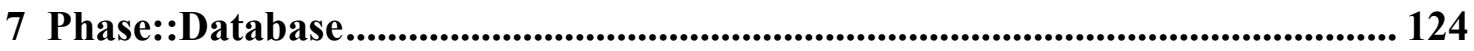

8 Phase::Database::PostgreSQL ...................................................................................... 126

9 Phase::EquationofState::AasbergPetersen ............................................................... 127

10 Phase::EquationofState::Generic.......................................................................... 129

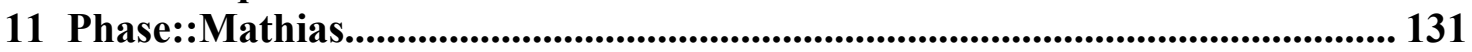

12 Phase::EquationofState::PRKS .............................................................................. 133

13 Phase::EquationofState::VanderWaals.............................................................. 135

14 Phase::Flash .............................................................................................................. 137

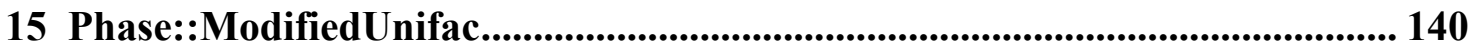

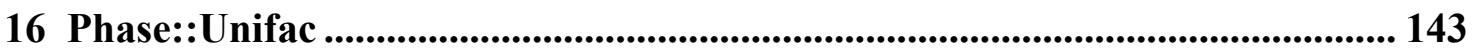




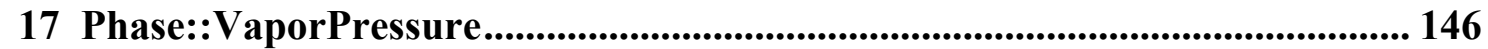

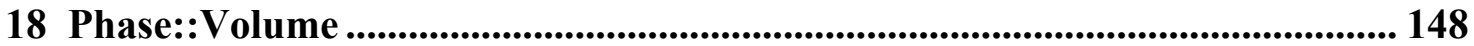

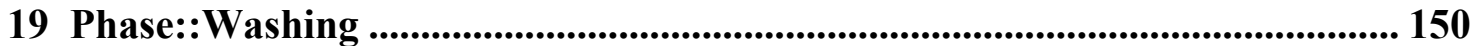

20 Phase::WilsonK ................................................................................................... 152

21 Standard::Function ............................................................................................... 153

22 Standard::List ............................................................................................................ 154

23 Viscosity::Chung........................................................................................................ 159

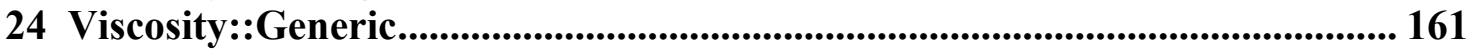

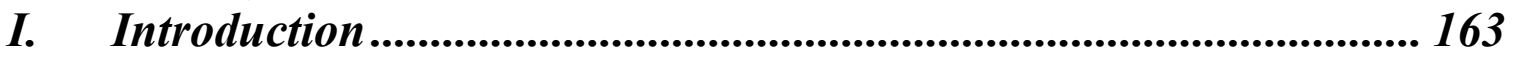

III. Getting Started .................................................................................. 166

A. File Structure .................................................................................................................... 166

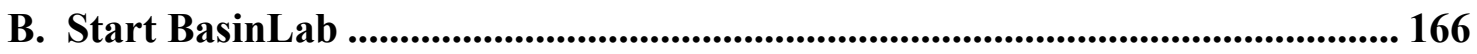

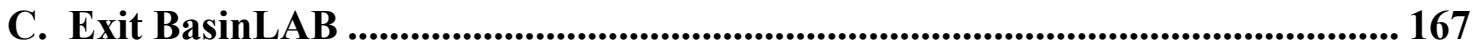

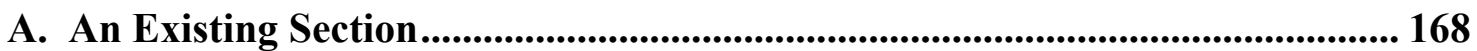

B. New Interpretation............................................................................................... 171

V. An Example Illustrating Advanced Chemistry Capabilities.............. 179

A. Geological Input................................................................................................................ 179

B. Physical Processing ............................................................................................. 180

C. Determining and Verifying the Heat Flow History ................................................ 182

D. Pseudo 2D Hydrocarbon Generation................................................................... 187

E. Finite Element Modeling ............................................................................................. 189

F. Selecting And Modifying Kinetic Maturation Models............................................ 195

G. 2D Maturation and Migration...................................................................... 201

H. Overview of Petroleum Generation and Migration ............................................. 202

I. Macro-Chemistry of the Petroleum System ............................................................. 205

J. Defining the Petroleum Micro-Chemistry ......................................................... 214

K. Micro-Chemistry of the Petroleum System ..................................................... 223

L. Phase State and Gas Washing in the Model Petroleum System ................ 225

M. Phase State and Gas Washing of Specific Petroleum .................................... 230

VI. Reconstructing the Physical History of a Basin ........................ 231

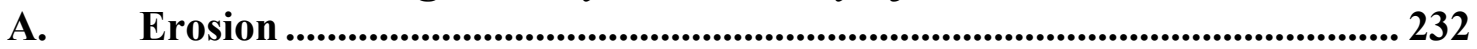

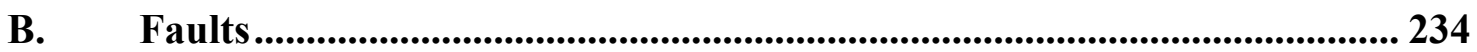

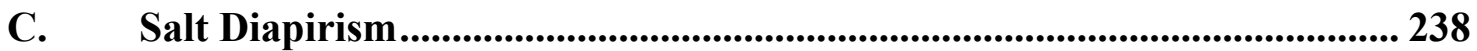

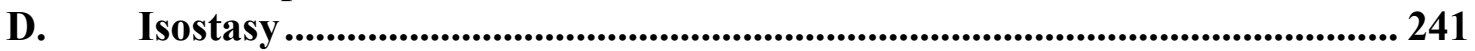

E. Rift Heatflow and Water Depth Corrections .................................................. 243

F. Water Depth ................................................................................................ 249

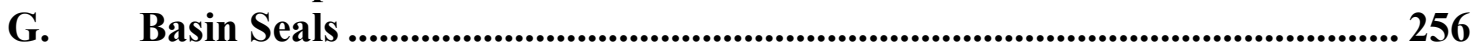

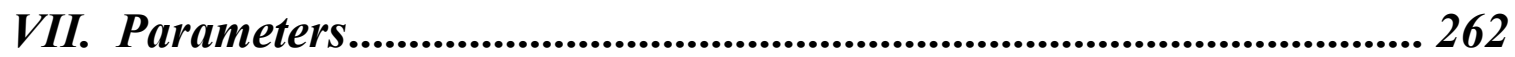

A. Geohistory Parameters and Porosity ............................................................... 263

B. Material Properties............................................................................................. 264

2. Salt and Seal Permeability ........................................................................... 267

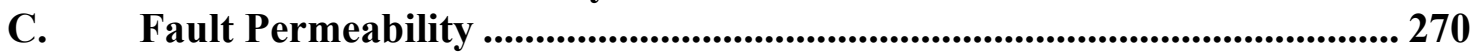

D. Kinetic Maturation Models..................................................................... 274

E. Temperature Boundary Conditions .................................................................... 280

F. Controls for 1D Thermal and Migration Calculations................................ 283

G. Saving Control Parameters....................................................................... 284 
VIII. Verifying the Basin Heatflow History ........................................ 285

A. Time-temperature History Confirmation................................................................... 285

B. Hydrocarbon Maturation .............................................................................................. 292

IX. 2D Fluid Flow and Temperature Equation Solutions ...................... 292

A. Flow in a Simple Fault-Antithetic Fault System ............................................. 294

B. Brief Comments on Finite Methods ................................................................... 308

X. Petroleum Migration.......................................................................... 310

A. Primary Migration...................................................................................... 310

B. Secondary Migration ......................................................................................... 314

C. Comments on Secondary Migration Methods.................................................... 321

XI. Petroleum Composition and Phase Chemistry.................................. 323

A. Petroleum Composition ............................................................................... 323

B. Petroleum Decomposition and Phase Chemistry ............................................. 327

C. Washing a Standard Petroleum............................................................................. 335

XII. Public Web Site and Future Planned Developments ..................... 338

XIII. Appendices ........................................................................................ 339

A. User Input Files.......................................................................................................... 339

B. Variable Names Recognized in Sflat ................................................................. 340

C. Sketch Derivation of Equations .................................................................................. 341

XIV. References Cited.............................................................................. 346

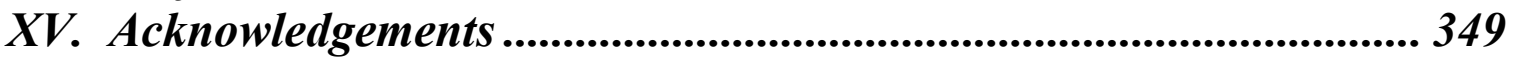

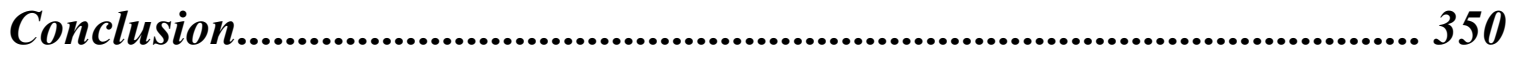




\section{List of Graphical Figures}

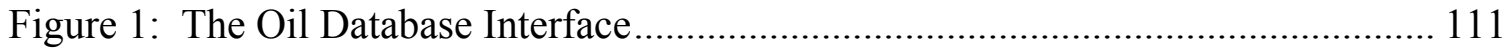

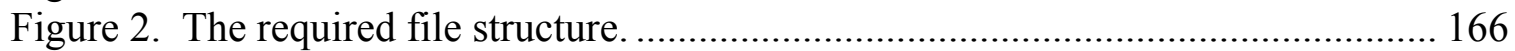

Figure 3. Select the blcases folder to establish the path to the desired file structure..... 167

Figure 4. A resized version of the Basin $L A B$ workspace. A 2D section is processed using the menus on the top toolbar of the workspace.......................................... 167

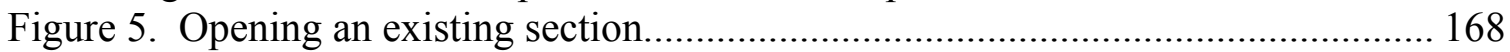

Figure 6. Selecting an existing *.dxf file will bring up that graphics file and the digitizing wells from the last case. To get the Digiwells for the current case (if it has been interpreted previously), select the Edit Previous Interpretation menu, close the forms that appear, and select the Edit Previous Digiwell Positions menu. Alternatively you can simply erase the wells and add the two shown above................................... 170

Figure 7. Paths to existing files for the case selected in Figure 5. An SFLAT and dxf file exists for the case, but not a GoCAD section. .................................................. 171

Figure 8. Canvas sketch of basin. The image consists of a red bounding box and 6 horizons (the base of the box to the purple horizon just below the top. The top of the bounding box is normally considered to be sea level. The horizons are all simple lines except for the third horizon from the top, which is a polyline representing a reef. The Canvas image is saved as a Carb_Reef.dxf file, which is read into Basin $L A B$.

Figure 9. The sketch is read into BasinLAB by selecting File: New Section Project and browsing to the Carb_Reef.dxf file saved above. The title, description, and domain dimensions are added as indicated. Depressing the OK button reads the selected *.dxf file and opens a digitizing control form and a BasinLAB image of the section as shown in Figure 10.

Figure 10. Position the digitizing wells (light blue vertical lines) over the sides of the reef using the digitizing well command form to the left. Digitizing wells can be selected using the top spinner. Once selected, they can be erased, or moved, or nodes in the digitizing well can be individually moved. Digitizing wells can be erased and added. Hints displayed on the status bar at the bottom of the workspace are provided when the mouse is positioned over a button, label or any feature of a form.

Figure 11. Depressing the Digitize button (under the mouse) digitizes the section shown in Figure 10. Depressing the adjacent Adjust Grid and Properties button allows lithologies, strata, and well names to be assigned as shown in Figure 12.

Figure 12. Complete section digitization by depressing the Adjust Grid and Properties.

The result is a control form and the digitized section as shown.

Figure 13. Assign initial strata properties by depressing the Initial Strata Properties button as shown, and dialing in the appropriate lithology for each strata in the section. Note that at this stage the strata have generic names. The lithology control on the left is a portion of the control form shown in Figure 12. Note that the third strata from the base has been assigned carbonate lithology.

Figure 14. The lithology of the outlined element has been modified by navigating to that element and changing the lithology as shown to the left. The color is the carbonate fraction (top combo window). The element outlined in black was initially red, 
indicating it was $100 \%$ coarse carbonate. However, it has now been modified to $100 \%$ silicate and $45 \%$ sand. Also the strata names have been changed to Base_Carbonate and Top_carbonate as shown in the Navigate Elements grouping in the control form. Strata ages and well names can be similarly modified. 175

Figure 15. Lithology pattern plot of the SFLATorig.txt section created as described above. Notice that the base of the section is at $200 \mathrm{~m}$ depth and the section is $500 \mathrm{~m}$ wide, and that the seafloor is below sea level by the amount indicated in the Canvas sketch (Figure 8). More lithologic details are inherent in the Lithology Color plot where shale is set to blue, sand to red, and carbonate to green. 176

Figure 16. The first few lines of a standard GoCAD section file. Files of this style are read by BasinLAB using the Files: Open New Project menu command with the GoCAD option selected as shown in Figure 17. The initial processing steps are discussed in the following two figures. The remaining steps are the same as in processing *.DXF files and were discussed above.

Figure 17. Read in a GoCAD section by selecting the GoCAD option for the input data source in Files: New Section Project. This brings up the form to the left. Clicking OK results in the graphics shown in Figure 18.

Figure 18. The base graphics show the GoCAD input file read from the GoCAD folder in the blcases $\backslash$ Examples.BL $\backslash$ GoCAD_exmpl.2d $\backslash$ case folder. The blue digitizing wells ("Digiwells") have been added by using the Add New Well button and clicking with the right mouse button along the well (top to bottom) and finishing with a right mouse button click. NOTE: When the section first comes up, the DigiWells are from the previously digitized graphic. This is intentional, as it greatly facilitates digitizing 3D sections. If a previous graphic interpretation has been made, the Digiwells for the current section can be obtained by using the Graphic Input: Read and edit Previous Interpretation command and then returning to the Edit Previous Digiwell Positions command.

Figure 19. The McBride_1 ${ }^{\text {st }}$ third.2d section, plotted using Geology: Edit/Plot Spreadsheet:

Figure 20. Control form obtained from GeoHistory: Create Geohistory:

Figure 21. Geologic history of section shown in Figure 20 that was constructed using form in that figure. Notice how salt pod at $10.5 \mathrm{Ma}$ is loaded and redistributed. Note changes in sea level. Vertical axis is elevation in km; horizontal axis is distance along the section in km. Plots are created by GeoHistory: Plot and Edit: Plot menu command. Axis labels are turned off in View: Plot Controls: Show Axis Titles.

Figure 22. Heatflow evaluation form obtained from Heatflow and Maturation: Evaluate Heatflow:

Figure 23. Temperature and $\log 10$ biomarker Re are plotted as functions of depth in well

5. Temperature data are shown as small boxes. The computed temperature profile in (a) is shown as a solid line; 10, 20 and $30 \mathrm{C} / \mathrm{km}$ profiles are shown as dashed lines for comparison. The near-surface temperature gradient is $\sim 20 \mathrm{C} / \mathrm{km}$. In (b) the $22 S / 22 S+22 R$ C32 Hopane ratio conversion index is converted to its vitrinite reflectance equivalent, Re, using a regression of the Burnham and Sweeney vitrinite model with the calculated conversion index for all nodes in the well. The Re values are plotted at the depths the oil samples were collected $(+)$. In addition the vitrinite 
reflectances computed by the Burnham and Sweeney (solid line) and two custom vitrinite models (dotted and dashed lines) are shown. The vitrinite reflectance model used to compute the Re is the Burnham and Sweeney model. The black part of that curve shows the range of Re over which the 22S/22S+22R C32 Hopane maturity measure is valid.

Figure 24 . The R28TA/R28TA+aR29MA_triaromatic_steroid biomarker maturity marker is added to the $22 S / 22 S+22 R$ C32 Hopane maturity marker in Figure 24 by adding this biomarker to the combo box selection (by control shift selection). The plotting conventions are the same as in Figure 24.

Figure 25. Regression between the $22 S / 22 S+22 R$ C 32 Hopane conversion index and the calculated Burnham and Sweeney vitrinite Ro maturity for all points in well 1. This regression is calculated and displayed using the portion of the form in Figure 22 shown.

Figure 26. Depressing the surace Tenp...6. in the Figure 22 form causes the following form to appear:

Figure 27. Forms for evaluating hydrocarbon maturation called forth with the Heatflow and Maturation: Evaluate Maturation menu command.

Figure 28. Computed from Figure 27 by moving the element marker down to the Jurassic source strata using the navigate spinners, and depressing the Compute Maturation Button. The simulated maturation shows that oil has been generated and cracked to methane. Only methane is being generated at the present time.

Figure 29. Comparison of methane and $\mathrm{C} 15+$ generation for the North Sea and Cameo Coal kerogens at the Jurassic source location in Well 5. Kerogen 1 is the North Sea Kerogen and is plotted with bold lines. Kerogen 2 is the Cameo Coal kerogen and it is plotted with thinner lines.

Figure 30. Selecting Reset Controls from the Solve Equations menu as shown will bring up the form on the left, asking for selection of the equations to be solved. In this case we request only the temperature equation be solved.

Figure 31. The default solution controls initiated in Figure 31 are shown here. Only the temperature equation is solved. It is solved once $($ NDelt $=1)$ after each stratum is deposited. The spinners can be used to increase the number of timesteps steps taken after a stratum is deposited. For example we could solve for $\mathrm{T}$ in 5 timesteps after the deposition of a strata by toggling NDelt to 5 as shown for the strata whose deposition ends at 40Ma. The timesteps can be of equal size (if $\mathrm{FxDt}=1$ ), or can exponentially increase $(\mathrm{FxDt}>1)$ with timestep. The first and last $\mathrm{Dt}$ are shown. For the deposition ending at $30 \mathrm{Ma}, \mathrm{FxDT}=1.4$ allows the initial timestep to be $0.91 \mathrm{Ma}$ and the last 3.5, rather than a uniform 2 Ma. Temperature in the first layer is assumed to be the steady-state temperature. Hence timesteps do not apply and they are set to zero.

Figure 32. Finite element parameters that control the $10^{\text {th }}$ timestep (which ends at $30 \mathrm{Ma}$. Form arises when first column entry in Figure 31 form is doubleclicked. The transient temperature equation is solved implicitly $(\mathrm{TH}=1)$ with a maximum of 2 subtimesteps (Nsubt=-2) and one iteration. No variables are tracked. The hint bar for this form is shown in Figure 33

Figure 33. The hint bar that appears at the botton of the workspace when the mouse is positioned over the form in Figure 32 
Figure 34. Cross-section display of the Log 10 permeability in the 2D temperature solution. The temperature at the mouse tip is $83.2^{\circ} \mathrm{C}$. All physical parameters are available to plot or track, although many have zero values because the controlling equations were not selected for solution (for example the flow variables are zero because neither the pressure nor the convection equations were solved).

Figure 35. T Shade Control is used to highlight the oil window $\left(80<\mathrm{T}<120^{\circ} \mathrm{C}\right)$ in green.

Figure 36. Plot of $\mathrm{T}_{1 \mathrm{D}}-\mathrm{T}_{2 \mathrm{D}}$ obtained by selecting $1 \mathrm{D}-2 \mathrm{D}$ in the $\mathrm{T}[$ nodes] control box as shown. The caption, grayscale, and positioning the mouse as shown all indicate the differences in temperature can be $+/-20^{\circ} \mathrm{C}$. The $1 \mathrm{D}$ solutions temperatures are much too low under the salt dome (vertical dark band) and much too high adjacent to this band.

Figure 37. Thermal conductivity profile through the first vertical set of elements as indicated in the insert. Element navigator was used to select the first vertical element profile (vertical because vertical is selected in the profile group of the form), and the Plot Profile button in the Element Variables group depressed. Any variable could be selected. In this case we have selected thermal conductivity (K[tcu] in Element Variables group of Section Profiles form above left).

Figure 38. View the 2D solution (Solve Equations: View Calculated Solutions), select the t-series form (depress toolbar t-series button), and use the resulting form to navigate to the $144 \mathrm{Ma}$ Jurassic Kerogen element as illustrated. Depressing the Maturation Comparison button brings up the form in Figure 39.

Figure 39. Depressing the Calculate Maturation button and then (sequentially) the Plot

Ker1 and Plot Ker2 buttons results in the plots on the right. Note Tstart is $100^{\circ} \mathrm{C}$ and Tfinish $400^{\circ} \mathrm{C}$. The maturation is for the element selected in Figure 38. Maturation models for Type II kerogens can clearly be very different.

Figure 40. Comparison of the II_YT B684F maturation model to a Kimmeridge Clay maturation model for the element selected in Figure 39. Comparison is made by sequentially depressing the Sel Ker2, Calculate Maturation, Plot Kerl and Plot Ker2 buttons on the form in Figure 39.

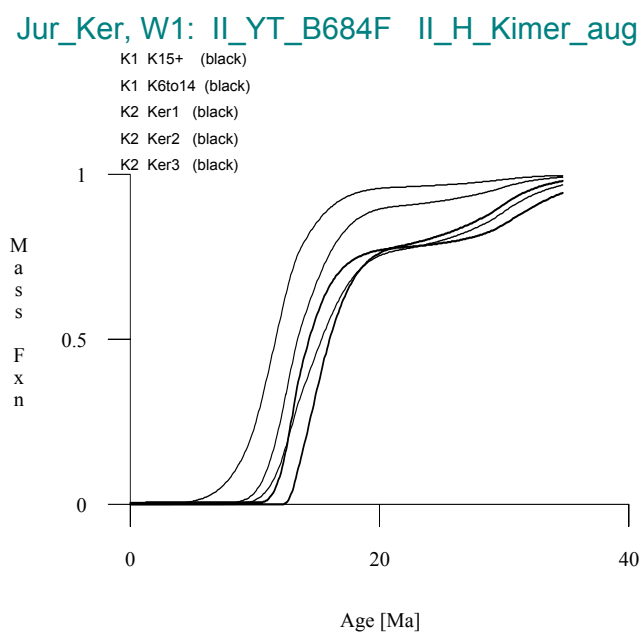

Figure 41. Comparison of particular maturation components in II_YT_B684F and II_H_Kimer_aug models shown in 
Figure 40. Components are selected in the combo boxes in the Display section of the form in Figure 39 and plotted with the Plot Comparison button. The YT kerogens are converted slightly sooner than the Kimmeridge kerogen. The graph on the right is calculated with the Calculate and Normalize button, which normalized all reactions to their maximum value. This facilitates comparing the timing of conversion. We focus on the kerogen conversion reactions because these are not affected by migration.

Figure 42. Portions of the spreadsheet containing the Activation Energy and Weights for the II_YT_B684F maturation model. Changing the K15+ parameters to the values shown in Figure 43 and applying them to the maturation calculations results in the shift of the K15+ conversion curve shown in Figure 44. 200

Figure 43. Modified II_YT_B684F kinetics. Note changes in K15+ activation energy and weights relative to Figure 42. The pre-exponential factor for the K15+ maturation reaction was also changed from $10^{14} \mathrm{sec}^{-1}$ to $10^{15} \mathrm{sec}^{-1}$. The maturation of $\mathrm{K} 15+$ is compared to H_Kimer_K15+ and K1 in Figure 44. 201

Figure 44. Comparison of kinetic models shown in Figure 37 as modified in Figure 43. The K15+ maturation for the II_YT_B684F model now approximates the timing of the $\mathrm{K} 15+$ and $\mathrm{K} 1$ kerogen components of the Kimmerigian, although the mass fraction converted is quite different.

Figure 45. Petroleum mass balance in the entire 2D section. The black curve shows the total mass of petroleum that could be generated by maturation of the kerogen deposited in the $85 \mathrm{~km}$ long, $1 \mathrm{~km}$ wide section. The jogs at $144 \mathrm{Ma}$ represents the deposition of the Jurassic kerogen source bed and the jog at 53 Ma the deposition of the Eocene source strata. The red curve shows the petroleum expelled from the basin and the blue curve the petroleum (generated and potential) remaining in the basin. The flat top of the black total petroleum curve shows petroleum mass is conserved. This plot was produced using HCMassBal toolbar button.

Figure 46. The total mobile petroleum mass vented from and remaining in the section (assumed to be $1 \mathrm{~km}$ wide). The blue curves show the mass of generated and vented liquid petroleum components. The red and orange curves the total and vented mass of gaseous hydrocarbon components. Petroleum components with carbon number less than 6 are assumed to be gaseous. The curves show that most of the petroleum generated in the basin has been expelled into the ocean. This plot was produced using the toolbar $\mathrm{O} / \mathrm{G}$ Inventory button. The age range is set in View: Plot Controls: Set Age Range. 203

Figure 47. Same as Figure 46 but showing the vented and total mobile petroleum mass that has been expelled from the source strata. Almost all the petroleum that escapes the source strata escapes the basin. Restriction to expelled hydrocarbons is requested with the View menu as shown below. Age range is set with the menu item below that highlighted....

Figure 48. Statistics on hydrocarbon generation. Produced using Solve Equations: View Calculated Solution: Stats toolbar button. The numbers correspond to the $0 \mathrm{Ma}$ intercept of the curves in Figure 45 through Figure 47. The Multiply by spinner allows the width of the section to be increased from its $1 \mathrm{~km}$ default value. Its use is illustrated in the lower figure. It shows the same statistics on hydrocarbon generation that are shown in the top figure except the Multiply by spinner has been 
used to increase the section width to $125 \mathrm{~km}$. Table shows that if the section modeled is typical, a $125 \mathrm{~km} \mathrm{x} 85 \mathrm{~km}$ area of the basin has generated 58 Billion Tonnes of liquid hydrocarbon of which $80 \%$ has been vented, and about $7 \%$ retained in migration conduits in the basin. 205

Figure 49. Gas oil ratio of migrating (mostly supercritical) petroleum. Constructed by selecting FxK2Gas in the Plot Control (XS button) form. Since GOR is the tracking variable its value under the mouse tip is displayed on the toolbar. The GOR under the mouse in this case is $16.6 \mathrm{~kg}$ gas per $\mathrm{kg}$ oil. The generation of Jurassic gas is evident as a light band between $\sim 14$ and $12 \mathrm{~km}$ depth. ........................................ 206

Figure 50. The fraction Eocene (K2) gas in the migrating petroleum. Constructed by selecting FxK2Gas in the Plot Control (XS button) form. The gas component of the migrating petroleum is mostly derived from Jurassic (K1 sources)...................... 207

Figure 51. The fraction Eocene (K2) oil in the migrating petroleum. Constructed by selecting FxK2Oil in the Plot Control (XS button) form. The oil component of the migrating petroleum is mostly derived from Eocene (K2 sources)........................ 207

Figure 52. GOR profile through the elements to the right of Well 1. The No Kerogen and No Zeros Field Selection Controls have been checked to eliminate source strata and zero values (where strata have zero thickness) in the plot. The contrast between high GOR in the interval between the Jurassic and Eocene sources, and the lower GORs in above the Eocene source $(\sim 12 \mathrm{~km})$, which is generating oil, is evident. 208

Figure 53. The incremental petroleum additions to each element in a vertical section adjacent to Well 1 at $\mathrm{t}=0$. The generation in the Eocene Source $(\sim 12 \mathrm{kmbsl})$ is about $33 \%$ that in the Jurassic Strata $(\sim 14 \mathrm{kmbsl})$. The increase starting at $\sim 3.3 \mathrm{~km}$ depth is due to compaction. Plot generated using the Section Profiles form (obtained with Prof toolbar button). 208

Figure 54. The gas mass flux just above the Jurassic source and right of well 1. Plotted with Solve Equations: View Calculated Solution: t-series: Navigate Elements: check Normlze (Dt,Dx): Plot. Ranges of age scale set in View: Plot Controls: Set Age Range. Notice that the IncGMF indicated in the Tracking Variables section of the form is $3.25 \times 10^{-4}$. This is the mass of gas transported across the selected element over the last timestep. This incremental mass addition divided by the element width $(13.2 \mathrm{~km})$ and the timestep $(0.25 \mathrm{Ma})$ yields the gas mass flux shown in the figure at $\mathrm{t}=0\left(1 \times 10^{-4} \mathrm{Bt} / \mathrm{km}^{2}-\mathrm{Ma}\right)$. 209

Figure 55. Change in the composition of Jurassic maturation products in the element just above this source bed (see Figure 50 for location). Plotted using Time Series form, Mobile K1 Prods combo selection. 210

Figure 56. Evolution of GOR (gas/oil mass ratio) in element shown in Figure 50. Plotted using Time Series form, GOR combo selection. 210

Figure 57. Instantaneous and cumulative hydrocarbon gas mass flux through an element $3.5 \mathrm{~km}$ below the surface as illustrated in the insert. Temperature in the element exceeds $65^{\circ} \mathrm{C}$ at ages left of the vertical yellow line. The figure is plotted using the combo entry in the Time Series form (Figure 54) illustrated below.

Figure 58. The HC Venting and Select Products forms are displayed when the VntProf button is depressed.

Figure 59. Fluxes of the hydrocarbon components across the H_144 age horizon (at the top of the Jurassic source bed). The total gas flux through the first element in this 
profile is shown in Figure 54. This plot shows that only the ends of the Jurassic source bed in our section are generating hydrocarbon at the present day. The sum of the gas component fluxes equals $10^{-4} \mathrm{Bt} / \mathrm{km}^{2}-\mathrm{Ma}$, which is the same gas flux shown in Figure 54.

Figure 60. The cumulative (time integrated) flux of Jurassic kerogen components across the $\mathrm{H}_{-} 144$ age horizon from the start of simulation to the present day.

Figure 61 . Flux of Jurassic methane across the $H_{-} 144$ horizon over the last $\sim 1 \mathrm{Ma}$. The bottom (red) profile corresponds to the profile in Figure 58. The forms used to compute this case are shown to the right of the graph.

Figure 62. Form summoned by Flash: Select Petroleum Model. We have selected the 61 molecule Meulbroek Thesis decomposition model. Construction of decomposition models is done within the Chemical Data.dba database and is described in a separate part of this report.

Figure 63. Some of the molecular components that comprise the Meulbroek Thesis (model 80) petroleum decomposition model. Form is for information purposes only and cannot be used to change the petroleum decomposition. It is summoned by Flash: View Molecular Components of Selected Model as illustrated above. The type of molecule is indicated by the Type flag ( $1=$ n-alkane, $2=$ cyclic alkane, $3=$ branched alkane, and $4=$ aromatic).

Figure 64. The scratchpad form used to design the decomposition of maturation macrocomponents into detailed molecular description of the petroleum. Form is summoned by Flash: Scratchpad: Design Petroleum Decomposition as illustrated above.

Figure 65. Distribution of n-alkanes specified by the upper portion of the form in Figure 64 if the Kissen slope is determined by the masses in the two highest carbon number cuts. The Kissen slope is the natural log slope. The $\log 10$ slope $=-0.1 / \ln 10=-$ 0.043. The Kissen slope posted is that of the last (highest $C_{n}$ ) cut.

Figure 66. Distribution of n-alkanes specified by the upper portion of the form in Figure 64 if the Kissen of the two highest carbon number cuts is set at -0.14 . The Kissen slope is the natural log slope, s.

Figure 67. Petroleum composition plotted by depressing Make and Plot total HC on the

Figure 64 form. ............................................................................... 220

Figure 68. Same as Figure 67 except the mole fractions in carbon number cut 3 have

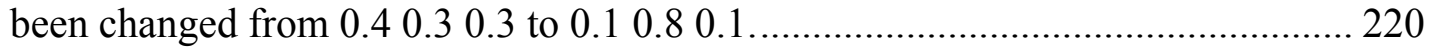

Figure 69. The Evaluate Kinetics form is selected with the Parameters: Evaluate kinetics scratchpad menu. Kerogen maturation models are selected from the Custom Kinetics folder. Some are provided with this distribution. Using the buttons on the top of the form we have selected two type II kerogen models. These are matured at a constant heating rate of $2^{\circ} \mathrm{C} / \mathrm{Ma}$. The maturation of the two kerogens can be compared as illustrated in Figure 27 and following figures. Here we use the maturation results to examine the hydrocarbon compositions as a function of temperature from 70 to $300^{\circ} \mathrm{C}$ using the Design Hydrocarbon Composition form shown in Figure 64.

Figure 70. Molecular composition of petroleum produced by heating two different type II kerogens from 70 to $300^{\circ} \mathrm{C}$ at $2^{\circ} \mathrm{C} / \mathrm{Ma}$. The maturation histories of the two kerogens was calculated using the form shown in Figure 69. The hydrocarbon 
compositions are displayed as a function of maturation temperature using the form in Figure 64. Temperature and depth increases from top to bottom. Caption now augmented to contain this information.

Figure 71. Form called by menu command Solve Equations: View Calculated Solution followed by depressing the PetCompsn button. With the Plot as Shift box checked, navigating the red element up (button under arrow) with the $K 1+K 2$ option selected causes the total petroleum composition to be plotted. The composition of just the kerogen 1 or kerogen 2 products can be plotted if the $K 1$ or $K 2$ options are selected. The total petroleum composition is for the element selected is shown in Figure 72.

Figure 72. The total petroleum composition of the element selected in Figure 71. It is plotted either by depressing the Plot button or, if the Plot as Shift box is checked, when the element is shifted to the one shown from any adjacent element using the navigator buttons. Notice that the (natural log) Kissen slopes f the heaviest (largest $\mathrm{C}_{\mathrm{n}}$ cut) are given for the products of both kerogens.

Figure 73. Vertical Kissen slope profile through well 1 elements produced by selecting the Kissen Vertical Profile option in the Figure 71 form and depressing the plot button as shown below. The discontinuity in the profiles at $\sim 10 \mathrm{~km}$ depth occurs where alkanes $>\mathrm{C} 15$ first appear and the Kissen slope shifts to the heaviest cut. Below $10 \mathrm{~km}$ the heavy n-alkanes have cracked to lighter fragments................... 224

Figure 74. Setting the $\mathrm{Wt} \% \mathrm{n}$-alkane fraction in cut 5 to zero for Kerogen 1 in the Flash: Design Petroleum Composition form (Figure 64) changes the profile shown in Figure 73 to change as shown. The Kissen slope is determined by the $\mathrm{C}_{2-3}$ and $\mathrm{C}_{6-14}$ fractions rather than the $\mathrm{C}_{6-14}$ and $\mathrm{C}_{15+}$ fractions. The Apply wtpct n-alk button must be depressed to apply the changes. 225

Figure 75. Plot of phase state of element using procedure: Solve Equations: View Calculated Solution: PetComposn button: navigate to element: choose Phase State option: push Plot button. The immediately underlying element is single phase. Hence the phase boundary in the Tiger Shoals area of the offshore Louisiana Gulf of Mexico is predicted to lie at $\sim 2.4 \mathrm{~km}$ depth. Notice that we have selected the PRKS (predictive Redlich-Kwong-Souave) equation of state for the phase state flash calculation. 226

Figure 76. Gas washing using the procedure: Solve Equations: View Calculated Solution: PetComposn button: navigate to element: choose Gas Wash Element: push Plot button. Here we wash element 615 at $2.91 \mathrm{kmbsl}$ (positioning mouse over element as shown gives depth and element on toolbar). We wash 1 mole of petroleum with the composition in element 615 with 10 moles of CH4 in 20 washing steps. Each washing aliquot is 1.15 times larger than the last. The first aliquot is 0.098 moles, the last is 1.389 moles. Results are plotted by selecting Plot Gas Wash Results and are shown in Figure 77-Figure 80. Results are written to a *.csv file in the case Metafiles folder if the Write *.csv button is depressed. .......................... 227

Figure 77. Plotting results of wash in Figure 76 with the procedure: select Plot Gas Wash Results option, select plot type (here Alkane Xn vs n), depress Plot. The log plot option is checked, so the plot is $\log \mathrm{Xn}$ vs $\mathrm{Cn}$.

Figure 78. Plot of moles of liquid petroleum remaining vs. moles of wash gas (CH4) interacted with the petroleum. Over $90 \mathrm{Wt} \%$ of the original oil is removed by 10 
moles of methane washing 1 mole of original petroleum (read y value from toolbar for position of mouse- 0.092 moles of petroleum remaining at 10 moles wash)... 228

Figure 79. Mole fraction gas in each flash calculation as a function of washing........... 229 Figure 80. Moles of component $\mathrm{n}$ divided by the moles of component $\mathrm{n}$ in the initial 1 mole of petroleum. The proportion of methane (pink curve) in the residual oil remains roughly constant. Higher carbon number alkanes are less depleted. Color intensity (pink to red) increases with carbon number. The caption indicates molecular type ( $1=$ alkane, $2=$ cyclic alkane, $3=$ isoalkane, and $4=$ aromatic $)$ as well as molecular weight. Any set of components can be selected in the combo box (cntrl key adds components) and plotted.

Figure 81. Erosion history specified in the blcases: Examples.BL: Erosion.2d example case, called up with the GeoHistory: Add Erosion: Add/Edit Erosion menu command. The form on the left shows the uncompacted thickness of all strata; the form on the right shows the uncompacted erosion that has been restored and then removed for the strata highlighted. Edits are transferred between forms using the transfer buttons. It is not appropriate in this case, but in cases where erosion has removed more than one stratum, the erosion process can occur over more than one time interval, e.g., more than two stratigraphic intervals can be highlighted on the left form, and modified by erosion on the right.

Figure 82. Figure at right shows section of the blcases: Examples.BL: Erosion.2d example at the present day. The first panel shows how $0.97 \mathrm{~km}$ has been added to the second pseudo-well, $1.34 \mathrm{~km}$ to the third, and 0.97 to the fourth. The second panel shows how these additions have been removed by erosion. Subsequent strata are deposited and the erosion surface buried until the final (present day) panel is reached. Notice that deposition can occur at a horizon that is also eroding. The carbonate (yellow box pattern) is deposited at the same time the shale (blue dashes) is eroding. Sand is indicated by red dots.

Figure 83. The Askii erosionfile.txt file. Files are automatically documented by comments demarcated by \# symbols as shown.

Figure 84. Fault_code for the blcases: Examples.BL: Fault_test.2d case. The overlay selects the Fault_Code. The value edit boxes in the Location and Nodal Values group indicates the values of the Fault code at the 4 corners of the element selected (dark outline). The colored corner indicates the node that shades the elements in the section. Navigating the elements shows that the first and last pseudowell have Fault_code flags of 0, the left side of the fault has Fault_code flags of 3, and the right side of the fault flags of 5. The flags can be set for a whole well by toggling the Display selection to Well, or for a single element if the Display is set to element as above. The Value is changed for the variable selected in the Overlay Information group by changing the value in the edit box and depressing the Apply button...... 235

Figure 85. Geologic history of blcases: Examples.BL: Fault_test.2d case with (left) and without (right) detachment slip allowed. Right boundary is required to be vertical. Faulting is modeled by requiring that the right side of the fault maintain a fixed distance from the left side, as sediment layers are backstripped and decompacted. 
Figure 86. Section from the Niger Delta analyzed by (Cathles, Colling et al. 2003) shows how a complex set of faults can be captured with the fault codes described above.

Figure 87. Section in Paris basin prepared by Alain Raboute that illustrates a section with a complex fault system and erosion. The Fault_code flags for each pseudowell are shown in the $4^{\text {th }}$ panel.

Figure 88. GeoHistory: Create Geohistory with no salt diapirism selected. Figure 89 shows the history with two options of salt diapirism. Axes labels are turned off in plot by unchecking View: Plot Controls: Show Axes Titles. Case is blcases/Examples.BL/MiniBasin.2d.

Figure 89. GeoHistory:Create Geohistory with salt diapirism selected. Left shows history with

Figure 90. Water depth is predicted for the $1050 \mathrm{~km}$ long section from the ArkansasLouisiana border to the Sigsbee Knoles shown to the left using the GeoHistory: Create Geohistory menu command with the Geohistory Controls in Figure 88 checked as shown. Case is blcases/Examples.BL/Louisiana_Line.2d....

Figure 91. Comparison of water depths predicted from present sea level by backstripping, decompacting, and assuming isostatic equilibrium on a $10 \times 10^{23} \mathrm{~N}-\mathrm{m}$ lithosphere (top figure) and on a $1 \times 10^{23} \mathrm{~N}-\mathrm{m}$ (lower figure). Figures here were created by writing a word metafile (*.wmf) image of the GeoHistory: Plot and Edit: Plot menu command, selecting color as the type of plot, using the time spinner on the workspace toolbar (left) to move back in time to $16.5 \mathrm{Ma}$, and then using the file button to place the image displayed in the Metafiles folder of the blcases/Examples.BL/Louisiana_Line. $2 d$ case. The *.wmf images placed in the Metafiles folder are labeled with the type of plot, picture number, and timestamp: GeoHistColorXS_13\#19_(10_1_19h34m).WMF. This indicates the image is the $13^{\text {th }}$ color frame of the geohistory saved as session picture number 19 at 5:34 pm on October 1 . In this case the *.wmf images were imported to Adobe Illustrator, simplified, and modified slightly.

Figure 92. Sea level history shown in Figure 90 corrected for thermal expansion using the GeoHistory: Rift HF and Water Depth): Estimate HF/Water Depth plate model. Notice the water depths after rifting are greatly reduced in the early stages of basin evolution. The case folder is: blcases/Examples.BL/Louisiana_Line. $2 d$. 244

Figure 93. Basal and surface heat flow profiles across the section. $\overline{\text { Plot using }}$ GeoHistory: Rift HF and Water Depth):Plot Basal and Surface Heatflow. Heatflow measurements along the section are automatically plotted if a heatflowdata.txt file is in the case folder. Its format is: 246

Figure 94 . Heat flow history inferred by the plate model in the blcases/Examples.BL/Louisiana_Line.2d. The heat flow history is examined using Geology: Edit/Plot Spreadsheet, selecting the HF(hfu) column and depressing the Edit Highlighted Column button. 246

Figure 95. The form and scratchpad summoned by depressing the Lithosphere and Mantle Param button on the GeoHistory: Rift HF and Water Depth): Estimate $H F /$ Water Depth form. A stretching factor entered in the Beta edit box (in this case 4) can be used to calculate the initial subsidence of a water- or sediment-filled basin by depressing the Subsidence from Beta button as indicated. Alternatively the 
stretching factor can be estimated from the initial or ultimate (total) subsidence of the basin by entering values in the appropriately-labeled boxes and pressing the Compute Beta from Subsidence button. Different parameter values can be entered in the top portion of the form and they will be used in subsequent calculations... 247 Figure 96. Geology: Graphic Edit menu command for the blcases/Examples.BL/Minibasin. $2 d$ case. 250

Figure 97. Geology: Graphic Edit form. Uniform water depth is added to each stratum by selecting that strata Display radio button, the Wat_depth overlay, and navigating to the desired strata. The value in the Overlay Information group is then changed from 0 to 2 (as shown) and the Apply button depressed. When this is done the color indicating water depth immediately changes to a shade of red. Water depth is carried for each node by the upper node of a highlighted element, as indicated by the red shading in the value box of the Location and Nodal Values group, and by the check marks in the Overlay Information group (two in this case because the entire strata is selected). Selecting Elements in the Display group and navigating reveals the nodal values that have been applied to each element and allows different sea levels to be assigned to the nodes of a stratum. This can be done in the Location and Nodal Values group. Here we have assigned a water depth of 5 to the top of the deepest (non-salt) strata, a value of 4 to the next strata up, etc. The water depth at of the top strata (the present water depth) is $1 \mathrm{~km}$. Figure 98 shows the water depths assigned, and Figure 99 the Geohistory that results when the case is processed with these assigned water depths.

Figure 98. The table of water depths created by graphic interaction with the SFLAT file described in Figure 97. This form is obtained by Geology: Edit/Plot Spreadsheet: selecting the water depth column: depressing the Edit Highlighted Column button. The water depths could, as an alternative to the graphics methods of Figure 97, be modified using this spreadsheet. 251

Figure 99. The geologic history of the blcases/Examples.BL/MiniBasin.2d case with sea levels assigned as indicated above. This case should be compared to that shown in the left panel of Figure 89. Notice how the top of the section changes elevation (water depth) with time. 252

Figure 100. Definition of the parameters in paleodepth file, the parameters in the paleodepth file for the blcases/Examples.BL/GoM_Shelf.2d, and a diagram illustrating parameters in the file. All lines in the file contain NS entries, where NS is the number of strata in the present day section (in this case 21). The first line of the paleodepth.txt file contains only 4 entries (the rest are 0 ). Wslope is the width of the slope in kilometers. $D s b$ is the depth of the slope break below seal level in kilometers. MaxWD is the maximum water depth in kilometers. The final entry on the first line is a code that is 1 if the shoreline is to the left, and -1 if it is to the right. The second line of the file contains 3 entries: Sshelf, the slope of the shelf in $\mathrm{km} / \mathrm{km}$ from the shelf break and negative because water depth decreases shoreward of the break. Sslope is the slope of the slope in $\mathrm{km} / \mathrm{km}$, which is positive because water depth increases seaward of the slope break. Socean is the slope of the ocean floor in $\mathrm{km} / \mathrm{km}$. The final line gives the distance of the shelf break in $\mathrm{km}$ from the end of the section, $X s b(t)$ at the time each age horizon is deposited. Only the distances for the strata actually deposited in the model (11 in this case) are given. The other 10 entries can have any value and are usually given the value $999 .$. 253 
Figure 101. Frames from the blcases/Examples.BL/GoM_Shelf.2d GeoHistory model with water depths defined by the paleodepth file illustrated in Figure 100.

Figure 102. SFLAT file of blcases/Examples.BL/GoM_Shelf. $2 d$ case obtained with menu command Geology: Edit/Plot Spreadsheet shows how a transgressing seal is defined by inserting horizons with horizon numbers of 0 (here called TRANSSEAL). The plot is made by depressing the Lithology

Pattern button at the top of the spreadsheet. The transgressing seal is shown by the yellow line.. 256

Figure 103. Migrating seal elements and pressure under them at the present day and 3.7 Ma before present for the blcases/Examples.BL/GoM_Shelf. $2 d$ case. Seal was defined in the SFLAT file as shown in Figure 102 and processed (to infer the geological history of the section) as a migrating seal (see Figure 88). The images above are obtained from GeoHistory: Plot and Edit: Check Grid and Graphic Edit. Note that the graphic image is placed in the clipboard only when the File button on the workspace toolbar is depressed. A *.wmf file is also placed in the Metafiles folder when this is done.

Figure 104. Editing the finite element grid using Solve Equations: View FE Grid allows the definition of a seal to be improved. Double clicking a seal element removes the seal flag; double clicking a non-seal element turns on the seal flag. This procedure can be used to improve the form of the seal as illustrated.

Figure 105. Parameters controlling how salt, seals, hydrofracturing, and aquathermal and the maturation of bulk organic matter will affect the calculated brine flow. Form summoned by Parameters: Permeability (+scrpad) and Pressure: Salt, Seals, Hydrofx, Aquathml, Org. Bkgnd as shown.

Figure 106. Flow calculated at two different times for the

blcases/Examples.BL/GoM_Shelf. $2 d$ case with the salt and seal controls set as shown in Figure 105. Because special assignment of salt permeability is not turned on, salt has its lithologic permeability of $10^{-9}$ millidarcies. Brine flow moves around salt bodies. Because the seal permeability is not turned on, the 2D brine flow calculations do not treat seal elements specially, and these elements have their normal lithologically-specified permeability. Figure 107 shows the flow at $8.3 \mathrm{Ma}$ if the salt permeability is set at $10^{-4} \mathrm{md}$ rather than $10^{-9} \mathrm{md}$.

Figure 107. Flow at 8.3 Ma in the blcases/Examples.BL/GoM_Shelf.2d case shown in the lower figure in Figure 106, but with the salt permeability set to $10^{-4} \mathrm{md}$ rather that $10^{-9}$. With this higher salt permeability the flow passes through the salt rather than moving 100's of kilometers to avoid this transit. Calculation of this flow pattern was achieved using the restart button 1 -step Solve on the toolbar that appears when a case is examined graphically using Solve Equations: View Calculated Solution... 261

Figure 108. Same case as the upper figure in Figure 106 but the seal has been turned on with a base permeability of $10^{-4} \mathrm{md}$ and a decrease with elevation of $-0.5 \log$ units per kilometer below sea level.

Figure 109. GeoHistory parameters form produced by menu command Parameters: GeoHistory Parameters.

Figure 110. Material properties form produced by menu command Parameters: Material Properties. 264

Figure 111. The properties of the matrix permeability algorithms can be explored efficiently with the Parameters: Permeability $(+$ scrpad): Explore Matrix Algorithms form. Plots for the porosity and the harmonic combo models are shown. 
The Calculate button calculates the permeability at the porosity, lithology, and hydrocarbon saturation specified. 266

Figure 112 . The permeabilities calculated by the various permeability algorithms for the current section can be examined using the Parameters: Permeability (+scrpad): Examine Section Permeability menu command. The Navigate Elements section moves the red element indicator, and the edit boxes of that group show the strataparallel and perpendicular permeabilities (kh and kv) and the anisotropy. The Strata Permeability combo box allows the different matrix algorithms to be selected. The image immediately changes when this is done. The Material Properties table can be edited using the button below this combo box. The edited properties are then used in subsequent displays and calculations. 266

Figure 113. Thermal conductivity form produced by menu command Parameters: Thermal Conductivity (+scrpad).

Figure 114. A sediment can be designed by assigning its mineralogy, the solid volume fraction of those minerals, the porosity, and the oil and gas saturation fractions. This is done as described in the text using the lower group of the Thermal Conductivity Scratchpad form shown in Figure 113. Here we show a portion of the mineral selection and volume fractions assigned for the Abu Gabra shale. Depressing the Calculate button for the parameter selections shown gives the result shown in the third panel.

Figure 115. Radioactive Heat Generation form produced by menu command Parameters: Heat Generation (+scrpad). Heat generation is specified by specifying the $\mathrm{Wt} \%$ potassium and the ppm uranium and thorium in a basin's sediments. Depressing the Calculate button then calculates the heat generation in cgs heat generation (HGU) and mks units. If a basin depth is indicated, the radiogenic heatflow in HFU and $\mathrm{mW} / \mathrm{m} 2$ is also calculated. If the skin depth (the depth below the basin surface) is zero radiogenicity is assumed not to decay with depth. Otherwise the radiogenicity is decreased with depth such that it is $1 / \mathrm{e}$ at the skin depth specified. The heat generation expected from the gamma tool response in API units can be estimated by dialing in an average gamma tool reading. Alternatively the gamma tool response expected for a particular heat generation rate can be determined by spinning the heat generation spinner in the Heat Generation group. The relations used are from (Bucker and Rybach 1996)................................... 270

Figure 116. Parameters for fault permeability are defined in the figure....................... 271

Figure 117. Fault permeability of the fault in the blcases/Examples.BL/MiniBasin.2d case illustrated in the left panel of Figure 89. Fault permeability is examined using the Parameters: Permeability (+scrpad): Examine Section Permeability form. The Adjacent Fraction Coarse fault property has been selected. Its profile down the fault is shown in the insert (obtained by depressing the Plot Flt Prof button. The adjacent fraction coarse is 0 for the first three elements, and zero at the top. It is maximum at the second element down from the top. The base of this element lies at $-1.5 \mathrm{~km}$ (x2 Tracking Variable in the form). The fraction coarse there is 0.67 (x value on the toolbar). Figure 118 shows the Adjacent Fraction Coarse and Sliprate fault permeabilities for this case.

Figure 118. Adjacent Fraction Coarse and Sliprate permeability of the fault in the blcases/Examples.BL/MiniBasin.2d case shown in Figure 113. The Sliprate 
permeability is low because the fault slip rate is small $(<0.26 \mathrm{~km} / \mathrm{Ma})$. We have pasted the fault model selected on the cross section images of this figure. Profile plots show how permeability varies with depth along the fault and are created using the Plot Flt Prof button on the Examine Section Permeability form in, for example, Figure 117. 274

Figure 119. Green River shale Kinetic maturation file. The file is documented with comments enclosed in pound (\#) signs. The first line gives the number of reactants, products, and the (maximum) number of activation energy weights. The second and third lines give the names of the reactants and products. The carbon number range of the products is given in the name connected by an underscore. This convention communicates the range to BasinLAB. BasinLAB uses units of Ma in its maturation calculations. Hence the $4^{\text {th }}$ line gives the conversion from $1 /$ seconds to Ma. The $5^{\text {th }}$ line gives the pre-exponential for all 7 reactions in $1 /$ seconds. The $6^{\text {th }}$ line can be ignored; it communicates variables to be written to output files in workstation calculations. The $7^{\text {th }}$ line gives the maximum possible mass of the reactant or product. It is the initial value of the kerogen (mass fraction of the total) and the total products that could be produced if all decomposition reactions were turned off. These values are computed in BasinLAB and can be copied into the kinetics file from there. The $8^{\text {th }}$ line gives the density of the solid and the average molecular weights of the gaseous products. Basin $L A B$ uses it to distinguish solid from liquid products (liquid petroleum is assumed to have a density $<1$ or $>2$ ). The $9^{\text {th }}$ and $10^{\text {th }}$ lines are specific to the workstation and of no concern here. The $12^{\text {th }}$ line gives conversion factors; the $13^{\text {th }}$ initial reaction masses. The remaining lines give the stoichiometric decomposition matrix and the activation energies and their weights.

Figure 120. Holding the mouse over the Select or Edit kinetic menus shows the kinetics currently in the workspace. Choices can be changed by selecting a different kinetic file using the navigator in Select. The selected kinetics files can be changed using the Edit menu (see Figure 121). The changes apply to the session only. The kinetic files must be edited in Excel (and saved as tab-delimited text files, and then the " inserted by Excel removed using the replace function in Notepad) to make any changes permanent

Figure 121. Edit version of the Green River kinetics file shown in Figure 119. Any of the data can be modified and applied to the current case. The Calculate Norm Factors button calculates the maximum possible amounts of all species as discussed in the text 279

Figure 122. Comparison of two Type II kinetic models using the Parameters: Evaluate Kinetics menu command. The products are normalized to their maximum possible buttons because the maturation was computed using the Calculate and Normalize button (under mouse). The two models differ mainly in the timing of K15+ conversion to $\mathrm{C} 15+$. The production curves for these kerogens at the depth of the Jurassic Source in our Gulf of Mexico section is shown in Figure 39. A pseudo 2D example is shown in Figure 28 and Figure 29.

Figure 123. The maximum timestep allowed in maturation calculations is set in the Parameters: Thermal Calculation Controls form. The time series is linearly interpolated and sub-divided to meet this criterion. 
Figure 124. The Parameters: Thermocline and Basal HF: Define Thermocline form allows design of an ocean thermocline that is either linear or exponential. In this case an exponential thermocline starts at $80 \mathrm{~m}$ water depth and exponentially approaches a deep water temperature of $4.5^{\circ} \mathrm{C}$ :

Figure 125. The heatflow.txt and waterdepth.txt files have the same format. An example of a heatflow.txt file is shown above. The first line of the file gives the number of pseudowells and strata at which heatflow is defined. The next two lines gives the number of the pseudowells (from the left) and strata (from the top) at which heatflow is defined. The next remaining lines are a table of heatflow data nwell columns and nstrata rows. The heatflow.txt and waterdepth.txt files are read by the Parameters: Thermocline and Basal HF: Read Heatflow and Water Depth menu command as illustrated. The resulting form allows the files to be read, and the data consequently placed into the SFLAT file to be read and edited as illustrated in Figure 94.

Figure 126. This form from Parameters: Thermal Calculation Controls mainly sets controls for 1D thermal calculations in a single well, but also allows radioactive heat generation to be turned off, and controls the refinement of the T-t timeseries in maturation calculations by setting the maximum timestep allowed in them. The number of iteration in a timestep refers to the iteration loop, which converges thermal conductivity with temperature. Sub-timesteps are the number of intervals each timestep is subdivided into for calculation purposes.

Figure 127. This form from Parameters: Set Migration Controls sets the pore saturation required for hydrocarbons to migrate from source stratum, and the hydrocarbon saturation required to migrate through stratum above the source stratum. It also sets the kerogen cutoff grade. Kerogen maturation is not computed below this amount of kerogen.

Figure 128. First two wells of the temperaturedata.txt file in the gri_Analysis.BL/McBride_ ${ }^{\text {st }}$ third.2d case. The first line indicates whether the depth data are in meters of feet, the temperatures in $\mathrm{C}$ or $\mathrm{F}$, and allows the temperature and depth data to be modified by multiplying by or adding a constant. The next line gives the total number of wells with data. The following lines give the pseudowell number, the number of data points in that well and the name of the well (enclosed in $\$$ signs, not \# signs). The total vertical depth below seal level and the temperatures are then listed.

Figure 129. The r0vitrinitedata.txt file for the gri_Analysis.BL/McBride_ ${ }^{\text {st }}$ third.2d case. Same comments as in Figure 128 apply.

Figure 130. Header and part of the first two wells in the Biomarker.txt file of the gri_Analysis.BL/McBride_ $1^{\text {st }}$ third. $2 d$ case. The first line gives the number of biomarker types, the next lines their names. The names must be exactly the same as in Table 13. The remaining lines are similar to those described in Figure 128, except there are three columns of biomarker ratio data. Notice that if there is no data of a particular biomarker type at a particular depth, the data is input as -999 . The bio287

Figure 131. Example of vitrinite kinetic data. The pre-exponential constant is always measured in $\mathrm{sec}^{-1}$

Figure 132. Canvas sketch of a fault-antithetic fault system. The model consists of a bounding box, several age-stratigraphic horizons (including the top surface) that cut horizontally across the bounding box, and, optionally, faults that cut vertically across the bounding box. The input Canvas file is placed in the GoCad folder of the Antithetic_Fault.2d case. For input to BasinLAB it must be saved as a *.dxf file. The options shown are chosen when it is saved in Canvas 
Figure 133. Antithetic.dxf file is read in using File: New Section Project. The title, short description, and dimensions (in $\mathrm{km}$ ) of the section are input and the $O K$ button depressed. This brings up the graphics and form shown in Figure 134......

Figure 134. The Canvas sketch as imported to BasinLAB when OK in Figure 133 is depressed. The form allows pseudowells to be moved and adjusted. Since the faults appear in reasonable positions already, we can immediately depress the Digitize button (at mouse position on toolbar). Depressing this button and then the Adjust Grid and Properties button to its right results in the graphics shown in Figure 135.

Figure 135. Digitized version of graphic shown in Figure 134. Initial strata properties are most easily input by depressing the Initial Strata Properties button as shown. This brings up the spreadsheet shown in Figure 136.

Figure 136. Initial Strata Properties spreadsheet form requested in Figure 135. We have selected an all silicate section except for a salt layer at the base. The sand fraction increases upwards, however. The lithologies are applied by depressing the Apply button. After this is done the lithology can be verified by selecting sand or shale in the Overlay Information combo in Figure 135.

Figure 137. Faults can be assigned by selecting the Fault, Shift Nodes option of the Select Function group. Fault codes are assigned by selecting the Fault_flag overlay and labeling the faults with 5 on their right side, 3 on their left, and 9 for nodes that will be attracted to the left side of the system where the antithetic fault merges with the main fault (see V.B). For example the Location and Nodal Values group shows the fault flags at the corners of the black element are 3's (left side main fault) and 9's (nodes to be pasted to this side of the fault). Toggling to the Faults, Shift Nodes option of the Select Function group allows the fault assignments to be checked... 297

Figure 138. Depressing the Display Fault Width button adjusts the sides of the faults as dictated by the fault flags. The Fault Width $[\mathrm{km}]$ spinner adjusts the width of the fault. The graphics interpretation should be saved using Graphic Input: Save Interpretation or by taking option when form is closed.

Figure 139. Graphic Input: Place into SFLAT and Save transfers the graphic interpretation to an SFLATorig.txt file in the Antithetic Fault.2d case folder. The Geology: Graphic Edit menu can now be used to refine the grid, and insert a source strata. This is done first by dialing the DX Max spinners in the Refinements group to the desired refinement. Since this has been done in the image above the results are shown (13 pseudowells by 14 age horizons). Refinement is done separately for X1 (pseudowells) and X2 (age horizons) followed by pressing the Refine button. The source strata is inserted as a horizon by setting the Locn to -0.1 , typing in the name of the inserted strata ("Source_strata") and depressing the $\mathrm{H}+$ button, as shown. The grade of the source strata is initially assumed to be zero. This is changed by selecting Ker1(wtpct) in the Overlay Information combo, navigating to the source strata with the Strata toggle in the Display group selected, and changing the Value in the Overlay Information group to 4 (for $4 \mathrm{wt} \%$ ). A $100 \mathrm{~m}$ thick layer of $4 \mathrm{wt} \%$ TOC has been inserted. Finally a similar approach is used to insert sands into the shale section. The lithologic fractions must sum to 1 . The final section is shown in Figure 140. 299 
Figure 140. The final antithetic fault section after three sands and a source strata (just above the basal salt) have been inserted into SFLAT file shown in Figure 139. This is the root SFLAT file that now is processed to determine the geologic history of the fault system. First, however, the fault flags must be corrected as shown in Figure 141

Figure 141. The fault flags have been improperly interpolated. They have been partially corrected in this image, and will be fully corrected when the 9's in the Location and Nodal Values edit boxes (corresponding to the base of the black-outlined element) are changed to 3's, the nodes on the right side of the antithetic fault are changed to 5's, and the extra 9's above where the antithetic fault joins the main fault have been changed to 3's. The properly modified SFLAT file is shown in Figure 142........ 300

Figure 142. Proper distribution of the fault code. Element color is controlled by the lower right hand node. Fault flags for all nodes are shown in the insert table. See Section VI.B (p. 225) for definition of fault codes.

Figure 143. Geologic history of the SFLAT file shown in Figure 142 processed with GeoHistory: Create GeoHistory as shown. GeoHistory plots are produced using GeoHistory: Plot and Edit GeoHistory: Plot. The final step is to assure that the grid is not twisted, as discussed in Figure 144. 301

Figure 144. Check the grid using the GeoHistory: Plot and Edit Graphics: Grid Check and Graphic Edit as shown above. The 11-16 ${ }^{\text {th }}$ frames have bad grid. Depressing the Check Grid button at the top of the toolbar shows where the grid is bad in the displayed frame, and Figure 145 shows how it can be fixed.

Figure 145. Depressing the Check Grid button on the top toolbar shows that the grid is bad in well 9, horizons (from the base) 6,7, and 10. The problem is that the main fault is not straight and the grid is twisted at irregularities. The easiest fix is to straighten the fault. This is done by selecting the main fault by moving the well marker to the left side of the fault $\left(9^{\text {th }}\right.$ well) using the Well spinner at the top of the Shift Nodes in Well group. The red line in the image shows the pre-straightened position of this pseudowell. Then select the top and bottom of the well in the Straighten Segment group (as shown) and depress the Straighten button under the mouse arrow. In some cases multiple depressions of this button are needed to fully straighten the well. With the left hand side of the well, straightened, depressing the Display Fault Width button adjusts the right side of the fault and produces the image shown above. Depressing the Check Grid button at the top toolbar shows that the grid is fully fixed. The modifications are then saved to the GEOGRID file using the Save Modifications button on the top toolbar. Figure 146 shows how the slight jog in the pseudowells between the main and antithetic faults just above where they join can be corrected.

Figure 146. The jog mentioned in Figure 145 can be corrected by navigating the red marker well to the $7^{\text {th }}$ and $8^{\text {th }}$ wells, depressing the Edit Psw button, selecting the node using the Horizon No. spinner, and using the move buttons to shift the node's position. Movement can be parallel to the Geo-Grid or to the vertical and horizontal Axes depending on the toggle settings in the Move Parallel to Group. This figure shows a zoom of the area between the antithetic and main faults. The red line shows the old position of pseudowell 7. The arrow shows the new position of the "boxed" node. Grid can be refreshed using the Grid button at the top of the Figure 145 form. 
Finer movements are achieved by depressing the control key when moving the nodes. Remember to Save Modification (button on toolbar at top of the workspace form). Unzoom by depressing the shift key while pressing the Zoom button. ...... 303

Figure 147. Default solution controls from Solve Equations: Equation Controls: Edit Controls.

Figure 148. Pressing the Age(endstep) button of any timestep in Figure 147 shows the internal controls for computation of that timestep. The default controls for the timestep at $0.5 \mathrm{Ma}$ is shown. The pressure equation is solved as a steady state equation (the only option currently supported), the temperature equation as a transient equation. For the temperature equation a maximum of 1 subtimesteps (Nsubt) is taken in the solution of each refined timestep. If the number were -1 the minimum number of sub-timesteps would be one and the maximum would be set such that the temperature change is less than a specified value. These sub-timesteps are inside the parameter loop and so are carried out for constant material and fluid properties. One iteration (Nit) is taken across each set of Nsubt sub-timesteps for each equation. The iteration loop includes material property changes and thus iterations converge temperature-dependent parameters such as thermal conductivity. Parameters use time-average (across the iteration loop timestep) state variables (e.g., temperature). Subtimesteps and iterations can be defined individually for each equation but are always 1 for steady state solutions. TH specifies whether the solution is solved implicitly $(=1)$ or explicitly $(=0)$ or in between $(0<\mathrm{TH}<1)$. Trk specifies the node at which the solution is tracked. The value of the variable is written into a text file that is placed in the Metafiles folder of the case, if this variable is not zero. The node number can be determined from Solve Equations: View FE Grid or, if the case has been calculated previously, from View Calculated Solution graphics by positioning the mouse over the node. Note, primary equation solution variables are nodal variables.

Figure 149. Parameters: Permeability (+Scrpad): Examine Section Permeabilty form.

The empirical model in which permeability is a function of porosity and the fraction coarse lithology (Section VI.B.1) has been selected for the matrix permeability, and the adjacent lithology model (Section VI.C) has been selected for fault permeability. The colors in the above figure indicate permeability... 305

Figure 150. Plot created with Solve Equations: View Calculated Solution: select Int$m f[\mathrm{~kg} / \mathrm{cm} 2-a]$ and Vector Style Mag. The shading is controlled by the log integrated mass flux $[\mathrm{kg} / \mathrm{cm} 2]$. The log range is from -1 to 3 . Flow is indicated by wind flags whose flag length is proportional to the mass flux. The flag anchor is at the center of each element. Flags are not displayed when the flux is small. The length of the flag can be adjusted in a number of ways (constant length to show direction only, magnitude, and log magnitude). The brine flow is significantly channeled by the main and antithetic faults. The sands low in the section significantly channel brine flow. Most of the brine flow is driven by compaction in the upper $\sim 3 \mathrm{~km}$ of the section. The integrated mass flux is lower in more recently deposited sediments because there has not been as much time for fluids to pass through them. Since Tracking is checked for the integrated mass flux the edit window on the graphic toolbar shows that the integrated mass flux in the main fault at the position of the arrow (element 563 ) is $307 \mathrm{~kg} / \mathrm{cm}^{2}$ 
Figure 151. Default solutions controls can be set to default values for all equations and timesteps using Solve Equations: Equation Controls: Set Default Solution Parameters. In this case we have set the number of iterations for the temperature equation to 2. The Criterion column specifies, in the case of the temperature equation, the maximum temperature change allowed for a sub-timestep solution. If the temperature change anticipated from the sediment deposition over a sub-timestep exceeds this value, the subtimestep is subdivided until this is not the case. If the min button is selected, the sub-refined timestep will be 1or this value, whichever is greatest. If the max button is selected, the sub-refined timestep will be 1 .

Figure 152. Scratchpad for evaluating the rate of capillary expulsion of petroleum from a source stratum. Access through Primary Migration: Scratchpad. 310

Figure 153. The relation between dead oil (gas free viscosity) temperature and API gravity, and between gas-saturated viscosity and dead oil viscosity and GOR..... 311

Figure 154. The relative permeability curves used in the hydrocarbon expulsion simulations shown in the following figures. Obtained using the Primary Migration: Plot RelPerm Curves menu.

Figure 155. The time required to expel petroleum from a $100 \mathrm{~m}$ thick source bed if the grain size is 0.5 microns. The initial hydrocarbon saturation is set at 0.6 , the generation rate to 0 , and saturations are calculated every 100,000 yrs for $1 \mathrm{Ma}$ (Number of Timesteps $=10$ ). After $1 \mathrm{Ma}$ the saturation has dropped from 0.6 to $\sim 0.44$. Useful statistics are given on the form as shown below.

Figure 156. The petroleum saturation is $\sim 0.6$ and the expulsion and generation rates are approximately equal after $1 \mathrm{Ma}$ for the $100 \mathrm{~m}$ thick source strata discussed above. For this calculation the Generation Rate was set to $0.1 \mathrm{Ma}^{-1}$. The Length of Simulation is $1 \mathrm{Ma}$. The Number of Timesteps to 10. This petroleum would bleed from the source strata for millions of years after petroleum generation had ceased (see Figure 155).

Figure 157. The retention of petroleum under the conditions of Figure 156 but with a $10 \mathrm{~m}$ thick source strata are reached in $\sim 200 \mathrm{ka}$ rather that in $\sim 1 \mathrm{Ma}$ and the retention is $\sim 0.3$ rather than 0.6 .

Figure 158. Methane distribution at $1.833 \mathrm{Ma}$ for (a) petroleum migration parallel to brine flow (0\% vertical) and (b) 1\% vertical. Calculated with Supercritical Migration: Mobile Phase Maturation/Migration. The red wind flags show brine flow with the flag length proportional to the brine flux. The log petroleum mass transfer (IncTMF) between elements (in billions of tones) over the timestep is shown in grayscale. The grayscale range is from $10^{-7}$ to $10^{-1}$.

Figure 159. Methane distribution at $0 \mathrm{Ma}$ for (a) petroleum migration parallel to brine flow ( $0 \%$ vertical), and (b) $1 \%$ vertical. Calculated with Supercritical Migration: Mobile Phase Maturation/Migration. The conventions are the same as in Figure 158, except the age of the section has been changed using the toolbar spinner. Petroleum generation begins at about $0.67 \mathrm{Ma}$ in the right half of the section and is near maximum at the present day.

Figure $160 . \mathrm{C}_{6-14}$ venting in $10^{9}(\mathrm{Bt})$ tones $/ \mathrm{km}^{2}$-Ma at past times for the $1 \%$ buoyancy rotation. Plotted using Solve Equations: View Calculated Solution: VntProf button: selecting either a single product and multiple ages or multiple products and a single minimum age: and using the $\mathrm{v}$ button (for present venting). 320 
Figure 161. The black curve shows the total petroleum-generating potential of the section (and $+/-0.5 \mathrm{~km}$ to either side), the red curve the petroleum expelled from the basin (to the ocean) and the blue curve the petroleum (generated and potential remaining in the basin). The black curve is the sum of the red and blue. Its flat top shows that petroleum mass has been faithfully conserved in the simulation. Plot is for $1 \%$ buoyancy rotation. The pulses of petroleum generation and migration on the left and right of the section are evident in the balance curves. Curves are generated by depressing the HCMassBal button when the Solve Equations: View Calculated

Solutions has been activated.

Figure 162. Sedimentation history for the

BasinModels $\mid$ blcases $\mid$ Examples.BL $\backslash$ Antithetic_Fault. $2 d \mid$ case discussed above. Obtained by Solve Equations: View Calculated Solution: Prof button: navigate to desired well: depress Sedimentation Rate[t] button. Sedimenation Rate[t]. 321

Figure 163. The instantaneous and cumulative mass flux of petroleum vertically through the element just above the source strata at the left side of the section can be obtained with Solve Equations: View Calculated Solution: $t$ series button: navigate to element: select the Inst+Cum Total mass flux from the Std Choices group of the $t$ series form and depress the plot button as shown. 323

Figure 164. The distribution of $\mathrm{C} 1$ in the section. Plot obtained by Solve Equations: View Calculated Solution: XM Plot Control form with selections made as shown to the right. $\mathrm{C} 1$ has been selected (spreadsheet is scrolled up so it is the top entry). Grams of hydrocarbon $(\mathrm{gmHC})$ have been selected as the Shade Normalization so that the mass fraction $\mathrm{C}_{1}$ is displayed. The log Transformation is turned on with limits of $10^{-1}$ and $10^{-7}$ so that the full distribution of $\mathrm{C}_{1}$ is evident. A blue Vector Color has been selected so that the brine mass flux vectors show against the gray and black background. Figure 165 shows a profile of hydrocarbon compositions in the middle left portion of the section.

Figure 165. Profile through the section in the position shown obtained using Solve Equations: View Calculated Solution: Prof form: navigating to position shown: selecting HC Composition: depressing Plot Profile. The $k 1$ mass fraction has also been selected. Methane dominates the deep section; oil the shallow.

Figure 166. A time series plot of hydrocarbon composition at the location of the main fault shown by the red outline and arrow is obtained with Solve Equations: View Calculated Solution: $t$ series. The Mobile K1 Products standard choice has been selected. The hydrocarbon composition at first becomes more gas-rich, but this tendency is overcome by the later generation of oil in the right half of the section. Figure 167 shows a similar time series plot for hydrocarbons leaving the source strata in the left side of the section.

Figure 167. Petroleum composition time series for a position just above the source strata at the left side of the section. Conventions and plotting options are the same as in Figure 166. Only the position of the time probe has been changed. The $\mathrm{C}_{3-5}$ component reaches a maximum mass fraction of $\sim 0.47$ (as shown bythe arrow). . 326

Figure 168. Comparison of maturation of two type II kerogen models for the location shown in Figure 167. The figure is computed using the $t$-series probe described in Figure 167, except that the Maturation Comparison button is pressed. This brings up the Evaluate Maturation form shown to the right. The maturation is calculated 
by depressing the Calculate Maturation button, and the comparison is selected and

plotted as indicated.

Figure 169. Selection of an element for detailed chemical investigation of the petroleum migrating though it is achieved via the Solve Equations: View Calculated Solution: Petroleum Composition form summoned with the PetCompsn button on the section toolbar. This figure shows that we have used the navigate buttons to select an element in the upper part of the main fault (arrow location). The mouse positioned over the element shows that the element (\# 659) lies $1.2 \mathrm{~km}$ below the surface and has a temperature of about $50^{\circ} \mathrm{C}$. The Tracking Variables are set to the mobile decay products of the first kerogen. Depressing the Plot button (with the Petroleum Composn radio selected), produces the plot in Figure 170.

Figure 170. With the forms in Figure 169 open, check the decomposition scheme by executing Flash: Design Petroleum Decomposition. This overlays the form shown above on the Petroleum Composition form. It shows the mass fractions of kerogen 1 products in the element selected in Figure 169. The percent n-alkanes in each carbon number cut (green numbers in first column on the top) can be changed to produce a reasonable kissen slope. The distribution is saved for future use using the Save wtpct $n$-alk button. The form functions as described in the discussion associated with Figure 64. As discussed there the alkane kissen slope is determined by their mass of the heaviest two carbon number cuts, with lighter cuts attached to the heavier $\mathrm{C}_{\mathrm{n}}$ with slopes adjusted to accommodate their mass. The non-alkanes are then distributed as shown in the lower group. The oil decomposition allows components to be assigned where the numbers are red. The red numbers must sum to 1 in each vertical column. A uniform distribution is provided by depressing the Guess button. This distribution can be changed using the spinners, but be sure the numbers sum to 1 in each vertical column. The total oil composition is plotted by depressing the Plot Total HC button. The result is shown on the right. The impact of changing the red non-alkane distribution numbers can be evaluated by hitting the Plot Total HC button after a change is made. The distribution can be applied to the current case (and saved for subsequent cases unless altered) by depressing the redlettered Save Cut Distrib button. Once the hydrocarbon decomposition is designed save the Cut and wtpct distributions using the color-lettered buttons and close the Design Hydrocarbon Distribution form. Depressing the Plot button on the Petroleum Composition form (Figure 169) then shows the same plot as above. ... 330

Figure 171. The phase state of the petroleum in the element selected in Figure 169 can be determined by selecting the Phase State toggle and depressing the Plot button. The phase calculation takes $\sim 25$ seconds to compute for the Mathias equation of state. If the petroleum is two phases at the average pressure and temperature in the selected element, the n-alkane compositions of both phases are plotted. If the petroleum is single phase only the liquid n-alkane composition is plotted. Moving down the main fault we find that the phase boundary is at $\sim 1.5 \mathrm{~km}$ depth $\left(57^{\circ} \mathrm{C}\right)$.

Figure 172. Gas washing can be calculated for the petroleum in element 659 (Figure 169) by selecting the Gas Wash Element radio and specifying the moles and type of washing gas (20 moles $\mathrm{CH} 4)$, and the ratio between washing aliquots, and depressing the Compute button. In this case we know the petroleum is above the phase boundary so we start with 0 moles of methane added to the oil. If the 
petroleum is below the phase boundary, we find the phase boundary by adding a number of aliquots of washing gas, starting with an aliquot whose size is indicated under the Pre-wash Start label and increasing at the moles indicated under the Moles label for a total of Nbr aliquots. This range of aliquots should be designed to span the phase boundary. The program finds the two wash gas additions that span the phase boundary and subdivides this interval 4 times to hone in on the boundary. This amount of washing gas is then added to the petroleum, and the washing sequence started. Then the phase boundary is determined the number of moles of washing gas needed to reach it is posted in the edit box at the bottom of the form. A washing case can be written to the Metafiles folder as a comma separated variable file labeled Wash_History_pic\#_(date and time stamp).csv. This file can be read and processed in Excel and is self-documented as shown in. This file can also be read by Basin $L A B$ at any later time and plotted (see Figure 174). It format is indicated in Figure 173.

Figure 173. First few lines of the Washing_History.csv file. The first lines give information on the location in the section that was washed. The case path is given. The cumulative moles of washing gas is given, as well as the moles of liquid remaining (not removed by the washing gas) and the mole fraction of liquid removed by each wash. The remainder of the table gives the composition of the liquid fraction as a after each aliquot of washing.

Figure 174. Plotting of a gas washing case is achieved by selecting the Plot Gas Wash Results in the Petroleum Composition form. Previous Wash_History files can be read from the metafiles folder of any case and displayed, or the case just computed can be displayed. A number of plot styles are available as shown in Figure 175-Figure 176. The conventional style shown above shows that the washing break number is $\sim 17$ in this case. Note that the equation of state shown in the image is irrelevant; the equation of state used in the calculation is controlling. For recent discussions of methane washing see Losh et. al., (2002).

Figure 175. Other plotting options can be selected from the Plot Components combo. For example the petroleum removal can be plotted relative to the original n-alkane distribution Alkane $X_{n} / X_{\text {nno }}-{ }_{\text {as }}$ shown in the figure on the left. The remaining moles of petroleum can be plotted using the Moles Liq vs. Moles Wash Gas option. This plot (where we have unchecked the log checkbox) shows that about 60 mole percent of the original petroleum has been removed by a combination of phase separation and gas washing. 333

Figure 176. The mole fraction of wash gas in the phase separates declines as washing progresses. This plot is obtained using the Mlfxn gas vs. Moles Wash Gas option shown in the caption of Figure 175. The plot to the left tracks the washing of some of the principle n-alkane components. It is described in Figure 177.

Figure 177. Finally changes in the abundance of any of the components in the oil can be plotted as a function of washing. This is done using the removal of selected components section of the plotting Petroleum Composition form. In Figure 177 we have plotted the selection of components shown selected above. In the figure to the right we have plotted the log depletion of a set of alkane (pink to red with type =1) and branched alkane (green with type $=3$ ) components (log checkbox checked). The caption gives the component name, the chemical formula, the structure type (1=alkane, $2=$ cyclic alkane, $3=$ branched alkane, $4=$ aromatic), and the molecular 
weight. This kind of plot could allow the identification of components that are diagnostic of washing.

Figure 178. The composition of a petroleum whose composition was determined in the laboratory from Peter Meulbroek's PhD thesis is plotted using Flash: Flash/Wash Decomposed Petroleum: Petroleum Composn toggle: Plot button. The numbers in the caption are the mole and mass fractions of each component type in the petroleum.

Figure 179. The phase properties of the Petroleum shown in Figure 178 can be determined with the Phase State toggle. The Set $\mathrm{P}$ and T portion of this form defines pressure and temperature as a function of depth for a sealed basin of the user's design. The Specify $P(z), T(z)$ button defines the pressure and temperature profile in the basin in terms of the $\mathrm{P}$ and $\mathrm{T}$ at its surface, the $\mathrm{P}$ and $\mathrm{T}$ gradients above and below the seal, the temperature gradient in the seal and its rupture pressure, and the thickness of the seal. This supports plots of $\mathrm{P}$ vs. depth, $\mathrm{T}$ vs. depth, and $\mathrm{P}$ vs. $\mathrm{T}$ (shown above). The spinner on the form sets $\mathrm{P}$ and $\mathrm{T}$ according to depth using the defined relationship. Here we have selected a depth of $1.5 \mathrm{~km}(\mathrm{~T}=30$ bars, $\mathrm{P}=150$ bars). Alternatively we could have specified the $\mathrm{P}$ and $\mathrm{T}$ directly with the $\mathrm{T}$ and $\mathrm{P}$ spinners.

Figure 180. The phase state of the Thesis petroleum is determined for the $\mathrm{P}$ and $\mathrm{T}$ specified by depressing the Plot button in Figure 179. This procedure shows that the petroleum becomes single phase only above $\sim 0.9 \mathrm{~km}$ depth. This is because the petroleum contains less $\mathrm{CH}_{4}$ than it probably should. Addition of $\mathrm{CH}_{4}$ can cause the petroleum to phase separate at greater (even much greater) depths.

Figure 181. The petroleum can be washed with $\mathrm{CO} 2$ as well as methane. Here we select a washing depth of $2 \mathrm{~km}$ (55C and 210 bars) and have specified pre-wash additions starting at 0.1 moles $\mathrm{CO}_{2}$ and increasing by 0.1 moles for 8 test aliquots. The prewash testing shows that 0.16 moles of $\mathrm{CO}_{2}$ are sufficient to bring the petroleum to the phase boundary. The Figure on the right shows how petroleum components are removed by washing with 2 moles of $\mathrm{CO}_{2} \cdot \mathrm{CO}_{2}$ is a much more potent washing agent than methane; 2 moles does more than 20 moles of $\mathrm{CH}_{4}$ 


\section{Introduction}

A basin model is a software application designed to simulate the evolution of a sedimentary basin, to provide insights and risk management during the petroleum exploration process. Today's state-of-the-art sedimentary basins models simulate basin evolution in four steps:

1. The physical evolution of the basin is reconstructed. This requires detailed information on the structure and stratigraphy of the basin.

2. The heat flow and thermal history of the basin is established. This can be calculated with stretching models, calibrated with paleo-thermometer biomakers

3. The history of hydrocarbon maturation is calculated. What is required is chemical kinetics for kerogen source rock, and calibration using the current thermal maturity of source rock.

4. Hydrocarbon migration is estimated.

At the onset of the DOE Advanced Basins Project in 1999, all available sedimentary basin simulators suffered from the following shortcomings

- They were expensive. A typical $2 \mathrm{D}$ package cost $\sim \$ 80,000$ to buy and required a dedicated expert to run. This is beyond the means of most small independent companies, and certainly beyond the means of most academics. 3D packages, if available, were beyond the reach of all but the largest multinational exploration companies.

- Basin models ignored most chemical information. This included basin-specific maturity indicator kinetics, and the chemical and physical properties of the hydrocarbons generated and migrating within the basin,

- Basin models did not have the flexibility to explore the physical flow controls (both stratigraphic and fault seals and traps), which can vary by region or basin.

To remedy this situation and to bring the powerful modeling techniques of basin modeling to smaller exploration firms and academia, the Advanced Chemistry Basins Project was proposed to build a powerful, open source chemically-aware basin simulator, and make it available to the general public. The goals of the project were to create a simple and capable modeling package that can

1. Determine and play back the physical evolution of a basin,

2. Calibrate the heat flow history to maturation indicators and present temperature,

3. Predict hydrocarbon maturation and the initial chemistry of the hydrocarbons,

4. Predict the migration of these hydrocarbons,

5. Take into account the phase fractionation and mixing that results, and 
6. Take into account, parametrically, the effects of different ratios of buoyancy to hydrodynamic drive and seal effectiveness on oil and gas migration so that the predicted pattern of hydrocarbon composition can be explored as a function of various migration scenarios.

At the end of the four-year project, we have completed this goal, as detailed in this report. The model produced is set of applications that add advanced chemical information to a new basin model. To allow the model to be broadly applicable, a large body of basic research into the chemical kinetics of maturation indicators (necessary for model calibration), and into the kinetics of kerogen maturation (necessary to predict the type and composition of generated hydrocarbons) has been completed, and is reported here.

\section{Organization}

The original project was proposed with 7 tasks, divided among four institutions. The seven tasks are

- "Paleo-Heat Flow Calibration Using Chemical Maturity indicators", and "Compositional Modeling of Hydrocarbon Generation and Destruction", both implemented at the Petroleum, Energy, and Environmental Research Center (PEER) at Caltech;

- "Physical Properties of Hydrocarbon Mixtures", implemented at the Materials and Process Research Center (MSC) at Caltech;

- "Building and Adding to the Conventional Basin Model", implemented at the Geology Department at Cornell University,

- "Maturation Kinetics and Primary Expulsion", implemented jointly between Cornell and PEER,

- "Phase Fractionation, Mixing, Gas Washing", implemented jointly between Cornell and the MSC, and

- "Packaging of User-friendly Basin Modeling and Analysis System", implemented jointly between Cornell and GeoGroup, Inc.

This report is divided into three parts, reflecting the three research thrusts within the project. In the first section, "", the basic chemical parameters necessary to calibrate paleo-temperature and hydrocarbon maturity (tasks 1,2, and 5) are reported as provided by PEER. The second section, "HCToolkit-Manual", describes the software implemented at the MSC to predict the phase behavior of complex chemical mixtures (Tasks 3 and 6). The final section, "Processing Geological Cross-sections", details the completed basin model (fulfilling tasks $4-7$ ), as implemented at Cornell and GeoGroup.

\section{Model Distribution}

The final Model is available for download at several locations. The HCToolkit and EOSInterface (the chemical models) can be found at http://ruby.wag.caltech.edu.

The basin model can be found at http://www.eas.cornell.edu/eas/research/GeoModeling/BasinLAB/ 


\section{Results and Discussion}

\section{Paleo-Heat Flow Calibration Using Chemical Maturity indicators}

Assessing the occurrence, quantity, and timing of hydrocarbon generation requires an accurate thermal history model for source rocks and coals within a sedimentary basin. Although the present-day heat flux can be calculated accurately from down-hole temperature measurements, the paleoheat flux, can only be assessed by models using thermal indicators. A thermal indicator is defined as an indicator whose value changes only with time and temperature. The quality of reconstructed paleoheat flux depends on the number and location of the measured values, and the type of thermal indicator. For validity the thermal indicator used should change significantly over the observed maturity range. Examples of such indicators are the vitrinite reflectance (Sweeney and Burnham, 1989, Tang et al, 1996, Hunt, 1979; Tissot and Welte, 1984; Waples, 1981), biomarkers (such as methylphenanthrene, dibenzothiophene or the pristane and pristene), apatite fission tracks, and smectite to illite conversion. The proposed research will provide users with easy access to a variety of different thermal indicators and the ability to input custom kinetics for these indicators. The indicators we propose to include are discussed below.

\section{Vitrinite Reflectance}

Vitrinite reflectance is a popular thermal maturity indicator that is a widely used to calibrate thermal history models in basin analysis for petroleum exploration (Hunt, 1979; Tissot and Welte, 1984; Waples, 1981). Unfortunately, due to the variability of kerogen and coal properties, vitrinite reflectance data show a large scatter. Part of the problem is that the maturation of some vitrinites is suppressed. Vitrinite with suppressed reflectance cannot be differentiated from normal vitrinite by standard petrographic analyses In addition, models of vitrinite reflectance do not generally allow users to specify basin- or kerogen-specific kinetics. Sweeney et al (1990) proposed using a vitrinite reflectance conversion (VCI). VCI can be calculated by using a set of distribution activation energies. Since VCI kinetics is affected significantly by kerogen type, HI, and TOC values, it is very important to allow user to input or select different vitrinite kinetics for different type of organic matter.

Vitrinite reflectance kinetics database

Vitrinite reflectance $\left(R_{\mathrm{o}}\right)$ kinetics have been determined for six (6) different kerogens. Pyrolysis experiments were carried out in sealed gold tubes at two different heating rates.
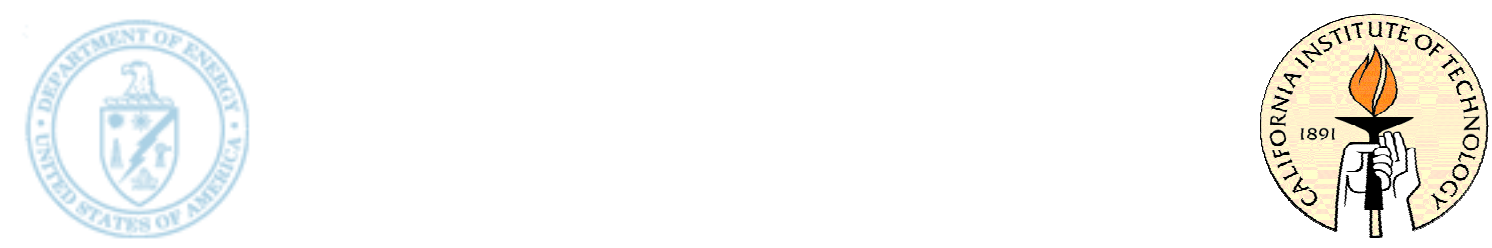
Vitrinite suppression

$R_{\mathrm{o}}$ kinetics have been derived for liptinite-rich type II and type III kerogens. By combining $R_{0}$-temperature data for these two kerogen end members, we can now correct for vitrinite suppression in mixed type II-III kerogens.

\section{MethyIPhenanthrene Index (MPI)}

MethylPhenanthrene Index (MPI) was developed by (Radke et al., 1982; Boreham et al., 1988) $: \mathrm{MPI}=1.5(2-\mathrm{MP}+3-\mathrm{MP}) /(\mathrm{P}+1-\mathrm{MP}+9-\mathrm{MP})$ where $\mathrm{P}$ and $\mathrm{x}-\mathrm{MP}$ are respectively phenanthrene and $\mathrm{X}$-methylphenanthrene.

\section{Proposed work:}

1. Evaluate MPI parameter and document when and how this parameter should be used.

2. Derive kinetic parameters for this indicator by artificially maturing kerogen samples.

3. Integrate the MPI indicator into our basin modeling system so that data of this kind can contribute to heat flow calibration and maturity comparison.

\section{MethylDibenzothiophene Index (MDI)}

Of the four methyldibenzothiophene isomers, 1- methyldibenzothiophene shows the lowest thermal stability. Because the stability of this isomer is much lower than that of others, the methyldibenzothiophene ratio (MDI) has been introduced as a maturity indicator (Radke et al., 1986). The MDI thus relies on the same chemical basis as the MPI : MDI=(4-MDBT)/(1-MDBT).

\section{Proposed work:}

Evaluate MDI parameter and document to user when and how to this parameter.

Through artificial maturation of kerogen samples derive $n$ member of kinetic parameter for this indicator.

Integrated into basin modeling for heat flow calibration and maturity comparison.

\section{Hopane and Sterane ratios}

Molecular parameters, based on hopane and sterane ratios, are used to constrain the thermal evolution of basins in the temperature range of 50 to $150{ }^{\circ} \mathrm{C}$ (Mackenzie, 1984). The most important parameter (Bein et al., 1987; Goodarzi et al., 1989; Waples et al., $1991)$ is the $\mathrm{C}_{29}$ sterane isomerization defined as $(20 \mathrm{~S} / 20 \mathrm{~S}+20 \mathrm{R})$.

\section{Homohopane isomerization}

The degree of isomerization at the $\mathrm{C}-22$ position in the $\mathrm{C}_{31}-\mathrm{C}_{35}$ extended $17 \alpha(\mathrm{H}), 21 \beta(\mathrm{H})$ hopanes (Ensminger et al., 1977) is a widely used measure of maturity for oils and bitumens (Peters and Moldowan, 1993). With increasing thermal input, the biologically produced $22 \mathrm{R}$ configuration (5) is gradually converted to an equilibrium mixture of $22 \mathrm{R}$ 
and $22 \mathrm{~S}(6)$ diastereomers (Figure 1). Typically, $22 \mathrm{~S} /(22 \mathrm{~S}+22 \mathrm{R})$ ratios are calculated from relative abundances of the $\mathrm{C}_{31}$ or $\mathrm{C}_{32}$ homohopane epimers. Studies of several petroliferous basins have shown that steady state values of $c a$. 0.6 (0.57-0.62; Seifert and Moldowan, 1986) are usually reached early in the oil window before significant petroleum generation has taken place (e.g., Seifert and Moldowan, 1980; Mackenzie et al., 1980; Schoell et aI., 1983).
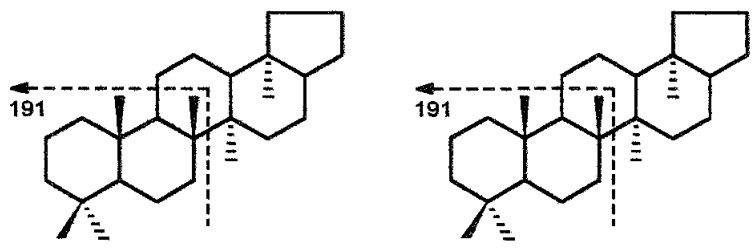

(5)

$$
\mathrm{x}=\mathrm{n}-\mathrm{CnH} 2 \mathrm{n}+1 . \mathrm{n}=2.3, \ldots, 6
$$

Figure 1. A reaction scheme showing the equilibration between biological $22 \mathrm{R}$ and geological22S epimers of the $\mathrm{C} 3 \mathrm{rC35}$ homohopanes with increasing thermal maturity in sediments.

Changes in relative abundances of the $22 \mathrm{R}$ and $22 \mathrm{~S}$ diastereomers of the $\mathrm{C}_{31}$ and $\mathrm{C}_{32}$ $17 \alpha(\mathrm{H}), 21 \beta(\mathrm{H})$-homohopanes $[22 \mathrm{~S} /(22 \mathrm{~S}+22 \mathrm{R})]$ have been advocated as indicators of palaeotemperatures for use in basin models (Mackenzie and McKenzie, 1983; Mackenzie et al., 1984). A number of workers have employed this parameter for the evaluation of thermal histories of sediments, usually in combination with other maturation indices such as the $\mathrm{C}_{29} 20 \mathrm{~S} /(20 \mathrm{~S}+20 \mathrm{R})$ sterane and $\mathrm{TA} /(\mathrm{TA}+\mathrm{MA})$ aromatic steroid hydrocarbon ratios (e.g., Beaumont et al., 1985; Hong et al., 1986; Marzi and Rullkotter, 1992). As for the sterane parameter (8ection 1.1.1), the change in the $22 \mathrm{~S} /(22 \mathrm{~S}+22 \mathrm{R})$ diastereomer ratio is assumed to follow a simple unidirectional "reaction", with initial and final values set at zero and 0.61, respectively. Based on a calibration with geological samples subjected to different levels of thermal stress, Mackenzie and McKenzie (1983) proposed pseudo-first order kinetic constants for the $\mathrm{C}_{32} 17 \alpha(\mathrm{H}), 21 \beta(\mathrm{H})$-29-bishomohopane epimer parameter $\left(E a=91 \mathrm{~kJ} \mathrm{~mol}^{-1}, A=2.5 \times 10^{-2} \mathrm{~s}^{-1}\right)$. Interestingly, however, these values could not be reconciled with observations from laboratory heating experiments (ref). Marzi and RullkOtter (1992) (?) subsequently recommended adjustments to the apparent activation energy and frequency factor for the $\left(\mathrm{C}_{31}\right) 22 \mathrm{~S} /(22 \mathrm{~S}+22 \mathrm{R})$ homohopane transformation in accordance with both natural and artificially matured sample suites $\left(E_{a}=168 \mathrm{~kJ} \mathrm{~mol}^{-1}, A=8.1 \times 10^{8} \mathrm{~s}^{-1}\right)$, although the authors expressed some reservations about the values obtained because separate treatment of the two data sets yielded significantly different kinetic parameters. 
Caution is required in the use of the $22 \mathrm{~S} /(22 \mathrm{~S}+22 \mathrm{R})$ parameter in basin modeling studies since certain other factors such as the lithology of the host sediment also appear to affect the extent of $17 \alpha(\mathrm{H})$-homohopane isomerization in some instances. Moldowan et al. (1992), for example, reported the presence of equilibrated $22 \mathrm{R} / 22 \mathrm{~S}$ mixtures in very immature carbonate rocks from the Adriatic Basin. Interestingly, laboratory simulations of natural thermal evolution have indicated that free homohopanes in bitumens isomerize more rapidly than their kerogen-bound counterparts (Peters et al., 1990). Many extracts from shallow sediments deposited under hypersaline conditions show mature hopane signatures (ten Haven et aI., 1986), suggesting the existence of unusual diagenetic pathways in such systems.

\section{S/20R diastereomer ratios}

Changes in the relative abundances of the $\mathrm{C}-20$ diastereomers of the $\mathrm{C}_{27}-\mathrm{C}_{29}$ steranes have been routinely employed for the assessment of thermal maturities of petroleum source rocks and crude oils (Peters and Moldowan, 1993). With increasing levels of thermal stress, proportions of the biological 20R diastereomers decrease monotonically in relation to the corresponding $20 \mathrm{~S}$ geosteranes. In practice, ratios are generally calculated from the measured abundances of the $5 \alpha(\mathrm{H}), 14 \alpha .(\mathrm{H}), 17 \alpha .(\mathrm{H})-24$-ethylcholestane (C29) epimers (1 and 2) (Figure 1.1), and expressed as either $20 \mathrm{~S} /(20 \mathrm{~S}+20 \mathrm{R}), \% 20 \mathrm{~S}$ or 20S/20R. $20 \mathrm{~S} /(20 \mathrm{~S}+20 \mathrm{R})$ values normally increase from near zero in shallow immature sediments to $c a$. 0.5 (0.52-0.55) in mature source rocks (Seifert and Moldowan, 1986). In some cases, however, ratios have been observed to decline in overmature sediments (e.g., Strachan et al., 1989).

It is important to note that a number of studies concerned with mechanistic aspects of thermally-induced changes in relative abundances of the 20R and 20S C29 $\alpha \alpha \alpha$-sterane epimers (e.g., Peters et al., 1990; Abbott et al., 1990) have suggested that, in contrast to previously held views (e.g., Mackenzie et al., 1980), interconversion and equilibration between the two diastereomers is unlikely. According to Abbott et al. (1990), observed maturational trends in this ratio can be better explained as the net result of a complex set of processes involving different rates of release/formation and destruction for the 20R and $20 \mathrm{~S}$ compounds. Indeed, Requejo (1992) has shown that measured 20S/(20S + 20R) values for a group of produced oils were inversely proportional to the epimer concentrations, implying a thermal stability control on this parameter in natural samples. Proposed kinetic constants for the 20R/20S C29 $\alpha \alpha \alpha$-sterane release/degradation processes (Abbott et a/., 1990) do not, however, appear to conform to geologic observations (Marzi, 1992).

In spite of these uncertainties, 20S/20R diastereomer ratios remain a valid indicator of sediment maturity. It should be emphasized though that other factors apart from maturational effects may also influence relative epimer abundances. For example, processes responsible for changes in the $20 \mathrm{~S} /(20 \mathrm{~S}+20 \mathrm{R})$ ratio appear to be less favored in coals than in shales under most sediment heating conditions (e.g., Strachan et al., 1989). In another study, Grantham (1986) reported unusually low values in crude oils derived from Tertiary source rocks. Widely variable ratios have also been encountered in extracts from sediments located in early diagenetic zones (Moldowan et a/., 1986; Peakman and Maxwe11, 1988). 
Mackenzie and McKenzie (1983) first published pseudo-first order kinetic constants for the C29 20S/(20S + 20R) parameter in a study of sedimentary sequences from the North Sea and Pannonian Basin (Hungary) for which temperature and burial histories had been inferred (i.e., $\mathrm{E}_{\mathrm{a}}=91 \mathrm{~kJ} \mathrm{~mol}^{-1}, A=6 \times 10^{-3} \mathrm{~s}^{-1}$ ) The approach used assumes that the observed change in the sterane epimer ratio can be described in terms of a simple unidirectional "reaction", with initial and final values set at zero and 0.54, respectively.

More physicochemically reasonable activation energy and frequency factor values were determined by Rullkotter and Marzi (1988) from hydrous pyrolysis experiments performed on Toarcian shale samples from northern Germany $\left(E_{\mathrm{a}}=170 \mathrm{~kJ} \mathrm{~mol}^{-1}, \mathrm{~A}=6\right.$ $\left.\mathrm{x} 10^{8} \mathrm{~s}^{-1}\right)$. Marzi and Rullkotter (1992) later used kinetic parameters calculated by combining these two data sets $\left(E_{a}=169.0 \mathrm{~kJ} \mathrm{~mol}^{-1}, \mathrm{~A}=4.86 \times 10^{8} \mathrm{~s}^{-1}\right)$, in conjunction with measured $20 \mathrm{~S} /(20 \mathrm{~S}+20 \mathrm{R})$ ratio to assess the thermal history of sediments from the Michigan Basin, USA. In view of the above discussion concerning the validity of some of the fundamental assumptions regarding interconversion and equilibration of the $20 \mathrm{R} / 20 \mathrm{~S}$ diastereomers, however, care should be exercised when using $\mathrm{C}_{29} \alpha \alpha \alpha$-sterane ratios in basin modeling computations.

\section{Apatite Fission-Track}

Spontaneous fission-tracks in apatite can provide quantitative information on the thermal evolution of sedimentary basins in the temperature range of $20-150{ }^{\circ} \mathrm{C}$ (Gleadow et al., 1983; Green et al., 1989). Numerous recent studies have used apatite fission-tracks to reconstruct the thermal history of sedimentary basins (Naeser, 1993; Omar et al., 1994; Arne et al., 1994; Gallagher, 1995; Rohrman et al., 1996; Kohn et al., 1997). The advantage of this thermal indicator is that is provides information not only about paleotemperatures (in the range above), but also about their variation through time.

\section{Proposed work:}

1. Integrate the apatite fission track maturity indicator into our basin modeling system so that this kind of data can contribute to - heat flow calibration and maturity comparison.

\section{Smectite/IIIite}

The transformation of smectite into illite-smectite (I-S), generally through the formation of intermediate, randomly inter-stratified $(\mathrm{Ra})$ and ordered $(\mathrm{Rl})$ mixed-layer I-S phases occurs under many conditions. For example smectite-illite transformation can occur during burial diagenesis, during regional metamorphism, during hydrothermal alteration, as a result of thrust sheet burial, and increased thermal gradients (Eberl, et al., 1993; Elliott and Matisoff, 1996). While increased temperatures appear to be a common factor for triggering I-S transformation, ionic activities of potassium and other cations $\left(\mathrm{Ca}^{2+}\right.$, $\mathrm{Na}^{+}$and $\mathrm{Mg}^{2+}$ ) also appear to affect the rate of transformation. Time is the least understood and constrained factor affecting the rate of this transformation (Elliott and Matisoff, 1996). Additionally, the stoichiometry and driving mechanisms of the 
transformation are still unknown. Aluminum conserved, and non-aluminum conserved reactions have been proposed to describe this transformation (Elliott and Matisoff, 1996):

Al-conserved:

$\left(3.93 \mathrm{~K}^{+}\right)+(1.57$ Smectite $) \rightarrow($ Illite $)+\left(1.57 \mathrm{Na}^{+}\right)+\left(3.14 \mathrm{Ca}^{2+}\right)+\left(4.28 \mathrm{Mg}^{2+}\right)+\left(4.78 \mathrm{Fe}^{3+}\right)+\left(24.66 \mathrm{Si}^{4+}\right)$

$+\left(57 \mathrm{O}^{2+}\right)+\left(11.40 \mathrm{OH}^{-}\right)+\left(15.7 \mathrm{H}_{2} \mathrm{O}\right)$

Non Al-conserved:

$\left(4.5 \mathrm{~K}^{+}\right)+\left(8 \mathrm{Al}^{+3}\right)+($ Smectite $) \rightarrow($ Illite $)+\left(\mathrm{Na}^{+}\right)+\left(2 \mathrm{Ca}^{2+}\right)+\left(2.5 \mathrm{Fe}^{3+}\right)+\left(2 \mathrm{Mg}^{2+}\right)+\left(3 \mathrm{Si}^{4+}\right)+\left(10 \mathrm{H}_{2} \mathrm{O}\right)$

Within Al-conserved transformation (Equation [1]) 1.57 moles of smectite react to form only 1 mole of illite. Due to the loss of silica and water this leads to an illite volume that is considerably less than that which the smectite occupied. The non Al-conserved reaction (Equation [2]) can also be expressed as:

$\mathrm{K}^{+}+\mathrm{X}-$ Smectite $\rightarrow$ Illite-Smectite + Quartz $+\mathrm{X}^{+}$

Additionally the I-S transformation could also occur as a consequence of the reduction of silica activity at the onset of quartz precipitation (Equation [4]), for example:

$\mathrm{K}$-feldspar $+\mathrm{K}$-smectite $\rightarrow 2$ Illite $+4 \mathrm{SiO}_{2}(\mathrm{aq})$

The rate of transformation depends upon the activity of potassium and temperature (Pytte and Reynolds, 1989; Velde and Vasseur, 1992; Eberl et at. 1993; Huang et at. 1993; Elliott and Matisoff, 1996). However, it would appear that these models do not account for the activities of $\mathrm{Al}, \mathrm{Si}$, and $\mathrm{p}(\mathrm{H} 2 \mathrm{O})$, or for the simultaneous dissolution of K-feldspar. Eberl et al. (1996) modeled reactions at high $\mathrm{pH}$. They concluded that I-S transformation could occur at Earth surface temperatures $\left(30^{\circ} \mathrm{C}-60^{\circ} \mathrm{C}\right)$ and at higher $\mathrm{pH}$ values $(12.4$ 13.5) found within cement porewaters.

Using XRD smectite transforms to illite as a solid-state transformation on an intact aluminosilicate lattice via a series of inter-stratified phases of I-S. The type of stacking, the order of mixed layer I-S, and the percentage of illite layers in I-S (RO and Rl respectively), have been used as semi-quantitative geothermometers for basins of Mesozoic age or younger (Elliott and Matisoff, 1996). Using SEM smectite has been shown to transform to 3 distinct crystalline illite structures e.g. flakes, laths, and hexagonal plates. The occurrences of these differing forms of illite suggest that the transformation is composed of at least 2 sequential reactions (see Equations [1-4]) (Elliott and Matisoff, 1996).

Various parameters have been used to generate models of the I-S transformation from differing geological settings. These are compared in Table 1.

Table 1: Parameters used by various authors for the kinetic modeling of the Illite-Smectite transformation. 


\begin{tabular}{|c|c|c|c|c|}
\hline Reference & Geological Setting & $\begin{array}{c}\text { Temperature } \\
\text { Range }\end{array}$ & $R_{0}$ values & Comments \\
\hline Eberl et al., (1993) & $\begin{array}{l}\text { Experimental } \\
\text { (Wyoming Clay) } \\
\text { (Kinney Bentonite) }\end{array}$ & $35^{\circ} \mathrm{C}$ to $60^{\circ} \mathrm{C}$ & - & $\begin{array}{l}\text { High } \mathrm{pH} \text { conditions } \\
(\mathrm{pH} 12.4-\mathrm{pH} 13.5)\end{array}$ \\
\hline $\begin{array}{l}\text { Hillier et al., } \\
\text { (1995) }\end{array}$ & $\begin{array}{l}\text { Modeled } \\
\text { Vienna Basin Great } \\
\text { Hungarian Plain } \\
\text { Transcarpathian } \\
\text { Basin }\end{array}$ & $\begin{array}{l}25^{\circ} \mathrm{C} / \mathrm{km} \\
35^{\circ} \mathrm{C} / \mathrm{km} \\
55^{\circ} \mathrm{C} / \mathrm{km}\end{array}$ & $\begin{array}{c}0.4 \%-0.6 \% \\
0.3 \%-0.9 \% \\
0.25 \%-2.5 \%\end{array}$ & $\begin{array}{l}\text { Heat flow } \\
50 \mathrm{mWm}^{-2} \\
80-100 \mathrm{mWm}^{-2} \\
105 \mathrm{mWm}^{-2}\end{array}$ \\
\hline $\begin{array}{l}\text { Verajao and } \\
\text { Meunier, (1996) }\end{array}$ & $\begin{array}{l}\text { Modeled } \\
\text { Sergipe-Alagoas } \\
\text { Basin, Brazil }\end{array}$ & $32^{\circ} \mathrm{C} / \mathrm{km}$ & $0.11 \%-1.3 \%$ & - \\
\hline $\begin{array}{l}\text { Elliott et al., } \\
\text { (1991) }\end{array}$ & $\begin{array}{l}\text { Modeled } \\
\text { Denver Basin, USA }\end{array}$ & $\begin{array}{c}\text { Geothermal } \\
\text { Gradient } \\
36^{\circ} \mathrm{C} \text { to } 51^{\circ} \mathrm{C} / \mathrm{km}\end{array}$ & $0.7 \%-1.5 \%$ & - \\
\hline $\begin{array}{l}\text { Velde and Lanson, } \\
\text { (1993) }\end{array}$ & $\begin{array}{l}\text { Modeled } \\
\text { Salton Sea, CA, } \\
\text { USA } \\
\text { Paris Basin, France }\end{array}$ & $\begin{array}{c}200^{\circ} \mathrm{C} \text { to } \\
400^{\circ} \mathrm{C} / \mathrm{km} \\
30^{\circ} \mathrm{C} \text { to } 280^{\circ} \mathrm{C} / \mathrm{km}\end{array}$ & $\begin{array}{l}0.2 \%-4.0 \% \\
0.6 \%-3.0 \%\end{array}$ & - \\
\hline
\end{tabular}

With increasing depth of burial in sedimentary basins there is a progressive conversion of smectite to illite and the extent of this reaction is frequently used as an indicator of diagenetic grade (Hillier et al., 1995). Besides the increase of temperature with depth, many other factors may influence the progress of this reaction, and thus complicate attempts to use the I-S transformation as a geothermometer.

However, when the models of Pytte and Reynolds (1989), Ve1de and Vasseur (1992) and Huang et al., (1993) are applied to data from the Paris Basin, Denver Basin, Salton Sea and Gulf Coast, it can be shown that quite differing results for the amount of illite (\%) generated can result (Figure2). 


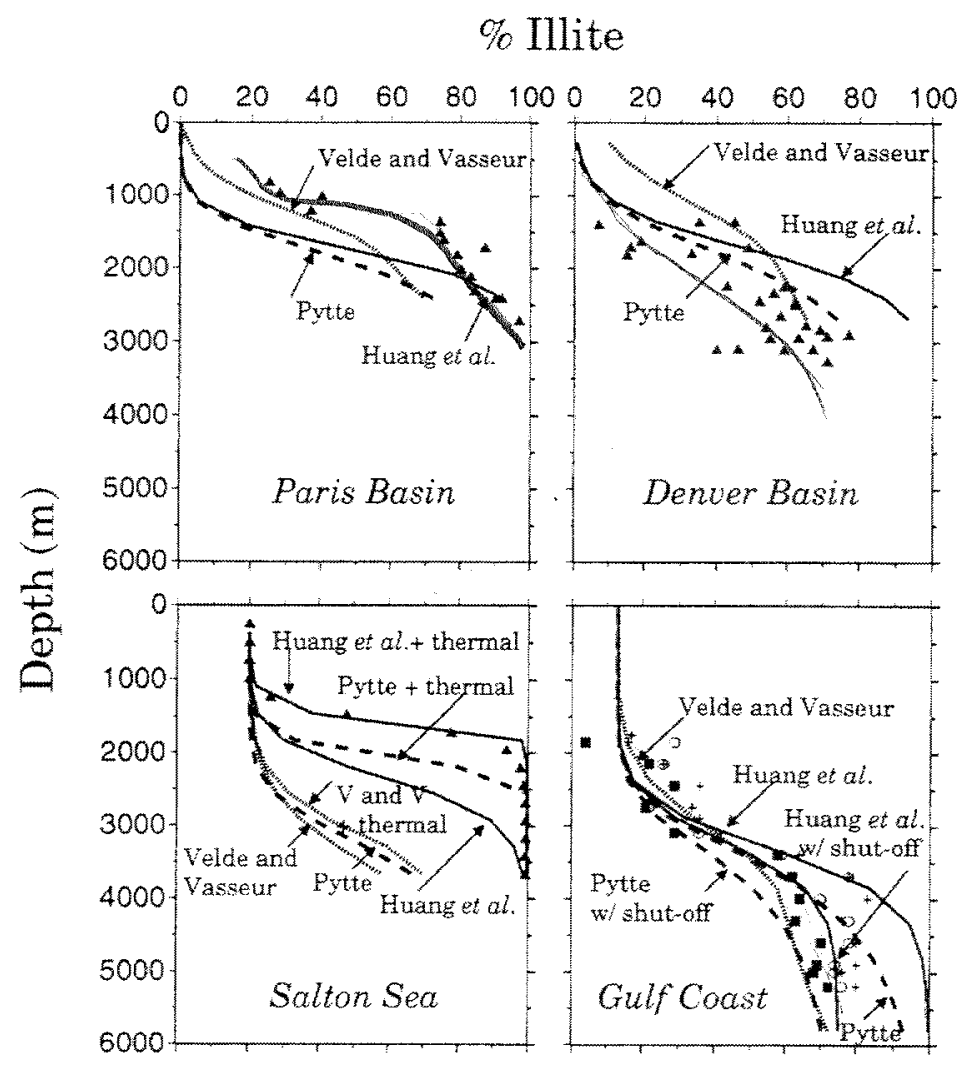

Figure 2: The variation of illite (\%) with depth from 4 different geological settings with regard to 3 models of illite generation. (After Elliott and Matisoff, 1996).

Using these models and other parameters (Table 2) the mean age of I-S transformation can be calculated (Elliott and Matisoff, 1996).

Table 2: Parameters specific to geological settings.

\begin{tabular}{|lcccc|}
\hline & Denver Basin & Gulf Coast & Salton Sea & Paris Basin \\
\hline $\mathrm{K}^{+}(\mathbf{p p m})$ & $\mathrm{K}^{+} / \mathrm{Na}^{+}=0.1$ & 200 & 3200 & - \\
\hline$\Delta \mathbf{t}(\mathbf{M a})$ & 1 & 0.1 & 0.001 & 1 \\
\hline Geothermal gradient $\left({ }^{\circ} \mathbf{C} / \mathbf{k m}\right)$ & 25 & 25 & 35,72 & $32.5,45$ \\
\hline
\end{tabular}

The mean age of I-S transformation can be calculated using Equation [5]:

Mean Age $=\sum_{i=1}^{S A}\left[\left(\frac{I_{i}}{I_{\text {Total }}}\right) \cdot(S A-i)\right]$

Where:

$\mathrm{SA}=$ stratigraphic age 
$\mathrm{I}_{\mathrm{i}}=$ fraction of illite (\% illite in I-S) formed at each time step i (Ma) from i $=1$ to SA

$\mathrm{I}_{\text {Total }}=$ total amount of illite formed.

The mean age of I-S transformation is the sum of the product of the fraction of illite formed at each step, and the age of that time step. The product $\left[\left(\mathrm{I}_{\mathrm{i}} / \mathrm{I}_{\text {Total }}\right) \cdot(\mathrm{SA}-\mathrm{i})\right]$ is defined as the $\mathrm{K} / \mathrm{Ar}$ age increment as it represents the contribution to the $\mathrm{K} / \mathrm{Ar}$ age from the illite formed during the $i$ th time step. This is best shown in Figure 3. The calculated $\mathrm{K} / \mathrm{Ar}$ ages for maximum burial (Ma) are shown in Table 3.

Figure 3: The variation of $\mathrm{K} / \mathrm{Ar}$ age increment as a function of depth for various geologic basins. The mean age of I-S transformation is equivalent to the area under the curve, defined by plotting the $\mathrm{K} / \mathrm{Ar}$ age increment at each time step versus the stratigraphic depth at each time step. This area is directly comparable to the measured K/Ar age of I-S (After Elliott and Matisoff, 1996).

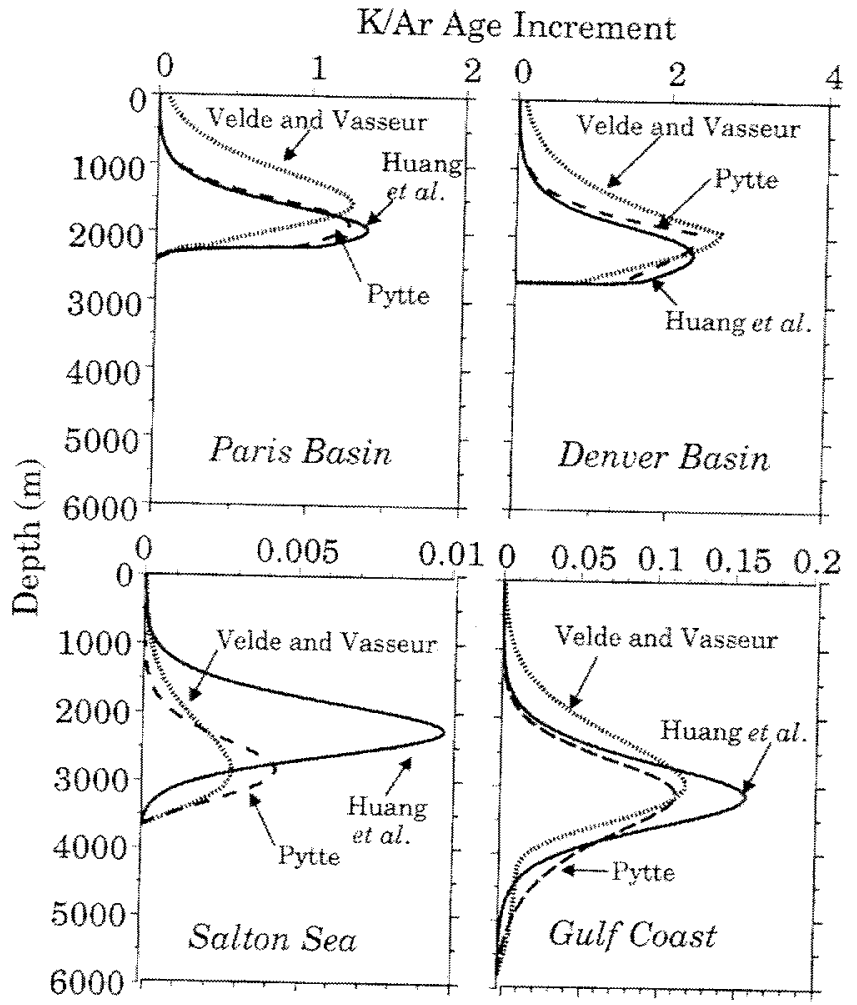




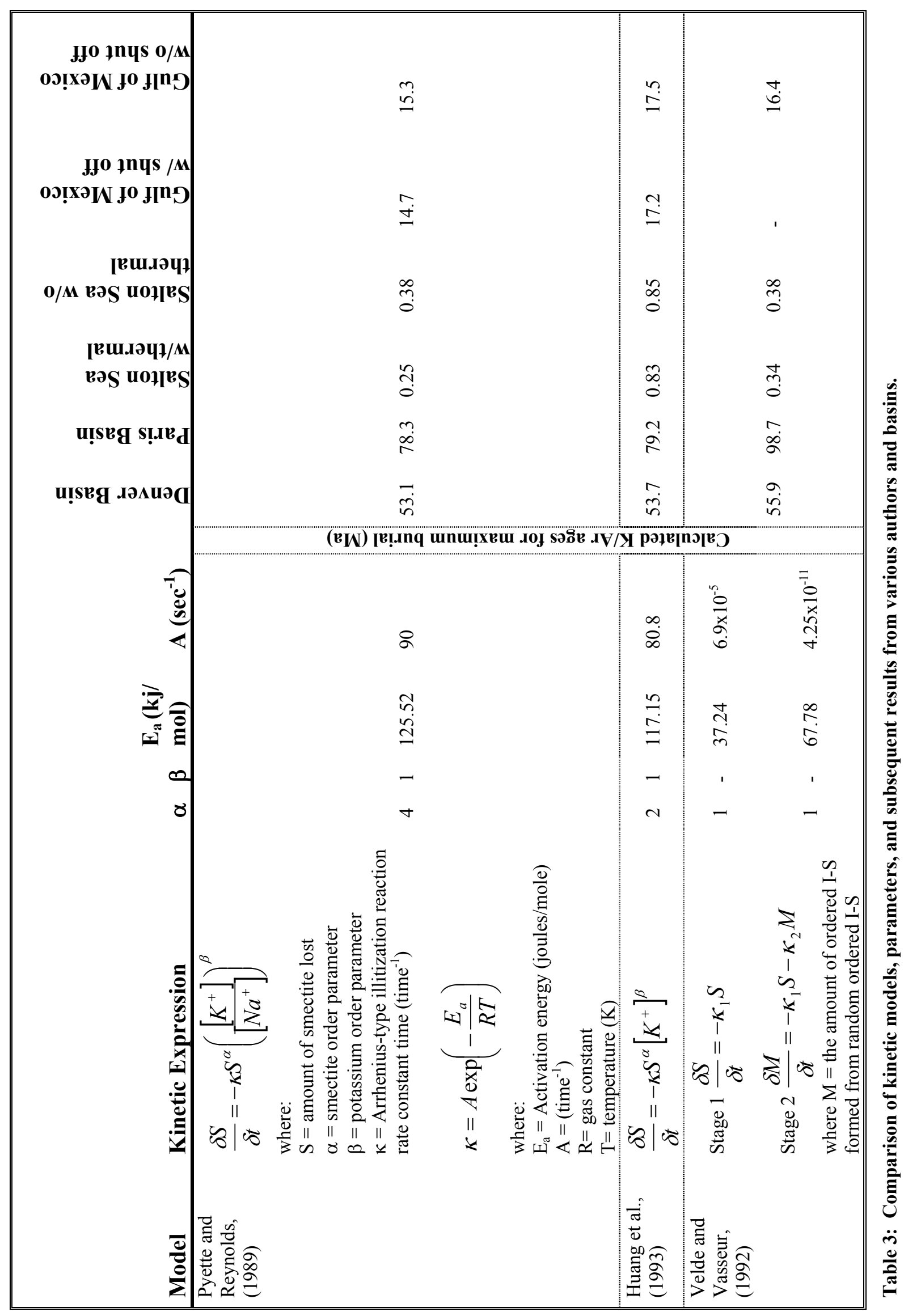




\section{Concluding remarks}

- None of the kinetic expressions found for the simulations of I-S transformation can be universally applied to all sedimentary basins, this may be due to:

○ improperly defined transformation mechanism

○ improperly chosen activation energies

$\circ$ no consideration of ion activities of $\mathrm{K}^{+}$and other ions

- Once a model is formulated, the model can be used to study a basin's thermal history, past geothermal gradient, and $\mathrm{K}$ concentration

- The models can be further calibrated by comparing the calculated and measured ages of I-S transformation and the K/Ar ages of I-S

- Calculated ages of I-S transformation can be used to indicate the timing at which source rocks are being heated to form oil and gas

- These kinetic expressions cannot be used to determine whether or not I-S transformation occurs as a direct result of dissolution or reprecipitation mechanisms.

\section{Compositional Modeling of Hydrocarbon Generation and Destruction}

Current commercial basin modeling software for hydrocarbon generation mainly use kinetics derived from Rock-Eval pyrolysis to predict oil generation (Tissot and Welte, 1984; Sweeney et al, 1989; Burnham, 1987; Ungerer and Petet, 1987). Although this technique has been proved very useful, its limitations are becoming increasingly appreciated. A new MCTP-GC technique overcomes many of these limitations (Tang and Stauffer, 1994). The primary difference between this technique and Rock-Eval pyrolysis is that MCTP-GC can provide compound-specific generation information, while Rock-Eval only gives bulk chemical information. With the MCTP-GC technique, one can obtain kinetic parameters for different classes of compounds such as $\mathrm{CO}_{2}$, hydrocarbon gas, light and heavy saturate hydrocarbons, aromatics etc. This allows prediction of the gas to oil ratio, physical properties of hydrocarbon phases (see the next proposed the section), and better modeling of primary and secondary hydrocarbon migration and entrapment.

\section{Proposed work:}

1) Integrate MCTP-GC hydrocarbon generation kinetics into our basin modeling software.

2) By coupling with a reaction network of oil cracking, one can predict:

a) the location of gas and oil windows

b) gas/oil ratio 
c) properties of the generated oil such as condensate content, wax percentage, viscosity and etc.

3) The hydrocarbon gas and fluid chemistry will combined with an equation of state in order to model primary and secondary migration, and phase separation due to the pressure and temperature changes.

\section{Technical Approach}

Our model incorporates thermodynamic properties for four distinct but interacting phases: solid phase (kerogen, asphaltene and pyrobitumen), oil, water, and gas. A generalized form of the Peng-Robinson equation-of-state (EOS) has been used, and Henry's constants were used to estimate the solubility of solutes in the aqueous phase. Representative molecular species of the solid and oil phases were modeled in some detail, and the form and parameters of the EOS, viscosities, and densities were extended to pseudo-components representing the chemical composition for crude oils of commercial interest. The associated equations-of-state (EOS) have proven highly accurate and reliable when compared to experimental measurements over a wide range of temperatures and pressures.

\section{Compositional Modeling of Hydrocarbon Generation and Destruction}

For many years, Rock-Eval pyrolysis has been used in oil exploration to predict the hydrocarbon generating potential of source rocks (Tissot and Welte, 1984; Sweeney et al., 1989; Burnham et al., 1987; Ungerer and Pelet, 1987). Since the products generated from Rock-Eval pyrolysis are a mixture of compounds, activation energies derived with this technique are poorly defined. The various products have quite different generation rates and activation energies, and it is impossible to obtain accurate kinetic molecular parameters from Rock-Eval information alone. Therefore, activation energies derived from Rock-Eval pyrolysis are only an averaged number. Although useful in oil exploration, these averaged numbers can lead to errors in defining the oil window or the timing of oil generation. An activation energy difference of a few kilocalories will shift the oil window significantly over geological time; this makes the accurate measurement of activation energies for predicting hydrocarbon generation essential. Mathematical models based on the evolution of hydrocarbon classes $\left(\mathrm{C}_{1}, \mathrm{C}_{2}-\mathrm{C}_{5} \mathrm{C}_{6}-\mathrm{C}_{15}, \mathrm{C}_{15+}\right)$ measured by Rock-Eval pyrolysis do provide more accurate data on hydrocarbon formation but are still based on bulk data and are not compound-specific (Espitalie et al., 1988). Attempts to determine compound-specific kinetic parameters by pyrolysis-triple quadrupole mass spectrometry have generally been limited to light hydrocarbon species (Braun et al., 1992; Reynolds et al., 1991); furthermore, with this technique it is difficult to obtain quantitative data for the individual compounds due to ion-neutral reactions in the ionization chamber (matrix effects).

For a narrow activation energy distribution, one can assume the same frequency factor for different reactions. However, the transition state theory of unimolecular reactions dictates a linear relationship between the logarithm of the frequency factor and activation energy. This implies that the assumption of a single frequency factor for kerogens with a 
broad activation energy distribution may lead to significant errors for kerogens with a broad S2 peak (e.g. Type III).

The products generated from Rock-Eval pyrolysis have a broad range of activation energies. For instance, the isoprenoid compounds are generated much earlier than the normal alkanes, and the heavy molecular weight alkanes are generated earlier than the lighter alkanes such as methane and ethane. Therefore, the generation of light hydrocarbon gases requires higher activation energies than for oil generation, and the calculation of the activation energy of a gas-prone kerogen can be difficult.

To avoid the limitations of the Rock-Eval pyrolysis technique and improve the accuracy of activation energy measurement for hydrocarbon generation, we developed the Multiple Cold Trap Pyrolysis GC (MCTP-GC) technique (Tang and Stauffer, 1994). The primary difference between this technique and Rock-Eval pyrolysis is that MCTP-GC can provide compound-specific generation information while Rock-Eval only gives bulk information. In contrast to conventional pyrolysis $\mathrm{GC}$, this technique provides time-resolved information on product evolution. With MCTP-GC, one can derive kinetic models for oil generation based on detailed molecular data. This improves our ability to predict not only the timing of oil generation, but also the oil composition at different stages of kerogen conversion. 


\section{Methods and Materials}

\section{Kerogen Sources and Types}

The kerogen samples used in this study are listed in Table 4. Model development primarily relied on experimental pyrolysis data generated from the Green River Shale, Kimmeridge Clay, and Cameo Coal as representative samples of Type I, II, and III kerogens respectively. Model results were later verified by comparison with experimental data generated from the remain kerogen samples. These three kerogens were selected because they have been widely studied are generally accepted as being characteristic of their respective kerogen types. The three primary kerogen samples used in this study are briefly described below.

Table 4: Kerogens used in this study, their locations, and kerogen type.

\begin{tabular}{|lccc|}
\hline Name & Location & Kerogen Type & H/C Ratio \\
\hline Green River Shale & Utah, USA, & Type I & 1.51 \\
\hline Kimmeridge Clay & North Sea, UK & Type II & 1.22 \\
\hline Cameo Coal & Colorado, USA & Type III & 0.99 \\
\hline FN2 & China & Type I & 1.61 \\
\hline B648 & China & Type II & 1.28 \\
\hline Devonian Shale & Western Canada & Type II & 1.25 \\
\hline Cape Range & Australia & Type II/III & 1.13 \\
\hline
\end{tabular}

Green River Shale

The Green River Formation was deposited in several lake basins that formed in response to Laramide tectonics in the Western U.S. over a 10 to 23 Ma period from the Paleocene into the Eocene (Franczyk et al., 1990). The formation is primarily composed of organicrich dolomitic oil shales interbedded with evaporitic deposits. Total organic carbon content of the Green River Formation ranges from 0.2 to $33.7 \mathrm{wt} \%$ with a modal value of $\sim 3.0 \%$. The total pyrolytic yield (Rock-Eval S1+S2) for samples with TOC greater than $0.5 \%$ ranges from 3.0 to $370.6 \mathrm{mg} \mathrm{HC} / \mathrm{g}$ rock (Katz, 1995). In general the Green River Formation can be considered an excellent petroleum source rock $(>6 \mathrm{mg} \mathrm{HC} / \mathrm{g}$ rock; Tissot and Welte, 1984). Green River Shale organic matter is composed of approximately $80 \%$ kerogen (Tissot et al., 1978) and was used to generated the type I kerogen maturation curve for the van Krevelen and modified van Krevelen diagrams (Tissot et al. 1974; Espitalié et al., 1977). Pyrolysis-gas chromatography (PGC) of the Green River kerogen shows a domination by normal alkanes and alkenes, which is typical of alginate (Katz, 1995; Larter and Douglas, 1980). Green River Formation organic residues have been found to contain the remains of bacteria, cyanobacteria, chlorophyte algae, euglenophytes, fungi, moss, fern spores, and pollens (Bradley, 1931), which may indicate that Green River organic matter is largely derived from algal or bacteria mats (Bucheim and Surdam, 1977). Most of the Green River Formation is thermally immature with pyrolysis $\mathrm{T}_{\max }$ values less than $440^{\circ} \mathrm{C}$, which is considered the onset temperature for petroleum generation (Bissada, 1982). The main phase of oil generation within the Uinta basin is estimated to have occurred at $2600 \mathrm{~m}$ depth (Anders and Gerrild, 1984), which is consistent with the calculated activation energy of $52.4 \mathrm{kcal} / \mathrm{mol}$ for 
hydrocarbon generation determined from thermal modeling of Green River kerogen (Sweeney et al., 1986).

\section{Kimmeridge Clay}

The Kimmeridge Clay Formation was deposited during the Late Jurassic-Early Cretaceous period (approximately 150 mya) in a shallow $(<80 \mathrm{~m})$ marine environment, and it is the most important source of petroleum hydrocarbons in the North Sea (Cooper, et al., 1995). Typically the formation is composed of dark grey, black, or dark brown claystone with infrequent carbonate bands and varies in thickness from $<50 \mathrm{~m}$ to $>1250 \mathrm{~m}$ (Barnard and Barstow, 1992). Mineralogically the oil "shales" within the Kimmeridge Clay Formation are generally composed of $40-50 \%$ organic matter, $10-30 \%$ carbonate, $10-15 \%$ quartz and feldspar, $5-10 \%$ pyrite, and $5-15 \%$ clay minerals (O'Brien and Slatt, 1990). Locally clay contents can reach as high as 45\% (Shaw and Primmer, 1991), consequently references to the Kimmeridge Shale that commonly occur in the literature are incorrect, and this lithology should properly be considered a claystone (Cooper et al., 1995). Total organic carbon content in the Kimmeridge Clay Formation mainly ranges from $<0.5$ to $>40.0 \%$ and averages $\sim 8.0 \%$ (Cooper et al., 1995), and the organic matter is composed of a variable amount (10-90\%) of amorphous material with the remainder being equally divide among phytoclasts and exinites (Tyson, 1989). Hydrogen index values derived from Rock Eval pyrolysis data show that Kimmeridge Clay samples vary from $<100$ to $>1000 \mathrm{mg} \mathrm{HC} / \mathrm{g}$ OC and average between 500 and $600 \mathrm{mg} \mathrm{HC} / \mathrm{g} \mathrm{OC}$ (Cooper et al., 1995). In the majority of samples studied, amorphous kerogen of type II constituted $>90 \%$ of the kerogen in the Kimmeridge Clay with minor amounts type I kerogen of low to medium $\mathrm{T}_{\max }$ values (Barnard et al., 1981; Cooper and Barnard, 1984; Cooper, 1990). Microscopic examination of Kimmeridge Clay organic matter shows that it is composed of discrete particles deposited in ultralaminae composed largely of cell wall material of Chlorophycean algae and lacking filamentous structures that would indicate the previous presence of mat-forming bacteria (Largeau et al., 1990).

\section{Cameo Coal}

The Cameo Coal is a major coalbed methane target within the Piceance Basin located in northwestern Colorado, USA, which formed in latest Cretaceous through Eocene times in response to Laramide compressional tectonics (Baars et al., 1988). The Cameo Coal is one of several organic-rich members of the Williams Fork Formation within the Campanian age Mesaverde Group. The Williams Fork Formation consists primarily of marine shales interbedded with sandstones and coal beds. These sediments were deposited in a prograding shoreline environment characterized by wave-dominated beach, lagoonal, and freshwater swamp sub-environments (Lorenz, 1989). Within the CameoWheeler-Fairfield coal zone the thickness of individual coal seams is as great as $11 \mathrm{~m}$, and the net coal thickness varies from $<6 \mathrm{~m}$ to $>18 \mathrm{~m}$ (Collins, 1976). The coals in the Cameo-Wheeler-Fairfield coal zone range in rank from high-volatile A bituminous $\left(\mathrm{R}_{\mathrm{o}}=\right.$ $1.0 \%)$ to low-volatile bituminous rank $\left(\mathrm{R}_{\mathrm{o}}=1.8 \%\right)$ (Johnson and Rice, 1990). The organic matter in the Cameo coal is largely composed of vitrinite $(>75 \%)$ with lesser amounts of exinites and inertinite (Collins, 1976; Law et al., 1989) and the kerogen is typical of type III kerogen (Johnson and Rice, 1990). 


\section{Analytical Methods}

\section{Multiple Cold Trap Pyrolysis Gas Chromatography}

Instrument Description. In the MCTP-GC technique, the evolution of each pyrolysis product with time and temperature is measured. A sample such as a source rock, isolated kerogen, coal, or oil shale is heated (isothermal or at a linear temperature ramp) and the evolving pyrolysis products are collected in the multiple cold trap system at predetermined temperature or time setpoints. After the pyrolysis is completed, the contents of each trap are analyzed by gas chromatography. Each chromatogram represents a fingerprint of the pyrolysis products generated in a given temperature interval-a "thermal slice" of the pyrolysate. Combining the quantitative GC data over the whole temperature range allows construction of evolution profiles for individual pyrolysis products. Therefore, in a single pyrolysis experiment, a wealth of compoundspecific information can be gained. Figures 1 and 2 illustrate the thermal slicing concept.

The MCTP-GC instrument has been described elsewhere (Tang and Stauffer, 1994). In brief, the system consists of a commercial pyrolysis GC (Chemical Data Systems, model 820GS; Oxford, PA, U.S.A.) linked to the multiple cold trap unit. Ten cryogenic traps are arranged radially around a 22-port high temperature rotary valve (Valco, Houston, TX). The traps cycle between $-195^{\circ} \mathrm{C}$ (liquid nitrogen) when trapping the pyrolysate fractions, and $300^{\circ} \mathrm{C}$ in the detrapping/analysis mode. The remainder of the system (transfer lines, valve) is continually maintained at $300^{\circ} \mathrm{C}$ to avoid condensation of higher boiling compounds.

\section{Hydrous Pyrolysis Experimentation}

Gold Tube Preparation. The gold tubing used in the pyrolysis experiments measured 4 $\mathrm{mm}$ outer diameter and $3.62 \mathrm{~mm}$ inner diameter. The gold tubing was cut with a scalpel into $5.0 \mathrm{~cm}$ lengths and cleaned first by washing in boiling $6 \mathrm{~N} \mathrm{HCl}$ and then rinsing 3 times with dichloromethane and methanol, respectively. The gold tube was then annealed at $9000 \mathrm{C}$ for $8-12$ hours.

Sample Loading and Gold Tube Welding. After annealing, one end of the $5.0 \mathrm{~cm}$ tubes was crimped using smooth faced needle nose pliers, clipped to produce a straight edge, and then welded shut using an acetylene torch. The weld was then inspected for blemishes using a 6x microscope and rewelded if necessary. Approximately $100 \mathrm{mg}$ of coal was then loaded into a gold tube in a glove box containing an argon atmosphere. After sample loading, the open end of the gold tube was crimped parallel to the first weld and weighed. The mass of coal in the gold tube was determined by difference. The unwelded edge of the gold tube was then clipped as described above and welded using an arc welder. During arc welding, the bottom of the gold tube was placed in a beaker of water and a drop of water is placed around the edge to be welded to keep temperature inside the gold tube to a minimum during welding. The sealed gold tube capsule was weighed again after the second welding for comparison with the capsule weight after the experiment.

Bombs. The bombs utilized in the hydrothermal laboratory are $31 \mathrm{~cm}$ long, have a diameter of $3.2 \mathrm{~cm}$, and a bore diameter of $7 \mathrm{~mm}$, are constructed of Rene (nickel alloy), and have a steel cap. Water pressure (up to 3,000 bars) is applied through the cap via a 
1/8" o.d. stainless steel line with a cone-in-cone fit to the bomb housing. The read thermocouple from the control unit is inserted into a port in the back of the bomb for temperature monitoring. Heavy Duty Electric Company (Milwaukee, WI) brand, 13" length $\times 6.5 "$ diameter split furnaces are used in all experiments.

Temperature Control. Furnace temperature was controlled and monitored by a custom built 40 channel controller unit manufactured at UCLA. Each furnace was equipped with an American National Standards Institute Type K chromel/alumel (nickel-chromium versus nickel aluminum) thermocouple used by the controller unit to adjust and control the furnace temperature. Each furnace was also equipped with a Type K thermocouple for temperature read out which was monitored at the control unit. The type $\mathrm{K}$ thermocouple has a range of $-500^{\circ} \mathrm{C}$ to $12,000^{\circ} \mathrm{C}$, an accuracy of $+/-2.20^{\circ} \mathrm{C}$ or $.75 \%$ (whichever is greater) and a readout resolution of $1.00^{\circ} \mathrm{C}$. Temperature was read on an Omega Engineering Incorporated Model 115 digital readout with a resolution of $10^{\circ} \mathrm{C}$. In order to minimize experimental error, temperatures for all furnaces were calibrated with a single thermocouple.

Pressure Control. Water pressure was manually controlled using a pressure pump (Owatonna Tool Company Model A Pressure Intensifier, Owatonna, MN). Pressure was monitored using a pressure gauge (Heisse, $+/-0.1 \%$ ) which can be read to $+/-1$ bar.

Pyrolysis. Experiments were conducted for 72 hours, isothermally at $3000^{\circ} \mathrm{C}$ and $3400^{\circ}$ $\mathrm{C}\left(+/ .2 .50^{\circ} \mathrm{C}\right)$ and pressures ranging from 70 bars to 2000 bars. No water was added. The experimental times and temperatures were chosen based on the work of Lewan (1985) to approximate vitrinite maturation near the beginning and end of oil generation. One gold tube containing sample was placed in each bomb followed by a solid stainless steel rod (4 mm o.d.) which occupied essentially all the remaining void space. The bomb was then filled with water, the bomb cap screwed in place, and the bomb placed in a furnace. Ceramic spacers of constant length were used to insulate the end of each bomb and to ensure that the position of the bomb in the furnace was always the same. The read thermocouple was inserted through a hole in the ceramic spacer and into a port in the end of the bomb (Figure X) for monitoring temperature at the controller unit (Figure X). Temperature was raised incrementally over a period of 90 minutes such that the target temperature was never exceeded. Pressure was maintained near the pressure of interest, but not in excess, until the target temperature was reached. The pressure of interest was then set. Temperature and pressure were monitored at least twice daily, and adjusted as needed, until the experiments were completed.

After the experiments were complete, the bombs were cooled in an air stream for approximately ten minutes and the gold tubes then removed from the bombs, weighed, and the contents analyzed. If the weight of the gold tube after the experiment was within $+/-0.25 \mathrm{mg}$ of the weight of the gold tube before the experiment, the run was considered successful.

Gas Product Analysis. Product analysis was performed at Chevron Petroleum Technology Company and included analysis of the non hydrocarbon and hydrocarbon gases, hydrocarbon liquids and residual solids. Gas collection was initiated by puncturing the gold tube under vacuum. The pyrolysate gas was initially exposed to the dry ice/acetone trap $\left(\mathrm{T}=-770^{\circ} \mathrm{C}\right)$ for $4-5$ minutes. The gas sample was then expanded into 
the liquid nitrogen trap $\left(\mathrm{T}=-196^{\circ} \mathrm{C}\right)$ for $4-5$ minutes. The liquid nitrogen noncondensable gases $\left(\mathrm{N}_{2}, \mathrm{CH}_{4}, \mathrm{CO}\right.$ and $\left.\mathrm{H}_{2}\right)$ were collected in the gas burette using a Toepler pump, the total fraction quantified, and introduced directly onto a gas chromatograph (GC). After $\mathrm{GC}$ analysis of the liquid nitrogen noncondensable gases had begun, the liquid nitrogen bath was removed and the $\mathrm{CO}_{2}$ and $\mathrm{C}_{2}-\mathrm{C}_{5}$ fraction was collected, quantified and frozen into a Pyrex tube.

The liquid nitrogen non-condensable gases were analyzed with a two channel United Technologies/Packard 43SA gas chromatograph equipped with thermal conductivity detectors (TCD). Hydrogen and helium were analyzed on one channel using a $1 / 8^{\prime \prime} \times 6^{\prime}$ 60/80 mesh Hayesep Q and 1/8" x 3' 60/80 mesh Mol Siev 13x columns in series/bypass configuration with a 1/8" x 18', 45/60 mesh Mol Siev 5A column. Nitrogen carrier gas flow was $15 \mathrm{ml} / \mathrm{min}$ and analysis was done isothermally at $35^{\circ} \mathrm{C}$ in less than 4 minutes. Oxygen + argon, nitrogen, methane and carbon monoxide were analyzed on the other channel using a 1/8" x 6' 80/100 mesh PoroPak N column in series/bypass configuration with 1/8" x 6' 80/100 mesh Mol Siev 13x and 1/8" x 1.3' 80/100 mesh Mol Siev 5A columns. Helium carrier gas flow was $30 \mathrm{ml} / \mathrm{min}$ and analysis was done isothermally at $35^{\circ} \mathrm{C}$ in less than 9.5 minutes.

The liquid nitrogen-condensable $\mathrm{CO}_{2}$ and $\mathrm{C}_{2}-\mathrm{C}_{5}$ fraction was analyzed on a two channel Hewlet Packard 5890 Series II gas chromatograph equipped with a TCD and a flame ionization detector (FID). A 1/8"x 12' 60/80 mesh Hayesep R packed column was used in conjunction with the TCD for analysis of $\mathrm{CO}_{2}, \mathrm{~N}_{2}, \mathrm{O}_{2}, \mathrm{Ar}$, and $\mathrm{C}_{1}-\mathrm{C}_{3}$. Helium carrier gas flow was $30 \mathrm{ml} / \mathrm{min}$. A $50 \mathrm{~m} \times .53 \mathrm{~mm} \times 5 \mu \mathrm{m}$ methyl silicone column was used in conjunction with the FID to analyze $\mathrm{C}_{1}-\mathrm{C}_{12}$ hydrocarbons. Helium carrier gas flow was $15 \mathrm{ml} / \mathrm{min}$. Oven temperature for both channels was programmed from $0^{\circ} \mathrm{C}$ to $50^{\circ} \mathrm{C}$ at $10^{\circ} \mathrm{C} / \mathrm{min}$ and $50^{\circ} \mathrm{C}$ to $200^{\circ} \mathrm{C}$ at $15^{\circ} \mathrm{C} / \mathrm{min}$ followed by a 5 minute hold. Analysis was complete in 20 minutes.

For the $340^{\circ} \mathrm{C}$ experiments, gas yields were high enough to permit stable carbon isotope ratio measurements on methane, ethane, propane and carbon dioxide. Individual compounds were isolated and converted to carbon dioxide gas using a Finnigan-MAT semi-automated gaschromatography-combustion system. In brief, approximately $2 \mathrm{ml}$ of pyrolysis gas was injected onto a $6 \mathrm{ft} \times 1 / 8$ in stainless steel column packed with $80 / 100$ mesh Poropack $\mathrm{N}$ that was temperature programmed from $35^{\circ} \mathrm{C}$ to $165^{\circ} \mathrm{C}$ at $10^{\circ} \mathrm{C} / \mathrm{min}$ with a helium flow rate of $30 \mathrm{ml} / \mathrm{min}$. Combustion was achieved in $8 \times 3 / 8$ in o.d. sections of Vycor tubing packed with cupric oxide pellets and held at $850^{\circ} \mathrm{C}$. Carbon dioxide was separated from water of combustion using standard cryogenic techniques and analyzed for ${ }^{13} \mathrm{C} /{ }^{12} \mathrm{C}$ ratios with a Finnigan-MAT 251 isotope ratio mass spectrometer. Results are reported in a notation relative to PDB standard and were calibrated assuming $\delta^{13} \mathrm{C}(\mathrm{NBS}-22)=-29.81 \%$ (Burnham et al., 1989).

Liquid Product Analysis. Following gas analysis, the dry ice/acetone bath was removed and the trap rinsed 3 times with pentane to obtain the $\mathrm{C}_{6}-\mathrm{C}_{12}$ gas condensate fraction. This was added to the total bitumen extract. The remaining gold tube was cut open with a scalpel and the residual coal extracted 3 times by sonication in a $4: 1$ dichloromethane/methanol solvent mixture to obtain the bitumen fraction. Following centrifugation, the extract was decanted and passed through a $0.45 \mu \mathrm{m}$ nylon filter. Bitumen samples were analyzed on a Hewlett Packard Model 5880 GC equipped with an 
on-column injector, a DB-1 30 meter, $.32 \mathrm{~mm}$ i.d., .25 $\mu \mathrm{m}$ methyl silicone capillary column and a flame ionization detector.

Solid Product Analysis. Coal samples were prepared for vitrinite reflectance analysis by first grinding in an agate mortar, mixing the powder with epoxy resin and mounting the mixture on a Plexiglas slide in a predrilled $5 \mathrm{~mm}$ well. After hardening, the coal/epoxy surface was wet sanded with 600 grit sandpaper and polished successively with $1.0 \mu \mathrm{m}$ and $0.5 \mu \mathrm{m}$ alumina.

Vitrinite reflectance (\% Ro) was measured at $546 \mathrm{~nm}$ wavelength using a Zeiss reflected light microscope. The percent reflected light from fifty telocollinite maceral fragments per sample was measured. The microscope was equipped with a Hewlett Packard 300 computer for statistical manipulation of the data.

Elemental analysis (\% C, H, N, S, 0) of the residual coals were determined at Huffman Laboratories, Inc. in Golden, Colorado using standard methods.

\section{Computational Methods}

\section{Reaction Network for the Kerogen Cracking}

Kerogen is the most abundant component of sedimentary organic matter in petroleum source rocks. As sediments are buried, kerogen undergoes thermal maturation that leads to petroleum generation. Further thermal heating of kerogen and/or cracking of petroleum produces gaseous compounds. Due to the limitations of current technologies, accurate knowledge of both the nature and the absolute amount of all chemical bonds in the kerogen network is not possible. Although the chemical structure of kerogen is not known, several kinetic models are available in the literature for describing its thermal behavior (primary cracking). These models assume either parallel reactions or successive reactions but it is impossible to discriminate between them, because an accurate distribution of the chemical bonds in kerogen is not yet available. The thermal decomposition of oil is very complicated in terms of the kinetics due to the large number of different reactions involved. Kinetic parameters are usually determined from experimental data of artificial maturation of kerogen either in open or closed pyrolysis systems in the temperature range of $\sim 250-600^{\circ} \mathrm{C}$. Assuming that the rate constant dependence follows the Arrhenius equation,

$$
\mathrm{k}=\mathrm{A}_{\mathrm{f}} \exp \left(-\mathrm{E}_{\mathrm{a}} / \mathrm{RT}\right)
$$

calculations can be performed through mathematical optimizations which allow the determination of various sets of kinetic parameters for the activation energies $\left(E_{a}\right)$ and frequency factors $\left(\mathrm{A}_{\mathrm{f}}\right)$ based on a nonlinear least-squares fitting. A very broad range of values for $E_{a}$ and $A_{f}$ is available in the literature (Ungerer, 1989) leading to a great uncertainty in predictions of the depth of the oil window in geological conditions. Consequently, the validity of the kinetic parameters available in the literature depends on the reaction scheme assumed to describe kerogen cracking, the choice of the experimental system for simulating the thermal decomposition of the kerogen, and the set of $\mathrm{E}_{\mathrm{a}} / \mathrm{A}_{\mathrm{f}}$ given by the mathematical optimization procedures.

To establish a reaction network for kerogen cracking, we have adopted a kinetic model based on a distribution of activation energies (Eddy and Rightmire, 1982). The rate of the 
cracking reaction is defined by the rate of oil to gas transformation. By quantifying the percentage of remaining normal paraffin, we can define an oil cracking conversion parameter. Distribution energy kinetic parameters were fitted to our experimental data of oil conversion using a deconvolution procedure described by Sundararaman et al. (1992). Our approach involved three basic assumptions: First, we grouped hydrocarbons according to their molecular weights into the following groups: $\mathrm{C}_{1}, \mathrm{C}_{2}, \mathrm{C}_{3}-\mathrm{C}_{5}, \mathrm{C}_{6}-\mathrm{C}_{14}$ and $\mathrm{C}_{15}+$, and assumed each group of hydrocarbon has the same thermal cracking behaviors. Therefore the reaction network of kerogen cracking takes form of the following:

$$
\begin{array}{ll}
\text { Kerogen } & \rightarrow a_{11} C_{15}++a_{12} C_{6}-C_{14}+a_{13} C_{3}-C_{5}+a_{14} C_{2}+a_{15} C_{1} \\
C_{15}+ & \rightarrow a_{21} C_{6}-C_{14}+a_{22} C_{3}-C_{5}+a_{23} C_{2}+a_{24} C_{1}+a_{25} \text { coke } \\
C_{6}-C_{14} & \rightarrow a_{31} C_{3}-C_{5}+a_{32} C_{2}+a_{33} C_{1}+a_{34} \text { coke } \\
C_{3}-C_{5} & \rightarrow a_{41} C_{2}+a_{42} C_{1}+a_{43} \text { coke } \\
C_{2} & \rightarrow a_{51} C_{1}+a_{52} \text { coke }
\end{array}
$$

Where $\mathrm{a}_{\mathrm{ij}}$ denotes the coefficients that would be optimized.

Second, we have assumed a certain frequency factor range from $10^{14} 1 / \mathrm{s}$ to $10^{15} 1 / \mathrm{s}$ for each cracking reaction. The frequency factors of $1 \times 10^{14}$ for kerogen cracking to $\mathrm{C}_{15+}, 2$ $\mathrm{x} 10^{14}$ for kerogen cracking to $\mathrm{C}_{6}-\mathrm{C}_{14}$, and $5 \times 10^{14}$ for kerogen cracking to $\mathrm{C}_{3}-\mathrm{C}_{5}$, were used, and a fixed frequency factor of $1 \times 10^{15}$ was chosen for all other hydrocarbon cracking. Third, we elected to use a Weibull distributed activation energy model to describe the thermal decomposition behavior of the $C_{n}$ normal paraffins under the conditions that the laboratory pyrolysis experiments were performed. In this model, each single reaction uses a subset (typically 5 to 9 values) of the distributed activation energies that range from $40 \sim 60 \mathrm{kcal} / \mathrm{mol}$, while the values of activation energies and their coefficients are fitted into the experimental data.

This mathematical treatment is especially suited to working with complex reaction systems, and yields a smooth conversion (fraction reacted) versus temperature profile when extrapolating to typical geological heating regimes. The optimization and fitting of the reaction network to the experimental data are, however, not trivial, considering a large set of coefficients are needed to be determined. An algorithm for the non-linear fitting, namely 'the simplex method' is utilized in our program to provide the satisfactory fitting.

\section{The Simplex Method}

The simplex method was invented in the early 1960's (Spendley, et al., 1962), and it has been used to solve a variety of problems ranging from shimming the gradient and curvature setting in NMR instruments to optimizing the solvent composition in HPLC by the "seven solvent" system (Ernst, 1963; Morgan and Demming, 1974; Ritter et al., 1975; Lam et al., 1976; Jurs and Isenhour, 1975). As the results presented below indicate, simplex can also be used to obtain rate constants and other important data from transient absorption decay curves by a completely unbiased analysis of the experimental data. The simplex procedure can be applied directly to the raw data and the unintegrated rate expressions for the scheme in question, and thus minimizes the subjective manipulation of the data. In an experiment designed to test the generality and limitations of the simplex method, ten different kinetic schemes have been analyzed using "data" 
synthesized for each scheme. The results prove the generality of the simplex method and demonstrate the ease with which even complex kinetic data can be analyzed in a direct, straightforward manner to obtain a reliable result. In some kinetic schemes multiple solutions can be found using simplex which exactly overlay the decay curve. In all of the cases studied, the false solutions could be differentiated from true solutions using chemical intuition based on "reasonable" values for rate constants, but this observation demonstrates the need for caution in interpreting kinetic data analyzed by any method.

Although flash spectroscopy is confined to the study of relatively simple reactive molecules, the processes and products formed can be exceedingly complex. This leads to complicated absorption decay curves which can best be interpreted only through the application of numerical methods. Numerical analysis by the method shown above was useful but the procedure is labor intensive and provides ample opportunities for arithmetic errors. Thus the simplex method is far preferable. In spite of the absence of a rigorous theoretical expression derived from the chemistry of the intermediates involved in the observed decay, the simplex method can be used to find the best fit between the experimental data and a series of postulated kinetic schemes, whether or not the scheme being analyzed is appropriate. It is then the responsibility of the experimenter to decide whether the curve generated from the calculated constants matches the shape of the experimental decay.

Simplex is an algorithm which systematically reduces the error between the calculated and experimental curves by iteration. Initial sets of values are guessed based on chemical intuition, and the corresponding curves are calculated based on the hypothesized scheme. Sufficient sets of data must be chosen that there is at least one more set of parameters than the number of actual variables. In subsequent iterations the worst set of parameters is replaced by a new set and the process is continued until the match between the two curves falls within a given tolerance. These are then taken as the "best" values possible for the assumed scheme.

Figure 1 shows three iterations in a two dimensional case. In the original set of guesses $(\mathrm{ABC})$, point $\mathrm{A}$ has the lowest response and is discarded, leaving point $\mathrm{B}$ and $\mathrm{C}$. Reflection of point $\mathrm{A}$ across the face $\mathrm{BC}$ generates points $\mathrm{D}$ and forms the second set (BCD). Reflection of $\mathrm{C}$ point gives the third set (BDE). Finally, the set (DEF) is formed after eliminating point $\mathrm{B}$.

The original simplex technique has no provision for acceleration and adaptation to fit the particular response surface being studied. Figure 2 illustrates how a two dimensional modified simplex works where two parameters $\mathrm{k}_{1}$ and $\mathrm{k}_{2}$ are to be found. Suppose $\mathrm{C}$ is the correct set of values, and $\mathrm{B}, \mathrm{N}$ and $\mathrm{W}$ are the sets of values in the initial guesses. After evaluation of each point one can discern that $\mathrm{B}$ yields the best response, $\mathrm{N}$ is the next to the best and $\mathrm{W}$ is the worst point. $\mathrm{P}$ is the centroid of the hyperface $\mathrm{BN}$. Reflection of $\mathrm{W}$ across $\mathrm{BN}$ generates point $\mathrm{R}$. There are three possibilities to be considered for the response at point $\mathrm{R}$. The response at $\mathrm{R}$ is more desirable than the response at $\mathrm{B}$. If the point $\mathrm{E}$ is better than the point $\mathrm{B}$, then $\mathrm{BNE}$ is the new basis for the simplex analysis. If $E$ is not more desirable than $B$, then $B N R$ is the new simplex. If $R$ is neither better than B nor worse than the response at N, then BNR is the new basis set. If $\mathrm{R}$ is worse than $\mathrm{N}$, but better than $\mathrm{W}$, then BNCR will be used. If the point $\mathrm{R}$ is worse than the point $\mathrm{W}$, then the $\mathrm{BNCW}$ will be selected. 
The method of evaluating each point is that the calculated and observed decay curves are compared point by point with the experimental decay curves and the absolute difference between the two curves is summed and that value used to evaluate the fit. The set of constants leading to the poorest fit is the one which has the largest residue. 


\section{References}

Abbott G. D., Wang G. Y., Eglinton T. I., Home A. K., and Petch G. S. (1990) The kinetics of sterane biological marker release and degradation processes during the hydrous pyrolysis of vitrinite kerogen. Geochim. Cosmochim. Acta 54, 2451-2461.

Anders, D. E., and Gerrild, P. M. (1984) Hydrocarbon generation in lacustrine rocks of Tertiary age, Uinta Basin, Utah - organic carbon, pyrolysis yield, and light hydrocarbons. In Hydrocarbon source rocks of the greater Rocky Mountain region (Edited by J. Woodward, F. F. Meisnner, and J. L. Clayton). Rocky Mt. Assoc. Geol., Denver, pp. 513-529.

Baars, D. L., Bartelson, B. L., Chapin, C. E., Curtis, B. F., De Voto, R. H., Everett, J. R., Johnson, R. C., Molenaar, C. M., Peterson, F., Schenk, C. J., Love, J. D., Merin, I. S., Rose, P. R., Ryder, R. T., Waechter, N. B., and Woodward, L. A. (1988) Basins of the Rocky Mountain Region. In Sedimentary Cover - North American Craton, US (Edited by L. L. Sloss). Geologic Society of America, Decade of North American Geology, v. D-2, p. 109-220.

Barnard, P. C., Collins, A. G., and Cooper, B. S. (1981) Identification and distribution of kerogen facies in a source rock horizon - examples from the North Sea basin. In Organic maturation studies and fossil fuel exploration (Edited by J. Brooks). Academic Press, London, pp 271-282.

Barnard, P. C., and Barstow, M. A. (1992) Hydrocarbon generation, migration, alteration, entrapment, and mixing in the Central and Northern North Sea. In Petroleum Migration (Edited by W. A. England and A. J. Fleet), Geol. Soc. London, Spec. Publ. 59: 167-190.

Beaumont C., Boutilier R, Mackenzie A. S. and Rullkotter J. (1985) Isomerization and aromatization of hydrocarbons and the paleothermometry and burial history of Alberta Foreland Basin. Am. Assoc. Petrol. Geol. Bull. 69, 546-566.

Bissada, K. K. (1982) Geochemical constraints on petroleum generation and migration - 
A review. Proc. $2^{\text {nd }}$ ASCOPE Conf. Manila, Oct., 1981, pp. 69-87.

Bradley, W. H. (1931) The origin of the oil shale and its microfossils of the Green River Formation of Colorado and Utah. U. S. Geol. Survey Prof. Paper 496-A:86.

Braun, R. L., Burnham, A. K., and Reynolds, J. G. (1992) Oil and evolution kinetics for oil shale and petroleum source rocks determined from pyrolysis -TQMS data at two heating rates. Energy and Fuels, 6, 468-474.

Bucheim, H. P., and Surdam, R. C. (1977) Fossil catfish and the depositional environment of the Green River Formation Wyoming. Geology, 5: 196-198.

Burnham, A. K., Braun, R. L. Gregg, H. R., and Samoun, A. M. (1987) Comparison of methods for measuring kerogen pyrolysis rates and fitting kinetic parameters. Energy and Fuels, 1, 452-458.

Burnham, A. K., Oh, M. S., and Crawford, R. W. (1989) Pyrolysis of Argonne Premium Coals: activation energies and related chemistry. Energy and Fuels, 3, 42-55.

Collins, B. A. (1976) Coal deposits of the Carbondale, Grand Hogback, and Southern Danforth Hills coal fields, eastern Piceance Basin, Colorado. Quarterly of the Colorado School of Mines, v. 71, no. 1, 138 p.

Cooper, B. S. (1990) Practical Petroleum Geochemistry. Robertson Sci. Publ., London, $174 \mathrm{pp}$.

Cooper, B. S., and Barnard, P. C., (1984) Source rocks and oils of the central and northern North Sea. In Petroleum Geochemistry and Basin Evaluation (Edited by G. Demaison and R. J. Murris), Am. Assoc. Petrol. Geol., Tulsa, Mem 35: 303-314.

Cooper, B. S., Barnard, P. C., and Telnaes, N. (1995) The Kimmeridge Clay Formation of the North Sea. In Petroleum Source Rocks (Edited by B. J. Katz), SpringerVerlag, Berlin, 89-110.

Eberl, D.D., Velde, B. and McCormick, T. (1993) Synthesis of illite-smectite from smectite at Earth surface temperatures and high pH. Clay Minerals, 28, 49-60. 
(Eddy and Rightmire, 1982)

Elliott, W.C., Aronson, J.L., Matisoff, G. and Gautier, D.L. (1991) Kinetics of the Smectite to Illite Transformation in the Denver Basin: Clay Mineral, K-Ar Data, and Mathematical Model Results. Bulletin o/the American Association o/Petroleum Geologists, 75(3), 436462.

Elliott, W.C. and Matisoff, G. (1996) Evaluation of kinetic models for the smectite to illite transformation. Clays and Clay Minerals, 44(1), 77-87.

Ensminger A., Albrecht P., Ourisson G. and Tissot B. (1977) Evolution of polycyclic hydrocarbons under the effect of burial (Early Toarcian shales, Paris Basin). In Advances in Organic Geochemistry 1975 (Edited by Campos R. and Goni J.), pp. 45-52. Enadisma, Madrid.

Ernst, R. R. (1963) Rev. Sci. Instrum., 39, 998.

Espitalié, J., Laporte, L. J., Madec, M., Marquis, F., Leplat, P. J., Paulet, J., and Boutedeu, A. (1977) Méthode rapide de charactérization des roches mères de leur potential pétrolier et de leur degree d'évolution. Rev. Inst. Français du Pétrole, 32: $32-42$.

Espitalié, J., Ungerer, P., Irwin, I., and Marquis, F. (1987) Primary cracking of kerogens. Experimenting and modeling C1, C2-C5, C6-C15+ classes of hydrocarbons formed. In Advances in Organic Geochemistry 1987 (edited by Mattavelli, L. and Novelli, L.). Org. Geochem., 13, 893-899.

Franczyk, K. J., Pitman, J. K., and Nichols, D. J. (1990) Sedimentology, mineralogy, palynology, and depositional history of some Uppermost Cretaceous and Lowermost Tertiary rocks along the Utah Book and Roan Cliffs east of the Green River. U.S. Geol. Survey Bull., 1787:27.

Grantham P. J. (1986) Sterane isomerization and moretane/hopane ratios in crude oils derived from Tertiary source rocks. Org. Geochem. 9, 293-304. 
ten Haven H. L., de Leeuw J. W., Peakman T. M. amd Maxwell J. R (1986) Anomalies in steroid and hopanoid maturity indices. Geochim. Cosmochim. Acta 50, 853-855.

Hillier, S., Matyas, J., Matter, A. and Vasseur, G. (1995) Illite/Smectite diagenesis and its variable correlation with vitrinite reflectance in the Pannonian Basin. Clays and Clay Minerals, 43(2), 174-183.

Hong Z.-H., Li H.-Z., Rullkotter J. and Mackenzie A. S. (1986) Geochemical application of sterane and triterpane biological marker compounds in the Linyi Basin. In Advances in Organic Geochemistry 1985 (Edited by Leythaeuser D. and Rullkotter J.). Org. Geochem. 10, 433-439.

Huang, W-L., Longo, J.M. and Pevear, D.R. (1993) An experimentally derived kinetic model for smectite-to-illite conversion and its use as a geothermometer. Clays and Clay Minerals, 41, 162-177.

Johnson, R. C., and Rice, D. D. (1990) Occurrence and geochemistry of natural gases, Piceance Basin, northwest Colorado. Am. Assoc. Petr. Geol. Bull. 74(6), 805829.

Jurs, P. C. and Isenhour, T. L. (1975) Chemical Applications of Pattern Recognition, John Wiley Sons, New York.

Katz, B. J. (1995) The Green River Shale: an Eocene carbonate lacustrine source rock. In Petroleum Source Rocks (Edited by B. J. Katz), SpringerVerlag, Berlin, 309324.

Lam, T. F., Wilkins, C. L., Branner, T. R., Soltzberg, L. J., and Kaberline, S. L. (1976) Anal. Chem., 48, 1786.

Largeau, C., Derenne, S., Clarray, C., Casadevall, E., Raynaud, J. F., Lugardou, B., Berkaloff, C., Corolleur, M., and Posseau, B. (1990) Characterization of various kerogens by Scanning Electron Microscopy (SEM), and Transmission Electron Microscopy (TEM) - morphological relationships with resistant outer walls in extant microorganisms. Meded. Rijks. Geol. Dienst. 45: 91-101. 
Larter, S. R., and Douglas, A. G. (1980) A pyrolysis-gas chromatographic method for kerogen typing. In Advances in Organic Geochemistry, 1979 (Edited by A. G. Douglas and J. R. Maxwell), Pergamon Press, New York, pp. 579-583.

Law, B. E., Nuccio, V. F., and Stanton, R. W. (1989) Evaluation of source rock characteristics, thermal maturation, and pressure history of the Upper Cretaceous Cameo coal zone, Deep Seam well, Piceance basin, Colorado. 1989 Coalbed Methane Symposium Proceedings, p. 341-353.

Lewan, M. D. (1985) Evaluation of petroleum generation by hydrous pyrolysis experimentation. Phil. Trans., R. Soc. Lon. 315, 124-134.

Lorenz, J. C. (1989) Reservoir sedimentology of rocks of the Mesaverde Group, multiwell experiment site and east-central Piceance Basin, northwest Colorado. In Geology of Tight Gas Reservoirs in the Pinedale Anticline Area, Wyoming, and at the Multiwell Experiment Site, Colorado (Edited by B. E. Law and C. W. Spencer), US Geological Survey Bulletin 1886, p. K1-K24.

Mackenzie A. S. and McKenzie D. P. (1983) Aromatization and isomerization of hydrocarbons in sedimentary basins formed by extension. Geol. Mag. 120, 417-470.

Mackenzie A. S., Patience R L, MaxweU J. R, Vandenbroucke M. and Durand B. (1980) Molecular parameters of maturation in the Toarcian Shales, Paris Basin, France. I. Changes in the configurations of acyclic isoprenoid alkanes, steranes, and triterpanes. Geochim. Cosmochim. Acta 44, 1709-1721.

Marzi R (1992) Comment on "The kinetics of sterane biological marker release and degradation processes during the hydrous pyrolysis of vitrinite kerogen" by G. D. Abbott, G. Y. Wang, T. I. Eglinton, A. K. Home and G. S. Petch. Geochim. Cosmochim. Acta 56, 533-534.

Marzi R. and RuUkotter J. (1992) Qualitative and quantitative evolution and kinetics of biological marker transformations - Laboratory experiments and application to the Michigan Basin. In Biological Markers in Sediments and Petroleum (Edited by Moldowan 1. M., Albrecht P. and Philp R. P.), pp. 18-41. Prentice-Hall, Englewood 
Cliffs, New Jersey.

Moldowan J. M., Sundararaman P. and Schoell M. (1986) Sensitivity of biomarker properties to depositional environment and/or source input in the Lower Toarcian of S.W. Germany. Org. Geochem. 10, 915-926.

Moldowan J. M., Sundararaman P., Salvatori T., Alajbeg A., Gjukic B., Lee C. Y. and Demaison G. J. (1992) Source correlation and maturity assessment of select oils and rocks from the Central Adriatic Basin (Italy and Yugoslavia). In Biological Markers in Sediments and Petroleum (Edited by Moldowan J. M., Albrecht P. and Philp R P.), pp. 370-401. Prentice-Hall, Englewood Cliffs, New Jersey.

Morgan, S. L., and Demming, S. N. (1974) Anal. Chem., 46, 1170.

O’Brien, N. R., and Slatt, R. M. (1990) Argillaceous Rock Atlas. Springer-Verlag, Berlin, $141 \mathrm{pp}$.

Peakman T. M. and Maxwell J. R (1988) Early diagentic pathways of steroid alkanes. Org. Geochem. 13, 583-592.

Peters K. E. and Moldowan J. M. (1993) The Biomarker Guide: Interpreting Molecular Fossils in Petroleum and Ancient Sediments, 363 pp. Prentice-Hall, Englewood Cliffs, New Jersey.

Peters K. E., MoIdowan J. M. and Sundararaman P. (1990) Effects of hydrous pyrolysis on biomarker thermal maturity parameters: Monterey phosphatic and siliceous members. Org. Geochem. 15, 249-265.

Pytte, A.M. and Reynolds, R.C. (1989) The thermal transformation of smectite to illite. In: Naeser, N.D. and McCulloh, T.H (Eds.) Thermal history of sedimentary basins. SpringerVerlag, Berlin. 133-140.

Requejo A. G. (1992) Quantitative analysis of triterpane and sterane biomarkers: methodology and applications in molecular maturity studies. In Biological Markers in Sediments and Petroleum (Edited by Moldowan J. M., Albrecht P. and Philp R 
P.), pp. 222-240. Prentice-Hall, Englewood Cliffs, New Jersey.

Reynolds, J. G., Crawford, R. W., and Burnham, A. K. (1991) Analysis of oil shale and petroleum source rock pyrolysis by triple quadrupole mass spectrometry: comparisons of gas evolution at the heating rate of $10^{\circ} \mathrm{C} / \mathrm{min}$. Energy and Fuels, 5 , 507-523.

Ritter, G. L., Owry, S. R., Wilkins, C. L., and Isenhour, T. L. (1975) Anal. Chem., 47, 1951.

Rullkotter J. and Marzi R (1988) Natural and artificial maturation of biological markers in a Toarcian shale from northern Germany. In Advances in Organic Geochemistry 1987 (Edited by Mattavelli L. and Novelli L.). Org. Geochem. 13, 639-645.

Schoen M., Teschner M., Wehner H., Durand B. and Oudin J. L (1983) Maturity related biomarker and stable isotope variations and their application to oil/source rock correlation in the Mahakam Delta, Kalimantan. In Advances in Organic Geochemistry 1981 (Edited by Bjorey M. etal.), pp. 156-163.

Seifurt W. K. and Moldowan J. M. (1980) The effect of thermal stress on source rock quality as measured by hopane stereochemistry. Phys. Chem. Earth 12, 229-237.

Seifurt W. K. and Moldowan J. M. (1986) Use of biological markers in petroleum exploration. In Biological Markers in the Sedimentary Record (Edited by Johns R B.), pp. 261-290. Elsevier, Amsterdam.

Shaw, D. A. V., and Primmer, T. J. (1991) Diagenesis of mudrocks from the Kimmeridge Clay Formation of the Brae area, UK North Sea. Mar. Petrol. Geol., 8: 270-277.

Spendley, W., Hext, G. R., and Himsworth, F. R. (1962) Thechnometrics 4, 441.

Strachan M. G., Alexander R., Kagi R. I., Subroto E. D. and van Bronswijk W. (1989) Constraints on the use of ethylcholestane diastereomer ratios as maturity indicators for petroleum. Org. Geochem. 14, 423-432. 
Sundararaman et al. (1992)

Sweeney, J. J., Burnham, A. K., and Braun, R. L. (1986) A model for hydrocarbon maturation in the Uinta Basin, Utah. In Thermal modeling in sedimentary basins (Edited by J. Burus), Éditions Technip., Paris, pp. 547-561.

Sweeney, J., Talukdar, S., Burnham, A. and Vallejus, C. (1989) Pyrolysis kinetics applied to prediction of oil generation in the Maracaibo Basin. Org. Geochem., 16, $1-8$.

Tang, Y. and Stauffer, M. (1994) Development of multiple cold trap pyrolysis. J. Appl. Anal. Pyrolysis, 28, 167-174.

Tissot, B. P., Durand, B., Espitalié, J., and Combaz, A. (1974) Influence of nature and diagenesis of organic matter in formation of petroleum. Am. Assoc. Petrol. Geol. Bull., 58: 499-506.

Tissot, B. P., and Welte, D. H. (1984) Petroleum Formation and Occurrence, $2^{\text {nd }}$ Ed. pp. 699. Springer Verlag, Berlin.

Tyson, R. V. (1989) Late Jurassic palynofacies trends, Piper and Kimmeridge Clay Formations, UK onshore and northern North Sea. In Northwest European Micropaleontology and Palynology (Edited by D. J. Butler, and M. C. Keen), Ellis Horwood, Chichester, pp 135-172.

Ungerer, P. (1989) Org. Geochem., 16(1-3), 470-501.

Ungerer, P. and Pelet, R. (1987) Extrapolation of the kinetics of oil and gas from laboratory experiments to sedimentary basin. Nature, 327, 52-54.

Velde, B. and Lanson, B. (1993) Comparison of I/S transformation and maturity of organic matter at elevated temperatures. Clays and Clay Minerals, 41(2), 178-183.

Velde, B. and Vasseur, G. (1992) Estimation of the diagenetic smectite-to-illite transformation in time-temperature space. American Mineralogist, 77, 967-976. 
Verajao, A. and Meunier, A. (1996) Burial and thermal conditions of diagenesis in the Lower Cretaceous Barra de Itiuba Shale Formation, Sergipe-Algagoas Basin, Brazil. Bulletin de la Societe Geologique de France, 167(5), 597-607. 
Appendix 1: Data Tables 
Table 5: Kinetic data for Easy $\% R_{0}$ (Sweeney and Burnham, 1990).

\begin{tabular}{|c|c|c|c|}
\hline \multirow{2}{*}{ Frequency factor, $A\left(\mathrm{~s}^{-1}\right)$} & & $\begin{array}{c}\text { Activation } \\
\text { energy } E_{i}(\text { Kcal } \\
\left.\text { mol }^{-1}\right) \\
\end{array}$ & $\begin{array}{c}\text { Weight } W_{i} \\
(\%)\end{array}$ \\
\hline & $1.00 E+13$ & 34 & 3.00 \\
\hline \multirow{2}{*}{\multicolumn{2}{|c|}{$\begin{array}{l}\text { Vitrinite conversion index* } \\
\mathrm{VCI}=\left[\ln \left(\% R_{0}\right)-\mathrm{C} 2\right] / \mathrm{C} 1\end{array}$}} & 35 & 0.00 \\
\hline & & 36 & 3.00 \\
\hline \multirow{36}{*}{$\begin{array}{l}\mathrm{C} 1 \\
\mathrm{C} 2\end{array}$} & 3.70 & 37 & 0.00 \\
\hline & -1.60 & 38 & 4.00 \\
\hline & & 39 & 0.00 \\
\hline & & 40 & 4.00 \\
\hline & & 41 & 0.00 \\
\hline & & 42 & 5.00 \\
\hline & & 43 & 0.00 \\
\hline & & 44 & 5.00 \\
\hline & & 45 & 0.00 \\
\hline & & 46 & 6.00 \\
\hline & & 47 & 0.00 \\
\hline & & 48 & 4.00 \\
\hline & & 49 & 0.00 \\
\hline & & 50 & 4.00 \\
\hline & & 51 & 0.00 \\
\hline & & 52 & 7.00 \\
\hline & & 53 & 0.00 \\
\hline & & 54 & 6.00 \\
\hline & & 55 & 0.00 \\
\hline & & 56 & 6.00 \\
\hline & & 57 & 0.00 \\
\hline & & 58 & 6.00 \\
\hline & & 59 & 0.00 \\
\hline & & 60 & 5.00 \\
\hline & & 61 & 0.00 \\
\hline & & 62 & 5.00 \\
\hline & & 63 & 0.00 \\
\hline & & 64 & 4.00 \\
\hline & & 65 & 0.00 \\
\hline & & 66 & 3.00 \\
\hline & & 67 & 0.00 \\
\hline & & 68 & 2.00 \\
\hline & & 69 & 0.00 \\
\hline & & 70 & 2.00 \\
\hline & & 71 & 0.00 \\
\hline & & 72 & 1.00 \\
\hline
\end{tabular}


Table 6: Kinetic data for Brown Shale (Type I).

\begin{tabular}{|c|c|c|c|}
\hline & & $\begin{array}{c}\text { Activation energy } \\
E_{i}\left(\mathrm{kcal} \mathrm{mol}^{-1}\right)\end{array}$ & Weight $W_{i}(\%)$ \\
\hline Frequency factor, $A\left(\mathrm{~s}^{-1}\right)$ & $1.33 E+14$ & 34 & 0.00 \\
\hline Vitrinite conversion index & & 35 & 0.00 \\
\hline $\mathrm{VCl}=\left[\ln \left(\% R_{\mathrm{o}}\right)-\mathrm{C} 2\right] / \mathrm{C} 1$ & & 36 & 0.00 \\
\hline C1 & 1.629 & 37 & 0.00 \\
\hline $\mathrm{C} 2$ & -0.693 & 38 & 0.00 \\
\hline & & 39 & 0.00 \\
\hline & & 40 & 0.00 \\
\hline & & 41 & 0.00 \\
\hline & & 42 & 5.40 \\
\hline & & 43 & 0.00 \\
\hline & & 44 & 0.00 \\
\hline & & 45 & 0.00 \\
\hline & & 46 & 8.50 \\
\hline & & 47 & 0.00 \\
\hline & & 48 & 6.23 \\
\hline & & 49 & 7.75 \\
\hline & & 50 & 2.28 \\
\hline & & 51 & 2.14 \\
\hline & & 52 & 9.99 \\
\hline & & 53 & 1.18 \\
\hline & & 54 & 14.06 \\
\hline & & 55 & 1.13 \\
\hline & & 56 & 1.02 \\
\hline & & 57 & 10.11 \\
\hline & & 58 & 4.27 \\
\hline & & 59 & 1.39 \\
\hline & & 60 & 4.15 \\
\hline & & 61 & 7.11 \\
\hline & & 62 & 2.12 \\
\hline & & 63 & 1.20 \\
\hline & & 64 & 2.25 \\
\hline & & 65 & 3.32 \\
\hline & & 66 & 4.39 \\
\hline & & 67 & 0.00 \\
\hline & & 68 & 0.00 \\
\hline & & 69 & 0.00 \\
\hline & & 70 & 0.00 \\
\hline & & 71 & 0.00 \\
\hline & & 72 & 0.00 \\
\hline
\end{tabular}


Table 7: Kinetics data for Barnett Shale (Type IIa).

\begin{tabular}{|c|c|c|c|}
\hline & & $\begin{array}{c}\text { Activation energy } E_{i} \\
\left(\mathrm{kcal} \mathrm{mol}^{-1}\right)\end{array}$ & Weight $W_{i}(\%)$ \\
\hline Frequency factor, $A\left(s^{-1}\right)$ & $2.63 E+14$ & 34 & 0.00 \\
\hline Vitrinite conversion index ${ }^{*}$ & & 35 & 0.00 \\
\hline $\mathrm{VCl}=\left[\ln \left(\% R_{\mathrm{o}}\right)-\mathrm{C} 2\right] / \mathrm{C} 1$ & & 36 & 0.00 \\
\hline $\mathrm{C} 1$ & 1.631 & 37 & 0.00 \\
\hline $\mathrm{C} 2$ & -0.799 & 38 & 0.00 \\
\hline & & 39 & 0.00 \\
\hline & & 40 & 0.00 \\
\hline & & 41 & 0.00 \\
\hline & & 42 & 0.16 \\
\hline & & 43 & 0.69 \\
\hline & & 44 & 2.67 \\
\hline & & 45 & 2.29 \\
\hline & & 46 & 0.30 \\
\hline & & 47 & 0.01 \\
\hline & & 48 & 0.06 \\
\hline & & 49 & 12.81 \\
\hline & & 50 & 0.00 \\
\hline & & 51 & 0.00 \\
\hline & & 52 & 14.92 \\
\hline & & 53 & 0.08 \\
\hline & & 54 & 17.94 \\
\hline & & 55 & 0.08 \\
\hline & & 56 & 18.89 \\
\hline & & 57 & 0.00 \\
\hline & & 58 & 2.45 \\
\hline & & 59 & 11.14 \\
\hline & & 60 & 1.16 \\
\hline & & 61 & 0.00 \\
\hline & & 62 & 8.34 \\
\hline & & 63 & 1.36 \\
\hline & & 64 & 0.33 \\
\hline & & 65 & 0.19 \\
\hline & & 66 & 4.13 \\
\hline & & 67 & 0.00 \\
\hline & & 68 & 0.00 \\
\hline & & 69 & 0.00 \\
\hline & & 70 & 0.00 \\
\hline & & 71 & 0.00 \\
\hline & & 72 & 0.00 \\
\hline
\end{tabular}


Table 8: Kinetic data for Red Water (Type IIb).

\begin{tabular}{|c|c|c|c|}
\hline & & $\begin{array}{l}\text { Activation energy } \\
E_{i}\left(\mathrm{kcal} \mathrm{mol}^{-1}\right) \\
\end{array}$ & Weight $W_{i}(\%)$ \\
\hline Frequency factor, $A\left(s^{-1}\right)$ & $8.75 E+13$ & 34 & 0.00 \\
\hline \multirow{2}{*}{\multicolumn{2}{|c|}{$\begin{array}{c}\text { Vitrinite conversion index* } \\
\mathrm{VCl}=\left[\ln \left(\% R_{0}\right)-\mathrm{C} 2\right] / \mathrm{C} 1\end{array}$}} & 35 & 0.00 \\
\hline & & 36 & 0.00 \\
\hline $\mathrm{C} 1$ & 1.946 & 37 & 0.00 \\
\hline \multirow[t]{35}{*}{$\mathrm{C} 2$} & -1.204 & 38 & 0.00 \\
\hline & & 39 & 0.00 \\
\hline & & 40 & 0.00 \\
\hline & & 41 & 1.79 \\
\hline & & 42 & 0.87 \\
\hline & & 43 & 0.12 \\
\hline & & 44 & 8.88 \\
\hline & & 45 & 1.70 \\
\hline & & 46 & 0.34 \\
\hline & & 47 & 7.70 \\
\hline & & 48 & 2.24 \\
\hline & & 49 & 10.22 \\
\hline & & 50 & 8.23 \\
\hline & & 51 & 0.67 \\
\hline & & 52 & 11.56 \\
\hline & & 53 & 22.61 \\
\hline & & 54 & 0.20 \\
\hline & & 55 & 13.20 \\
\hline & & 56 & 0.06 \\
\hline & & 57 & 1.90 \\
\hline & & 58 & 4.74 \\
\hline & & 59 & 0.89 \\
\hline & & 60 & 0.00 \\
\hline & & 61 & 0.13 \\
\hline & & 62 & 0.23 \\
\hline & & 63 & 0.80 \\
\hline & & 64 & 0.91 \\
\hline & & 65 & 0.00 \\
\hline & & 66 & 0.00 \\
\hline & & 67 & 0.00 \\
\hline & & 68 & 0.00 \\
\hline & & 69 & 0.00 \\
\hline & & 70 & 0.00 \\
\hline & & 71 & 0.00 \\
\hline & & 72 & 0.00 \\
\hline
\end{tabular}


Table 9: Kinetics data for Dongfang (Type III).

\begin{tabular}{|c|c|c|c|}
\hline & & $\begin{array}{c}\text { Activation energy } E_{i} \\
\left(\mathrm{kcal} \mathrm{mol}^{-1}\right)\end{array}$ & Weight $W_{i}(\%)$ \\
\hline Frequency factor, $A\left(s^{-1}\right)$ & $8.07 E+13$ & 34 & 0.00 \\
\hline \multirow{2}{*}{\multicolumn{2}{|c|}{$\begin{array}{l}\text { Vitrinite conversion index } \\
\mathrm{VCl}=\left[\ln \left(\% R_{0}\right)-\mathrm{C} 2\right] / \mathrm{C} 1\end{array}$}} & 35 & 0.00 \\
\hline & & 36 & 0.00 \\
\hline $\mathrm{C} 1$ & 1.792 & 37 & 0.00 \\
\hline \multirow[t]{35}{*}{$\mathrm{C} 2$} & -0.693 & 38 & 0.00 \\
\hline & & 39 & 0.00 \\
\hline & & 40 & 0.00 \\
\hline & & 41 & 0.00 \\
\hline & & 42 & 0.00 \\
\hline & & 43 & 3.90 \\
\hline & & 44 & 2.96 \\
\hline & & 45 & 2.51 \\
\hline & & 46 & 1.95 \\
\hline & & 47 & 4.10 \\
\hline & & 48 & 8.59 \\
\hline & & 49 & 3.21 \\
\hline & & 50 & 0.00 \\
\hline & & 51 & 5.16 \\
\hline & & 52 & 8.45 \\
\hline & & 53 & 7.90 \\
\hline & & 54 & 2.72 \\
\hline & & 55 & 3.05 \\
\hline & & 56 & 7.62 \\
\hline & & 57 & 1.88 \\
\hline & & 58 & 4.60 \\
\hline & & 59 & 4.22 \\
\hline & & 60 & 2.76 \\
\hline & & 61 & 2.80 \\
\hline & & 62 & 2.92 \\
\hline & & 63 & 4.71 \\
\hline & & 64 & 2.04 \\
\hline & & 65 & 1.56 \\
\hline & & 66 & 6.43 \\
\hline & & 67 & 3.95 \\
\hline & & 68 & 0.00 \\
\hline & & 69 & 0.00 \\
\hline & & 70 & 0.00 \\
\hline & & 71 & 0.00 \\
\hline & & 72 & 0.00 \\
\hline
\end{tabular}


Table 10: Kinetics data for Fushan (Coal a).

\begin{tabular}{|c|c|c|c|}
\hline & & $\begin{array}{c}\text { Activation energy } \\
E_{i}\left(\mathrm{kcal} \mathrm{mol}^{-1}\right)\end{array}$ & Weight $W_{i}(\%)$ \\
\hline Frequency factor, $A\left(s^{-1}\right)$ & $7.63 E+13$ & 34 & 0.00 \\
\hline \multirow{2}{*}{\multicolumn{2}{|c|}{$\begin{array}{l}\text { Vitrinite conversion index } \\
\mathrm{VCl}=\left[\ln \left(\% R_{0}\right)-\mathrm{C} 2\right] / \mathrm{C} 1\end{array}$}} & 35 & 0.00 \\
\hline & & 36 & 8.54 \\
\hline $\mathrm{C} 1$ & 1.897 & 37 & 0.00 \\
\hline \multirow[t]{35}{*}{$\mathrm{C} 2$} & -1.204 & 38 & 0.00 \\
\hline & & 39 & 3.26 \\
\hline & & 40 & 3.38 \\
\hline & & 41 & 3.12 \\
\hline & & 42 & 4.02 \\
\hline & & 43 & 1.94 \\
\hline & & 44 & 10.10 \\
\hline & & 45 & 0.00 \\
\hline & & 46 & 0.00 \\
\hline & & 47 & 16.58 \\
\hline & & 48 & 2.17 \\
\hline & & 49 & 0.00 \\
\hline & & 50 & 4.85 \\
\hline & & 51 & 9.36 \\
\hline & & 52 & 0.00 \\
\hline & & 53 & 11.15 \\
\hline & & 54 & 0.00 \\
\hline & & 55 & 0.00 \\
\hline & & 56 & 13.82 \\
\hline & & 57 & 0.88 \\
\hline & & 58 & 0.00 \\
\hline & & 59 & 0.00 \\
\hline & & 60 & 6.84 \\
\hline & & 61 & 0.00 \\
\hline & & 62 & 0.00 \\
\hline & & 63 & 0.00 \\
\hline & & 64 & 0.00 \\
\hline & & 65 & 0.00 \\
\hline & & 66 & 0.00 \\
\hline & & 67 & 0.00 \\
\hline & & 68 & 0.00 \\
\hline & & 69 & 0.00 \\
\hline & & 70 & 0.00 \\
\hline & & 71 & 0.00 \\
\hline & & 72 & 0.00 \\
\hline
\end{tabular}


Table 11: Kinetic data for Twin Arrow (Coal b).

\begin{tabular}{|c|c|c|c|}
\hline & & $\begin{array}{l}\text { Activation energy } \\
E_{i}\left(\mathrm{kcal} \mathrm{mol}^{-1}\right)\end{array}$ & Weight $W_{i}(\%)$ \\
\hline Frequency factor, $A\left(\mathrm{~s}^{-1}\right)$ & $4.25 E+11$ & 34 & 0.00 \\
\hline \multirow{2}{*}{\multicolumn{2}{|c|}{$\begin{array}{l}\text { Vitrinite conversion index } \\
\mathrm{VCl}=\left[\ln \left(\% R_{\mathrm{o}}\right)-\mathrm{C} 2\right] / \mathrm{C} 1\end{array}$}} & 35 & 0.00 \\
\hline & & 36 & 0.00 \\
\hline \multirow{36}{*}{$\begin{array}{l}\mathrm{C} 1 \\
\mathrm{C} 2\end{array}$} & 1.569 & 37 & 10.90 \\
\hline & -0.693 & 38 & 4.08 \\
\hline & & 39 & 1.08 \\
\hline & & 40 & 0.00 \\
\hline & & 41 & 10.23 \\
\hline & & 42 & 0.12 \\
\hline & & 43 & 0.00 \\
\hline & & 44 & 0.03 \\
\hline & & 45 & 10.02 \\
\hline & & 46 & 21.09 \\
\hline & & 47 & 4.59 \\
\hline & & 48 & 0.50 \\
\hline & & 49 & 8.90 \\
\hline & & 50 & 6.98 \\
\hline & & 51 & 3.81 \\
\hline & & 52 & 0.16 \\
\hline & & 53 & 6.92 \\
\hline & & 54 & 0.30 \\
\hline & & 55 & 4.74 \\
\hline & & 56 & 0.52 \\
\hline & & 57 & 0.00 \\
\hline & & 58 & 4.79 \\
\hline & & 59 & 0.24 \\
\hline & & 60 & 0.00 \\
\hline & & 61 & 0.00 \\
\hline & & 62 & 0.00 \\
\hline & & 63 & 0.00 \\
\hline & & 64 & 0.00 \\
\hline & & 65 & 0.00 \\
\hline & & 66 & 0.00 \\
\hline & & 67 & 0.00 \\
\hline & & 68 & 0.00 \\
\hline & & 69 & 0.00 \\
\hline & & 70 & 0.00 \\
\hline & & 71 & 0.00 \\
\hline & & 72 & 0.00 \\
\hline
\end{tabular}


Table 12: Kinetics data for C29 sterane $20 \mathrm{~S} /(20 \mathrm{~S}+20 \mathrm{R})$ diastereomer ratio.

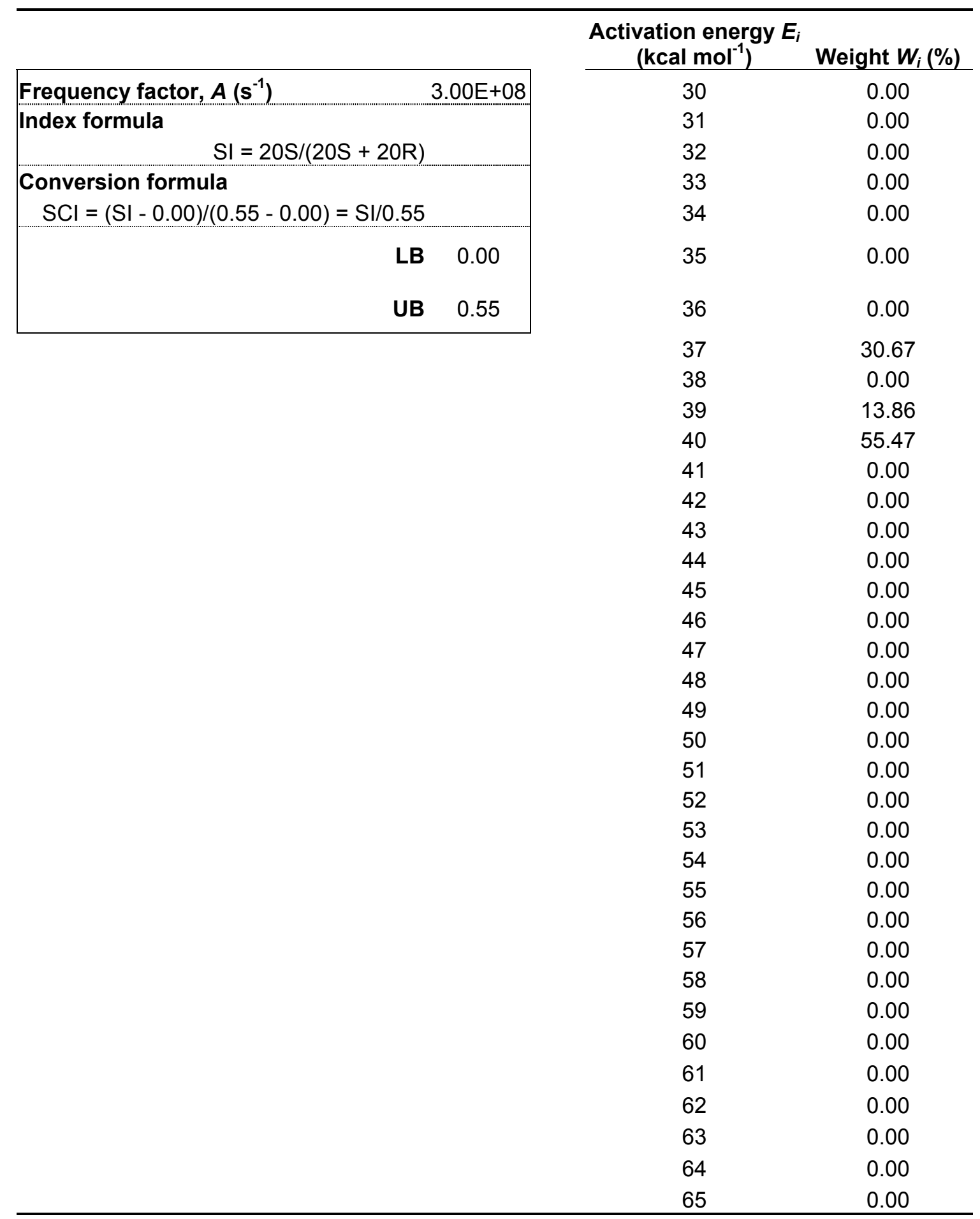


Table 13: Kinetic data for Homohopane 22S/(22S + 22R) diastereomer ratio.

\begin{tabular}{|c|c|c|c|}
\hline & \multirow[b]{2}{*}{$6.92 \mathrm{E}+08$} & \multirow{2}{*}{$\begin{array}{c}\begin{array}{c}\text { Activation energy } E_{i} \\
\left(\mathrm{kcal} \mathrm{mol}^{-1}\right)\end{array} \\
30\end{array}$} & \multirow{2}{*}{$\frac{\text { Weight } \boldsymbol{W}_{\boldsymbol{i}}(\mathbf{\%})}{0.00}$} \\
\hline Frequency factor, $A\left(s^{-1}\right)$ & & & \\
\hline Index formula & & 31 & 0.00 \\
\hline \multicolumn{2}{|l|}{$\mathrm{HI}=22 \mathrm{~S} /(22 \mathrm{~S}+22 \mathrm{R})$} & 32 & 0.00 \\
\hline \multirow{2}{*}{\multicolumn{2}{|c|}{$\begin{array}{l}\text { Conversion formula } \\
\mathrm{HCl}=(\mathrm{HI}-0.00) /(0.58-0.00)=\mathrm{HI} / 0.58\end{array}$}} & 33 & 1.07 \\
\hline & & 34 & 0.77 \\
\hline LB & 0.00 & 35 & 9.11 \\
\hline UB & 0.58 & 36 & 0.00 \\
\hline & & 37 & 65.74 \\
\hline & & 38 & 21.25 \\
\hline & & 39 & 2.06 \\
\hline & & 40 & 0.00 \\
\hline & & 41 & 0.00 \\
\hline & & 42 & 0.00 \\
\hline & & 43 & 0.00 \\
\hline & & 44 & 0.00 \\
\hline & & 45 & 0.00 \\
\hline & & 46 & 0.00 \\
\hline & & 47 & 0.00 \\
\hline & & 48 & 0.00 \\
\hline & & 49 & 0.00 \\
\hline & & 50 & 0.00 \\
\hline & & 51 & 0.00 \\
\hline & & 52 & 0.00 \\
\hline & & 53 & 0.00 \\
\hline & & 54 & 0.00 \\
\hline & & 55 & 0.00 \\
\hline & & 56 & 0.00 \\
\hline & & 57 & 0.00 \\
\hline & & 58 & 0.00 \\
\hline & & 59 & 0.00 \\
\hline & & 60 & 0.00 \\
\hline & & 61 & 0.00 \\
\hline & & 62 & 0.00 \\
\hline & & 63 & 0.00 \\
\hline & & 64 & 0.00 \\
\hline & & 65 & 0.00 \\
\hline
\end{tabular}


Table 14: Kinetic data for Aromatic steroids TA/(TA + MA) ratio.

\begin{tabular}{|c|c|c|c|}
\hline & \multirow[b]{2}{*}{$1.02 E+14$} & \multirow{2}{*}{$\begin{array}{c}\begin{array}{c}\text { Activation energy } E_{i} \\
\left(\mathrm{kcal} \mathrm{mol}^{-1}\right)\end{array} \\
30\end{array}$} & \multirow{2}{*}{$\frac{\text { Weight } \boldsymbol{W}_{\boldsymbol{i}}(\mathbf{\%})}{0.00}$} \\
\hline Frequency factor, $A\left(\mathrm{~s}^{-1}\right)$ & & & \\
\hline Index formula & & 31 & 0.00 \\
\hline \multicolumn{2}{|l|}{$A S I=T A /(T A+M A)$} & 32 & 0.00 \\
\hline \multirow{2}{*}{\multicolumn{2}{|c|}{$\begin{array}{l}\text { Conversion formula } \\
\mathrm{ASCI}=(\mathrm{ASI}-0.00) /(1.00-0.00)=\mathrm{ASCl}\end{array}$}} & 33 & 0.00 \\
\hline & & 34 & 0.00 \\
\hline \multirow{31}{*}{$\begin{array}{l}\text { LB } \\
\text { UB } \\
\end{array}$} & 0.00 & 35 & 0.00 \\
\hline & 1.00 & 36 & 0.00 \\
\hline & & 37 & 0.00 \\
\hline & & 38 & 0.00 \\
\hline & & 39 & 0.00 \\
\hline & & 40 & 0.00 \\
\hline & & 41 & 0.00 \\
\hline & & 42 & 0.00 \\
\hline & & 43 & 8.00 \\
\hline & & 44 & 1.02 \\
\hline & & 45 & 0.00 \\
\hline & & 46 & 90.98 \\
\hline & & 47 & 0.00 \\
\hline & & 48 & 0.00 \\
\hline & & 49 & 0.00 \\
\hline & & 50 & 0.00 \\
\hline & & 51 & 0.00 \\
\hline & & 52 & 0.00 \\
\hline & & 53 & 0.00 \\
\hline & & 54 & 0.00 \\
\hline & & 55 & 0.00 \\
\hline & & 56 & 0.00 \\
\hline & & 57 & 0.00 \\
\hline & & 58 & 0.00 \\
\hline & & 59 & 0.00 \\
\hline & & 60 & 0.00 \\
\hline & & 61 & 0.00 \\
\hline & & 62 & 0.00 \\
\hline & & 63 & 0.00 \\
\hline & & 64 & 0.00 \\
\hline & & 65 & 0.00 \\
\hline
\end{tabular}


Table 15: Kinetic data for Methyl Phenanthrene Index (MPI).

\begin{tabular}{|c|c|c|c|}
\hline & & $\begin{array}{c}\text { Activation energy } E_{i} \\
\left(\mathrm{kcal} \mathrm{mol}^{-1}\right)\end{array}$ & Weight $W_{i}(\%)$ \\
\hline Frequency factor, $A\left(s^{-1}\right)$ & $1.00 E+14$ & 30 & 0.00 \\
\hline Index formula & & 31 & 0.00 \\
\hline $1.5 \times(2-M P+3-M P)$ & & 32 & 0.00 \\
\hline $\overrightarrow{(P+1-M P+9-M P)}$ & & 33 & 0.00 \\
\hline Conversion formula & & 34 & 0.00 \\
\hline \multirow[t]{31}{*}{$\mathrm{MPI} / 4.5$} & & 35 & 0.00 \\
\hline & & 36 & 0.00 \\
\hline & & 37 & 0.00 \\
\hline & & 38 & 0.00 \\
\hline & & 39 & 0.00 \\
\hline & & 40 & 0.00 \\
\hline & & 41 & 0.00 \\
\hline & & 42 & 0.00 \\
\hline & & 43 & 0.30 \\
\hline & & 44 & 0.00 \\
\hline & & 45 & 1.25 \\
\hline & & 46 & 2.55 \\
\hline & & 47 & 0.51 \\
\hline & & 48 & 3.44 \\
\hline & & 49 & 0.00 \\
\hline & & 50 & 2.91 \\
\hline & & 51 & 0.14 \\
\hline & & 52 & 5.35 \\
\hline & & 53 & 0.00 \\
\hline & & 54 & 7.92 \\
\hline & & 55 & 0.00 \\
\hline & & 56 & 9.88 \\
\hline & & 57 & 0.03 \\
\hline & & 58 & 11.48 \\
\hline & & 59 & 1.54 \\
\hline & & 60 & 10.83 \\
\hline & & 61 & 6.27 \\
\hline & & 62 & 5.42 \\
\hline & & 63 & 16.61 \\
\hline & & 64 & 0.85 \\
\hline & & 65 & 12.73 \\
\hline
\end{tabular}


Table 16: Kinetic data for Phenanthrene Alkylation Index (PAI).

\begin{tabular}{|c|c|c|c|}
\hline & & $\begin{array}{c}\text { Activation energy } E_{i} \\
\left(\mathrm{kcal} \mathrm{mol}^{-1}\right)\end{array}$ & Weight $W_{i}(\%)$ \\
\hline Frequency factor, $A\left(s^{-1}\right)$ & $1.00 E+14$ & 30 & 0.00 \\
\hline \multirow{2}{*}{\multicolumn{2}{|c|}{$\begin{array}{l}\text { Index formula } \\
\qquad P A 1=([1-M P]+[2 M P]+[3 M P]+[9 M P]) /[P])\end{array}$}} & 31 & 0.00 \\
\hline & & 32 & 0.00 \\
\hline \multirow{33}{*}{$\begin{array}{r}\text { Conversion formula } \\
\mathrm{PAl} / 2.8 \\
\end{array}$} & & 33 & 0.00 \\
\hline & & 34 & 0.00 \\
\hline & & 35 & 0.00 \\
\hline & & 36 & 0.00 \\
\hline & & 37 & 0.00 \\
\hline & & 38 & 0.00 \\
\hline & & 39 & 0.00 \\
\hline & & 40 & 0.00 \\
\hline & & 41 & 0.00 \\
\hline & & 42 & 0.00 \\
\hline & & 43 & 0.00 \\
\hline & & 44 & 0.00 \\
\hline & & 45 & 2.47 \\
\hline & & 46 & 6.28 \\
\hline & & 47 & 2.52 \\
\hline & & 48 & 12.24 \\
\hline & & 49 & 0.58 \\
\hline & & 50 & 9.98 \\
\hline & & 51 & 2.09 \\
\hline & & 52 & 18.39 \\
\hline & & 53 & 0.00 \\
\hline & & 54 & 31.76 \\
\hline & & 55 & 13.68 \\
\hline & & 56 & 0.00 \\
\hline & & 57 & 0.00 \\
\hline & & 58 & 0.00 \\
\hline & & 59 & 0.00 \\
\hline & & 60 & 0.00 \\
\hline & & 61 & 0.00 \\
\hline & & 62 & 0.00 \\
\hline & & 63 & 0.00 \\
\hline & & 64 & 0.00 \\
\hline & & 65 & 0.00 \\
\hline
\end{tabular}


Table 17: Kinetic data for Methyl-Adamantane Index (MAI) .

\begin{tabular}{|c|c|c|}
\hline & $\begin{array}{c}\text { Activation energy } \\
E_{i}\left(\mathrm{kcal} \mathrm{mol}^{-1}\right)\end{array}$ & $\begin{array}{c}\text { Weight } W_{i} \\
(\%)\end{array}$ \\
\hline Frequency factor, $A\left(\mathrm{~s}^{-1}\right)$ & 30 & 0.00 \\
\hline Index formula & 31 & 0.00 \\
\hline MAI $=100^{*} \quad$ (1-Methyl-Adamantane) & 32 & 0.00 \\
\hline (1-Methyl-Adamantane + 2-Methyl-Adamantane) & 33 & 0.00 \\
\hline Conversion formula & 34 & 0.00 \\
\hline \multirow[t]{31}{*}{$\mathrm{MAI} / 100$} & 35 & 0.00 \\
\hline & 36 & 0.00 \\
\hline & 37 & 0.00 \\
\hline & 38 & 0.00 \\
\hline & 39 & 0.00 \\
\hline & 40 & 0.00 \\
\hline & 41 & 0.00 \\
\hline & 42 & 0.00 \\
\hline & 43 & 0.00 \\
\hline & 44 & 0.00 \\
\hline & 45 & 0.00 \\
\hline & 46 & 0.00 \\
\hline & 47 & 0.00 \\
\hline & 48 & 0.79 \\
\hline & 49 & 0.00 \\
\hline & 50 & 0.00 \\
\hline & 51 & 0.97 \\
\hline & 52 & 7.73 \\
\hline & 53 & 1.65 \\
\hline & 54 & 12.32 \\
\hline & 55 & 0.41 \\
\hline & 56 & 16.10 \\
\hline & 57 & 0.74 \\
\hline & 58 & 15.60 \\
\hline & 59 & 2.31 \\
\hline & 60 & 11.81 \\
\hline & 61 & 8.09 \\
\hline & 62 & 2.60 \\
\hline & 63 & 16.03 \\
\hline & 64 & 2.84 \\
\hline & 65 & 0.00 \\
\hline
\end{tabular}


Table 18: Kinetic data for Methyl-Dimantane Index (MDI).

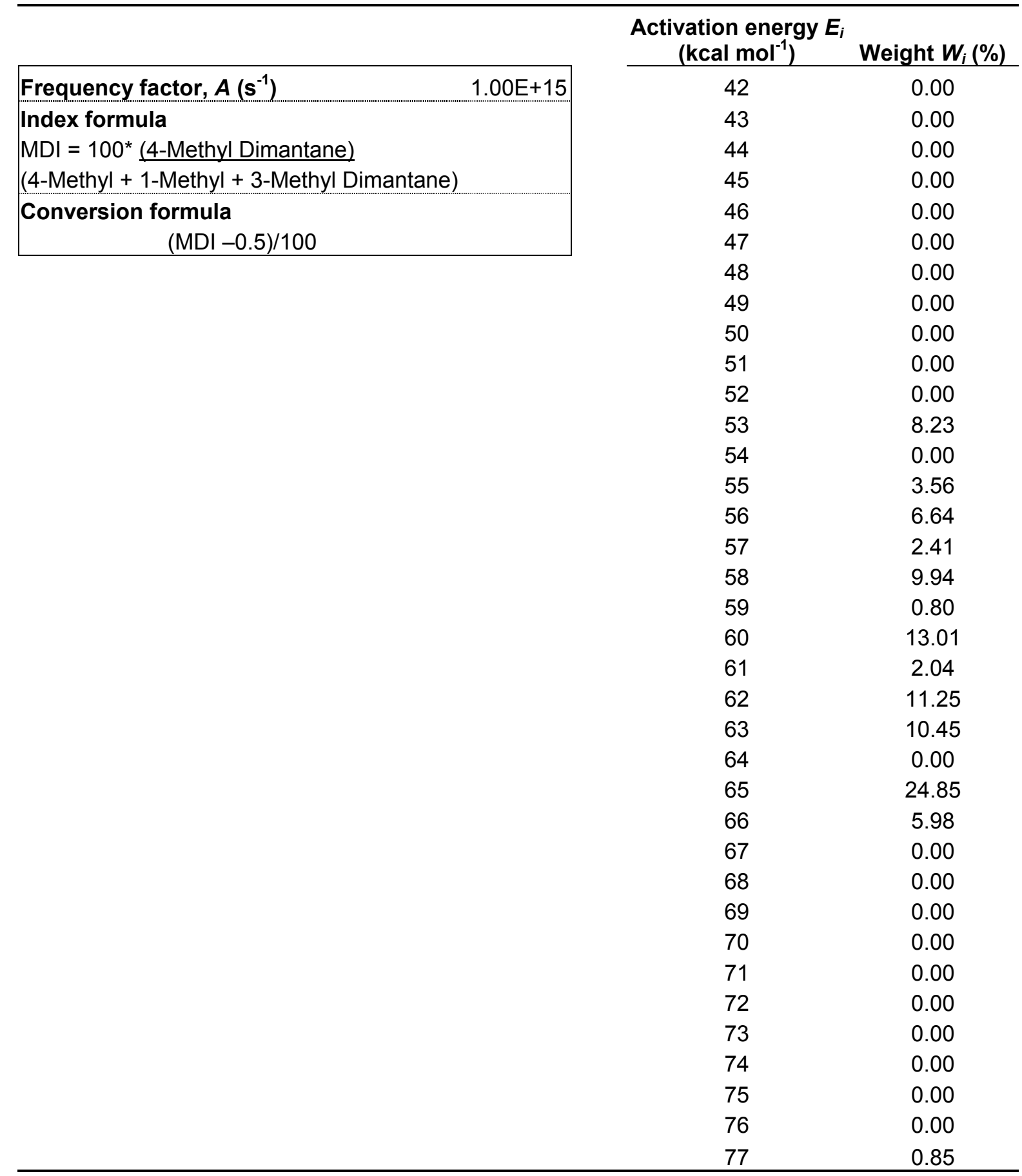


Table 19: Kinetics data for cracking of Green River kerogen and thermal maturation products.

\begin{tabular}{|c|c|}
\hline \multicolumn{2}{|c|}{ Green River Kerogen $\Psi$ C15+ } \\
\hline Frequency factor, $A\left(s^{-1}\right)$ & $1.0 E+14$ \\
\hline $\begin{array}{l}\text { Activation energy } E_{i} \\
\left(\mathrm{kcal} \mathrm{mol}^{-1}\right)\end{array}$ & Weight $W_{i}(\%)$ \\
\hline 44.88 & 0.66 \\
\hline 48.18 & 19.42 \\
\hline 50.96 & 59.84 \\
\hline 53.27 & 19.42 \\
\hline 58.46 & 0.66 \\
\hline \multicolumn{2}{|c|}{ C15+ Cracking } \\
\hline Frequency factor, $A\left(\mathrm{~s}^{-1}\right)$ & $1.0 E+15$ \\
\hline $\begin{array}{l}\text { Activation energy } E_{i} \\
\left(\mathrm{kcal} \mathrm{mol}^{-1}\right)\end{array}$ & Weight $W_{i}(\%)$ \\
\hline 56.98 & 0.66 \\
\hline 58.01 & 19.42 \\
\hline 60.54 & 59.84 \\
\hline 65.69 & 19.42 \\
\hline 73.42 & 0.66 \\
\hline
\end{tabular}

\begin{tabular}{|c|c|}
\hline \multicolumn{2}{|c|}{ Green River Kerogen $\Psi$ C6-C14 } \\
\hline Frequency factor, $A\left(\mathrm{~s}^{-1}\right)$ & $1.0 E+14$ \\
\hline $\begin{array}{c}\text { Activation energy } E_{i} \\
\left(\mathrm{kcal} \mathrm{mol}^{-1}\right)\end{array}$ & Weight $W_{i}(\%)$ \\
\hline 47.97 & 0.66 \\
\hline 41.78 & 19.42 \\
\hline 44.21 & 59.84 \\
\hline 51.93 & 19.42 \\
\hline 58.69 & 0.66 \\
\hline
\end{tabular}

\begin{tabular}{|c|c|}
\hline \multicolumn{2}{|c|}{ C6-C14 Cracking } \\
\hline Frequency factor, $A\left(\mathrm{~s}^{-1}\right)$ & $1.0 E+15$ \\
\hline $\begin{array}{c}\text { Activation energy } E_{i} \\
\left(\mathrm{kcal} \mathrm{mol}^{-1}\right)\end{array}$ & Weight $W_{i}(\%)$ \\
\hline 56.42 & 0.66 \\
\hline 59.21 & 19.42 \\
\hline 64.00 & 59.84 \\
\hline 66.79 & 19.42 \\
\hline 67.58 & 0.66 \\
\hline
\end{tabular}

C3-C5 Cracking

\begin{tabular}{|c|c|c|c|}
\hline Frequency factor, $A\left(\mathrm{~s}^{-1}\right)$ & $1.0 E+15$ & Frequency factor, $A\left(s^{-1}\right)$ & $1.0 E+15$ \\
\hline $\begin{array}{l}\text { Activation energy } E_{i} \\
\left(\mathrm{kcal} \mathrm{mol}^{-1}\right)\end{array}$ & Weight $W_{i}(\%)$ & $\begin{array}{l}\text { Activation energy } E_{i} \\
\left.(\mathrm{kcal} \mathrm{mol})^{-1}\right)\end{array}$ & Weight $W_{i}(\%)$ \\
\hline 63.05 & 0.66 & 63.02 & 0.66 \\
\hline 64.03 & 19.42 & 64.01 & 19.42 \\
\hline 65.00 & 59.84 & 65.00 & 59.84 \\
\hline 65.98 & 19.42 & 66.99 & 19.42 \\
\hline 66.95 & 0.66 & 67.98 & 0.66 \\
\hline
\end{tabular}


Table 20: Kinetics data for cracking of North Sea Kerogen and thermal maturation products.

\begin{tabular}{|c|c|c|c|}
\hline \multicolumn{2}{|c|}{ North Sea Kerogen $\Psi$ C15+ } & \multicolumn{2}{|c|}{ North Sea Kerogen $\Psi$ C6-C14 } \\
\hline Frequency factor, $A\left(\mathrm{~s}^{-1}\right)$ & $1.0 E+14$ & Frequency factor, $A\left(\mathrm{~s}^{-1}\right)$ & $1.0 E+14$ \\
\hline $\begin{array}{c}\text { Activation energy } E_{i} \\
\left(\mathrm{kcal} \mathrm{mol}^{-1}\right)\end{array}$ & Weight $W_{i}(\%)$ & $\begin{array}{c}\text { Activation energy } E_{i} \\
\left(\mathrm{kcal} \mathrm{mol}^{-1}\right)\end{array}$ & Weight $W_{i}(\%)$ \\
\hline 41.00 & 0.66 & 47.32 & 0.66 \\
\hline 45.50 & 19.42 & 49.66 & 19.42 \\
\hline 50.00 & 59.84 & 52.00 & 59.84 \\
\hline 54.54 & 19.42 & 54.34 & 19.42 \\
\hline 59.00 & 0.66 & 56.68 & 0.66 \\
\hline \multicolumn{2}{|c|}{ C15+ Cracking } & \multicolumn{2}{|c|}{ C6-C14 Cracking } \\
\hline Frequency factor, $A\left(\mathrm{~s}^{-1}\right)$ & $1.0 E+15$ & Frequency factor, $A\left(\mathrm{~s}^{-1}\right)$ & $1.0 E+15$ \\
\hline $\begin{array}{c}\text { Activation energy } E_{i} \\
\left(\mathrm{kcal} \mathrm{mol}^{-1}\right)\end{array}$ & Weight $W_{i}(\%)$ & $\begin{array}{c}\text { Activation energy } E_{i} \\
\left(\mathrm{kcal} \mathrm{mol}^{-1}\right)\end{array}$ & Weight $W_{i}(\%)$ \\
\hline 53.69 & 0.66 & 58.24 & 0.66 \\
\hline 56.35 & 19.42 & 61.12 & 19.42 \\
\hline 59.00 & 59.84 & 64.00 & 59.84 \\
\hline 61.66 & 19.42 & 66.88 & 19.42 \\
\hline 64.31 & 0.66 & 69.76 & 0.66 \\
\hline \multicolumn{2}{|c|}{ C3-C5 Cracking } & \multicolumn{2}{|c|}{ C2 Cracking } \\
\hline Frequency factor, $A\left(\mathrm{~s}^{-1}\right)$ & $1.0 E+15$ & Frequency factor, $A\left(\mathrm{~s}^{-1}\right)$ & $1.0 E+15$ \\
\hline $\begin{array}{c}\text { Activation energy } E_{i} \\
\left(\mathrm{kcal} \mathrm{mol}^{-1}\right)\end{array}$ & Weight $W_{i}(\%)$ & $\begin{array}{c}\text { Activation energy } E_{i} \\
\left(\mathrm{kcal} \mathrm{mol}^{-1}\right)\end{array}$ & Weight $W_{i}(\%)$ \\
\hline 63.05 & 0.66 & 62.98 & 0.66 \\
\hline 64.03 & 19.42 & 64.99 & 19.42 \\
\hline 65.00 & 59.84 & 67.00 & 59.84 \\
\hline 65.98 & 19.42 & 69.01 & 19.42 \\
\hline 66.95 & 0.66 & 71.02 & 0.66 \\
\hline
\end{tabular}


Table 21: Kinetics data for cracking of Cameo coal kerogen and thermal maturity products.

\begin{tabular}{|c|c|c|c|}
\hline \multicolumn{2}{|c|}{ Cameo Coal Kerogen $\Psi$ C15+ } & \multicolumn{2}{|c|}{ Cameo Coal Kerogen $\Psi$ C6-C14 } \\
\hline Frequency factor, $A\left(\mathrm{~s}^{-1}\right)$ & $1.00 E+14$ & Frequency factor, $A\left(s^{-1}\right)$ & $2.00 \mathrm{E}+14$ \\
\hline $\begin{array}{c}\text { Activation energy } E_{i} \\
\left(\mathrm{kcal} \mathrm{mol}^{-1}\right)\end{array}$ & Weight $W_{i}(\%)$ & $\begin{array}{c}\text { Activation energy } E_{i} \\
\left(\mathrm{kcal} \mathrm{mol}^{-1}\right)\end{array}$ & Weight $W_{i}(\%)$ \\
\hline 47.00 & 4.59 & 43.00 & 0.33 \\
\hline 50.00 & 1.92 & 47.00 & 5.15 \\
\hline 52.00 & 5.03 & 50.00 & 0.84 \\
\hline 54.00 & 26.35 & 52.00 & 13.29 \\
\hline 55.00 & 23.69 & 54.00 & 28.10 \\
\hline 57.00 & 20.48 & 55.00 & 23.53 \\
\hline 62.00 & 14.95 & 57.00 & 15.76 \\
\hline 63.00 & 1.97 & 62.00 & 10.47 \\
\hline 67.00 & 1.02 & 67.00 & 2.53 \\
\hline \multicolumn{2}{|c|}{ Cameo Coal Kerogen $\Psi$ C3-C5 } & \multicolumn{2}{|c|}{ C15+ Cracking } \\
\hline Frequency factor, $A\left(\mathrm{~s}^{-1}\right)$ & $5.00 \mathrm{E}+14$ & Frequency factor, $A\left(s^{-1}\right)$ & $1.00 E+15$ \\
\hline $\begin{array}{c}\text { Activation energy } E_{i} \\
\left(\mathrm{kcal} \mathrm{mol}^{-1}\right)\end{array}$ & Weight $W_{i}(\%)$ & $\begin{array}{c}\text { Activation energy } E_{i} \\
\left(\mathrm{kcal} \mathrm{mol}^{-1}\right)\end{array}$ & Weight $W_{i}(\%)$ \\
\hline 47.00 & 19.97 & 53.69 & 0.66 \\
\hline 52.00 & 25.10 & 56.35 & 19.42 \\
\hline 53.00 & 3.49 & 59.00 & 59.84 \\
\hline 54.00 & 35.24 & 61.66 & 19.42 \\
\hline 56.00 & 11.34 & 64.31 & 0.66 \\
\hline 57.00 & 2.57 & & \\
\hline 60.00 & 0.49 & & \\
\hline 61.00 & 1.80 & & \\
\hline \multicolumn{2}{|c|}{ C6-C14 Cracking } & \multicolumn{2}{|c|}{ C3-C5 Cracking } \\
\hline Frequency factor, $A\left(\mathrm{~s}^{-1}\right)$ & $1.00 \mathrm{E}+15$ & Frequency factor, $A\left(\mathrm{~s}^{-1}\right)$ & $1.00 \mathrm{E}+15$ \\
\hline $\begin{array}{c}\text { Activation energy } E_{i} \\
\left(\mathrm{kcal} \mathrm{mol}^{-1}\right)\end{array}$ & Weight $W_{i}(\%)$ & $\begin{array}{c}\text { Activation energy } E_{i} \\
\left(\mathrm{kcal} \mathrm{mol}^{-1}\right)\end{array}$ & Weight $W_{i}(\%)$ \\
\hline 58.24 & 0.66 & 63.05 & 0.66 \\
\hline 61.12 & 19.42 & 64.03 & 19.42 \\
\hline 64.00 & 59.84 & 65.00 & 59.84 \\
\hline 66.88 & 19.42 & 65.98 & 19.42 \\
\hline 69.76 & 0.66 & 66.95 & 0.66 \\
\hline \multicolumn{2}{|c|}{ C2 Cracking } & & \\
\hline Frequency factor, $A\left(s^{-1}\right)$ & $1.00 \mathrm{E}+15$ & & \\
\hline $\begin{array}{l}\text { Activation energy } E_{i} \\
\left(\mathrm{kcal} \mathrm{mol}^{-1}\right)\end{array}$ & Weight $W_{i}(\%)$ & & \\
\hline 62.98 & 0.66 & & \\
\hline 64.99 & 19.42 & & \\
\hline 67.00 & 59.84 & & \\
\hline 69.01 & 19.42 & & \\
\hline 71.02 & 0.66 & & \\
\hline
\end{tabular}


Table 25: Kinetics data for cracking of Redwater kerogen and thermal maturity products.

\begin{tabular}{|c|c|c|c|}
\hline \multicolumn{2}{|c|}{ Redwater Kerogen $\Psi$ C15+ } & \multicolumn{2}{|c|}{ Redwater Kerogen $\Psi$ C6-C14 } \\
\hline Frequency factor, $A\left(\mathrm{~s}^{-1}\right)$ & $1.0 \mathrm{E}+14$ & Frequency factor, $A\left(\mathrm{~s}^{-1}\right)$ & $2.0 E+14$ \\
\hline $\begin{array}{c}\text { Activation energy } E_{i} \\
\left(\mathrm{kcal} \mathrm{mol}^{-1}\right)\end{array}$ & Weight $W_{i}(\%)$ & $\begin{array}{c}\text { Activation energy } E_{i} \\
\left(\mathrm{kcal} \mathrm{mol}^{-1}\right)\end{array}$ & Weight $W_{i}(\%)$ \\
\hline 50.00 & 32.16 & 51.00 & 39.13 \\
\hline 51.00 & 17.72 & 52.00 & 0.12 \\
\hline 53.00 & 8.06 & 54.00 & 4.38 \\
\hline 54.00 & 4.72 & 55.00 & 10.99 \\
\hline 58.00 & 26.38 & 59.00 & 32.67 \\
\hline \multirow[t]{3}{*}{59.00} & 10.96 & 60.00 & 5.39 \\
\hline & & 63.00 & 6.62 \\
\hline & & 64.00 & 0.7 \\
\hline \multicolumn{2}{|c|}{ Redwater Kerogen $\Psi$ C3-C5 } & \multicolumn{2}{|c|}{ C15+Cracking } \\
\hline Frequency factor, $A\left(s^{-1}\right)$ & $5.0 \mathrm{E}+14$ & Frequency factor, $A\left(\mathrm{~s}^{-1}\right)$ & $1.0 \mathrm{E}+15$ \\
\hline $\begin{array}{c}\text { Activation energy } E_{i} \\
\left(\mathrm{kcal} \mathrm{mol}^{-1}\right)\end{array}$ & Weight $W_{i}(\%)$ & $\begin{array}{c}\text { Activation energy } E_{i} \\
\left(\mathrm{kcal} \mathrm{mol}^{-1}\right)\end{array}$ & Weight $W_{i}(\%)$ \\
\hline 52.00 & 18.18 & 53.69 & 0.66 \\
\hline 53.00 & 0.71 & 56.35 & 19.42 \\
\hline 55.00 & 10.36 & 59.00 & 59.84 \\
\hline 56.00 & 9.57 & 61.66 & 19.42 \\
\hline 60.00 & 11.75 & 64.31 & 0.66 \\
\hline 61.00 & 25.82 & & \\
\hline 65.00 & 23.61 & & \\
\hline \multicolumn{2}{|c|}{ C6-C14 Cracking } & \multicolumn{2}{|c|}{ C3-C5 Cracking } \\
\hline Frequency factor, $A\left(s^{-1}\right)$ & $1.0 \mathrm{E}+15$ & Frequency factor, $A\left(\mathrm{~s}^{-1}\right)$ & $1.0 \mathrm{E}+15$ \\
\hline $\begin{array}{c}\text { Activation energy } E_{i} \\
\left(\mathrm{kcal} \mathrm{mol}^{-1}\right)\end{array}$ & Weight $W_{i}(\%)$ & $\begin{array}{c}\text { Activation energy } E_{i} \\
\left(\mathrm{kcal} \mathrm{mol}^{-1}\right)\end{array}$ & Weight $W_{i}(\%)$ \\
\hline 58.24 & 0.66 & 63.05 & 0.66 \\
\hline 61.12 & 19.42 & 64.03 & 19.42 \\
\hline 64.00 & 59.84 & 65.00 & 59.84 \\
\hline 66.88 & 19.42 & 65.98 & 19.42 \\
\hline 69.76 & 0.66 & 66.95 & 0.66 \\
\hline \multicolumn{2}{|c|}{ C2 Cracking } & & \\
\hline Frequency factor, $A\left(s^{-1}\right)$ & $1.0 E+15$ & & \\
\hline $\begin{array}{c}\text { Activation energy } E_{i} \\
\left(\mathrm{kcal} \mathrm{mol}^{-1}\right)\end{array}$ & Weight $W_{i}(\%)$ & & \\
\hline 62.98 & 0.66 & & \\
\hline 64.99 & 19.42 & & \\
\hline 67.00 & 59.84 & & \\
\hline 69.01 & 19.42 & & \\
\hline 71.02 & 0.66 & & \\
\hline
\end{tabular}


Table 26: Kinetics data for cracking of Cape Range kerogen and thermal maturity products.

\begin{tabular}{|c|c|}
\hline \multicolumn{2}{|c|}{ Cape Range Kerogen $\Psi$ C15+ } \\
\hline Frequency factor, $A\left(\mathrm{~s}^{-1}\right)$ & $1.0 \mathrm{E}+14$ \\
\hline $\begin{array}{c}\text { Activation energy } E_{i} \\
\left(\mathrm{kcal} \mathrm{mol}^{-1}\right)\end{array}$ & Weight $W_{i}(\%)$ \\
\hline 40.00 & 3.60 \\
\hline 53.00 & 44.43 \\
\hline 54.00 & 21.36 \\
\hline 55.00 & 27.09 \\
\hline 56.00 & 2.80 \\
\hline 64.00 & 0.72 \\
\hline
\end{tabular}

Cape Range Kerogen $\Psi$ C3-C5

Frequency factor, $\boldsymbol{A}\left(\mathrm{s}^{-1}\right) \quad 5.0 \mathrm{E}+14$

Activation energy $E_{i}$

\begin{tabular}{cc}
$\left(\mathbf{k c a l ~ m o l}^{-1}\right)$ & Weight $\boldsymbol{W}_{\boldsymbol{i}}(\mathbf{\%})$ \\
\hline 46.00 & 3.40 \\
52.00 & 3.38 \\
53.00 & 0.64 \\
55.00 & 11.60 \\
56.00 & 14.56 \\
58.00 & 35.71 \\
59.00 & 24.16 \\
70.00 & 6.55 \\
\hline
\end{tabular}

C6-C14 Cracking

Frequency factor, $\boldsymbol{A}\left(\mathbf{s}^{-1}\right) \quad 1.0 \mathrm{E}+15$

Activation energy $E_{i}$ $\left(\mathrm{kcal} \mathrm{mol}^{-1}\right)$

Weight $W_{i}(\%)$

\begin{tabular}{|c|c|c|c|}
\hline$\left(\mathrm{kcal} \mathrm{mol}^{-1}\right)$ & Weight $W_{i}(\%)$ & $\left(\mathrm{kcal} \mathrm{mol}^{-1}\right)$ & Weight $W_{i}(\%)$ \\
\hline 58.24 & 0.66 & 63.05 & 0.66 \\
\hline 61.12 & 19.42 & 64.03 & 19.42 \\
\hline 64.00 & 59.84 & 65.00 & 59.84 \\
\hline 66.88 & 19.42 & 65.98 & 19.42 \\
\hline 69.76 & 0.66 & 66.95 & 0.66 \\
\hline \multicolumn{4}{|c|}{ C2 Cracking } \\
\hline Frequency factor, $A\left(\mathrm{~s}^{-1}\right)$ & $1.0 \mathrm{E}+15$ & & \\
\hline $\begin{array}{l}\text { Activation energy } E_{i} \\
\left(\mathrm{kcal} \mathrm{mol}^{-1}\right)\end{array}$ & Weight $W_{i}(\%)$ & & \\
\hline 62.98 & 0.66 & & \\
\hline 64.99 & 19.42 & & \\
\hline 67.00 & 59.84 & & \\
\hline 69.01 & 19.42 & & \\
\hline 71.02 & 0.66 & & \\
\hline
\end{tabular}

C3-C5 Cracking

Frequency factor, $\boldsymbol{A}\left(\mathbf{s}^{-1}\right) \quad 1.0 \mathrm{E}+15$

Activation energy $E_{i}$ 
Table 27: Kinetics data for cracking of FN2 kerogen and thermal maturity products.

\begin{tabular}{|c|c|c|c|}
\hline \multicolumn{2}{|c|}{ FN2 Kerogen $\Psi$ C15+ } & \multicolumn{2}{|c|}{ FN2 Kerogen $\Psi$ C6-C14 } \\
\hline Frequency factor, $A\left(s^{-1}\right)$ & $1.0 E+14$ & Frequency factor, $A\left(s^{-1}\right)$ & $2.0 E+14$ \\
\hline $\begin{array}{l}\text { Activation energy } E_{i} \\
\left(\mathrm{kcal} \mathrm{mol}^{-1}\right)\end{array}$ & Weight $W_{i}(\%)$ & $\begin{array}{l}\text { Activation energy } E_{i} \\
\left(\mathrm{kcal} \mathrm{mol}^{-1}\right)\end{array}$ & Weight $W_{i}(\%)$ \\
\hline 40.00 & 4.39 & 49.00 & 5.51 \\
\hline 49.00 & 6.29 & 50.00 & 3.90 \\
\hline 50.00 & 6.08 & 51.00 & 0.93 \\
\hline 53.00 & 55.92 & 53.00 & 13.68 \\
\hline 54.00 & 19.29 & 54.00 & 46.09 \\
\hline 55.00 & 0.76 & 56.00 & 27.64 \\
\hline 56.00 & 6.15 & 57.00 & 0.80 \\
\hline \multirow[t]{2}{*}{64.00} & 1.12 & 60.00 & 0.16 \\
\hline & & 67.00 & 1.29 \\
\hline \multicolumn{2}{|c|}{ FN2 Kerogen $\Psi$ C3-C5 } & \multicolumn{2}{|c|}{ C15+ Cracking } \\
\hline Frequency factor, $A\left(s^{-1}\right)$ & $5.0 E+14$ & Frequency factor, $A\left(s^{-1}\right)$ & $1.0 E+15$ \\
\hline $\begin{array}{l}\text { Activation energy } E_{i} \\
\left(\mathrm{kcal} \mathrm{mol}^{-1}\right)\end{array}$ & Weight $W_{i}(\%)$ & $\begin{array}{l}\text { Activation energy } E_{i} \\
\left(\mathrm{kcal} \mathrm{mol}^{-1}\right)\end{array}$ & Weight $W_{i}(\%)$ \\
\hline 50.00 & 7.39 & 53.69 & 0.66 \\
\hline 54.00 & 21.86 & 56.35 & 19.42 \\
\hline 55.00 & 16.41 & 59.00 & 59.84 \\
\hline 57.00 & 11.43 & 61.66 & 19.42 \\
\hline 58.00 & 17.19 & 64.31 & 0.66 \\
\hline 60.00 & 6.36 & & \\
\hline 61.00 & 8.78 & & \\
\hline 68.00 & 6.01 & & \\
\hline 69.00 & 4.57 & & \\
\hline \multicolumn{2}{|c|}{ C6-C14 Cracking } & \multicolumn{2}{|c|}{ C3-C5 Cracking } \\
\hline Frequency factor, $A\left(\mathrm{~s}^{-1}\right)$ & $1.0 \mathrm{E}+15$ & Frequency factor, $A\left(s^{-1}\right)$ & $1.0 \mathrm{E}+15$ \\
\hline $\begin{array}{c}\text { Activation energy } E_{i} \\
\left(\mathrm{kcal} \mathrm{mol}^{-1}\right)\end{array}$ & Weight $W_{i}(\%)$ & $\begin{array}{c}\text { Activation energy } E_{i} \\
\left(\mathrm{kcal} \mathrm{mol}^{-1}\right)\end{array}$ & Weight $W_{i}(\%)$ \\
\hline 58.24 & 0.66 & 63.05 & 0.66 \\
\hline 61.12 & 19.42 & 64.03 & 19.42 \\
\hline 64.00 & 59.84 & 65.00 & 59.84 \\
\hline 66.88 & 19.42 & 65.98 & 19.42 \\
\hline 69.76 & 0.66 & 66.95 & 0.66 \\
\hline \multicolumn{2}{|c|}{ C2 Cracking } & & \\
\hline Frequency factor, $A\left(\mathrm{~s}^{-1}\right)$ & $1.0 \mathrm{E}+15$ & & \\
\hline $\begin{array}{c}\text { Activation energy } E_{i} \\
\left(\mathrm{kcal} \mathrm{mol}^{-1}\right)\end{array}$ & Weight $W_{i}(\%)$ & & \\
\hline 62.98 & 0.66 & & \\
\hline 64.99 & 19.42 & & \\
\hline 67.00 & 59.84 & & \\
\hline 69.01 & 19.42 & & \\
\hline 71.02 & 0.66 & & \\
\hline
\end{tabular}


Table 28: Kinetics data for cracking of B648 kerogen and thermal maturity products.

\begin{tabular}{|c|c|c|c|}
\hline \multicolumn{2}{|c|}{ B648 Kerogen $\Psi$ C15+ } & \multicolumn{2}{|c|}{ B648 Kerogen $\Psi$ C6-C14 } \\
\hline Frequency factor, $A\left(s^{-1}\right)$ & $1.0 E+14$ & Frequency factor, $A\left(\mathrm{~s}^{-1}\right)$ & $2.0 E+14$ \\
\hline $\begin{array}{c}\text { Activation energy } E_{i} \\
\left(\mathrm{kcal} \mathrm{mol}^{-1}\right)\end{array}$ & Weight $W_{i}(\%)$ & $\begin{array}{c}\text { Activation energy } E_{i} \\
\left(\mathrm{kcal} \mathrm{mol}^{-1}\right)\end{array}$ & Weight $W_{i}(\%)$ \\
\hline 52.00 & 7.95 & 51.00 & 3.67 \\
\hline 53.00 & 13.34 & 52.00 & 14.36 \\
\hline \multirow[t]{4}{*}{54.00} & 78.71 & 55.00 & 53.24 \\
\hline & & 56.00 & 27.33 \\
\hline & & 57.00 & 0.76 \\
\hline & & 67.00 & 0.64 \\
\hline \multicolumn{2}{|c|}{ B648 Kerogen $\Psi$ C3-C5 } & \multicolumn{2}{|c|}{ C15+ Cracking } \\
\hline Frequency factor, $A\left(s^{-1}\right)$ & $5.0 E+14$ & Frequency factor, $A\left(\mathrm{~s}^{-1}\right)$ & $1.0 E+15$ \\
\hline $\begin{array}{l}\text { Activation energy } E_{i} \\
\left(\mathrm{kcal} \mathrm{mol}^{-1}\right)\end{array}$ & Weight $W_{i}(\%)$ & $\begin{array}{l}\text { Activation energy } E_{i} \\
\left(\mathrm{kcal} \mathrm{mol}^{-1}\right)\end{array}$ & Weight $W_{i}(\%)$ \\
\hline 52.00 & 3.33 & 53.69 & 0.66 \\
\hline 53.00 & 11.43 & 56.35 & 19.42 \\
\hline 56.00 & 22.56 & 59.00 & 59.84 \\
\hline 57.00 & 29.60 & 61.66 & 19.42 \\
\hline 59.00 & 30.66 & 64.31 & 0.66 \\
\hline 60.00 & 0.62 & & \\
\hline 68.00 & 1.22 & & \\
\hline 69.00 & 0.58 & & \\
\hline \multicolumn{2}{|c|}{ C6-C14 Cracking } & \multicolumn{2}{|c|}{ C3-C5 Cracking } \\
\hline Frequency factor, $A\left(s^{-1}\right)$ & $1.0 \mathrm{E}+15$ & Frequency factor, $A\left(\mathrm{~s}^{-1}\right)$ & $1.0 \mathrm{E}+15$ \\
\hline $\begin{array}{c}\text { Activation energy } E_{i} \\
\left(\mathrm{kcal} \mathrm{mol}^{-1}\right)\end{array}$ & Weight $W_{i}(\%)$ & $\begin{array}{c}\text { Activation energy } E_{i} \\
\left(\mathrm{kcal} \mathrm{mol}^{-1}\right)\end{array}$ & Weight $W_{i}(\%)$ \\
\hline 58.24 & 0.66 & 63.05 & 0.66 \\
\hline 61.12 & 19.42 & 64.03 & 19.42 \\
\hline 64.00 & 59.84 & 65.00 & 59.84 \\
\hline 66.88 & 19.42 & 65.98 & 19.42 \\
\hline 69.76 & 0.66 & 66.95 & 0.66 \\
\hline \multicolumn{2}{|c|}{ C2 Cracking } & & \\
\hline Frequency factor, $A\left(s^{-1}\right)$ & $1.0 E+15$ & & \\
\hline $\begin{array}{c}\text { Activation energy } E_{i} \\
\left(\mathrm{kcal} \mathrm{mol}^{-1}\right)\end{array}$ & Weight $W_{i}(\%)$ & & \\
\hline 62.98 & 0.66 & & \\
\hline 64.99 & 19.42 & & \\
\hline 67.00 & 59.84 & & \\
\hline 69.01 & 19.42 & & \\
\hline 71.02 & 0.66 & & \\
\hline
\end{tabular}


Table 29: Theoretical reaction network for thermal maturation of kerogen B648.

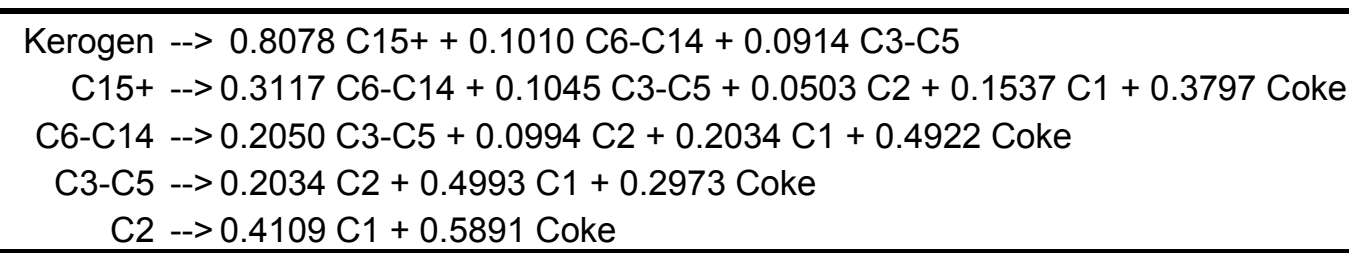

Table 30: Theoretical reaction network for thermal maturation of Cape Range kerogen.

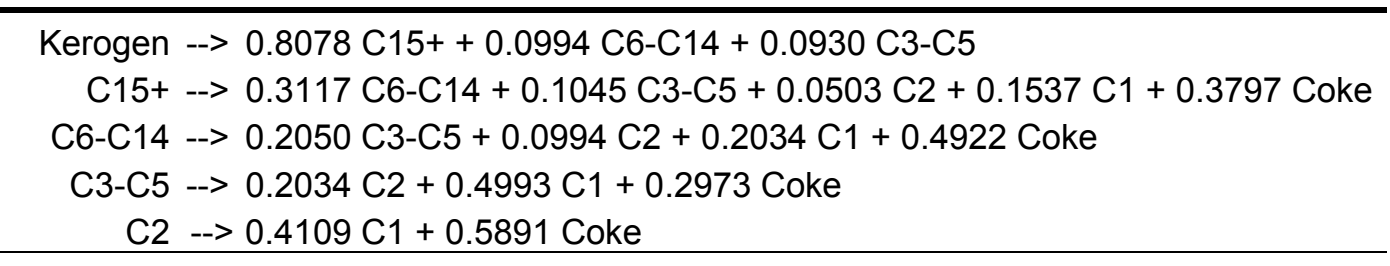

Table 31: Theoretical reaction network for thermal maturation of kerogen FN2.

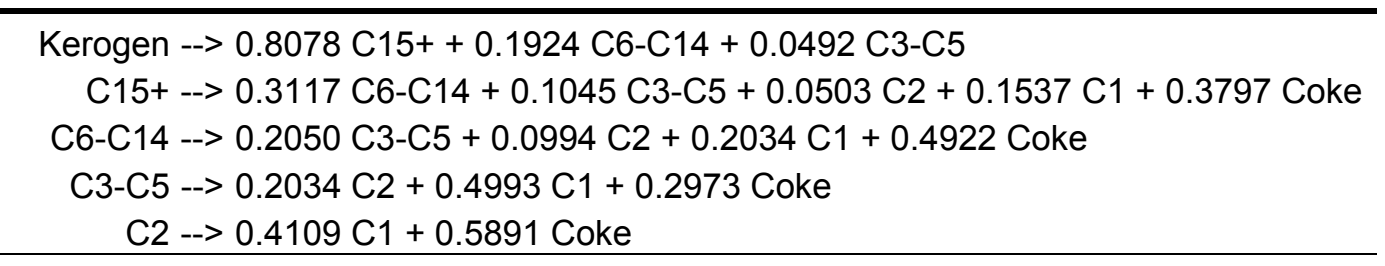

Table 32: Theoretical reaction network for thermal maturation of Green River kerogen.

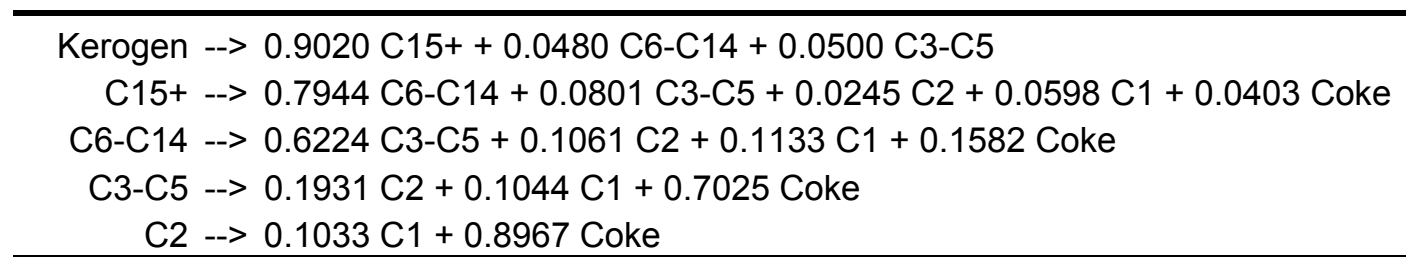

Table 33: Theoretical reaction network for thermal maturation of North Sea kerogen.

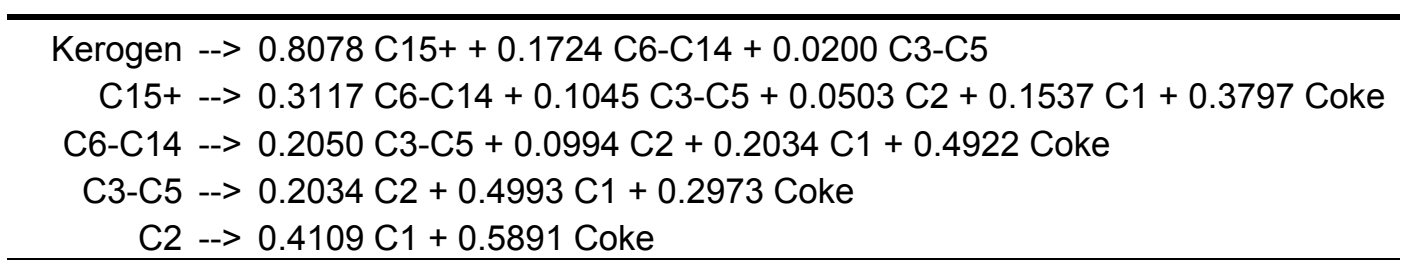


Table 34: Theoretical reaction network for thermal maturation of Redwater kerogen.

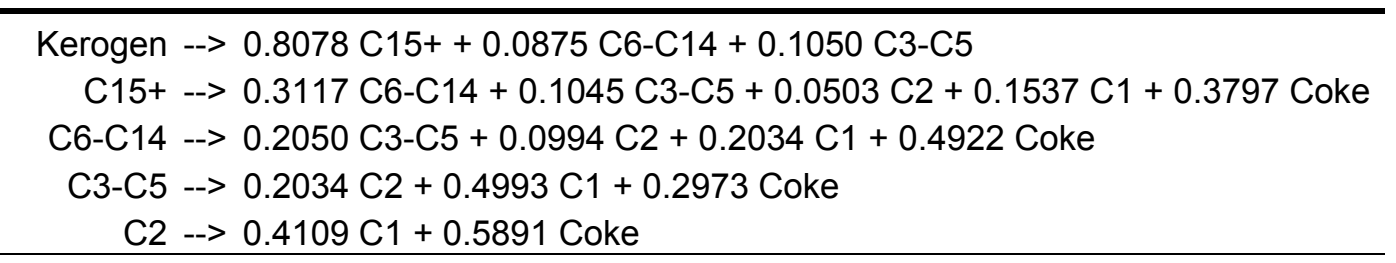

Table 35: Theoretical reaction network for thermal maturation of Cameo coal.

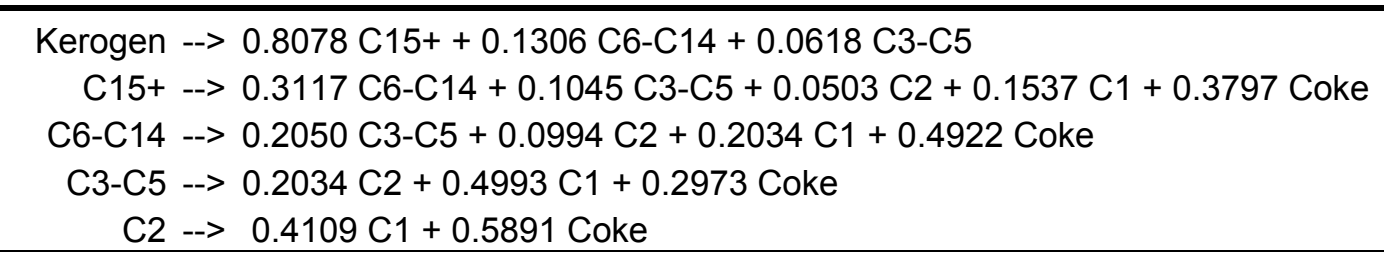


Appendix 2: Experimental and theoretical data plots 


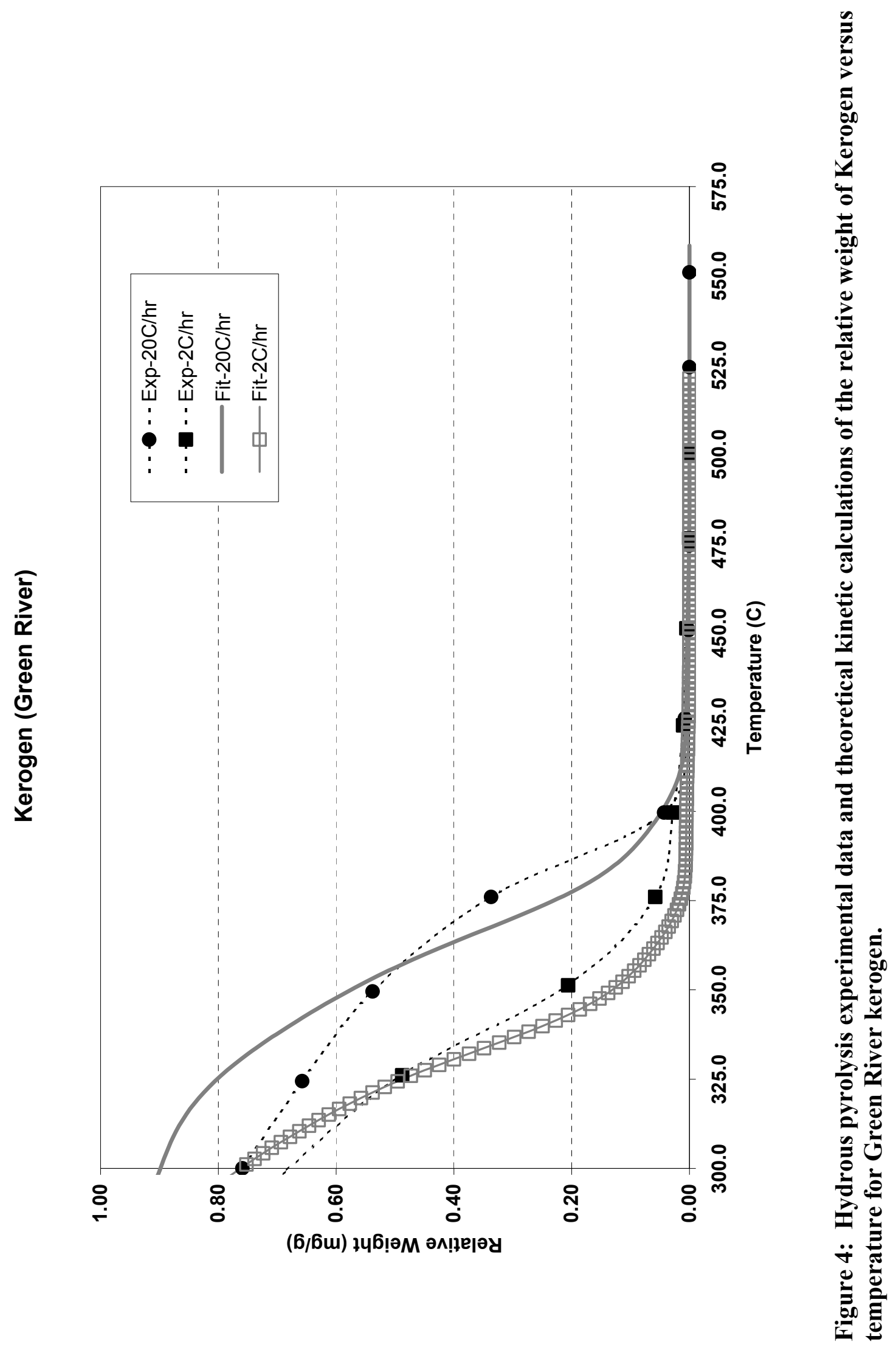




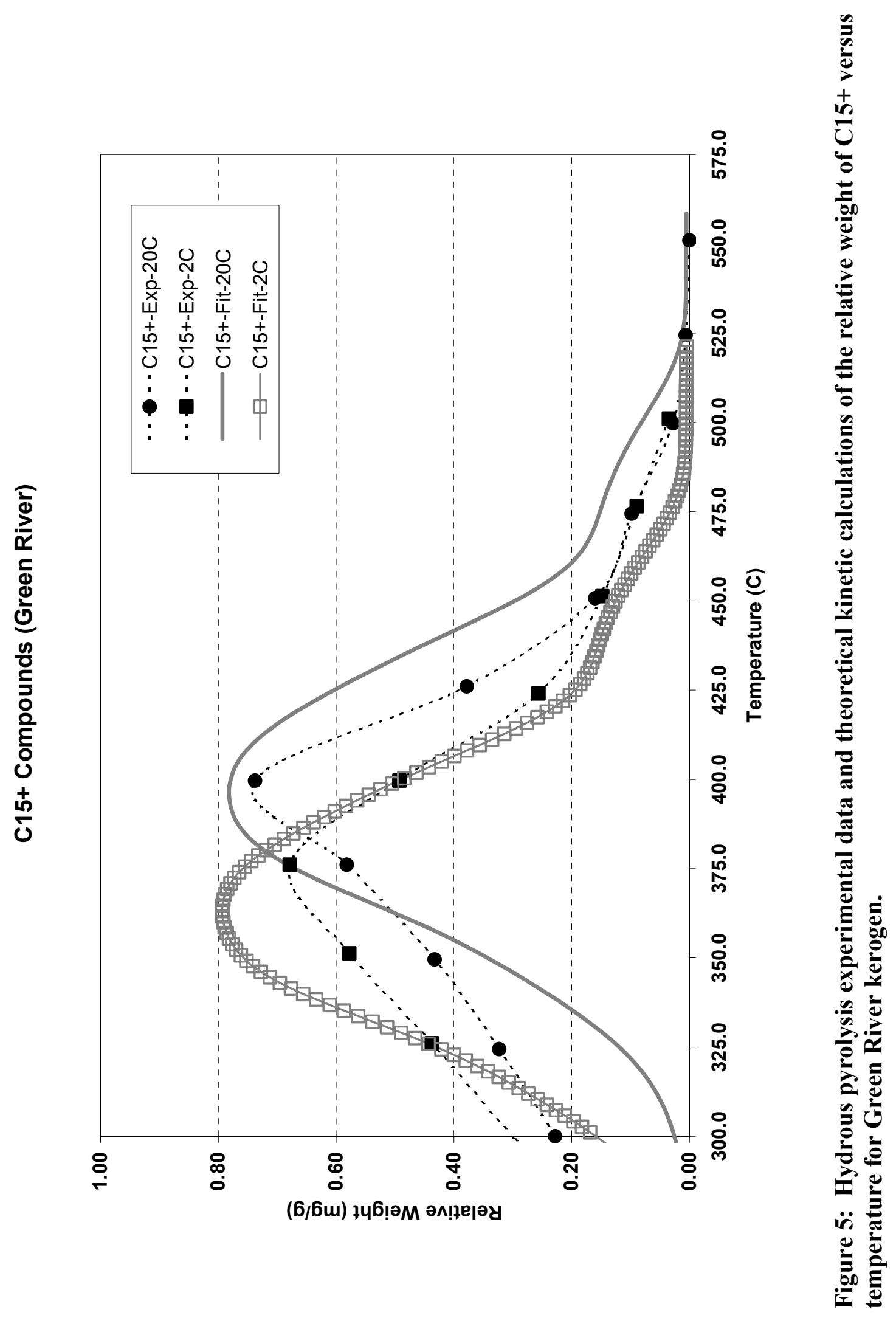



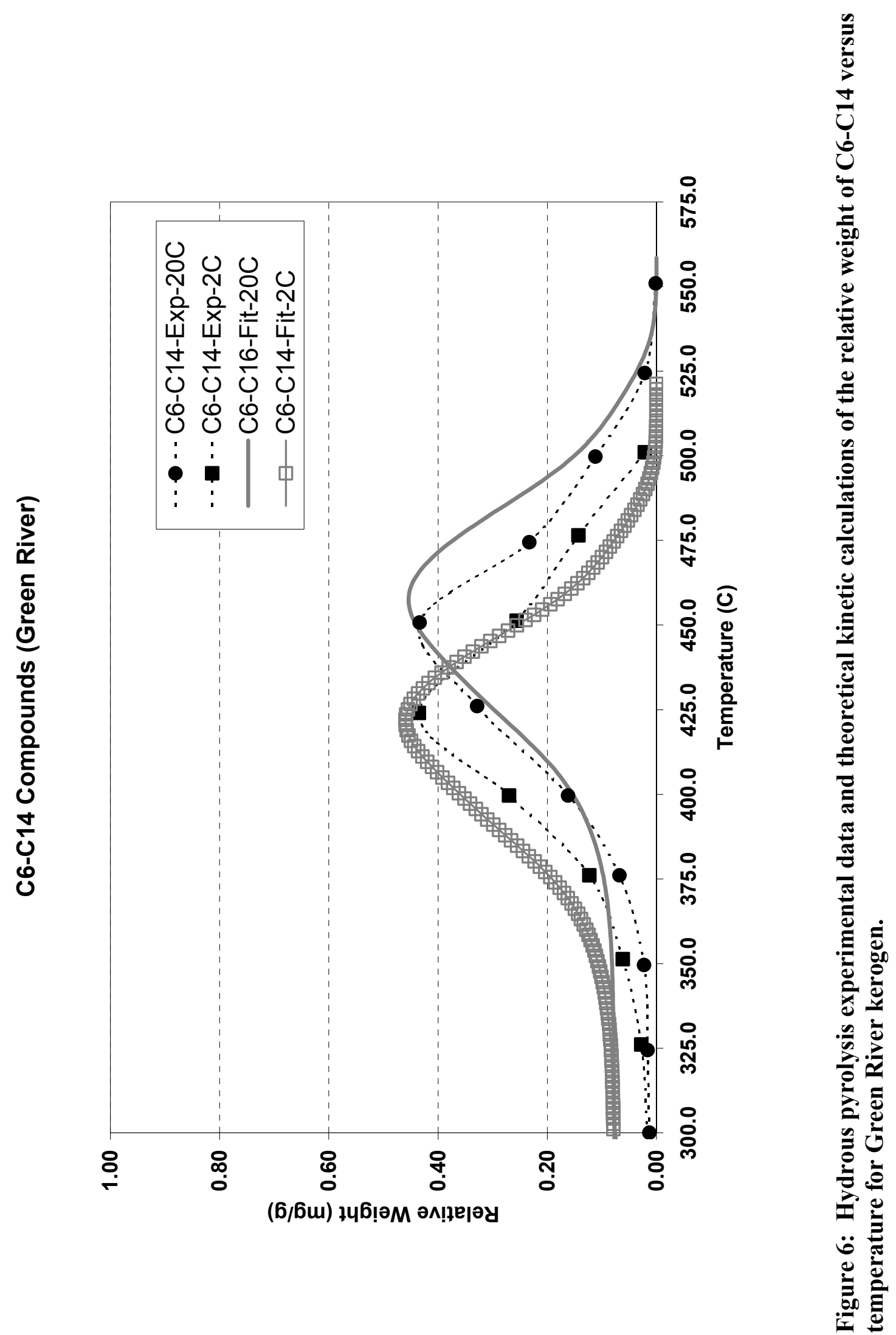

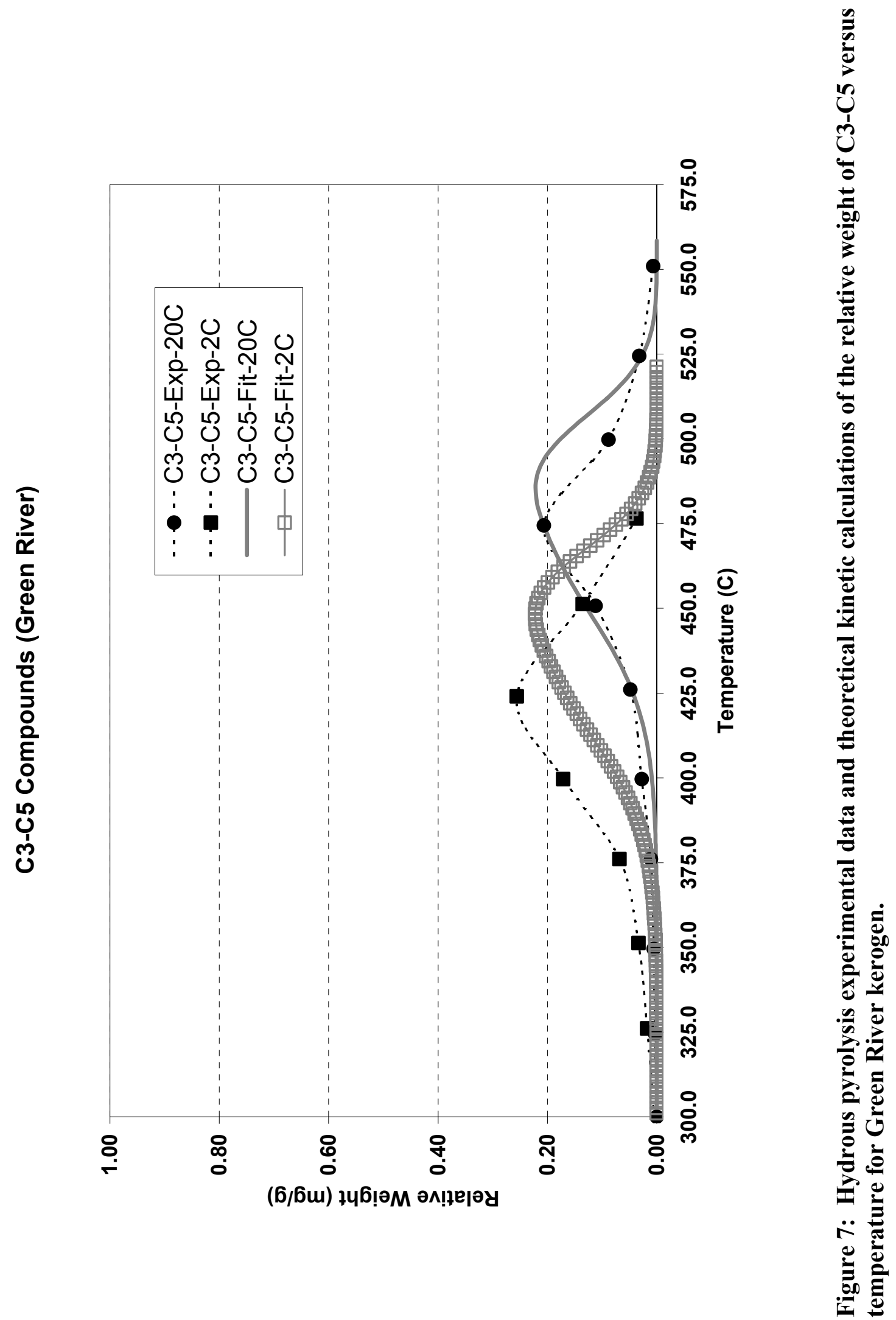

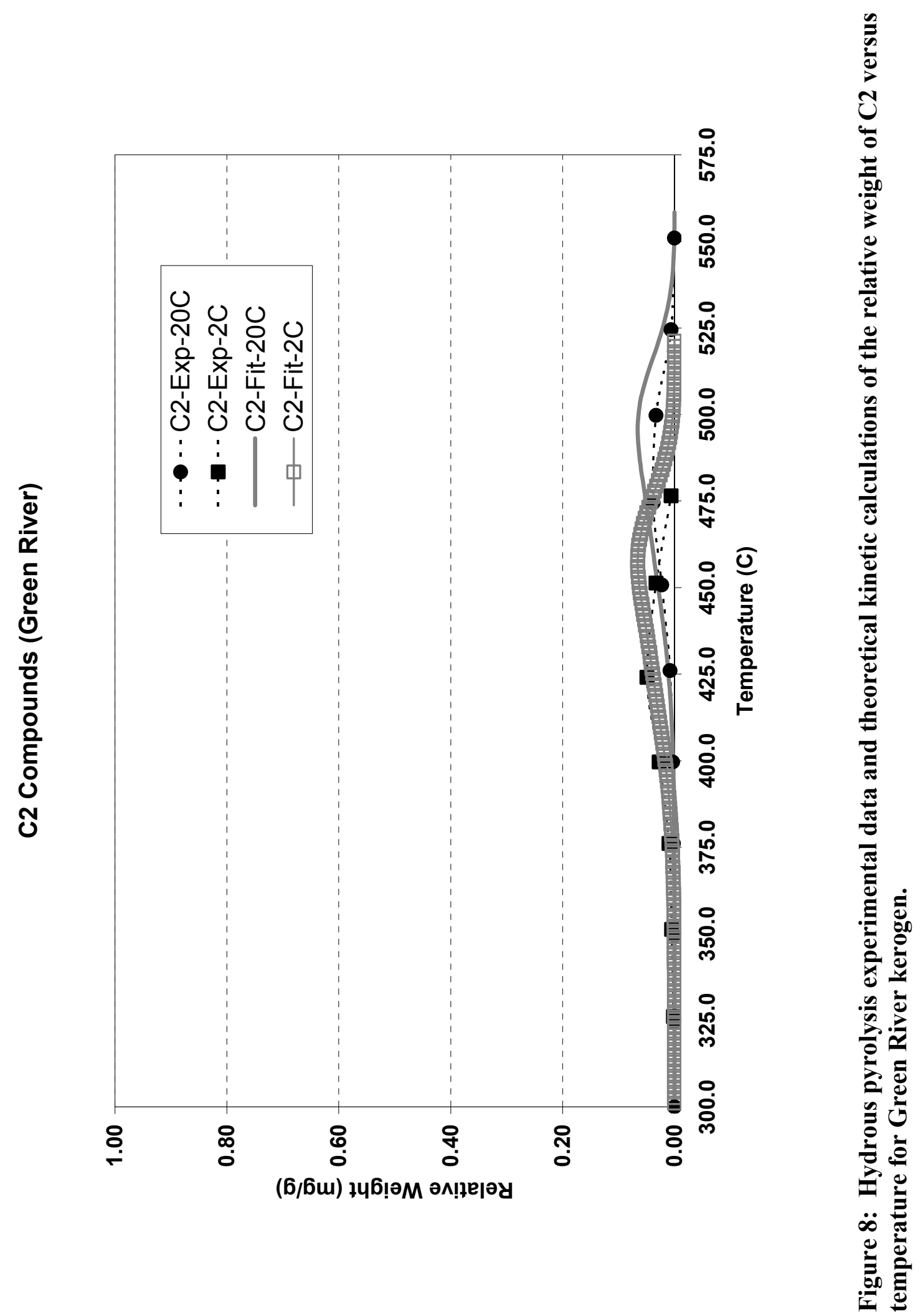

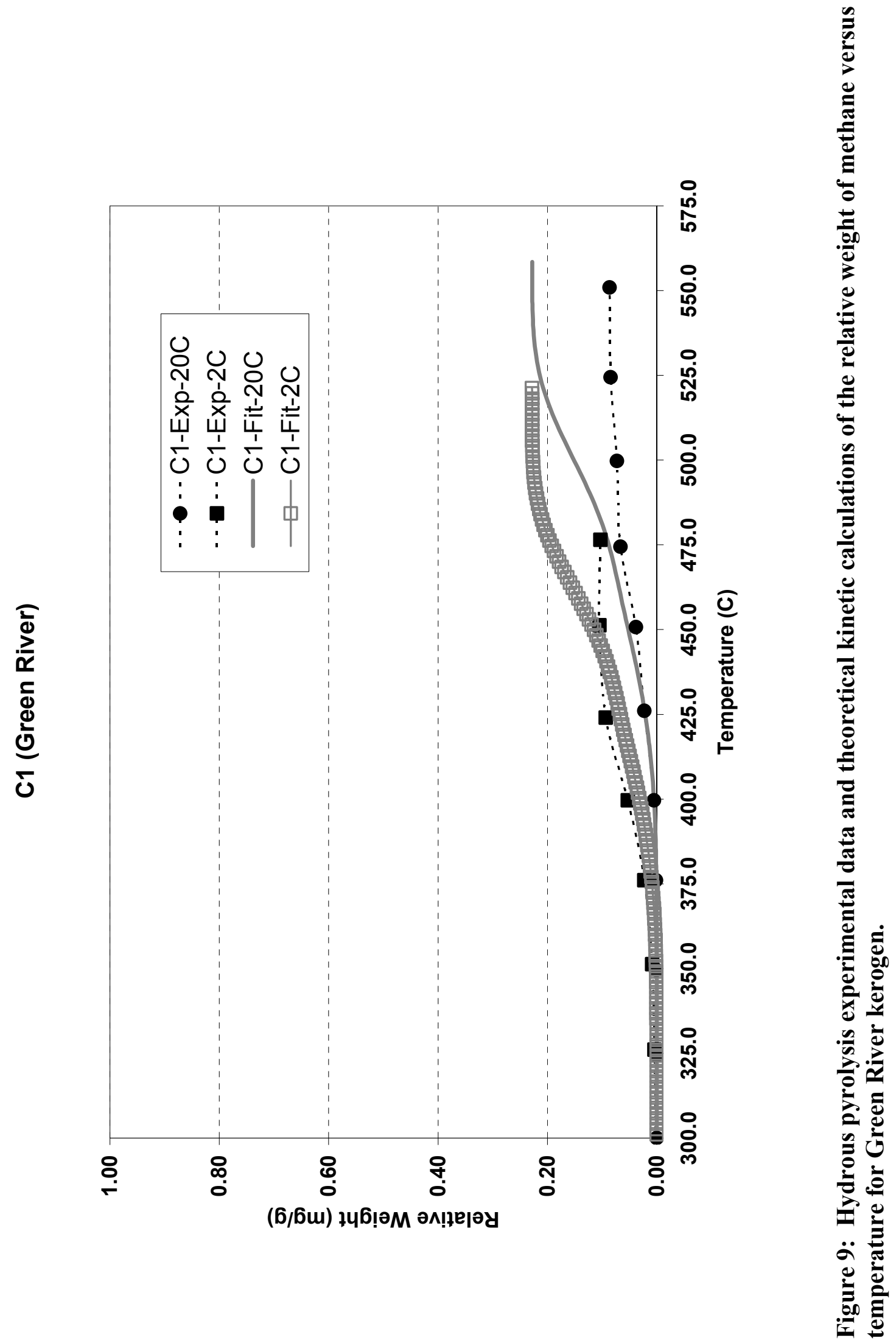

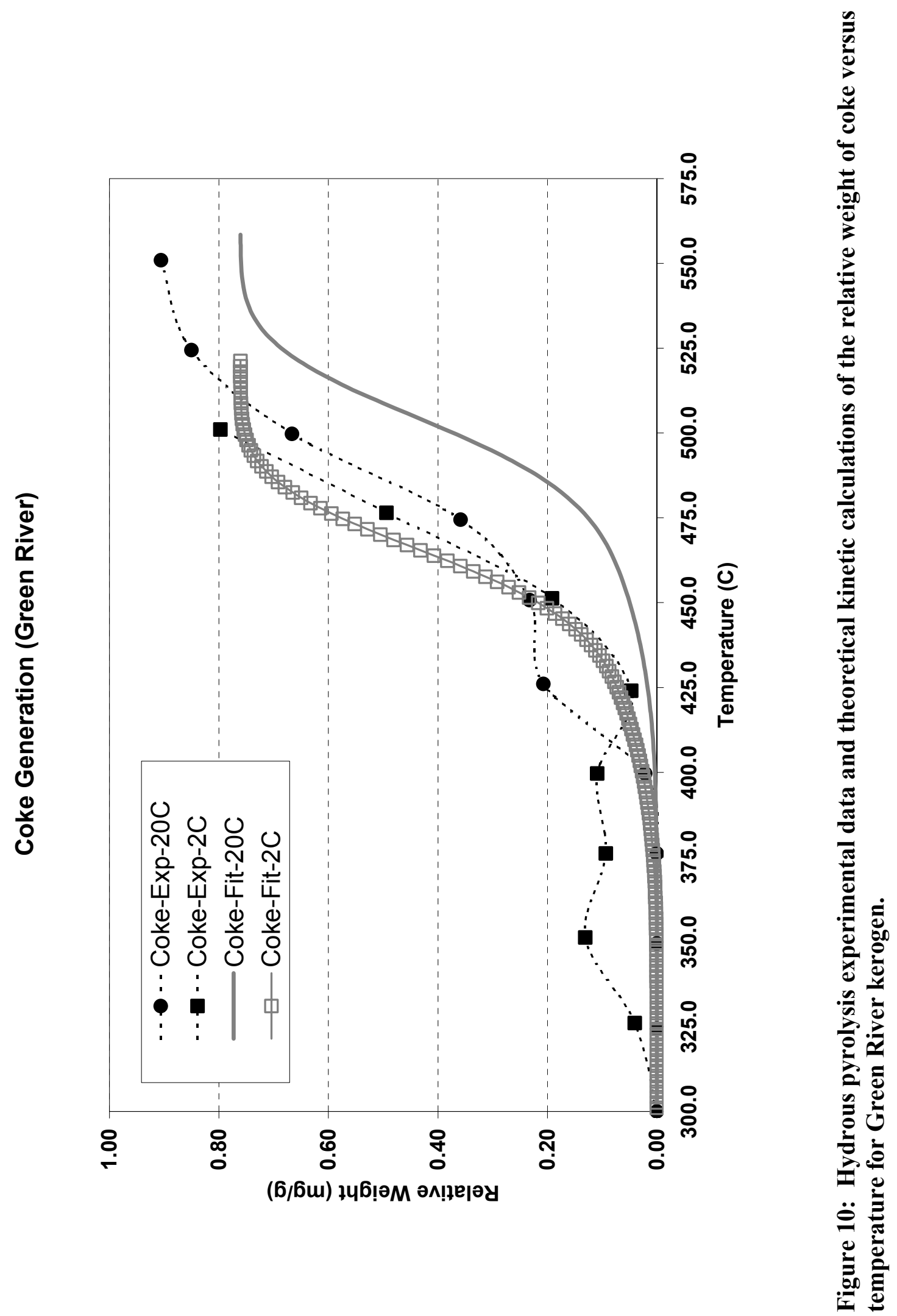

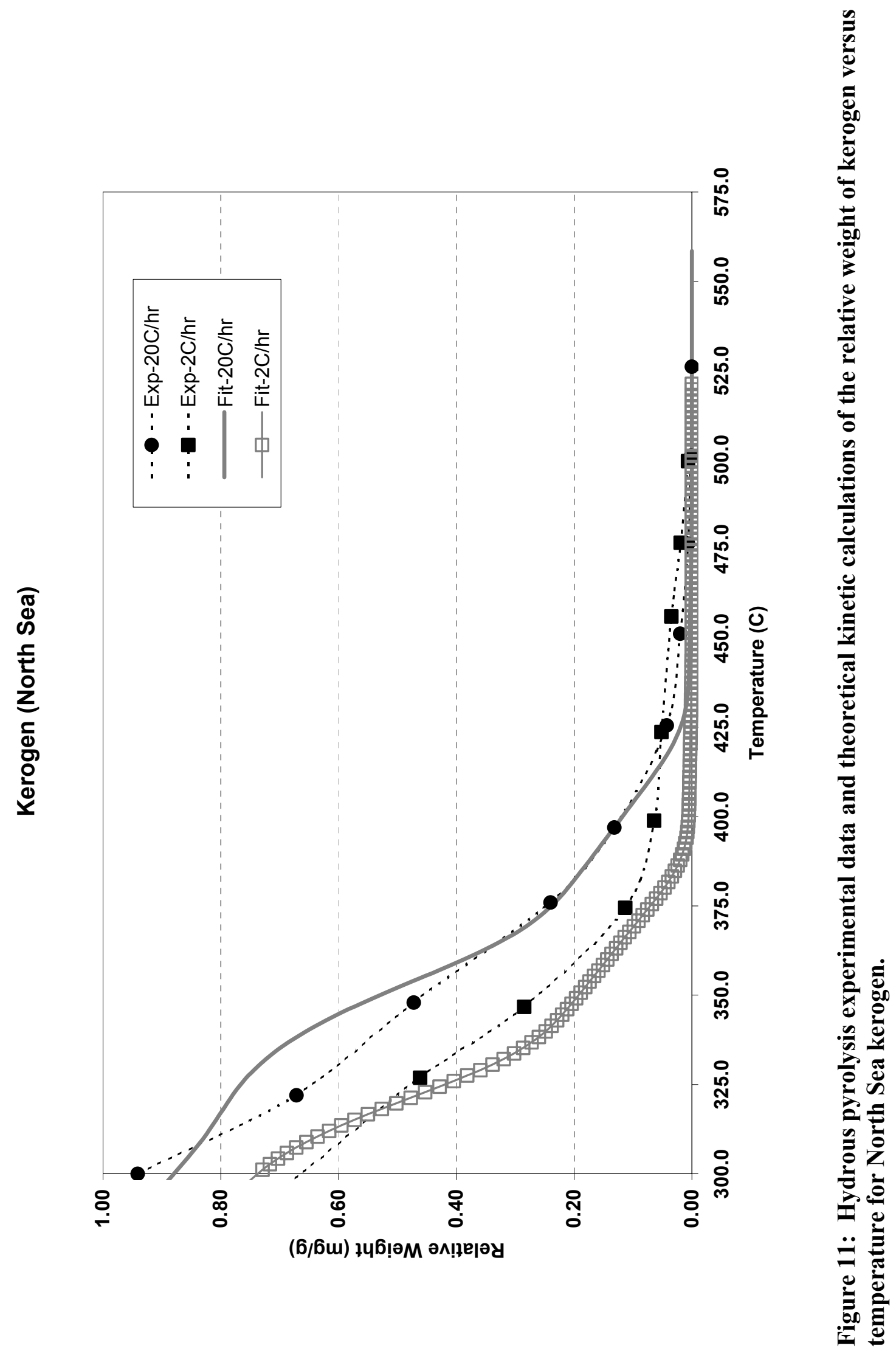


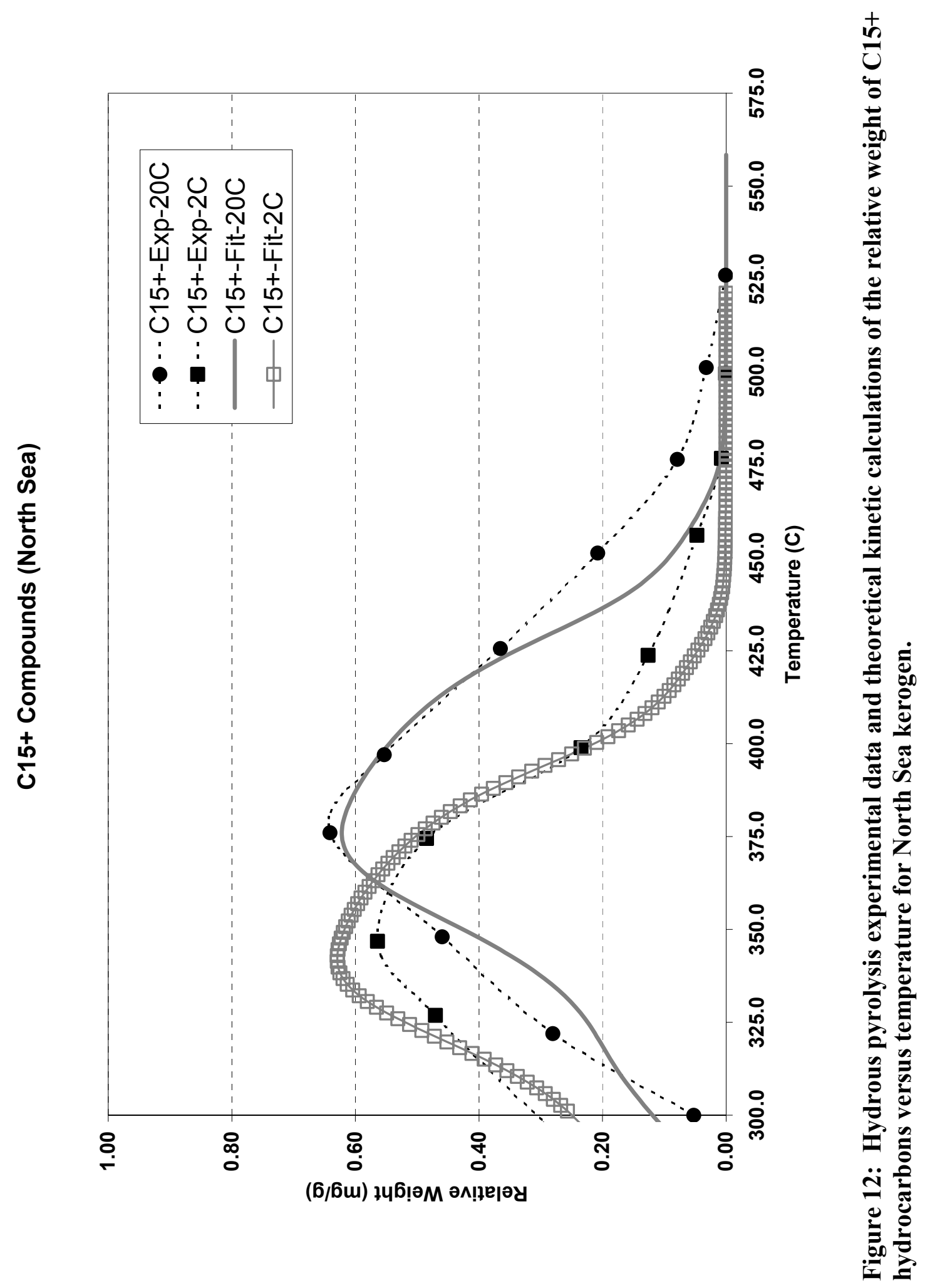



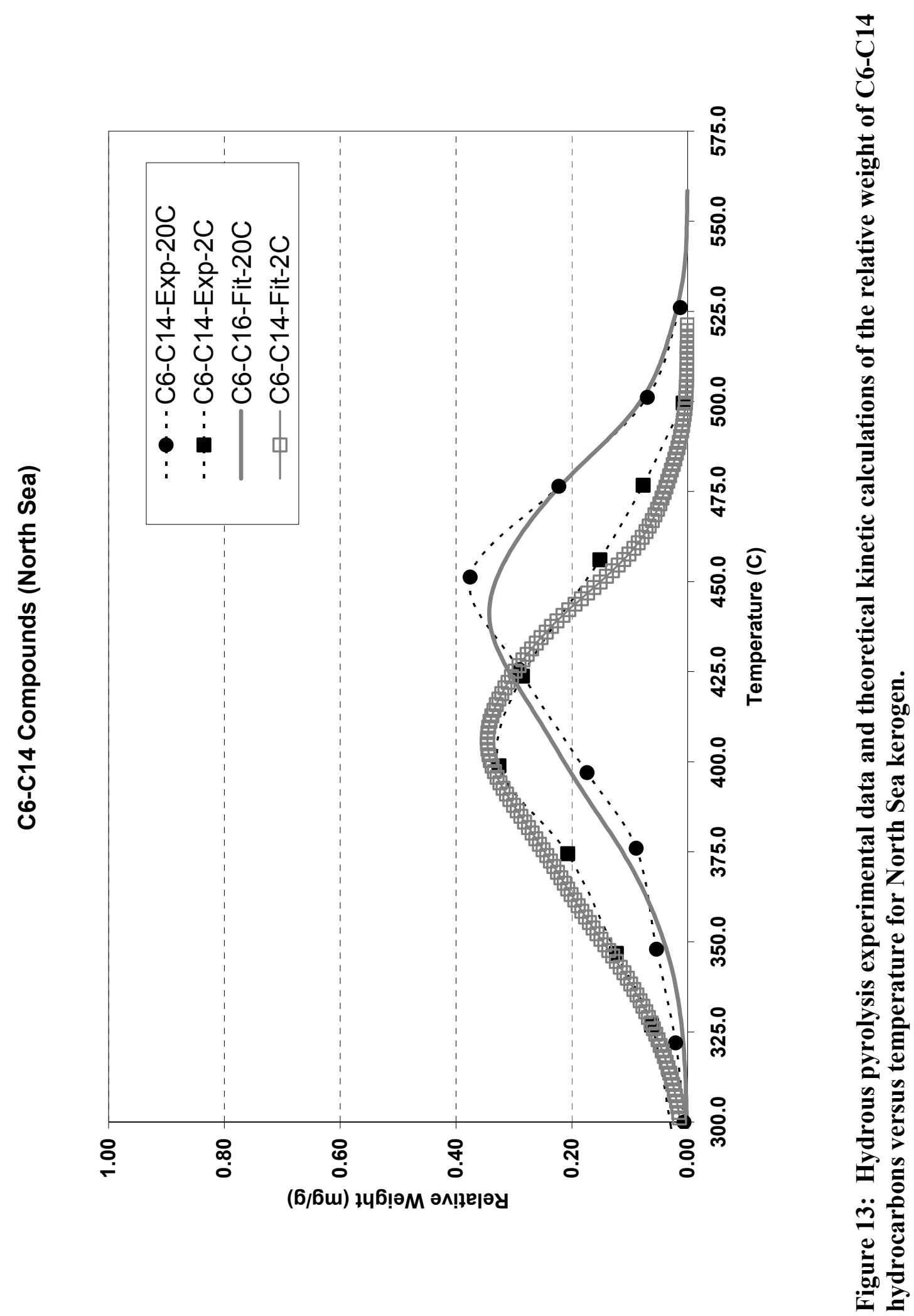

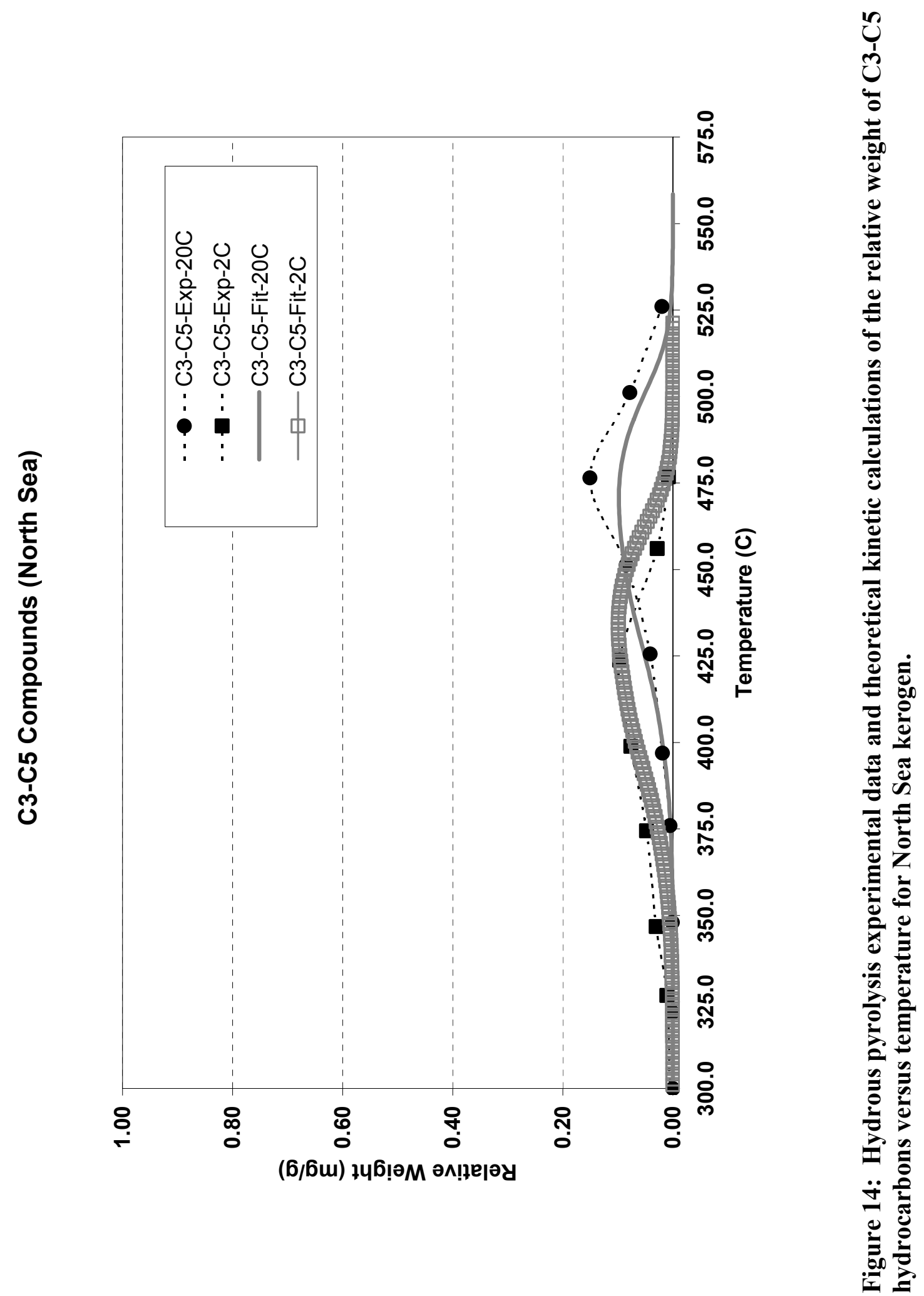

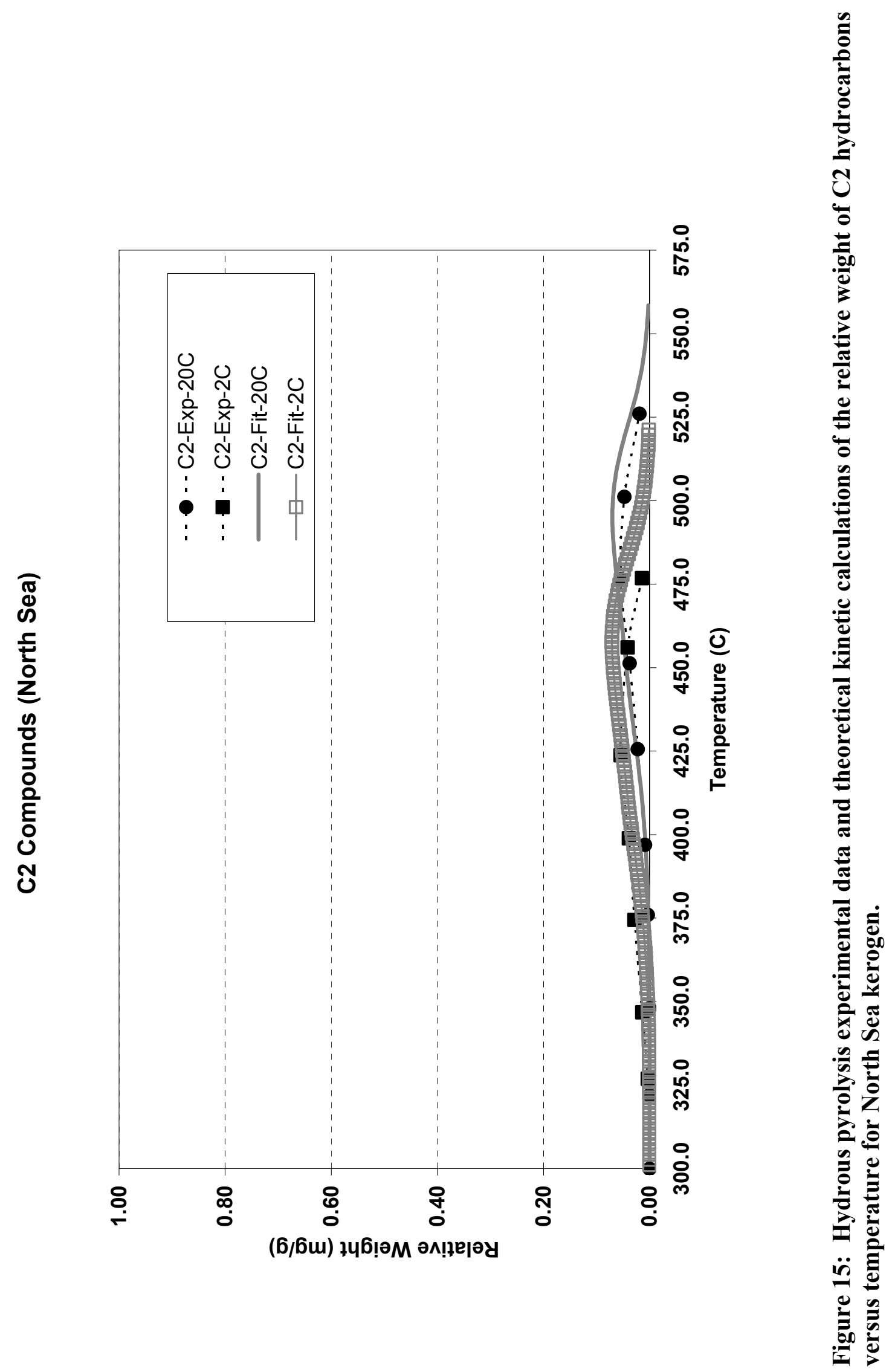

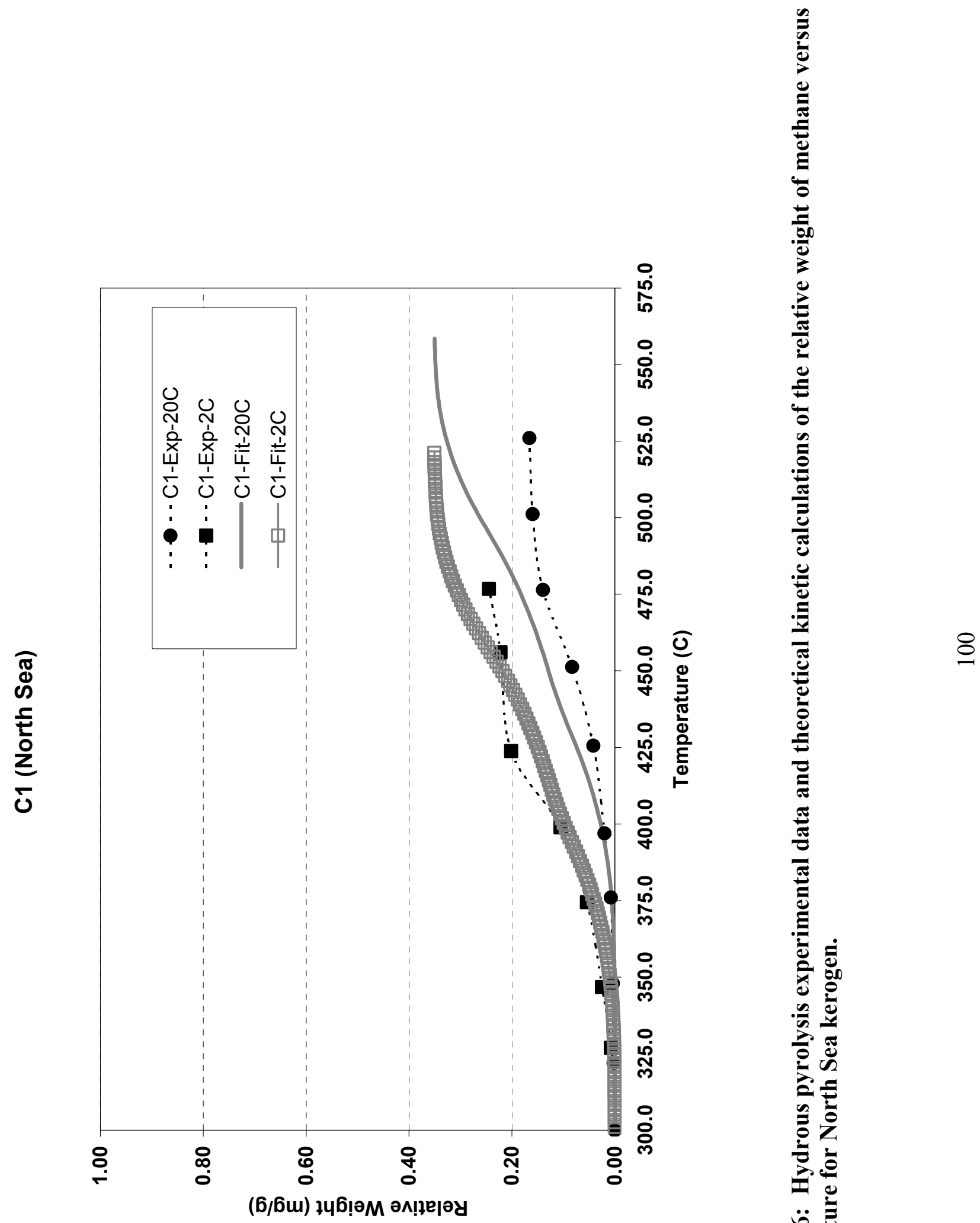

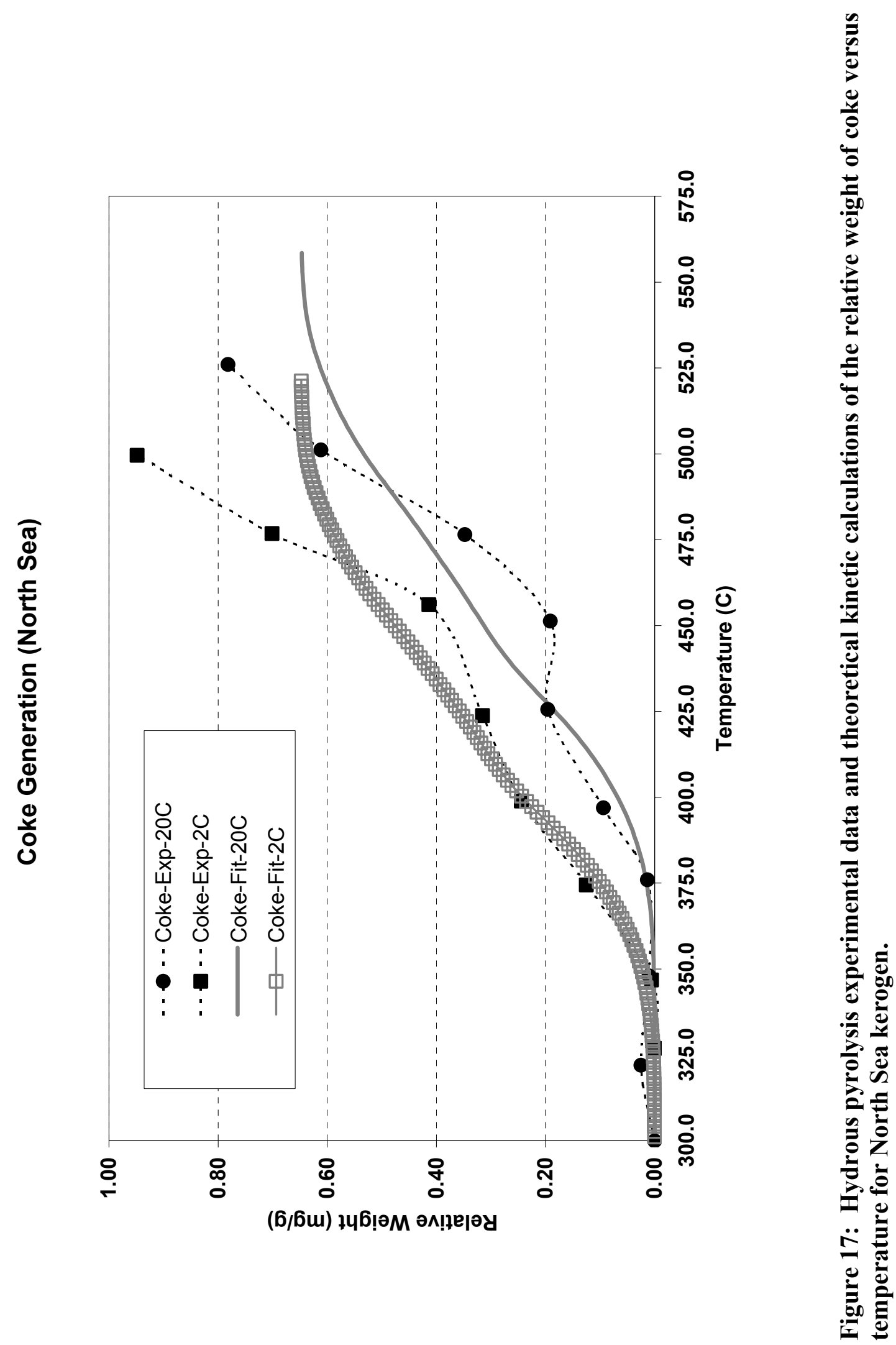


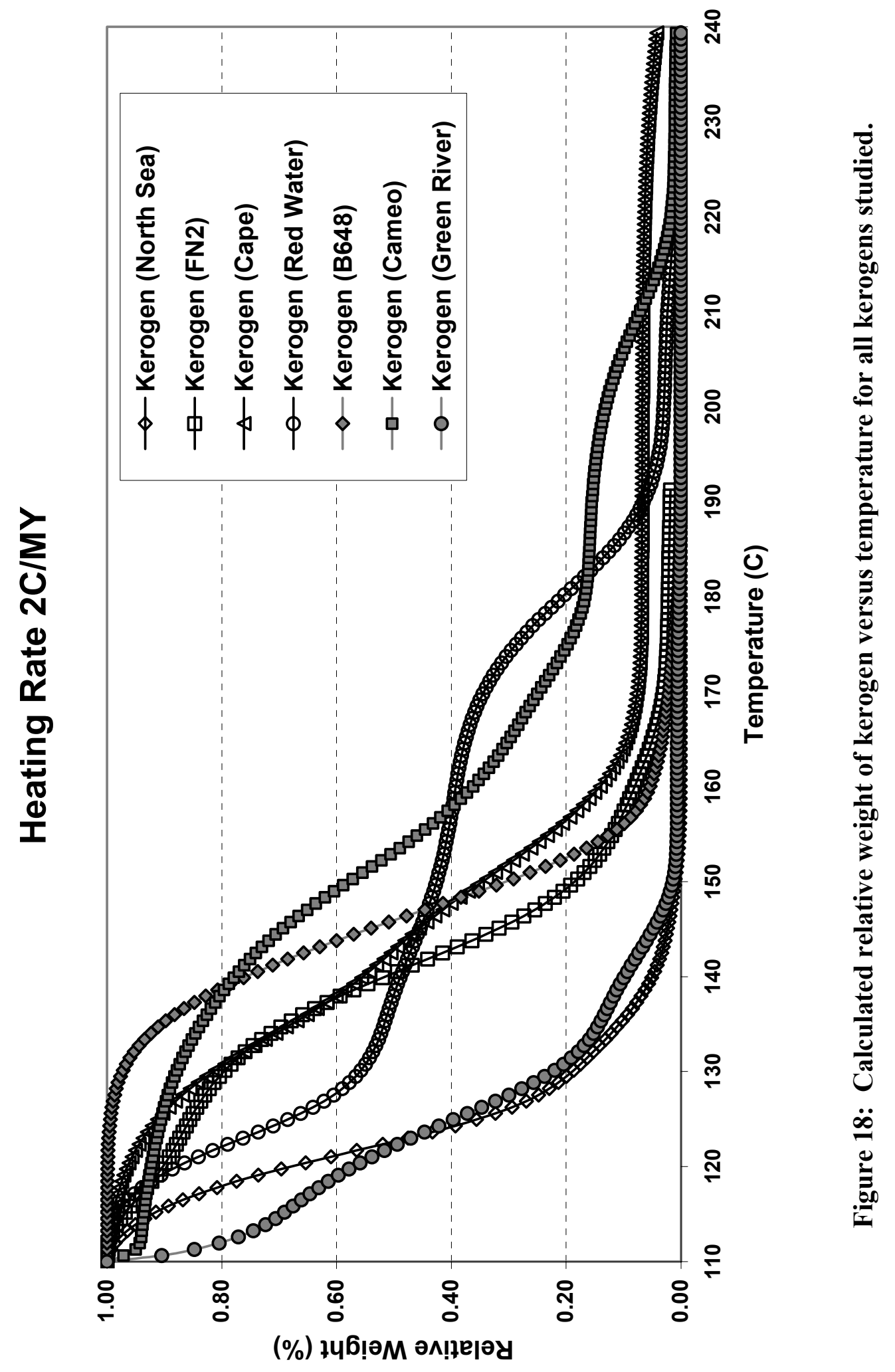




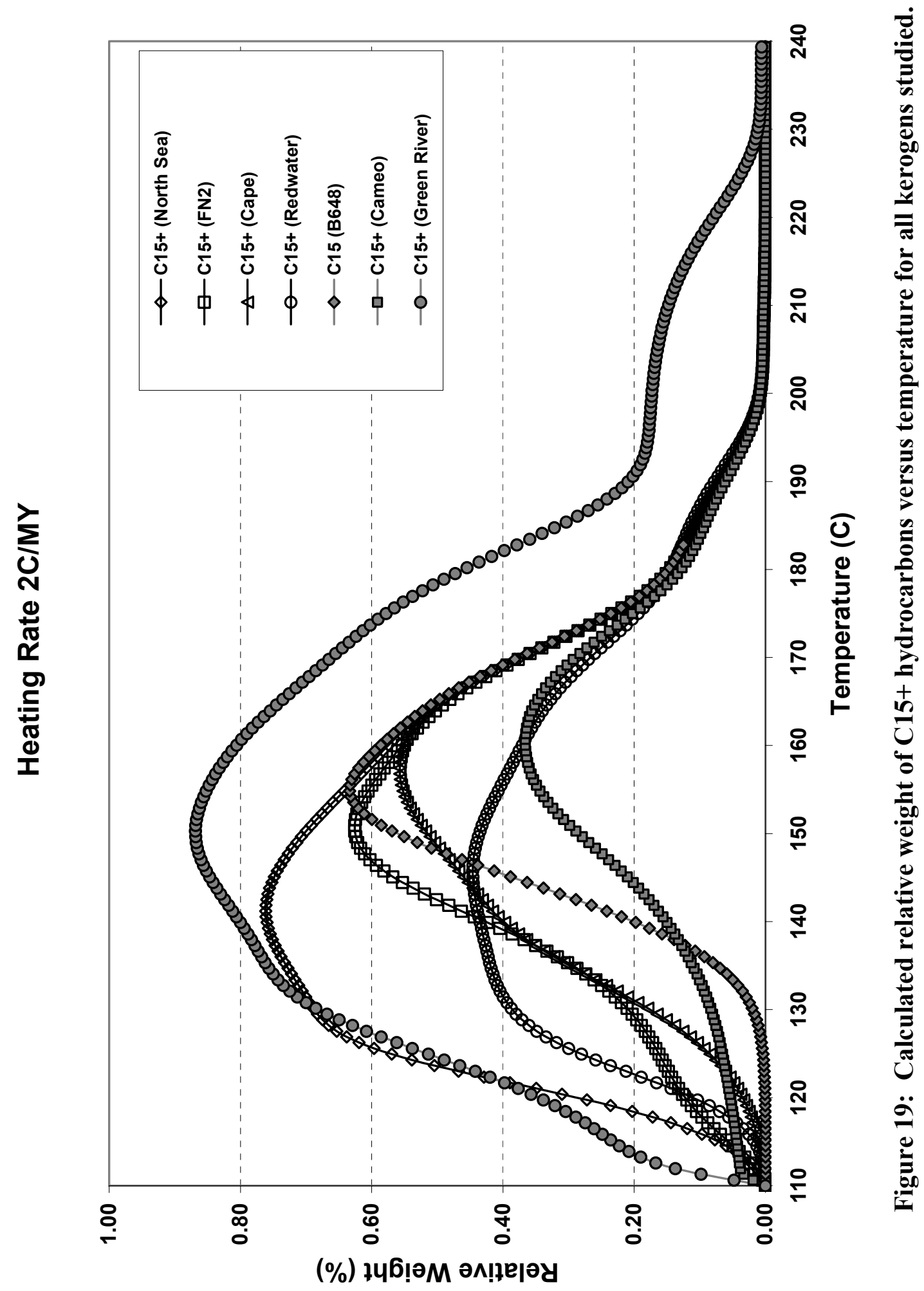




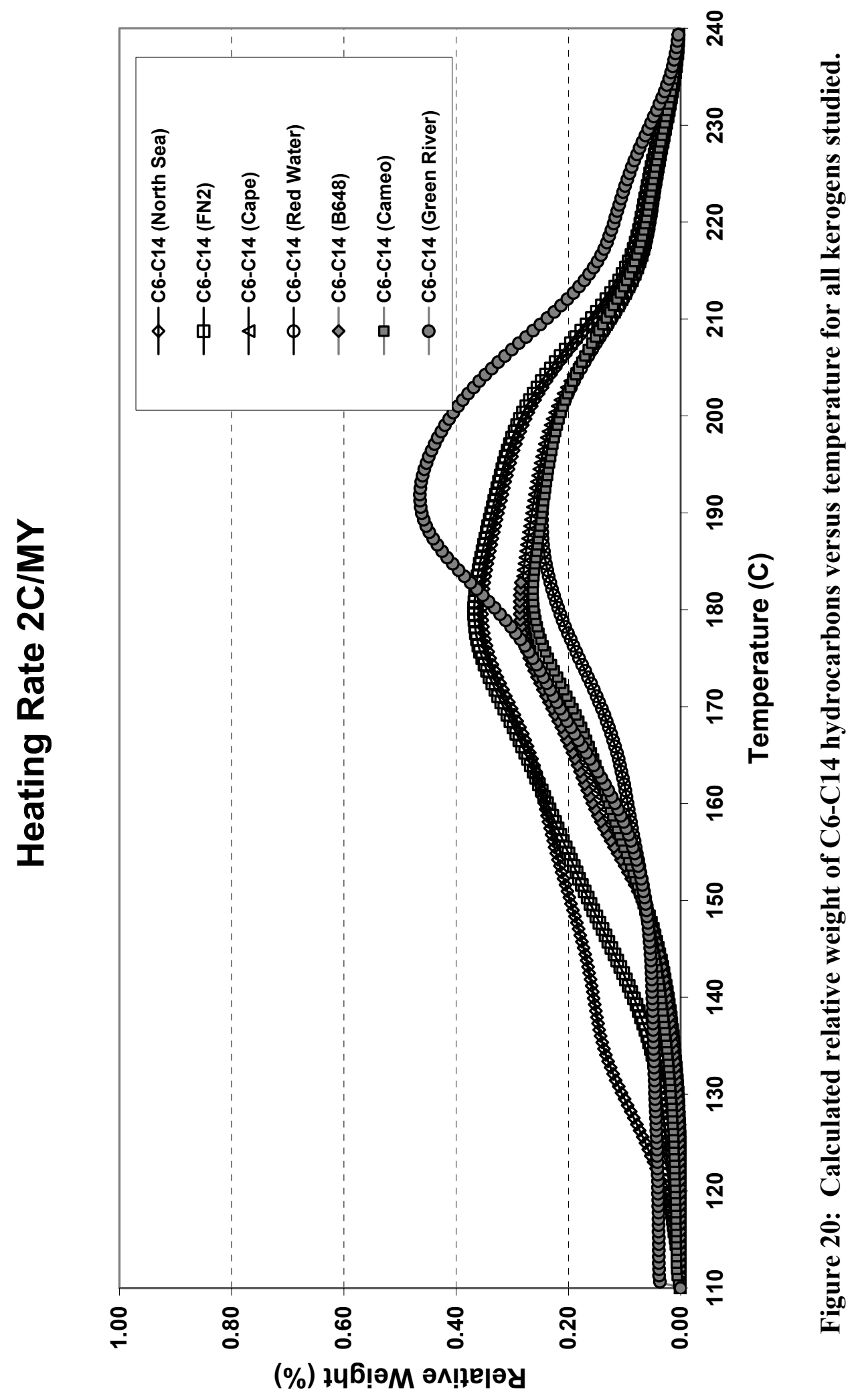




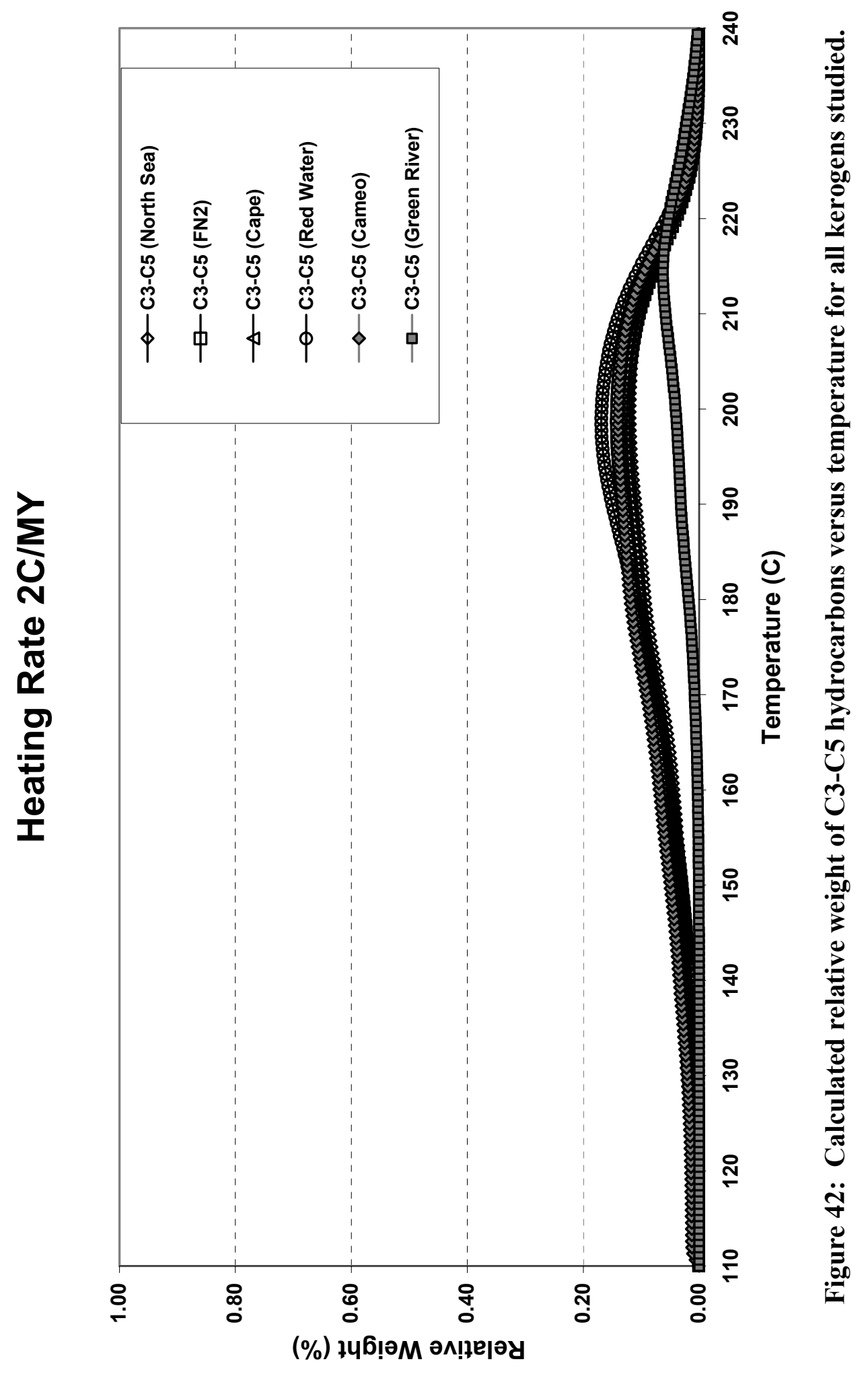




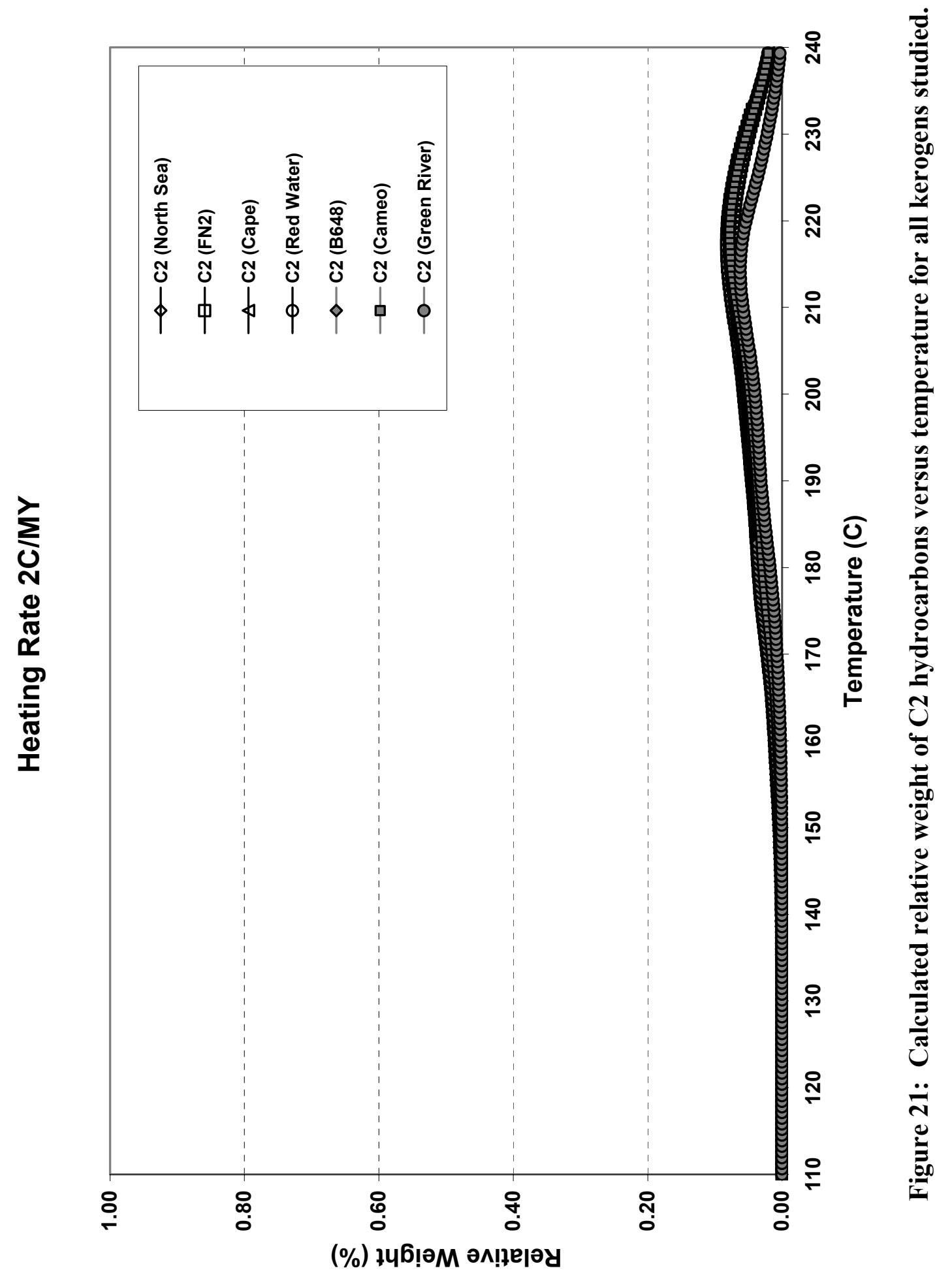




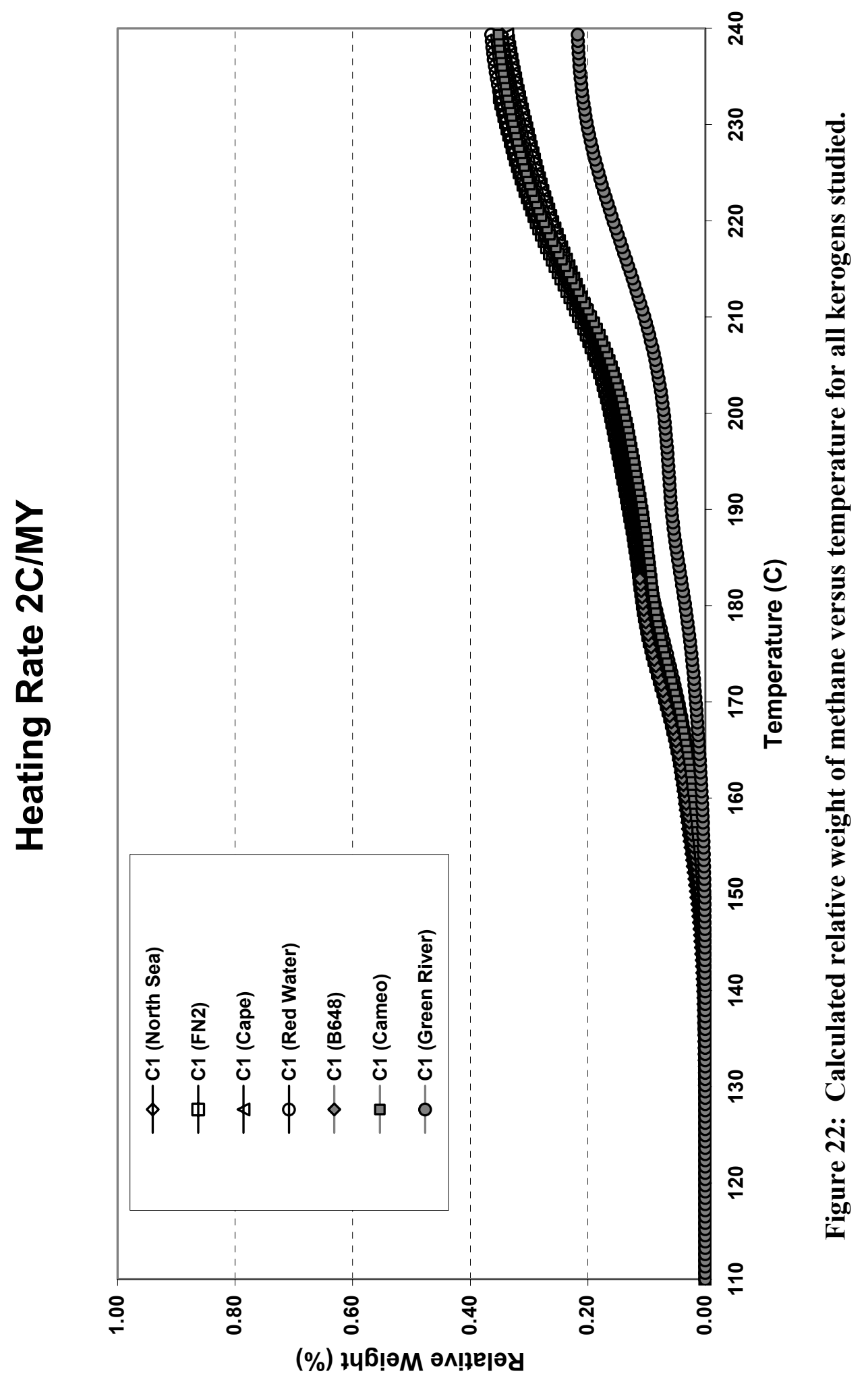

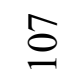




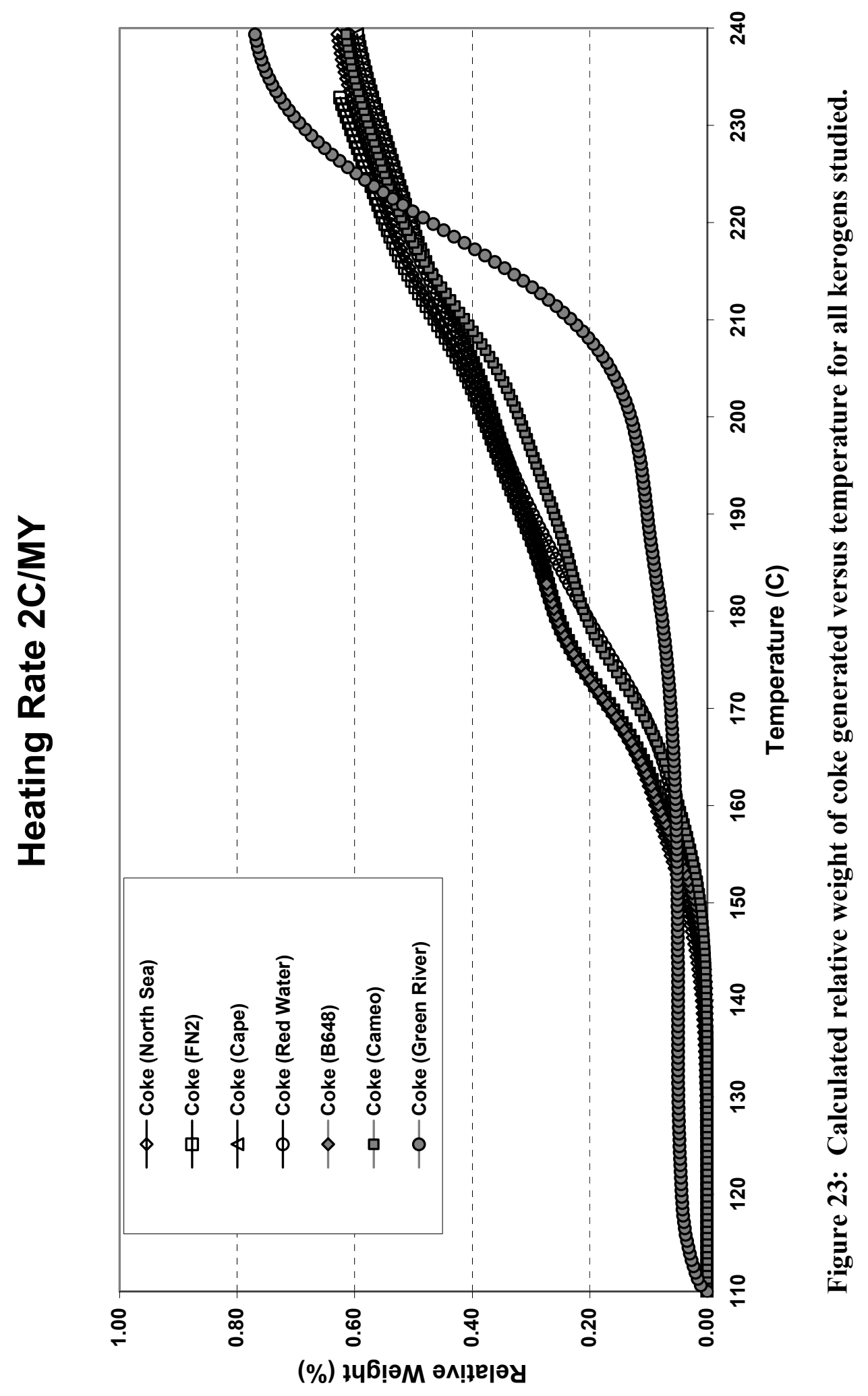




\section{Modeling Phase Behavior}

A major goal for the Advanced Chemistry Basin Model project has been to be able to simulate the phase behavior of complex hydrocarbon mixtures (oils) throughout the normal range of conditions found in sedimentary basins. The goals were

- To create a phase behavior prediction engine that can integrate into the basin model simulator

- To create a database of component information that spans the most relevant hydrocarbon species

- To create a flexible, extensible software package that can be used for this project, and later leveraged for other work

- To remain as platform independent as possible, so that the engine is not tied to a specific operating system or hardware.

These goals were achieved by creating two software applications: the HCToolkit, and the EOSInterface. The HCToolkit is a series of perl $^{1}$ modules that can be used to simulate phase behavior of arbitrarily-complex mixtures. These can be used as stand-alone code on any machine on which perl runs (which covers almost all hardware and software systems today, from desktops to supercomputers). The EOSInterface is a COM object written using Activestate's PerlCOM ${ }^{2}$ application, build on top of the HCToolkit engine. COM technology (specific to the MS Windows platform) allows different components to fit together.

The HCToolkit is designed in a general fashion to allow arbitrary mixtures to calculate phase behavior using a variety of user-specified methods. The list of methods provided is not exclusive; with a minimum of coding, a user can add a new method. Current methods supplied include four equatiosn of state (Van der Waals, Mathias, Aasberg-Petersen, and PSRK), two methods of calculating viscosity (Chung and _), two excess Gibbs Free Energy calculators (UNIFAC and modified UNIFAC), several flash methods (LL, LV), and connections to three database servers (postgresql, mysql, and odbc).

The HCToolkit consists of two distinct pieces: a database, built using one of a number of common database engines (postgresql, mysql, or MS Access [depreciated]), and a set of perl modules that access molecular and mixture properties from the database and calculate such common mixture properties as single-phase molar volume, density, phase separation and composition as a function of $(\mathrm{P}, \mathrm{T})$, and viscosity.

\section{Database}

The database for the HCToolkit is designed to be platform and engine-independent; all versions contain the same data and functionality. The database consists of a set of tables, half of which contain basic single-compound and compound interaction data, the other half of which contain user-provided information on mixtures. There is also a utility designed to aid in specification of mixtures, the "Oil Interface" (OI.pl, see Figure 1), which allows the user to quickly construct fluids, add them to the database, modify them, or delete them.

\footnotetext{
${ }^{1} \mathrm{http}: / / \mathrm{www} \cdot$ perl.com

${ }^{2}$ http://www.activestate.com
} 


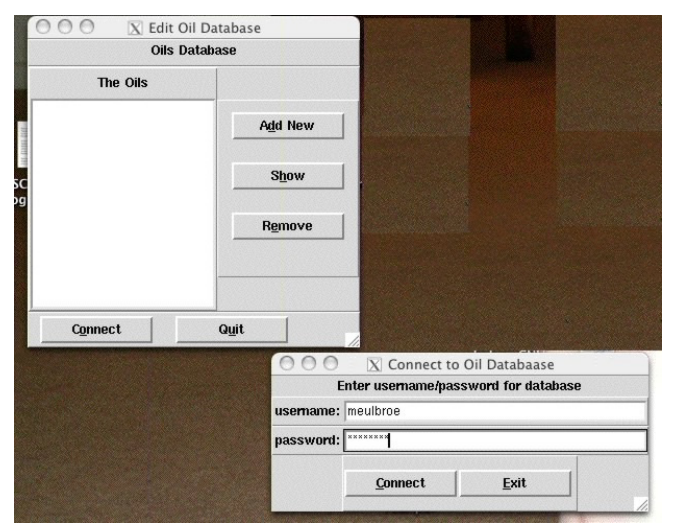

Figure 1: The Oil Database Interface

\section{Tables}

The heart of a relational database is the table layout in which data is stored. Tables in the HCToolkit database are divided between user-specified information and data tables.

The primary user-specified information table is the "Oils" table, which indexes the mixtures available to users of the HCToolkit. This table forms a placeholder for each mixture (though no compositional information is stored here).

Table 1: Table "Oils"

\begin{tabular}{|l|l|l|l|}
\hline \multicolumn{1}{|c|}{ Name } & \multicolumn{1}{c|}{ Type } & Default Value & \multicolumn{1}{c|}{ Meaning } \\
\hline ID & Integer & $\begin{array}{l}\text { Auto } \\
\text { increment }\end{array}$ & A unique ID \\
\hline Name & Text & --- & The name of the fluid \\
\hline Description & Text & --- & $\begin{array}{l}\text { A short description of the } \\
\text { fluid }\end{array}$ \\
\hline
\end{tabular}

The "OilComponents" table contains the actual component specification for each mixture.

Table 2: OilComponents

\begin{tabular}{|l|l|l|l|}
\hline Name & Type & $\begin{array}{l}\text { Default } \\
\text { Value }\end{array}$ & Meaning \\
\hline ID & Integer & $\begin{array}{l}\text { Auto } \\
\text { increment }\end{array}$ & A unique ID \\
\hline OilNumber & Integer & --- & $\begin{array}{l}\text { Reference to the Oil } \\
\text { number (ID in the "Oils" } \\
\text { table) }\end{array}$ \\
\hline ComponentNumber & Integer & --- & $\begin{array}{l}\text { Reference to the } \\
\text { component number (ID in } \\
\text { the "Data" table) }\end{array}$ \\
\hline WhichComponent & Integer & --- & $\begin{array}{l}\text { Index that allows } \\
\text { components of the oil to } \\
\text { be (re)numbered }\end{array}$ \\
\hline
\end{tabular}


The "Data" table contains thermochemical data on individual species. This data was collected from a number of sites on the web, and its accuracy is NOT guaranteed. If you want to make sure of accuracy, put together your own table! Note that there are a number of legacy fields in the table (not listed here) whose existence is depreciated.

Table 3: The Data Table

\begin{tabular}{|l|l|c|l|}
\hline \multicolumn{1}{|c|}{ Name } & \multicolumn{1}{c|}{ Type } & Default Value & \multicolumn{1}{c|}{ Meaning } \\
\hline ID & Integer & Auto increment & A unique ID \\
\hline Name & text & --- & Chemical Name \\
\hline Formula & text & --- & Molecular Formula \\
\hline MOLEWT & double & --- & Molecular weight \\
\hline TCRITK & double & --- & Critical temperature \\
\hline PCRITBAR & double & --- & Critical pressure \\
\hline VCRITCM3 & double & --- & Critical Volume \\
\hline ZCRIT & double & --- & Critical Compressibility \\
\hline ACENTRIC & double & --- & Acentric Factor \\
\hline DIPOLE & double & --- & Dipole Moment \\
\hline TMELTK & double & --- & Melting Temperature \\
\hline TBOILK & double & --- & Boiling Temperature \\
\hline Family & double & --- & Reference to Family Table \\
\hline TriplePtTK & double & --- & Triple Point Temperature \\
\hline TriplePtPBar & double & --- & Triple Point Pressure \\
\hline
\end{tabular}

The "families" table allows for characterization of data along broad categories (e.g., "alkanes"). This is primarily for future extensibility.

Table 4: Families

\begin{tabular}{|l|l|l|l|}
\hline Name & Type & Default Value & Meaning \\
\hline ID & Integer & $\begin{array}{l}\text { Auto } \\
\text { increment }\end{array}$ & A unique ID \\
\hline Type & Text & -- & $\begin{array}{l}\text { The family } \\
\text { name }\end{array}$ \\
\hline
\end{tabular}

An important part of the modeling is support for UNIFAC, an excess Gibbs Free Energy calculation model. In addition to the thermochemical data equations of state use, UNIFAC requires a number of group additivity parameters. Each species is treated as a 'bag' of functional groups. Properties of these groups are stored in the table "UnifacGroups".

Table 5: UnifacGroups

\begin{tabular}{|l|l|c|l|}
\hline \multicolumn{1}{|c|}{ Name } & \multicolumn{1}{c|}{ Type } & Default Value & \multicolumn{1}{c|}{ Meaning } \\
\hline ID & Integer & Auto increment & A unique ID \\
\hline MainID & integer & --- & The main group ID \\
\hline MainName & text & --- & Main group name \\
\hline SubName & text & --- & Subgroup name \\
\hline Q & Double & --- & Q factor \\
\hline R & Double & --- & R factor \\
\hline
\end{tabular}


UNIFAC parameters take two forms: individual functional group components (specified above), and group interaction parameters, specified in the table "UnifacInteractions". Though this table is nominally a matrix of $(\mathrm{i}, \mathrm{j})$ interactions, it is a sparse table, due to the availability of published data.

Table 6: UnifacInteractions

\begin{tabular}{|l|l|c|l|}
\hline Name & Type & Default Value & \multicolumn{1}{c|}{ Meaning } \\
\hline ID & Integer & Auto increment & A unique ID \\
\hline I & Integer & --- & ith index \\
\hline J & Integer & --- & jth index \\
\hline Aij & Real & --- & Interaction parameter \\
\hline
\end{tabular}

The UNIFAC calculations that include a species require molecular structure data (connectivity) for each species. Though this information is available for all species in the database, it turns out to take some time and specialized knowledge to determine the connectivity (this is very hard to do automatically), so the table is populated 'as needed'. The table that includes species connectivity data is called "DataGroups", and it details the number and type of functional groups in each species

Table 7: DataGroups

\begin{tabular}{|l|l|c|l|}
\hline Name & Type & Default Value & \multicolumn{1}{c|}{ Meaning } \\
\hline ID & Integer & Auto increment & A unique ID \\
\hline DataID & integer & --- & A reference to the species ID (from the Data table) \\
\hline Name & text & --- & Name of species (redundant) \\
\hline gID & integer & --- & Reference to the UnifacData table \\
\hline gCount & integer & --- & Number of this group in this molecule \\
\hline
\end{tabular}

It is a burden to maintain a distributed database without advanced tools such as LDAP, but also beyond the scope of this project to create a database distribution mechanism. As a stopgap, there is a mechanism for introducing updates to the database using the perl script "updateDatabase.pl", found in the $<$ root $>$ /database directory. To avoid duplications, this script records all updates to the table "Updates".

Table 8: Updates Table

\begin{tabular}{|c|c|c|c|}
\hline Name & Type & Default Value & Meaning \\
\hline ID & Integer & $\begin{array}{l}\text { Auto } \\
\text { increment }\end{array}$ & A unique ID \\
\hline FixID & integer & $-\overline{--}$ & $\begin{array}{l}\text { The ID of the fix (if } \\
\text { needed) }\end{array}$ \\
\hline ApplicationDate & timestamp & now & $\begin{array}{l}\text { When the fix was } \\
\text { applied }\end{array}$ \\
\hline Description & text & --- & A description of the fix \\
\hline
\end{tabular}

Finally, there are some simple test mixtures distributed with the database. These are included purely as examples; their relevance to any given location or situation is not guaranteed. 
Table 9: Test Oils

\begin{tabular}{|l|l|l|}
\hline ID & Name & Description \\
\hline 27 & TwoComponent & Simple test oil \\
\hline 43 & AllAlkanes & Oil of 20 alkanes \\
\hline 49 & cyclohexane-test & test oil of pure cyclohexane \\
\hline 50 & n-decane-test & test oil of pure n-decane \\
\hline 51 & ethyl-benzene-test & test oil of pure ethyl-benzene \\
\hline 62 & BasinOil & A test realistic oil \\
\hline 63 & CibOp & Vermilion Oil \\
\hline 72 & UnifacOil & Oil to test Unifac \\
\hline 73 & ViscosityOil & test for the viscosity calculations \\
\hline 74 & PressureViscosityOil & test oil for positive pressure \\
\hline 75 & PhaseTest & $\begin{array}{l}\text { Test of the Phase Equilibria prediction of } \\
\text { the EOS }\end{array}$ \\
\hline 76 & PhaseTest2 & second test of the EOS \\
\hline 78 & LLtest & test for liquid-liquid flash module \\
\hline 79 & water-ethane & water and ethane mix for solubility \\
\hline 80 & water-H2S & H20-H2S mix for solubility test \\
\hline 77 & water-methane & test oil to study aqueous methane solubility \\
\hline 81 & water-CO2 & test mix \\
\hline 82 & test-heavy & test the heaviest component \\
\hline 86 & phil-test & temp test oil for phil \\
\hline 87 & thesis & my thesis oil \\
\hline 88 & UNOCAL & oil from unocal files \\
\hline 89 & UNOCAL-methane & oil from unocal files \\
\hline
\end{tabular}

\section{Perl Scripts and Modules}

The perl scripts and modules are the heart of the toolkit. The modules are calculating engines that provide functionality, and the scripts are user interfaces that call upon the computational engines. The following is a file-by-file description of each of the major modules (.pm files) and scripts (.pl files). 


\section{PT-PhaseDiagram.pl}

SYNOPSIS
PT-PhaseDiagram.pl - $\mathrm{t}<$ num_t_pts $>-\mathrm{p}<$ num_p_pts $>$ [other options...]
DESCRIPTION

A program to create a $(\mathrm{P}, \mathrm{T})$ Phase diagram for a given fluid

VERSION

\$Author: meulbroek \$. \$Id: PT-PhaseDiagram.pl,v 1.9 2004/03/02 01:09:30 meulbroek Exp \$

-OilNumber

OPTIONS

the number of the fluid to get data on

$-h$

print brief help information

- man

full documentation

-model

the equation of state to use (defaults to vdw)

-t|-NumTPoints

number of temperature points to use in the phase field

$-\mathrm{p} \mid-$ NumPPoints

number of pressure points to use in the phase field

$-\operatorname{MinT}$

Minimum temperature of the phase field, in K. Optional, defaults to 273

- MaxT

Maximum temperature of the phase field, in K. Optional, defaults to 373.

- MinP

Minimum pressure of the phase field, in bars (optional, defaults to 0.05 )

$-\mathrm{MaxP}$

Maximum pressure of the phase field, in bars (optional, defaults to 120)

-f or-MoleFractionFile

optional file from which to read composition of the mix. Compositions can also be passed via the $-\mathrm{X}$ parameter, or via stdin. Note that the precedince is $-\mathrm{X},-\mathrm{f}, \mathrm{STDIN}$

- vfFile

(optional) name of the file to write the vapor fraction output. The format of the file is ascii tab delimited, and the fields are (P, T, vf). There is one record per line. For historical reasons, this defaults to "vdw.dat".

- lmfFile

(optional) the name of the file to write liquid compositions. The format of the file is ascii tab delimited, and the fields are $(\mathrm{P}, \mathrm{T}, \mathrm{x} 1, \mathrm{x} 2, \ldots)$. There is one record per line. Note that if this option is not specified, the liquid composition is not saved. 
-vmfFile

(optional) the name of the file to write vapor compositions. The format of the file is ascii tab delimited, and the fields are $(\mathrm{P}, \mathrm{T}, \mathrm{y} 1, \mathrm{y} 2, \ldots)$. There is one record per line. Note that if this option is not specified, the vapor composition is not saved.

$-\mathrm{X}$

the composition of the mix to simulate (a string of comma-offset floats; e.g., "0.2, 0.8"). These can instead be read from stdin (either comma or newline delimited)

-on, -OilNumber (optional) the number of the fluid from the database. Note that the composition specified under option (X) MUST have the correct number of components for the specified oil;

-MaxIterations (optional) the number of iterations the calculation will try before returning. Note that the calculation will return early if converged. Default is 30 .

-Precision

(optional) the precision required for a calculation to converge. This is specified as a floating point number, scientific notation is ok. Default is 1e-9

CHANGES

\$Log\$

AUTHOR

Peter Meulbroek, meulbroek@wag.caltech.edu

COPYRIGHT

copyright 2001 Peter Meulbroek, Richard P. Muller, William Goddard III

SEE ALSO

perl(1), Phase::Flash(1) 


\section{PX-PhaseDiagram.pl}

SYNOPSIS
PX-PhaseDiagram.pl -x $<$ num_z_pts $>$ - $\mathrm{p}<$ num_ $\_$_ pts $>$[other options...]
DESCRIPXION

A program to create a $(\mathrm{P}, \mathrm{X})$ Phase diagram for a given fluid

OPTIONS

$-\mathrm{h}$

print detailed help information

$-\mathrm{x} \mid$ NumZPoints

number of Composition points to use in the phase field

$-\mathrm{p} \mid$ NumPPoints

number of pressure points to use in the phase field

$-\operatorname{Min} X$

(optional) Minimum Composition of the phase field. Defaults to 0.01

-MaxX

(optional) Maximum composition of the phase field. Defaults to 0.9.

-MinP

(optional) Minimum pressure of the phase field, in bars (defaults to 0.05)

-MaxP

(optional) Maximum pressure of the phase field, in bars (defaults to 120)

$-\mathrm{Z}$

the composition of the mix to simulate (a string of comma-offset floats; e.g., "0.2, 0.8 ")

-on, OilNumber

(optional) the number of the fluid from the database. Note that the composition specified under option (X) MUST have the correct number of components for the specified oil;

-T, temperature

(optional) the temperature at which to draw the phase diagram. Defaults to $273.14 \mathrm{~K}$

CHANGES

\$Log\$

AUTHOR

Peter Meulbroek, meulbroek@wag.caltech.edu

COPYRIGHT

copyright 2001 Peter Meulbroek, Richard P. Muller, William Goddard III

perl(1), Phase::Flash(1)

\section{SEE ALSO}




\section{3 getIDs.pl}

Program to create an oil based on a text file of names

$$
\begin{gathered}
\text { getIDS.pl }[-\mathrm{i}<\text { txtfile }>][-\mathrm{o}<\text { outfile }>] \\
\text { DESCRIPTION }
\end{gathered}
$$

program that reads through a tab-delimited text file, extracts names and mole fractions, then creates an oil based on those names. It finally creates a file with ids and mole fractions.

\section{EXPORT}

None by default.

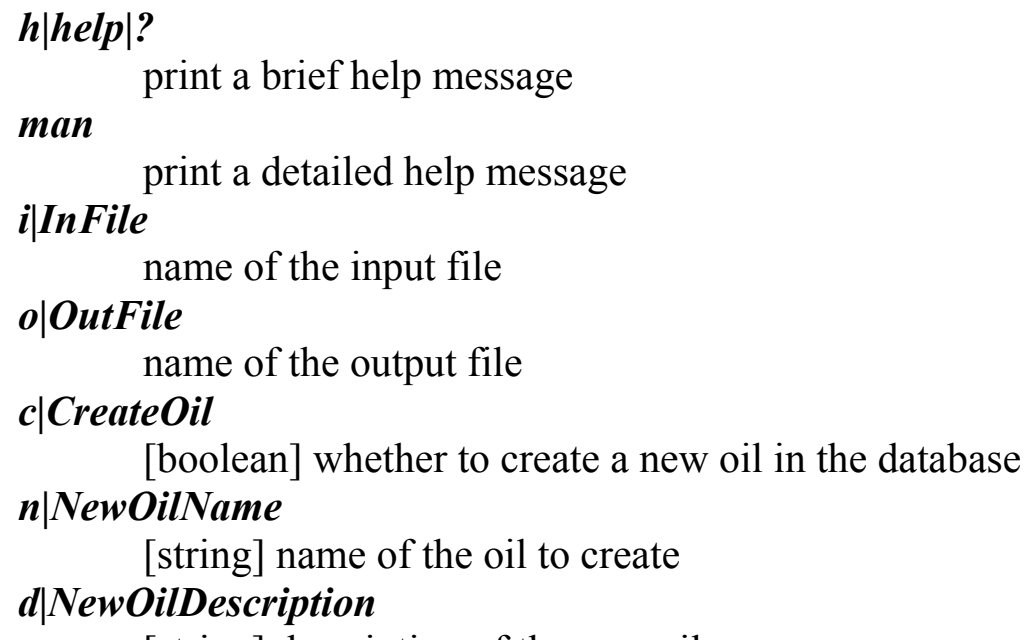




\section{4 creatComponent.pl}

createComponent [options]

SYNOPSIS

Options:

-help brief help message

-man full documentation

-Filename use the component file $<$ Filename $>$

$-<$ no $>$ IncrementVersion

increment the component minor version

DESCRIPTION

creatComponent.pl - creating the activeX component

Peter Meulbroek, meulbroek@wag.caltech.edu

AUTHOR

COPYRIGHT

copyright 2003 Peter Meulbroek, William Goddard III

SEE ALSO

$\operatorname{perl}(1)$. 


\section{EOSInterface}

use EOSInterface;

\section{SYNOPSIS}

my $\$$ ei $=\$$ EOSInterface->new(); \# calls initialize

\$ei->flash $([[\$ p, \$ t, \$ z]]) ;$

DESCRIPTION

EOSInterface forms the basis for the COM object, EOSInterface. It is turned from a class into an object using the ActiveState PerlCOM application. Though the class can be used stand-alone (i.e., within perl), this is generally not recomended, as it has limited functionality.

The component contains as private members all objects needed for flashing (equilibrating a mixture at a given $\mathrm{P}, \mathrm{T}$, separating into liquid and vapor phases if thermodynamically desireable)

It is based on the HCToolkit, and thus follows all the strictures of that set of modules. Briefly, it relies on a database (accessed by Phase::Database) of component properties, and flashes a mixture (collection of species) defined in that database. The user utilizes the component by first initializing it, then flashing mixtures.

\section{GLOBAL VARIABLES}

In order to control a COM object, the object itself can have properties. These corespond to global (package) variables in the underlying perl module. Note that the variables can either be lexical or not; PerlCOM does not seem to care...

These variables include

\section{Initialized}

(Boolean) Whether the object has called the 'Initialize' method

\section{DoDebug}

(Boolean) Whether to use the debug function to print debugging information. Note that this only works on windows platforms

\section{FUNCTIONS}

The following are methods of the Object:

\section{debug}

print debugging information in a popup dialog note that this only works on Micro\$oft windoze!

\section{Flash}

'Flash' the mixture, setting various return values.

\section{Syntax}

Flash $([\$ p, \$ t, \$ z, \$ x, \$ y] \ldots])$, where

\section{$\$ p$}

is pressure (in bars)

\$t

is temperature (in Kelvin) 
$\mathbf{\$ z}$

is composition (array of mole fractions)

\$x

is the best 'guess' for liquid composition (optional)

$\$ y$

is the best 'guess' for vapor composition (optional)

Note that the call takes a reference to an array of references of values, so that it can be used with a matrix of 'flash' points.

i.e.,:

Flash $([$

$[\mathrm{p} 1, \mathrm{t} 1,[\mathrm{z} 11, \mathrm{z} 12, \ldots],[\mathrm{x} 11, \mathrm{x} 12, \ldots],[\mathrm{y} 11, \mathrm{y} 12, \ldots]]$,

$[\mathrm{p} 2, \mathrm{t} 2,[\mathrm{z} 21, \mathrm{z} 22, \ldots],[\mathrm{x} 21, \mathrm{x} 22, \ldots],[\mathrm{y} 21, \mathrm{y} 22, \ldots]]$,

]

)

return values

[Vapor Fraction, liquidMW, vaporMW, liqMolarVolume vapMolarVolume, liqViscosity, vapViscosity, liqInterfacial Tension, vapInterfacial tension] note that the last 2 are zero...

\section{side-effects}

This sets the global arrays \$X and \$Y (liquid and vapor composition, respectively). Note that these are references to arrays of array references, in the same shape as the original call matrix. In fact, this is the way that calcuated phase compositions are returned to the caller

\section{Requirements}

Flash can only be called after initialize.

\section{Initialize}

Initialize sets up the component to flash mixtures. It defines flash-independant quantities, such as the EOS model, the database connection, and the mixture number.

Syntax

Initialize(\$mixtureID, \$EOSModel)

where $<$ \$mixtureID $>$ defaults to 27, \$EOSModel defaults to VanderWaals.

new

new is the object constructor, which is only needed if the package is called from perl (not as a

COM object). This is a wrapper for Initialize

$\%$ TypeLib $=($ PackageName $=>$ 'EOSInterface', Constructor $=>$ 'new', TypeLibGUID $=>$ '\{717DB150-5794-11D5-8C49-FFFFFF000000\}', ControlGUID $=>$ '\{717DB151-5794-11D58C49-FFFFFF000000 $\}$ ', DispInterfaceIID=> ' 7 17DB152-5794-11D5-8C49-FFFFFF000000 $\}$ ', ControlName $=>$ 'Perl.EOSInterface', ControlVer $=>3.10$, \# increment if new object with same ProgID \# create new GUIDs as well ProgID $=>$ 'Perl.EOSInterface', DefaultMethod => 'Initialize', Methods $\Rightarrow\{$ 'Initialize' $\Rightarrow$ RetType $\Rightarrow$ VT_I4, TotalParams $\Rightarrow 2$, 
NumOptionalParams $=>$ 1, ParamList $=>$ [ 'OilID' $=>$ VT_I4, 'EOSModel' $=>$ VT_BSTR ], $\}$, 'Flash' $\Rightarrow>$ RetType $=>$ VT_VARIANT, TotalParams $=>1$, NumOptionalParams $=>0$, ParamList $=>$ [ 'PropertyMatrix' $\Rightarrow$ VT_VARIANT, ], $\}\}$, \# end of 'Methods' Properties $=>\{$ 'Names' $\Rightarrow$ Type $=>$ VT_VARIANT, ReadOnly $=>1$,$\} , 'DoDebug' =>\{$ Type $=>$ VT_I4, ReadOnly $=>0$,$\} , 'EOSModel' =>\{$ Type $=>$ VT_BSTR, ReadOnly $=>0\},,{ }^{\prime} X^{\prime}=>\{$ Type $=>$ VT_VARIANT, ReadOnly $=>0,\},{ }^{\prime} Y^{\prime}=>\{$ Type $=>$ VT_VARIANT, ReadOnly $=>0$,$\} ,$ 'MaxIterations' $=>\{$ Type $=>$ VT_I4, ReadOnly $=>0$,$\} , 'InitialVolume' =>\{$ Type $=>$ VT_R8, ReadOnly $=>0$,$\} , 'MaxDeltaV' \Rightarrow$ Type $=>$ VT_R8, ReadOnly $=>0$,$\} , 'ActualIterations' =>$ $\{$ Type $=>$ VT_I4, ReadOnly $=>1\}$,$\} , \# end of 'Properties' ); \# end of \%TypeLib$ 


\section{Phase::Database::Access}

Perl front-end to a database of chemical properties

SYNOPSIS

use Phase::Database::Access; my $\$ \mathrm{f}=$ Phase::Database::Access->new;

DESCRIPTION

\section{Summary}

\section{Package Version \$Revision\$}

\section{Copyright}

Copyright 2000, Peter Meulbroek

This program is free software; you can redistribute it and/or modify it under the terms of the GNU General Public License as published by the Free Software Foundation; either version 2 of the License, or (at your option) any later version.

This program is distributed in the hope that it will be useful, but WITHOUT ANY WARRANTY; without even the implied warranty of MERCHANTABILITY or FITNESS FOR A PARTICULAR PURPOSE. See the GNU General Public License for more details.

You should have received a copy of the GNU General Public License along with this program; if not, write to the Free Software Foundation, Inc., 59 Temple Place, Suite 330, Boston, MA 02111-1307 USA

\section{CreateID}

CreateID is difficult to do in access; though there is the idea of indexes, there is no idea of sequences. Hence, we can not just grab the next sequence value. Instead, we do an (expensive) search for the maximum ID, then add 1 to it. THIS IS NOT SAFE

\section{Database specific}

Microsoft access is not quote-happy, so we do not have to quote anything

Peter Meulbroek, meulbroek@wag.caltech.edu

$$
\text { AUTHOR }
$$

COPYRIGHT

copyright 2001 Peter Meulbroek, Richard P. Muller, William Goddard III

SEE ALSO

$\operatorname{perl}(1)$. 


\section{Phase::Database}

Perl front-end to a database of chemical properties

SYNOPSIS

used in the Phase::Flash/Viscosity family of packages

use Phase::Database;

my $\$ \mathrm{f}=$ Phase::Database- $>$ new;

\section{DESCRIPTION}

\section{Package Version}

0.2

\section{Copyright}

Copyright 2000, Peter Meulbroek

This program is free software; you can redistribute it and/or modify it under the terms of the GNU General Public License as published by the Free Software Foundation; either version 2 of the License, or (at your option) any later version.

This program is distributed in the hope that it will be useful, but WITHOUT ANY WARRANTY; without even the implied warranty of MERCHANTABILITY or FITNESS FOR A PARTICULAR PURPOSE. See the GNU General Public License for more details.

You should have received a copy of the GNU General Public License along with this program; if not, write to the Free Software Foundation, Inc., 59 Temple Place, Suite 330, Boston, MA 02111-1307 USA

\section{History}

00/02/14 16 00:20

created package

01/02/02 11:41:42

ported to perl

08/29/02

expanded to allow multiple database types by subclass

$11 / 14 / 02$ added support for UNIFAC

\section{Keywords}

\section{Subclassing}

The Phase::Database class does nothing right out of the box; it must be sub-classed to define drivers for specific databases. Even though all database access is via DBI, the sql syntax for individual databases unfortunately tends to be a little different; enough that statements have to be individually crafted. Any subclass inherits the class structure of this class. To instanciate a subclass, the callback function 'init' can be used to define necessary data structures. At minimum, a hash ( $\$ \mathrm{self}-\mathrm{SQL}>$ ) needs to be filled with correct syntax for statements with the following keys (each of which references a sql statement):

\section{'CreateOil'}


creates a new oil. Takes three parameters (ID, Name, Description).

'CreateComponent'

creates a member of an oil. Takes 4 parameters (ID, OilNumber, ComponentNumber, WhichComponent)

\section{'DeleteComponents'}

removes all components from an oil. Takes 1 parameter (OilNumber)

\section{'DeleteOil'}

deletes an oil. Takes one parameter (ID)

\section{'SelectComponents'}

selects all components from an oil. Takes 1 parameter (OilNumber)

Option SQL statements can be added to sub-divide the data into classes. These include

\section{'SelectFamilies'}

select all 'Families'; arbitrary grouping of data into non-overlapping categories

\section{'SelectFamilyData'}

select data from all members of a family; Takes 1 parameter (ParameterID)

\section{'SelectUData'}

select component functional groups. Takes 1 parameter (speciesID), returns multiple rows

\section{'SelectUGroups'}

selects the unifac group parameters (main group ID, Q, R) for a unifac subgroup given the subgroup ID

\section{'SelectAij'}

select the interaction coefficient between two unifac main groups, given their IDs (in order).

\section{Required Functions}

There are also several functions that have to be implemented as well. They include:

\section{CreateID}

creates an ID for a given table- Takes 1 parameter (tablename)

\section{init}

callback from the super-class 'new' functions. This function must, at minimum, initialize data structures (see above), three class properties (UserName, Password, and ConnectionString [a legal DBI string]), and call the superclass init (i.e., \$self->SUPER::init);

Peter Meulbroek, meulbroek@wag.caltech.edu

$$
\text { AUTHOR }
$$

COPYRIGHT

copyright 2001 Peter Meulbroek, Richard P. Muller, William Goddard III

SEE ALSO

$\operatorname{perl}(1)$. 


\title{
8 Phase::Database::PostgreSQL
}

Perl front-end to a database of chemical properties

use Phase::Database;

SYNOPSIS

my $\$ \mathrm{f}=$ Phase::Database->new;

\section{DESCRIPTION}

\section{Summary}

Package Version \$Revision\$

Copyright

Copyright 2000, Peter Meulbroek

This program is free software; you can redistribute it and/or modify it under the terms of the

GNU General Public License as published by the Free Software Foundation; either version 2 of the License, or (at your option) any later version.

This program is distributed in the hope that it will be useful, but WITHOUT ANY

WARRANTY; without even the implied warranty of MERCHANTABILITY or FITNESS

FOR A PARTICULAR PURPOSE. See the GNU General Public License for more details.

You should have received a copy of the GNU General Public License along with this program; if not, write to the Free Software Foundation, Inc., 59 Temple Place, Suite 330, Boston, MA 02111-1307 USA

\section{NOTES}

postgresql is unique among the supported databases in that quoting of names and variables is quite important (by default, all names are lower case). This means that all the sql statements have to be properly quoted, else weird errors occur.

$$
\text { AUTHOR }
$$

Peter Meulbroek, meulbroek@wag.caltech.edu

\section{COPYRIGHT}

copyright 2001 Peter Meulbroek, Richard P. Muller, William Goddard III

\author{
SEE ALSO
}

$\operatorname{perl}(1)$. 


\section{Phase::EquationofState::AasbergPetersen}

The Aasberg-Petersen and Stenby CEOS

\section{SYNOPSIS}

Prediction of Thermodynamic Properties of Oil and Gas Condensate Mixtures

my $\$ \mathrm{db}=$ Phase::Database->new;

\$db->LoadOilByName("TwoComponent");

my $\$$ EOS $=$ Phase::EquationofState::AasbergPetersen->new(

$$
\text { ' } \mathrm{T} \text { ' => 200, }
$$$$
' P^{\prime}=>10,
$$

' $Z$ ' $=>$ Standard::List- $>$ new $(.5, .5)$,

'Database' $=>\$ d b$,

$$
) \text {; }
$$

DESCRIPTION

An new method for using CEOS to predict PVT properties based on the Aasberg-Petersen Stenby

\section{Algorithm Author}

Kim Aasaberg-Petersen and Erling Stenby

\section{Copyright}

Copyright 1999, 2001 Peter Meulbroek

This program is free software; you can redistribute it and/or modify it under the terms of the GNU General Public License as published by the Free Software Foundation; either version 2 of the License, or (at your option) any later version.

This program is distributed in the hope that it will be useful, but WITHOUT ANY WARRANTY; without even the implied warranty of MERCHANTABILITY or FITNESS FOR A PARTICULAR PURPOSE. See the GNU General Public License for more details.

You should have received a copy of the GNU General Public License along with this program; if not, write to the Free Software Foundation, Inc., 59 Temple Place, Suite 330, Boston, MA 02111-1307 USA

\section{History}

$\$ \log \$$

\section{0/01/30 17:03:30:}

Modified package for distribution. Note that the UNIFAC symbols were ripped out (and put in the UNIFAC package)

01/01/16 09:16:17

Ported the package to perl

\section{Keywords}

UNIFAC, activity, Phase Equilibrium, thermodynamics 


\section{FUNCTIONS}

MaxCarbonNumber

MaxCarbonNumber calculates the maximum carbon number required to fix the distribution calculated by pseudodistribution and that satisfies the conditions $\operatorname{mf}[\mathrm{n}]>=10 \hat{(-6)}$ and $\mathrm{n}<=$ 80 .

Syntax

MaxCarbonNumber(\$Alpha1, \$Alpha2, \$CnPlus)

\section{MoleFractionDistribution}

MoleFractionDistribution returns the mole fraction distribution that uses the parameters calculated by PseudoDistribution and MaxCarbonNumber.

Syntax

MoleFractionDistribution(\$Alpha1, \$Alpha2, \$CnPlus, \$MaxCn)

\section{MolecularWeightDistribution}

MolecularWeightDistribution calculates the molecular weights of the pseudo-components.

Syntax

MolecularWeightDistribution(\$CnPlus, \$MaxCn)

Am

Am calculates the AasbergPetersen mixture attraction parameter.

Syntax

\$EOS->Am()

Bm

Bm calculates the AasberPetersen mixture incompressible volume.

Syntax

\$EOS->Bm( $)$

LnPhi

LnPhi calculates the $\log$ [fugacity coefficient] of the mixture.

Syntax

\$EOS->LnPhi(\$v)

AUTHOR

Peter Meulbroek, meulbroek@wag.caltech.edu COPYRIGHT

copyright 2001 Peter Meulbroek, Richard P. Muller, William Goddard III

perl(1).

SEE ALSO 


\section{Phase::EquationofState::Generic}

\section{SYNOPSIS}

use Phase::EquationofState::Generic;

\section{DESCRIPTION}

Phase::EquationofState::Generic - common elements to cubic equations of state

The base class for all cubic equations of state. The class defines a couple of abstract methods (that must be implemented in derived classes)

- $\mathrm{Bm}$

The incompressible volume mix rule

Syntax: $\$ E O S->B m()$, where $\$ E O S$ is a valid, instanciated

Phase::EquationofState::Generic object.

- $\mathrm{Am}$

The attraction factor mixing rule

Syntax: \$EOS->Am (), where \$EOS is a valid, instanciated

Phase::EquationofState::Generic object.

- 1Coef

sets the Coefficients array

The Coefficients array is defined as $(\$ \mathrm{u}, \$ \mathrm{w})$, where $\mathrm{P}=\mathrm{R} \mathrm{T} /(\mathrm{v}-\mathrm{b})-\mathrm{a} /(\mathrm{v} * * 2$

$+u v b+w b * * 2)$

- LVolume

calculates the specific volume $\left(\mathrm{cm}^{* * 3} / \mathrm{mol}\right)$ of the liquid phase

- VVolume

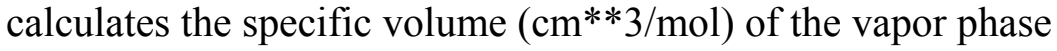

- LiquidInflection

Local minima (optional)

Liquid Inflection finds the smaller of the roots of $\mathrm{dP} / \mathrm{dv}$ equation (there are 0,1 or 2 of these roots) that is, the local minima of the $\mathrm{P}$ equation. This corresponds to the superheating maximum

- VaporInflection

Local maxima (optional)

Vapor Inflection finds the larger of the roots of $\mathrm{dP} / \mathrm{dv}$ equation (there are 0,1 or 2 of these roots) that is, the local maxima of the $\mathrm{P}$ equation. This corresponds to the supercooling minimum

- Pressure

Pressure, as a function of $\mathrm{t}, \mathrm{v}$, and $\mathrm{z}$

Syntax \$EOS->Pressure (), where \$EOS is a valid, instanciated Phase: : EquationofState: : Generic object.

- $\mathrm{K}$ 


\section{Equilibria coefficients}

$\mathrm{K}$ returns the equilibria coefficents (at equilibria, @y/@x, or @phiL/@phiV, where phi is the fugacity coefficient for each species in the mix

\section{FUNCTIONS}

- ChangeConditions

Call to change $\mathrm{P}$ (pressure), $\mathrm{T}$ (temperature), or $\mathrm{Z}$ (composition vector).

Syntax

\$EOS->ChangeConditions $(\{\mathrm{P}=\$ \mathrm{p}, \mathrm{T}=>\$ \mathrm{t}, \mathrm{Z}=>\$ \mathrm{z}\})>$

- avgMW

The average molecular weight of the mixture, calculated as @Z . @MW.

Syntax

\$EOS->avgMW()

AUTHOR

Peter Meulbroek, meulbroek@wag.caltech.edu COPYRIGHT

copyright 2001 Peter Meulbroek, Richard P. Muller, William Goddard III

$\operatorname{perl}(1)$.

SEE ALSO 


\section{Phase::Mathias}

Paul Mathias"s "Versatile Phase Equilibrium Equation of State"

SYNOPSIS

use Phase::EquationofState::Mathias;

use Phase::Database;

my $\$ \mathrm{db}=$ Phase::Database->new;

\$db->LoadOilByName("TwoComponent");

my \$EOS = Phase::EquationofState::Mathias->new(

' $\mathrm{T}$ ' $=>200$, $' P^{\prime}=>10$,

' $Z$ ' => Standard::List->new $(.5, .5)$,

'Database' $=>\$ d b$,

DESCRIPTION

Name

Phase::EquationofState::Mathias

Usage

Mathias implements the Mathias Equation of State

Limitation

Limited mixing rule

Package Author

Peter Meulbroek, 2000

Algorithm Author

Original Model by Paul Mathias,

Mathias Paul M., "A Versatile Phase Equilibrium Equation of State”, Industrial Engineering Chemical Process Design Development, 22(6), 385-391 (1983)

\section{Copyright}

Copyright 1997, 2000 Peter Meulbroek

This program is free software; you can redistribute it and/or modify it under the terms of the GNU General Public License as published by the Free Software Foundation; either version 2 of the License, or (at your option) any later version.

This program is distributed in the hope that it will be useful, but WITHOUT ANY WARRANTY; without even the implied warranty of MERCHANTABILITY or FITNESS FOR A PARTICULAR PURPOSE. See the GNU General Public License for more details. 
You should have received a copy of the GNU General Public License along with this program; if not, write to the Free Software Foundation, Inc., 59 Temple Place, Suite 330, Boston, MA 02111-1307 USA

\section{History}

\$Log\$

- $10 / 27 / 93$

created: all volumes check against STRAPP w.i. a few percent

- 11/17/97 22:32:50:

Modified original Package to include a new mixing rule for A (based on the unifac paramters. Note that this does not necessarily need the unifac package

- $\quad$ 00/01/30 17:03:30:

Modified package for distribution. Note that the UNIFAC symbols were ripped out (and put in the UNIFAC package)

- $\quad<01 / 01 / 16$ 09:16:17:>

Ported the package to perl

- $03 / 01 / 19$

moved from Standard::Matrix to Standard::List

- Keywords

UNIFAC, activity, Phase Equilibrium, thermodynamics

$$
\text { FUNCTIONS }
$$

\section{Am}

Am is the Mathais mix A

\section{Bm}

$\mathrm{Bm}$ is the Mathias mix B

\section{LnPhi}

LnPhi calculates the natural log of the fugacity coefficients of a multicomponent fluid, and returns an array of $\{$ LnphiL, LnphiV\}.

Peter Meulbroek, meulbroek@wag.caltech.edu

$$
\text { AUTHOR }
$$

COPYRIGHT

copyright 2001 Peter Meulbroek, Richard P. Muller, William Goddard III

SEE ALSO

perl(1). 


\section{Phase::EquationofState::PRKS}

The Predictive Redlich Kwong Soave equation of state

SYNOPSIS

Prediction of Thermodynamic Properties of Oil and Gas Condensate Mixtures

my $\$ \mathrm{db}=$ Phase::Database->new;

\$db->LoadOilByName(“TwoComponent”);

my \$EOS = Phase::EquationofState::PRKS->new(

' $\mathrm{T}$ ' $=>200$,

$$
' \mathrm{P}^{\prime}=>10,
$$

'Z' => Standard::List->new $(.5, .5)$,

'Database' $=>\$ d b$,

$$
) \text {; }
$$

\section{Copyright}

DESCRIPTION

Copyright 2002 Peter Meulbroek

This program is free software; you can redistribute it and/or modify it under the terms of the GNU General Public License as published by the Free Software Foundation; either version 2 of the License, or (at your option) any later version.

This program is distributed in the hope that it will be useful, but WITHOUT ANY WARRANTY; without even the implied warranty of MERCHANTABILITY or FITNESS FOR A PARTICULAR PURPOSE. See the GNU General Public License for more details.

You should have received a copy of the GNU General Public License along with this program; if not, write to the Free Software Foundation, Inc., 59 Temple Place, Suite 330, Boston, MA 02111-1307 USA

\section{History}

$\$ \log \$$

02/12/04 : created package

\section{Keywords}

UNIFAC, activity, Phase Equilibrium, thermodynamics

$$
\text { FUNCTIONS }
$$

Am

Am calculates the PRKS mixture attraction parameter.

\section{Bm}

Bm calculates the PRKS mixture incompressible volume.

\section{LnPhi}

LnPhi calculates the Log[fugacity coefficient] of the mixture. 
AUTHOR

Peter Meulbroek, meulbroek@wag.caltech.edu

COPYRIGHT

copyright 2001 Peter Meulbroek, Richard P. Muller, William Goddard III

SEE ALSO

Phase::EquationofState::Generic, Phase::Volume, perl(1) 


\section{Phase::EquationofState::VanderWaals}

Perl version of THE first Cubic Equation of State

SYNOPSIS

use Phase::EquationofState::VanderWaals;

my $\$ \mathrm{db}=$ Phase::Database->new;

\$db->LoadOilByNumber(27);

my \$EOS = Phase::EquationofState::VanderWaals->new (

$$
P=>10,
$$

$\mathrm{T}=>100$,

$\mathrm{Z}=>$ Standard::List- $>$ new $(.5, .5)$,

Database $=>\$ d b$

$$
) \text {; }
$$

DESCRIPTION

VanderWaals implements the Van der Waals cubic equation of state. Note that this is for historic interest only! The equation is not nearly as accurate as later work!

\section{Limitation}

Limited mixing rule

\section{Source}

The following was taken from Reid, Properties of Gases and Liquids, chapter 3 (p. 42-43).

\section{Equation}

The VdW eos is: $\mathrm{P}==\left(-\left(\mathrm{a} / \mathrm{v}^{* *} 2\right)\right)+(\mathrm{R} * \mathrm{~T}) /(\mathrm{v}-\mathrm{b})$

\section{Copyright}

Copyright 2000 Peter Meulbroek

This program is free software; you can redistribute it and/or modify it under the terms of the GNU General Public License as published by the Free Software Foundation; either version 2 of the License, or (at your option) any later version.

This program is distributed in the hope that it will be useful, but WITHOUT ANY WARRANTY; without even the implied warranty of MERCHANTABILITY or FITNESS FOR A PARTICULAR PURPOSE. See the GNU General Public License for more details.

You should have received a copy of the GNU General Public License along with this program; if not, write to the Free Software Foundation, Inc., 59 Temple Place, Suite 330, Boston, MA 02111-1307 USA

\section{History}

$\$ \log \$$

00/04/04 13:11:56 meulbroek: created package

\section{Keywords}

Phase Equilibrium, thermodynamics 
Note that this is only applicable for individual species!

\section{Functions}

\section{Bm}

calcluates the incompressible volume of a mixture $\mathrm{Bm}==\$ \mathrm{~B} . \$ \mathrm{Z}$

Am

calculates the attractive mixing parameter

\section{Pressure}

Pressure calculates the equilibrium pressure of a mixture. Syntax:

Pressure (mixObject, Temperature, volume)

\section{LnPhi}

LnPhi calculates the Log Fugacity coefficient of the mixture. Syntax: LnPhi (mixObject, volume)

\$EOS->LnPhi(\$v)

AUTHOR

Peter Meulbroek, meulbroek@wag.caltech.edu COPYRIGHT

copyright 2001 Peter Meulbroek, Richard P. Muller, William Goddard III

$\operatorname{perl}(1)$.

SEE ALSO 


\section{Phase::Flash}

SYNOPSIS

Liquid-Vapor phase equilibirum using a single stage flash algorithm.

use Phase::Flash;

DESCRIPTION

perl version of a Liquid-Vapor equilibrium code

Title

Single Stage Flash Calculation

\section{Summary}

This file implements the flash model of Rijkers and Heidemann, "Convergence Behavior of Single-Stage Flash Calculations", Equations of State: Theories and Applications, American Chemical Society, 1985.

\section{Package Version}

1.5

\section{Copyright}

Copyright 2000, Peter Meulbroek

This program is free software; you can redistribute it and/or modify it under the terms of the GNU General Public License as published by the Free Software Foundation; either version 2 of the License, or (at your option) any later version.

This program is distributed in the hope that it will be useful, but WITHOUT ANY WARRANTY; without even the implied warranty of MERCHANTABILITY or FITNESS FOR A PARTICULAR PURPOSE. See the GNU General Public License for more details.

You should have received a copy of the GNU General Public License along with this program; if not, write to the Free Software Foundation, Inc., 59 Temple Place, Suite 330, Boston, MA 02111-1307 USA

History

$\$ \log \$$

00/02/14 16 00:20 created package

01/02/02 11:41:42 ported to perl

\section{Keywords}

\section{FUNCTIONS}

$X$

returns the composition of the liquid phase

Y

returns the composition of the Vapor phase

\section{ChangeConditions}

Change conditions of the flash calculation.

\section{Syntax}

\$flash->ChangeConditions (\%opts), where $\%$ opts contains any/all of the following keys: (P, T, Z, X, Y). $\mathrm{P}$ is the new pressure, $\mathrm{T}$ is the new temperature, $\mathrm{Z}$ is the new 
composition, $\mathrm{X}$ is a guess for the new liquid, composition, and $\mathrm{Y}$ is a guess for the new vapor composition. If any of the values are not included, the current value is preserved.

\section{Density}

calculates the average density of a multiphase mixture, and returns the an array of [avg density, liquid density, vapor density]

\section{Syntax}

\$Flash->Density(\%opts)

\section{NewtonRalphsen}

NewtonRalphsen is an implementation of the Newton-Ralphsen root-finding algorithm.

Syntax: NewtonRalphsen ( $\$ \mathrm{f}, \$ t 0, \frac{\circ}{\circ} \mathrm{pts}$ ), where $\$ \mathrm{f}$ is a function object, $\$ \mathrm{t} 0$ is the starting value, and \%opts are any/all of the following keys \%opts $==$ (lowerbound, upperbound, MaxIterations)

\section{Flash}

the Flash method flashes the system to reach equilibrium using a conventional successive substitution method. It returns the number of iterations necessary to converge

\section{Flash Options}

(also valid as options to the class, passed as Options(<list-of-options $>$ ) in constructor)

\section{InitialFraction}

InitialFraction is an option that specifies the vapor fraction 'guess' for Flash. Default: InitialFraction $=>0.5$

\section{Precision}

Precision is an option that specifies the maximum Vapor Fraction iteration error allowed for the system to be considered converged. Default: Precision $=>1 \mathrm{e}-8$,

\section{MaxIterations}

MaxIterations is the number of global sucessive substitution calls allowed before the system returns a value. Default: MaxIterations $=>10$

\section{VolumeMaxIterations}

VolumeMaxIterations is the number of iterations the internal solve for vapor fraction is allowed (this occurs within each step solving for chemical equilibria. Default: VolumeMaxIterations $=>10$

\section{CompositionPrecision}

CompositionPrecision is an option that allows the user to specify convergance in the composition of each phase. When passed a scalar, the sum of deviations between iterations of all components in all phases must be less than that scalar. E.g., for a two component mix, $\mathrm{x} 1,1-\mathrm{x} 1,0+\mathrm{y} 1,1-\mathrm{y} 1,0+\mathrm{x} 2,1-\mathrm{x} 2,0+\mathrm{y} 2,1-\mathrm{y} 2,0<\mathrm{C}$

Defaults to 1

$\boldsymbol{K}$

$\mathrm{K}$ returns a guess at the equilibrium ratio, $\mathrm{y} / \mathrm{x}==\mathrm{LnPhi} * \mathrm{~L} / \mathrm{LnPhi}^{*} \mathrm{~V}$ Note that the value is cached for optimium performance; if the phases are changed external to the Phase::Flash object, the Phase::Flash::IsDirty flag must be set. Syntax: \$self->K. 
AUTHOR

Peter Meulbroek, meulbroek@wag.caltech.edu

COPYRIGHT

copyright 2001 Peter Meulbroek, Richard P. Muller, William Goddard III

SEE ALSO

$\operatorname{perl}(1)$. 


\section{Phase::ModifiedUnifac}

SYNOPSIS

use Phase::Database;

my $\$ \mathrm{db}=$ Phase::Database->new(whatever)

use Phase::ModifiedUnifac;

my $\$$ un $=$ Phase::Unifac->new (

Database $=>\$ d b$,

$\mathrm{T}=>$ \$temperature,

$\mathrm{Z}=>$ \$molefraction

) ;

DESCRIPTION

Perl library of UNIFAC routines to predict activity coefficents The unifac model, as presented by Larsen, Rasmussen, and Fredenslund, "A Modified UNIFAC group-contribution model for prediction of Phase Equilibria and Heats of mixing", Indus. Eng. Chem. Res, v. 26, pp. 2274-2286 (1985)

Author

Peter Meulbroek, 2002

Summary

calculate the activity coefficient of a mixture, the excess Gibbs free energy of a mixture, and the derivative of these properties w.r.t. composition (n)

\section{Copyright}

This program is free software; you can redistribute it and/or modify it under the terms of the GNU General Public License as published by the Free Software Foundation; either version 2 of the License, or (at your option) any later version.

This program is distributed in the hope that it will be useful, but WITHOUT ANY WARRANTY; without even the implied warranty of MERCHANTABILITY or FITNESS FOR A PARTICULAR PURPOSE. See the GNU General Public License for more details.

You should have received a copy of the GNU General Public License along with this program; if not, write to the Free Software Foundation, Inc., 59 Temple Place, Suite 330, Boston, MA 02111-1307 USA

History

\$Log\$

March 8, 2000:

created package

Dec 10, 2001: 
ported to perl

Dec 26, 2002:

after considerable mathematica work, added D [Gamma, $\mathrm{n}$ ] to replace the old (wrong) $D[$ Gamma, x] (which did not include cross-terms)

Jan 11, 2003 :

moved to Modified Unifac from Unifac. All functions and values compared to mathematica and the examples in Reid et al. $\left(5^{\text {th }}\right.$ ed), and within acceptable tolerances.

\section{Keywords}

volume, CEOS, phase, thermodynamic equilibrium, flash

\section{Limitation}

does NOT deal well with pressure.

\section{FUNCTIONS}

The following is an implementation of UNIFAC from Larsen et at. al, IECR v 26. The functions herein correspond to the functions in that paper

\section{initialization}

in initialization, the following properties are automatically calculated:

$-\mathrm{W}$

(eqn 6), used in combinatorial activity coefficient.

$$
\mathrm{W}=\operatorname{Norm}(\hat{\mathrm{r}}(2 / 3) \mathrm{Z})
$$

$-\mathrm{r}$ and $\mathrm{q}$

(eqn 15 and 16), molar volume and and contact numbers

- tau

(eqn 10) Boltzmann factors

$-\operatorname{theta}(\mathrm{j}, \mathrm{i})$

(eqn 9), local area fractions

$-\operatorname{theta}(\mathrm{i})$

\section{set_T}

(eqn 4), surface area fractions

sets the temperature to a new value and sets all dependant values

set_Z

sets the composition to a new vector, and sets all dependant values

gE

excess gibbs free energy at standard pressure

$g E c$

the combinatorial activity coefficient. Note that this actually returns $g e 0 c /(R \star T)$. From equation 7 .

\section{$g E r$}

the residual activity coefficient. Note that this actually returns $g E r /(R * T)$. From equation 8. 
equation 8-10.49

lngamma $=$ lngammac + lngammar

equation 8-10.50

lngammac $=1+\log (\$ w / \$ Z)-\$ w / \$ Z$, where $\$ w$ is defined above

equation??

lngammar $=$ ? ?

\section{Gamma}

The residual group activity coefficient. This function is not meant to be called on its own; it is called by lngammaR, the mixture residual activity coefficient

\section{GammaK}

The pure fluid group activity coefficient. This returns a matrix of residual group activity coefficients where the ith row represents the group activity coefficient in the fluid consisting of pure species $i$.

AUTHOR

Peter Meulbroek,meulbroek@wag.caltech.edu COPYRIGHT

copyright 2001 Peter Meulbroek, Richard P. Muller, William Goddard III

$\operatorname{perl}(1)$.

SEE ALSO 


\section{Phase::Unifac}

Perl library of UNIFAC routines to predict activity coefficents

use Phase::Database;

SYNOPSIS

my $\$ \mathrm{db}=$ Phase::Database->new(whatever);

use Phase::Unifac;

my \$un $=$ Phase::Unifac->new (

Database $=>\$ d b$,

$\mathrm{T}=>$ \$temperature,

$\mathrm{Z}=>$ \$molefraction

\section{) ;}

DESCRIPTION

The unifac model, as presented by Reid et al.

\section{Author}

Peter Meulbroek, 2000

substantial revisions, 2002-3

Summary

calculate the activity coefficient of a mixture, the excess Gibbs free energy of a mixture, and the derivative of these properties w.r.t. composition (n)

\section{Copyright}

This program is free software; you can redistribute it and/or modify it under the terms of the GNU General Public License as published by the Free Software Foundation; either version 2 of the License, or (at your option) any later version.

This program is distributed in the hope that it will be useful, but WITHOUT ANY WARRANTY; without even the implied warranty of MERCHANTABILITY or FITNESS FOR A PARTICULAR PURPOSE. See the GNU General Public License for more details.

You should have received a copy of the GNU General Public License along with this program; if not, write to the Free Software Foundation, Inc., 59 Temple Place, Suite 330, Boston, MA 02111-1307 USA

\section{History}

$\$ \log \$$

March 8, 2000

created package

Dec 10, 2001

ported to perl 
Dec 26, 2002

after considerable mathematica work, added D[Gamma, $\mathrm{n}]$ to replace the old (wrong)

$\mathrm{D}[\mathrm{Gamma}, \mathrm{x}]$ (which did not include cross-terms)

\section{Keywords}

volume, CEOS, phase, thermodynamic equilibrium, flash

\section{Limitation}

does NOT deal well with pressure.

\section{Source}

taken from Reid et al, Properties of gases and Liquids $\left(4^{\text {th }}\right.$ ed), later updated to $5^{\text {th }}$ addition

\section{Implementation}

the following is an equation-by equation implementation of Reid et al.s equations. Note that equations are specified in mathematica format (the original derivation / syntax checking format)

\section{eqn 8-10.52}

$\mathrm{r}=\mathrm{uNuk}(\mathrm{i})$. R;

$\mathrm{q}=\mathrm{uNuk}(\mathrm{i}) \cdot \mathrm{uQ}$;

\section{equation 8-10.49}

lngamma $=$ lngammac + lngammar

equation 8-10.50

$\operatorname{lngammac}=\log (\mathrm{Phi} / \mathrm{Z})+0.5^{*} \mathrm{z} * \mathrm{q} * \log ($ Theta $/ \mathrm{Phi})+1-\mathrm{Phi} / \mathrm{Z} *(\mathrm{Z}$. 1)

\section{equation 8-10.55}

$\ln \mathrm{G}$ - the group activity coefficient. Note that this is in a mixture;

$\ln G=\mathrm{uQ}^{*}(1-\log ($ Theta . Psi $)$ - Psi . (Theta / (Psi . Theta $\left.\left.)\right)\right)$;

\section{equation 8-10.55b}

$\ln \mathrm{G} 0$ the group activity coefficients for each species in the mixture

returns - matrix ( $\mathrm{n} \times \mathrm{m})$

\section{eqn 8-10.54}

Lngammar is the residual activity coefficient

Lngammar $=\operatorname{Sum}\left[\mathrm{uNu}(\mathrm{k}, \mathrm{i})^{*}(\log \mathrm{G}(\mathrm{k})-\log \mathrm{G}(\mathrm{k}, \mathrm{i})),\{\mathrm{k}, \mathrm{n}\}\right]$

returns - Vector(m)

G0e -

excess gibbs free energy at standard pressure (1 bar). Produced by the formula

$\mathrm{G} 0 \mathrm{e}==\mathrm{R} \mathrm{T} \log (\mathrm{x}$. gamma $)$

\section{dlnGc}

function to produce the derivative of (the combinatorial part of the excess gibbs free energy) with respect to mole fraction $(\mathrm{x})$.

returns: a list of derivative components (\$m long) 


\section{dLogGamma}

function to return the derivative of the activity coefficient

$$
\text { AUTHOR }
$$

Peter Meulbroek, meulbroek@wag.caltech.edu

\section{COPYRIGHT}

copyright 2001 Peter Meulbroek, Richard P. Muller, William Goddard III

$\operatorname{perl}(1)$

SEE ALSO 


\section{Phase::VaporPressure}

Estimate the vapor pressure of a mixture

SYNOPSIS

use Phase::VaporPressure;

my $\$ \mathrm{db}=$ Phase::Database->new;

\$db->LoadOilByName(“TwoComponent”);

my \$VP = Phase::VaporPressure- $>$ new $($ database $=>\$ d b)$;

\$VP->P(200);

\section{DESCRIPTION}

VaporPressure returns a vector of the (pure substance) vapor pressure of the mixture.

Taken from Reid, Prausnitz and Poling, The Properties of Gases and Liquids, (4 ${ }^{\text {th }}$ ed) 1987, p.207. Their equation was originally derived by Lee and Kesler (AIChE 21., p. 510 [1975]).

The original equation took Tr and gave Pvpr, so I modified it... One note: Reid recomends that the $w$ used be calculated, not taken from a tabulated source, for consistancy with $P c$, etc. Since this is ONLY to be used as an inital guess for $K$, I am just going to use the $w$ I have, which are from experimental values...

\section{FUNCTIONS}

\section{new}

create a new Phase: : VaporPressure object. Called with a valid Phase: : Database object

\section{Syntax}

Phase::VaporPressure- $>$ new $($ database $=>\$ d b)$;

\section{$<$ Data Methods>}

each of these functions returns a data member:

Ac

The accentric factor vector (for each species)

$T c$

The critical temperature vector (for each species)

$P c$

The critical pressure vector (for each species)

\section{$P$}

calculate vapor pressure of a mixture at a given $\mathrm{T}$

\section{syntax}

\$VP->P(\$T) 
AUTHOR

Peter Meulbroek, meulbroek@wag.caltech.edu

COPYRIGHT

copyright 2001 Peter Meulbroek, Richard P. Muller, William Goddard III

SEE ALSO

$\operatorname{perl}(1)$. 


\section{Phase::Volume}

Calculate specific volumes for cubic equations of state

SYNOPSIS

use Phase::Volume;

DESCRIPTION

Title

Generic Volume Solution

Author

Peter Meulbroek, 2000

Summary

Calculate the solution to a generic cubic equation of state

\section{Context}

Phase'Volume'

\section{Package Version}

0.99

\section{Copyright}

Copyright 2000, Peter Meulbroek

This program is free software; you can redistribute it and/or modify it under the terms of the GNU General Public License as published by the Free Software Foundation; either version 2 of the License, or (at your option) any later version.

This program is distributed in the hope that it will be useful, but WITHOUT ANY WARRANTY; without even the implied warranty of MERCHANTABILITY or FITNESS FOR A PARTICULAR PURPOSE. See the GNU General Public License for more details.

You should have received a copy of the GNU General Public License along with this program; if not, write to the Free Software Foundation, Inc., 59 Temple Place, Suite 330, Boston, MA 02111-1307 USA

\section{History}

\$Log\$

Jan 4, 2000: created package

Keywords volume, CEOS, phase, thermodynamic equilibrium, flash

\section{Mathematica Version}

2.2

\section{Limitation:}

CEOS

To Solve the Cubic equation of state for volume with a 'traditional' solver (as opposed to using the built-in "Roots" function of mathematica), the function needs to be put into 
polynomial form. This section demonstrates that procedure for the generic equation. The solution to the generic can be applied to specific CEOS by specifying the $\{\mathrm{u}, \mathrm{w}\}$ pair.

\section{FUNCTIONS}

\section{LiquidVolume}

LiquidVolume returns the volume of the liquid phase

\section{Syntax}

\$self->LiquidVolume()

VaporVolume

VaporVolume returns the volume of the vapor phase

\section{Syntax}

\$self->VaporVolume()

\section{Coefficients}

Coefficients calculates the coefficients of a cubic EOS as a function of $\{\mathrm{u}, \mathrm{w}\}$, where $\mathrm{P}==$ $\mathrm{R} T /(\mathrm{v}-\mathrm{b})-\mathrm{a} /(\mathrm{v} * \star 2+\mathrm{uvb}+\mathrm{wb} * 2)$.

Syntax: Coefficients $(\$ \mathrm{u}, \$ \mathrm{w}, \$ \mathrm{P}, \$ \mathrm{~T}, \$ \mathrm{a}, \$ \mathrm{~b})$

\section{VRoots}

VRoots returns the volume predicted of a cubic equation of state as a function of the coefficients of that CEOS.

Syntax:

$\operatorname{VRoots}[\{\mathrm{c}[0], \mathrm{c}[1], \mathrm{c}[2]\}]$;

\section{Extrema}

Extrema returns the min and max of the CEOS fn when given the coefficients list produced by coefficients. This is useful to get two distinct volumes in the 1 real-root section.

Syntaxt:

Extrema (a0, a1, a2) where the cubic equation is

$\operatorname{Sum}[\mathrm{a}[\mathrm{i}] \times \hat{\mathrm{i}},\{\mathrm{i}, 0,3\}]$, and $\mathrm{a}[3]==1$

$\boldsymbol{K}$

$\mathrm{K}$ is the equilibrium ratio for the Van der Waals cubic equation of state.

Syntax:

$\mathrm{K}(\$ 1 \mathrm{EOS}, \$ \mathrm{vEOS})$

AUTHOR

Peter Meulbroek, meulbroek@wag.caltech.edu

COPYRIGHT

copyright 2001 Peter Meulbroek, Richard P. Muller, William Goddard III

$\operatorname{perl}(1)$.

SEE ALSO 


\section{Phase::Washing}

use Phase::Washing;

$$
\text { SYNOPSIS }
$$

\section{DESCRIPTION}

perl function to 'wash' a mixture with a fluid, a.k.a. "gas washing", "evaporative fractionation", etc.

Title

Washing: A module that encapsulates washing a fluid with another

Author

Peter Meulbroek, 2000

Summary

Given a fluid at a $\mathrm{P}$ and $\mathrm{T}$, wash that fluid with another, given fluid. Return the exiting compostion

History

\$Log\$

March 22, 2000:

2001/12/12:

created package

ported to perl

\section{Keywords}

volume, CEOS, phase, thermodynamic equilibrium, flash

\section{Copyright}

Copyright 2000, Peter Meulbroek

This program is free software; you can redistribute it and/or modify it under the terms of the GNU General Public License as published by the Free Software Foundation; either version 2 of the License, or (at your option) any later version.

This program is distributed in the hope that it will be useful, but WITHOUT ANY WARRANTY; without even the implied warranty of MERCHANTABILITY or FITNESS FOR A PARTICULAR PURPOSE. See the GNU General Public License for more details.

You should have received a copy of the GNU General Public License along with this program; if not, write to the Free Software Foundation, Inc., 59 Temple Place, Suite 330, Boston, MA 02111-1307 USA

$$
\text { FUNCTIONS }
$$

\section{Wash}

Wash calculates the constant volume equilibrization of a mixture with a flow, y, with outflow determined by a flow vector $\mathrm{g}$.

the system is washed with the material vector \$fluid_comp, changes the \$sys composition, and returns a vector of the removed material

\section{Syntax}

\$self->Wash(\$fluid_comp, \$flow_mask) 
AUTHOR

Peter Meulbroek, meulbroek@wag.caltech.edu

COPYRIGHT

copyright 2001 Peter Meulbroek, Richard P. Muller, William Goddard III

SEE ALSO

$\operatorname{perl}(1)$. 


\section{Phase::WilsonK}

Perl library for initial guess for a mixture"s equilibrium ratio

\section{SYNOPSIS \\ DESCRIPTION}

This function is taken from Wilson, "A Modified Redlich-Kwong EOS, Application to General Physical Data Calculations", paper 15C, presented at the Annual AIChE Meeting in Cleveland, Ohio, May 4 - 7, 1968

\section{Package Version}

0.1

\section{Copyright}

Copyright 2000, Peter Meulbroek

This program is free software; you can redistribute it and/or modify it under the terms of the GNU General Public License as published by the Free Software Foundation; either version 2 of the License, or (at your option) any later version.

This program is distributed in the hope that it will be useful, but WITHOUT ANY WARRANTY; without even the implied warranty of MERCHANTABILITY or FITNESS FOR A PARTICULAR PURPOSE. See the GNU General Public License for more details.

You should have received a copy of the GNU General Public License along with this program; if not, write to the Free Software Foundation, Inc., 59 Temple Place, Suite 330, Boston, MA 02111-1307 USA

\section{History}

- 00/02/10 meulbroek

Created package

- $01 / 11 / 27$

moved only function to Phase::WilsonK

\section{Keywords}

Phase Equilibrium, utilities

\section{Mathematica Version}

3.0

\section{WilsonK}

WilsonK gives a list of Wilson"s estimation for equilibrium K"s in hydrocarbon mixtures.

$$
\text { AUTHOR }
$$

Peter Meulbroek, meulbroek@wag.caltech.edu

$$
\text { COPYRIGHT }
$$

copyright 2001 Peter Meulbroek, Richard P. Muller, William Goddard III

SEE ALSO

$\operatorname{perl}(1)$. 


\section{Standard::Function}

use Standard::Function;

\section{SYNOPSIS}

my $\$$ sum $=\operatorname{sub}\left\{\operatorname{my} \$\right.$ tot $=0 ; \operatorname{map}\left\{\$\right.$ tot $\left.+=\$ \_\right\} @ ;$ return $\$$ tot; $\}$

my $\$$ fn $=$ Standard::Function->new $(\$ s u m) ;$

$\$ f n->f(2,3,4)==9$

\section{DESCRIPTION}

a package that forms an abstract parent class for a numeric function. It provides 2 methods: $\mathrm{f}$ and df, each of which take 1 argument, and return the function and the derivative of the function at the argument.

Note that this is used to pass 'functionals'

AUTHOR

Peter Meulbroek, meulbroek@wag.caltech.edu

\section{COPYRIGHT}

copyright 2001 Peter Meulbroek, Richard P. Muller, William Goddard III

perl(1).

SEE ALSO 


\section{Standard::List}

Perl extension that is an abstract heirarchical mathematical object

use Standard::List;

$$
\text { SYNOPSIS }
$$

my $\$ \mathrm{v}=$ Standard::List->new $(1,2,3)$; \# a row vector

my $\$ \mathrm{~m}=$ Standard::List->new $([1,2,3],[4,5,6],[7,8,9])$; \# matrix

DESCRIPTION

Standard::List is an extention of the concept of a perl list. It gives me the ability to create some member functions. Note that it is written in pure perl, and hence VERY, VERY slow. I have been meaning to re-write the class as

1. a $\mathbf{C}$ extension

2. a c++ class

3. a pdl (perl data language) wrapper

4. (your idea goes here)

for about 4 years.

Copyright Copyright 2000, Peter Meulbroek

This program is free software; you can redistribute it and/or modify it under the terms of the GNU General Public License as published by the Free Software Foundation; either version 2 of the License, or (at your option) any later version.

This program is distributed in the hope that it will be useful, but WITHOUT ANY WARRANTY; without even the implied warranty of MERCHANTABILITY or FITNESS FOR A PARTICULAR PURPOSE. See the GNU

General Public License for more details.

You should have received a copy of the GNU General Public License along with this program; if not, write to the Free Software

Foundation, Inc., 59 Temple Place, Suite 330, Boston, MA 02111-1307 USA

\section{History}

EXPORT

None by default. 


\section{FUNCTIONS}

\section{Overloaded operators.}

Though it probably adds some overhead, I overloaded most perl operators, so that things such as $\$ A+\$ B$ make sense. Note that all operators are recursive. These include:

,

stringize a List (see below)

'=='

compare Lists piecewise

'eq'

string-compare Lists piecewise

'+

add two lists

'neg'

negate each element of a list (as in, $-\$ b$ )

,-'

subtract one list from another

,*,

multiply lists

,

divide lists

',

calculate the dot (inner) product of two lists

'sqrt'

take the square root of the list $=$ item ${ }^{*} * *$,

raise a list to a (scalar) power

'exp'

take the natural exponent of the list

'log'

take the natural $\log$ of the list

'abs'

return the absolute value of the list

'<'

find out if one list is piecewise less than another. Note that, if $\$ a$ and $\$ b$ are lists, it may very well be the case that $\operatorname{not}(\$ a<\$ b)$ and not $(\$ b<\$ a)$ and not $(\$ a==\$ b)$

Note that a lot of the following functions are designed to produce results akin to Mathematica, which is kind of wierd how it handles lists.

\section{Functions}


create a new object, optionally given intialization information

examples

\section{Standard::List->new; \# create an empty tensor}

Standard::List->new $(1,2,3)$; \#create a 3 vector

Standard::List->new $([1,2,3])$; \#create a 3x1 matrix

Standard::List->new([1,2,3],[4,5,6]); \# create a 3x2 (row-major) tensor

Standard::List->new $([1,2,3],[4,5,6],\{$ Direction $=>1\})$; \# create a $3 \times 2$ (column-major) tensor

\section{Stringize}

stringize is a function that returns a string (printable) representation of a list. It is called recursively for nested lists

\section{IdentityMatrix}

create an identity matrix; i.e., where the most-major (leftwise) diagonal is one, all other entries are zero. E.g., $\mathrm{I}(2)==[[1,0],[0,1]], \mathrm{I}(2,3)==[[1,0,0],[0,1,0]]$

Wrap

add a layer around the current list (e.g., turn a vector into a $1 \mathrm{x} \mathrm{n}$ matrix). This is mainly used for correct matrix and vector operations

Unwrap

do the opposite; take out the outer layer of a matrix example

$$
\operatorname{Unwrap}([[1,2,3]])==([1,2,3])
$$

Unwrap $([[1,2,3],[4,5,6]])==[1,2,3]$ \# note that we've lost information!

\section{RowMatrix}

take a Row vector, and replicate it to be a matrix.

\section{E.g.}

RowMatrix $([[1,2,3]], 3)==[[1,2,3],[1,2,3],[1,2,3]]$. Note that without a count argument, RowMatrix will produce a symmetric matrix.

\section{ColumnMatrix}

take a column vector, and replicate it to be a matrix. E.g., ColumnMatrix $([[1,2,3]]$, $3)=[[1,1,1],[2,2,2],[3,3,3]]$. Note that without a count argument, ColumnMatrix will produce a symmetric matrix.

\section{Map}

like mathematica 'map': allows a function to be 'mapped' on a list at any level.

E.g.,

$\operatorname{Map}(\$ f n, \$ l i s t, 2)$, returns $[\& \$ f n(1$ ist $-[0]), \& \$ f n($ list- $>[1]) \ldots]>$

\section{Entry}

get an entry from a matrix. Entry $(\$ \mathrm{i}, \$ \mathrm{j})$ gives the ith row, jth column for a row-major matrix, jth row ith column for a column-major tensor

\section{Push}

add @_to the List, as in 
\$List->Push $(1,2,4)$

Col

helper function to return a column of a two-dimensional structure. Note that access is by copy, NOT by reference. We need to go to a more sophisticated scheme to do the latter..

\section{Dimensions}

dimensions of the List (assumes symmetry, i.e., that all rows are the same length)

\section{Length}

return the length of the outer list

\section{Rows}

return a list of rows in the matrix; note that these rows are referential; change them and change the original matrix!

Transpose

return a new List with exchanged row and columns

\section{Copy}

a recursive (deep) copy

\section{DeepEqual}

compare two List to see if they are identical. Note that ' $==$ ' is overloaded to DeepEqual

\section{Apply}

reduce the outer-most list by 'replacing' it with a function, as in the mathematica function

E.g

$\$ \mathrm{~m}=$ Standard::List- $>$ new $([1,2,3])$;

$\$ \mathrm{n}=\operatorname{Apply}($ plus, $\$ \mathrm{~m})==6$

Nest

apply a function to the lowest levels of two identically-shaped tensors.

Syntax:

Nest(\$lhs, \$rhs, \$fn) \#for a binary fn

Nest ( $\$ l h s, \$ f n)$ \# for a unary function

$v D o t$

Norm

returns a List where the sum of all elements is one

\section{Diagonal}

return the principle diagonal of the List supplied. If an integer argument is passed, return the offset diagonal.

$\$ \mathrm{f}=[$

$$
\text { E.g.: }
$$

$\left[\begin{array}{lll}1 & 2 & 3\end{array}\right]$

$\left[\begin{array}{lll}4 & 5 & 6\end{array}\right]$

$\left[\begin{array}{lll}7 & 8 & 9\end{array}\right]$ 
$\$ f->$ Diagonal $==\left[\begin{array}{lll}1 & 5 & 9\end{array}\right]$

$\$ f->\operatorname{Diagonal}(1)==\left[\begin{array}{ll}4 & 8\end{array}\right]$

$\$ f->$ Diagonal $(-1)==[26]$

AUTHOR

Peter Meulbroek, meulbroek@wag.caltech.edu

\section{COPYRIGHT}

copyright 2001 Peter Meulbroek, Richard P. Muller, William Goddard III

$\operatorname{perl}(1)$.

SEE ALSO 


\section{Viscosity::Chung}

Perl extension to calculate viscosity of a gas mixture using the Chung method

use Viscosity::Chung;

SYNOPSIS

use Phase::Database;

my $\$ \mathrm{db}=$ Phase::Database->new;

\$db->LoadOilByName(“TwoComponent”);

my $\$ E O S=$ Phase::Equationofstate::AaasbergPetersen->new(

$\mathrm{T}=>100$,

$$
P=>10,
$$

Database $=>\$ d b$

) ;

my $\$$ Visc $=$ Viscosity::Chung->new (

Database $=>\$ \mathrm{db}$, $\mathrm{T}=>100$,

$\mathrm{EOS}=>\$ \mathrm{EOS}$,

\section{DESCRIPTION}

\section{Title}

Chung et al. Viscosity Solution

This section adapts the work of Chung et al., as reported by Reid, Prausnitz, Properties of Gases and Liquids, $\left(4^{\text {th }}\right.$ ed) 1985.

\section{Copyright}

Copyright 2001, Peter Meulbroek

This program is free software; you can redistribute it and/or modify it under the terms of the GNU General Public License as published by the Free Software Foundation; either version 2 of the License, or (at your option) any later version.

This program is distributed in the hope that it will be useful, but WITHOUT ANY WARRANTY; without even the implied warranty of MERCHANTABILITY or FITNESS FOR A PARTICULAR PURPOSE. See the GNU General Public License for more details.

You should have received a copy of the GNU General Public License along with this program; if not, write to the Free Software Foundation, Inc., 59 Temple Place, Suite 330, Boston, MA 02111-1307 USA

\section{History}

- $01-11-28$ 
created package

- 03-01-20

ported to Standard::List

\section{FUNCTIONS}

\section{Viscosity}

Calculates the viscosity of a mixture.

\section{Syntax}

\$visc->Viscosity(\$volume)

$$
\text { AUTHOR }
$$

Peter Meulbroek, meulbroek@wag.caltech.edu

$$
\text { COPYRIGHT }
$$

copyright 2001 Peter Meulbroek, Richard P. Muller, William Goddard III

SEE ALSO

$\operatorname{perl}(1)$. 


\section{Viscosity::Generic}

Abstract Perl extension to calculate viscosity of a mixture

SYNOPSIS

use Viscosity::MyPackage;

DESCRIPTION

\section{Title}

Generic Viscosity Solution

In order to implement this package, use a derived instance (such as Viscosity::Chung)

\section{Copyright}

Copyright 2001, Peter Meulbroek

This program is free software; you can redistribute it and/or modify it under the terms of the GNU General Public License as published by the Free Software Foundation; either version 2 of the License, or (at your option) any later version.

This program is distributed in the hope that it will be useful, but WITHOUT ANY WARRANTY; without even the implied warranty of MERCHANTABILITY or FITNESS FOR A PARTICULAR PURPOSE. See the GNU General Public License for more details.

You should have received a copy of the GNU General Public License along with this program; if not, write to the Free Software Foundation, Inc., 59 Temple Place, Suite 330, Boston, MA 02111-1307 USA

\section{History}

Jan 4, 2000

created package

2001-11-14

ported to perl

Peter Meulbroek, meulbroek@wag.caltech.edu

AUTHOR

COPYRIGHT

copyright 2001 Peter Meulbroek, Richard P. Muller, William Goddard III

SEE ALSO

$\operatorname{perl}(1$ 


\section{Processing Geologic Cross Sections to Estimate Hydrocarbon Production, Migration, and Chemistry}

This manual was prepared by L. M. Cathles, Department of Earth and Atmospheric Sciences, Cornell University, Ithaca, NY, 14853. It describes software he has written over several years to process geologic data to determine the geologic history of an area, and to solve for the thermal, maturation, and fluid flow history of the section. Advanced chemical capabilities have been added under funding from the DOE and in collaboration with scientists at the California Institute of Technology. As agreed under the DOE Advanced Chemistry Basin Modeling Project Grant (DE-AC26-99BC15217) to Cal Tech, Cornell, and GeoGroup Inc., the software developed is hereby released to the public domain. This manual describes the operation of the software. We have tested it as extensively as time permitted, but there undoubtedly remain bugs that will need fixing. Please report any problems to cathles@geology.cornell.edu. Portions of this software are Copywright (C)1991-4 MicroAPL Ltd., July 3, 1996. The software will be maintained as described in Section XII (p.338)

\section{Introduction}

There is a need for models that operate on a PC, are simple to use, have advanced chemical capabilities, and have the ability to plot all the chemical variables in an efficient fashion. Such models allow more thorough investigation of chemical data, and hopefully will allow broader and better use of such data.

The $\boldsymbol{B a s i n} \boldsymbol{L} \boldsymbol{A} \boldsymbol{B}$ software described here provides a simple and efficient way to capture the present state of a sedimentary basin and to infer, from the observed sedimentation pattern, the way it evolved to reach its present state. The software provides tools to infer and test the thermal history of the section and calculate the volumes of petroleum generated. The chemistry of the petroleum and its phase state can also be computed.

Basin $L A B$ calculates:

- Isostasy

- $\quad$ Effective stress

- $\quad$ Porosity as a function of effective stress

- $\quad$ Salt diapirism (specified or inferred)

- $\quad$ Faulting

- Erosion

- Surface temperature as a function of water depth and the position of the ocean
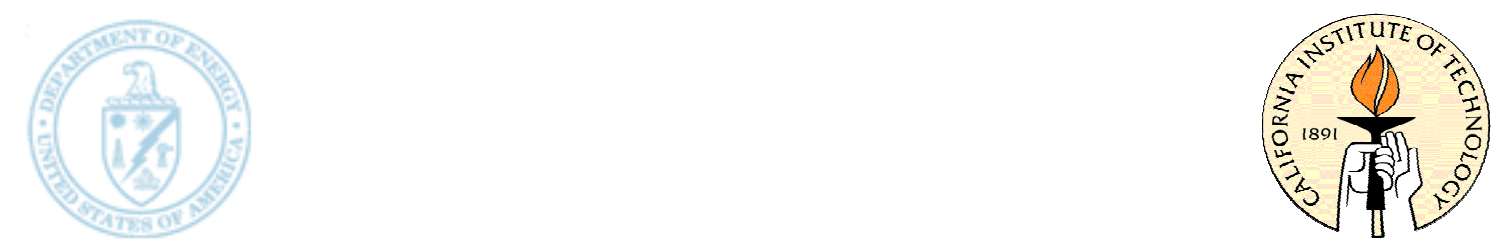
thermocline

- Heat flow from changes in sediment thickness and water depth

- Temperature as a function of sedimentation rate, heat flow, porosity, readiogenic heat generation, and pore fluid composition

- Vitrinite Ro reflectance or a number of vitrinite types

- $\quad$ Maturity from other indicators (hopane, sterand, diamondoid)

- Hydrocarbon maturation (fraction) and volumes of oil and gas generated (from TOC and kerogen type)

- The rate and chemistry of hydrocarbon generation

- $\quad$ Primary petroleum migration

- Secondary petroleum migration

- $\quad$ The phase state of migrating hydrocarbons

- Methane and $\mathrm{CO}_{2}$ washing

In addition $\boldsymbol{B a s i n} \mathbf{L} \boldsymbol{A B}$ :

- Compares observed temperature and vitrinite reflectance data to the calculated profiles

- Provides interactive editing of all model parameters and basin data

- Allows modifications and user specification of maturation kinetic parameters

- Includes extensive visualization tools

- Provides for archival and retrieval of all input and computed data

Special characteristics of $\boldsymbol{B} \boldsymbol{a} \sin \boldsymbol{L} \boldsymbol{A} \boldsymbol{B}$ include:

- $\quad$ Properties (such as thermal conductivity, permeability, heat generation, etc.) are related algorithmically to geological parameters that are known or inferred from the section description (such as lithology, fault location and movement, fault gauge ratio, etc).

- The geological history of the section (salt diapirism, fault movement, etc.) is inferred from the sedimentation pattern and user-specified erosion.

- Decompaction is based on user-specified of the top of overpressure location in the present-day section and it characterization as fixed or mobile.

- $\quad$ Estimates of fluid source terms due to compaction, maturation reactions, and heating are obtained from backstripping and a pseudo-2D determination of the thermal history of the section. These fluid source terms are then used in the 2D finite element solutions of the pressure equation.

- Convection (if requested) is calculated using a separate stream function equation.

- $\quad$ Scratchpads allow the user to investigate important material properties.

- An analysis is carried out in stages and saved as it is made.

- All variables are accessible to view in a flexible fashion (shades on the section, profiles through the section, timeseries at a point in the section, fluxes across surfaces, etc.) at all points of the analysis.

Five flow charts provide diagram Basin $\boldsymbol{L} \boldsymbol{A B}$ 's execution (pages 168, 232, 285, 293 and 315). Short descriptions with links to more extensive documentation of all user input 
files are provided in an appendix (p.339). 


\section{Getting Started}

To start, set up a file structure, and double click on the BasinL $\boldsymbol{A B}$ executable.

\section{A. File Structure}

The file structure is shown in Figure 2.

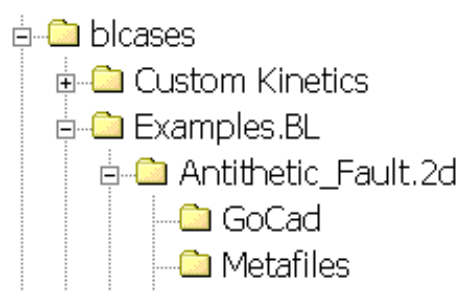

Figure 2. The required file structure.

The top-level directory is a folder called blcases. Within this folder there can be any number of $\boldsymbol{B a s i n L A B}\left({ }^{*}\right.$.BL) cases. Some of these can be a set of $2 \mathrm{D}$ section lines defining a 3D case. (BasinL $\boldsymbol{A B}$ has a 3D assembly capability, but this is not part of the DOE public domain release.) Alternatively a *.BL folder may simply contain an unrelated set of $2 \mathrm{D}$ sections. We will consider such a set of sections in the Examples.BL folder in this manual.

A GoCad folder in each *.2D line contains graphic input files. Sections are most easily input from graphic files. The graphics input files can be from *.dxf files from Canvas of GoCAD section files.

A Metafiles folder in each *.2D line holds all graphics produced during an analysis.

The Custom Kinetics folder in the blcases folder (conveniently placed at the same level as the *.BL cases) contains all maturation and maturity kinetic models.

\section{B. Start BasinLab}

Start Basin $\boldsymbol{L} \boldsymbol{A B}$ by double clicking the Basin $\boldsymbol{L} \boldsymbol{A B}$ icon. The system will request that you select the blcases folder to establish the path to the file structure you have just set up. Do so as illustrated in Figure 3.

The result will be the workspace shown (collapsed) in Figure 4. All section processing and modeling is carried out using menu commands on the toolbar at the top of this workspace. 


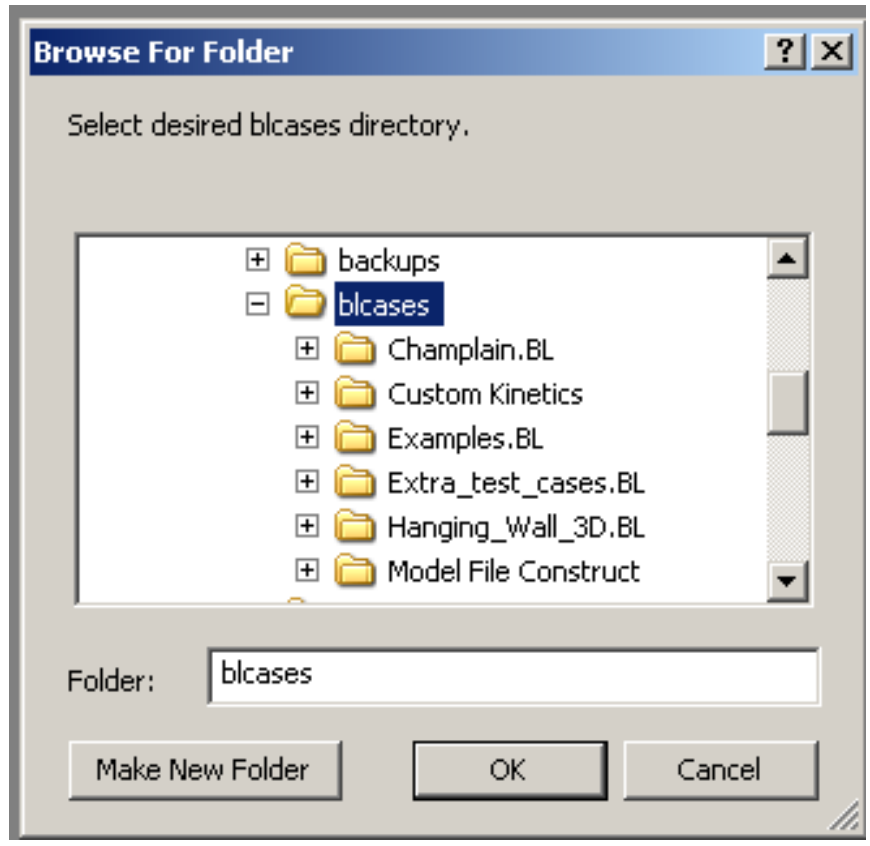

Figure 3. Select the blcases folder to establish the path to the desired file structure.

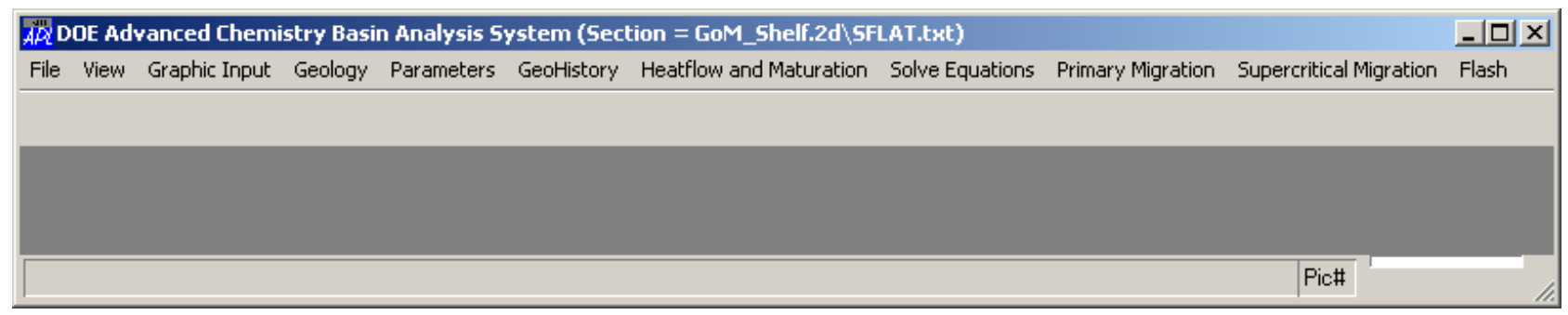

Figure 4. A resized version of the $\boldsymbol{B} \boldsymbol{a} \sin \boldsymbol{L} \boldsymbol{A} \boldsymbol{B}$ workspace. A $2 \mathrm{D}$ section is processed using the menus on the top toolbar of the workspace.

\section{Exit BasinLAB}

BasinL $\boldsymbol{A B}$ is exited using the File: Exit menu item.

\begin{tabular}{|l|}
\hline File View Graphic Input \\
New Section Project \\
Open Section Project \\
\hline Save CaseParameters \\
\hline Exit \\
\hline Set Path to blcases \\
Display all paths \\
\hline
\end{tabular}




\section{Section Input}

A $2 \mathrm{D}$ section may be input in several ways. The easiest way is from a Canvas *.dxf file. Input is also possible from a GoCAD section file. Once input, the section is digitized and lithologies added using $\boldsymbol{B a s i n} \boldsymbol{L} \boldsymbol{A B}$ tools. The file is stored as a "short flat file" or SFLAT.txt file. The SFLAT file is starting file for all processing and modeling.

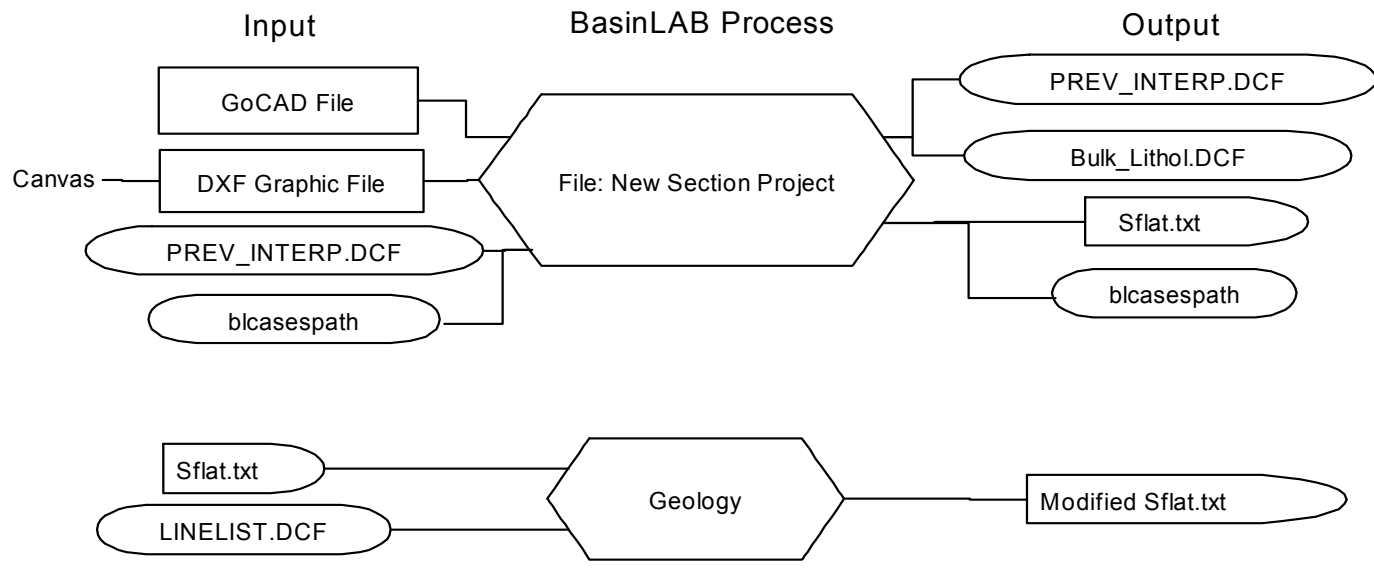

Chart 1. Input of a graphic section from a graphic input file or from an existing Sflat file. Tools provided (discussed below) allow graphic GoCAD or *.dxf files to be converted to the sflat file that is the basis of all further modeling. In this and all subsequent charts, rectangles enclose ascii files input by the user, rectangles with rounded edges indicate APL system files prepared (and readable) by BasinLab, and rectangles with one rounded side and a square left side indicate ascii files prepared by BasinLAB. Chart 2 is on page 232. Paths to all input files are recorded in blcasespath, an APL file that resides with the application.

\section{A. An Existing Section}

Distinction is made between a new and existing section project. If the Open Section Project menu item (Figure 5) is selected, the path to the case currently in the workspace is displayed as shown in Figure 6.

Figure 5. Opening an existing section.

\begin{tabular}{|l|}
\hline File View Graphic Input \\
New Section Project \\
\hline Poen Section Project \\
\hline Save CaseParameters \\
\hline Exit \\
\hline Set Path to blcases \\
Display all paths \\
\hline
\end{tabular}

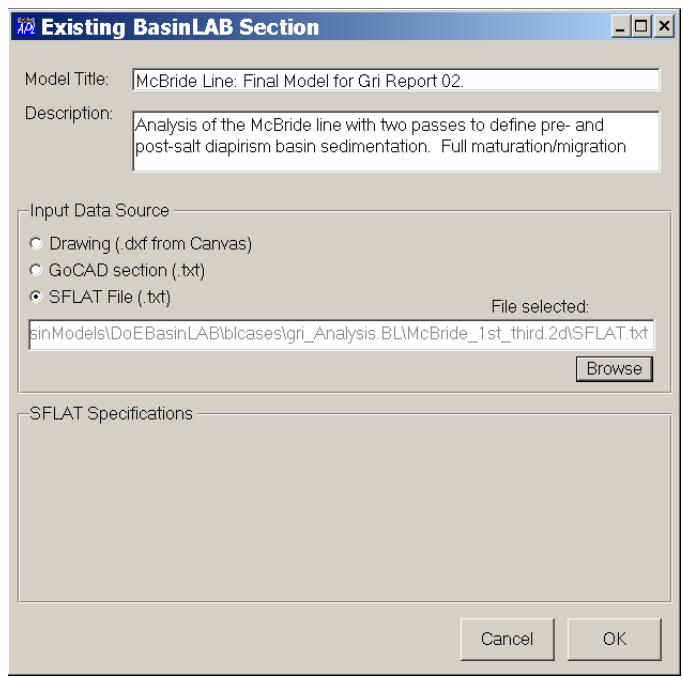


The existing file is the SFLAT file of the McBride line. If this is the desired section nothing further need be done. The case description could be changed. The changes will be saved if the form is closed using the OK button. Once the form is closed the section can be processed using the Geohistory menus, or examined using the Geology: Edit/Plot Spreadsheet or Geology: Graphic Edit menus.

A different (existing) case can be selected using the browse button in Figure 5. For example you could open a *.dxf input graphic for the Fault test.2d example. Select Drawing (*.dxf from Canvas) in Figure 5 as the Input Data Source, click the Browse button, and select Examples.BL|Faults_test.2d $\mid$ GoCad|Faultsslt.dxf file. The windows in Figure 6 will appear.

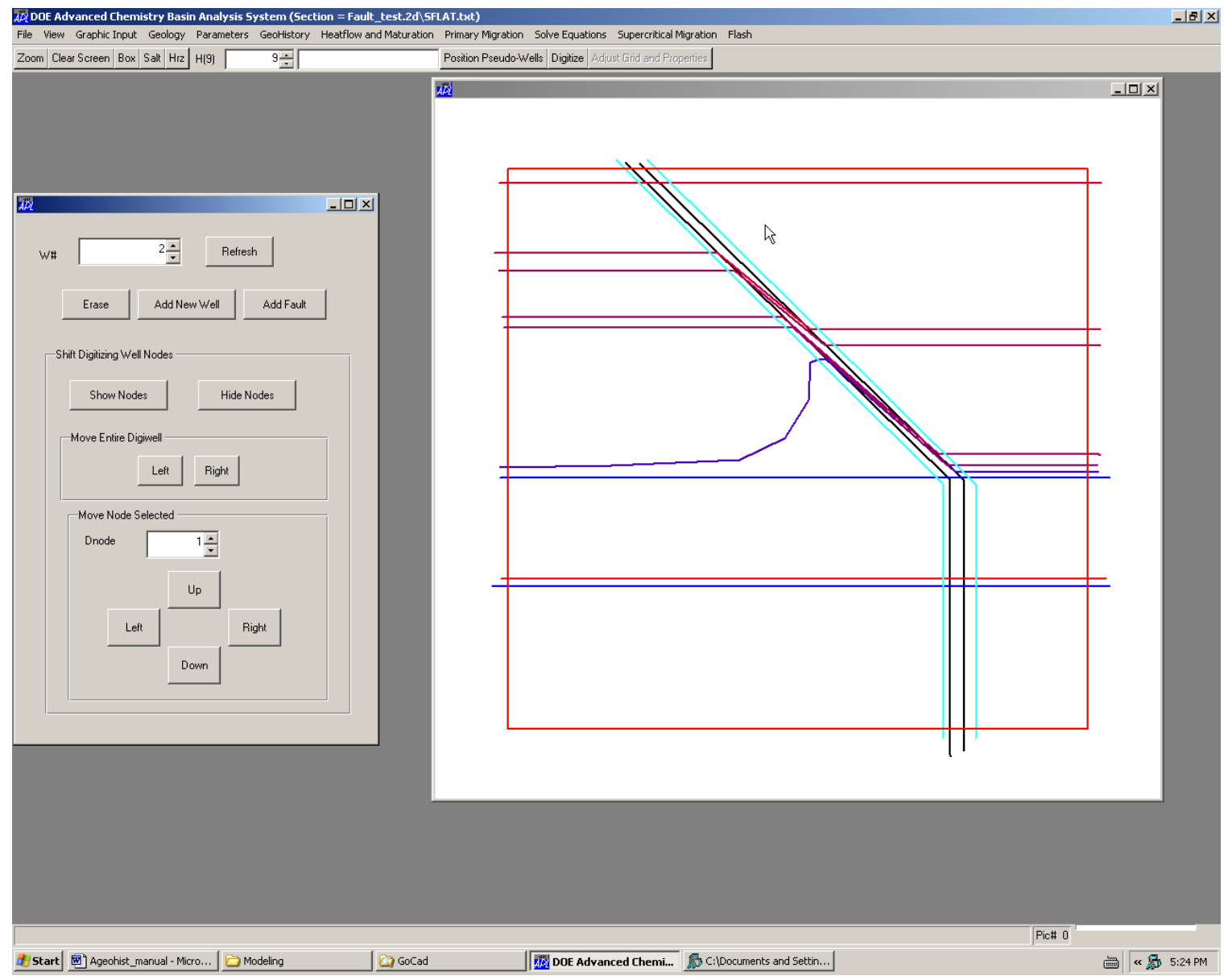

Figure 6. Selecting an existing *.dxf file will bring up that graphics file and the digitizing wells from the last case. To get the Digiwells for the current case (if it has been interpreted previously), select the Edit Previous Interpretation menu, close the forms that appear, and select the Edit Previous Digiwell Positions menu. Alternatively you can simply erase the wells and add the two shown above.

Selecting any input file retrieves the paths to all related input files. The paths to these files can be displayed using the File: Display all paths menu. The result is shown in Figure 7. This figure shows that an SFLAT file as well as a *.dxf file exists for this case, but there is no GoCad input file. The same set of paths would have been established if 
the SFLAT file for this case had been selected. Some information on each file can be obtained by toggling the input file buttons in the form shown in Figure 5.

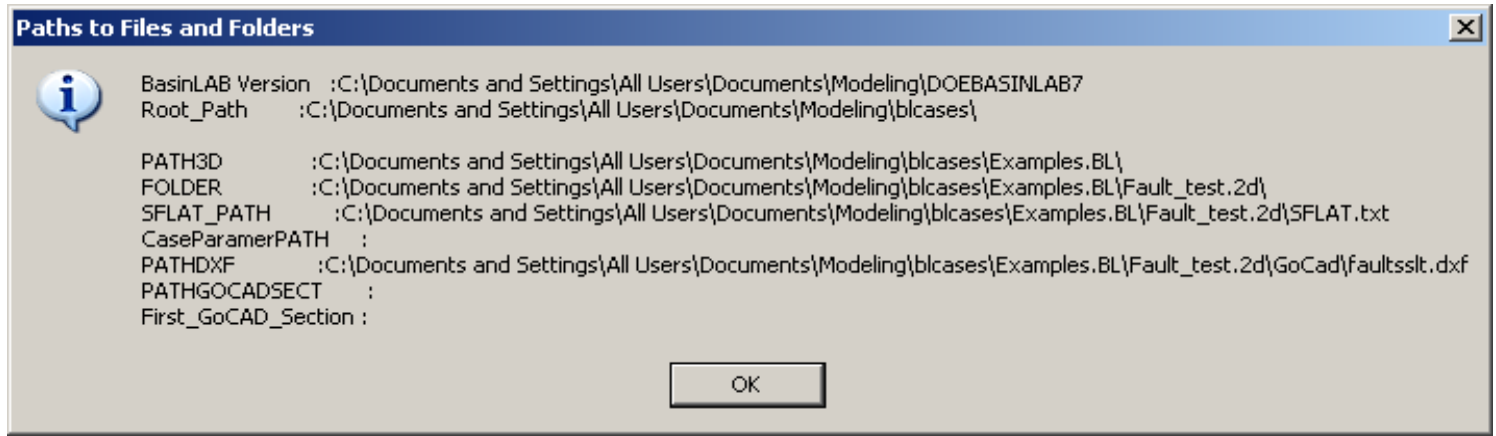

Figure 7. Paths to existing files for the case selected in Figure 5. An SFLAT and dxf file exists for the case, but not a GoCAD section.

\section{B. New Interpretation}

An SFLAT file can be constructed as a spreadsheet and exported as a tab-delimited text file (scrubbed using notepad of the extraneous "that Excel inserts) of constructed from a graphics file using tools provided by $\boldsymbol{B a s i n} \boldsymbol{L} \boldsymbol{A B}$. Interpretation of a graphics file is initiated using File: New Section Project (see Figure 5). The default graphics input file type is *.dxf. Default case parameters are assumed for a new case, and the dimensions of the graphics are presumed to be $10 \times 10 \mathrm{~km}$ deep. These dimensions should be changed to the real dimensions of the section. They are placeholder values only. The current title and description are the defaults for the new section. This is to facilitate construction of $3 \mathrm{D}$ cases and the cloning of an existing case. For a completely new section the user will want to change the title and description.

\section{An Example of *.DXF Graphic Input}

Consider the following example of graphic input to BasinL $\boldsymbol{A B}$. We start by sketching a cross section in Canvas. The sketch consists of a bounding box, and a series of lines or polylines that cut across the top and bottom box boundaries vertically or horizontally across both sides of the bounding box, as shown in Figure 8.

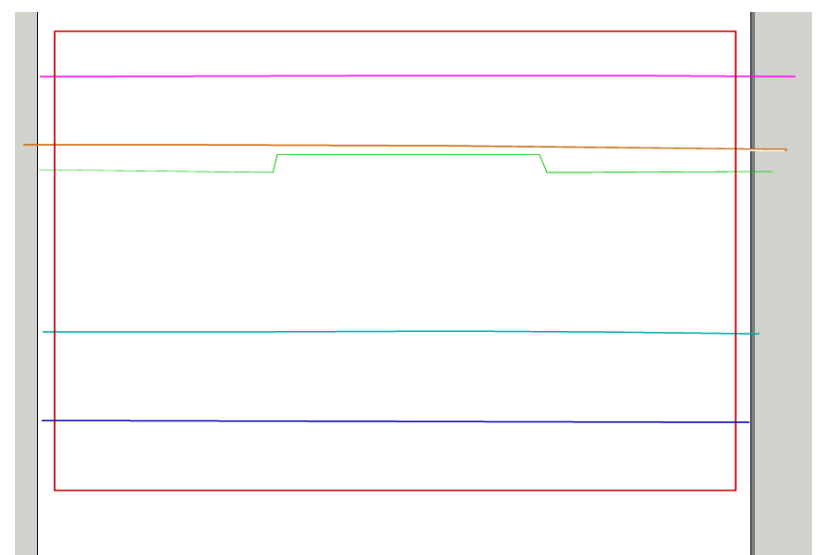

Carb_Reef.dxf file, which is read into $\boldsymbol{B a s i n} \boldsymbol{L} \boldsymbol{A B}$.
Figure 8. Canvas sketch of basin. The image consists of a red bounding box and 6 horizons (the base of the box to the purple horizon just below the top. The top of the bounding box is normally considered to be sea level. The horizons are all simple lines except for the third horizon from the top, which is a polyline representing a reef. The Canvas image is saved as a 


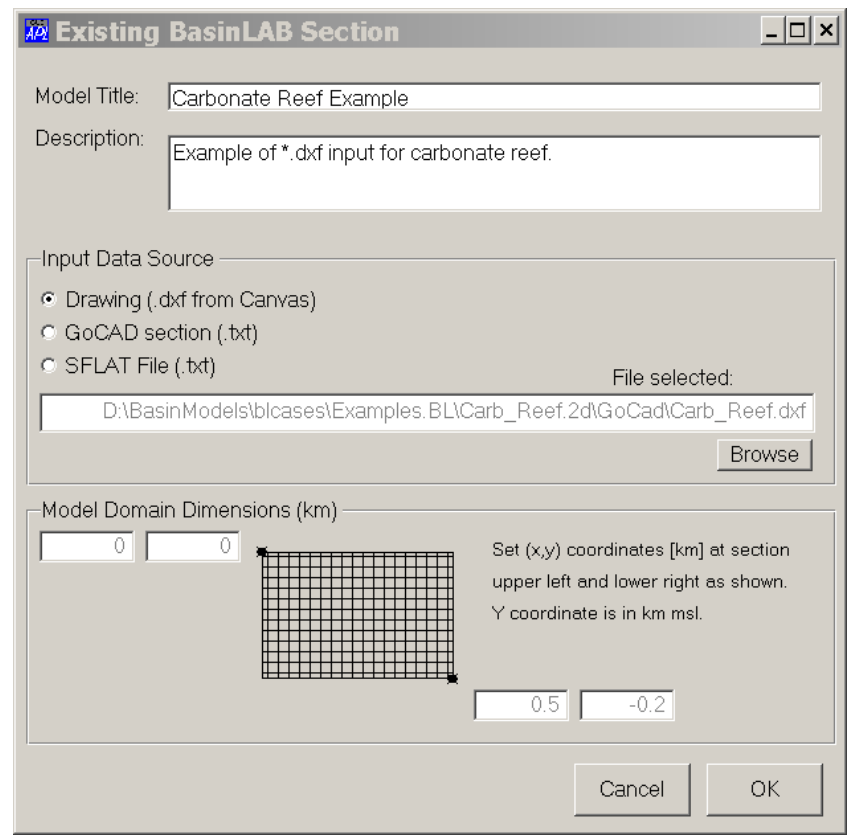

Figure 9. The sketch is read into BasinL $\boldsymbol{A B}$ by selecting File: New Section Project and browsing to the Carb_Reef.dxf file saved above. The title, description, and domain dimensions are added as indicated. Depressing the OK button reads the selected $*$.dxf file and opens a digitizing control form and a BasinLAB image of the section as shown in Figure 10.

The Canvas image shown in Figure 8 is output to a *.dxf file called, in this example, Carb_Reef.dxf and placed in the GoCad folder of the Carb_Reef.2D case folder. The Carb_Reef.dxf file is then read using the File: New Section Project menu command. The dimensions, title, and a brief description of the section are written into the window that appears when this is done, as indicated in Figure 9.

When the OK button in Figure 9 is depressed, the section appears with a digitizing control form and a set of digitizing wells from the previous case. This facilitates the digitization of 3D cases (where the earlier wells are just shifted in position), but this is not of concern to us here. Since the present case is entirely new, the previous digiwells are erased or modified to fit the section as shown in Figure 10. 


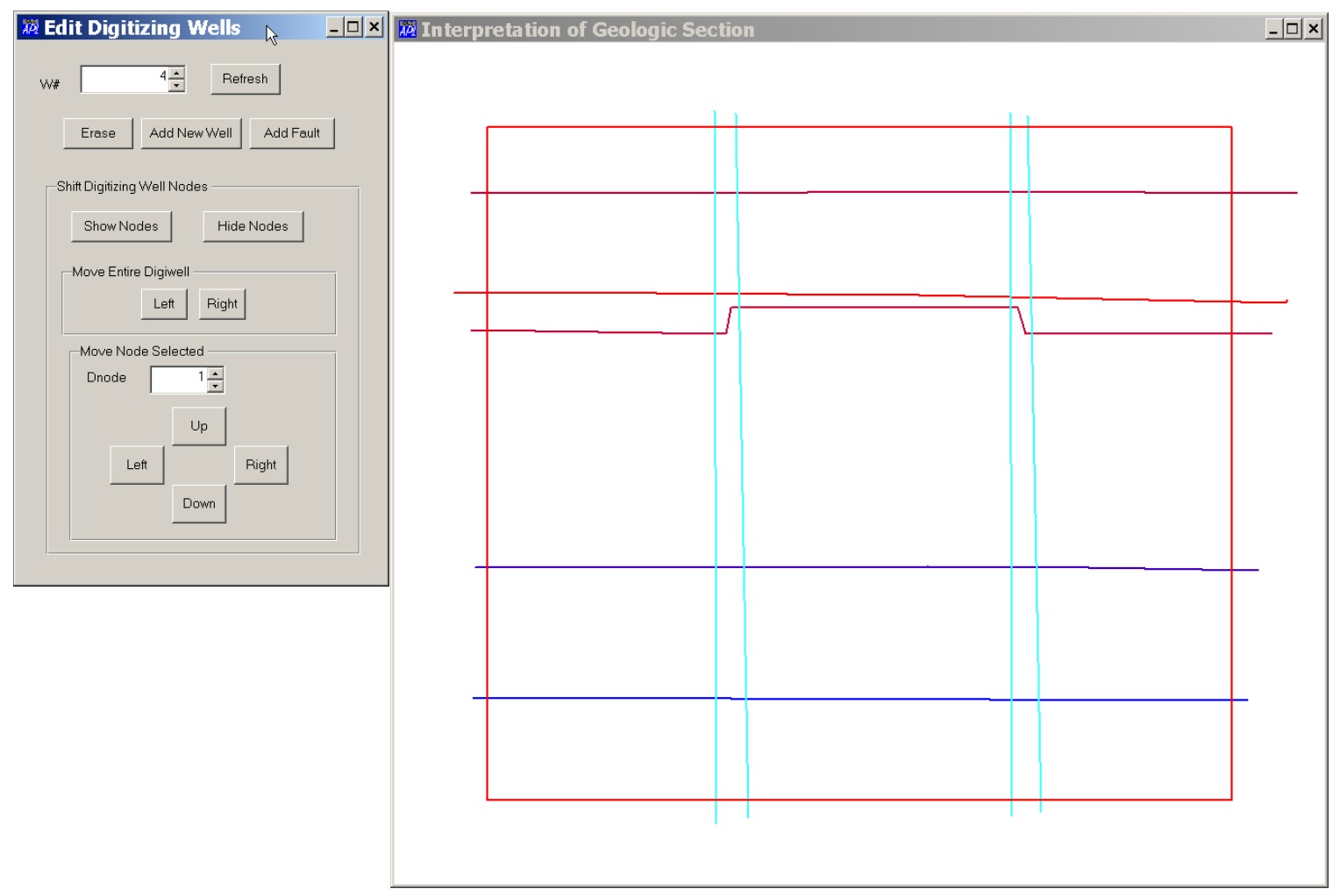

Figure 10. Position the digitizing wells (light blue vertical lines) over the sides of the reef using the digitizing well command form to the left. Digitizing wells can be selected using the top spinner. Once selected, they can be erased, or moved, or nodes in the digitizing well can be individually moved. Digitizing wells can be erased and added. Hints displayed on the status bar at the bottom of the workspace are provided when the mouse is positioned over a button, label or any feature of a form.

Once the digitizing wells are positioned appropriately, the section is digitized using the digitize button on the toolbar, as indicated by the mouse arrow in Figure 11. The result of depressing this button is shown in Figure 11.

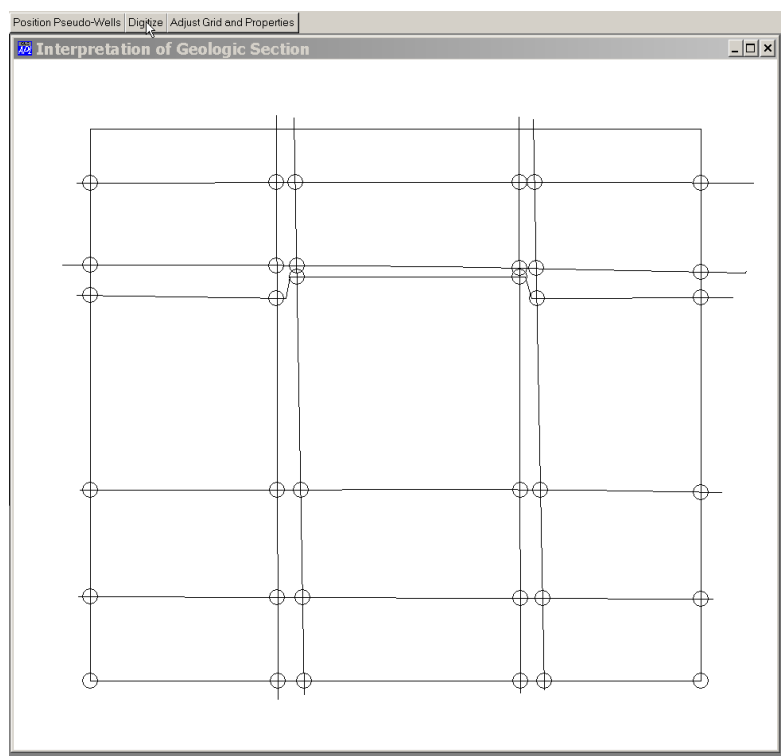

Figure 11. Depressing the Digitize button (under the mouse) digitizes the section shown in Figure 10. Depressing the adjacent Adjust Grid and Properties button allows lithologies, strata, and well names to be assigned as shown in Figure 12. 
Finally, depressing the Adjust Grid and Properties button (adjacent to the Digitize button under the mouse arrow in Figure 11) presents the digitized section and a control window that allows completion of the section, as shown in Figure 12.

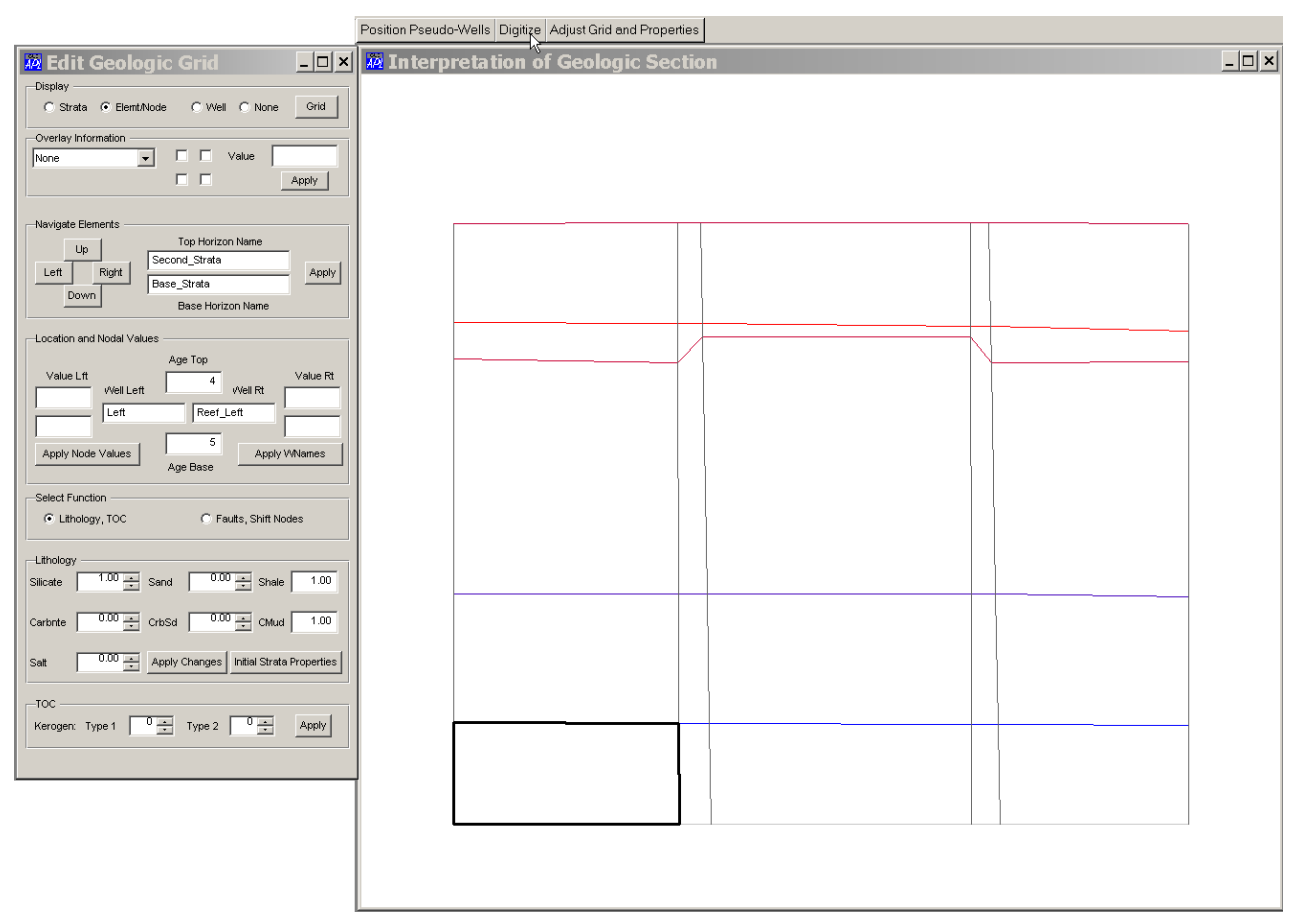

Figure 12. Complete section digitization by depressing the Adjust Grid and Properties. The result is a control form and the digitized section as shown.

Lithologies are assigned first to entire strata, and then modified. Strata Lithologies are assigned by depressing the Initial Strata Properties button in Figure 12 as shown in Figure 13.
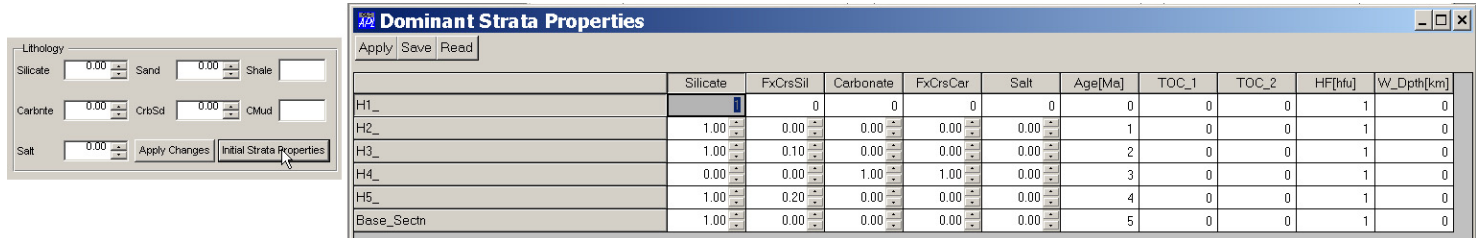

Figure 13. Assign initial strata properties by depressing the Initial Strata Properties button as shown, and dialing in the appropriate lithology for each strata in the section. Note that at this stage the strata have generic names. The lithology control on the left is a portion of the control form shown in Figure 12. Note that the third strata from the base has been assigned carbonate lithology. 
The final lithologic modifications are made by navigating to different regions of the section and modifying the lithology of individual elements as shown in Figure 14.

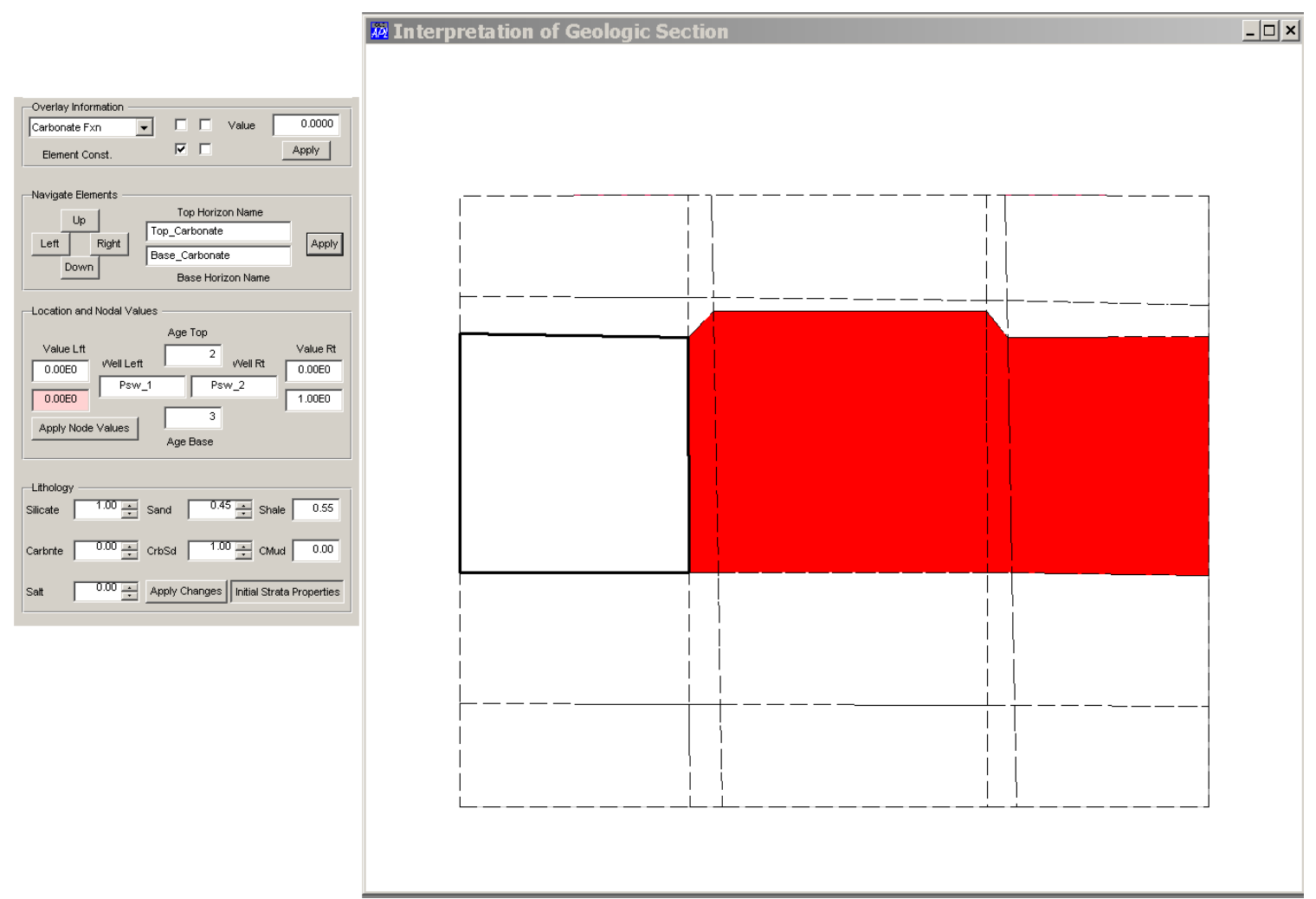

Figure 14. The lithology of the outlined element has been modified by navigating to that element and changing the lithology as shown to the left. The color is the carbonate fraction (top combo window). The element outlined in black was initially red, indicating it was $100 \%$ coarse carbonate. However, it has now been modified to $100 \%$ silicate and $45 \%$ sand. Also the strata names have been changed to Base_Carbonate and Top_carbonate as shown in the Navigate Elements grouping in the control form. Strata ages and well names can be similarly modified.

When the lithologic, strata and well name, and age assignments have been completed, the section is ready for output as an SFLAT file. This is done using the Graphic Input: Place into SFLAT and SAVE menu command. This creates a file called SFLATorig.txt in the case folder. When the SFLAT file is saved it is also read as the input SFLAT file. The SFLAT section can then be processed using the toolbar menus to the right of Graphic Edit (see Figure 4).

For example the section can be viewed using Geology: Edit/Plot Spreadsheet and clicking on the Lithology Pattern plot button on the toolbar of the spreadsheet that appears. This produces the section plot shown in Figure 15. 


\section{Section Plot}

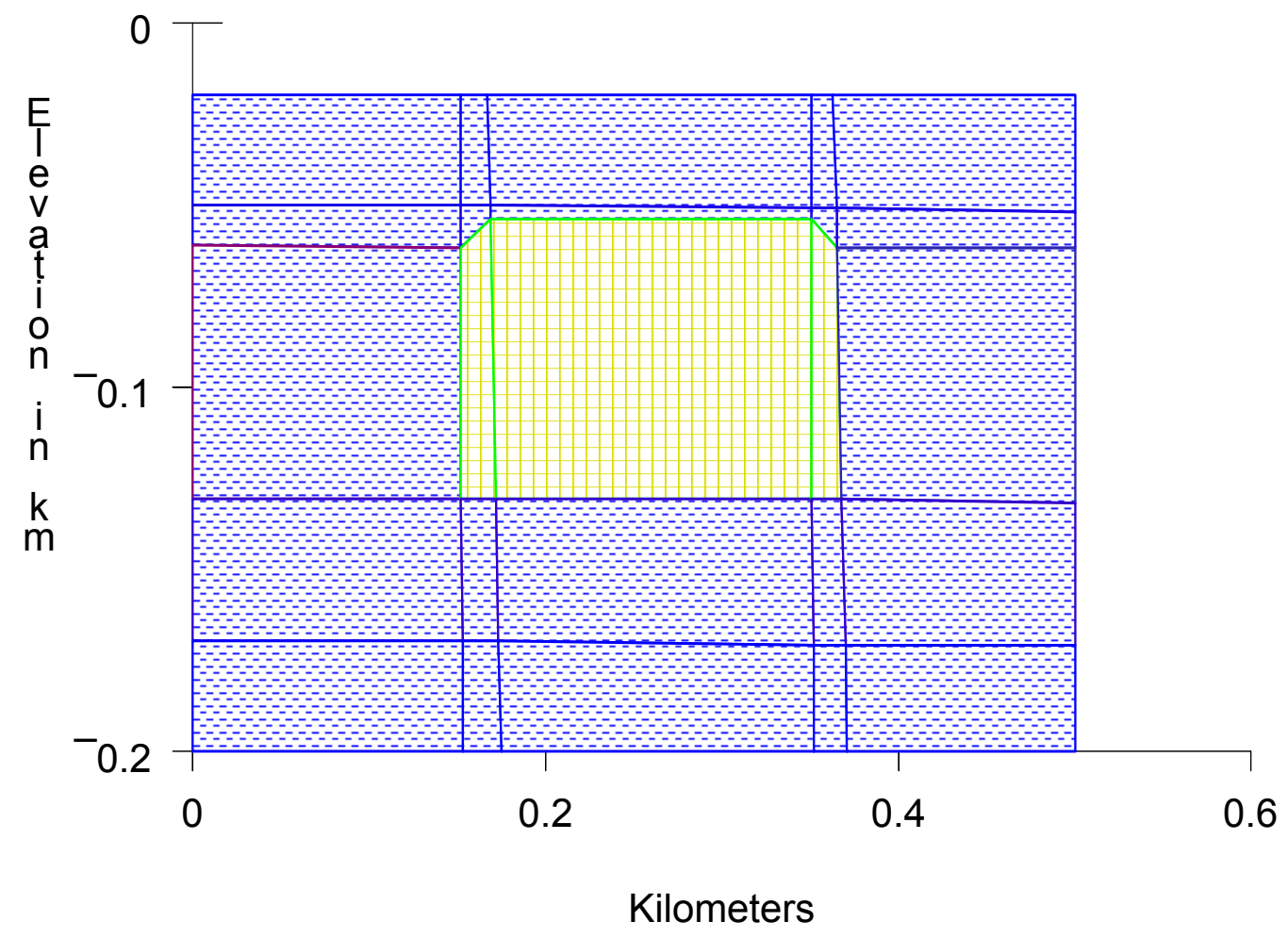

Figure 15. Lithology pattern plot of the SFLATorig.txt section created as described above. Notice that the base of the section is at $200 \mathrm{~m}$ depth and the section is $500 \mathrm{~m}$ wide, and that the seafloor is below sea level by the amount indicated in the Canvas sketch (Figure 8). More lithologic details are inherent in the Lithology Color plot where shale is set to blue, sand to red, and carbonate to green.

The SFLATorig.txt file itself has a specific format. The file is automatically annotated. A portion of this file is shown in Figure 15. Column names follow a strict convention that allows them to be recognized by BasinL $\boldsymbol{A B}$. The list of permitted column headings (useful if the Sflat file is constructed outside Basin LAB ) is given in Appendix B (p. 340). 


\begin{tabular}{|c|c|c|c|c|c|c|c|c|}
\hline 36 & 14 & \# Nbr Line & $\mathrm{es}, \mathrm{Nbr} \mathrm{Co}$ & olumns in sflat & & & & \\
\hline 6 & 0 & \# Nbr wells & Is, linear $(0$ & 0) or exponenti & $\operatorname{tial}(1)$ & ) compact & tion \# & \\
\hline 6 & 6 & \# Nbr wells & $\mathrm{Is}, \mathrm{Nbr} \mathrm{Hrz}$ & zns \# & & & & \\
\hline 1 & 1 1. & \# Scale $\mathrm{x}$ & Scale y \# & & & & & \\
\hline & W_Nbr & H_Nbr & $\mathrm{x} 1$ & X2(depth) & X3 F & Fx_Sd & Fx_Sh & Fx_Carb_Sd \\
\hline Left & 1 & 1 & 0 & -0.0197 & 0 & 0 & 1 & 0 \\
\hline Top_Carb_Cap & 1 & 2 & 0 & -0.0494 & 0 & 0 & 1 & 0 \\
\hline Top_Carbonate & 1 & 3 & 0 & -0.0604 & +0 & 0.1 & 0.9 & 0 \\
\hline Base_Carbonate & 1 & 4 & 0 & -0.131 & 0 & 0.55 & 0.45 & 0 \\
\hline Second_Strata & 1 & 5 & 0 & -0.1695 & 0 & 0.2 & 0.8 & 0 \\
\hline Base_Strata & 1 & 6 & 0 & -0.2 & 0 & 0 & 1 & 0 \\
\hline Reef_Left & 2 & 1 & 0.1522 & -0.0194 & 0 & 0 & 1 & 0 \\
\hline Top_Carb_Cap & 2 & 2 & 0.1524 & -0.0495 & 0 & 0 & 1 & 0 \\
\hline Top_Carbonate & 2 & 3 & 0.1524 & -0.0614 & 0 & 0.1 & 0.9 & 0 \\
\hline Base_Carbonate & 2 & 4 & 0.1529 & -0.1309 & 0 & 0 & 0 & 1 \\
\hline Second_Strata & 2 & 5 & 0.1531 & -0.1698 & 0 & 0.2 & 0.8 & 0 \\
\hline Base_Strata & 2 & 6 & 0.1533 & -0.2001 & 0 & 0 & 1 & 0 \\
\hline Reef_Ctr_Left & 3 & 1 & 0.1678 & -0.0194 & 0 & 0 & 1 & 0 \\
\hline Top_Carb_Cap & 3 & 2 & 0.169 & -0.0496 & 0 & 0 & 1 & 0 \\
\hline Top_Carbonate & 3 & 3 & 0.1692 & -0.0538 & 0 & 0.1 & 0.9 & 0 \\
\hline Base_Carbonate & 3 & 4 & 0.1723 & -0.1308 & 0 & 0 & 0 & 1 \\
\hline Second_Strata & 3 & 5 & 0.1739 & -0.1698 & \begin{tabular}{|l|}
3 \\
\end{tabular} & 0.2 & 0.8 & 0 \\
\hline Base_Strata & 3 & 6 & $\mid 0.1751$ & -0.2001 & $\mid$ & 0 & 1 & 0 \\
\hline
\end{tabular}

Table 10. A portion of the SFLATorig.txt file plotted in Figure 15. The column headers must have the exact form shown to be read successfully. Notice that the seafloor has been replaced by the well name. The column naming convention is given in Appendix B (p.340)

\section{An Example of GoCAD Graphic Input}

Basin $\boldsymbol{L} \boldsymbol{A B}$ also reads standard GoCAD section files. An example of the first few lines of such a file is shown in Figure 16.

GOCAD XSection 1

HEADER \{

name:ChampXS1

*Top_Region_1*solid*color:0.8 0.8 0.11 .0

bounding_pline:on

*Bathymetry_0_0_test_1*solid*color:0 011

*TopSalt6_0_test_1"solid*color:1 111

*134ma_top_source_1 ${ }^{*}$ solid"color:0.5 0.50 .751

*66_5ma_mcsb_surf_1*solid*color:0.392157 0.5843140 .9294121

*TopPliocene2_2_test_1*'solid*color:0.811765 0.1215690.564706 1

Figure 16. The first few lines of a standard GoCAD section file. Files of this style are read by BasinLAB using the Files: Open New Project menu command with the GoCAD option selected as shown in Figure 17. The initial processing steps are discussed in the 
following two figures. The remaining steps are the same as in processing *.DXF files and were discussed above.

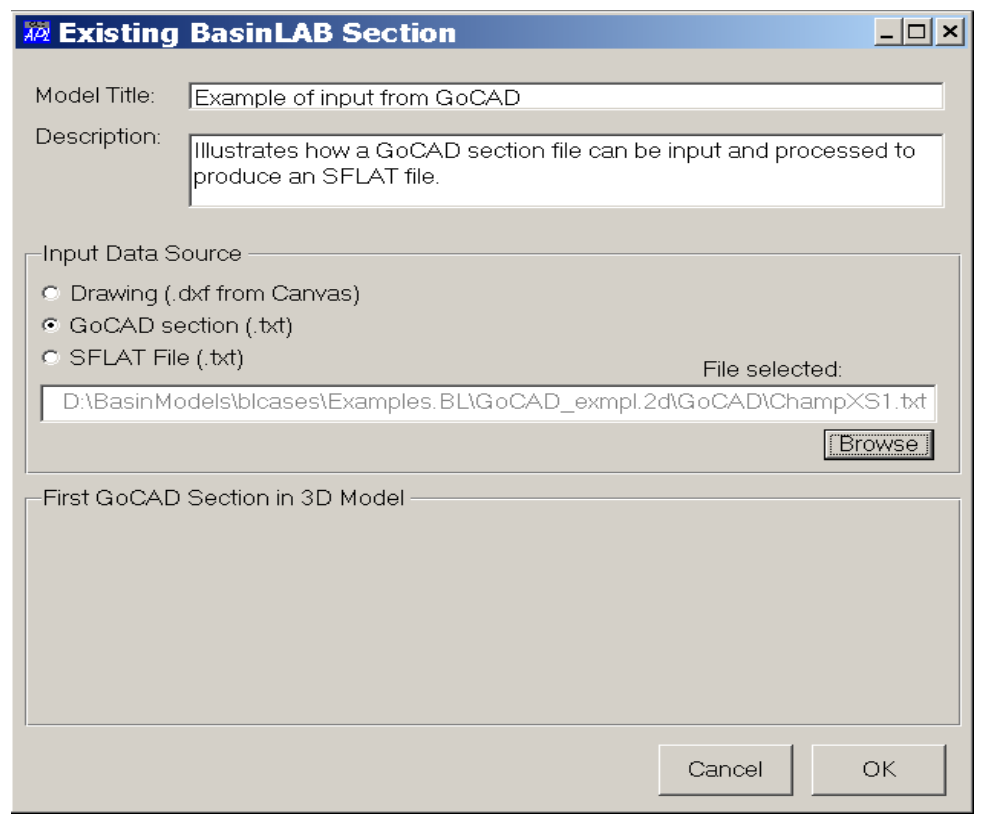

Figure 17. Read in a GoCAD section by selecting the GoCAD option for the input data source in Files: New Section Project. This brings up the form to the left. Clicking OK results in the graphics shown in Figure 18

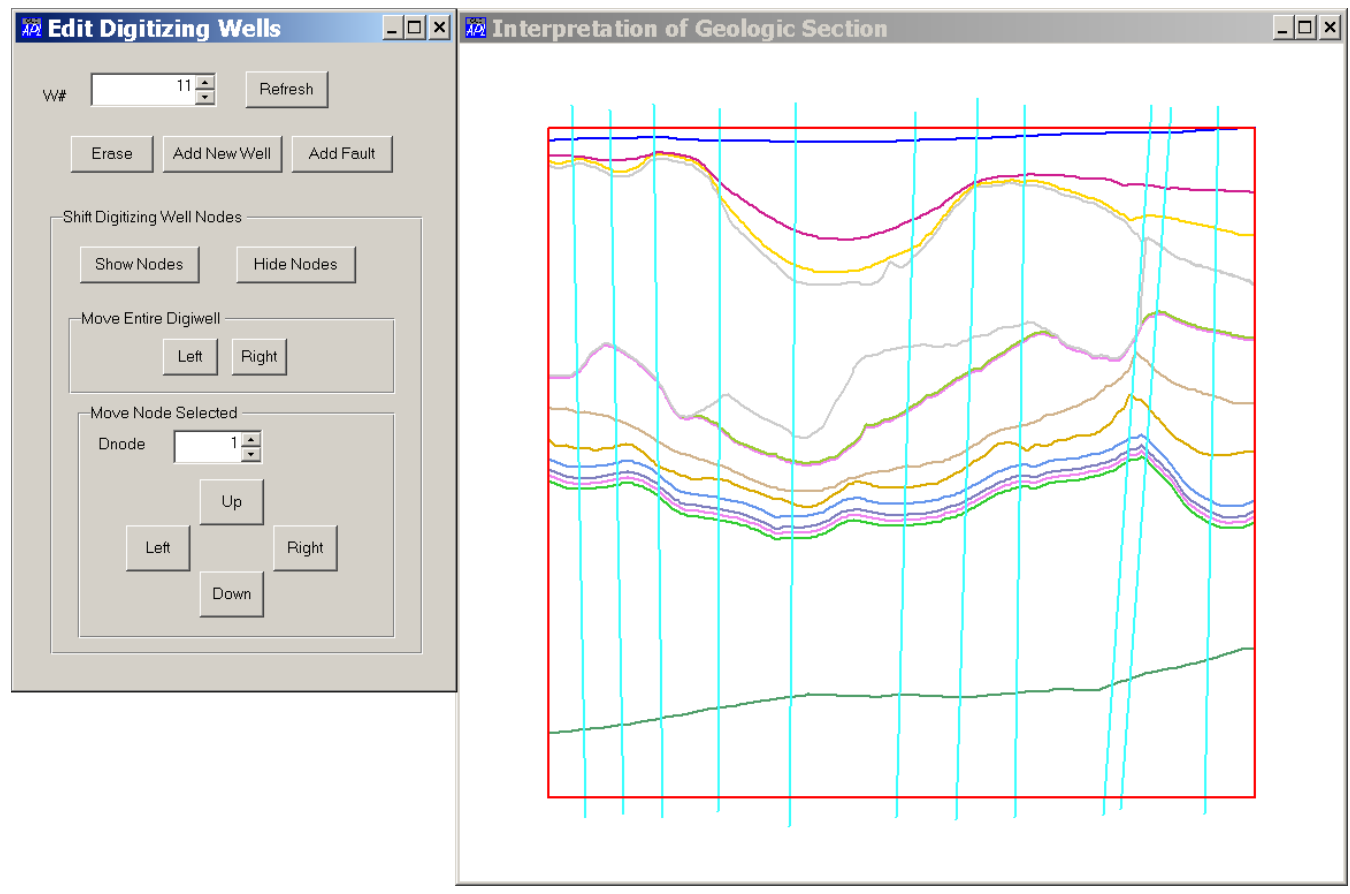

Figure 18. The base graphics show the GoCAD input file read from the GoCAD folder in the blcases $\backslash$ Examples.BL\GoCAD_exmpl.2d $\backslash$ case folder. The blue digitizing wells ("Digiwells") have been added by using the Add New Well button and clicking with the right mouse button along the well (top to bottom) and 
finishing with a right mouse button click. NOTE: When the section first comes up, the DigiWells are from the previously digitized graphic. This is intentional, as it greatly facilitates digitizing 3D sections. If a previous graphic interpretation has been made, the Digiwells for the current section can be obtained by using the Graphic Input: Read and edit Previous Interpretation command and then returning to the Edit Previous Digiwell Positions command.

\section{An Example Illustrating Advanced Chemistry Capabilities}

BasinL $\boldsymbol{A B}$ has a great many capabilities. Before describing them it is useful to gain an overview by considering an example section. This allows us to illustrate the most important chemical capabilities of $\boldsymbol{B a s i n} \boldsymbol{L} \boldsymbol{A B}$ without getting lost in too many details.

\section{A. Geological Input}

We will consider a $50 \mathrm{~km}$ long section running S from the Louisiana Coast. It is a portion of a longer section published by (McBride 1998) and it is fully described and modeled in a report available from the Gas Research Institute (Cathles 2002). The section is captured in an SFLAT file in the McBride $1^{\text {st }}$ third. $2 d$ folder in the gri_Analysis.BL folder provided as part of this release. Open the SFLAT file using menu command File: Open Section Project. Close the line number form that appears. You should be left with a clear workspace like that shown in Figure 4. Plot the section using Geology: Edit/Plot Spreadsheet menu and click the Lithology Pattern button on the spreadsheet toolbar. The section that results is shown in Figure 19. The advantage of this plot is that it shows the lithology and section dimensions clearly. Also clicking with the mouse near any of the strata gives the name of the strata, and clicking above the section near any of the pseudowells gives the name of the pseudowell.

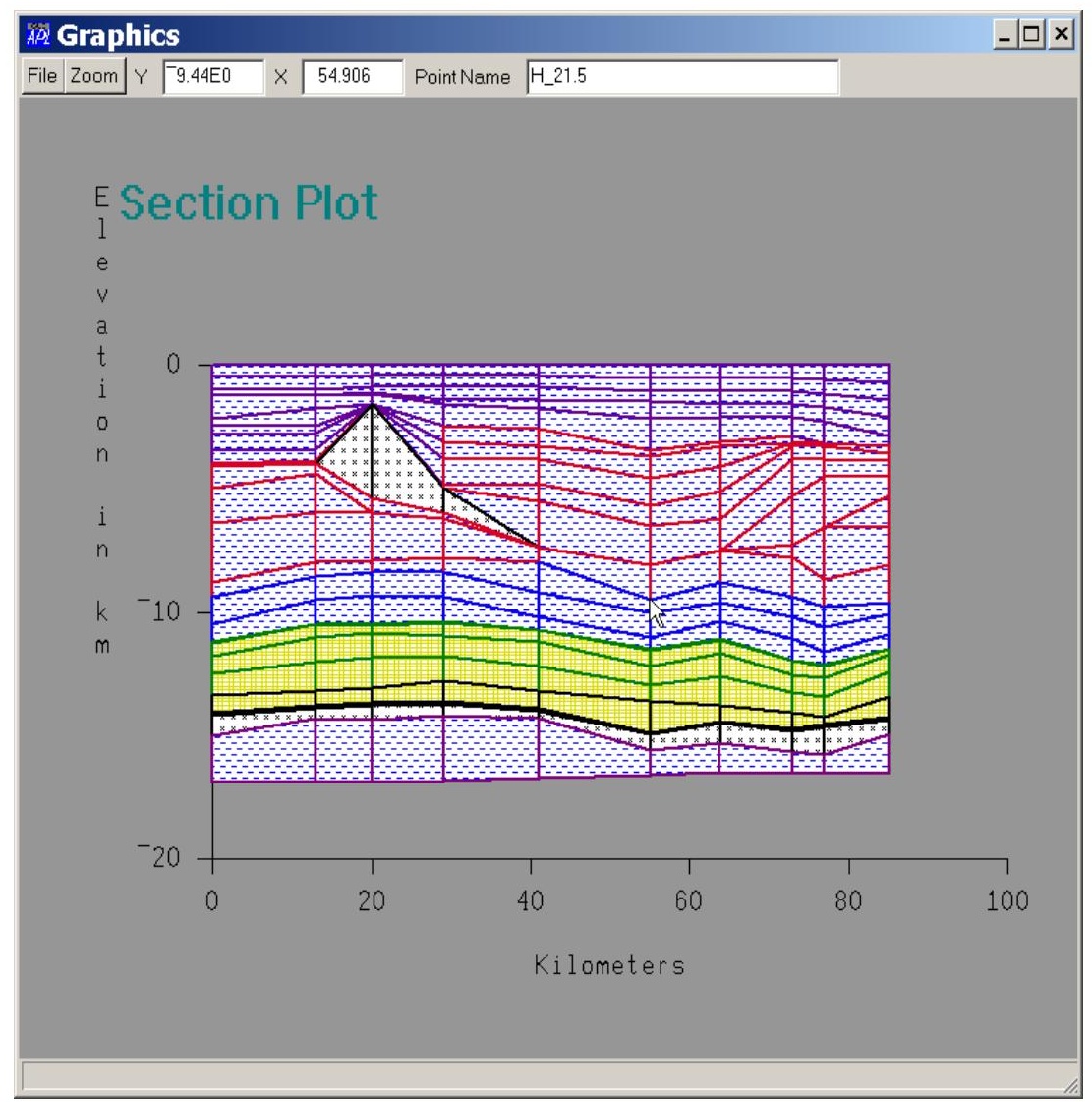

Figure 19. The McBride_1 ${ }^{\text {st }}$ third.2d section, plotted using Geology: Edit/Plot Spreadsheet:

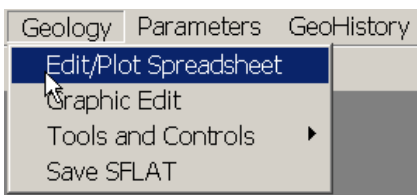

followed by clicking the Lithology Pattern button on the spreadsheet toolbar.

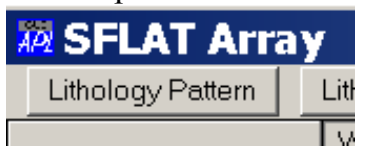

Depressing the mouse button at the position 
shown in the image to the left indicates that this is the $21.5 \mathrm{Ma}$ age horizon (point name on toolbar). The mouse tip is $9.44 \mathrm{~km}$ below sea level and $54.906 \mathrm{~km}$ from the left side of the section.

\section{B. Physical Processing}

The first step in processing is to determine the geologic history of the section. This is done by selecting the Geohistory: Create Geohistory menu control as shown in Figure 20 .

The button at the top of the form in Figure 20 allows the initial porosity and compressibility of the sediments to be examined. We will assume that the default properties are appropriate. The next control group allows a seal to be set. It can be a migrating of fixed seal. Its position is determined by seal flags in the SFLAT file. We will assume there is no seal in this case. Sedimentation can be started at any time. We select the maximum age possible. Because we want to simulate salt diapirism in the upper part of the section, but begin the sedimentation early, we check the Pass 1 box in the Late Salt Diapirism control group. After the first pass, we will check the Pass 2, box and process again. The salt diapirism box is checked, so that the redistribution of salt will be inferred from the pattern of sedimentation. We do not consider erosion, and sea level is specified in the SFLAT file. The Cinema review box is checked. The specified

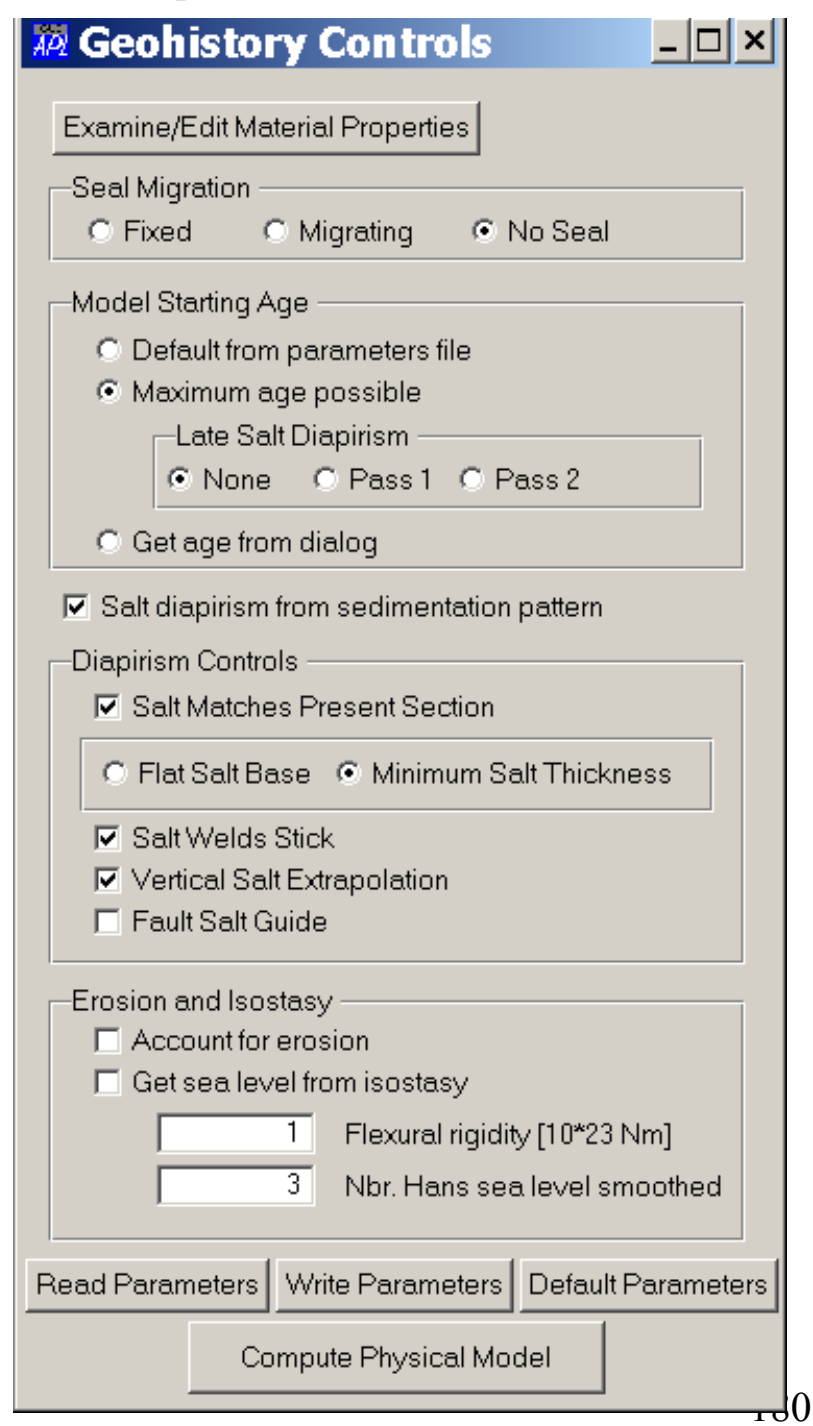
processing controls can be saved (or previously saved controls read) using the buttons at the base of the form. Finally the geologic history of the section is calculated by depressing the Compute Physical Model button at the bottom of the form.

Figure 20. Control form obtained from GeoHistory: Create Geohistory:

\begin{tabular}{l}
\hline GeoHistory Heatflow anc \\
Add Erosion \\
Create Geohistory \\
\hline Plot and Edit \\
\hline Tools and Controls \\
\hline
\end{tabular}

Process the SFLAT file to determine the geologic history of the section by checking the control boxes on this form and depressing the Compute Physical Model button.

The section is processed twice, once with the Pass 1 button selected, and then with the Pass 2 button selected. The result is the geologic history shown in Figure 21. 
The buttons on the bottom of the form read and write the Case Parameters file or obtain the default version of the Case Parameters.

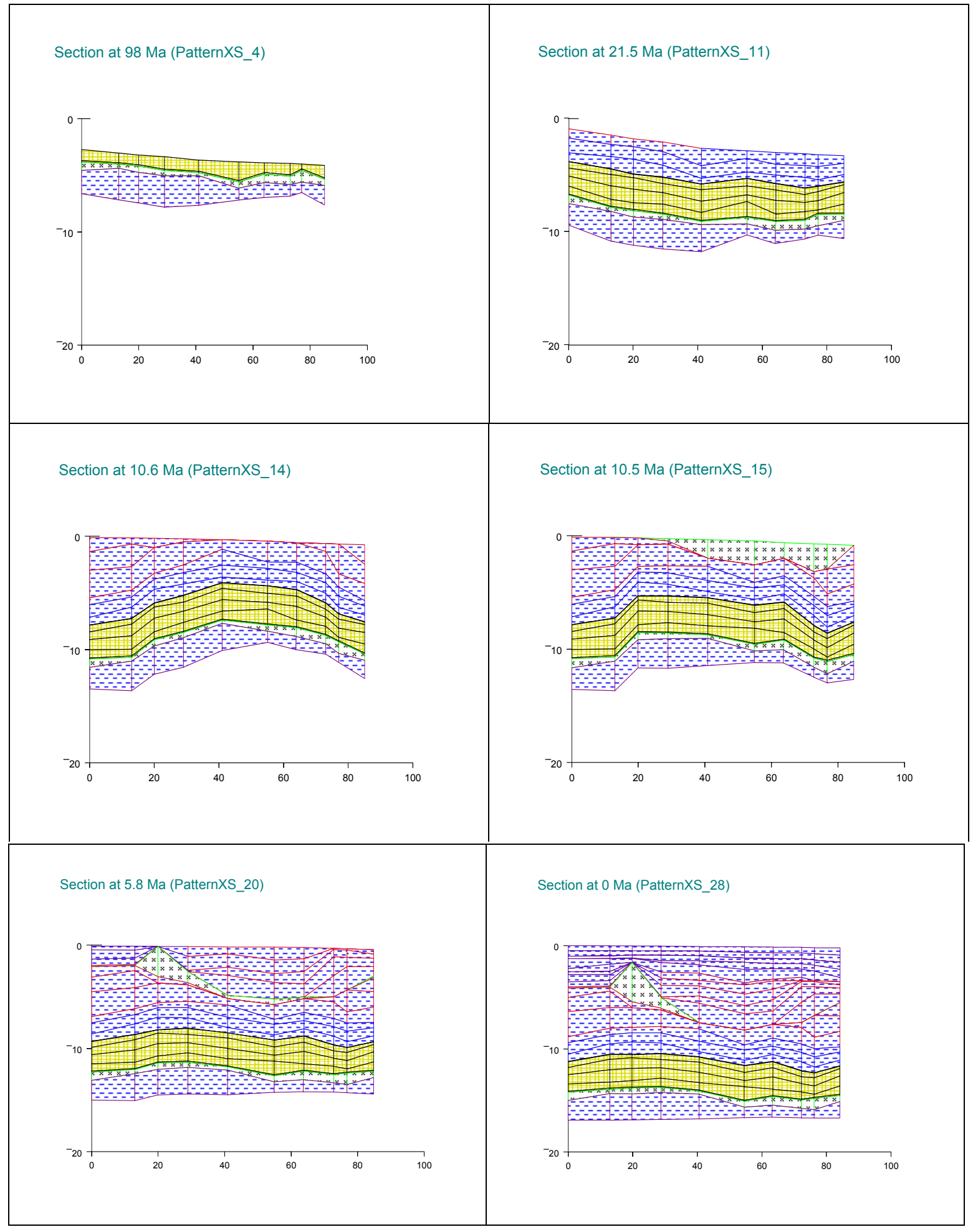


Figure 21. Geologic history of section shown in Figure 20 that was constructed using form in that figure. Notice how salt pod at $10.5 \mathrm{Ma}$ is loaded and redistributed. Note changes in sea level. Vertical axis is elevation in $\mathrm{km}$; horizontal axis is distance along the section in km. Plots are created by GeoHistory: Plot and Edit: Plot menu command. Axis labels are turned off in View: Plot Controls: Show Axis Titles.

\section{Determining and Verifying the Heat Flow History}

The next step after inferring the geologic evolution of the section is to infer and verify its temperature history. Heat flow is specified in the SFLAT file in this current case, having been inferred in a regional section (see (Cathles and Losh 2002)). Heat flow and the thermal conductivity algorithm determine temperature as a function of time. This history is verified by determining if the present day temperature profile matches that calculated, and by comparing computed and observed maturity indices. The temperature history is calculated in each pseudowell in a 1D calculation. The menu tool used is Heatflow and Maturation: Evaluate Heatflow. This brings up the form shown in Figure 22.

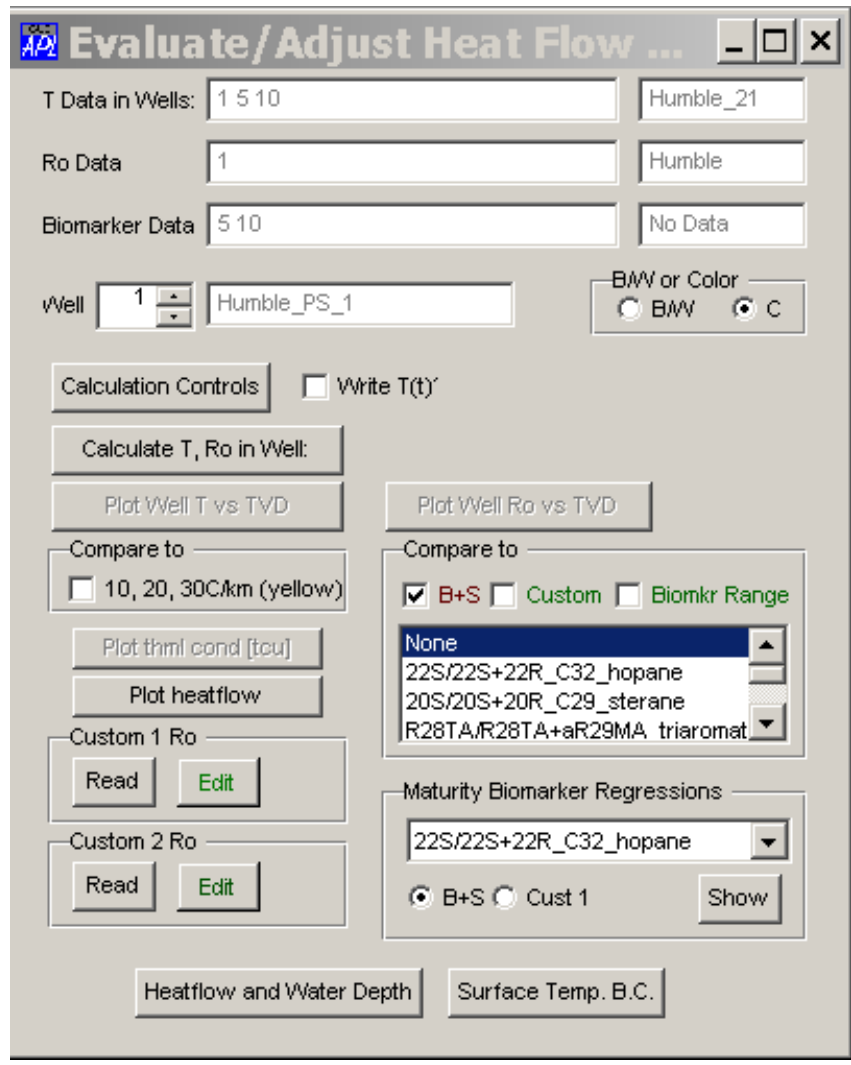

Figure 22. Heatflow evaluation form obtained from Heatflow and Maturation: Evaluate Heatflow:

\begin{tabular}{|c|}
\hline Heatflow and Maturation \\
\hline Evaluate Heatflow \\
\hline Evaluate Maturation \\
\hline
\end{tabular}

The form in Figure 22 shows that temperature data are available in pseudowells 1, 5, and 10 , vitrinite Ro data in pseudowell 1, and biomarker data in pseudowells 5 and 10. Use the spinner on the $4^{\text {th }}$ line to select the $5^{\text {th }}$ well, and depress the Calculate T, Ro in Well 
button. This calculates the $\mathrm{T}(\mathrm{t})$ history in the $5^{\text {th }}$ well. This temperature history is then used to calculate the vitrinite reflectance and the relationship between the biomarker ratios and vitrinite reflectance. The temperature calculation is $1 \mathrm{D}$ (for the $5^{\text {th }}$ well only). When the calculations are complete, the Plot Well T vs. TVD and Plot Well Ro vs. TVD buttons become active. TVD stands for total vertical depth (from the basin surface). The check boxes control what is plotted. The lowermost buttons allow heat flow, water depth, and the thermocline profile to be viewed and edited.

The purpose of the form in Figure 22 is of course to verify that the time-temperature history computed in well 5 agrees with maturity indicators in that well. Are present numerical well temperatures similar to those observed? Are biomarker maturities compatible with the time-temperature history in the numerical well? Figure 23 shows that this is the case.

The left figure plots computed present-day temperature as a function of depth, and compares it to data from various depths in the well. The right figure compares the maturities (Re values) of oils collected at various depths $(+)$ in the well to solid and dashed lines that show how the computed vitrinite maturity of sediments in the well (Rc values) change with depth. The methods used are described in more detail in Section 0.

\section{T[Z] Atlantic (T Well 5)}

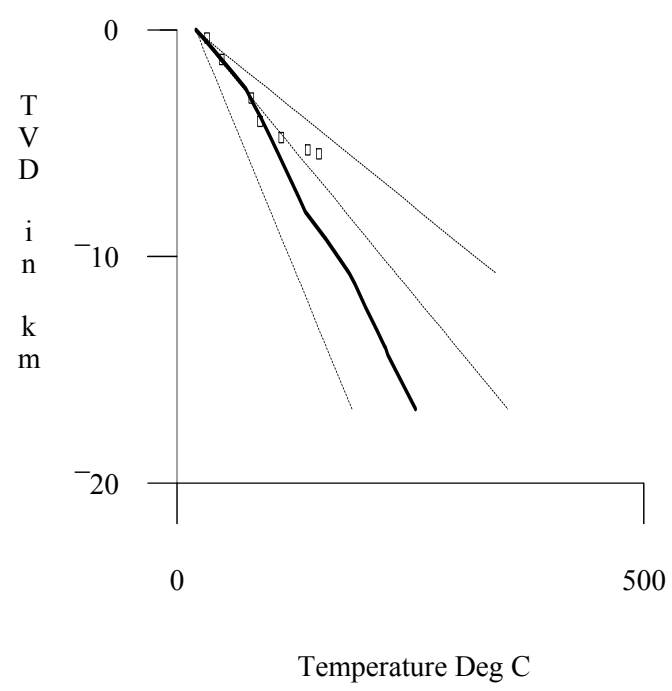

Ro[Z] Atlantic (W 5)

22S/22S+22R_C32_hopane (black plus)

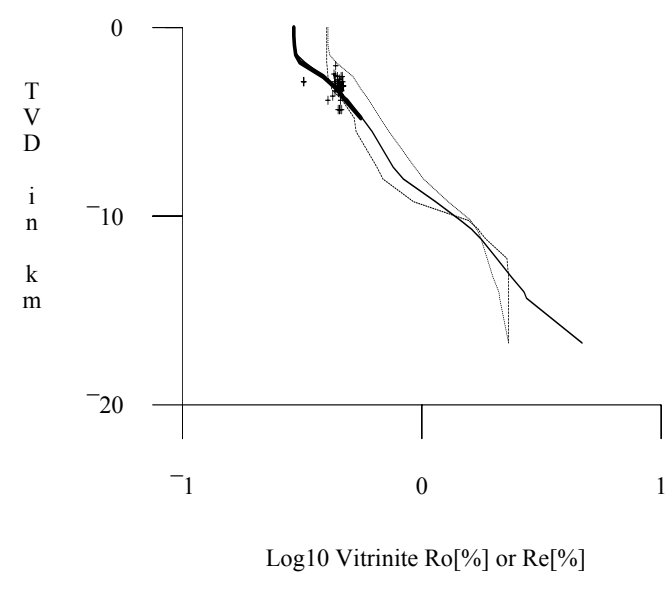

.


is shown as a solid line; 10,20 and $30 \mathrm{C} / \mathrm{km}$ profiles are shown as dashed lines for comparison. The near-surface temperature gradient is $\sim 20 \mathrm{C} / \mathrm{km}$. In (b) the $22 S / 22 S+22 R$ C32 Hopane ratio conversion index is converted to its vitrinite reflectance equivalent, Re, using a regression of the Burnham and Sweeney vitrinite model with the calculated conversion index for all nodes in the well. The Re values are plotted at the depths the oil samples were collected $(+)$. In addition the vitrinite reflectances computed by the Burnham and Sweeney (solid line) and two custom vitrinite models (dotted and dashed lines) are shown. The vitrinite reflectance model used to compute the Re is the Burnham and Sweeney model. The black part of that curve shows the range of Re over which the $22 S / 22 S+22 R$ C32 Hopane maturity measure is valid.

The black and white plot mode was selected. The plots displayed on the computer screen are automatically placed into the clipboard and so can immediately be pasted into a document.

积 Grapl the Zoom

In addition, the plots cold be saved as a ${ }^{*}$.wmf file in the metafiles folder by depressing the file button in the upper left corner of the plot form. The figure will be given the sequential pic\# Pic\# 0 indicated on the lower right corner of the workspace.

Figure 24 adds the R28TA/R28TA+aR29MA_triaromatic_steroid biomarker maturity indicator to the $22 S / 22 S+22 R$ C32 Hopane maturity indicator shown in Figure 23. Finally Figure 25 shows the regression between the calculated $22 S / 22 S+22 R C 32$ Hopane conversion index and the calculated Burnham and Sweeney vitrinite Ro for all points in well 5. 


\section{Ro[Z] Atlantic (W 1) \\ 22S/22S+22R_C32_hopane (black plus) \\ R28TA/R28TA+aR29MA_triaromatic_steroid (d brown square)}

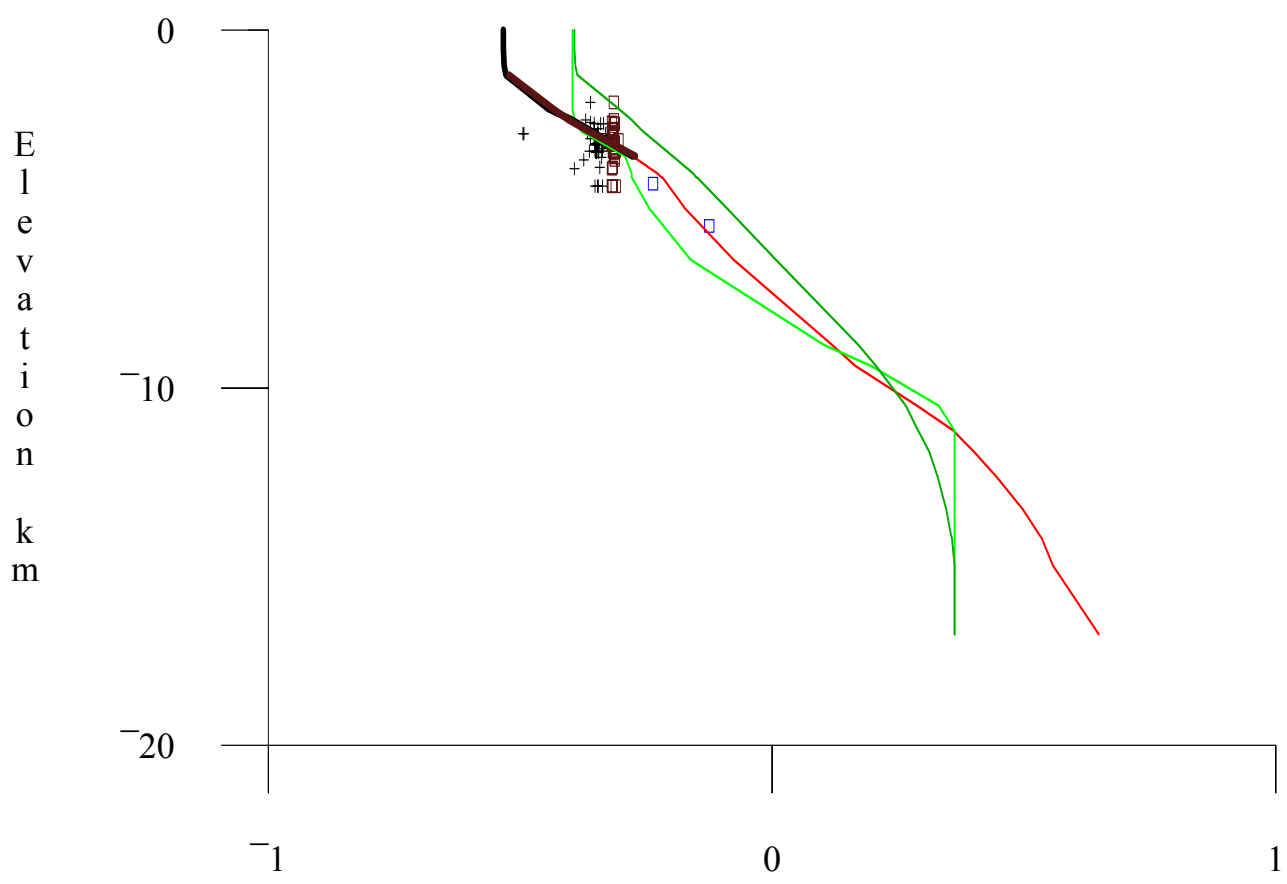

$\log 10$ Vitrinite $\operatorname{Ro}[\%]$ or $\operatorname{Re}[\%]$

Figure 24. The $R 28 T A / R 28 T A+a R 29 M A$ triaromatic_steroid biomarker maturity marker is added to the $22 S / 22 S+22 R C 32$ Hopane maturity marker in Figure 24 by adding this biomarker to the combo box selection (by control shift selection). The plotting conventions are the same as in Figure 24. 


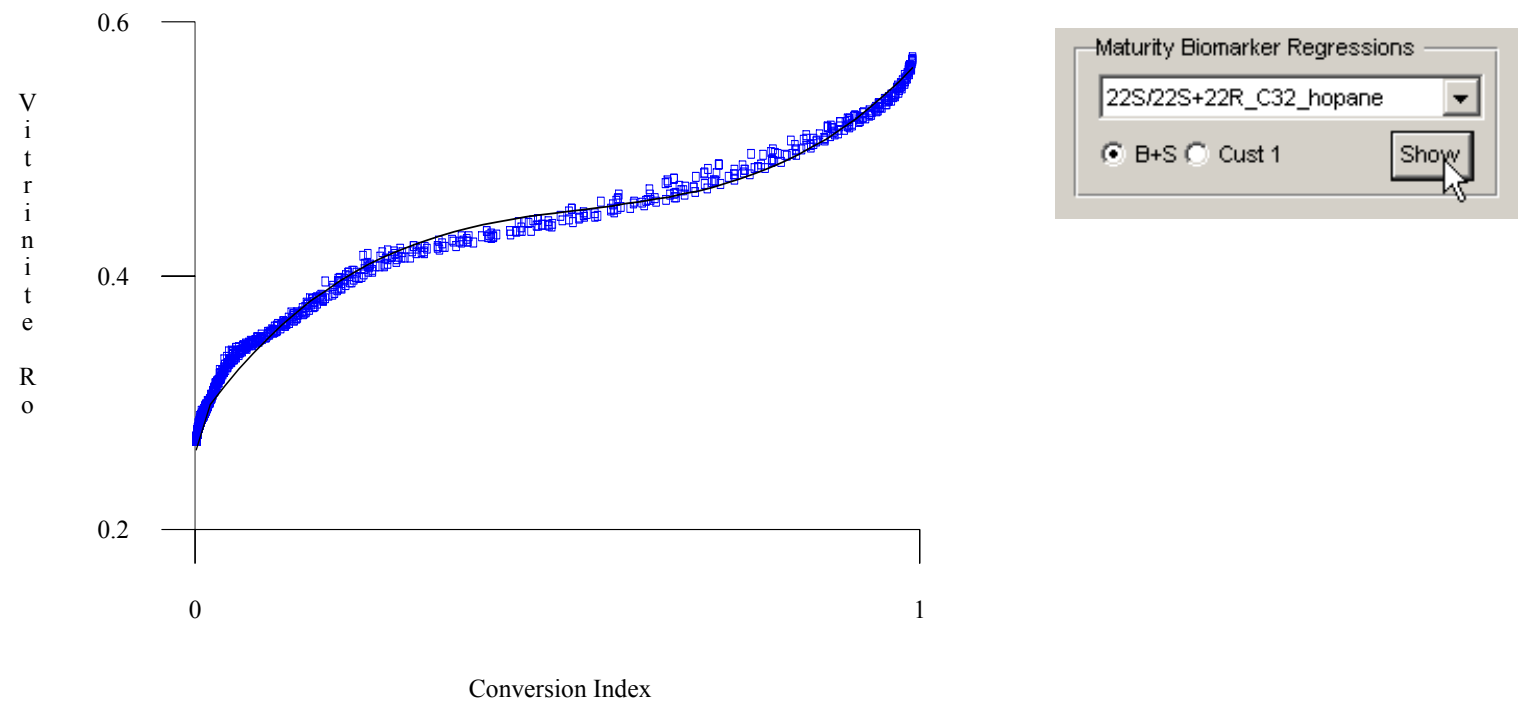

Figure 25. Regression between the $22 S / 22 S+22 R$ C 32 Hopane conversion index and the calculated Burnham and Sweeney vitrinite Ro maturity for all points in well 1 . This regression is calculated and displayed using the portion of the form in Figure 22 shown.

The heatflow evaluation form (Figure 22) allows changes to be made in the heatflow and water depth history of a well, and in the thermocline equation that converts water depth to seafloor temperature. The changes in water depth and heatflow are not permanent unless the SFLAT file is saved using the Geology: Save SFLAT menu. The thermocline equation changes are not permanent unless the Case Parameters file is saved using the File: Save Case Parameters menu. The changes affect the scratchpad calculation (and all subsequent workspace calculations) unless the case is opened again using File: Open Section Project. Figure 26 shows the thermocline curve and the form that allows its modification. 


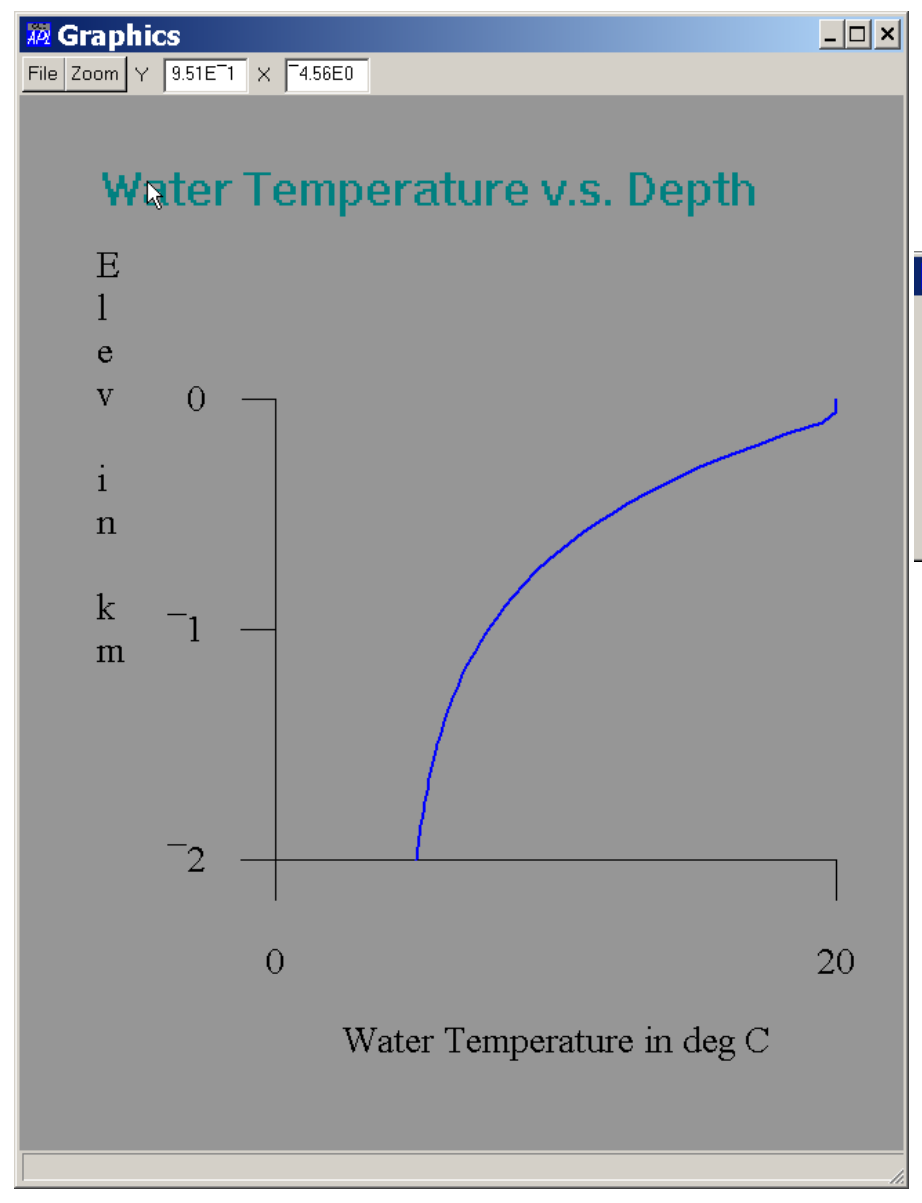

Figure 26. Depressing the Surface Temp. ...C. in the Figure 22 form causes the following form to

\begin{tabular}{|c|c|c|}
\hline API Thermal Surface Cond & & $-\square x$ \\
\hline Land Temperature $[\mathrm{C}]$ & 20 & -Thermocline \\
\hline Surface Water Temperature [C] & $\sqrt{20}$ & $c$ Linear \\
\hline Elevation of Top Thermocline $[\mathrm{km}] \Gamma$ & -0.08 & - Exponential \\
\hline Exponential Skin Depth [km] & -0.57 & \\
\hline Deep Water Temperature [C] & 4.5 & Apply and Plot \\
\hline
\end{tabular}

appear:

Depressing the Apply and Plot button of this form produces the graphic to the left. The thermocline equation (linear or exponential) and the constants that define it can be changed on the form and the results plotted and examined. The newly defined equation and constants will be used in subsequent calculations, but will only be saved permanently if this is

deliberately done using the File: Save Case Parameters menu.

\section{Pseudo 2D Hydrocarbon Generation}

Once the physical and thermal history of an area has been defined, the next questions

Heatflow and Maturation

Evaluate Heatflow

Evaluate Maturation usually regard the maturation of hydrocarbons and the degree to which this depends on the kerogen type and the maturation model chosen. These questions can be addressed in a preliminary way with the Heatflow and Maturation: Evaluate Maturation form. When this menu command is issued, the

form and graphic in Figure 27 appears. The maturation at a particular point can then be calculated by using the navigator to move the element marker to the desired location, in this case the intersection of pseudowell 5 with the Jurassic kerogen source bed, and depressing

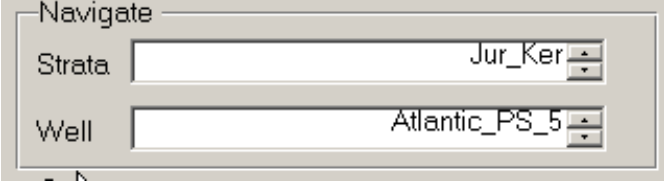


either the Calculate Maturation or Calculate and Normalize button. The difference is that the first calculates the mass fraction (of the original kerogen) is converted to various products, whereas the second normalizes each product to the maximum mass this product could attain (if it was produced and not cracked). This form supports a wide variety of plots (product as a function of time, temperature, vitrinite maturity, for example), and perhaps most importantly shows how a selected kerogen at a selected depth will mature for a particular kinetic model, and allows kinetic models to be easily compared. This is illustrated in Figure 28, Figure 29, and Figure 30.

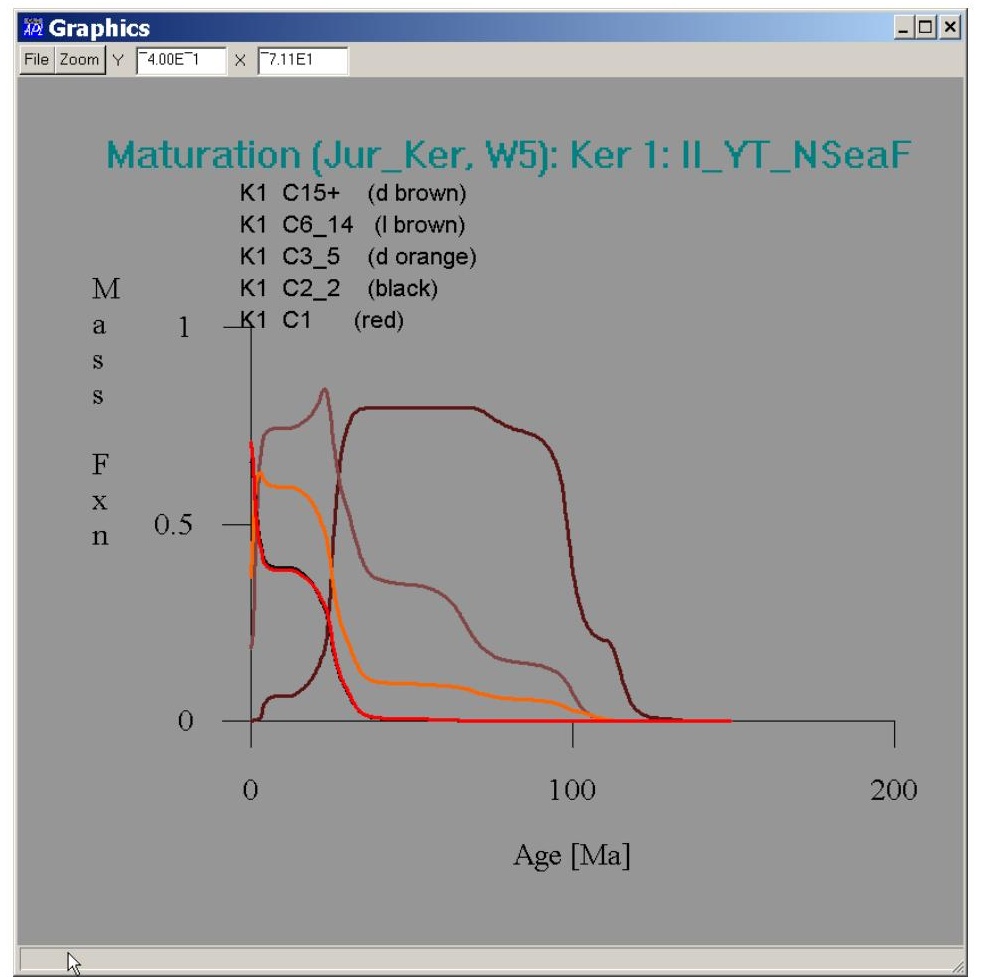

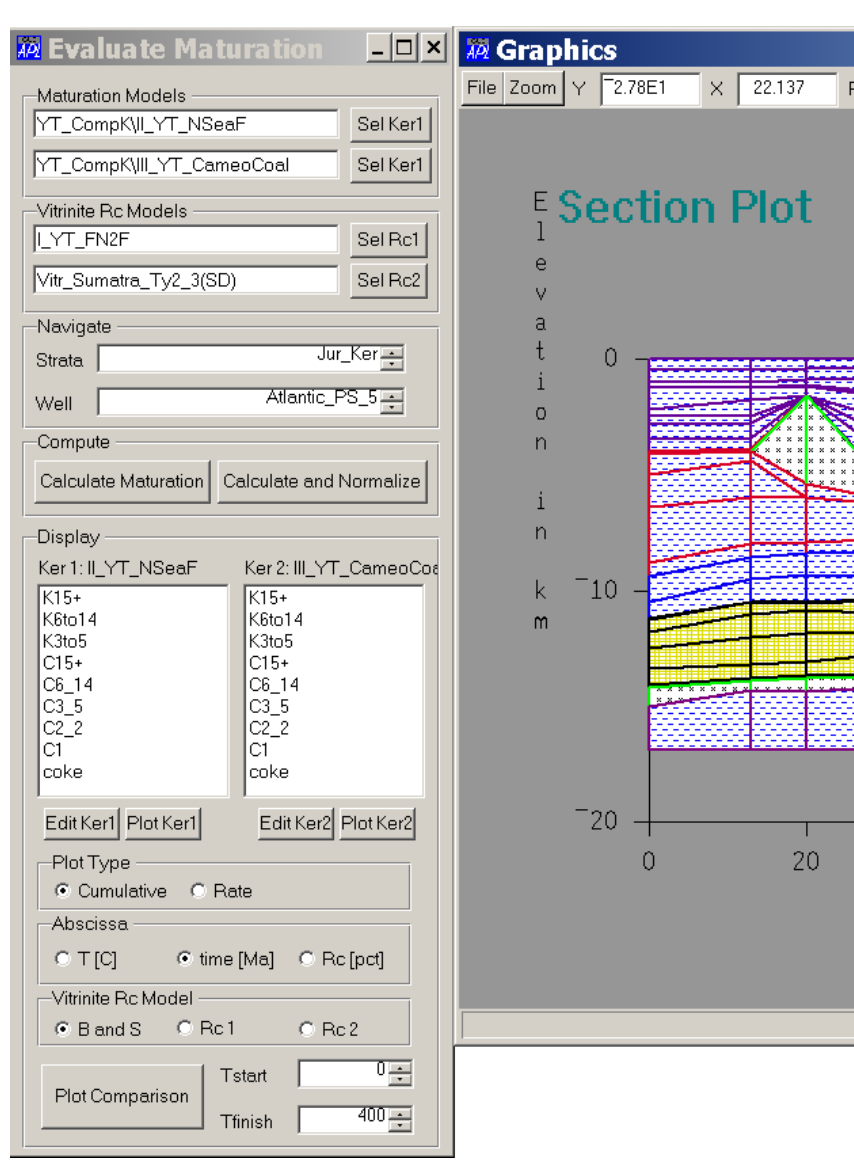

Figure 27. Forms for evaluating hydrocarbon maturation called forth with the Heatflow and Maturation: Evaluate Maturation menu command. 
Figure 28. Computed from Figure 27 by moving the element marker down to the Jurassic source strata using the navigate spinners, and depressing the Compute Maturation Button. The simulated maturation shows that oil has been generated and cracked to methane. Only methane is being generated at the present time.

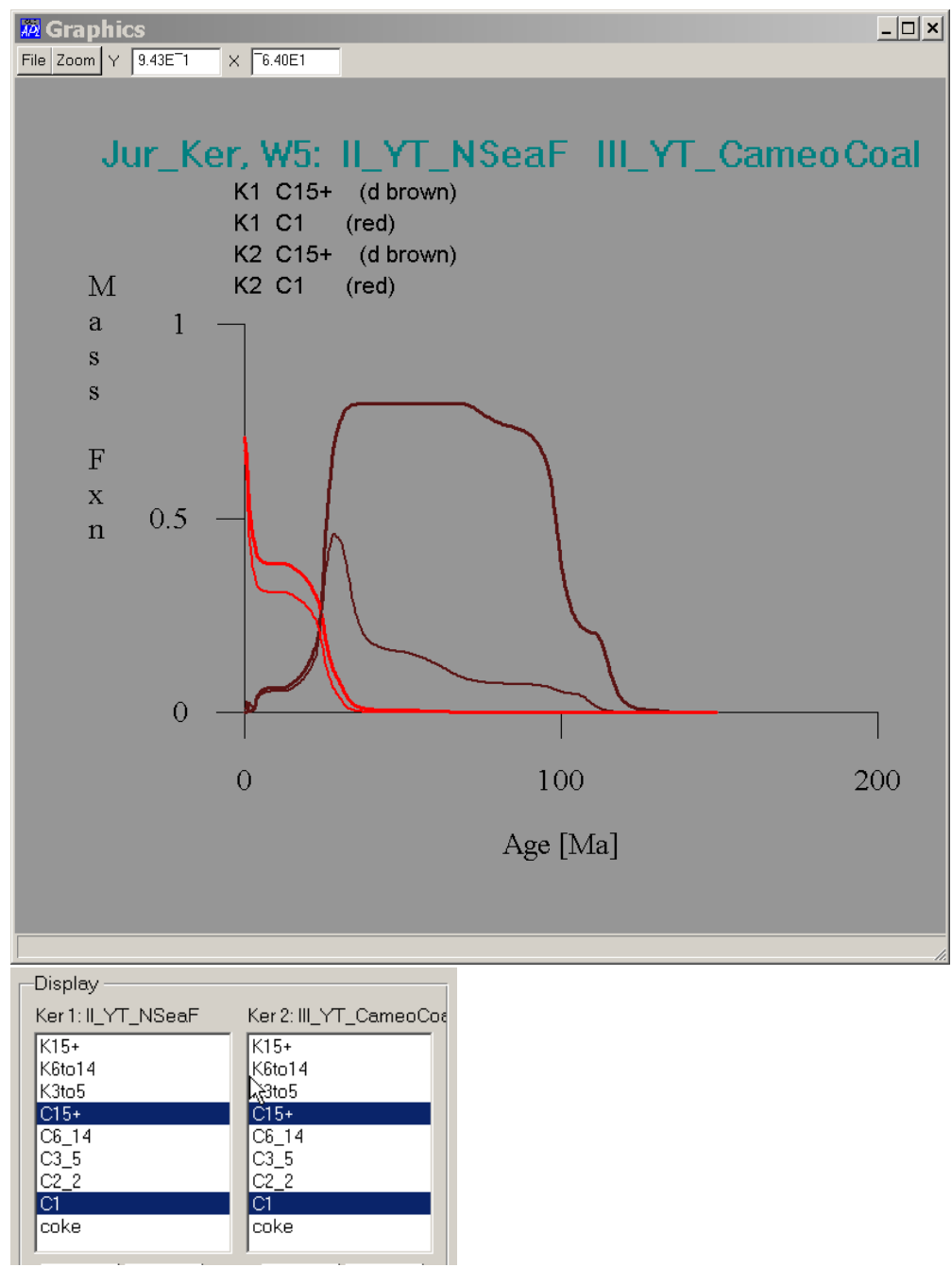

Figure 29. Comparison of methane and $\mathrm{C} 15+$ generation for the North Sea and Cameo Coal kerogens at the Jurassic source location in Well 5. Kerogen 1 is the North Sea Kerogen and is plotted with bold lines. Kerogen 2 is the Cameo Coal kerogen and it is plotted with thinner lines.

Figure is created by selecting the desired products in the combo boxes in the Figure 27 form and depressing the Plot Comparison Button

\section{E. Finite Element Modeling}

The final questions relate to how much hydrocarbon in generated in the section, how it migrates, and what the phase properties of the migrating hydrocarbons are in the section. 
Answering these questions requires creating a finite element grid, solving at least the temperature equation on it, and carrying out flash calculations on the migrating product phases.

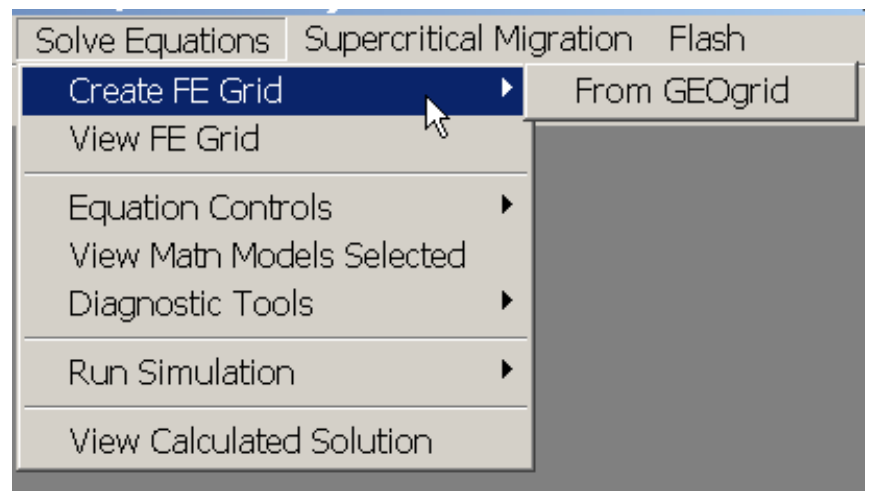

can be viewed, and this is useful when a seal is defined as will be seen in a later example, but this does not concern us here.

The next step is to select the equations to be solved and the frequency with which they are solved. This is done by resetting the equation controls as shown in Figure 30.

\begin{tabular}{|c|c|c|c|c|c|c|c|c|c|}
\hline \multicolumn{3}{|c|}{ API Eq... $-\square \times$} & \multirow{7}{*}{\multicolumn{7}{|c|}{$\begin{array}{l}\text { Figure 30. Selecting Reset Controls } \\
\text { menu as shown will bring up the form } \\
\text { selection of the equations to be solvec } \\
\text { the temperature equation be solved. }\end{array}$}} \\
\hline \begin{tabular}{|l|} 
Equation \\
\end{tabular} & \multicolumn{2}{|c|}{ Solve } & & & & & & & \\
\hline \multicolumn{2}{|l|}{ Pressure } & $\Gamma$ & & & & & & & \\
\hline \begin{tabular}{|l} 
Temperatu... \\
\end{tabular} & \multicolumn{2}{|c|}{$\sqrt{\nabla}$} & & & & & & & \\
\hline Convection & \multicolumn{2}{|r|}{$\Gamma$} & & & & & & & \\
\hline Salinity & \multicolumn{2}{|r|}{$\Gamma$} & & & & & & & \\
\hline $0+\operatorname{twa}=0$ & & 드 & & & & & & & \\
\hline \multicolumn{6}{|c|}{ Select Equations to be Solved } & \multicolumn{4}{|r|}{$-\square$} \\
\hline Age(endst... & $\mathrm{P}$ & $T$ & $P S$ & & TTrS... & NDelt & FxDt & $1 s t \mathrm{Dt}$ & last $D t$ \\
\hline $149.17 \mathrm{Ma}$ & $\Gamma$ & $\bar{v}$ & $\bar{\Gamma}$ & & $\mathrm{c}$ & $1 \div$ & $1.0 \div$ & 0.000000 & 0.000000 \\
\hline $144 \mathrm{Ma}$ & $\Gamma$ & $\bar{v}$ & $\Gamma$ & & $c$ & $1 \div$ & $1.0 \div$ & 5.170000 & 5.170000 \\
\hline $137.912 \mathrm{Ma}$ & $\Gamma$ & $\sqrt{v}$ & ᄃ & & $c$ & $1 \div$ & $1.0 \div$ & 6.088000 & 6.088000 \\
\hline $98 \mathrm{Ma}$ & $\Gamma$ & $\sqrt{v}$ & $\Gamma$ & & c & $1 \div$ & $1.0 \div$ & 39.912000 & 39.912000 \\
\hline $84 \mathrm{Ma}$ & $\Gamma$ & $\sqrt{v}$ & $\bar{\Gamma}$ & & $c$ & $1 \div$ & $1.0 \div$ & 14.00900 & 14.000000 \\
\hline $65 \mathrm{Ma}$ & $\Gamma$ & $\bar{v}$ & $\bar{\Gamma}$ & & $\mathrm{C}$ & $1 \div$ & $1.0 \div$ & 19.000 & 19.000000 \\
\hline $53 \mathrm{Ma}$ & $\Gamma$ & $\sqrt{v}$ & $\Gamma$ & & c & $1 \div$ & $1.0 \div$ & 12.000000 & 12.000000 \\
\hline $50 \mathrm{Ma}$ & $\Gamma$ & $\sqrt{v}$ & ᄃ & & $c$ & $1 \div$ & $1.0 \div$ & \begin{tabular}{|l|}
3.000000 \\
\end{tabular} & 3.000000 \\
\hline $40 \mathrm{Ma}$ & $\Gamma$ & $\sqrt{v}$ & $\bar{\Gamma}$ & & $c$ & $5 \div$ & $1.0 \div$ & 2.000000 & 2.000000 \\
\hline $30 \mathrm{Ma}$ & $\Gamma$ & $\bar{v}$ & $\bar{\Gamma}$ & & $\mathrm{C}$ & $5 \div$ & $1.4 \div$ & 0.913609 & 3.509721 \\
\hline $21.5 \mathrm{Ma}$ & $\Gamma$ & $\sqrt{v}$ & $\bar{\Gamma}$ & & c & $1 \div$ & $1.0 \div$ & 8.500000 & 8.500000 \\
\hline $13.8 \mathrm{Ma}$ & $\Gamma$ & $\bar{v}$ & $\bar{\Gamma}$ & & $\mathrm{c}$ & $1 \div$ & $1.0 \div$ & 7.700000 & 7.700000 \\
\hline $12.5 \mathrm{Ma}$ & $\Gamma$ & $\sqrt{v}$ & $\Gamma$ & & o & $1 \div$ & $1.0 \div$ & 1.300000 & 1.300000 \\
\hline $10.6 \mathrm{Ma}$ & $\Gamma$ & $\sqrt{v}$ & $\bar{\Gamma}$ & & $\mathrm{C}$ & $1 \div$ & $1.0 \div$ & 1.900000 & 1.900000 \\
\hline $10.5 \mathrm{Ma}$ & $\Gamma$ & $\bar{v}$ & г & & $c$ & $1 \div$ & $1.0 \div$ & 0.100000 & 0.100000 \\
\hline $10.1 \mathrm{Ma}$ & $\Gamma$ & $\bar{v}$ & $\Gamma$ & & $\mathrm{c}$ & $1 \div$ & $1.0 \div$ & 0.400000 & 0.400000 \\
\hline $8.8 \mathrm{Ma}$ & $\Gamma$ & $\bar{v}$ & I & & $\mathrm{c}$ & $1 \div$ & $1.0 \div$ & 1.300000 & 1.300000 \\
\hline $8.2 \mathrm{Ma}$ & $\Gamma$ & $\bar{v}$ & $\bar{\Gamma}$ & & $\mathrm{c}$ & $1 \div$ & $1.0 \div$ & 0.600000 & 0.600000 \\
\hline $6.3 \mathrm{Ma}$ & $\Gamma$ & $\sqrt{v}$ & I & & $c$ & $1 \div$ & $1.0 \div$ & 1.900000 & 1.900000 \\
\hline $5.8 \mathrm{Ma}$ & $\Gamma$ & $\bar{v}$ & $\bar{\Gamma}$ & & $\mathrm{c}$ & $1 \div$ & $1.0 \div$ & 0.500000 & 0.500000 \\
\hline $5.5 \mathrm{Ma}$ & $\Gamma$ & $\sqrt{v}$ & I & & $\mathrm{C}$ & $1 \div$ & $1.0 \div$ & 0.300000 & 0.300000 \\
\hline $4.2 \mathrm{Ma}$ & $\Gamma$ & $\bar{v}$ & ᄃ & & $\mathrm{c}$ & $1 \div$ & $1.0 \div$ & 1.300000 & 1.300000 \\
\hline $3 \mathrm{Ma}$ & $\Gamma$ & $\sqrt{v}$ & $\bar{\Gamma}$ & & $\mathrm{C}$ & $1 \div$ & $1.0 \div$ & 1.200000 & 1.200000 \\
\hline $1.4 \mathrm{Ma}$ & $\Gamma$ & $\sqrt{v}$ & $\Gamma$ & & $\mathrm{C}$ & $1 \div$ & $1.0 \div$ & 1.600000 & 1.600000 \\
\hline $0.95 \mathrm{Ma}$ & $\Gamma$ & $\sqrt{v}$ & I & & $\mathrm{C}$ & $1 \div$ & $1.0 \div$ & 0.450000 & 0.450000 \\
\hline $0.5 \mathrm{Ma}$ & $\Gamma$ & $\sqrt{v}$ & ᄃ & & $c$ & $1 \div$ & $1.0 \div$ & 0.450000 & 0.450000 \\
\hline $0.25 \mathrm{Ma}$ & $\Gamma$ & $\bar{v}$ & $\Gamma$ & & $\mathrm{c}$ & $1 \div$ & $1.0 \div$ & 0.250000 & 0.250000 \\
\hline $0 \mathrm{Ma}$ & $\Gamma$ & $\sqrt{v}$ & ᄃ & & $c$ & $1 \div$ & $1.0 \div$ & 0.250000 & 0.250000 \\
\hline
\end{tabular}

The finite element grid is set up using the Solve Equations: Create FE Grid menu: From GEOgrid command as illustrated to the left. This takes the frames in the geohistory movie and produces a triangular finite element mesh from them. Provided the GEOgrid is regular (not twisted), generation of the finite element mesh is automatic and need not concern the user. It 
here. Only the temperature equation is solved. It is solved once $($ NDelt $=1)$ after each stratum is deposited. The spinners can be used to increase the number of timesteps steps taken after a stratum is deposited. For example we could solve for $\mathrm{T}$ in 5 timesteps after the deposition of a strata by toggling NDelt to 5 as shown for the strata whose deposition ends at 40Ma. The timesteps can be of equal size (if $\mathrm{FxDt}=1$ ), or can exponentially increase $(\mathrm{FxDt}>1)$ with timestep. The first and last $\mathrm{Dt}$ are shown. For the deposition ending at $30 \mathrm{Ma}, \mathrm{FxDT}=1.4$ allows the initial timestep to be $0.91 \mathrm{Ma}$ and the last 3.5, rather than a uniform $2 \mathrm{Ma}$. Temperature in the first layer is assumed to be the steadystate temperature. Hence timesteps do not apply and they are set to zero.

Double clicking on any of the endstep ages (first column) gives information on the number of subtimesteps and iterations allowed. This is shown in Figure 32. As with all forms and buttons, the hint bar is useful in understanding the labels and function of the various parameters. The hint bar in for the form in Figure 32 is shown in Figure 33 as an example. The default parameters will normally serve to give a good solution, but in some cases it is useful to access them to fine tune a solution or investigate convergence.

\begin{tabular}{|c|c|c|c|c|c|c|c|c|}
\hline \multicolumn{8}{|c|}{ Aquation Solution Controls at timestep 10} & \multirow[t]{2}{*}{$-|\square| x$} \\
\hline Equation & Solve & $\# . s s$ & tnst & NS... & Nit & $\mathrm{TH}$ & Trk... & \\
\hline Pressure & $\Gamma$ & c & $c$ & 0 & 1 & 0 & 0 & \\
\hline Temperatu.. & $\sqrt{v}$ & 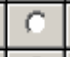 & 6 & -2 & 1 & 1 & 0 & \\
\hline Convection & $\Gamma$ & c & $c$ & 0 & 1 & 0 & 0 & \\
\hline Salinity & $\Gamma$ & $C$ & c & -2 & 1 & 1 & 0 & \\
\hline Pt-track Sa.. & $\pi$ & $c$ & $c$ & 0 & 1 & 0 & 0 & \\
\hline
\end{tabular}

Figure 32. Finite element parameters that control the $10^{\text {th }}$ timestep (which ends at $30 \mathrm{Ma}$. Form arises when first column entry in Figure 31 form is doubleclicked. The transient temperature equation is solved implicitly $(\mathrm{TH}=1)$ with a maximum of 2 subtimesteps $(\mathrm{Nsubt}=-2)$ and one iteration. No variables are tracked. The hint bar for this form is shown in Figure 33.

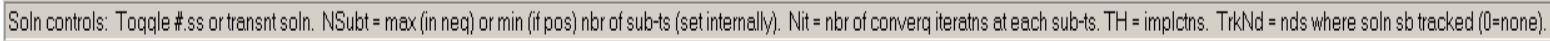

Figure 33. The hint bar that appears at the botton of the workspace when the mouse is positioned over the form in Figure 32.

\begin{tabular}{|l|l|}
\hline \hline Solve Equations Supercritical Migration Flash \\
Create FE Grid \\
View FE Grid \\
\hline $\begin{array}{l}\text { Equation Controls } \\
\text { View Matn Models Selected } \\
\text { Diagnostic Tools }\end{array}$ \\
\hline Run Simulation & All Selected 2D Equations \\
\hline Assign Kerogen Mass & \\
\hline Select Avg Ele T \\
\hline View Calculated Solution \\
\hline
\end{tabular}

Temperature is solved on the finite element grid when one of these two commands is executed. Either temperature is solved in all the wells (1D solution), and nodal results 
averaged over each element to give the element average temperature, or the temperature equation (and any other equation selected) is solved by finite element methods. In either case, temperature-dependent parameters are computed and all relevant parameters are placed in the SOLGRID file. Any of these variables can be subsequently viewed using the Solve Equations: View Calculated Solution menu (above). This is a good point to mention that the components of an analysis are preserved in system files as the analysis is made. For example the geologic history is stored in the GEOGRID file, the finite element grid in the FEGRID file, and the solution of the finite element equations, and subsequent migration rates, hydrocarbon chemistry, etc., in the SOLGRID file. This means that an analysis can always be picked up where it left off. No previous analysis steps need be repeated. These files are indicated in the flow charts (e.g., see p. 232, 293).

First run the Pseudo 2D Temperature solution by selecting the menu command as shown

Supercritical Migration Flash

Calculate Solid Phase Maturation

Set Migration Controls

$\checkmark$ Calculate Mobile Phase Maturation

just discussed. The 1D parameters are set in Parameters: 1D Thermal Calculation Controls.

Now solve the 2D temperature equation on the finite element grid by selecting All Selected 2D Equations above. The solution will take a couple of minutes to compute.

Examination of finite element calculations in a 2D section is always done with the

\begin{tabular}{l}
\hline Solve Equations Supercritical Mi \\
Create FE Grid \\
View FE Grid \\
\hline Equation Controls \\
View Matn Models Selected \\
Diagnostic Tools \\
\hline Run Simulation \\
\hline Assign Kerogen Mass \\
\hline Select Avg Ele T \\
\hline View Calculated Solution \\
\hline
\end{tabular}
Solve Equations: View Calculated Solution menu as shown to the left. This brings up the forms shown in Figure 34, which shows the results of the 2D temperature solution. The solution can be viewed at any of the 28 timesteps using the Frame spinner on the toolbar, and any of the solution variables can be examined. Figure 34 shows thermal conductivity as an example. Temperature has been selected as the tracking variable on the plot. The temperature under the mouse tip is shown on the Graphics toolbar. Any of the variables on the list on the form can be plotted by selecting that variable and depressing the Plot button. Also any variable can be selected as a tracking variable; it need not be the sane as the variable plotted. 


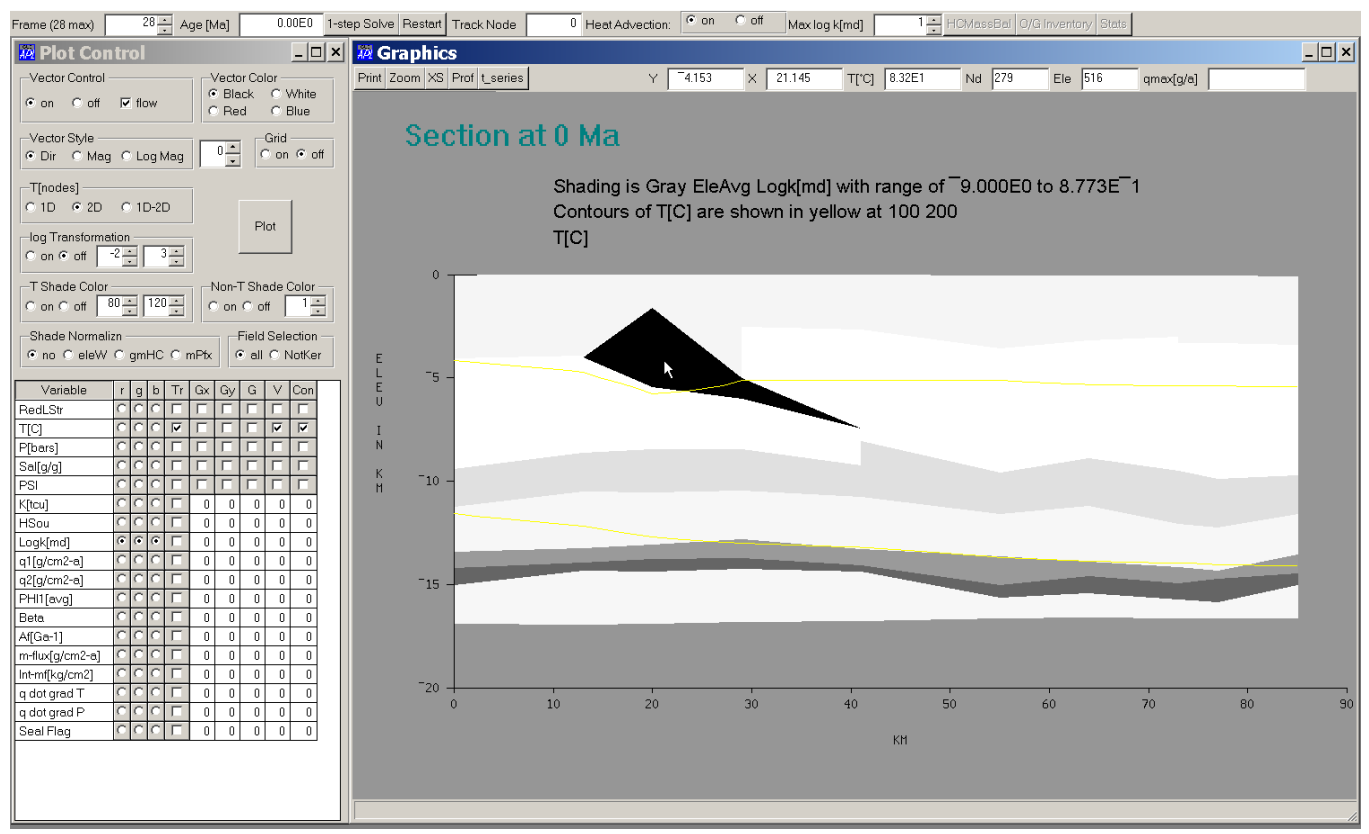

Figure 34. Cross-section display of the Log 10 permeability in the $2 \mathrm{D}$ temperature solution. The temperature at the mouse tip is $83.2^{\circ} \mathrm{C}$. All physical parameters are available to plot or track, although many have zero values because the controlling equations were not selected for solution (for example the flow variables are zero because neither the pressure nor the convection equations were solved).

Plot style can be controlled in a variety of ways using the $X S$ form shown in Figure 34. For example to depict the oil and gas windows, temperature could be shaded blue below $80^{\circ} \mathrm{C}$, green from 80 to $120^{\circ} \mathrm{C}$, and red above $120^{\circ} \mathrm{C}$ using the Thade Color control on the XS form. This is shown in Figure 35.

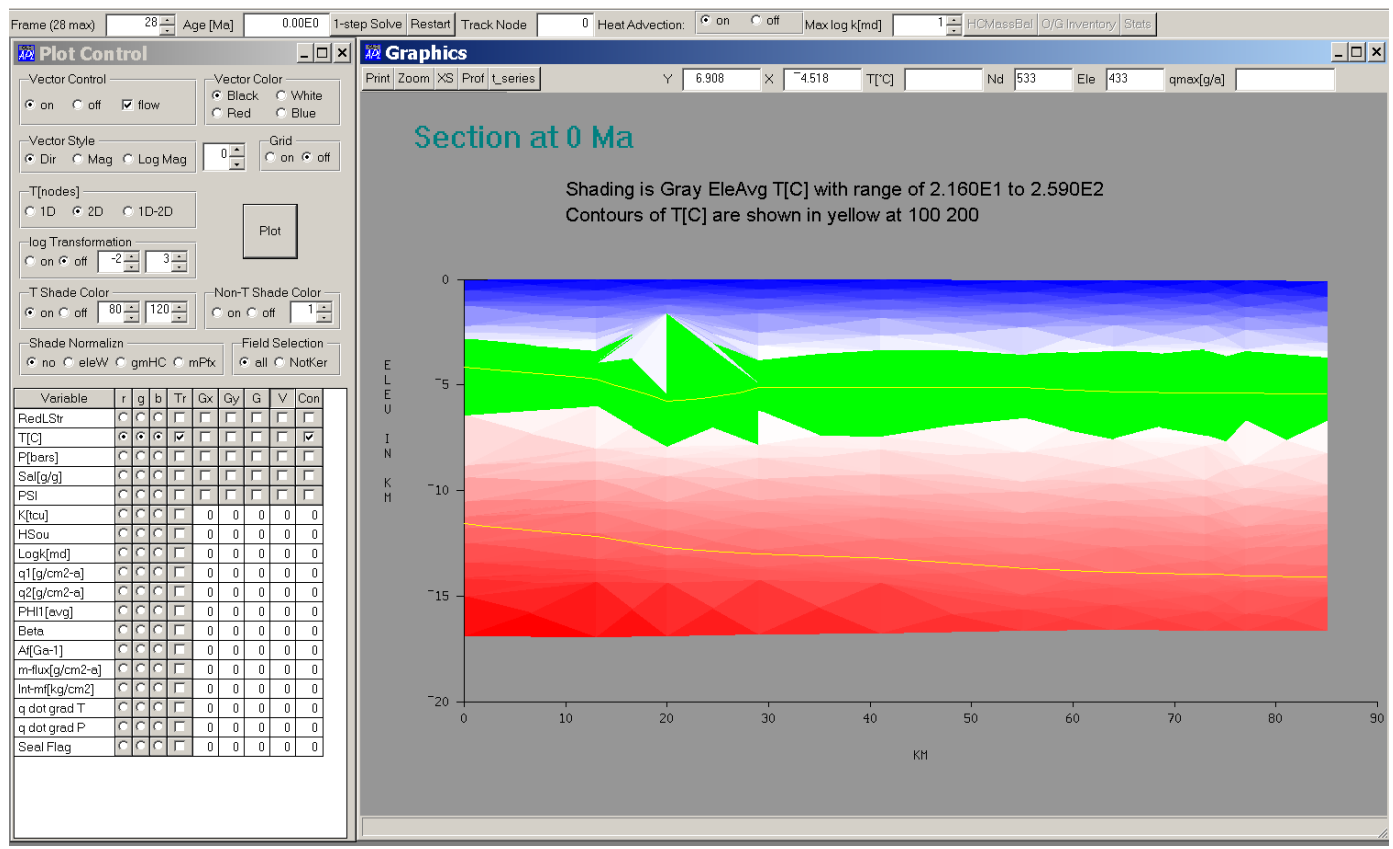


Figure 35. T Shade Control is used to highlight the oil window $\left(80<\mathrm{T}<120^{\circ} \mathrm{C}\right)$ in green.

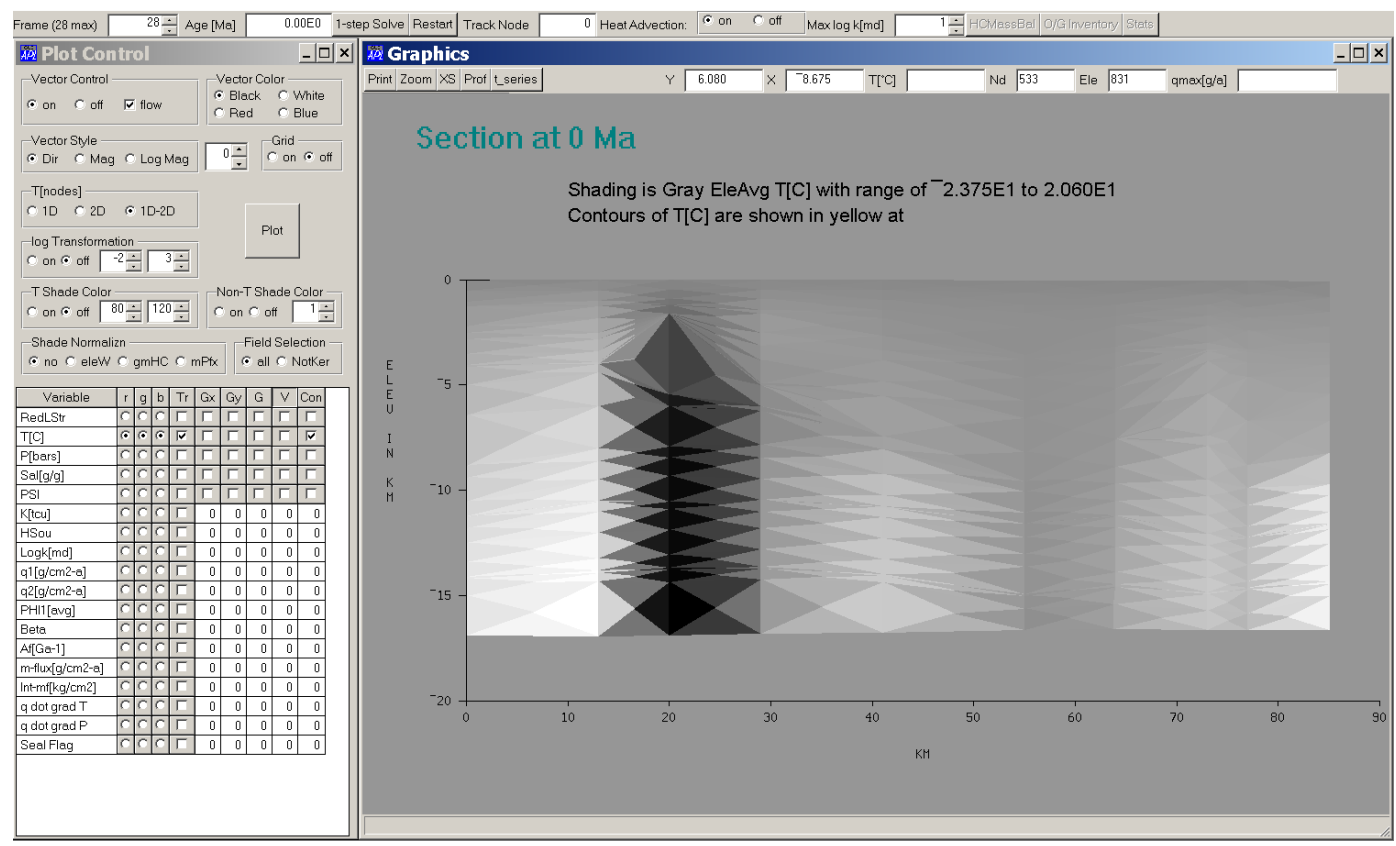

Figure 36. Plot of $\mathrm{T}_{1 \mathrm{D}}-\mathrm{T}_{2 \mathrm{D}}$ obtained by selecting $1 \mathrm{D}-2 \mathrm{D}$ in the $\mathrm{T}[$ nodes] control box as shown. The caption, grayscale, and positioning the mouse as shown all indicate the differences in temperature can be $+/-20^{\circ} \mathrm{C}$. The $1 \mathrm{D}$ solutions temperatures are much too low under the salt dome (vertical dark band) and much too high adjacent to this band.

The difference between the 1D and 2D temperature solutions is often of interest because maturity calculations may have been made with 1D calculations (as here). Figure 36 shows the difference between the 1D and 2D temperature solutions (e.g., plots $\left.\mathrm{T}_{1 \mathrm{D}}-\mathrm{T}_{2 \mathrm{D}}\right)$.

Strata and pseudowell-parallel profiles can be plotted using the Section Profiles control. Press the Prof button on the Graphics toolbar in Figure 36 to get this form. Figure 37 provides an example. 


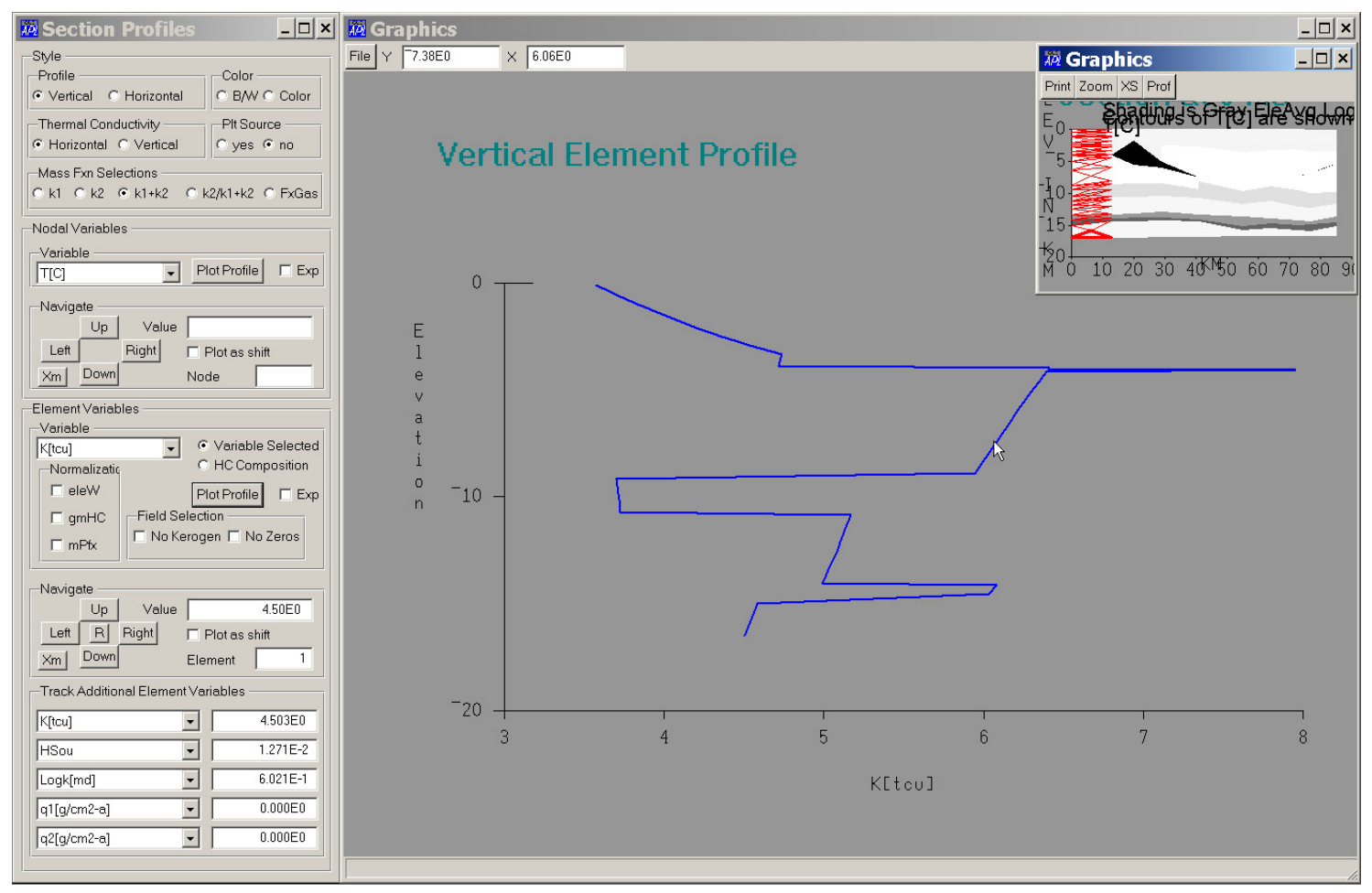

Figure 37. Thermal conductivity profile through the first vertical set of elements as indicated in the insert. Element navigator was used to select the first vertical element profile (vertical because vertical is selected in the profile group of the form), and the Plot Profile button in the Element Variables group depressed. Any variable could be selected. In this case we have selected thermal conductivity (K[tcu] in Element Variables group of Section Profiles form above left).

\section{F. Selecting And Modifying Kinetic Maturation Models}

The next step is to mature kerogen in the 2D section and migrate those hydrocarbons according to migration rules and criteria. The first step in this process is usually choosing an appropriate kinetic maturation model for the kerogen in the section. Migration results can be very sensitive to the choice of the maturation model.

Seemingly small differences in the maturation model can have big effects on the calculated pattern and chemistry of the migrating hydrocarbons.

Selection of a kerogen maturation model is facilitated with a tool similar to that shown in Figure 27. This tool uses the time-temperature history of a selected element to calculate, compare, and modify the kerogen conversion of two kinetic models. The modified kinetic model can then be used to calculate maturation and migration in the $2 \mathrm{D}$ section.

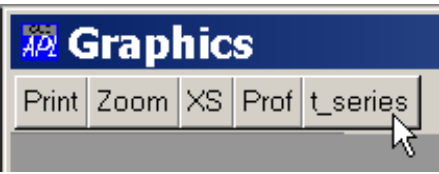

The maturation comparison tool is accessed through the $t$ series button on the toolbar of the Graphics form shown in 
Figure 34 to Figure 36 and illustrated to the left. All these figures used the $X S$ form, Figure 37 the Profform, and we will now use the $t$-series form. Figure 38 shows how this form can be used to select an element containing Jurassic Kerogen and plot useful time series in this element.

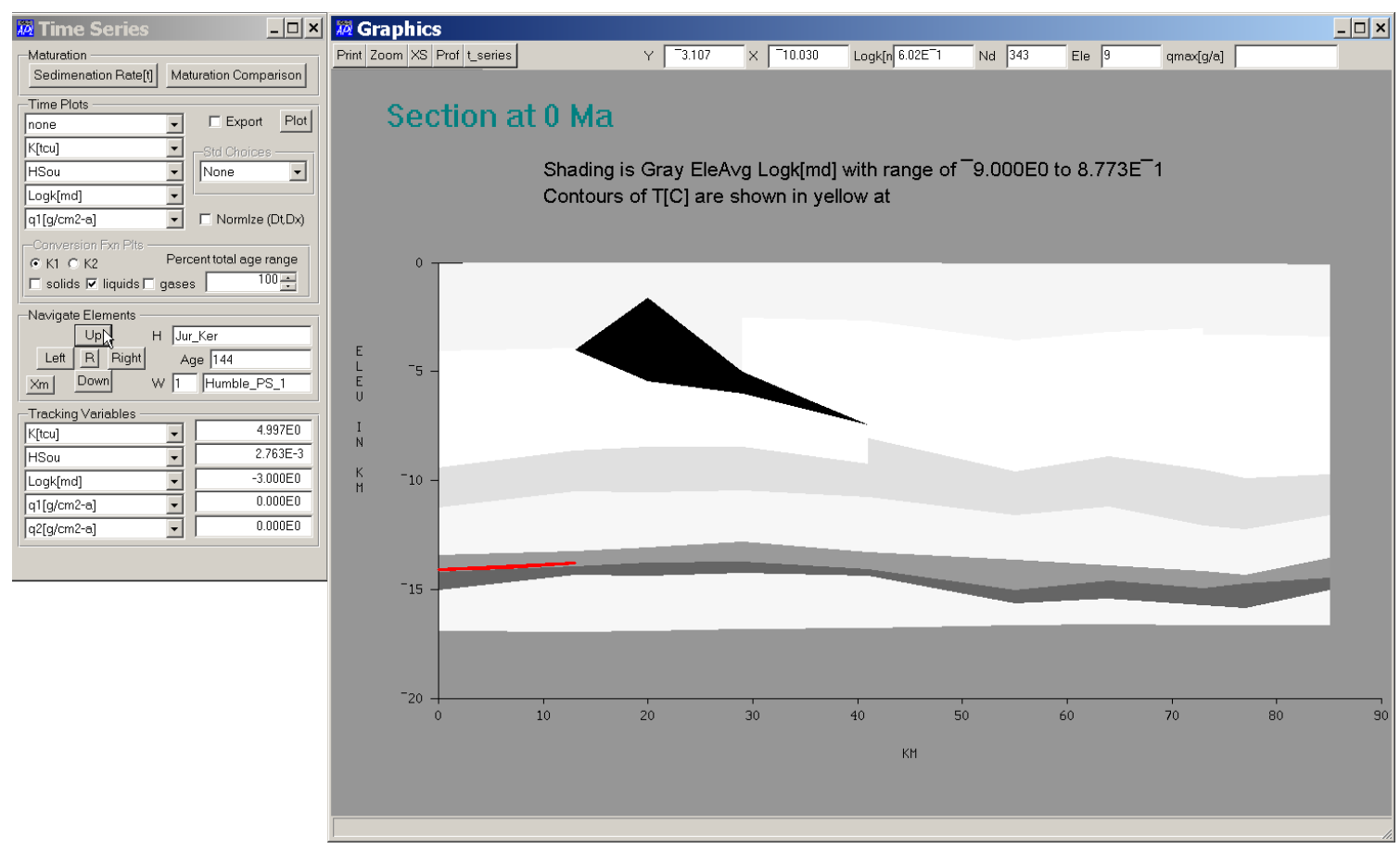

Figure 38. View the 2D solution (Solve Equations: View Calculated Solutions), select the $\mathrm{t}$-series form (depress toolbar t-series button), and use the resulting form to navigate to the 144 Ma Jurassic Kerogen element as illustrated. Depressing the Maturation Comparison button brings up the form in Figure 39. 

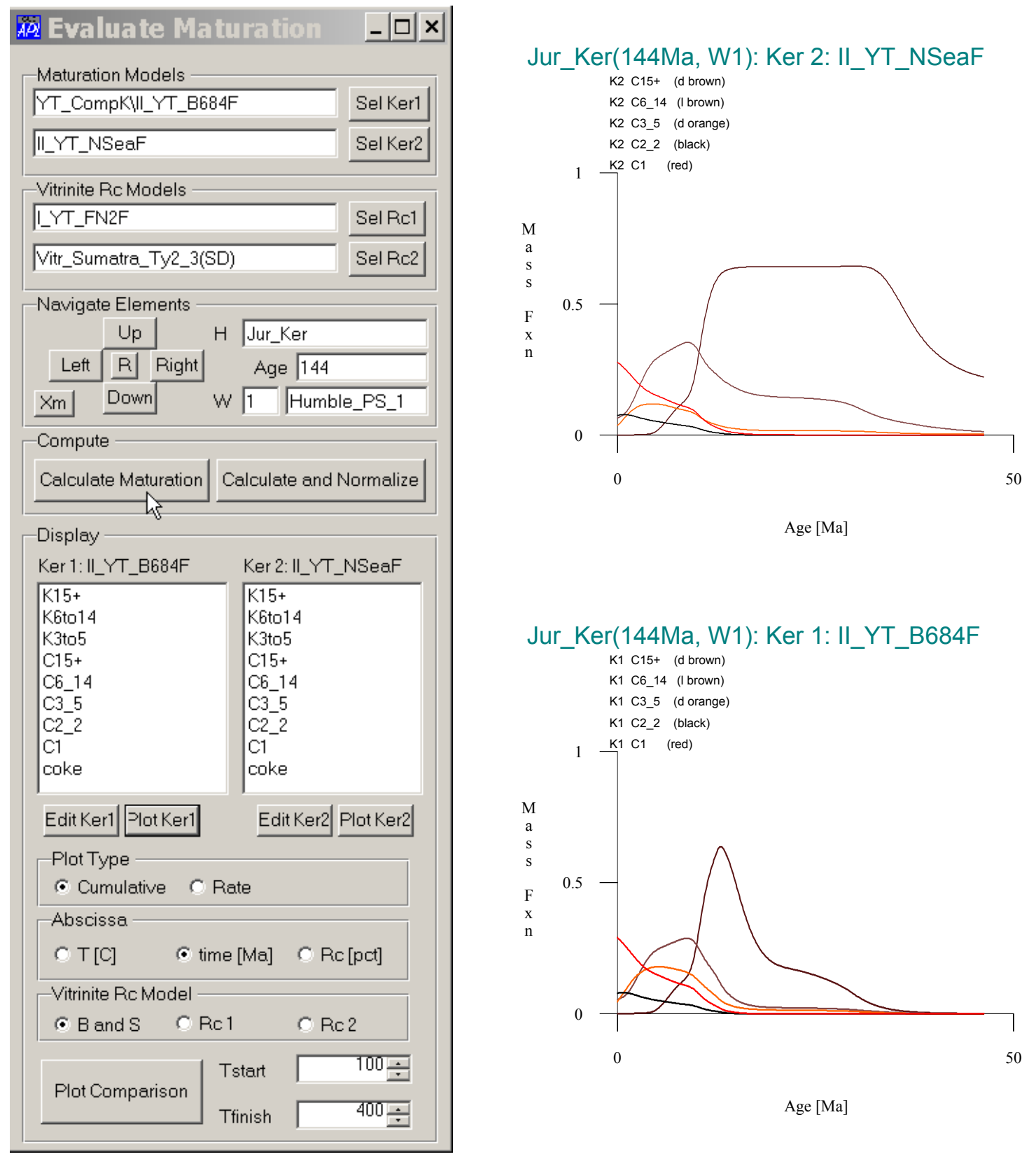

Age $[\mathrm{Ma}]$

Figure 39. Depressing the Calculate Maturation button and then (sequentially) the Plot Ker1 and Plot Ker2 buttons results in the plots on the right. Note Tstart is $100^{\circ} \mathrm{C}$ and Tfinish $400^{\circ} \mathrm{C}$. The maturation is for the element selected in Figure 38. Maturation models for Type II kerogens can clearly be very different.

Figure 40 compares the II_YT_B684F maturation model to a standard industry Kimmeridge Clay maturation model. Kinetic models are compared further in the next figure. In this case we have read in a different kinetic model (the II_H_Kimer_aug model) using the Sel Kerl button at the top of the form in Figure 39, 
and have recomputed the maturation history for these two models by depressing the Calculate Maturation button. 

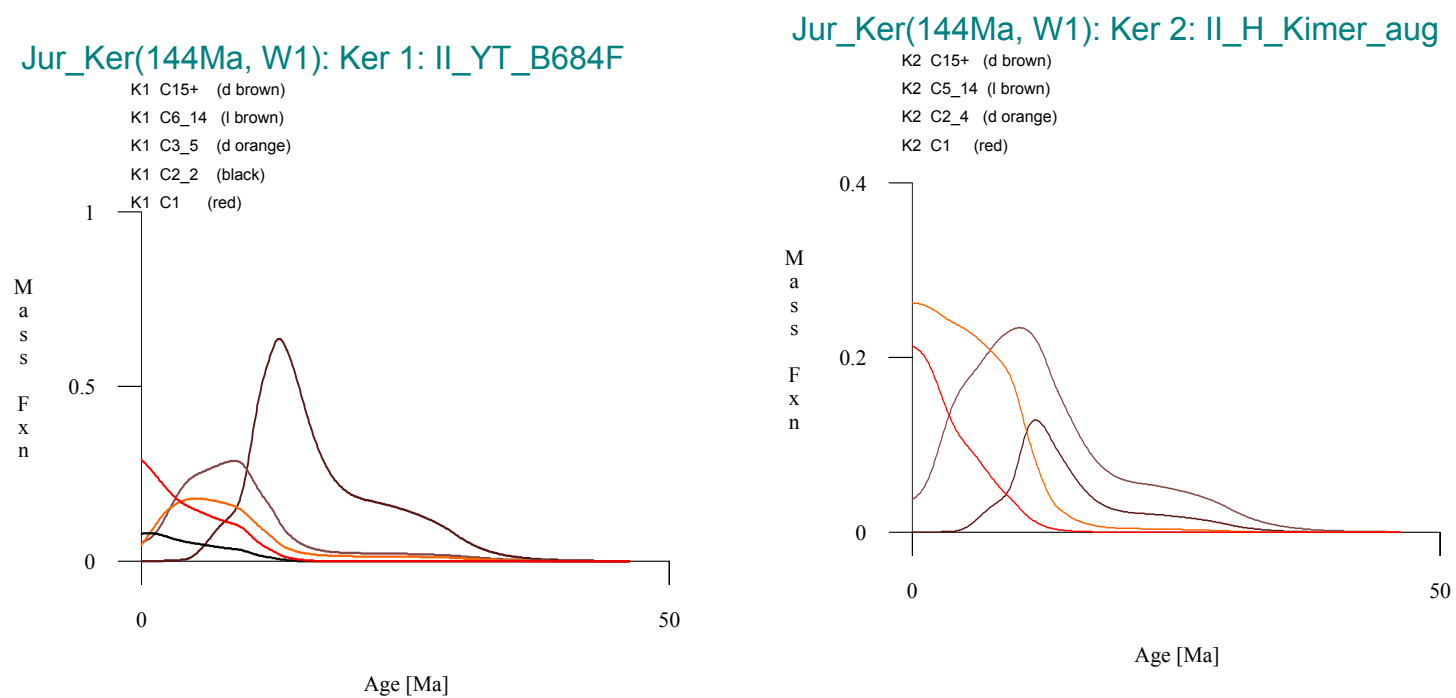

Figure 40. Comparison of the II_YT_B684F maturation model to a Kimmeridge Clay maturation model for the element selected in Figure 39. Comparison is made by sequentially depressing the Sel Ker2, Calculate Maturation, Plot Ker 1 and Plot Ker2 buttons on the form in Figure 39.
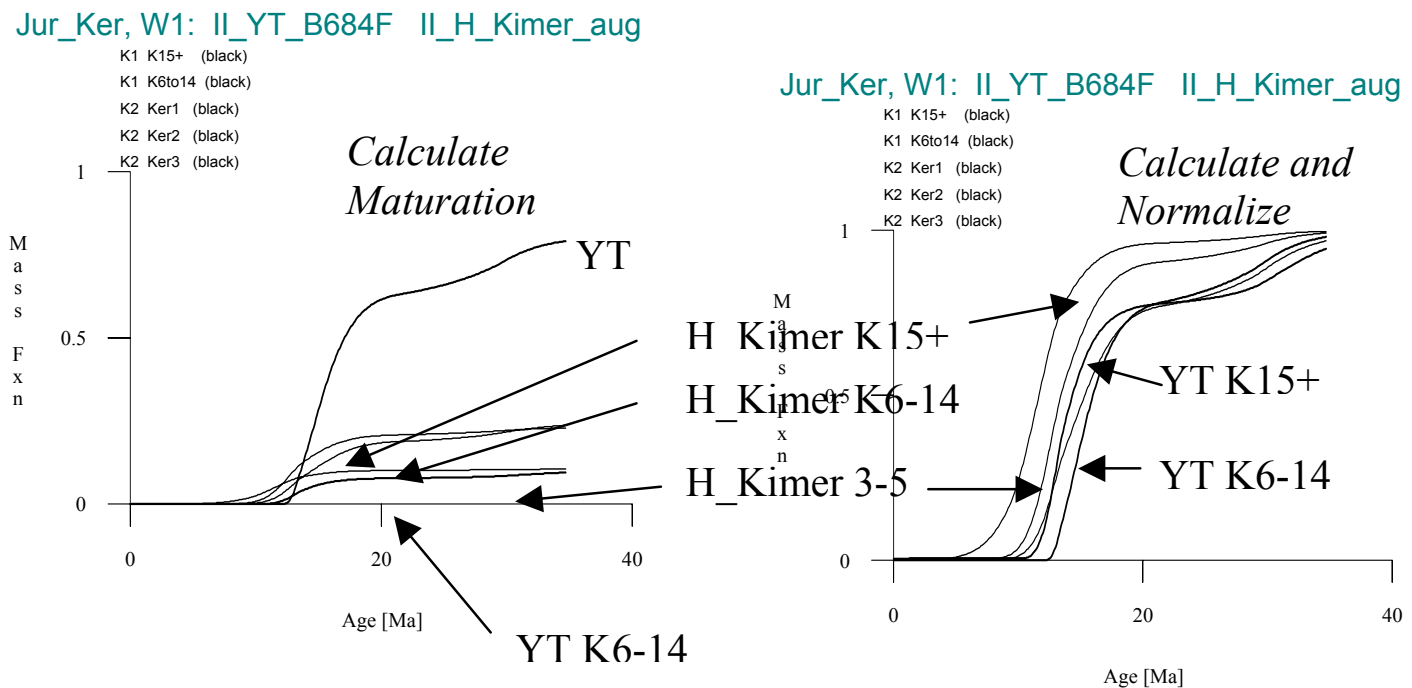

Figure 41. Comparison of particular maturation components in II_YT_B684F and II_H_Kimer_aug models shown in Figure 40. Components are selected in the combo boxes in the Display section of the form in Figure 39 and plotted with the Plot Comparison button. The YT kerogens are converted slightly sooner than the Kimmeridge kerogen. The graph on the right is calculated with the Calculate and Normalize button, which normalized all reactions to their maximum value. This 
facilitates comparing the timing of conversion. We focus on the kerogen conversion reactions because these are not affected by migration.

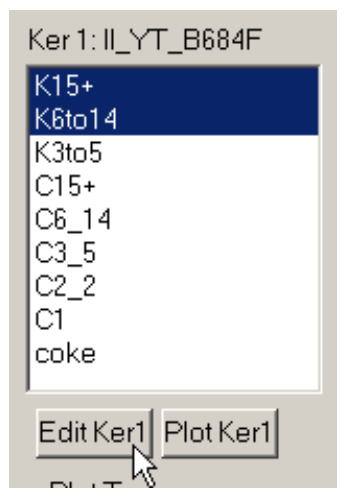

The II_YT_B684F kinetic model can be edited so that its maturation ends less abruptly by modifying kinetic parameters for $\mathrm{K} 15+$ conversion. The $\mathrm{K} 15+$ part of the kerogen in this model is by far the most important kerogen fraction, accounting for over 80 $\mathrm{Wt} \%$ of the hydrocarbon products produced. Editing is initiated by depressing the Edit Kerl button on the form shown in Figure 39 as illustrated to the left. This brings up spreadsheets containing all the kinetic parameters. Portions of the spreadsheet for the activation energy and weights is shown in Figure 42.

\begin{tabular}{|c|c|c|c|c|}
\hline \multicolumn{5}{|c|}{ AP Activ. Energy and Wts II_YT_B684F } \\
\hline \multicolumn{5}{|l|}{ Apply } \\
\hline & K15+ & K6to14 & K3to5 & C15+ \\
\hline 1 & 52000 & 51000 & 52000 & 53690 \\
\hline 2 & 53000 & 52000 & 53000 & 56350 \\
\hline 3 & 54000 & 55000 & 56000 & 59000 \\
\hline 4 & 0 & 56000 & 57000 & 61660 \\
\hline 5 & 0 & 57000 & 59000 & 64310 \\
\hline 6 & 0 & 67000 & 60000 & 0 \\
\hline 7 & 0 & 0 & 68000 & 0 \\
\hline 8 & 0 & 0 & 69000 & 0 \\
\hline
\end{tabular}

\begin{tabular}{r|r|r|}
\hline \multicolumn{1}{|c|}{ K15+ } & \multicolumn{1}{|c|}{ K6to14 } & \multicolumn{1}{|c|}{ K3to5 } \\
\hline 0.0795 & 0.0367 & 0.0333 \\
\hline 0.1334 & 0.1436 & 0.1143 \\
\hline 0.7871 & 0.5324 & 0.2256 \\
\hline 0 & 0.2733 & 0.296 \\
\hline 0 & 0.0076 & 0.3066 \\
\hline 0 & 0.0064 & 0.0062 \\
\hline 0 & 0 & 0.0122 \\
\hline 0 & 0 & 0.0058 \\
\hline
\end{tabular}

Figure 42. Portions of the spreadsheet containing the Activation Energy and Weights for the II_YT_B684F maturation model. Changing the K15+ parameters to the values shown in Figure $\mathbf{4 3}$ and applying them to the maturation calculations results in the shift of the K15+ conversion curve shown in Figure 44. 


\begin{tabular}{|r|r|r|r|}
\hline \multicolumn{1}{|c|}{ K15+ } & \multicolumn{1}{|c|}{ K6to14 } & \multicolumn{1}{c|}{ K3to5 } & \multicolumn{1}{c|}{ C15+ } \\
\hline 50000 & 51000 & 52000 & 53690 \\
\hline 55000 & 52000 & 53000 & 56350 \\
\hline 60000 & 55000 & 56000 & 59000 \\
\hline 65000 & 56000 & 57000 & 61660 \\
\hline 70000 & 57000 & 59000 & 64310 \\
\hline 0 & 67000 & 60000 & 0 \\
\hline 0 & 0 & 68000 & 0 \\
\hline 0 & 0 & 69000 & 0 \\
\hline
\end{tabular}

\begin{tabular}{|r|r|r|}
\hline \multicolumn{1}{|c|}{ C15+ } & \multicolumn{1}{c|}{ C6_14 } & \multicolumn{1}{c|}{ C3_5 } \\
\hline 0.0066 & 0.0066 & 0.0066 \\
\hline 0.1942 & 0.1942 & 0.1942 \\
\hline 0.5984 & 0.5984 & 0.5984 \\
\hline 0.1942 & 0.1942 & 0.1942 \\
\hline 0.0066 & 0.0066 & 0.0066 \\
\hline 0 & 0 & 0 \\
\hline 0 & 0 & 0 \\
\hline 0 & 0 & 0 \\
\hline
\end{tabular}

Figure 43. Modified II_YT_B684F kinetics. Note changes in K15+ activation energy and weights relative to Figure 42. The pre-exponential factor for the K15+ maturation reaction was also changed from $10^{14} \mathrm{sec}^{-1}$ to $10^{15} \mathrm{sec}^{-1}$. The maturation of $\mathrm{K} 15+$ is compared to H_Kimer_K15+ and K1 in Figure 44.

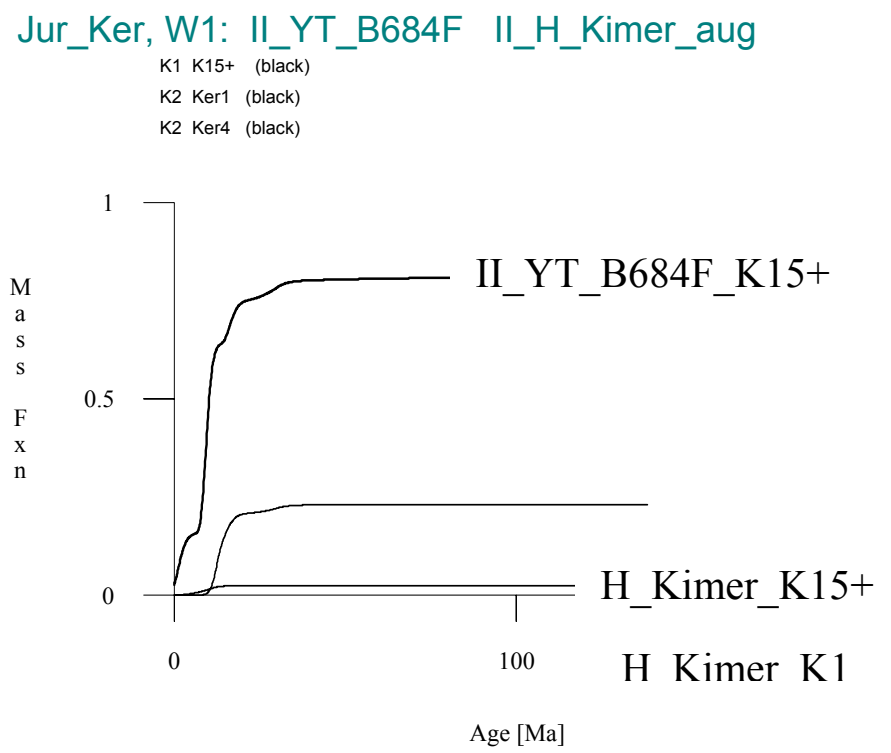

Figure 44. Comparison of kinetic models shown in Figure 37 as modified in Figure 43. The K15+ maturation for the II_YT_B684F model now approximates the timing of the $\mathrm{K} 15+$ and $\mathrm{K} 1$ kerogen components of the Kimmerigian, although the mass fraction converted is quite different.

\section{G. 2D Maturation and Migration}

We will choose the Jurassic Kerogen with the modified II_YT_B684F model shown in Figure 43 for the Jurassic source, and the III_YT_CameoCoal maturation model for the the Eocene kerogen. The next step is to mature these kerogens in the 2D section and migrate the hydrocarbons that are generated according to specified migration rules and criteria. 

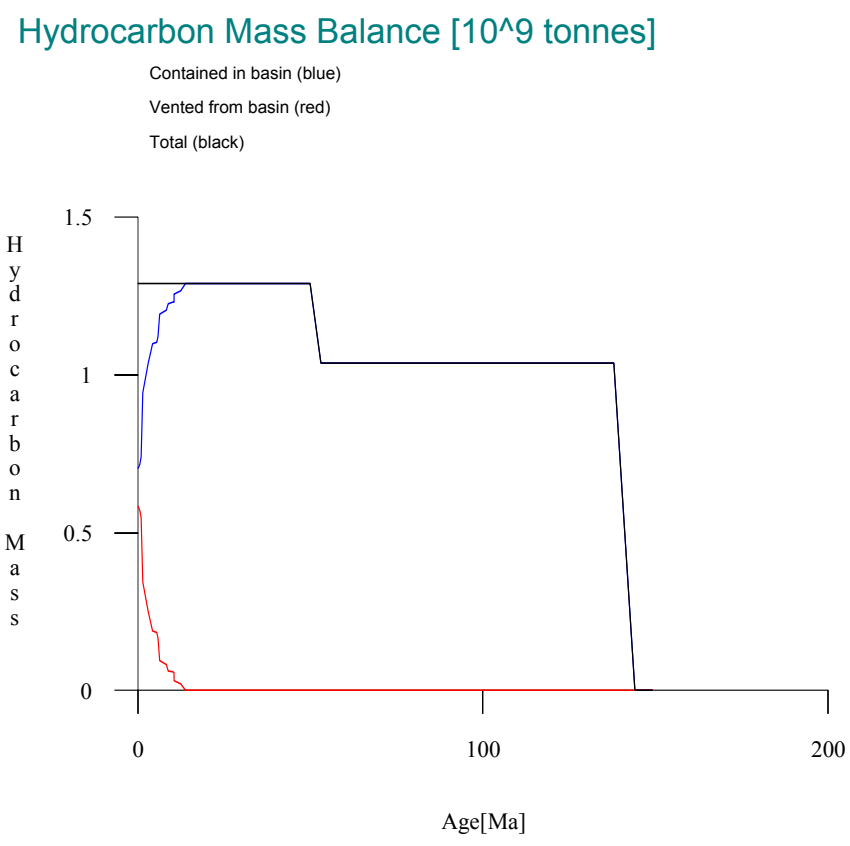

Figure 45. Petroleum mass balance in the entire 2D section. The black curve shows the total mass of petroleum that could be generated by maturation of the kerogen deposited in the $85 \mathrm{~km}$ long, $1 \mathrm{~km}$ wide section. The jogs at $144 \mathrm{Ma}$ represents the deposition of the Jurassic kerogen source bed and the jog at $53 \mathrm{Ma}$ the deposition of the Eocene source strata. The red curve shows the petroleum expelled from the basin and the blue curve the petroleum (generated and potential) remaining in the basin. The

flat top of the black total petroleum curve shows petroleum mass is conserved. This plot was produced using HCMassBal toolbar button.

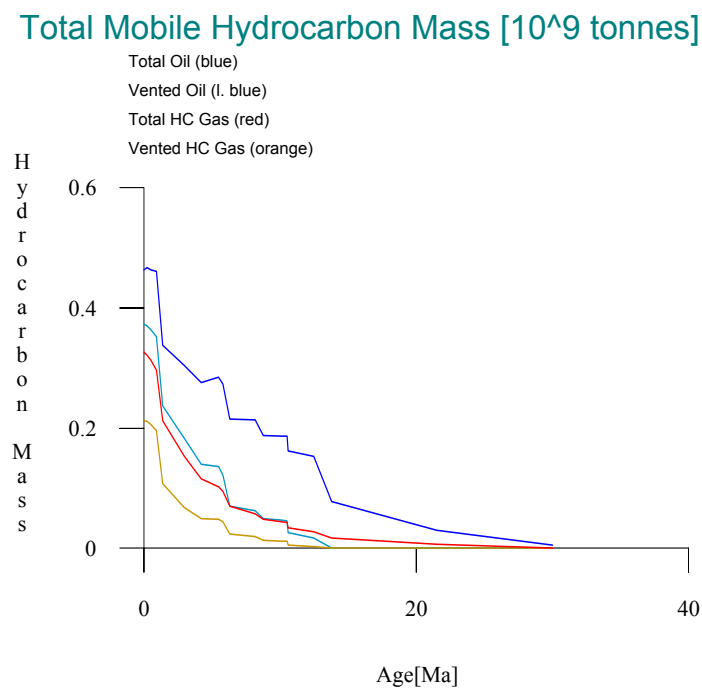

Figure 46. The total mobile petroleum mass vented from and remaining in the section (assumed to be $1 \mathrm{~km}$ wide). The blue curves show the mass of generated and vented liquid petroleum components. The red and orange curves the total and vented mass of gaseous hydrocarbon components. Petroleum components with carbon number less than 6 are assumed to be gaseous. The curves show that most of 
the petroleum generated in the basin has been expelled into the ocean. This plot was produced using the toolbar $O / G$ Inventory button. The age range is set in View: Plot Controls: Set Age Range.

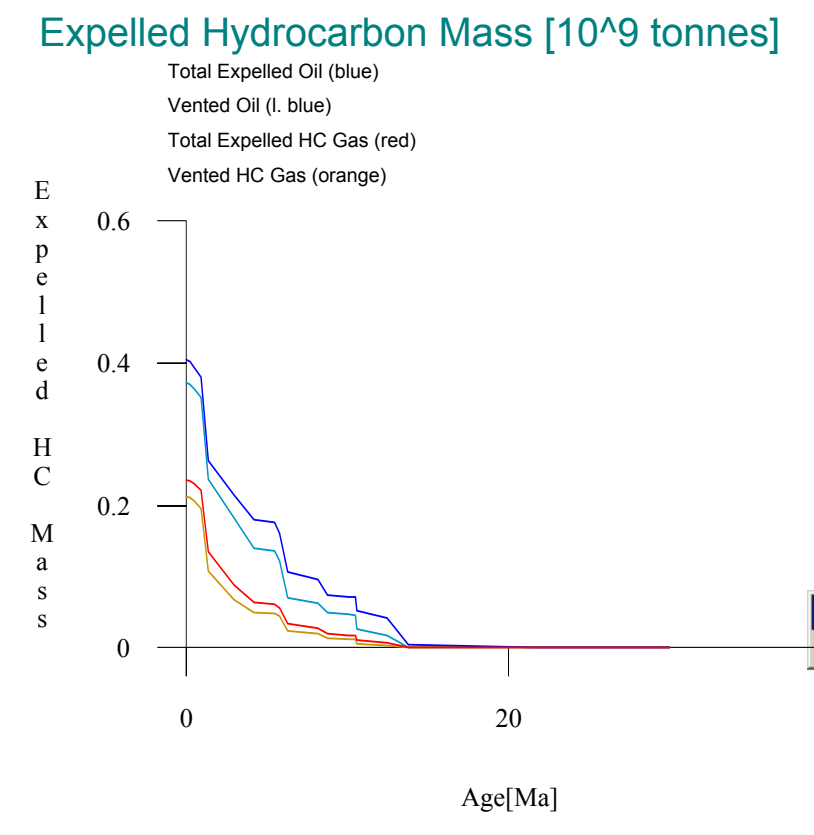

menu as shown below. Age range is set with the menu item below that highlighted.
Figure 47. Same as Figure 46 but showing the vented and total mobile petroleum mass that has been expelled from the source strata. Almost all the petroleum that escapes the source strata escapes the basin. Restriction to expelled hydrocarbons is requested with the View
Plot Controls $\checkmark \checkmark$ Show Titles Old ELEMT_PLTVARS $\checkmark$ Show Axes

40 Show Axes Titles Black and White Plots Negative Image

Manual Plot Close

$\checkmark$ Expelled Hydrocarbons Only Set Age Range 


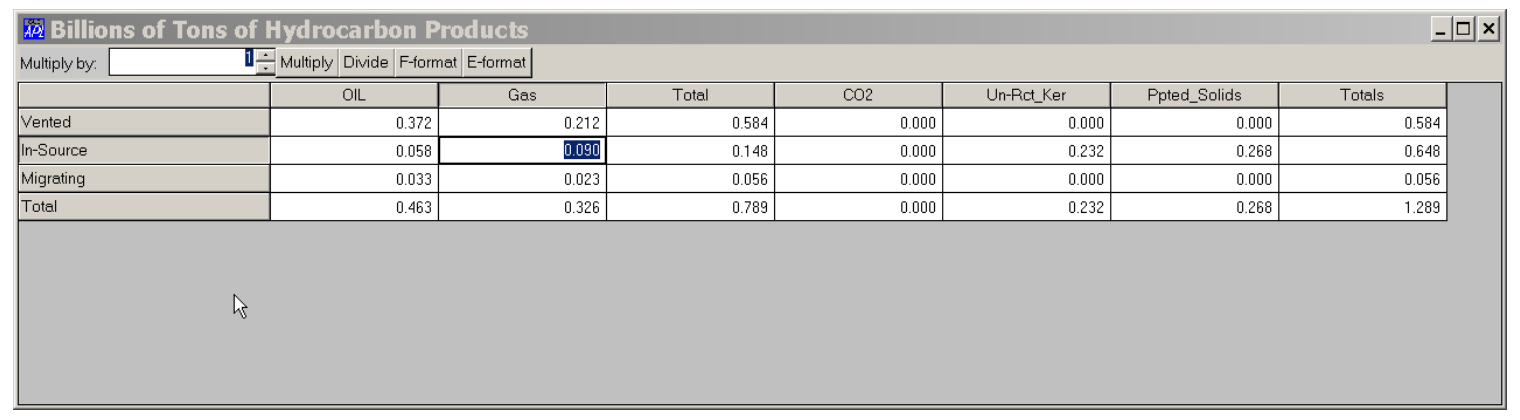

\begin{tabular}{|c|c|c|c|c|c|c|c|}
\hline \multicolumn{7}{|c|}{ Ail Billions of Tons of Hydrocarbon Products } & $-\square x$ \\
\hline \multirow[t]{2}{*}{ Multiply by: } & $125 \div$ Multiply Divide & F-format E-format & & & & & \\
\hline & OIL & Gas & Total & $\mathrm{CO} 2$ & Un-Rct_Ker & Ppted_Solids & Totals \\
\hline Vented & 46.496 & 26.553 & 73.049 & 0.000 & 0.000 & 0.000 & 73.049 \\
\hline In-Source & 7.261 & 11.262 & 18.523 & 0.000 & 29.049 & 33.441 & 81.014 \\
\hline Migrating & 4.105 & 2.926 & 7.032 & 0.000 & 0.000 & 0.000 & 7.032 \\
\hline Total & 57.863 & 40.742 & 98.604 & 0.000 & 29.049 & 33.441 & 161.095 \\
\hline & 4 & & & & & & \\
\hline
\end{tabular}

Figure 48. Statistics on hydrocarbon generation. Produced using Solve Equations: View Calculated Solution: Stats toolbar button. The numbers correspond to the $0 \mathrm{Ma}$ intercept of the curves in Figure 45 through Figure 47. The Multiply by spinner allows the width of the section to be increased from its $1 \mathrm{~km}$ default value. Its use is illustrated in the lower figure. It shows the same statistics on hydrocarbon generation that are shown in the top figure except the Multiply by spinner has been used to increase the section width to $125 \mathrm{~km}$. Table shows that if the section modeled is typical, a $125 \mathrm{~km} \mathrm{x} 85 \mathrm{~km}$ area of the basin has generated 58 Billion Tonnes of liquid hydrocarbon of which $80 \%$ has been vented, and about $7 \%$ retained in migration conduits in the basin.

\section{Macro-Chemistry of the Petroleum System}

The maturation generates what we call macro hydrocarbon products. All of these

\section{Alp Graphics}

Print Zoom XS Prof t_series VntProf PetCompsn

using the $X S$ form. The gas oil ratio ( $\mathrm{kg}$ gas per $\mathrm{kg}$ of oil) can be plotted by variety of ways using the buttons at the top of the main plot form shown at the left. For products can be examined visually in a example, all can be plotted on the 2D section

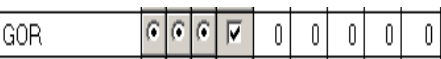

log Transformation c or $C$ off $-2 \div 3 \div$ scrolling to and selecting GOR as a variable as shown on the left (notice we have also checked the tracking box $(T r)$, and turned on the Log Transformation option. Depressing the Plot button results in the section in 
the section shown in Figure 49.

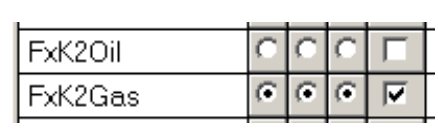

Figure 50 shows the fraction Eocene gas in the section, obtained by selecting FxK2Gas (for fraction gas derived from the maturation of kerogen (in this case a Cameo Coal proxy for the Eocene Wilcox coal gas). The gas component of the petroleum migrating in the upper parts of the section is mostly from the Jurassic K1 source. On the other hand, Figure 51 (constructed by selecting FxK2Oil) shows that oil component of the petroleum migrating in this area is mostly from Eocene sources. In constructing these plots the Log Transformation was turned off, and the plotted Field Selection excluded the kerogen source strata as shown to the left.

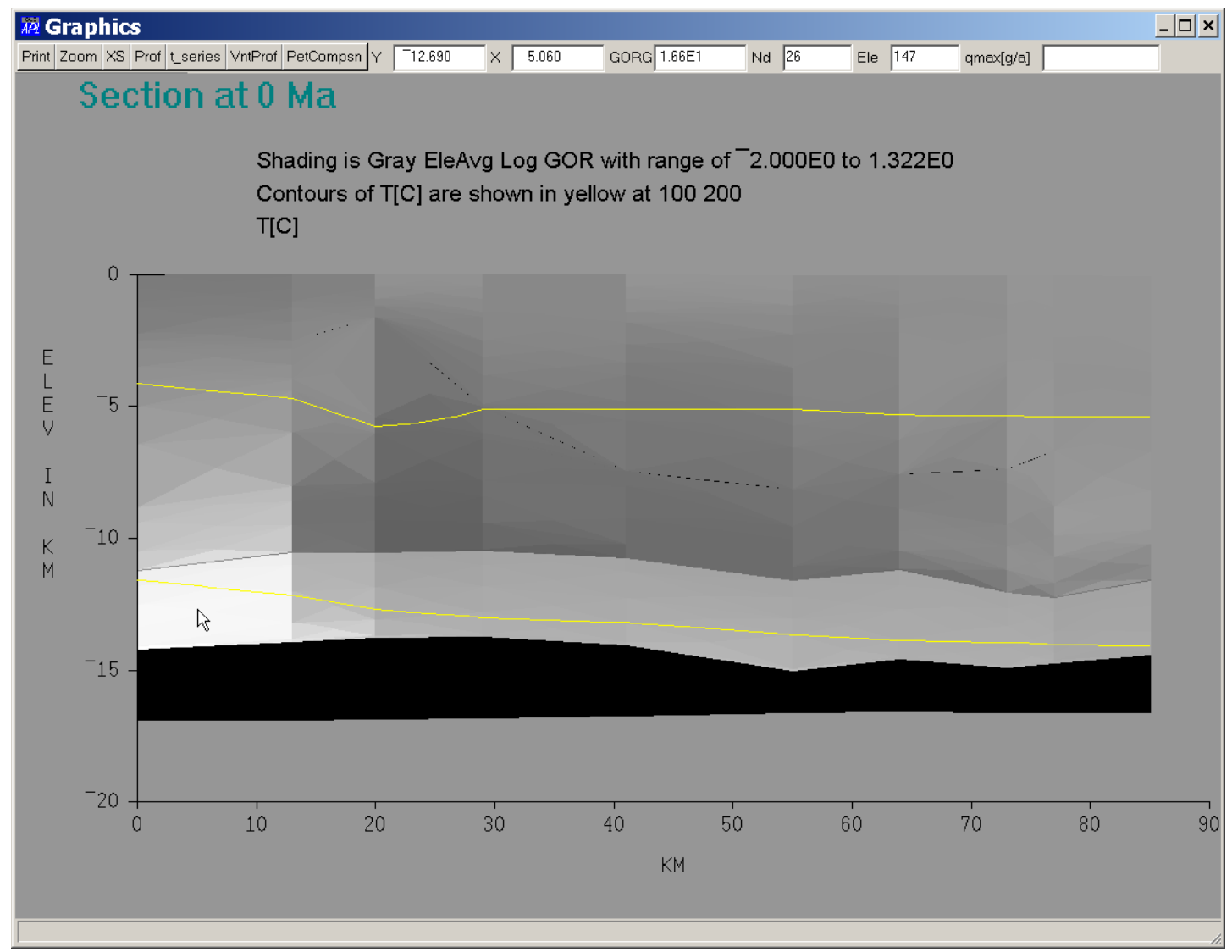

Figure 49. Gas oil ratio of migrating (mostly supercritical) petroleum. Constructed by selecting FxK2Gas in the Plot Control (XS button) form. Since GOR is the tracking variable its value under the mouse tip is displayed on the toolbar. The GOR under the mouse in this case is $16.6 \mathrm{~kg}$ gas per $\mathrm{kg}$ oil. The generation of Jurassic gas is evident as a light band between $\sim 14$ and $12 \mathrm{~km}$ depth. 


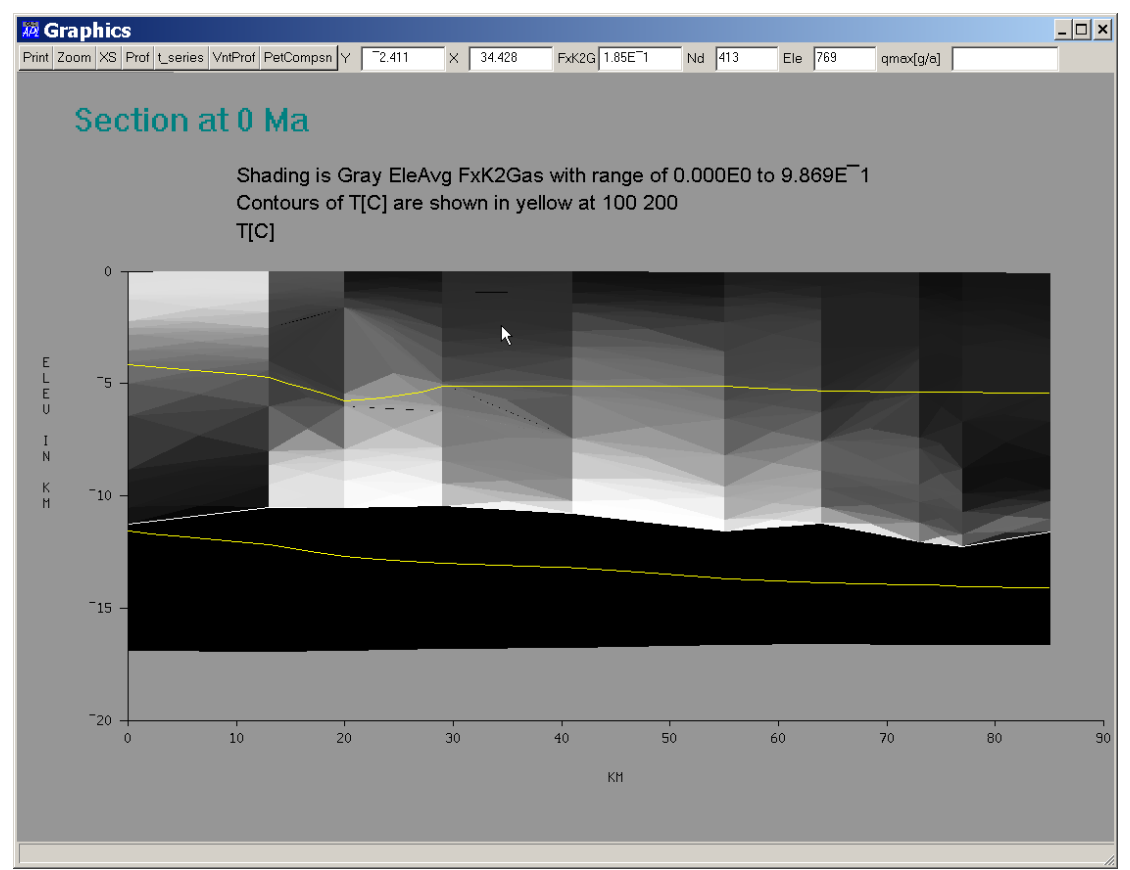

Figure 50. The fraction Eocene (K2) gas in the migrating petroleum. Constructed by selecting FxK2Gas in the Plot Control (XS button) form. The gas component of the migrating petroleum is mostly derived from Jurassic (K1 sources).

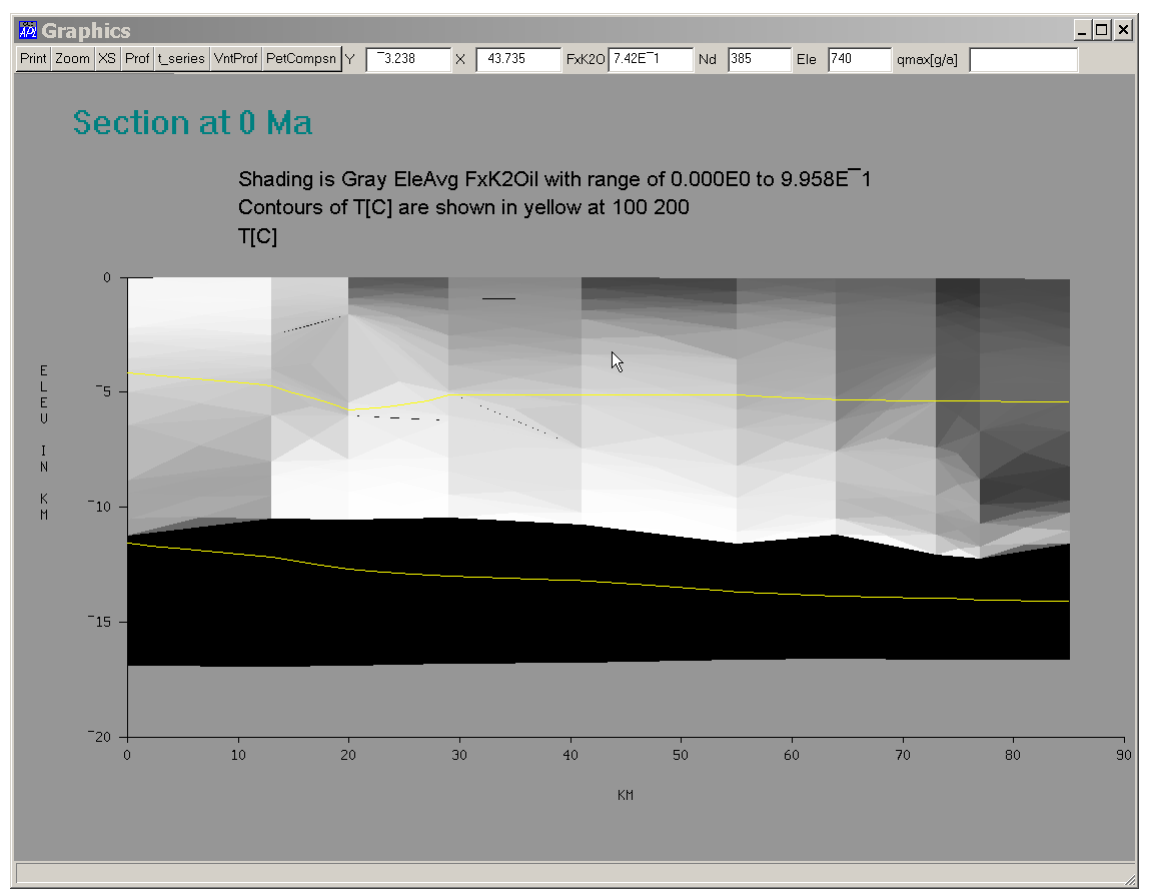

Figure 51. The fraction Eocene (K2) oil in the migrating petroleum. Constructed by selecting FxK2Oil in the Plot Control (XS button) form. The oil component of the migrating petroleum is mostly derived from Eocene (K2 sources). 


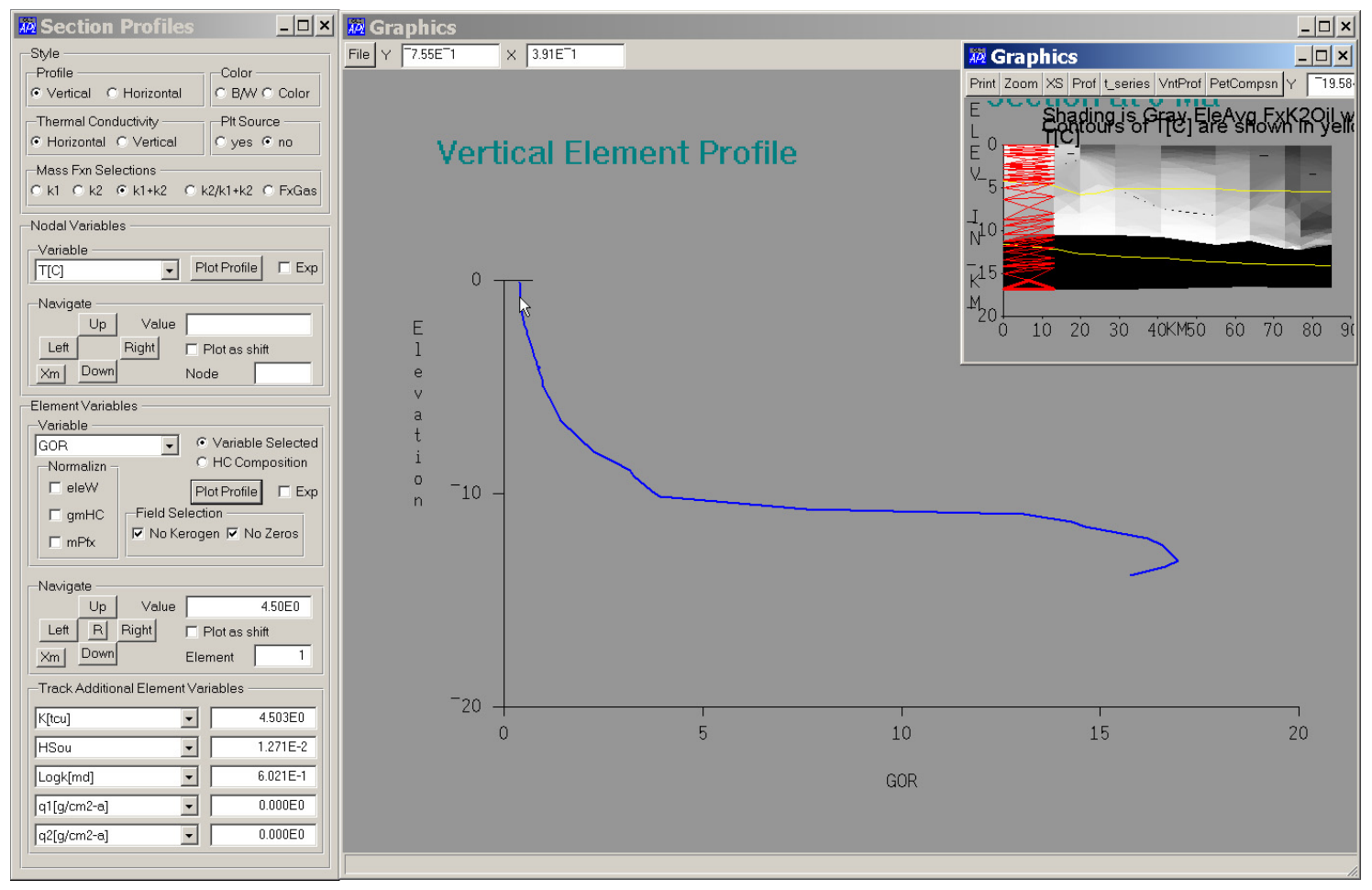

Figure 52. GOR profile through the elements to the right of Well 1. The No Kerogen and No Zeros Field Selection Controls have been checked to eliminate source strata and zero values (where strata have zero thickness) in the plot. The contrast between high GOR in the interval between the Jurassic and Eocene sources, and the lower GORs in above the Eocene source $(\sim 12 \mathrm{~km})$, which is generating oil, is evident.

The Section Profiles form is accessed by the Prof button. It allows construction of vertical and horizontal profiles of any variable or set of variables. Use of this form is illustrated in Figure 52. It shows a vertical GOR profile through the elements just to the left of Well 1. Figure 53 uses the same form to plot the total petroleum migration through the Well 1 elements in the last timestep of the simulation.

Vertical Element Profile

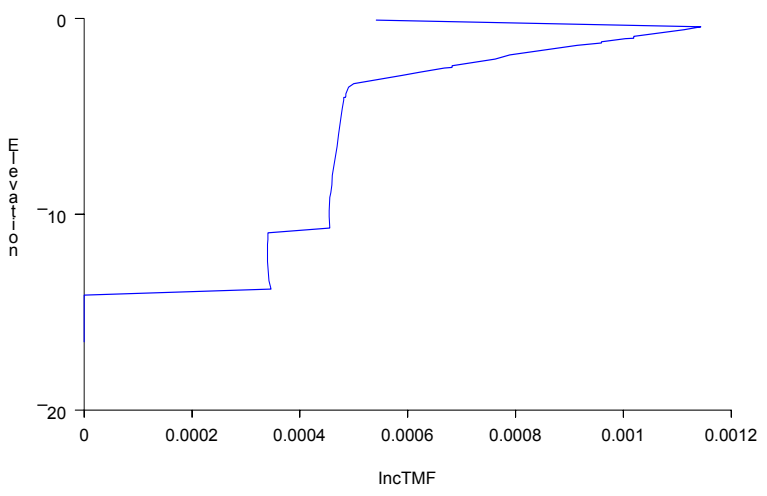

Figure 53. The incremental petroleum additions to each element in a vertical section adjacent to Well 1 at $\mathrm{t}=0$. The generation in the Eocene Source $(\sim 12 \mathrm{kmbsl})$ is about $33 \%$ that in the Jurassic Strata $(\sim 14$ $\mathrm{kmbsl})$. The increase starting at $\sim 3.3$ $\mathrm{km}$ depth is due to compaction. Plot generated using the Section Profiles form (obtained with Prof toolbar button). 


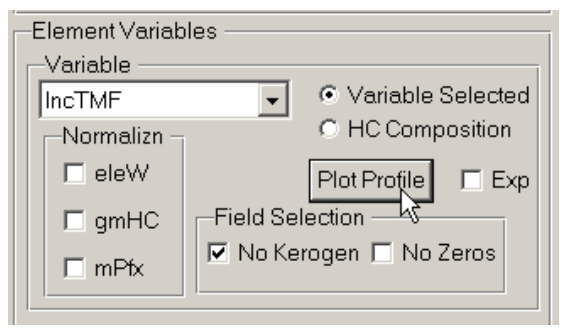

The history of gas venting can be obtained using the Time Series form. It is accessed through the $t$-series button on the Graphics form. The incremental gas mass additions to each element $(\operatorname{Inc} G M F)$ is converted to a true gas mass flux by checking Normlze $(D t, D x)$, and plotted by depressing the Plot button as shown in Figure 51 . This causes the gas mass additions to be divided by the timestep and the element width. We select the element just above the Jurassic Source for the gas mass flux time series (by using the Navigate Elements buttons). The location of the element selected is shown in the insert.

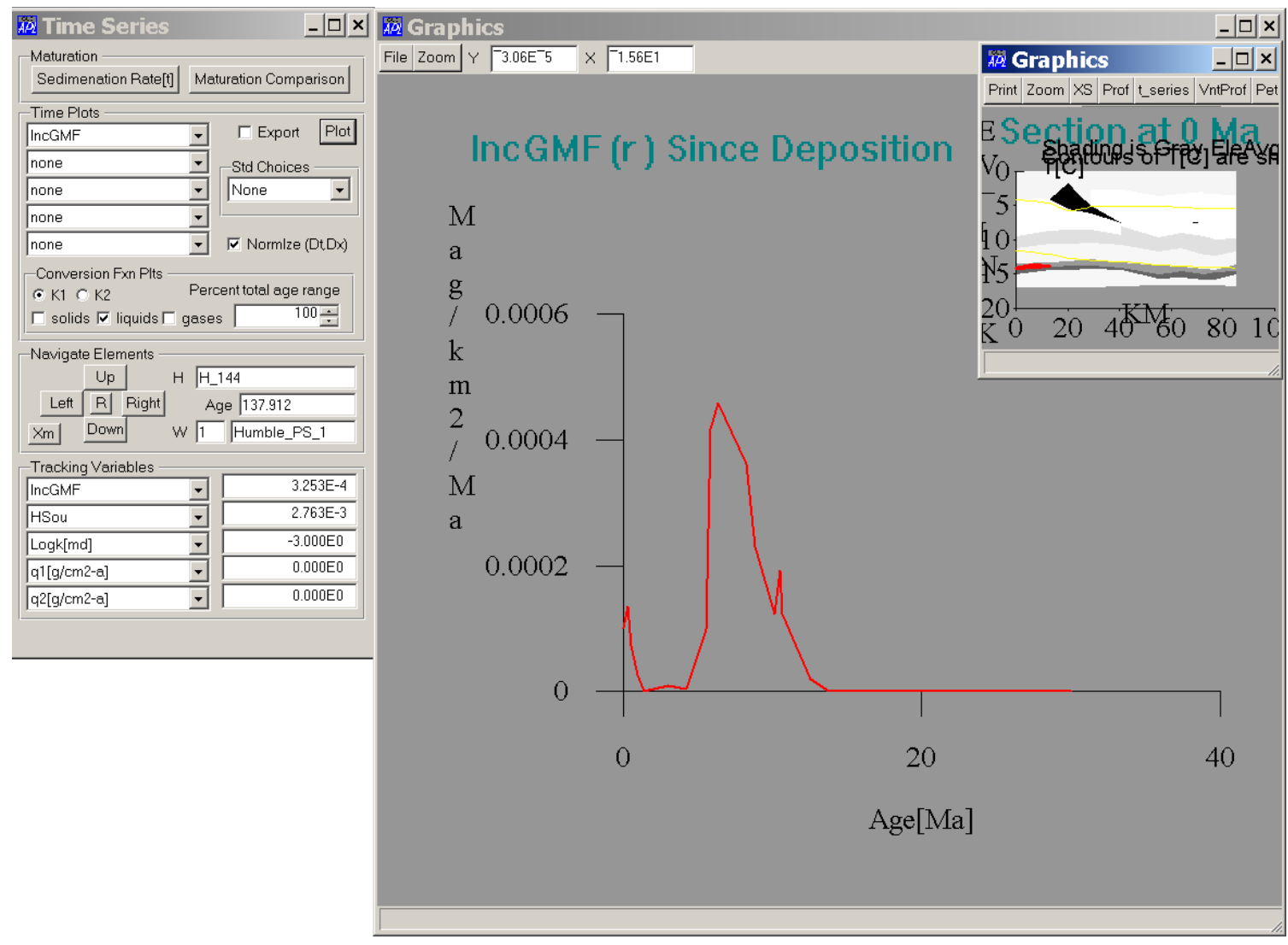

Figure 54. The gas mass flux just above the Jurassic source and right of well 1. Plotted with Solve Equations: View Calculated Solution: t-series: Navigate Elements: check Normlze (Dt,Dx): Plot. Ranges of age scale set in View: Plot Controls: Set Age Range. Notice that the IncGMF indicated in the Tracking Variables section of the form is $3.25 \times 10^{-4}$. This is the mass of gas transported across the selected element over the last timestep. This incremental mass addition divided by the element width $(13.2 \mathrm{~km})$ and the 
timestep $(0.25 \mathrm{Ma})$ yields the gas mass flux shown in the figure at $\mathrm{t}=0\left(1 \times 10^{-4} \mathrm{Bt} / \mathrm{km}^{2}-\right.$ Ma).

None

Mobile K1 Prods

Mobile K2 Prods

$\mathrm{K} 2 / \mathrm{K} 1+\mathrm{K} 2$

$\mathrm{GOR}$

Inst+Cum Total MF

Inst+Cum Gas MF

Inst+Cum Oil MF
Useful time history combinations can be selected from the Std Choices combo box of the Time Series form. For example Figure 55 shows how the composition of hydrocarbons in the element between the $1^{\text {st }}$ and $2 d$ well just above the Jurassic sourcebed (as shown in the insert in Figure 50 ) changed with time. Figure 56 shows the change in GOR in this element.

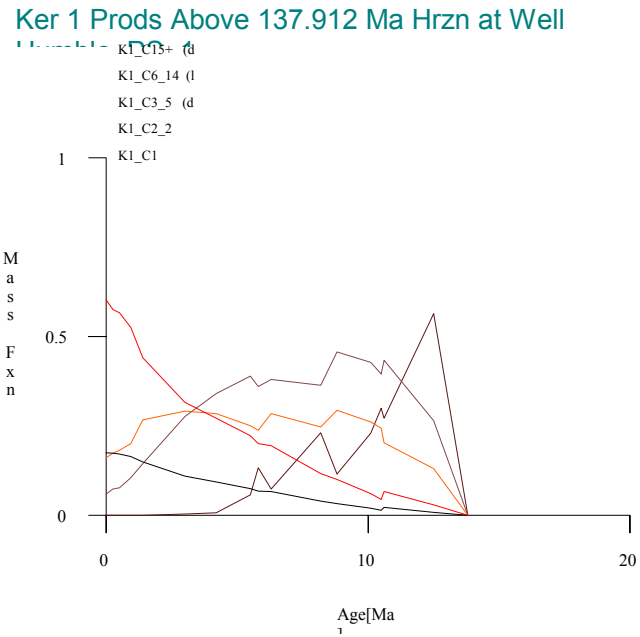

GOR Above 137.912 Ma Hrzn at Well Humble_PS_1

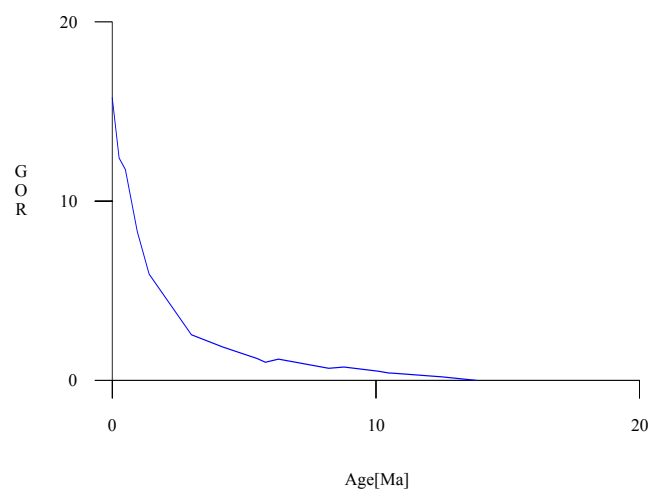

Figure 55. Change in the composition of Jurassic maturation products in the element just above this source bed (see Figure 50 for location). Plotted using Time Series form, Mobile K1 Prods combo selection.

Figure 56. Evolution of GOR (gas/oil mass ratio) in element shown in Figure 50. Plotted using Time Series form, GOR combo selection. 


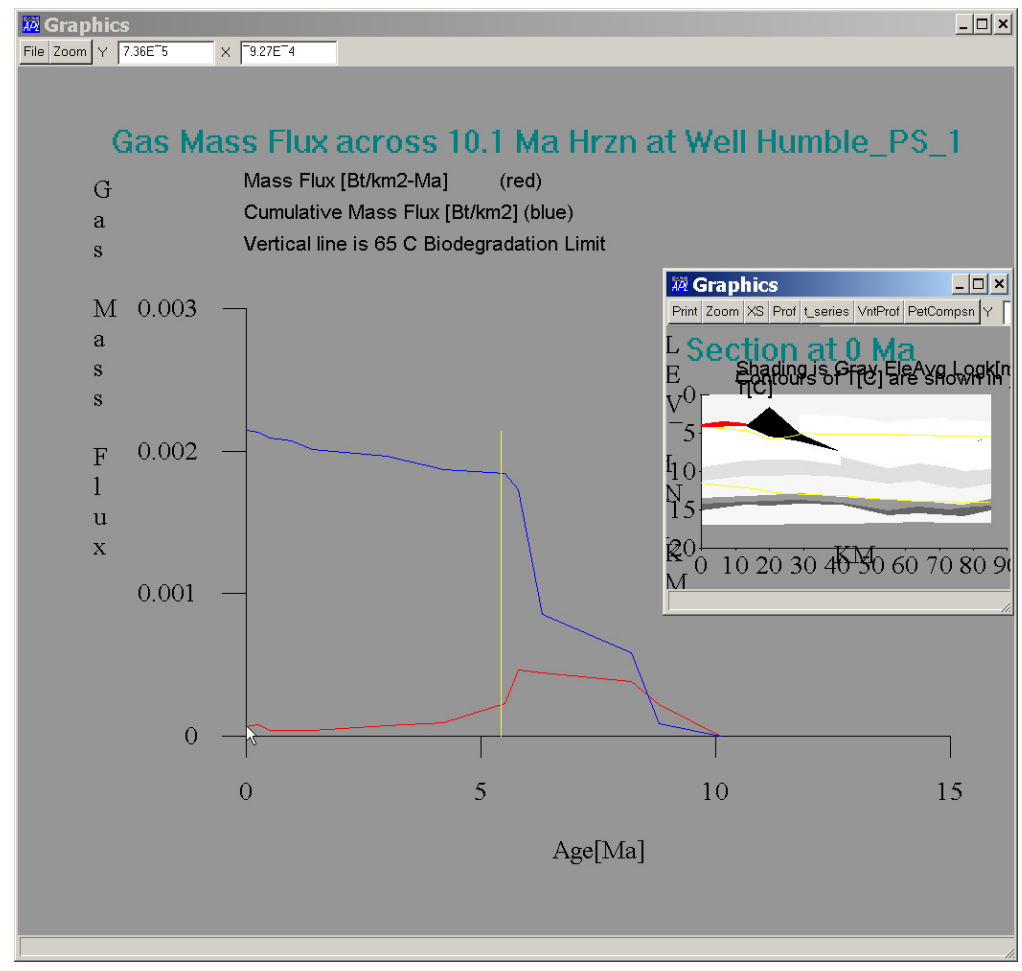

Figure 57. Instantaneous and cumulative hydrocarbon gas mass flux through an element $3.5 \mathrm{~km}$ below the surface as illustrated in the insert. Temperature in the element exceeds $65^{\circ} \mathrm{C}$ at ages left of the vertical yellow line. The figure is plotted using the combo entry in the Time Series form (Figure 54) illustrated below.

None

Mobile K1 Prods

Mobile K2 Prods

$\mathrm{K} 2 / \mathrm{K} 1+\mathrm{K} 2$

GOR

Inst+Cum Total MF

Inst+Cum Gas MF

Inst+Cum Oil MPY

A plot that is useful in evaluating when and how rapidly a reservoir could have filled is shown in Figure 57. Plotting the time at which an element reached $65^{\circ} \mathrm{C}$ (the temperature at which biodegradation ceases) shows the earliest time that un-biodegraded reservoirs could have filled. The hydrocarbon flux through the element allows estimation of the combination of aerial draw and filling time needed to fill reservoirs of a known volume (e.g., see (Cathles and Losh 2002)).

The VntProf button brings up a HC Venting and Select Products forms. These forms control the display of strata-parallel venting rate profiles. The VntProf form differs from the Prof forms in that they control the flux through a set of elements, not simply parameters in a set of elements. For example the Prof button form displays the mass of a macro maturation component in a profile of elements, the mass fraction of this component in the migrating hydrocarbon stream, the GOR, etc. The VntProf forms displays the flux of a macro component through a set of elements. 


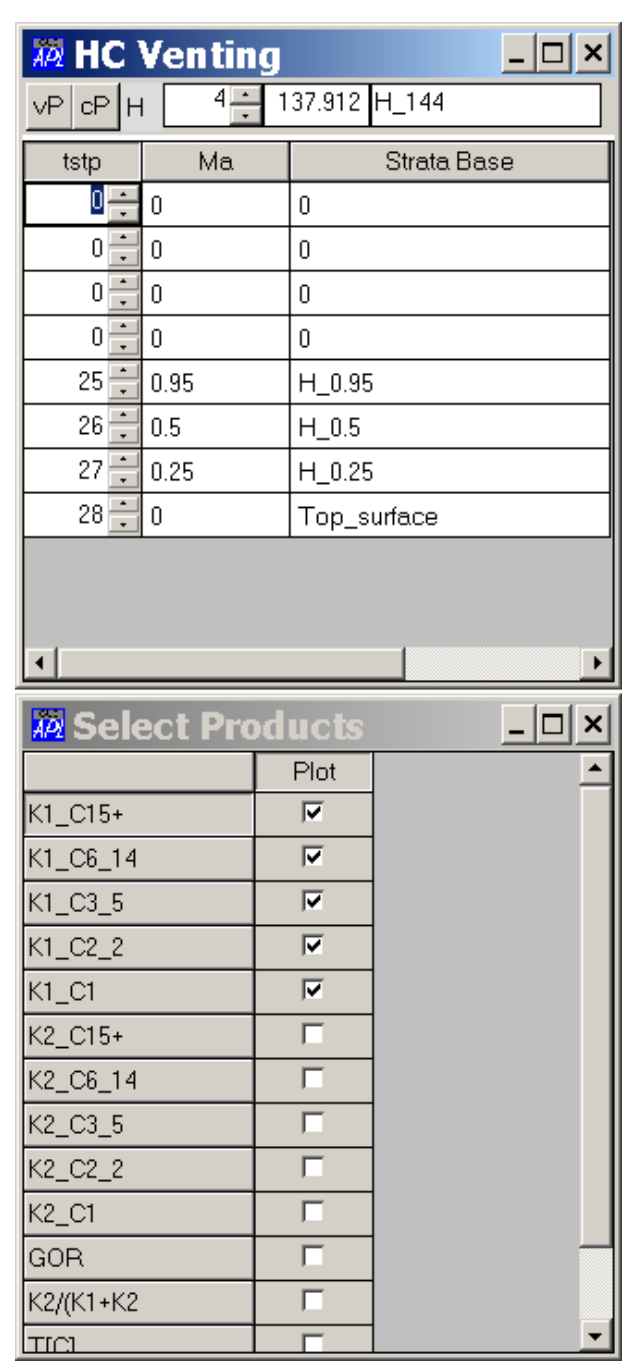

of the timestep selected).
Figure 58. The HC Venting and Select Products forms are displayed when the VntProf button is depressed.

The HC Venting and Select Products forms work together.

- If only one product is selected on the Select Products form, that variable is displayed for all the timesteps selected on the $H C$ Venting form.

- If more than one product is selected on the Select products form, then flux profiles for these products are displayed at the last timestep selected on the HC Venting form (the $28^{\text {th }}$ timestep ending at $0 \mathrm{Ma}$ in the case displayed).

- In either case the fluxes displayed are those crossing the horizon selected at the top of the HC Venting form (in this case the 137.9 Ma, H_144 horizon).

- Plots are created when the $\mathrm{vP}$ or $\mathrm{cP}$ buttons at the top of the HC Venting form are depressed.

- vP plots the venting rate profile over the selected timestep(s).

- cP plots the cumulative flux (e.g., the time integrated flux of selected component(s) that has crossed the selected horizon from the start of simulation to the time as the end

Figure 58 shows the plot that results when the $v P$ button in Figure 57 is depressed.

Figure 60 shows the plot that results when the

HC Flow through 137.912 Ma Hrzn (H_144) at 0 Ma $c P$ button is selected.

Figure 59. Fluxes of the hydrocarbon components across the H_144 age horizon (at the top of the Jurassic source bed). The total gas flux through the first element in this profile is shown in Figure 54. This plot 
shows that only the ends of the Jurassic source bed in our section are generating hydrocarbon at the present day. The sum of the gas component fluxes equals $10^{-4}$ $\mathrm{Bt} / \mathrm{km}^{2}-\mathrm{Ma}$, which is the same gas flux shown in Figure 54.

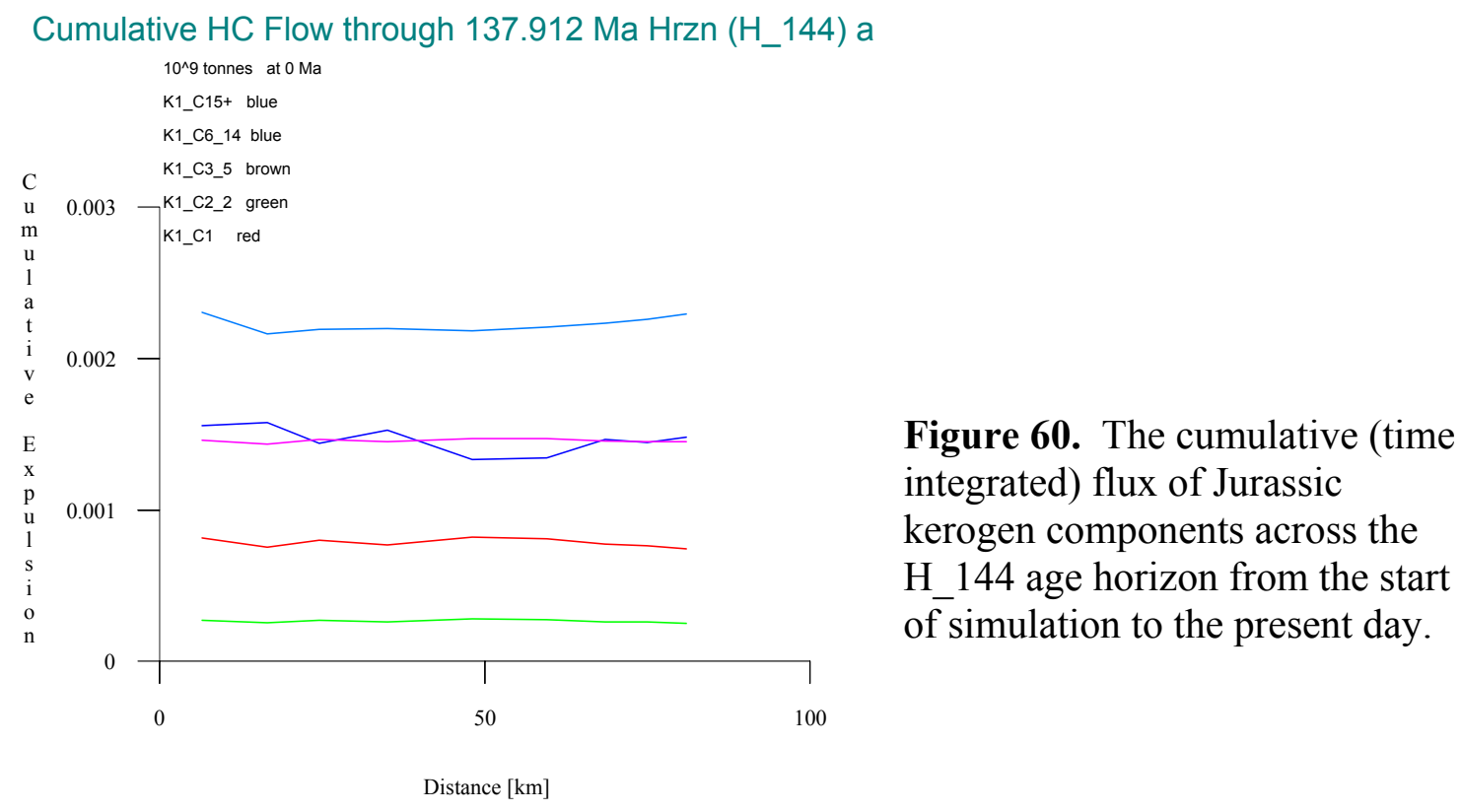

Figure 61 shows the flux of $\mathrm{C} 1$ across the $\mathrm{H} \_144$ horizon over the last $\sim 1 \mathrm{Ma}$.
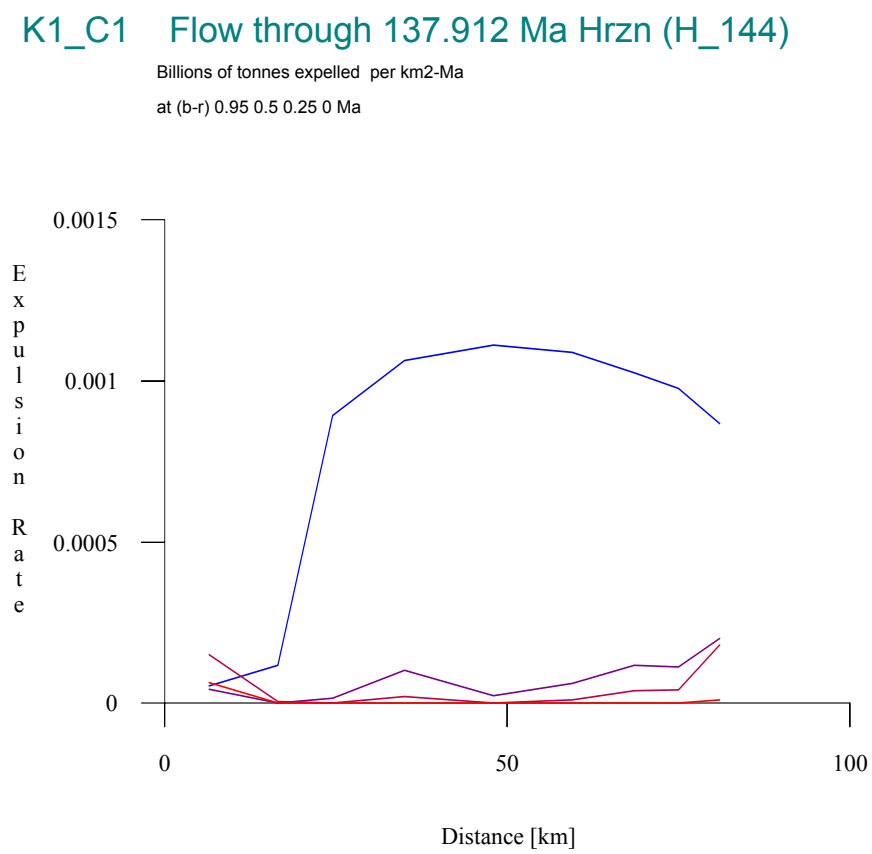


\begin{tabular}{|c|c|c|c|}
\hline \multicolumn{3}{|c|}{ AC He Venting } & $-\square x$ \\
\hline $\mathrm{VP} \mid \mathrm{CP} / \mathrm{H}$ & $4=$ & 37.912 & \\
\hline tstp & $\mathrm{Ma}$ & \multicolumn{2}{|c|}{ Strata Base } \\
\hline $0 \div$ & 0 & \multicolumn{2}{|l|}{0} \\
\hline $0 \div$ & 0 & \multicolumn{2}{|l|}{0} \\
\hline $0 \div$ & 0 & \multicolumn{2}{|l|}{0} \\
\hline $0 \div$ & 0 & \multicolumn{2}{|l|}{0} \\
\hline $25 \div$ & 0.95 & \multicolumn{2}{|l|}{ H_0.95 } \\
\hline $26 \div$ & 0.5 & \multicolumn{2}{|l|}{ H_0.5 } \\
\hline $27 \div$ & 0.25 & \multicolumn{2}{|l|}{ H_0.25 } \\
\hline $28 \div$ & 0 & \multicolumn{2}{|c|}{ Top_surface } \\
\hline \multicolumn{3}{|c|}{ AP: Select Productis } & $-\square x$ \\
\hline & & Plot & $\Delta$ \\
\hline K1_C15+ & & $\Gamma$ & \\
\hline K1_C6_14 & & $\Gamma$ & \\
\hline K1_C3_5 & & $\Gamma$ & \\
\hline $\mathrm{K} 1$ ${ }^{\mathrm{C} 2}$ _2 & & $\Gamma$ & \\
\hline K1_C1 & & $\sqrt{\nabla}$ & \\
\hline K2_C15+ & & $\Gamma$ & \\
\hline K2_C6_14 & & $\Gamma$ & \\
\hline K2_C3_5 & & $\Gamma$ & \\
\hline $\mathrm{K} 2$-C2_2 & & $\Gamma$ & \\
\hline $\mathrm{K} 2$ _C1 & & $\Gamma$ & \\
\hline GOR & & $\Gamma$ & \\
\hline $\mathrm{K} 2 /(\mathrm{K} 1+\mathrm{K} 2$ & & $\Gamma$ & \\
\hline Tirl] & & $\Gamma$ & 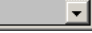 \\
\hline
\end{tabular}

Figure 61. Flux of Jurassic methane across the H_144 horizon over the last $\sim 1 \mathrm{Ma}$. The bottom (red) profile corresponds to the profile in Figure 58. The forms used to compute this case are shown to the right of the graph.

\section{J. Defining the Petroleum Micro-Chemistry}

The final step in the advanced chemistry investigation of a section is to break the migrating petroleum into molecular components, determine the phase state of the petroleum at various points in the basin, and evaluate how interactions of liquid petroleum with migrating gas might alter the liquid chemistry. This is done in two steps. First the decomposition of the maturation macro-components to a much larger set of molecular components is designed. Second this decomposition design is used to calculate the PVT properties of the migrating hydrocarbons in the section.

The first step in designing a molecular petroleum model is to select the molecular (or

\section{Select Petroleum Model \\ View Molecular Components of Selected Model \\ Design Petroleum Decomposition \\ Scratchpad: Flash/Wash Decomposed Petroleum}

lumped) components that will describe the petroleum. This is done with the Flash: Select Petroleum Model menu command. It brings up the form shown in Figure 62. A petroleum decomposition model can

be selected using this form. The decomposition candidates are prepared in a Chemical Data database prepared under this project, and provided with $\boldsymbol{B} \boldsymbol{a} \boldsymbol{a} \boldsymbol{s i n} \boldsymbol{L} \boldsymbol{A B}$. Connection to this database is automatic. 


\begin{tabular}{|c|c|c|c|}
\hline \multicolumn{4}{|c|}{ AOA Petroleum Decomposition Hadel $\quad-\square \mid x$} \\
\hline select & Nbr & Name & Descr. \\
\hline$C$ & 27 & TwoComponent & Simple test oil \\
\hline$C$ & 43 & AllAlkanes & Oil of 20 alkanes \\
\hline $\mathrm{C}$ & 44 & test1 & test oil \\
\hline $\mathrm{C}$ & 49 & cyclohexane-test & test oil of pure cyclohexane \\
\hline $\mathrm{C}$ & 50 & n-decane-test & test oil of pure n-decane \\
\hline $\mathrm{C}$ & 51 & ethyl-benzene-test & test oil of pure ethyl-benzene \\
\hline $\mathrm{C}$ & 52 & thesis & oil used in my thesis \\
\hline$C$ & 62 & BasinOil & A test "realistic" oil \\
\hline$C$ & 63 & CibOp & Vermilion Oil \\
\hline $\mathrm{C}$ & 72 & UnifacOil & Oil to test Unifac \\
\hline $\mathrm{C}$ & 1 & test & test Oil \\
\hline$C$ & 73 & ViscosityTest & test for the viscosity routines \\
\hline$C$ & 74 & PressureViscosityOil & test oil for positive pressure \\
\hline$C$ & 76 & PhaseTest & Test of the Phase Equilibria pred. o... \\
\hline $\mathrm{C}$ & 77 & PhaseTest2 & second test of the EOS \\
\hline$C$ & 78 & GWOil & \\
\hline $\mathrm{C}$ & 79 & TypicalOil & \\
\hline 6 & 80 & MeulbroekThesis & \\
\hline
\end{tabular}

Figure 62. Form summoned by Flash: Select Petroleum Model. We have selected the 61 molecule Meulbroek Thesis decomposition model. Construction of decomposition models is done within the Chemical Data.dba database and is described in a separate part of this report.

The molecular components that comprise decomposition model 80 (the Meulbroek Thesis model) can be viewed using the Flash: View Molecular Components of Selected Model

\section{Select Petroleum Model}

\section{View Molecular Components of Selected Model} Design Petroleum Decomposition

Scratchpad: Flash/Wash Decomposed Petroleum

Figure 63. Some of the molecular components that comprise the Meulbroek Thesis (model 80) petroleum decomposition model. Form is for information purposes only and cannot be used to change the petroleum decomposition. It is summoned by Flash: View Molecular Components of Selected Model as illustrated above. The type of molecule is indicated by the Type flag $(1=$ n-alkane, $2=$ cyclic alkane, $3=$ branched alkane, and 4=aromatic). menu, as shown in Figure 63. This form is for information purposes only. The molecular components cannot be altered using it.

\begin{tabular}{|l|l|r|r|r|}
\hline \multicolumn{1}{|c|}{ Name } & \multicolumn{1}{|c|}{ Formula } & Type & Mole Wt \\
\hline carbon dioxide & $\mathrm{CO} 2$ & \multicolumn{1}{|c|}{$\mathbf{x}$} & 44 \\
\hline methane & $\mathrm{CH} 4$ & 1 & 16.04 \\
\hline ethane & $\mathrm{C} 2 \mathrm{H} 6$ & 1 & 30.06 \\
\hline propane & $\mathrm{C} 3 \mathrm{H} 8$ & 1 & 44.09 \\
\hline isobutane & $\mathrm{C} 4 \mathrm{H} 10$ & 3 & 58.12 \\
\hline isopentane & $\mathrm{C} 5 \mathrm{H} 12$ & 1 & 72.15 \\
\hline n-butane & $\mathrm{C} 4 \mathrm{H} 10$ & 1 & 58.12 \\
\hline n-pentane & $\mathrm{C} 5 \mathrm{H} 12$ & 1 & 72.15 \\
\hline 2,2-dimethylbutane & $\mathrm{C} 6 \mathrm{H} 14$ & 3 & 86.17 \\
\hline cyclopentane_1 & $\mathrm{C} 5 \mathrm{H} 10$ & 2 & 70.13 \\
\hline 2-methylpentane & $\mathrm{C} 6 \mathrm{H} 14$ & 3 & 86.17 \\
\hline 3-methylpentane & $\mathrm{C} 6 \mathrm{H} 14$ & 3 & 86.17 \\
\hline n-hexane & $\mathrm{C} 6 \mathrm{H} 14$ & 1 & 86.17 \\
\hline methylcyclopentane & $\mathrm{C} 6 \mathrm{H} 12$ & 2 & 84.16 \\
\hline 2,2-dimethylpentane & $\mathrm{C} 7 \mathrm{H} 16$ & 3 & 100 \\
\hline cyclohexane & $\mathrm{C} 6 \mathrm{H} 12$ & 2 & 84.16 \\
\hline \hline
\end{tabular}


The Flash: Scratchpad: Design Petroleum Decomposition menu command is use to

Select Petroleum Model

View Molecular Components of Selected Model

Design Petroleum Decomposition

Scratchpad: Flash/Wash Decomposed 'setroleum design the decomposition of the macro components of a petroleum that are generated by maturation models. The Design form shown in Figure 64.

\section{Fesign Hydrocarbon Comm $-|\square| x \mid$ Macrocomponents and $n$-alkane Masses \\ C Ker 1 \\ C Ker 2 \\ $\mathrm{CO} 2$ \\ $0.000 \div$ \\ c generic \\ wt\% n-alk Cnf Cnl Mass n-Alk Petrol

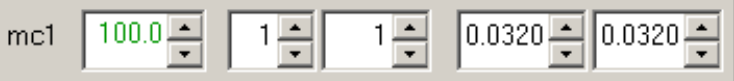

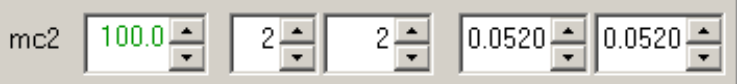 \\ mc3 $100.0 \div 3 \div 4 \div 0.1500 \div 0.1500 \div$

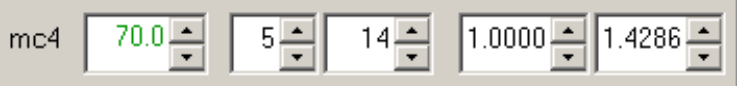 \\ mc5 $70.0 \div 15 \div 31 \div 1.0000 \div 1.4286 \div$}

Kissen Slope of Base Leg

Slope

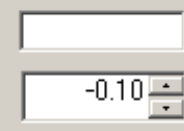

c Determine

C Specify

\begin{tabular}{l|l} 
Calculate and plot n-alkanes & $\mathrm{T}[\mathrm{C}]$ \\
Mole Fraction of Macro Components
\end{tabular}
molefx mc1 mc2 mc3 mc4 mc5 Iso $0.00 \sqrt{0.0 \pm 0.0 \div 0.0 \div 0} 0.0 \div 0$ Cycl $0.00 \sqrt{0.0 \div \sqrt{0.0 \div 0} \pm \sqrt{0.0 \div 0.0 \div 0}}$ Arom $0.00 \sqrt{0.0 \div 0.0 \div 0.0 \div 0} 0.000$ Asph $0.00 \sqrt{0.0 \pm \sqrt{0.0 \pm 0.0 \div 0} 0.0 \div 0}$ \begin{tabular}{ll|l|l|l|l} 
& 0.000 & 0.000 & 0.000 & 0.000 & 0.000 \\
\hline
\end{tabular} Guess Compute Save Cut Distrib Make and Plot Total HC $\mathrm { TCC } \longdiv { 5 0 \div } \Gamma \mathrm { Log }$
Figure 64. The scratchpad form used to design the decomposition of maturation macro-components into detailed molecular description of the petroleum. Form is summoned by Flash: Scratchpad: Design Petroleum Decomposition as illustrated above.

The petroleum is decomposed into the components shown in Figure 63 in two steps: First the n-alkane fraction is distributed among the n-alkane molecules in the petroleum. Then the remaining petroleum mass is 
distributed among the isoalkanes, cyclicalkanes, and aromatic molecules.

The input required to specify the $n$-alkane distribution is the mass of petroleum in each carbon number range (the last column in the top portion of the form) and the $\mathrm{Wt} \% \mathrm{n}$ alkanes in each of these cuts (the first column). The carbon number range $\left(\mathrm{C}_{\mathrm{nf}}\right.$ to $\left.\mathrm{C}_{\mathrm{nl}}\right)$ in the second and third columns can be specified by the user in the generic case, but the cut ranges are read from the maturation data base if a the petroleum masses derive from a maturation model.

In the generic case shown (generic toggle at the top of the form is selected), the first cut is methane, the second ethane, the third $\mathrm{C}_{3}-\mathrm{C}_{4}$, the fourth $\mathrm{C}_{5}-\mathrm{C}_{14}$, and the last $\mathrm{C}_{15+}$. The masses in these cuts are $0.32,0.52,0.15,1.43$ and 1.43 mass units respectively. The mass units are arbitrary because the relative mass is all that is important. We distribute whatever mass is in the carbon number cuts to molecular components. All of the first three carbon number cuts are assigned to $\mathrm{n}$-alkane molecules; $70 \mathrm{Wt} \%$ of the last two cuts.

The mole abundance of each n-alkane in the two highest carbon number cuts is assumed to be exponentially related to the others such that $C_{n}=b e^{s n}$, where $\mathrm{n}$ is the carbon number, $\mathrm{s}$ is the Kissen slope (usually a negative number), and $b$ is a constant. The $\mathrm{n}$ alkane mass in these cuts determines $b$ and $s$ if the determine toggle is on. The Kissen slope of the last (highest carbon number) cut is specified by the spinner if the specify toggle is on. The other n-alkane masses in the other carbon number cuts are determined by requiring that they are related by an exponential slope, s' (not necessarily $=\mathrm{s}$ ) and that mass of the first oil component in the cut is related to the mass of the last n-alkane in the underlying cut by a carbon number log slope of s'. The slope is then determined by the total mass in the cut. Figure 65 shows the distribution of n-alkane components for the above mass assignments if the Kissen slope is determined by the masses in the last two $\mathrm{C}_{\mathrm{n}}$ cuts; Figure 66 shows the distribution if the n-alkane distribution if the Kissen slope of the last (highest carbon number) legs is set at -0.14 .

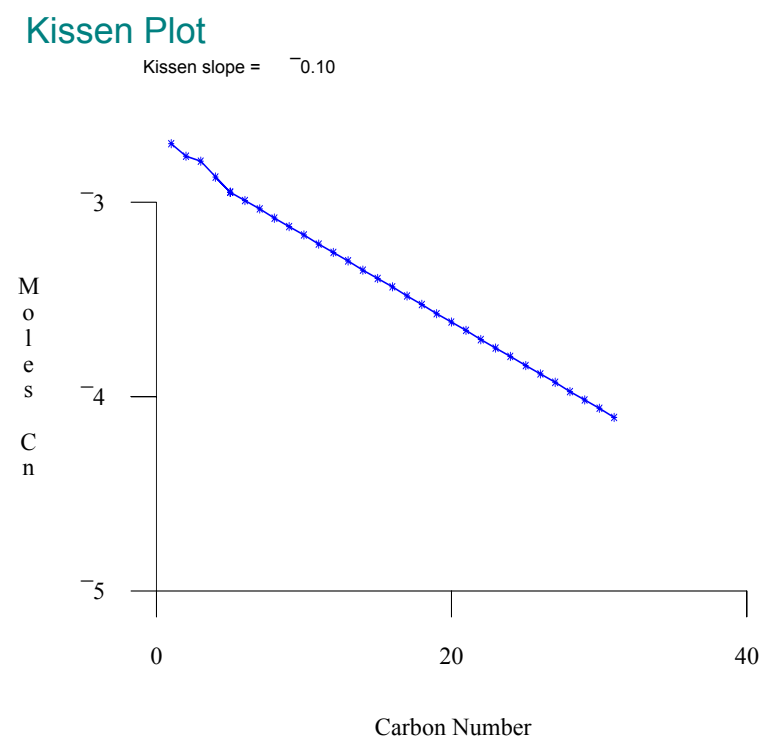

Figure 65. Distribution of nalkanes specified by the upper portion of the form in Figure 64 if the Kissen slope is determined by the masses in the two highest carbon number cuts. The Kissen slope is the natural log slope. The $\log 10$ slope $=-0.1 / \ln 10=-0.043$. The Kissen slope posted is that of the last (highest $C_{n}$ ) cut. 


\section{Kissen Plot}

Kissen slope $=-{ }^{-} 0.14$

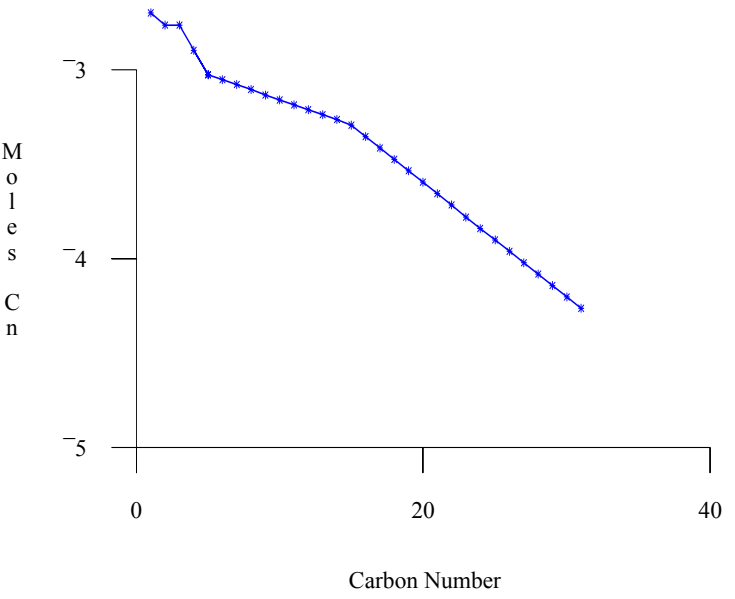

Figure 66. Distribution of n-alkanes specified by the upper portion of the form in Figure 64 if the Kissen of the two highest carbon number cuts is set at -0.14 . The Kissen slope is the natural log slope, s.

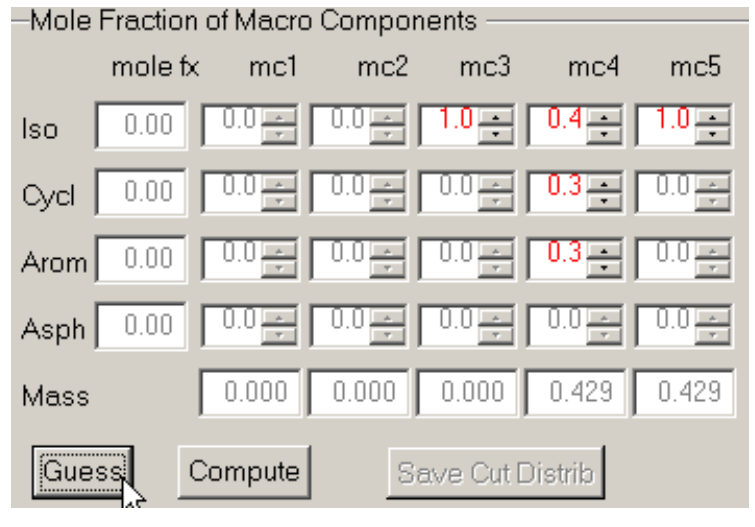

The non-n-alkane hydrocarbons are distributed using the lower portion of the Figure 64 form. Depressing the Guess button looks at the oil model (selected from the data base in Figure 62 and shown in Figure 63) to determine which non-nalkanes are present in which carbon number cuts, and distributes the moles of hydrocarbon evenly across these possibilities in each cut. In the case shown, isoalkanes are present in carbon number cuts 3, 4, and 5, cyclic and aromatic alkanes are present in cut 4 only. The current version of BasinLab does not distribute or identify asphaltine components. The bins (intersection of non-n-alkane type and carbon number cut) that contain components are red. The numbers are the mole fractions. They can be changes but they must sum to 1 in each vertical column. The bottom row shows the non-hydrocarbon mass assigned to each

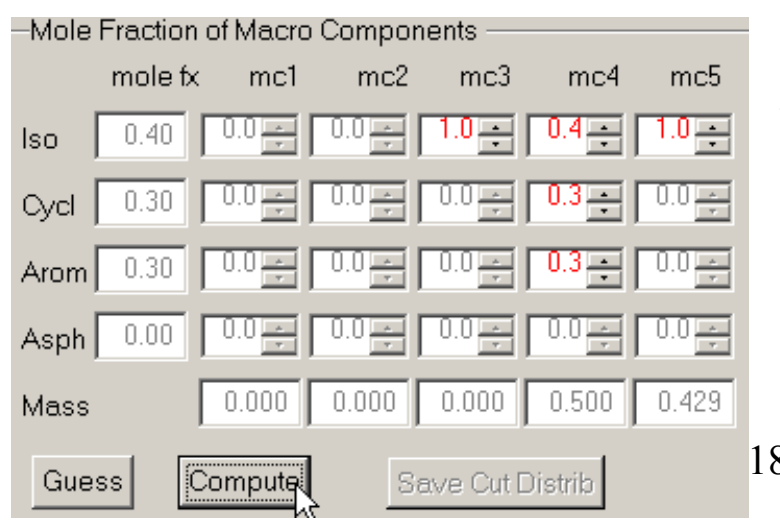
carbon number cut. There is no mass in cut 3 because its mass is $100 \%$ assigned to the n-alkane fraction (Figure 64). This assignment can be changed using the spinner in the upper part of the form. For example if the mass of petroleum in the $4^{\text {th }}$ carbon number cut is increased to 1.5 , the 
mass available for distribution is increased to 0.5 . Finally if the compute button is depressed the mole fraction of the various non-n-alkane components are computed and put in the first column as shown to the left. Finally the petroleum composition can be displayed by depressing the Make and Plot total $\mathrm{HC}$ button at the base of the form. If the $\log$ box is checked it is a $\log$ mole $C_{n}$ vs. $C_{n}$ molecular wt/14 ( carbon number) plot. This is shown in Figure 67. The mass fraction methane is increased from 0.032 to 0.0403 so the ordinate axis scales as shown.

The distribution of non-n-alkanes can be changed by changing their mole fraction distribution, or by increasing the non-n-alkane mass in a particular carbon number cut. For example, increasing the cyclic alkane mole fraction to 0.8 while reducing the isoalkane and aromatic mole fractions to 0.1 the $3^{\text {rd }}$ carbon number cut changes Figure 67 to Figure 68. 
Model Hydrocarbon Chromatogram

Kissen slope for Ker 1: II_H_Kimer_aug $=\quad{ }^{-} 0.10$

n-alkanes (blue *)

Isoalkanes (turquoise *)

cyclic alkanes (green square)

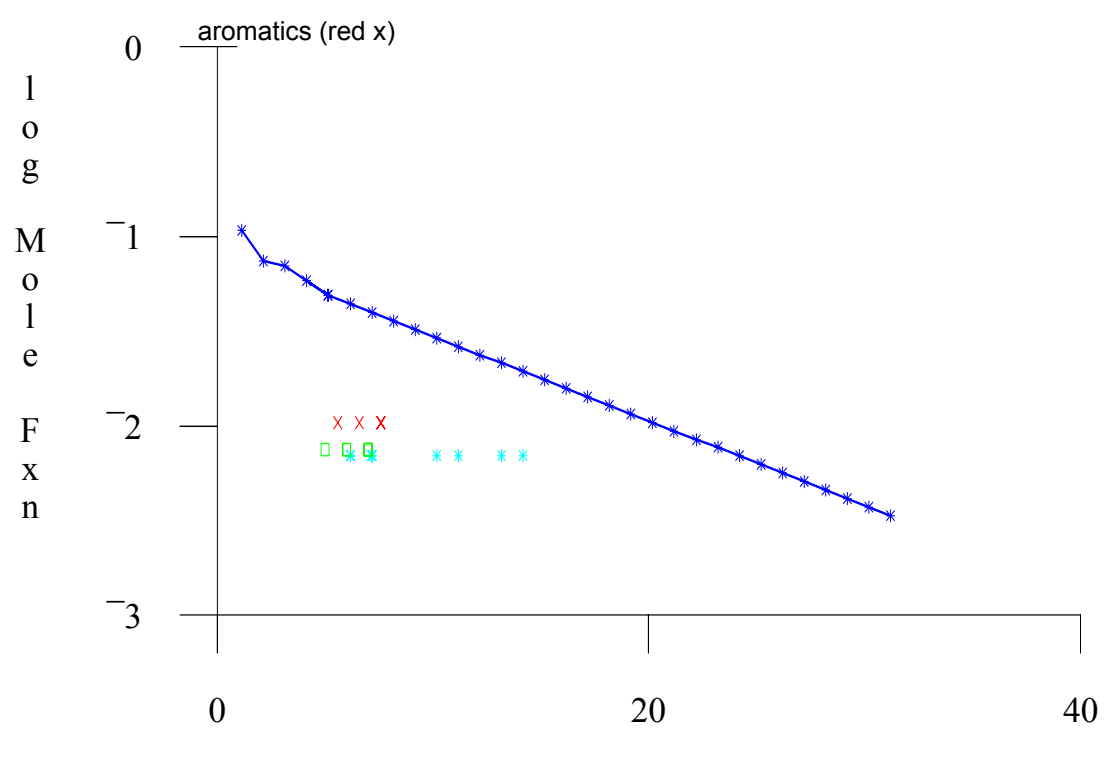

MolWt/14
Figure 67.

Petroleum composition plotted by depressing Make and Plot total $\mathrm{HC}$ on the Figure 64 form.

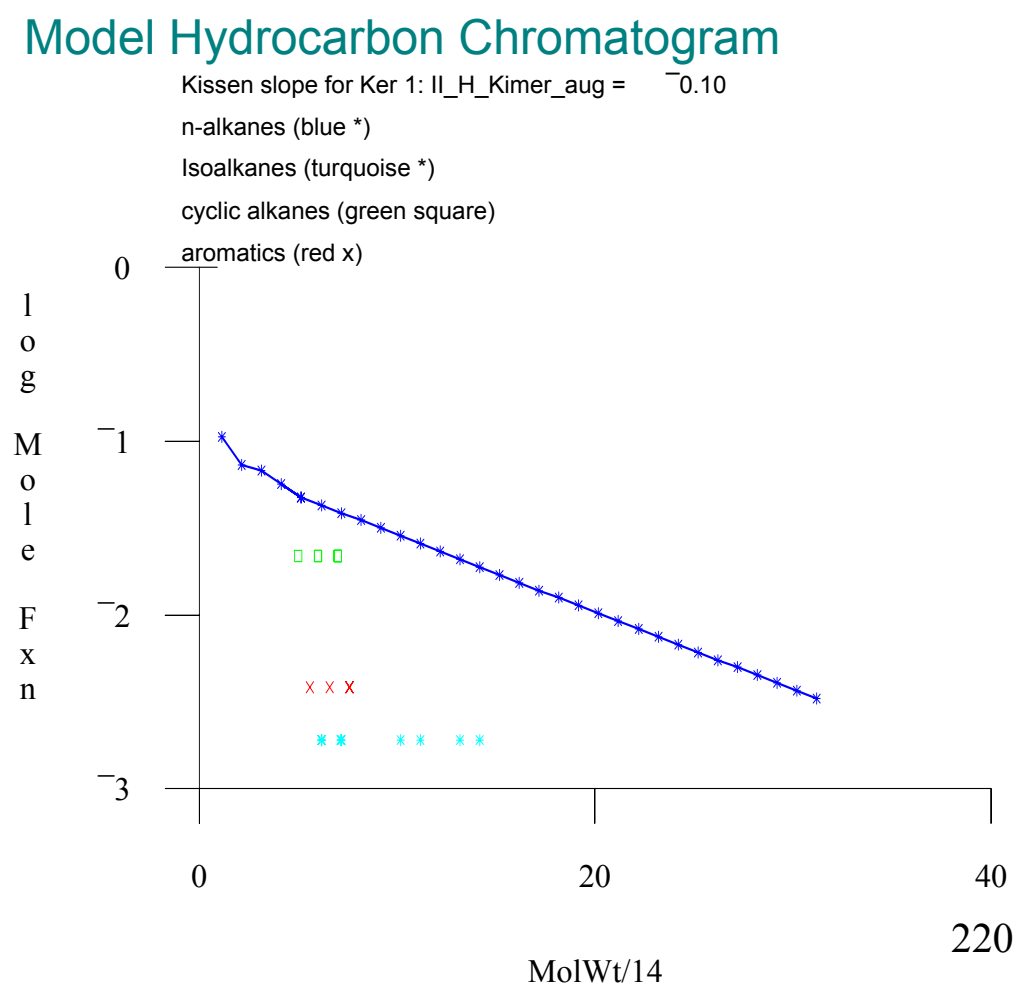

Figure 68. Same as Figure 67 except the mole fractions in carbon number cut 3 have been changed from 0.40 .30 .3 to 0.10 .80 .1 . 
Finally the Hydrocarbon Design form (Figure 64) is connected to the Evaluate Maturation scratchpad (Figure 27) and the Evaluate Kinetics scratchpad (Parameters: Evaluate kinetics scratchpad not yet discussed). Both of these forms allow kerogen maturation models to be selected and run for a particular time-temperature history. The first-mentioned scratchpad takes the $\mathrm{T}(\mathrm{t})$ history from a selected node in a pseudowell. The second-mentioned scratchpad allows computed maturation for a specified heating history. Selecting ker1 or ker2 in Figure 64 connects to the maturation for the most recent maturation history compute by these forms. When kerl is selected, the petroleum masses at the model temperature selected are placed in column 5 at the top of the Figure 64 form. The user can no longer modify columns 2-5 because these columns are specified the kinetic and maturation models. The user can define the $\mathrm{Wt} \%$ of the petroleum that is n-alkane (the first column), and the distribution of non-n-alkanes in the bottom portion of the form. Using the temperature $(\mathrm{T}[\mathrm{C}])$ spinner, the Kissen slopes and compositions of the oils derived from the two kerogens can be examined as a function of maturation (temperature).

For example we can select two type II kerogens and mature them at a heating rate of $2^{\circ} \mathrm{C}$ per Ma as shown in Figure 69, with the comparative results shown in Figure 70. The Kissen slopes are quite different for these two maturation models. Natural unaltered

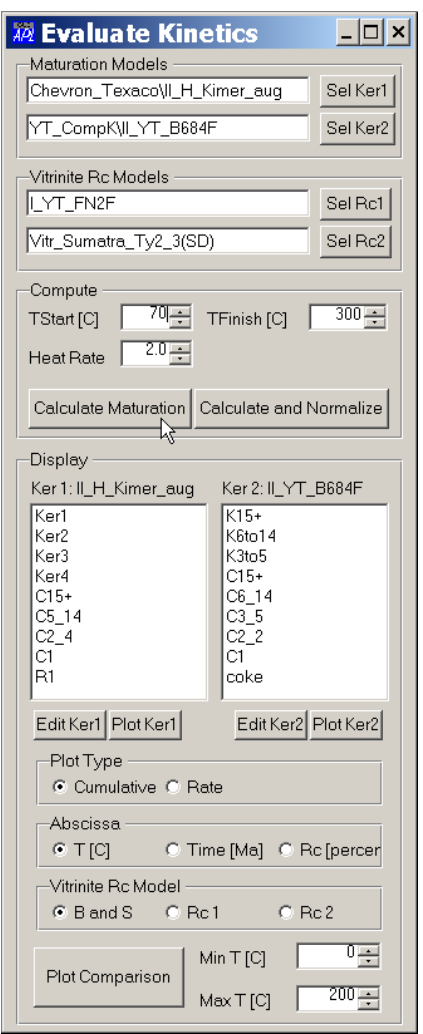
petroleums have $\log _{10}$ Kissen slopes between -0.04 (immature) and -0.25 (condensate). The corresponding $\log _{\mathrm{e}}$ Kissen slopes (reported in the figures) are -0.1 (immature) and -0.6 (condensate). The simple screening process illustrated in Figure 69 and Figure 70 would appear to be an effective way to evaluate the suitability of a particular maturation model for evaluating the potential phase state of petroleum migrating in a model 2D section.

Figure 69. The Evaluate Kinetics form is selected with the Parameters: Evaluate kinetics scratchpad menu. Kerogen maturation models are selected from the Custom Kinetics folder. Some are provided with this distribution. Using the buttons on the top of the form we have selected two type II kerogen models. These are matured at a constant heating 
rate of $2{ }^{\circ} \mathrm{C} / \mathrm{Ma}$. The maturation of the two kerogens can be compared as illustrated in Figure 27 and following figures. Here we use the maturation results to examine the hydrocarbon compositions as a function of temperature from 70 to $300^{\circ} \mathrm{C}$ using the Design Hydrocarbon Composition form shown in Figure 64.

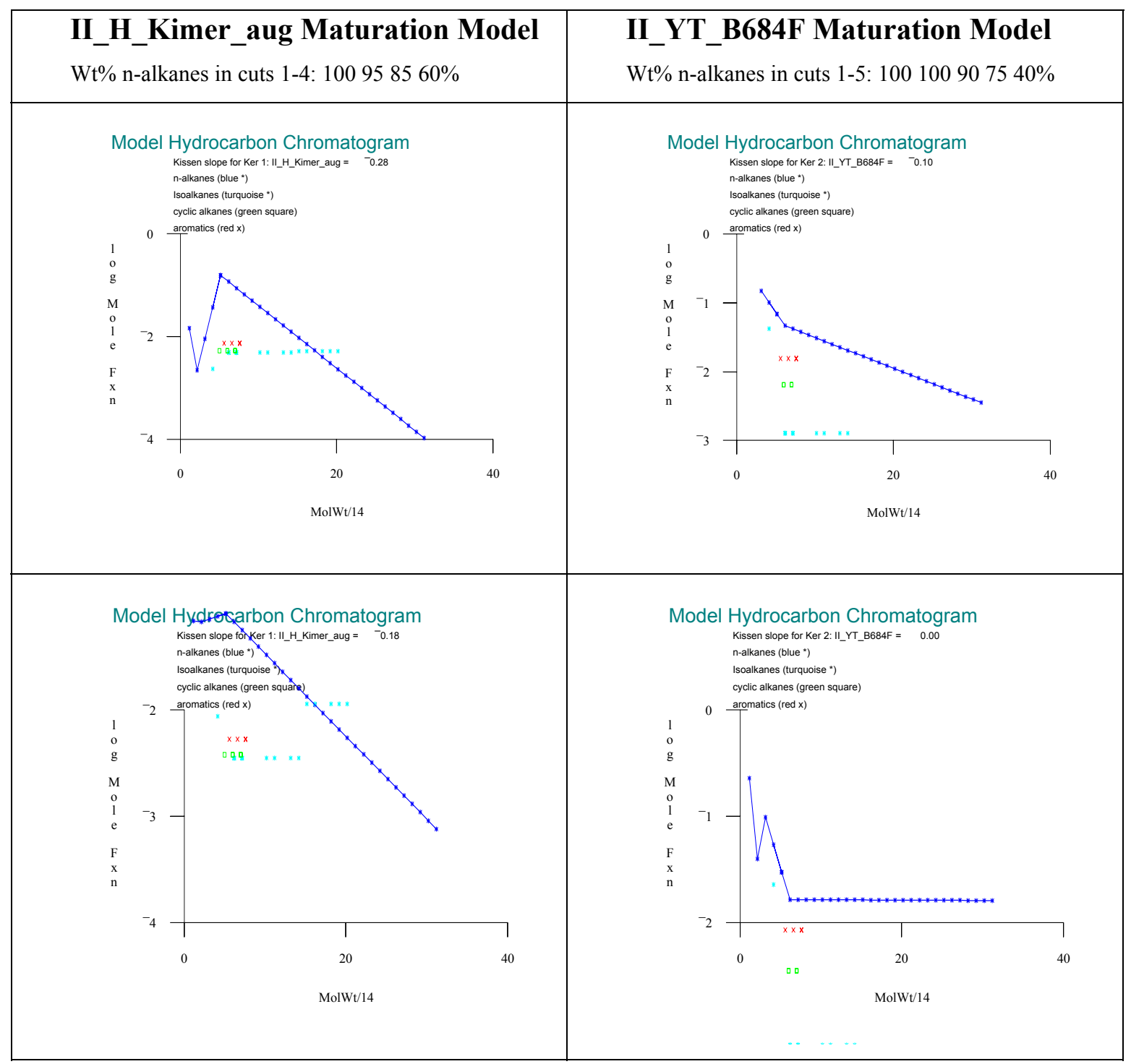




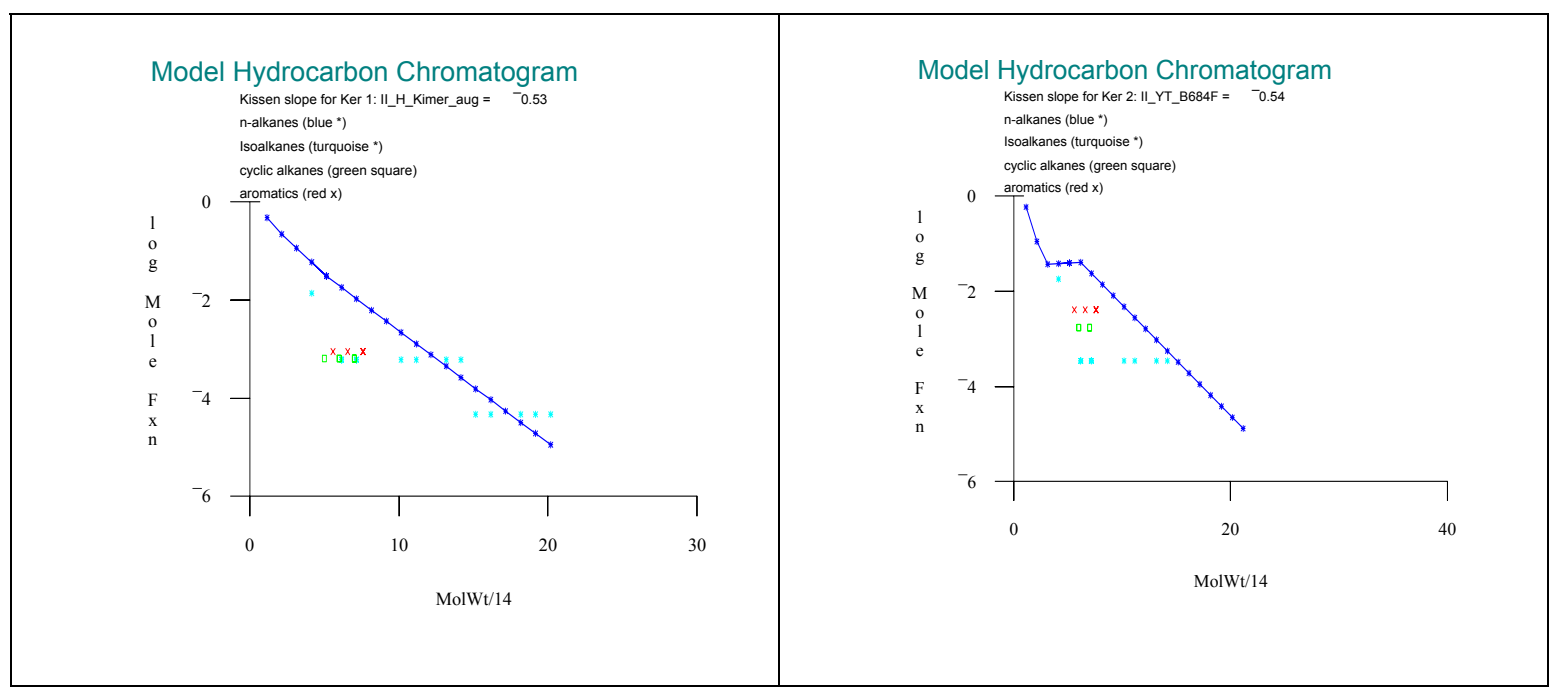

Figure 70. Molecular composition of petroleum produced by heating two different type II kerogens from 70 to $300^{\circ} \mathrm{C}$ at $2{ }^{\circ} \mathrm{C} / \mathrm{Ma}$. The maturation histories of the two kerogens was calculated using the form shown in Figure 69. The hydrocarbon compositions are displayed as a function of maturation temperature using the form in Figure 64.

Temperature and depth increases from top to bottom. Caption now augmented to contain this information.

\section{K. Micro-Chemistry of the Petroleum System}

The macrocomponent chemistry of the migrating hydrocarbons computed in section I above can be decomposed into molecular components following the rules established in the previous section. This can be viewed (as are all section variables) using the Solve Equations: View Calculated Solution menu command. This time depress the PetCompsn button on the top of the Graphics form. This summons the form shown in Figure 71.

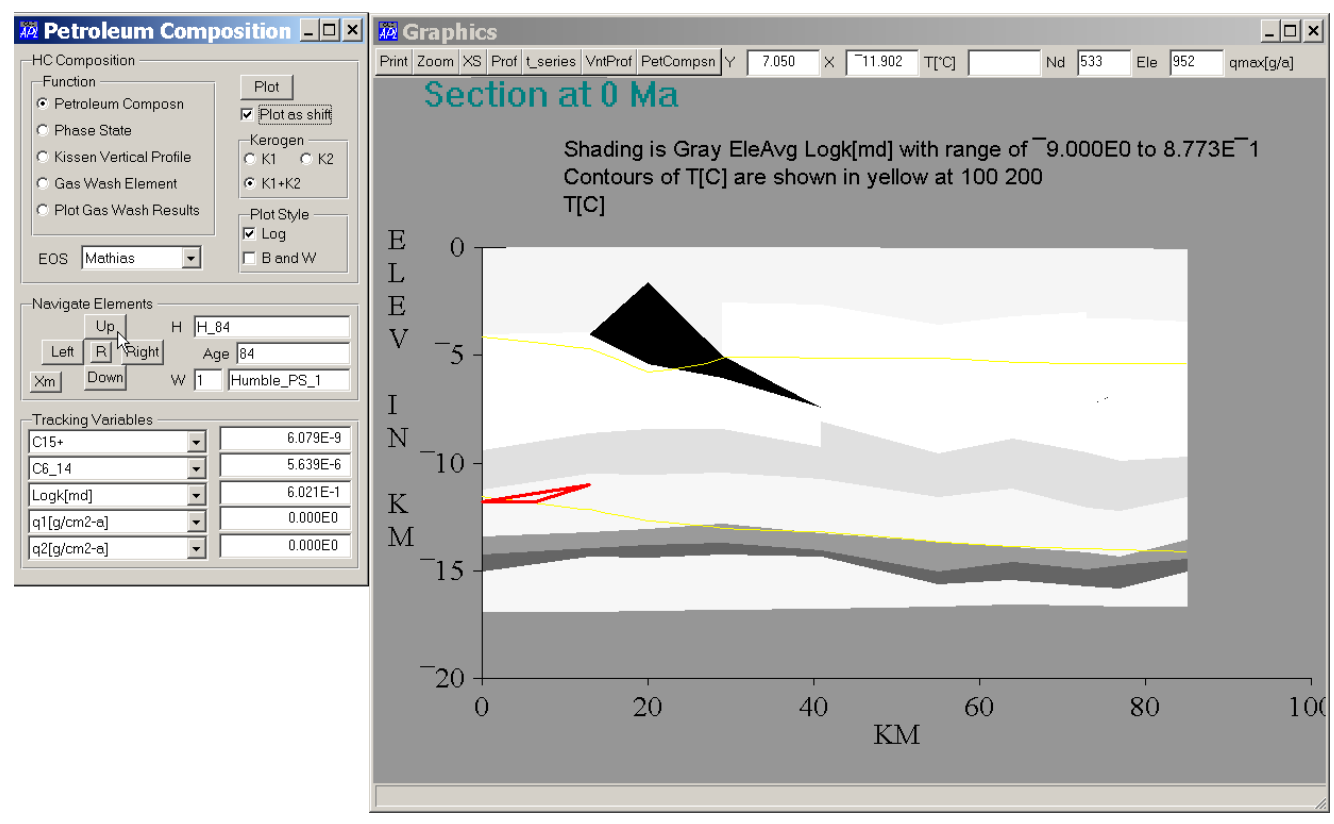


Figure 71. Form called by menu command Solve Equations: View Calculated Solution followed by depressing the PetCompsn button. With the Plot as Shift box checked, navigating the red element up (button under arrow) with the $K 1+K 2$ option selected causes the total petroleum composition to be plotted. The composition of just the kerogen 1 or kerogen 2 products can be plotted if the $K 1$ or $K 2$ options are selected. The total petroleum composition is for the element selected is shown in Figure 72.

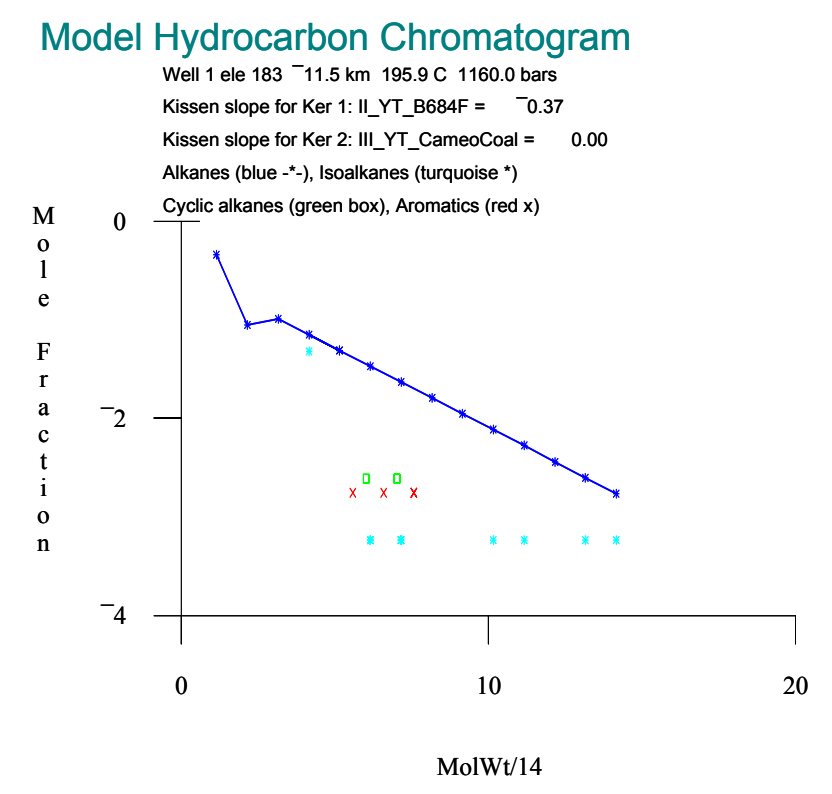

Figure 72. The total petroleum composition of the element selected in Figure 71. It is plotted either by depressing the Plot button or, if the Plot as Shift box is checked, when the element is shifted to the one shown from any adjacent element using the navigator buttons. Notice that the (natural $\log$ ) Kissen slopes $\mathrm{f}$ the heaviest (largest $C_{n}$ cut) are given for the products of both kerogens.

The Kissen slope profiles can be plotted for the vertical stack of elements containing the selected element if the Kissen vertical profile option is selected and the Plot button depressed. A vertical profile through the elements to the right of well 1 is shown in Figure 73.
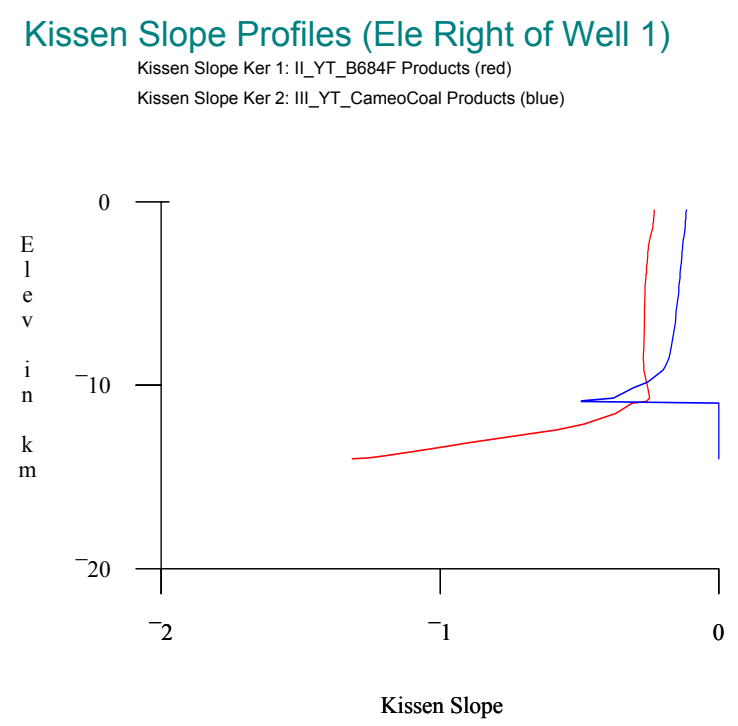

Figure 73. Vertical Kissen slope profile through well 1 elements produced by selecting the Kissen Vertical Profile option in the Figure 71 form and depressing the plot button as shown below. The discontinuity in the profiles at $\sim 10$ $\mathrm{km}$ depth occurs where alkanes $>\mathrm{C} 15$ first appear and the Kissen slope shifts to the heaviest cut. Below 10 $\mathrm{km}$ the heavy $\mathrm{n}$-alkanes have cracked to lighter fragments. 


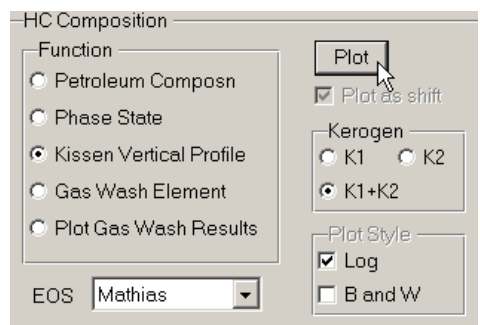

Kissen Slope Profiles (Ele Right of Well 1) Kissen Slope Ker 1: IIIYY__B684F Products (red) Kissen Slope Ker 2: III_YT_CameoCoal Products (blue)

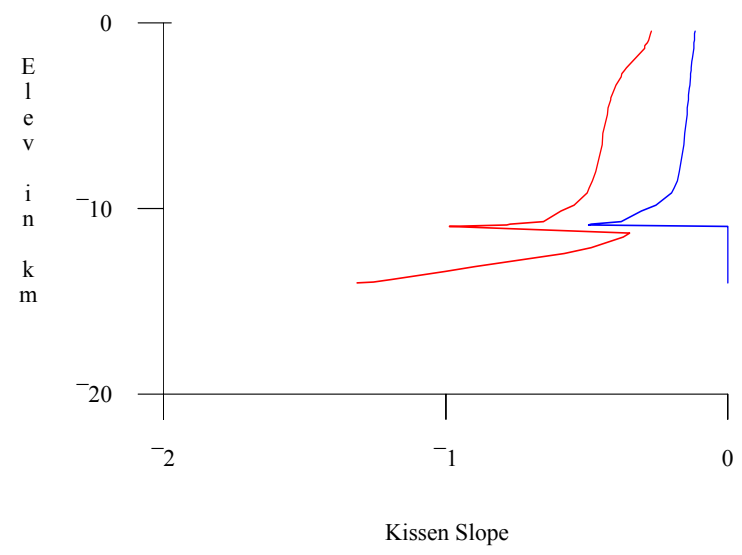

Figure 74. Setting the $\mathrm{Wt} \% \mathrm{n}$ alkane fraction in cut 5 to zero for Kerogen 1 in the Flash: Design Petroleum Composition form (Figure 64) changes the profile shown in Figure 73 to change as shown. The Kissen slope is determined by the $\mathrm{C}_{2-3}$ and $\mathrm{C}_{6-14}$ fractions rather than the $\mathrm{C}_{6-14}$ and $\mathrm{C}_{15+}$ fractions. The Apply wtpct nalk button must be depressed to apply the changes.

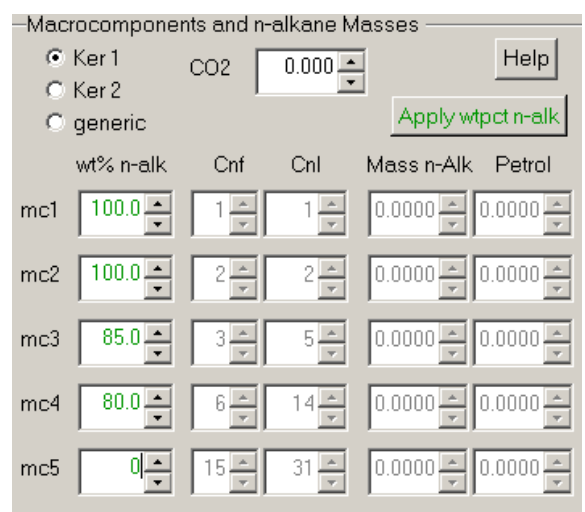

The hydrocarbon composition is determined using the rules specified in the Design Hydrocarbon Composition form shown in Figure 74. Changing these rules will change the nature of plots of the type shown in Figure 72 and Figure 73 . For example, setting the $\mathrm{Wt} \% \mathrm{n}$-alkane mass in carbon number cut 5 to zero for Kerogen 1 will cause the Kissen slope for Kerogen 1 to be determined from the $\mathrm{C}_{2-3}$ and $\mathrm{C}_{6-14}$ fractions, and will result in the profile shown in Figure 74.

\section{Phase State and Gas Washing in the Model Petroleum System}

The phase state of the decomposed (element composition) petroleum can be determined for any element by selecting the Phase State option on the Figure 71 form and depressing 
the plot button. The phase boundary can be found in this fashion, for example. Figure 75 shows how this can be done. The equation of state used in the calculations can be selected from four options. The pressure and temperature in the element is taken from the physical solution (Section E). The macro-chemical composition of the migrating petroleum is as was determined in Section $\boldsymbol{G}$. The decomposition to a molecular chemical description uses the rules specified in the Design form (Figure 64).

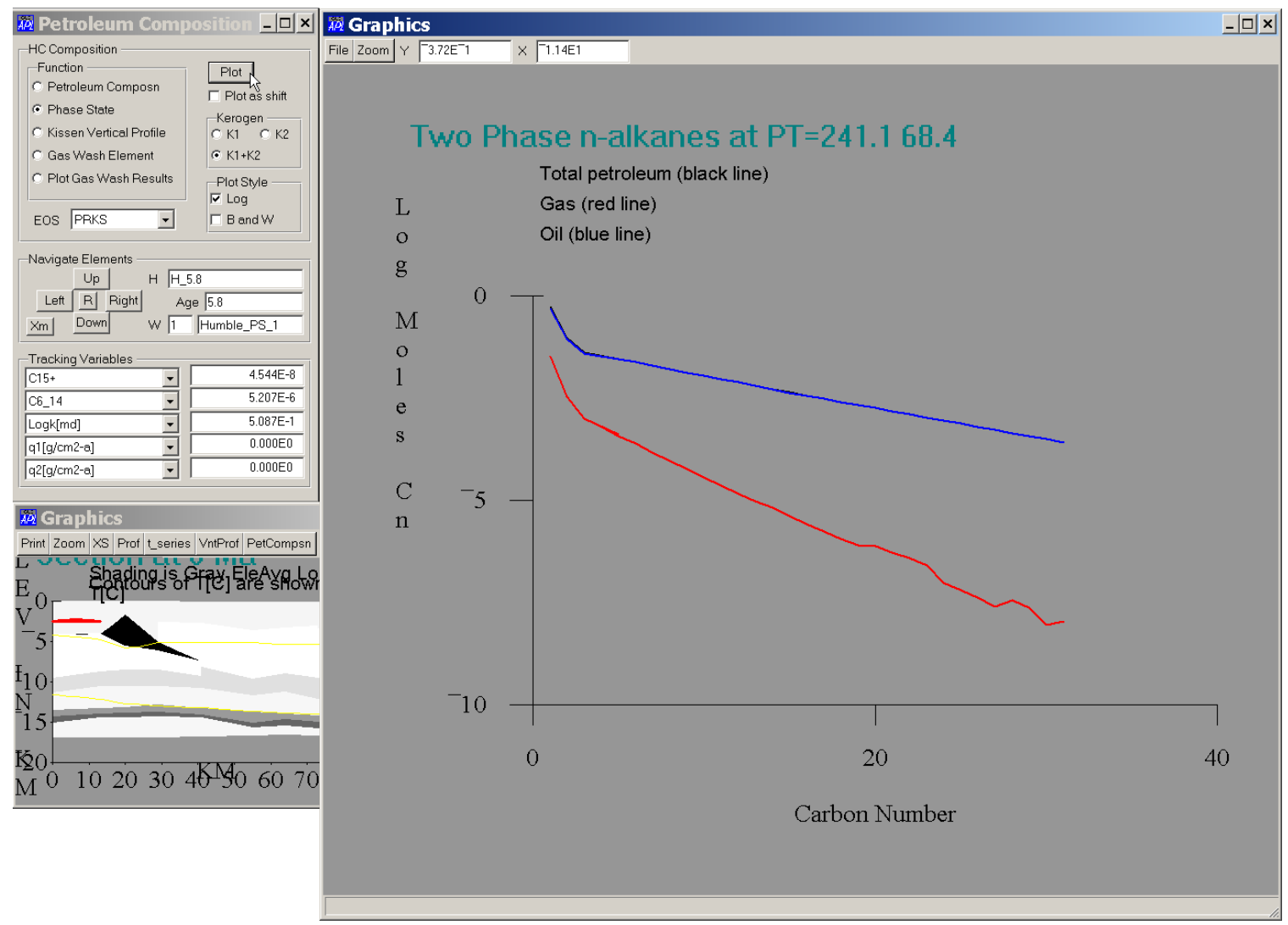

Figure 75. Plot of phase state of element using procedure: Solve Equations: View Calculated Solution: PetComposn button: navigate to element: choose Phase State option: push Plot button. The immediately underlying element is single phase. Hence the phase boundary in the Tiger Shoals area of the offshore Louisiana Gulf of Mexico is predicted to lie at $\sim 2.4 \mathrm{~km}$ depth. Notice that we have selected the PRKS (predictive Redlich-Kwong-Souave) equation of state for the phase state flash calculation.

The other choices on the Petroleum Composition form shown in Figure 71 and Figure 75 allow the petroleum in a selected element to be washed by dry gas $\left(\mathrm{CH}_{4}\right.$ or $\left.\mathrm{CO}_{2}\right)$. Selecting the Gas Wash Element option allows the petroleum in the element to be washed (and saved). Selecting the Plot Gas Wash Results allows the calculated washing history to be examined, or retrieved from file and examined. Figure 76 discusses options on the Gas Wash form. Figure 77-Figure 80 show some of the washing plots. 


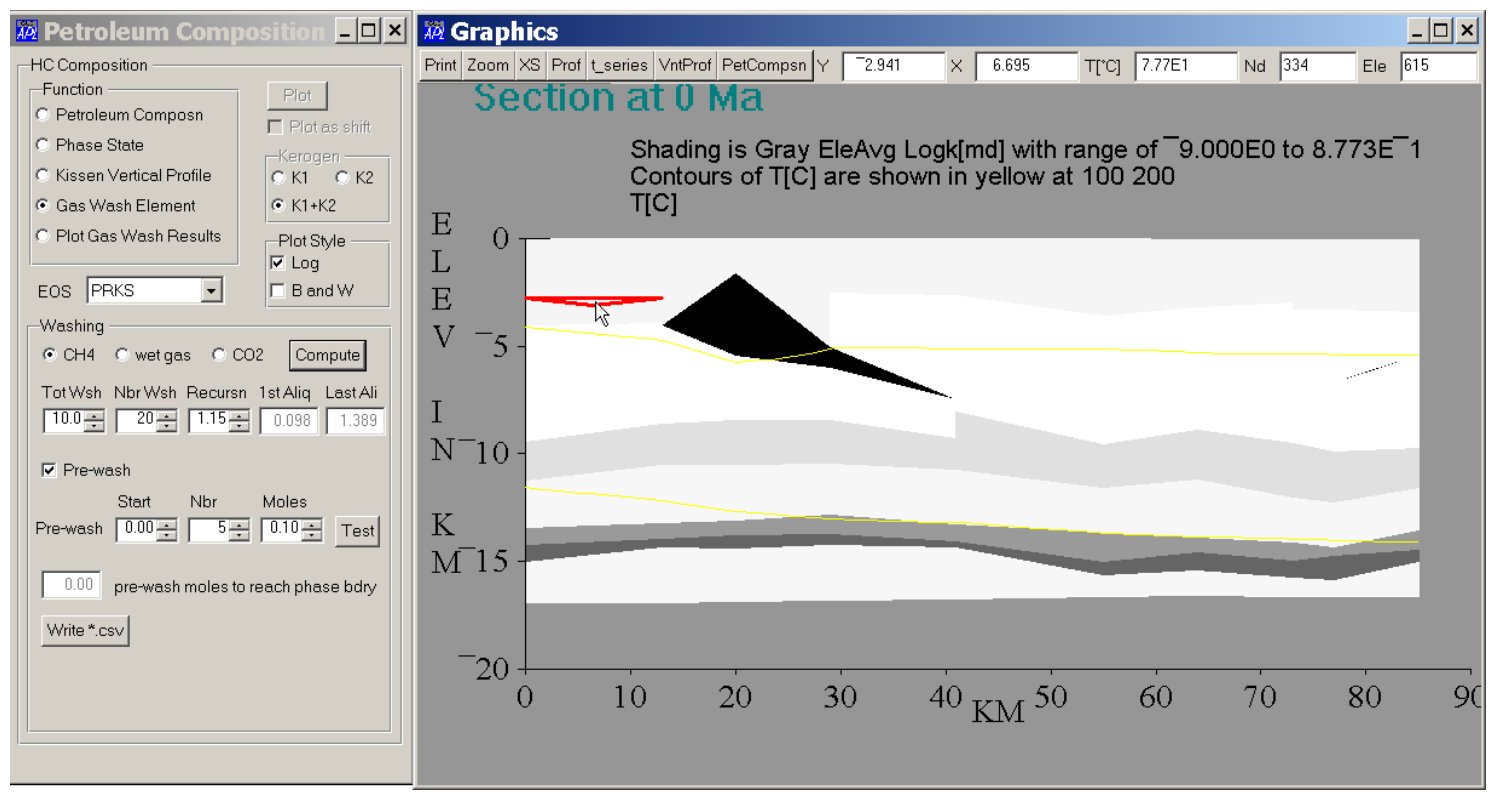

Figure 76. Gas washing using the procedure: Solve Equations: View Calculated Solution: PetComposn button: navigate to element: choose Gas Wash Element: push Plot button. Here we wash element 615 at $2.91 \mathrm{kmbsl}$ (positioning mouse over element as shown gives depth and element on toolbar). We wash 1 mole of petroleum with the composition in element 615 with 10 moles of CH4 in 20 washing steps. Each washing aliquot is 1.15 times larger than the last. The first aliquot is 0.098 moles, the last is 1.389 moles. Results are plotted by selecting Plot Gas Wash Results and are shown in Figure 77-Figure 80. Results are written to a *.csv file in the case Metafiles folder if the Write *.csv button is depressed. 


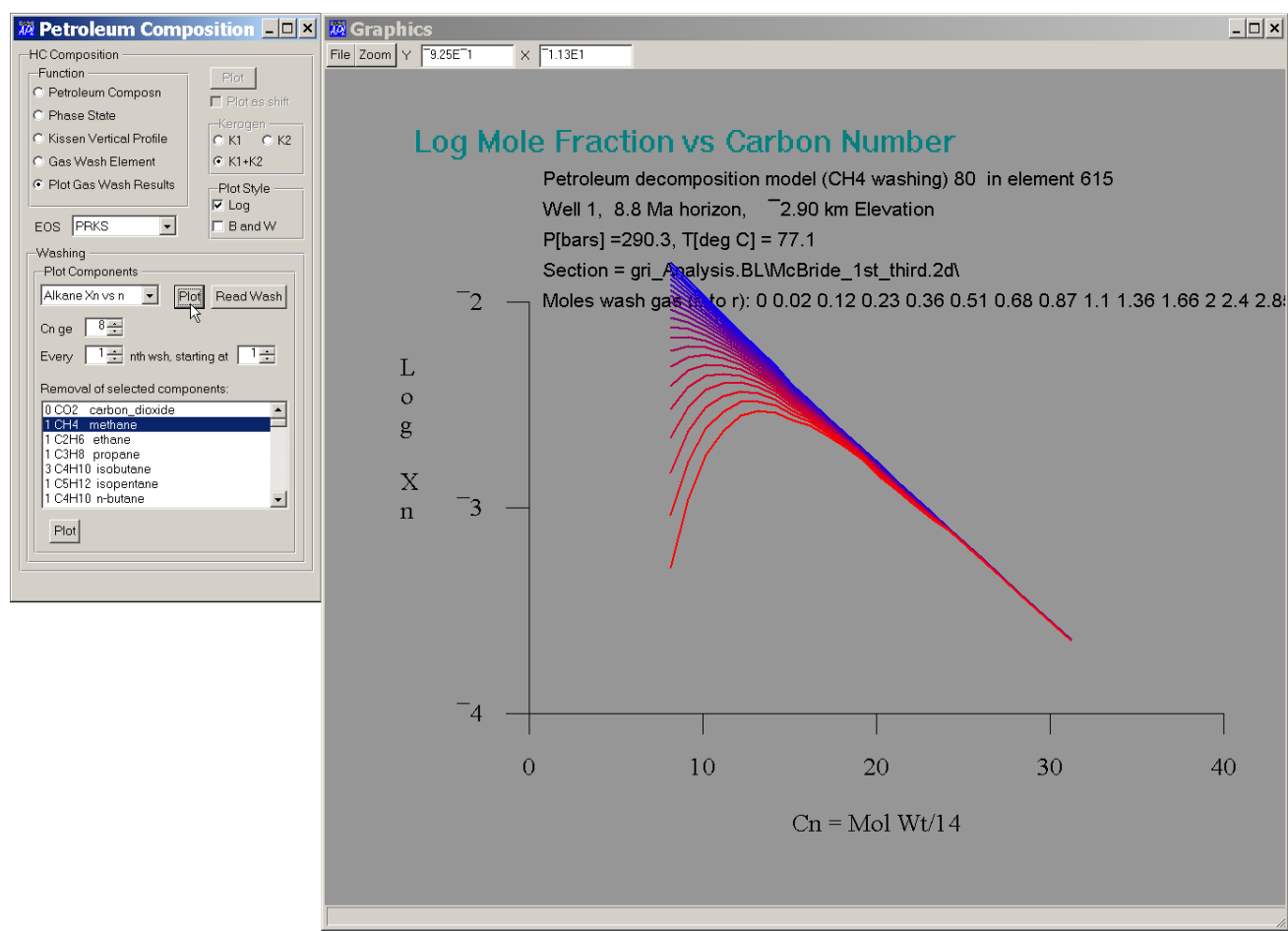

Figure 77. Plotting results of wash in Figure 76 with the procedure: select Plot Gas Wash Results option, select plot type (here Alkane Xn vs n), depress Plot. The log plot option is checked, so the plot is $\log \mathrm{Xn}$ vs $\mathrm{Cn}$.

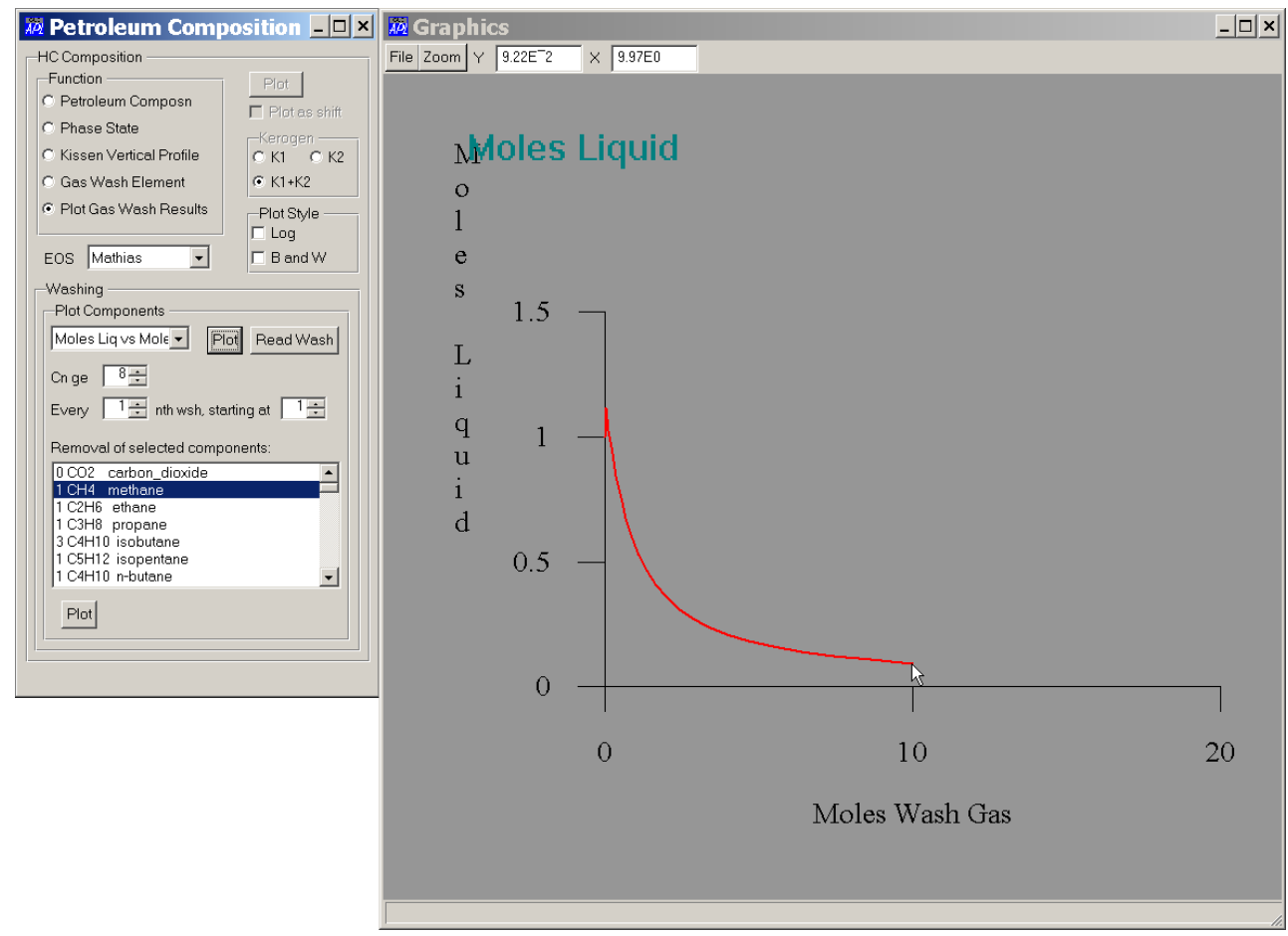

Figure 78. Plot of moles of liquid petroleum remaining vs. moles of wash gas (CH4) interacted with the petroleum. Over $90 \mathrm{Wt} \%$ of the original oil is removed by 10 moles 
of methane washing 1 mole of original petroleum (read y value from toolbar for position of mouse- 0.092 moles of petroleum remaining at 10 moles wash).

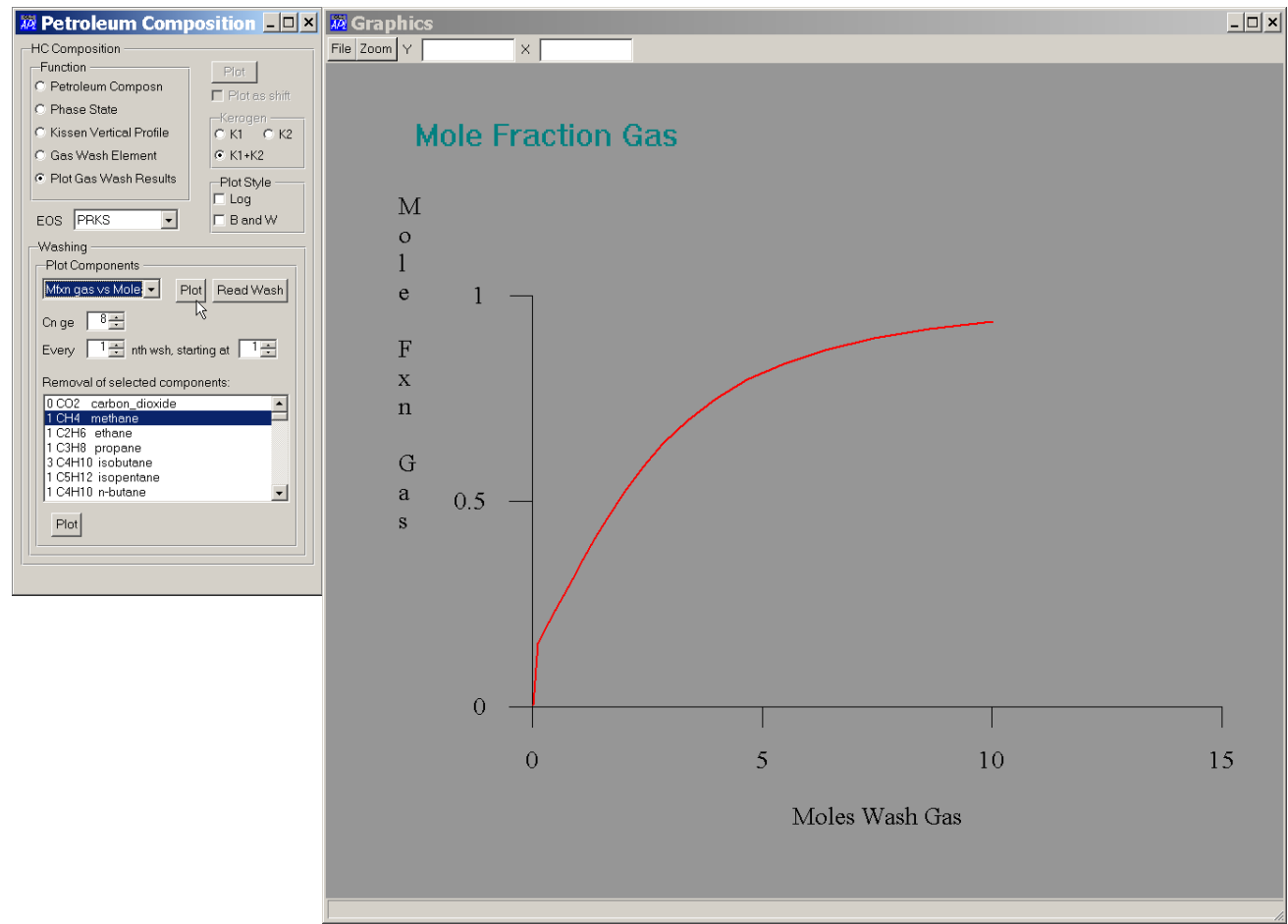

Figure 79. Mole fraction gas in each flash calculation as a function of washing.

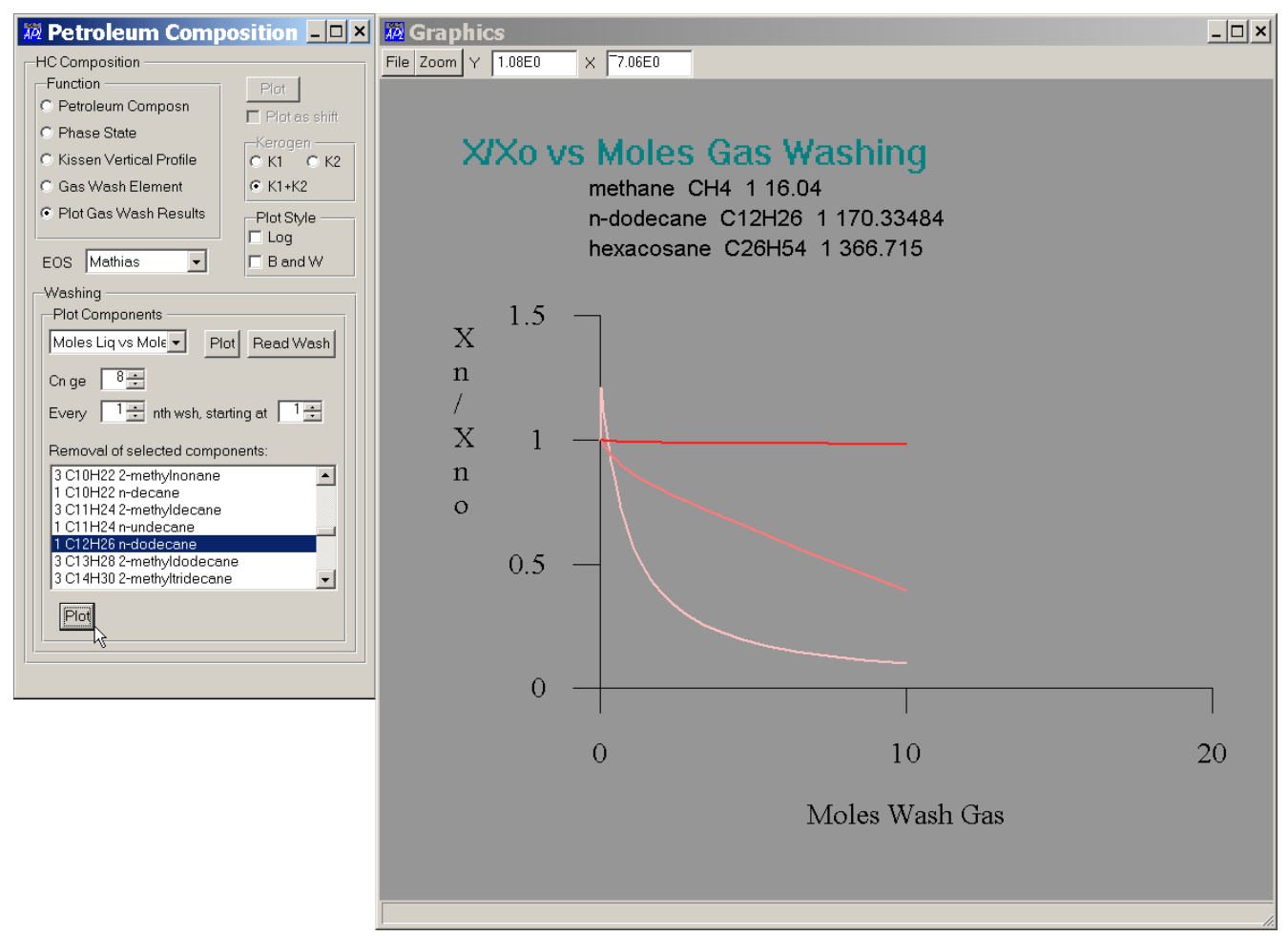


Figure 80. Moles of component $n$ divided by the moles of component $n$ in the initial 1 mole of petroleum. The proportion of methane (pink curve) in the residual oil remains roughly constant. Higher carbon number alkanes are less depleted. Color intensity (pink to red) increases with carbon number. The caption indicates molecular type (1=alkane, $2=$ cyclic alkane, $3=$ isoalkane, and $4=$ aromatic) as well as molecular weight. Any set of components can be selected in the combo box (cntrl key adds components) and plotted.

\section{Phase State and Gas Washing of Specific Petroleum}

quations Supercritical Migration Flash

Select Petroleum Model

View Molecular Components of Selected Model

Design Petroleum Decomposition

Scratchpad: Flash/Wash Decomposed Petroleum
The Flash: Scratchpad: Flash/Wash

Decomposed Petroleum menu allows a petroleum with defined composition to be flashed or washed at specified pressure and temperature. Being able to do this is useful in

evaluating the sensitivities of a decomposition scheme. This capability is described in Section XI.C (p.) of this manual. 


\section{Reconstructing the Physical History of a Basin}

The previous section provided an overview of the advanced chemical capabilities of BasinLAB. The next sections describe its functionality in more detail.

- Section VI (p.231) describes how BasinLAB reconstructs the past physical history of the basin from present strata thickness and fault locations.

- Section VII (p.262) describes how the physical and chemical parameters that control compaction, thermal conductivity, permeability, hydrocarbon maturation etc., are specified in $\boldsymbol{B a s i n} \boldsymbol{L} \boldsymbol{A B}$, and how they can be changed.

- Section VIII (p.285) describes how the heat flow history of the basin is inferred and verified.

- Section $I X$ (p. 292) discusses the solution of $2 D$ finite element equations defining basin temperature, fluid flow, and salinity transport.

- Section $X$ (p. 310) describes 2D hydrocarbon maturation, migration, generation statistics, chemistry, phase state, and washing interactions.

\section{In all these sections the following program features should be kept in mind:}

- Brief description of any button, form, edit windows, etc. is provided in the hint bar of the main workspace when the mouse is positioned over the item.

- The program functions proceed in a logical fashion across the menu bar of BasinLAB's workspace.

- A chart in each manual section shows the main files and functions in that section.

- Ascii input files provide program control or data input.

- System files keep track of an analysis as it is carried out. Analysis steps need not be repeated, although they can be. The analyst to pick up from where a previous analysis left off. The state of an analysis is indicated by whether or not menu commands are active.

- Examples that can be run by the user illustrate the manual. These examples are provided in the Examples. BL folder and are explicitly referenced in the discussion. 


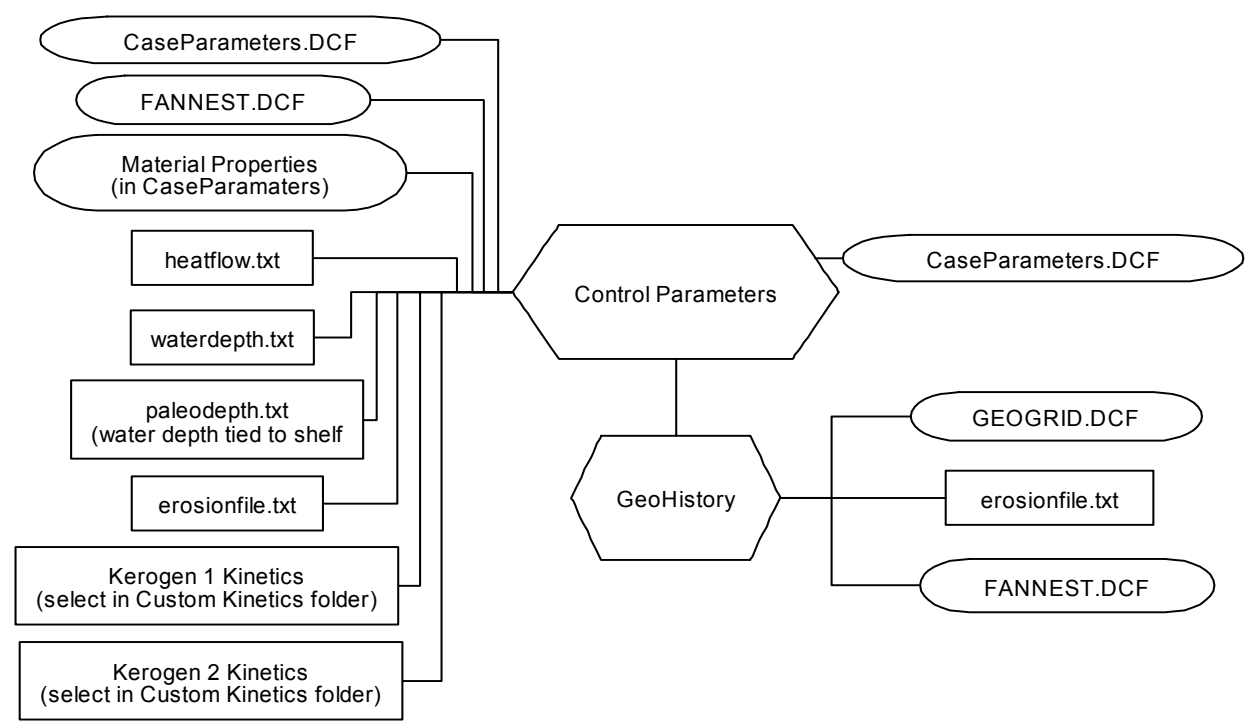

Chart 2. The input files, functions, and output files for inferring the geologic history of a 2D section. Box style conventions are the same as in Chart 1. Charts 3 and 1 are on pages 285 and 168 .

\section{A. Erosion}

The file structure and section input has been covered in Section II and III. The section is input as it appears today. However, erosion may have affected the section. The GeoHistory: Add Erosion menu command allows erosion to be input, modified and saved. The blcases: Examples.BL: Erosion.2d provides an example. Navigate to this example using the File: Open Section Project menu command, and then examine the erosion that is already specified in this case using the GeoHistory: Add Erosion: Add/Edit Erosion menu command. This brings up the forms shown in Figure 81.

\begin{tabular}{|c|c|c|c|c|c|c|c|c|c|c|}
\hline \multicolumn{5}{|c|}{ Hid Uncompacted Stratigraphic Isopachs } & \multicolumn{6}{|c|}{ 㣂 Add and Subtract (Uncompacted) Erosion } \\
\hline Apply & Update Erosion ->> & Help & & & $\ll$-Transfer & Help & & & & \\
\hline & WELL_1 & WELL_2 & WELL_3 & WELL_4 & & & WELL_1 & WELL_2 & WELL_3 & WELL_4 \\
\hline SAND_10 & 4426229508 & 1.639 & 1.639 & 1.639 & EROSION & & ] & -0.970 & -1.340 & -0.970 \\
\hline SHALE_12P5 & 0.769 & 0.769 & 0.769 & 0.769 & SDSH_25 & & 0.000 & 0.970 & 1.340 & 0.970 \\
\hline SHALE_13P75 & 0.385 & 0.385 & 0.385 & 0.385 & & & & & & \\
\hline SHALE_15 & 0.385 & 0.385 & 0.385 & 0.385 & & & & & & \\
\hline EROSION & 0.566 & -0.970 & -1.340 & -0.970 & & & & & & \\
\hline SDSH_25 & 1.587 & 1.446 & 1.343 & 1.446 & & & & & & \\
\hline SDSH_30 & 1.111 & 1.746 & 1.584 & 1.746 & & & & & & \\
\hline
\end{tabular}

Figure 81. Erosion history specified in the blcases: Examples.BL: Erosion.2d example case, called up with the GeoHistory: Add Erosion: Add/Edit Erosion menu command. The form on the left shows the uncompacted thickness of all strata; the form on the right shows the uncompacted erosion that has been restored and then removed for the strata highlighted. Edits are transferred between forms using the transfer buttons. It is not 
appropriate in this case, but in cases where erosion has removed more than one stratum, the erosion process can occur over more than one time interval, e.g., more than two stratigraphic intervals can be highlighted on the left form, and modified by erosion on the right.

Figure 82 shows the erosion history defined in Figure 81.

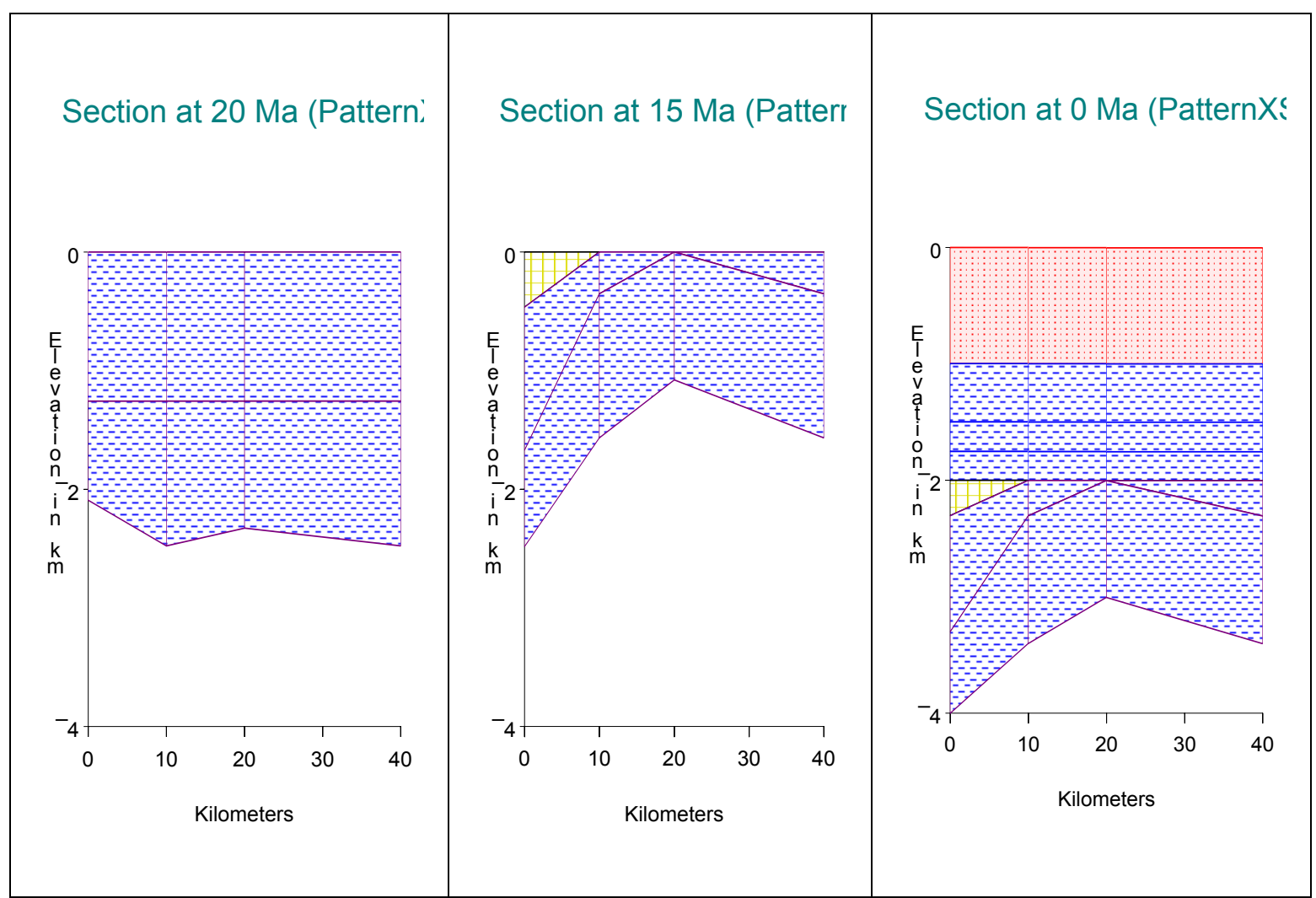

Figure 82. Figure at right shows section of the blcases: Examples.BL: Erosion.2d example at the present day. The first panel shows how $0.97 \mathrm{~km}$ has been added to the second pseudo-well, $1.34 \mathrm{~km}$ to the third, and 0.97 to the fourth. The second panel shows how these additions have been removed by erosion. Subsequent strata are deposited and the erosion surface buried until the final (present day) panel is reached. Notice that deposition can occur at a horizon that is also eroding. The carbonate (yellow box pattern) is deposited at the same time the shale (blue dashes) is eroding. Sand is indicated by red dots.

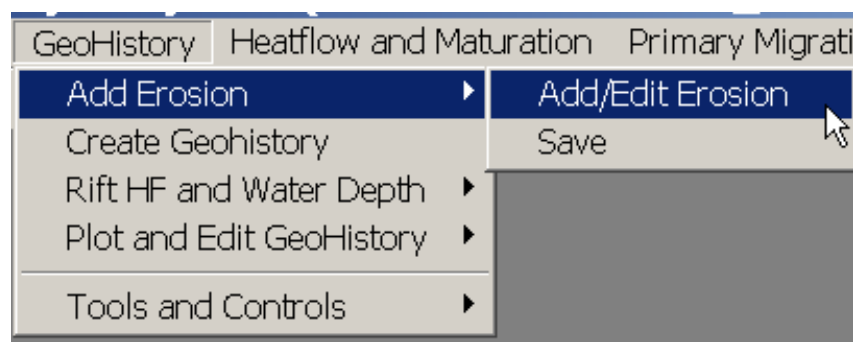

Modifications to the erosion history (or a newly defined erosion history) can be saved to the case folder using the GeoHistory: Add Erosion: Save menu command. The erosionfile.txt file is read when the case section is opened, and thus, once created, is a 
permanent part of the geologic definition of the case. The erosionfile.txt file has the format shown in Figure 83. The comments are added automatically when the file is created. This, and other askii files written by BasinLAB are self-documenting.

52 \# nbr rows in file (negl. this row), nbr strata (cols) affected by erosion\#

67 \# affected strata nbr counted down from top\#

$0 \quad 0 \quad$ \# km of uncompacted sediments added or removed at each well\#

$-0.970 .97$

$-1.341 .34$

$-0.970 .97$

Figure 83. The Askii erosionfile.txt file. Files are automatically documented by comments demarcated by \# symbols as shown.

\section{B. Faults}

Faults in BasinLAB are defined by pairs of pseudowells that are numerically held a set distance apart. They are defined by the Fault_code variable. The fault separation distance is set in the form summoned by the Parameters: GeoHistory Parameters form, and also in a number of other forms.

Fault flags are assigned as follows:

- 0 for left and right (edge) pseudowells in section

- 3 pseudowells fixed in space

- 4 pseudowells pasted on top of 3 to left

- 5 pseudowells fixed offset from 3 to left

- 7 pseudowells fixed offset from 3 to right

- 9 pseudowells pasted on top of 3 to the right

An example is provided by the blcases: Examples.BL: Fault test.2d case. Use the File: Open Section Project menu command to open the SFLAT file of this case, and use the Geology: Graphic Edit form to view the fault flags as illustrated in Figure 84. 


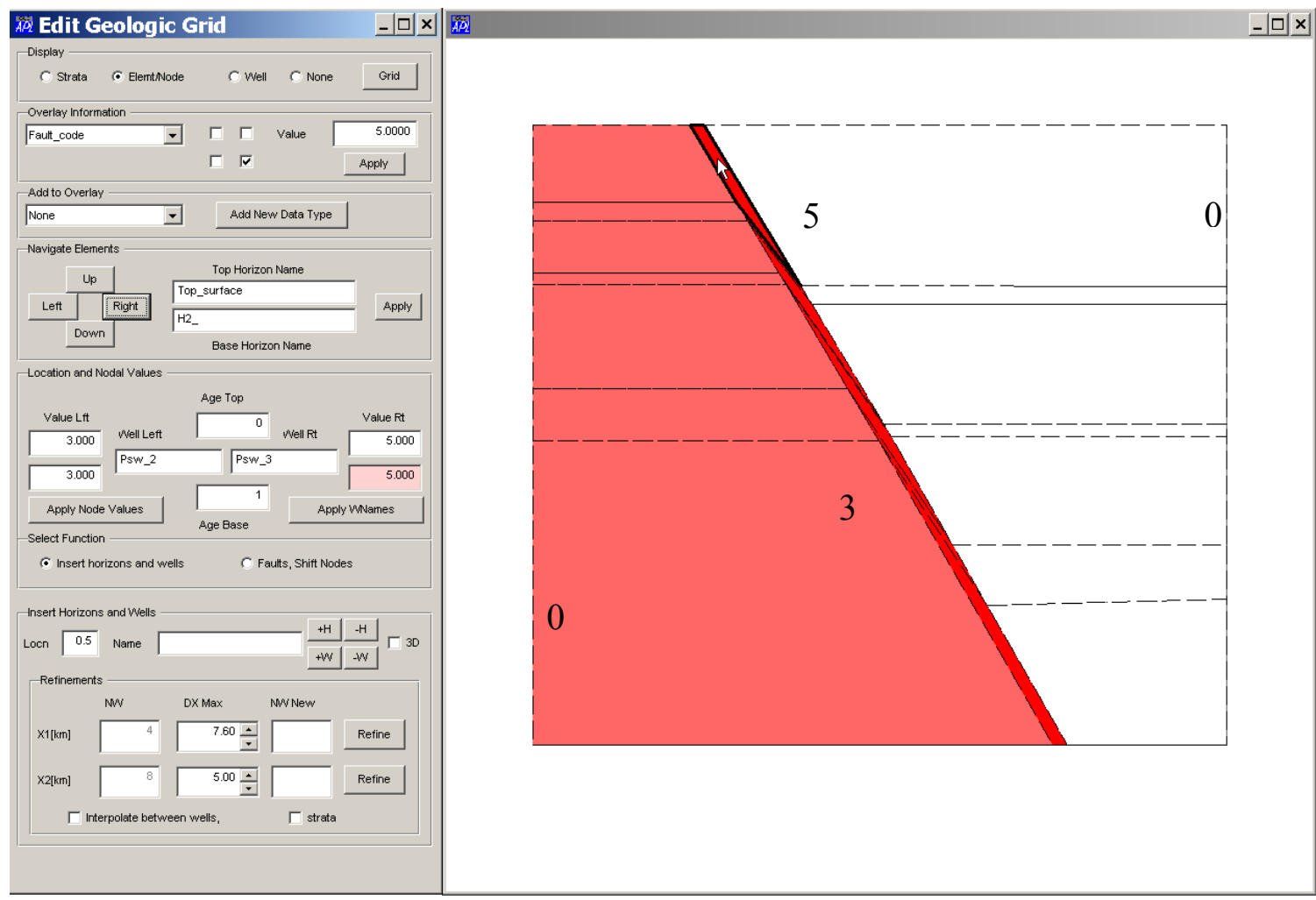

Figure 84. Fault_code for the blcases: Examples.BL: Fault_test. $2 d$ case. The overlay selects the Fault_Code. The value edit boxes in the Location and Nodal Values group indicates the values of the Fault_code at the 4 corners of the element selected (dark outline). The colored corner indicates the node that shades the elements in the section. Navigating the elements shows that the first and last pseudowell have Fault_code flags of 0 , the left side of the fault has Fault_code flags of 3, and the right side of the fault flags of 5. The flags can be set for a whole well by toggling the Display selection to Well, or for a single element if the Display is set to element as above. The Value is changed for the variable selected in the Overlay Information group by changing the value in the edit box and depressing the Apply button.

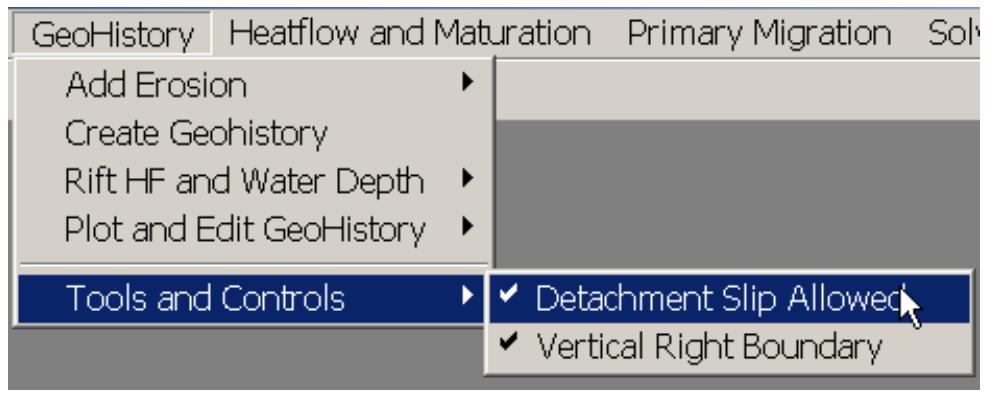

The geologic history for this case is calculated using the GeoHistory: Create GeoHistory menu command. Two control options can be selected as shown to the left. If Detachment Slip is not allowed, the wells to the right

are fixed in space at their present positions. If detachment slip is allowed their positions are not fixed. If the Vertical Right Boundary is on, the right boundary is vertical and at the position of the node with the smallest horizontal $x$ displacement. Figure 85 shows the geologic history of fault movement with and without detachment slip allowed. 


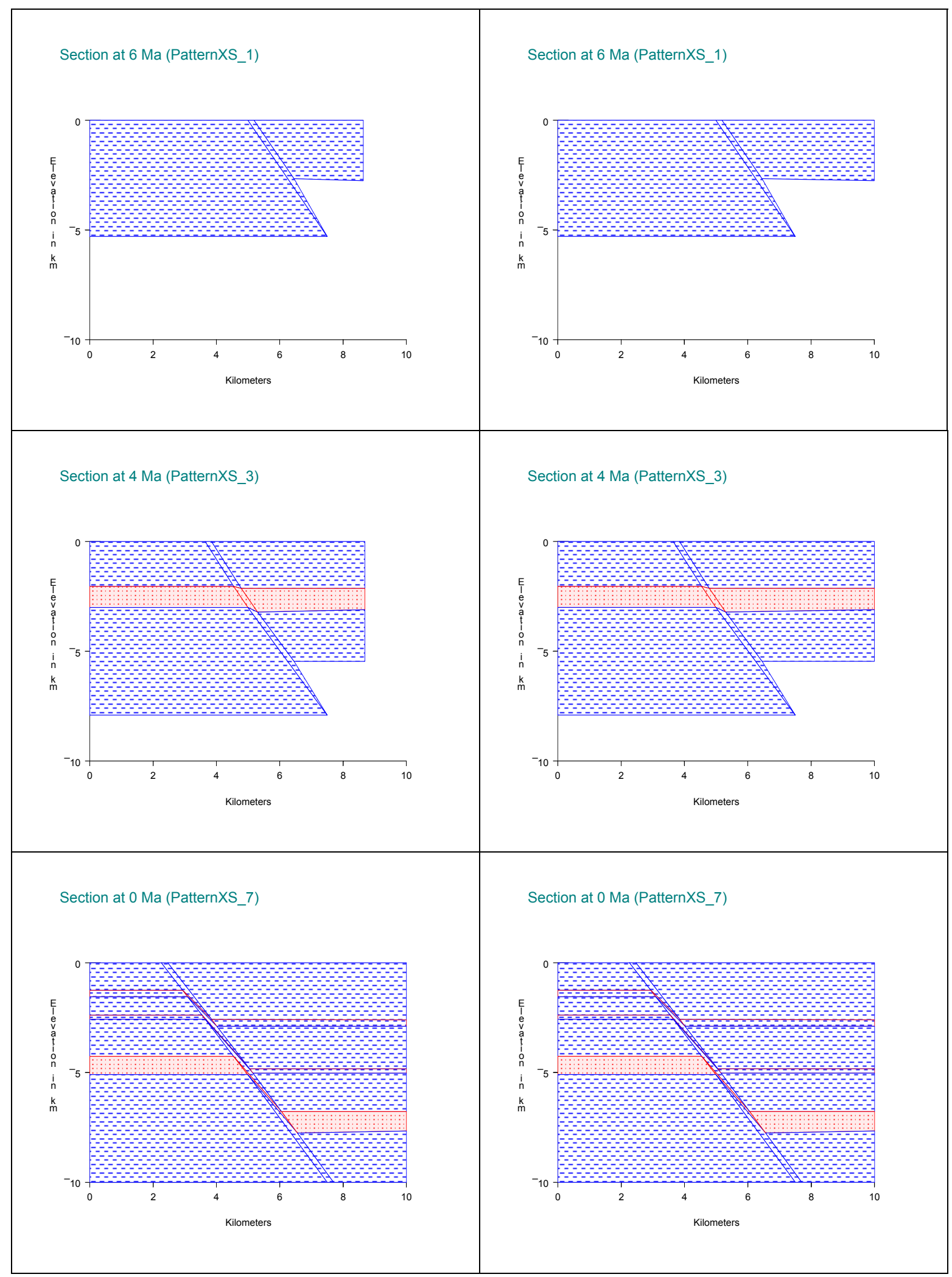


Figure 85. Geologic history of blcases: Examples.BL: Fault_test.2d case with (left) and without (right) detachment slip allowed. Right boundary is required to be vertical.

Faulting is modeled by requiring that the right side of the fault maintain a fixed distance from the left side, as sediment layers are backstripped and decompacted.

The fault controls allow modeling of quite complicated fault systems. Figure 86 shows a system of faults in offshore Nigeria (see (Cathles, Colling et al. 2003)). Figure 87 shows a complex set of fault in the Paris Basin where erosion is also included.
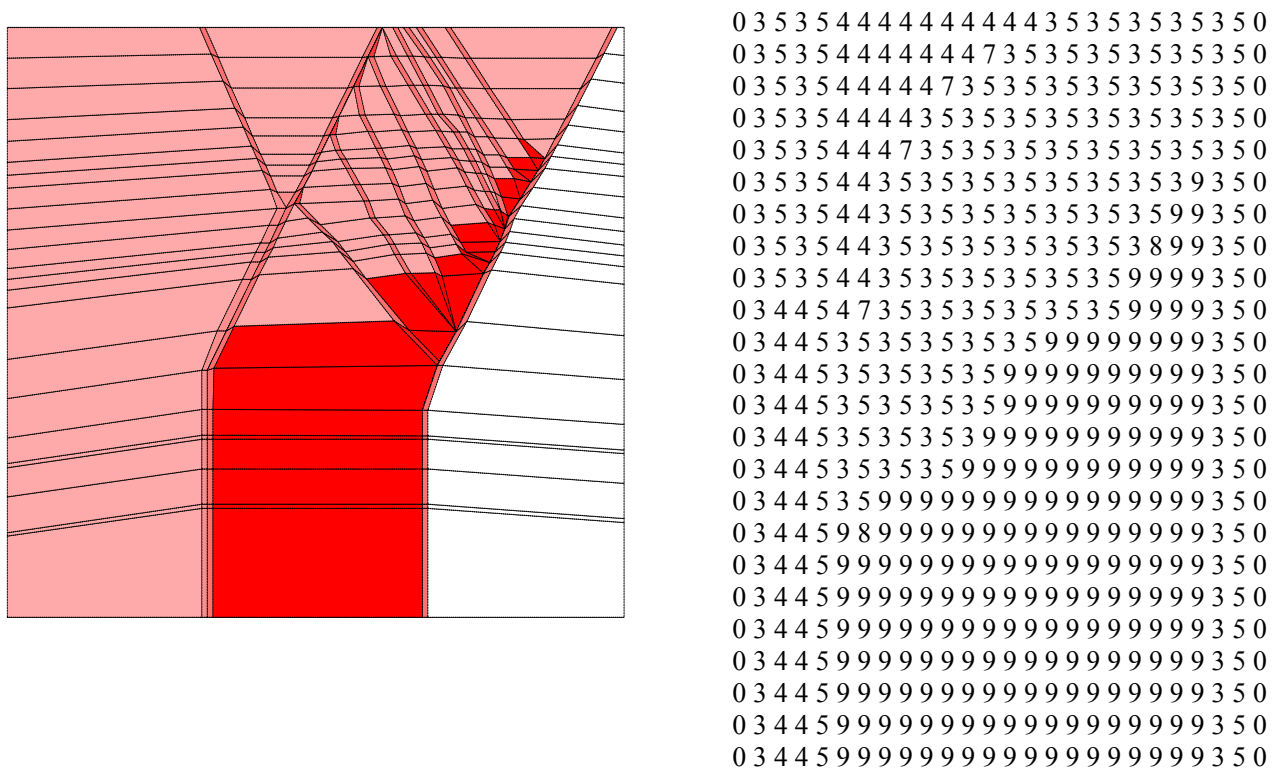

Figure 86. Section from the Niger Delta analyzed by (Cathles, Colling et al. 2003) shows how a complex set of faults can be captured with the fault codes described above. 

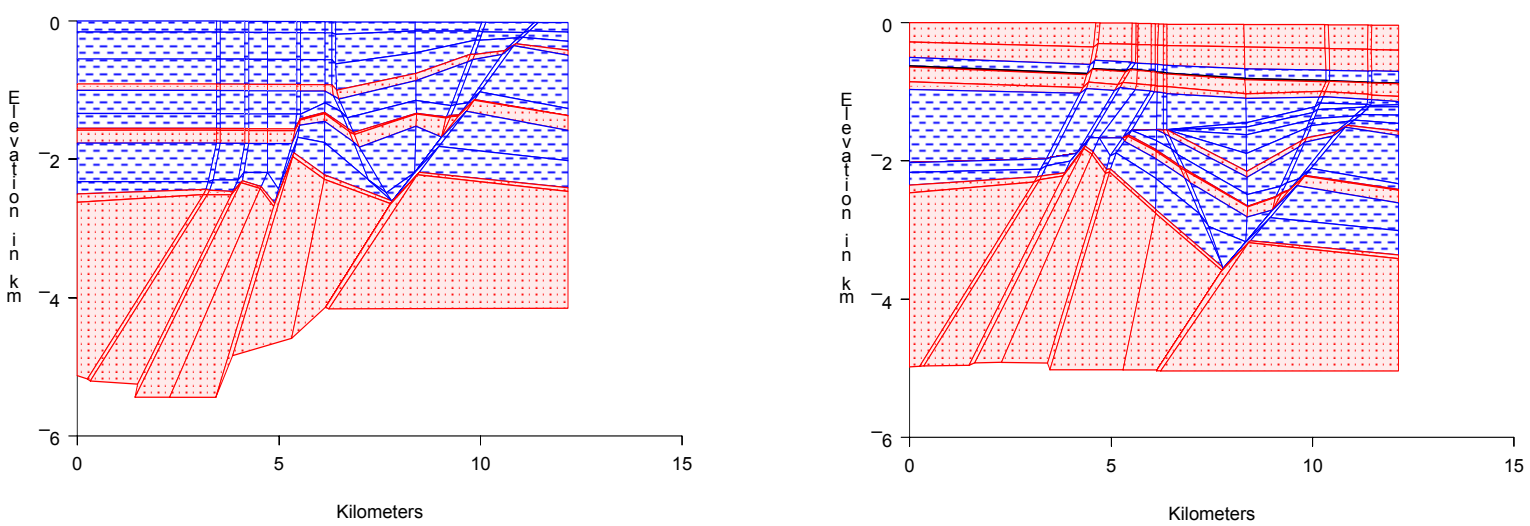

Section at $132 \mathrm{Ma}$ (PatternXS_13)

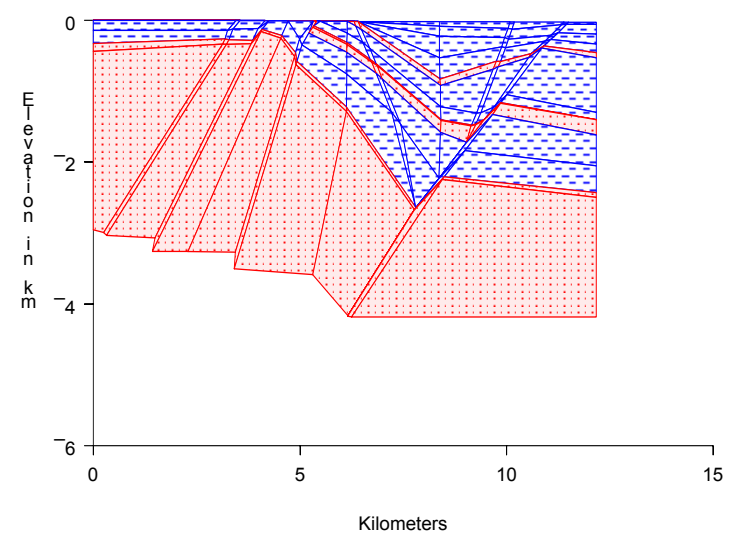

03535335335335350

03535335335335350

03535335335335350

03535335335335350

03535335335335350

03535335335335350

03535335335335350

03535335335335350

03535335335335350

03535335335335350

03535335335335350

03535335335335350

03535335335335350

03535335335335350

03535335335335350

03535335335335350

03535335335399350

03535335335999350

03535335399999350

03535335399999350

03535335399999350

Figure 87. Section in Paris basin prepared by Alain Raboute that illustrates a section with a complex fault system and erosion. The Fault_code flags for each pseudowell are shown in the $4^{\text {th }}$ panel.

\section{Salt Diapirism}

Salt movement provides accommodation space for sediment deposition. BasinLAB infers salt movements by relocating salt from areas with higher-than-average to areas less-than-average sediment deposition. The algorithm is presented, discussed, and evaluated against published interpretations in (Cornelius, Cathles et al. 2003). Salt diapirism is an option of the GeoHistory: Create Geohistory menu command. Figure 88 
shows the geologic history of a simple salt minibasin without salt diapirism. Figure 89 shows the same basin with two different options for calculating salt redistribution. Status field hints should be consulted for explanation of other options.
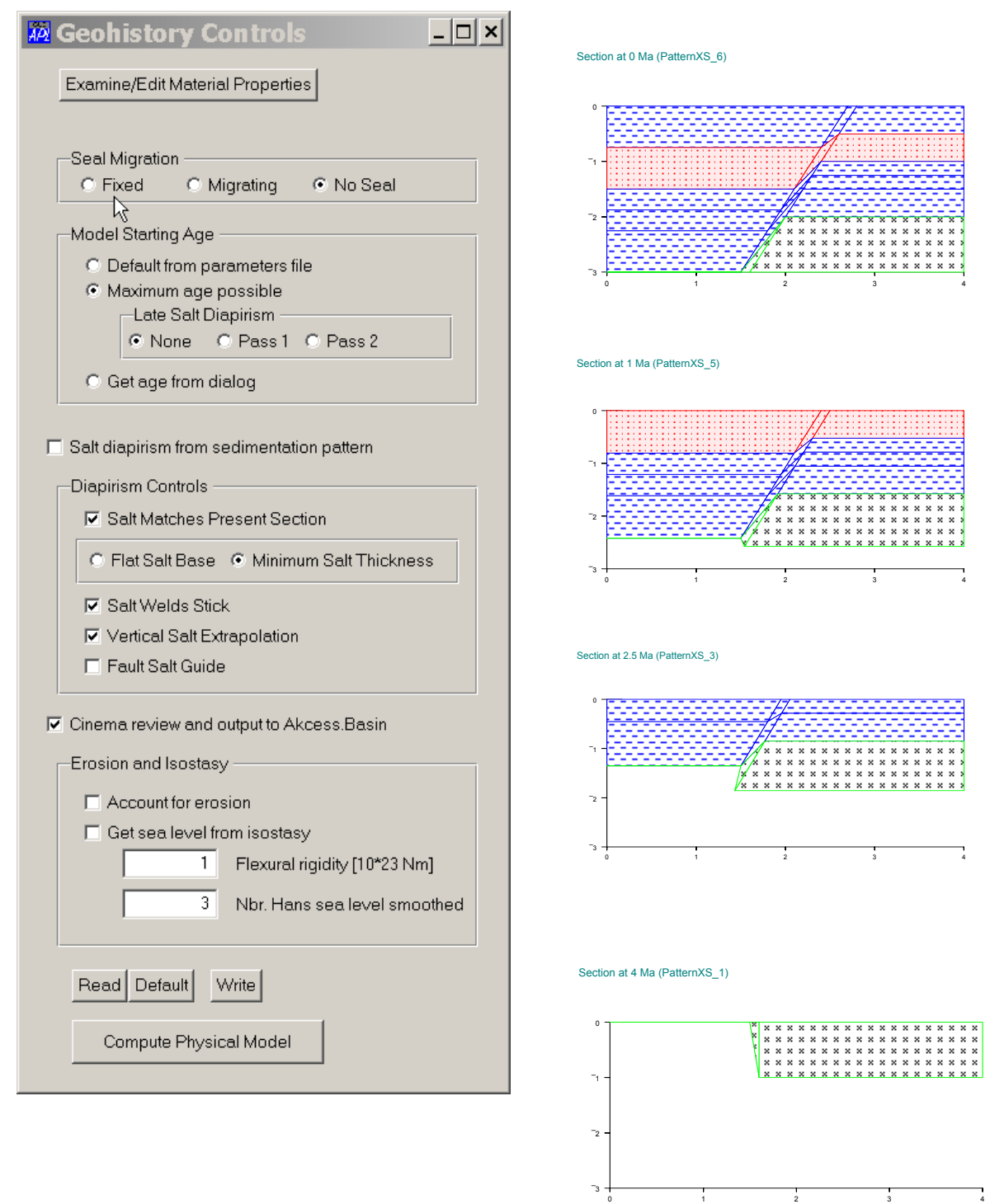

Figure 88. GeoHistory: Create Geohistory with no salt diapirism selected. Figure 89 shows the history with two options of salt diapirism. Axes labels are turned off in plot by unchecking View: Plot Controls: Show Axes Titles. Case is blcases/Examples.BL/MiniBasin.2d. 
Section at $0 \mathrm{Ma}$ (PatternXS_6)

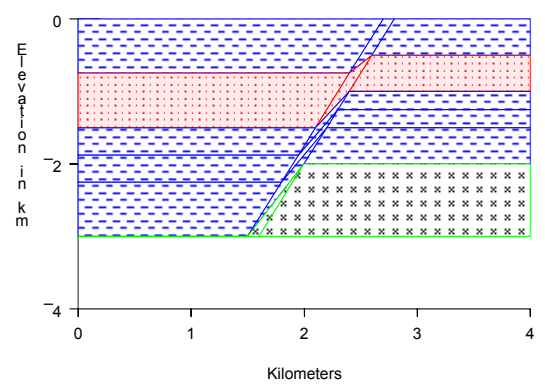

Section at 2.5 Ma (PatternXS_3)

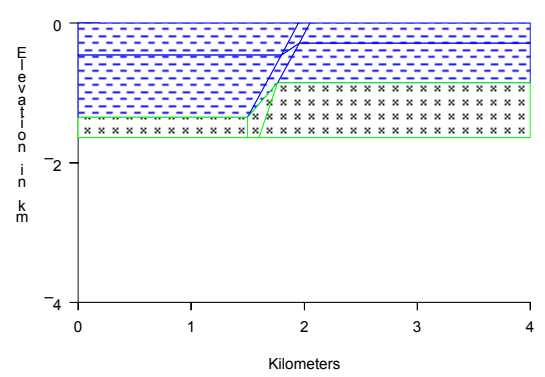

Section at $4 \mathrm{Ma}$ (PatternXS_1)

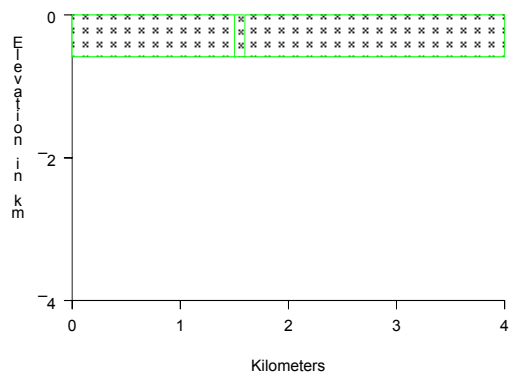

Section at 0 Ma (PatternXS_6)

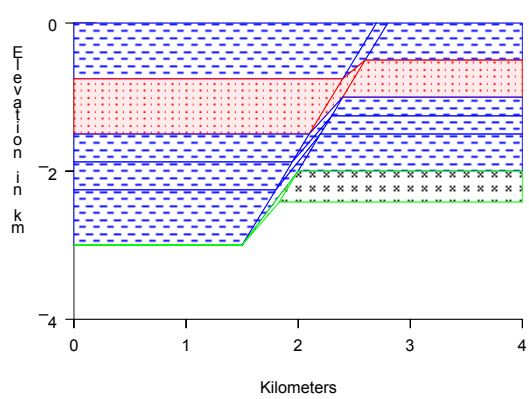

Section at 2.5 Ma (PatternXS_3)

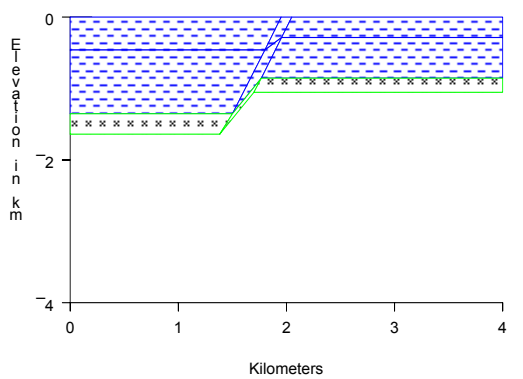

Section at $4 \mathrm{Ma}$ (PatternXS_1)

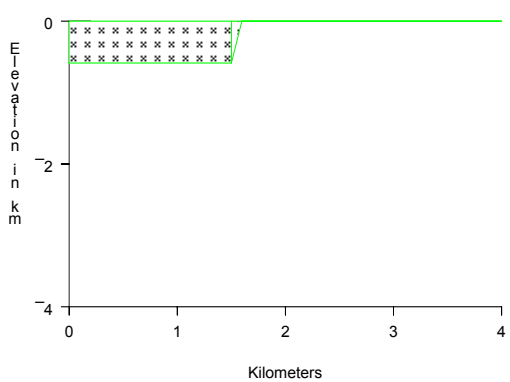

Figure 89. GeoHistory:Create Geohistory with salt diapirism selected. Left shows history with

\section{Salt Matches Present Section \\ - Flat Salt Base $C$ Minimum Salt Thickness}

Right shows history with Salt Matches Present Section unchecked, and Minimum Salt Thickness option selected.
Salt Matches Present Section, and Flat Salt Base options selected. Case is blcases/Examples.BL/MiniBasin.2d.

$$
\begin{aligned}
& \Gamma \text { Salt Matches Present Section } \\
& \qquad \text { Flat Salt Base Minimum Salt Thickness }
\end{aligned}
$$




\section{Isostasy}

For long ( $>$ about $200 \mathrm{~km}$ ) sections, isostatic depression of basement provides space for sediment accumulation. This is always calculated provided the flexural rigidity of the lithosphere has not been set to zero in the GeoHistory: Create Geohistory form (Figure 88). Water depth is assumed to be specified in the SFLAT input file (or by files and

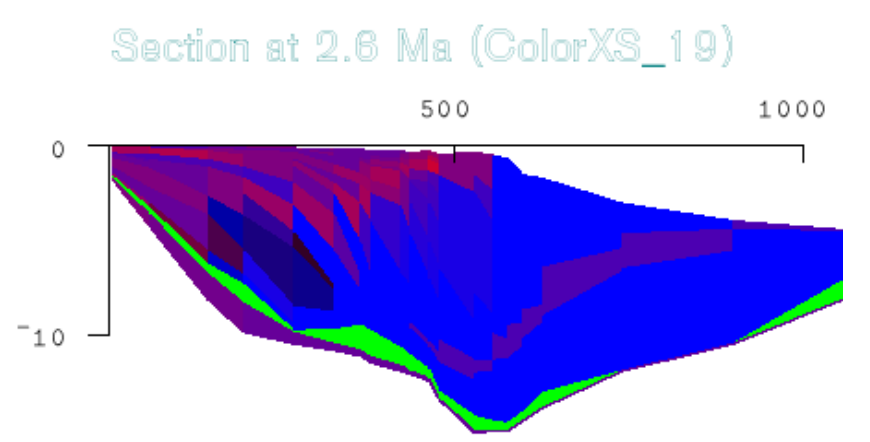

algorithms such as those discussed in the next section) unless the Get sea level from isostasy box is checked as shown in Figure 90. If this box is checked, water depth at past times is calculated from the present water depth by backstripping strata, decompacting the remaining strata, and calculating the isostatic elevation changes at the base of the basin that results from the unloading. The

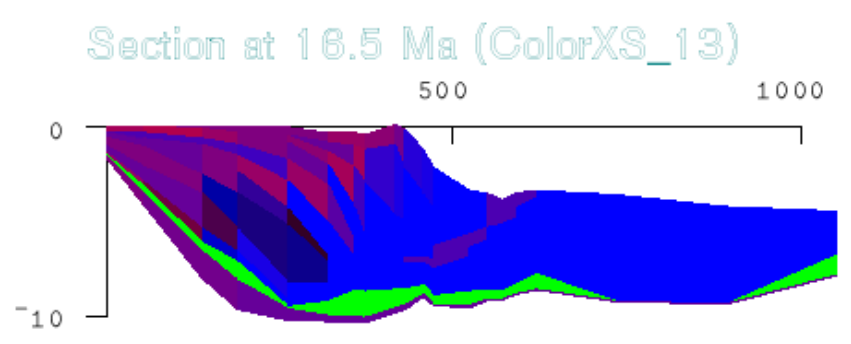
flexural rigidity of the lithosphere is taken into account. The predicted water depths are smoothed by haning the number of times indicated in the edit box labeled $\mathrm{Nbr}$. Hans sea level smoothed.

Figure 90. Water depth is predicted

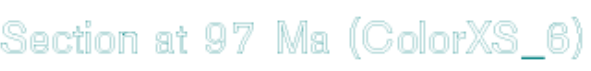

500
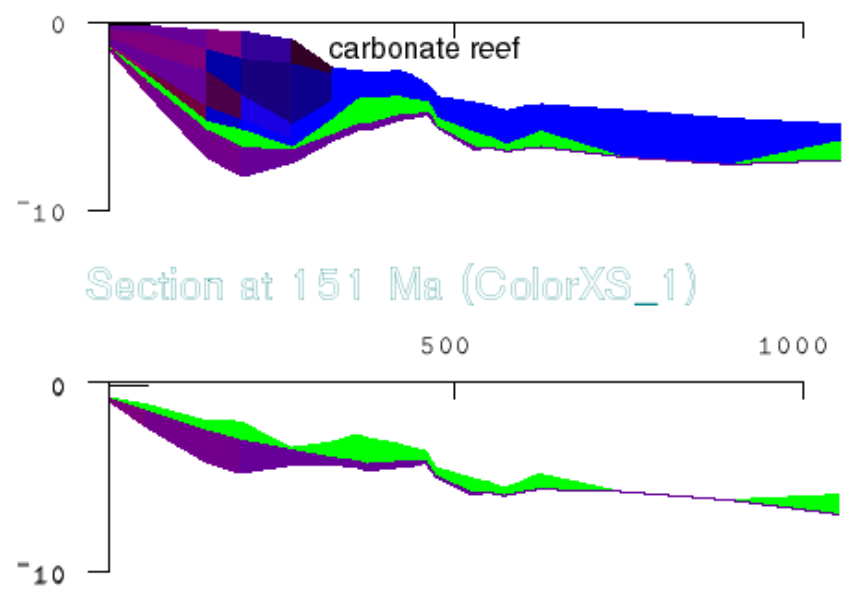

for the $1050 \mathrm{~km}$ long section from the Arkansas-Louisiana border to the Sigsbee Knoles shown to the left using the GeoHistory: Create Geohistory menu command with the Geohistory Controls in Figure 88 checked as shown. Case is blcases/Examples.BL/Louisiana_Line $.2 d$

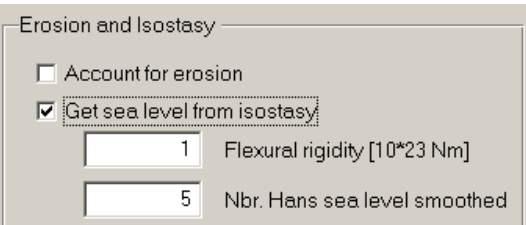

The water depths computed from isostasy for the Louisiana \#1 line are shown in Figure 90. The water depths are large when the Louanne salt was deposited at $151 \mathrm{ma}$, carbonate reefs at the shelf margin in a reasonable position at $97 \mathrm{ma}$, outer shelf is just at sea lever at 16.5 ma. As sediments replace water the basement subsides. For a flexural rigidity of 
$10^{23} \mathrm{~N}-\mathrm{m}$ the sea depths predicted as a function of time are reasonable over the entire basin history. If the flexural rigidity were significantly greater, the predicted shelf edge would stand significantly above sea level, especially around $16.5 \mathrm{Ma}$. Since we do not see extensive erosional features at this time and location in the Gulf section, the flexural rigidity must be significantly less than $10 \times 10^{23} \mathrm{~N}-\mathrm{m}$. This is illustrated in Figure 91 .

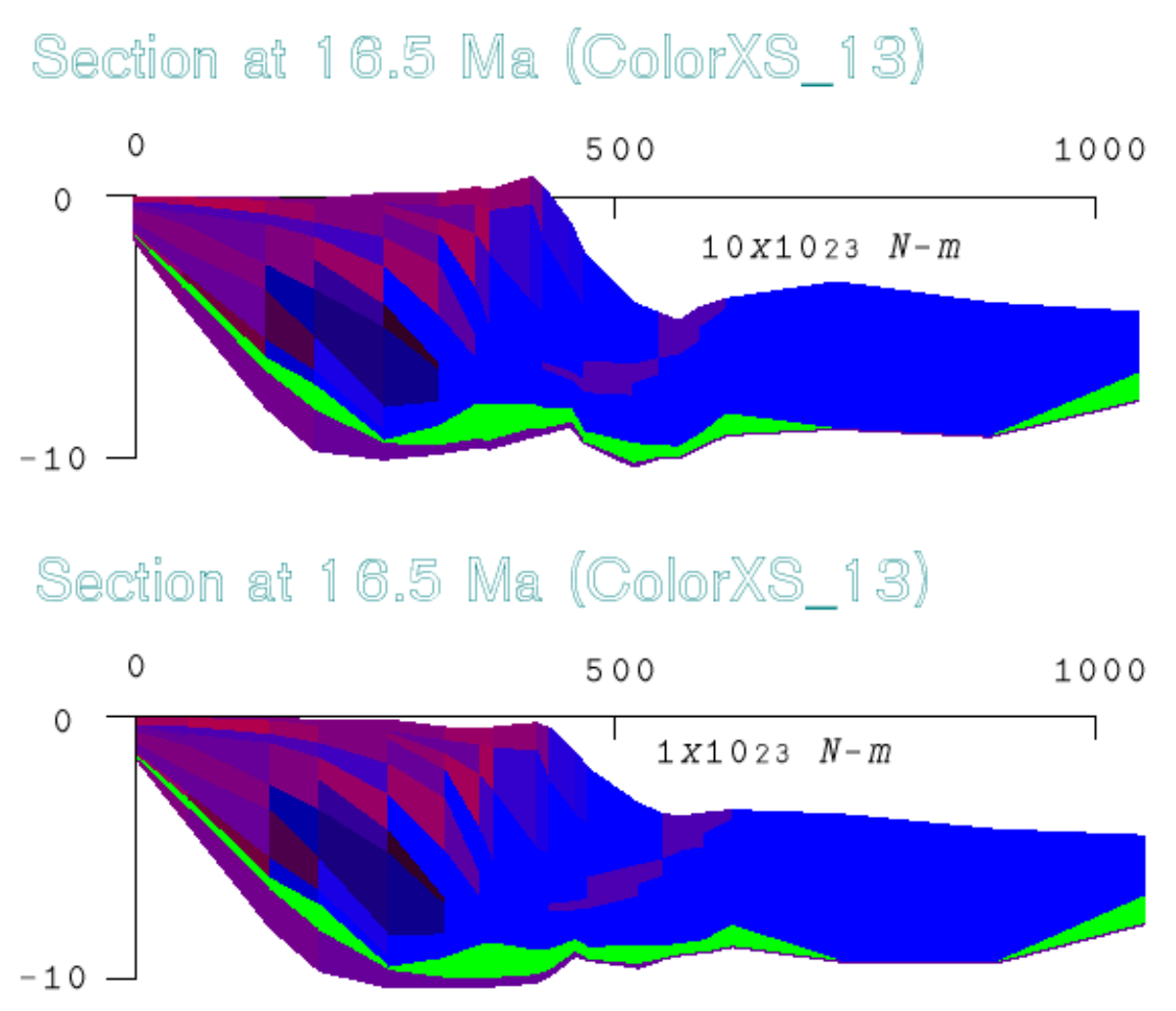

Figure 91. Comparison of water depths predicted from present sea level by backstripping, decompacting, and assuming isostatic equilibrium on a $10 \times 10^{23} \mathrm{~N}-\mathrm{m}$ lithosphere (top figure) and on a $1 \times 10^{23} \mathrm{~N}-\mathrm{m}$ (lower figure). Figures here were created Frame (2s 25 max) $25 \div$ by writing a word metafile (*.wmf) image of the

File Zoom GeoHistory: Plot and Edit: Plot menu command, selecting color as the type of plot, using the time spinner on the workspace toolbar (left) to move back in time to $16.5 \mathrm{Ma}$, and then using the file button to place the image displayed in the Metafiles folder of the blcases/Examples.BL/Louisiana_Line.2d case. The *.wmf images placed in the Metafiles folder are labeled with the type of plot, picture number, and timestamp: GeoHistColorXS_13\#19_(10_1_19h34m).WMF. This indicates the image is the $13^{\text {th }}$ color frame of the geohistory saved as session picture number 19 at $5: 34 \mathrm{pm}$ on October 1 . In this case the *.wmf images were imported to Adobe Illustrator, simplified, and modified slightly.

Note that water depth has been estimated by backstripping and decompacting the 
sediments and assuming isostasy only. We have not accounted for the thermal expansion of the lithosphere that would occur in the basin was initially formed by rifting. This is done in the next section.

\section{E. Rift Heatflow and Water Depth Corrections}

Heat flow into the base of a rift basin is determined by the radiogenicity of the sub-basin crust, the past history of sedimentation (which can cool the lithosphere), and the timing and extent of rifting. BasinLAB uses a 1D plate model to address these issues. The model first infers the thinning (stretching) of the crust and lithosphere from changes in water depth and sediment accumulation following the methods of (McKenzie 1978; Royden, Sclater et al. 1980; Watts 1981). The heat flow into the base of the accumulating sedimentary section is then calculated by thinning a model lithosphere and crust and depositing (and compacting) radiogenic sediments at a rate determined from the case section. The sub-basin crust is also radiogenic. The thickness of the total system of crust, lithosphere, and asthenosphere is $150 \mathrm{~km}$ - large enough to capture the long-term thermal transients associated with sedimentation. The methods used are similar to the finite difference model described in (Hutchison 1985). The plate model provides a good estimate of the heat flow to the base of a sedimentary basin and its thermal profile in the crust and lithosphere can be used, if this is appropriate, to correct the water depths estimated by backstripping and decompacting in the GeoHistory simulation described in the previous section. The plate model is appropriate only for rift basins. Other methods are provided for the input basal heatflow for rift and other types of basin (see parameters section below).

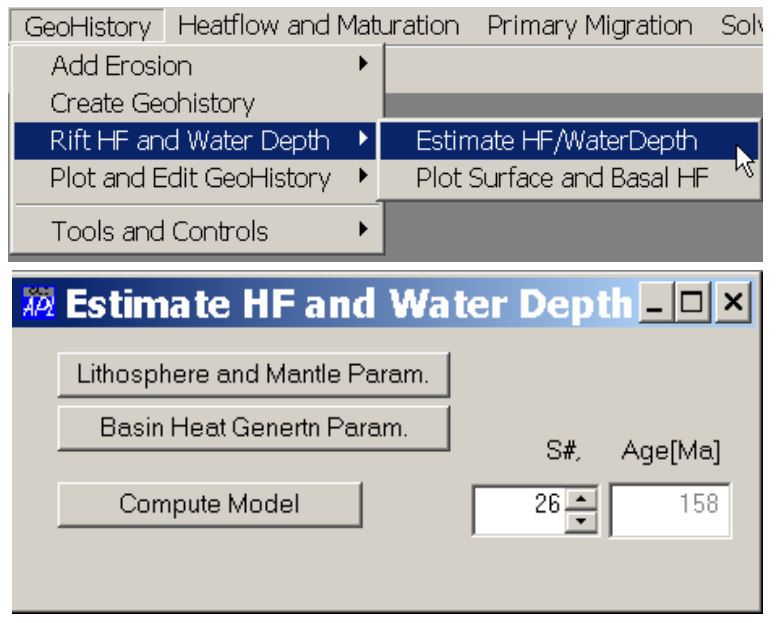

The plate model is executed using the GeoHistory: Rift HF and Water Depth): Estimate HF/Water Depth menu. This brings up the form on the lower left. Its spinner is used to select the age of rifting.

- The rift extension is calculated from the changes in water depth and sediment accumulation from the time of rifting to the present day (the full historical development of the basin).

- The plate parameters used in the calculation are presented for inspection and modification by depressing the Lithosphere and Mantle Param button. This form has useful scratchpad capabilities that illustrate the implications of the parameters for the model (see Figure 95).

- The sediment heat generation parameters are presented if the Basin Heat Genertn Param button is depressed.

- A case is processed by depressing the Compute Model button.

- The option to adjust water depth for the thermal expansion of the lithosphere is presented when the plate model has finished execution. 
- The surface and basal heatflow across the section can be plotted using the GeoHistory: Rift HF and Water Depth: Plot Surface and Basal HF menu.

- The heat flow evolution determined by the plate model can be viewed using the Geology: Edit/Plot Spreadsheet: Edit Highlighted Column button.

Figure 92 shows the sea levels in Figure 90 corrected for thermal expansion in this fashion. The basal and surface heat flow across the section is shown in Figure 93, and the heat flow history is shown in Figure 94. The heat flow is markedly depressed over the central parts of the section but is in good agreement with measured heat flows. Radiogenic decay in the basin sediments makes a substantial contribution to the total surface heatflow. The case is discussed in some detail in (Cathles and Losh 2002). A brief elaboration of the modeling methods and the parameters scratchpad is given at the end of this section.

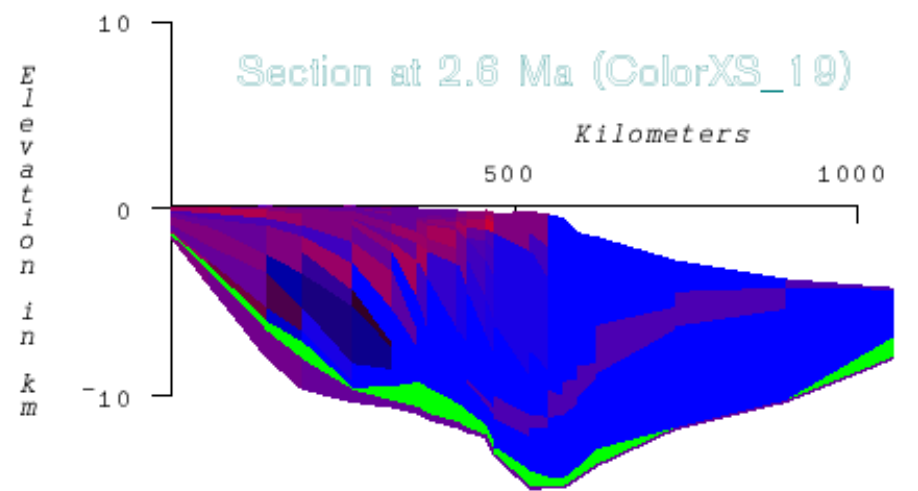

Figure 92. Sea level history shown in Figure 90 corrected for thermal expansion using the GeoHistory: Rift HF and Water Depth): Estimate HF/Water Depth plate model. Notice the water

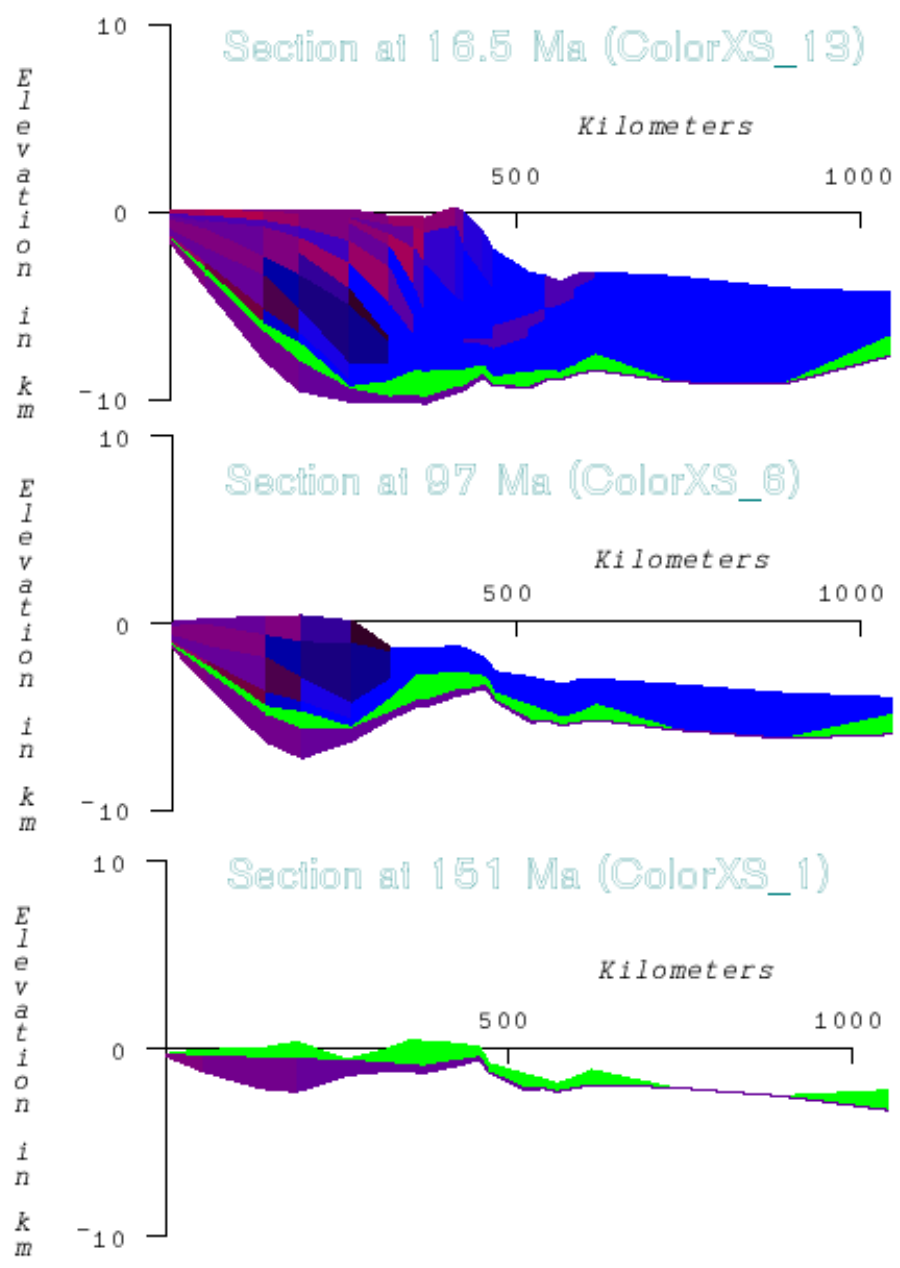
depths after rifting are greatly reduced in the early stages of basin evolution. The case folder is:

blcases/Examples.BL/Louisiana_L ine. $2 d$ 


\section{Heấ Flow from Basement [b] and Surface [I] (MFU)}

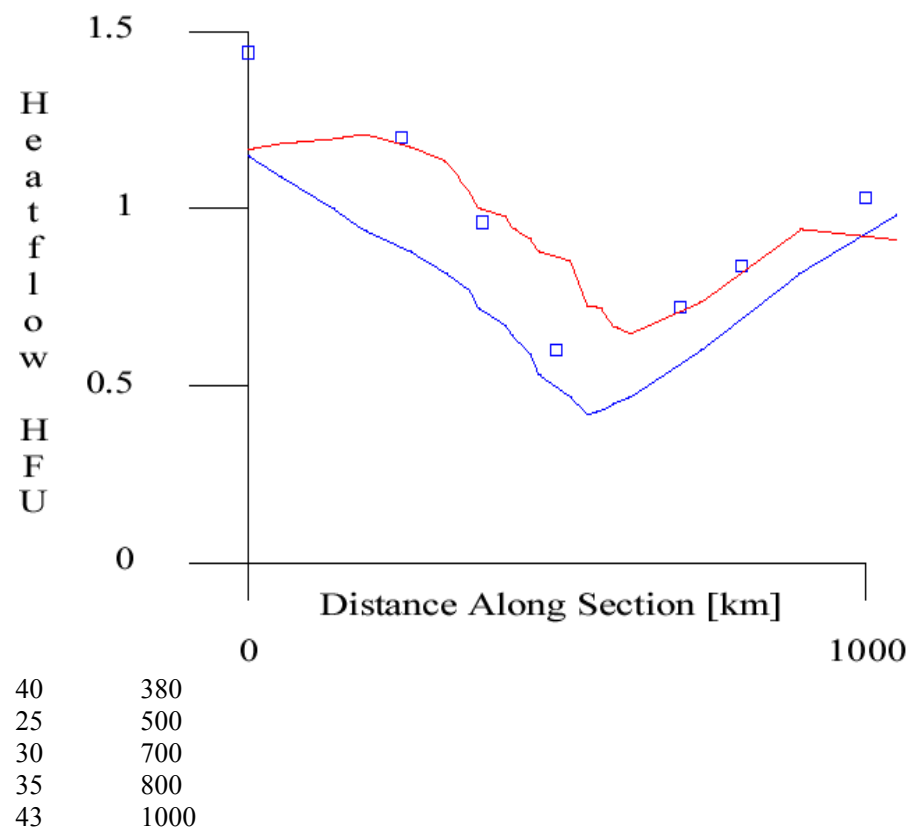

Figure 93. Basal and surface heat flow profiles across the section. Plot using GeoHistory: Rift HF and Water Depth):Plot Basal and Surface Heatflow. Heatflow measurements along the section are automatically plotted if a heatflowdata.txt file is in the case folder. Its format is:

.024 \#conversion to hfu, and $\mathrm{km}$ along section\# $60 \quad 0 \quad \#[\mathrm{hf}, \mathrm{x} 1]$ array giving hf along section\# $50 \quad 250$

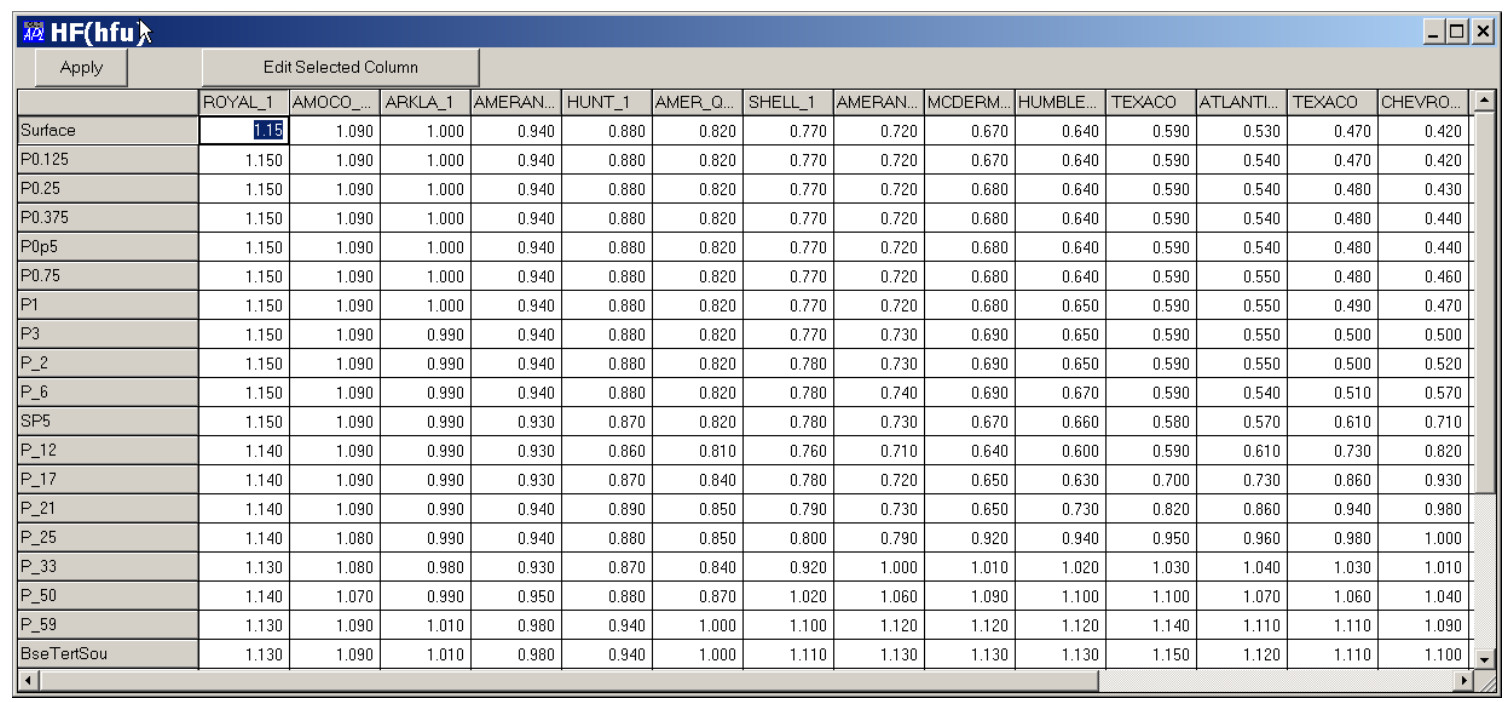

Figure 94. Heat flow history inferred by the plate model in the blcases/Examples.BL/Louisiana_Line.2d. The heat flow history is examined using Geology: Edit/Plot Spreadsheet, selecting the HF(hfu) column and depressing the Edit Highlighted Column button. 
The factor by which the crust and lithosphere have been extended in pure shear by rifting, the so-called beta factor, $\beta$, is inferred from changes in water depth, $\Delta \mathrm{W}_{\mathrm{D}}\left(\mathrm{t}_{\mathrm{r}}\right)$ and the sediment accumulation $\Delta S_{S}\left(t_{r}\right)$ that have occurred since the time of rifting, $t_{r}$ :

$$
1-\frac{1}{\beta}=\frac{\frac{\Delta S_{S}\left(t_{r}\right)}{m}+\Delta W_{D}\left(t_{r}\right)}{S_{I W}+S_{T W \infty}\left(1-\exp \left(-t_{r} / \tau\right)\right)}
$$

The parameters in equation (1) are defined in Table 10, and can be changed in BasinLAB using the Lithosphere and Mantle Param button on the GeoHistory: Rift HF and Water Depth): Estimate HF/Water Depth form shown in Figure 95. This form is also a scratchpad, which can be used to estimate the initial and ultimate subsidence of the rifted basin floor if the rift is filled with water only or with sediments.

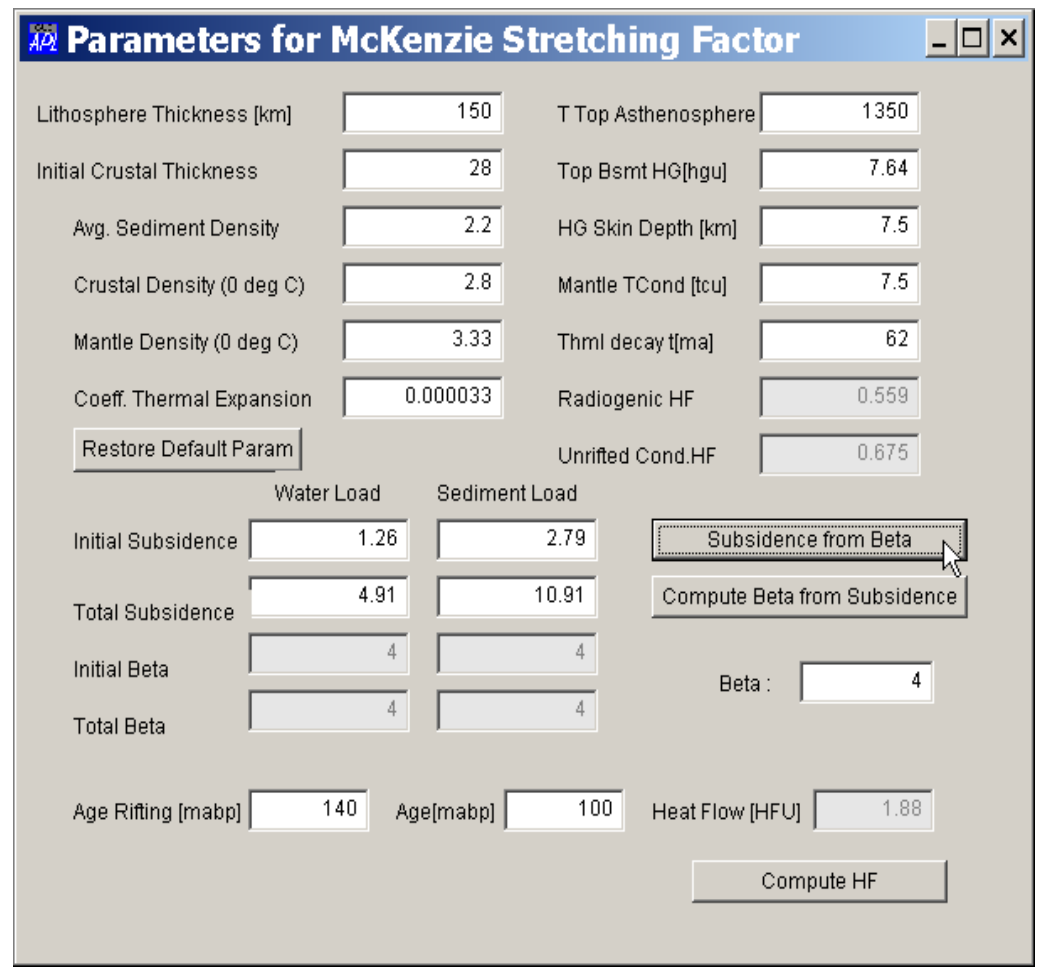

Figure 95. The form and scratchpad summoned by depressing the Lithosphere and Mantle Param button on the GeoHistory: Rift HF and Water Depth):

Estimate HF/Water Depth form. A stretching factor entered in the Beta edit box (in this case 4) can be used to calculate the initial subsidence of a water- or sediment-filled basin by depressing the Subsidence from Beta button as indicated. Alternatively the stretching factor can be estimated from the initial or ultimate (total) subsidence of the basin by entering values in the appropriately-labeled boxes and pressing the Compute Beta from Subsidence button. Different parameter values can be entered in the top portion of the form and they will be used in subsequent calculations.

The plate model parameters are defined in Table 11. 
Table 11. Definition of parameters in equation given above for the plate model. The parameter values listed are the Basin $\boldsymbol{L} \boldsymbol{A} \boldsymbol{B}$ default parameters and are from (Royden, Sclater et al. 1980; Sass, Brott et al. 1981; Watts 1981; Hutchison 1985). The parameters are used in the form shown in Figure 95.

\begin{tabular}{|c|c|c|}
\hline Variable & Definition & Value or Expression \\
\hline$\beta$ & $\begin{array}{l}\text { Crustal thinning: ratio of the pre-rifting thickness } \\
\text { of crust or lithosphere to the post-rifting thickness }\end{array}$ & Calculate from equation \\
\hline$\Delta S_{S}\left(t_{r}\right)$ & $\begin{array}{l}\text { Change in thickness of sedimentary section since } \\
\text { rifting at location where } \beta \text { is calculated }\end{array}$ & Observed value \\
\hline$\Delta W_{D}\left(t_{r}\right)$ & $\begin{array}{l}\text { Change in water depth since rifting at location } \\
\text { where } \beta \text { is calculated }\end{array}$ & Observed value \\
\hline $\mathbf{a}$ & Pre-rifting thickness of the crust plus lithosphere & $150 \mathrm{~km}$ \\
\hline $\mathbf{h}_{\mathbf{c}}$ & Pre-rifting thickness of the crust & $28 \mathrm{~km}$ \\
\hline$\rho_{\mathbf{w}}$ & Density of water & $1.03 \mathrm{~g} / \mathrm{cc}$ \\
\hline$\rho_{\mathbf{m o}}$ & Density of the mantle at $0^{\circ} \mathrm{C}$ & 3.33 \\
\hline$\rho_{\mathbf{c o}}$ & Density of the crust at $0^{\circ} \mathrm{C}$ & 2.8 \\
\hline$\alpha$ & Coefficient of thermal expansion & $3.3 \times 10^{-5}$ \\
\hline $\mathbf{T}_{\text {asth }}$ & Temperature at the top of the asthenosphere & $1350^{\circ} \mathrm{C}$ \\
\hline$\rho_{\mathbf{m}}$ & Density of the top of the asthenosphere & $\rho_{\mathrm{mo}}\left(1-\alpha \mathrm{T}_{1}\right)$ \\
\hline $\bar{\rho}_{c}$ & Average density of crust & $\rho_{c o}\left(1-\frac{\alpha T_{a s t h} h_{c}}{2 a}\right)$ \\
\hline $\bar{\rho}_{l}$ & Average density of lithosphere & $\rho_{c o}\left(1-\frac{\alpha T_{a s t h}}{2}\left(1+\frac{h_{c}}{a}\right)\right)$ \\
\hline $\mathbf{S}_{\text {IW }}$ & Initial water-loaded subsidence divided by & $a\left(\rho_{m}-\bar{\rho}_{l}\right)+h_{c}\left(\bar{\rho}_{l}-\bar{\rho}_{c}\right)$ \\
\hline$S_{T W \infty}$ & $\begin{array}{l}1-1 / \beta \\
\text { Ultimate thermal subsidence divided by } 1-1 / \beta\end{array}$ & $\begin{aligned} \quad \rho_{m}-\rho_{w} \\
\sim \frac{a \rho_{m} \alpha T_{a s t h}}{2\left(\rho_{m}-\rho_{w}\right)}\end{aligned}$ \\
\hline$\tau$ & Thermal decay time of the lithosphere & $62 \mathrm{Ma}$ \\
\hline $\mathbf{A}_{\text {crust }}$ & Heat generation at top of crust & $7.64 \times 10^{-13} \mathrm{cal} / \mathrm{cm}^{3}-\mathrm{sec}$ \\
\hline$\delta_{\text {crust }}$ & Skin Depth of radiogenicity in crust & $7.5 \mathrm{~km}$ \\
\hline $\mathbf{K}_{\text {lith }}$ & Thermal conductivity of crust and lithosphere & $7.5 \times 10^{-3} \mathrm{cal} / \mathrm{cm}-\mathrm{sec}-\mathrm{C}$ \\
\hline $\bar{\rho}_{l} \mathbf{c}$ & Heat capacity of crust and lithosphere & $0.89 \mathrm{cal} / \mathrm{cm}^{3}$ \\
\hline
\end{tabular}

The plate-cooling model is composed of three layers: the basin, the crust, and the lithosphere/asthenosphere below the crust. The asthenosphere is assumed to have constant temperature, $\mathrm{T}_{\text {asth }}$, of $1350^{\circ} \mathrm{C}$ (see Table 11). The lithosphere (solidified asthenosphere) is assumed to have no significant radioactive heat generation, but heat generation is included in the crust. 
The radiogenic heat generation at the top of the crust before rifting is $\mathrm{A}_{\text {crust. }}$. Radiogenic heat generation decays with depth in the crust such that $A(z)=A_{\text {crust }} \exp \left(-z / \delta_{\text {crust }}\right)$, where $\mathrm{z}$ is the depth below the top of the crust (positive downward), and $\mathrm{A}_{\text {crust }}$ is as defined in Table 1.

When rifting takes place the crust and lithosphere are reduced in thickness by a factor of $\beta$ (e.g., division by $\beta$ ). The exponential decrease of radiogenicity with depth in the crust is automatically adjusted so that the total heat generation in the thinned crust is reduced by a factor of $\beta$. The heat generation rate at the top of the crust is kept unchanged. The crust and mantle are treated as a common unit and have the same thermal properties except that the crust includes radiogenic heat production. Initial steady state temperature profiles are calculated in the rifted crust and lithosphere. Sediments are then added to the crust, and the warming of these sediments and cooling of the crust and lithosphere is calculated using finite element techniques. Compaction and heat production are accounted for in the basin sediments. The numerical methods are very similar to those used by(Hutchison 1985), except that we use a coordinate system attached to the solid matrix and finite element rather than finite difference methods of solution are used.

The cooling calculations are one dimensional. The finite element node spacing increases with depth below the top of the crust such that the spacing between each successive node increases by a factor of 1.5. The total number of nodes spanning the crust and mantle is 20. The lithosphere nodes span the full $150 \mathrm{~km}$ pre-rift thickness of the lithosphere. Changes in the thermal conductivity of the basin sediments that occur as they compact and are warmed are taken into account in the model, as is radiogenic heat generation in the basin sediments. Compaction is calculated assuming hydrostatic pore pressures. Standard finite element techniques (Baker and Pepper 1991) are used in the calculations. Two iterations are taken at each timestep to converge the temperature-dependent thermal conductivity in the basin sediments. The calculations have been verified against the conductive cooling of and initially isothermal halfspace (Carslaw and Jaeger 1959), and against the prior models of (Hutchison 1985). We also have verified that increasing the number of nodes and reducing the size of the timesteps taken do not change the computational results.

As indicated in Table 11 we assume in calculating the Louisiana section that the prerifting lithosphere thickness, a, in the Gulf of Mexico area was $150 \mathrm{~km}$ (Pollack and Chapman 1977). We take the pre-rift continental crust, $h_{c}$, to be $28 \mathrm{~km}$ thick. From (Pollack and Chapman 1977; Sass, Brott et al. 1981) we take the surface heat generation of the pre-rift crust to be $A_{\text {crust }}=3.2 \mathrm{~mW} / \mathrm{m} 3$ and the depth characterizing the exponential decay of the surface heat generation to be $\delta_{\text {crust }}=7.5 \mathrm{~km}$.

\section{F. Water Depth}

\section{Specified in SFLAT}

In most cases water depth will be specified directly in the SFLAT input file. It can also be set by assuming isostasy as in the previous section or by specifying the shoreline, shelf width and dip, and slope width and dip as discussed in the next section. This section illustrates how water depth is set in the SFLAT file of BasinLAB. 
Consider again the blcases/Examples.BL/MiniBasin.2d case shown in Figure 88. Water depth in that case was zero. There is no water depth column in the SFLAT file. However a water depth column can be added using the Geology: Graphic Edit: Edit Geologic Grid form as shown in Figure 96.

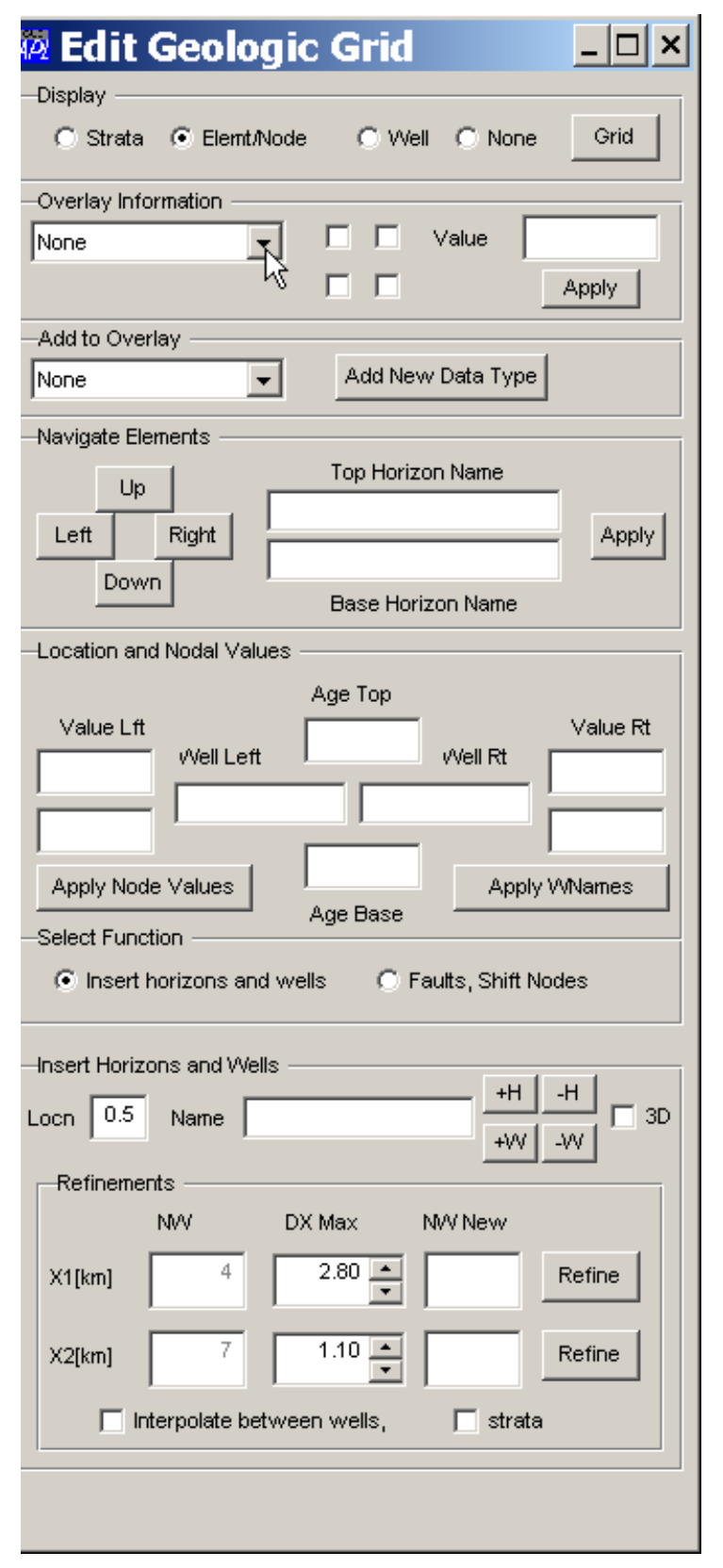

Figure 96. Geology: Graphic Edit menu command for the blcases/Examples.BL/Minibasin.2d case.

None
None
X2(depth)
X1
Fault_code
Fx_Sh
Age(Ma)
FX_Salt
X3
Fx_Sd
HF(htu)
Ker1(wtpct)
W_Nar
H_Nbr

The Overlay Information combo box shown to the left does not contain water depth as an entry. Because of this water depth was assumed to be zero in processing the geologic history of the case shown in Figure 88 and Figure 89.

The Add to Overlay combo box contains the SFLAT column entries that can be added. Water depth is on this list.

Selecting Wat_Depth in the Add to Overlay combo and pressing the Add New Data Type button adds water depth to the SFLAT file. The Wat_Depth entry is deleted from the Add to Overlay combo box and added to the Overlay Information combo box.

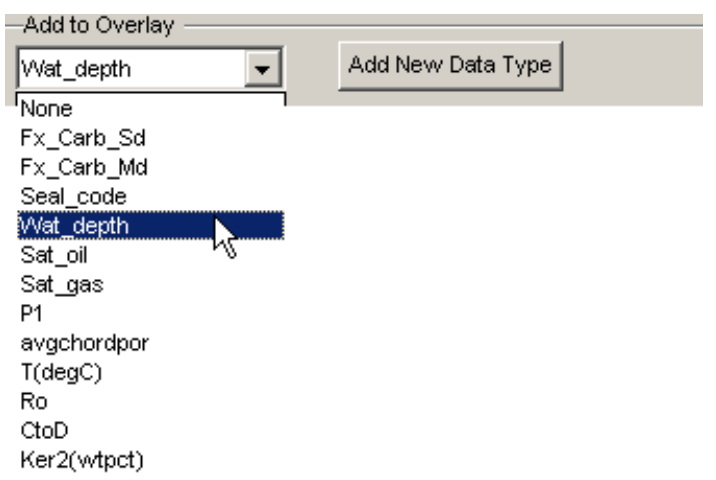

The default water depths in the SFLAT file are zero when Wat_Depth is added. Editing water depths in the blcases/Examples.BL/Minibasin.2d as shown in Figure 97 results in the geologic history shown in Figure 99. This figure should be compared to the left side of Figure 89. 


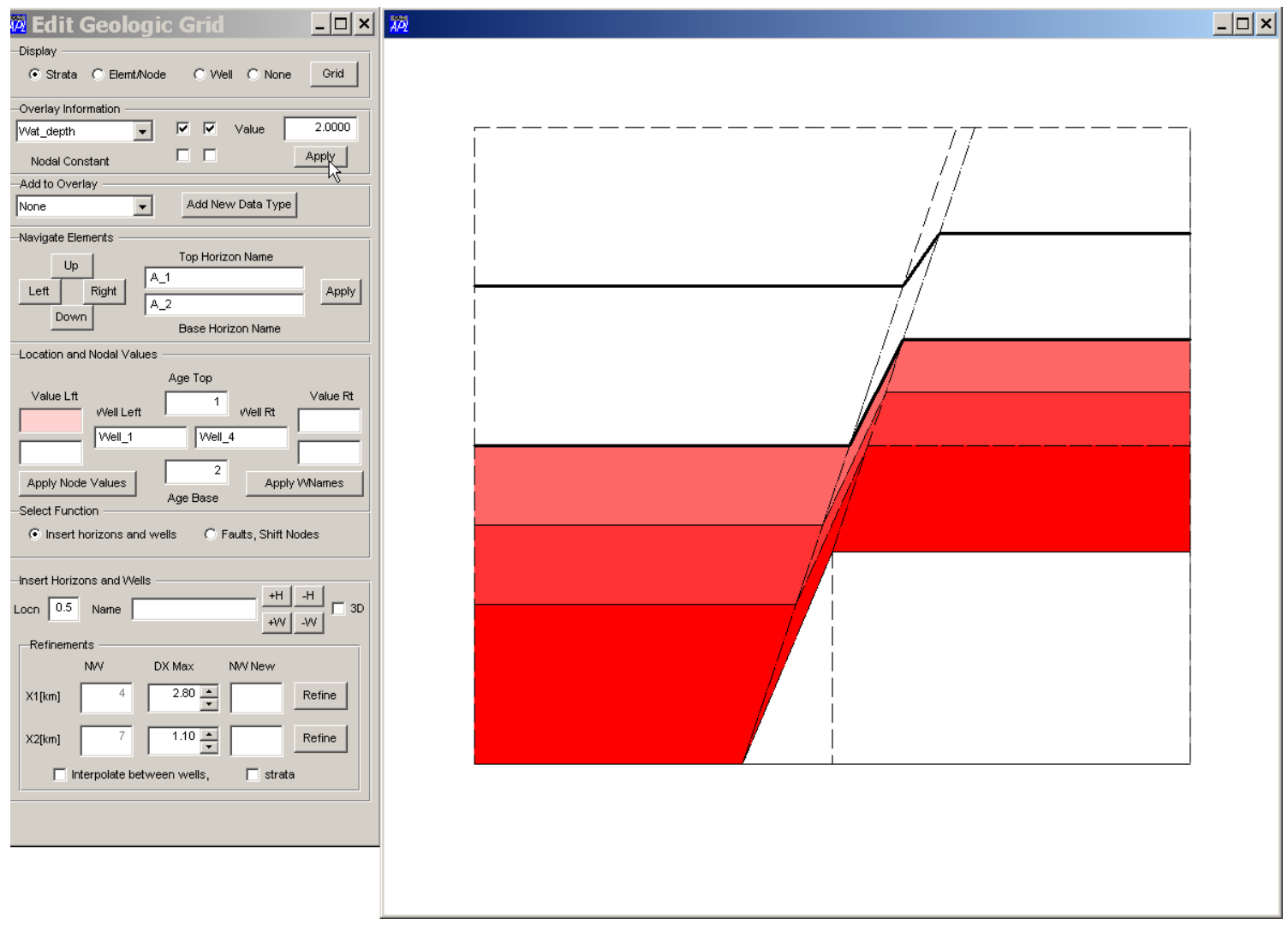

Figure 97. Geology: Graphic Edit form. Uniform water depth is added to each stratum by selecting that strata Display radio button, the Wat_depth overlay, and navigating to the desired strata. The value in the Overlay Information group is then changed from 0 to 2 (as shown) and the Apply button depressed. When this is done the color indicating water depth immediately changes to a shade of red. Water depth is carried for each node by the upper node of a highlighted element, as indicated by the red shading in the value box of the Location and Nodal Values group, and by the check marks in the Overlay Information group (two in this case because the entire strata is selected). Selecting Elements in the Display group and navigating reveals the nodal values that have been applied to each element and allows different sea levels to be assigned to the nodes of a stratum. This can be done in the Location and Nodal Values group. Here we have assigned a water depth of 5 to the top of the deepest (non-salt) strata, a value of 4 to the next strata up, etc. The water depth at of the top strata (the present water depth) is $1 \mathrm{~km}$. Figure 98 shows the water depths assigned, and Figure 99 the Geohistory that results when the case is processed with these assigned water depths.

\begin{tabular}{|c|c|c|c|c|}
\hline \multicolumn{5}{|c|}{ Ä Wat_depth } \\
\hline \multirow[t]{2}{*}{ Apply } & \multicolumn{4}{|c|}{ Edit Selected Column } \\
\hline & Well_1 & Well_2 & Well_3 & Well_4 \\
\hline Surface & 1 & 1.000 & 1.000 & 1.000 \\
\hline A__1 & 2.000 & 2.000 & 2.000 & 2.000 \\
\hline A_2 & 3.000 & 3.000 & 3.000 & 3.000 \\
\hline A2.5 & 4.000 & 4.000 & 4.000 & 4.000 \\
\hline A_ 3 & 5.000 & 5.000 & 5.000 & 5.000 \\
\hline A_4 & 0.000 & 0.000 & 0.000 & 0.000 \\
\hline A_5 & 0.000 & 0.000 & 0.000 & 0.000 \\
\hline
\end{tabular}

Figure 98. The table of water depths created by graphic interaction with the SFLAT file described in Figure 97. This form is obtained by Geology: Edit/Plot Spreadsheet: selecting the water depth column: depressing the Edit 
Highlighted Column button. The water depths could, as an alternative to the graphics methods of Figure 97, be modified using this spreadsheet.

Section at 0 Ma (PatternXS_6)

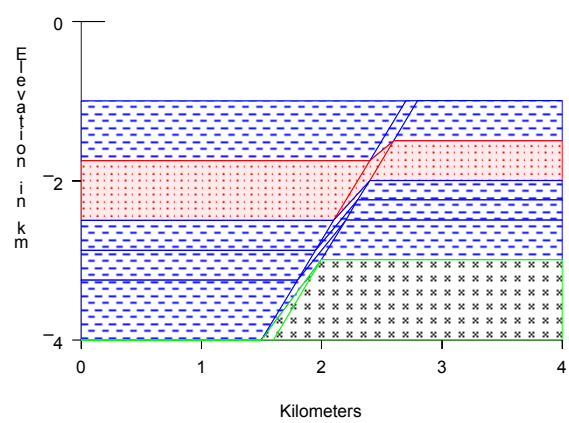

Section at $2 \mathrm{Ma}$ (PatternXS_4)

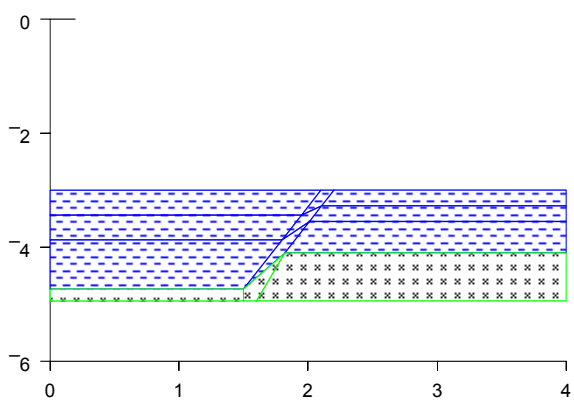

Section at $3 \mathrm{Ma}$ (PatternXS_2)

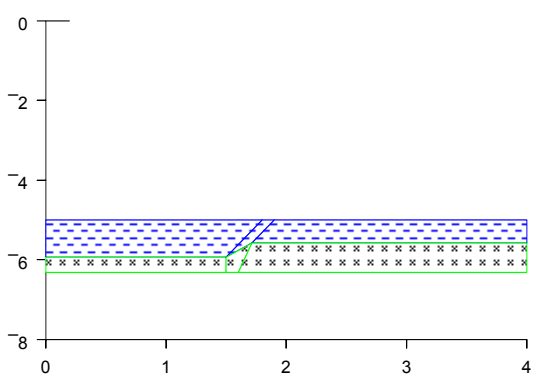

Section at $1 \mathrm{Ma}$ (PatternXS_5)

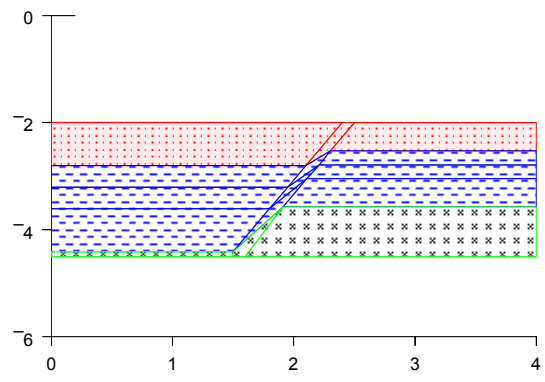

Section at 2.5 Ma (PatternXS_3)

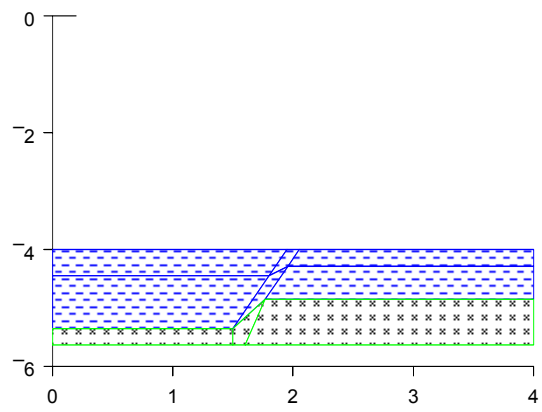

Section at $4 \mathrm{Ma}$ (PatternXS_1)

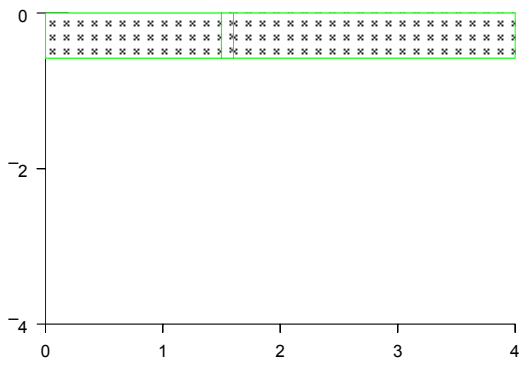

Figure 99. The geologic history of the blcases/Examples.BL/MiniBasin.2d case with sea levels assigned as indicated above. This case should be compared to that shown in the 
left panel of Figure 89. Notice how the top of the section changes elevation (water depth) with time.

\section{Water Depth From the paleodepth.txt file}

Entering water depth data (either graphically or in a spreadsheet) for all strata can be a tedious process. The paleodepth.txt file provides an efficient way to specify water depths in a $2 \mathrm{D}$ section.

The paleodepth file for the blcases/Examples.BL/GoM_Shelf.2d case is shown and defined in Figure 100. The geohistory of this case is shown in Figure 101. The methods and the section are discussed at length in (Cornelius, Cathles et al. 2003).

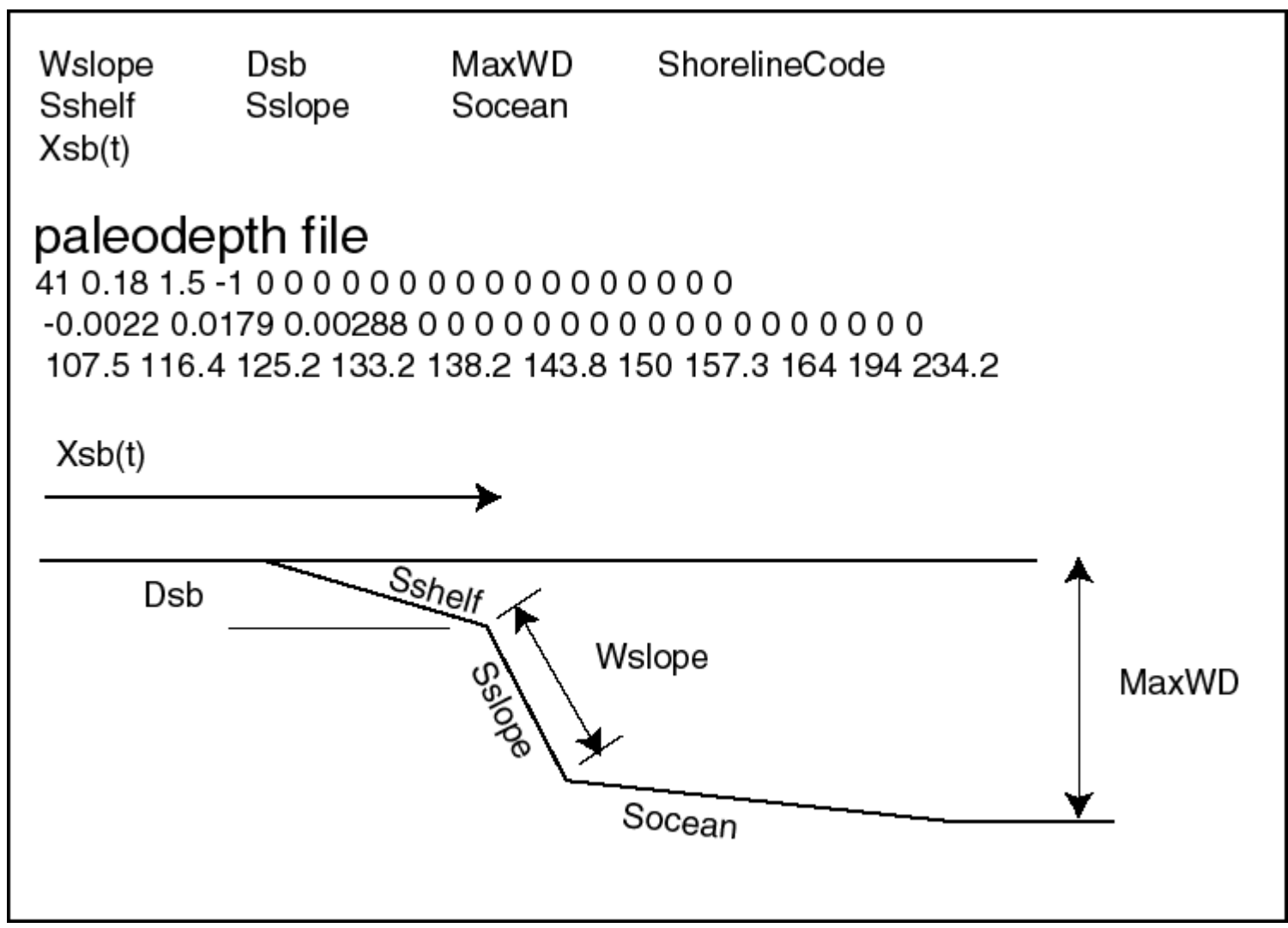

Figure 100. Definition of the parameters in paleodepth file, the parameters in the paleodepth file for the blcases/Examples.BL/GoM_Shelf.2d, and a diagram illustrating parameters in the file. All lines in the file contain NS entries, where NS is the number of strata in the present day section (in this case 21). The first line of the paleodepth.txt file contains only 4 entries (the rest are 0). Wslope is the width of the slope in kilometers. Dsb is the depth of the slope break below seal level in kilometers. MaxWD is the maximum water depth in kilometers. The final entry on the first line is a code that is 1 if the shoreline is to the left, and -1 if it is to the right. The second line of the file contains 3 entries: Sshelf, the slope of the shelf in $\mathrm{km} / \mathrm{km}$ from the shelf break and negative because water depth decreases shoreward of the break. Sslope is the slope of the slope in $\mathrm{km} / \mathrm{km}$, 
which is positive because water depth increases seaward of the slope break. Socean is the slope of the ocean floor in $\mathrm{km} / \mathrm{km}$. The final line gives the distance of the shelf break in $\mathrm{km}$ from the end of the section, $X s b(t)$ at the time each age horizon is deposited. Only the distances for the strata actually deposited in the model (11 in this case) are given. The other 10 entries can have any value and are usually given the value 999 . 

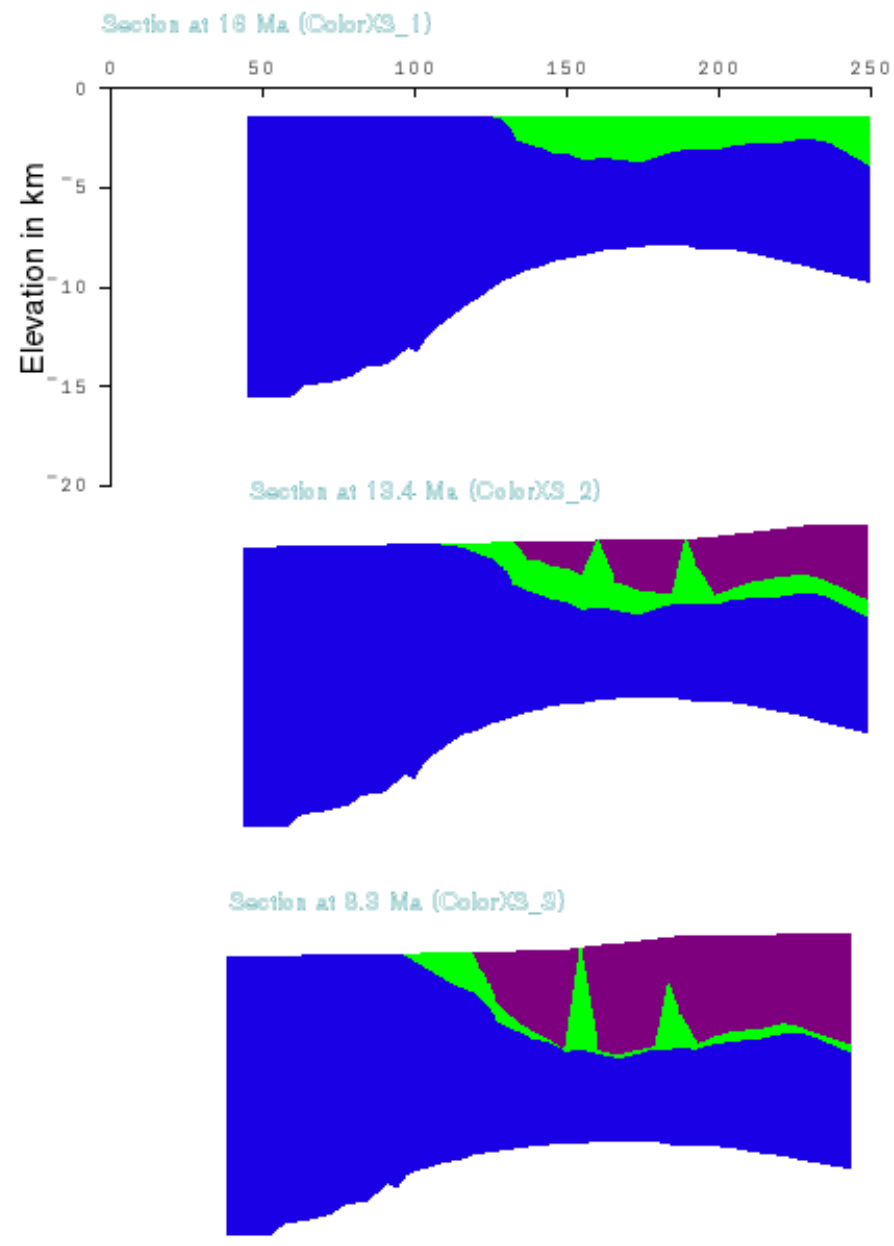

Section as $9.7 \mathrm{Ma}$ (Color) 8 _6)

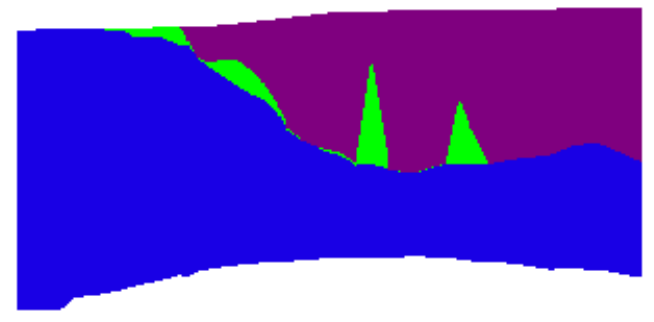

Section ar O Ma (Color)es_12)

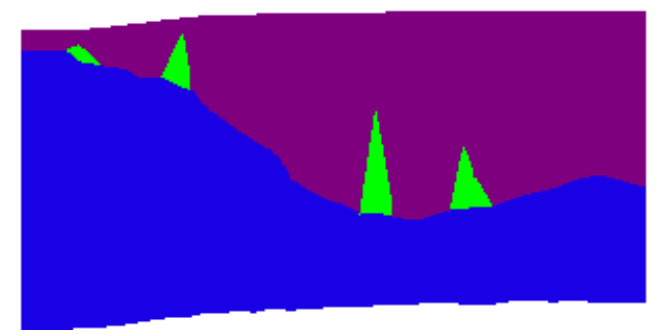

Figure 101. Frames from the blcases/Examples.BL/GoM_Shelf.2d GeoHistory model with water depths defined by the paleodepth file illustrated in Figure 100. 


\section{G. Basin Seals}

Seals can be defined in two ways in $\boldsymbol{B a s i n} \boldsymbol{L} \boldsymbol{A} \boldsymbol{B}$. The permeability of particular elements can be made small in parts of the basin. Alternatively elements may be labeled as seal elements. In this case their permeability can be automatically reduced. Seal elements also affect the initial processing of a section in several appropriate ways.

The blcases/Examples.BL/GoM_Shelf.2d case presented in the previous section contains a transgressing seal. This seal was input through strata in the SFLAT file that have horizon numbers of zero. Examination of the SFLAT file of this case using Geology: Edit/Plot Spreadsheet shows how this is done (Figure 102).

\begin{tabular}{|c|c|c|c|c|c|c|c|c|c|}
\hline \multirow[t]{2}{*}{ Lithology Pattern } & Litholology Color & \multicolumn{3}{|c|}{ Plot Selected Column } & \multicolumn{2}{|c|}{ Edit Isopachs } & \multicolumn{2}{|c|}{ Edit Highlited Column } & \multirow{2}{*}{$\begin{array}{l}\text { Apply Change } \\
\text { Seal_cod... }\end{array}$} \\
\hline & W_Nbr & H_Nbr & $\times 1$ & X2(depth) & $\times 3$ & Fx_Sd & Fx_Sh & Fx_Salt & \\
\hline TRANSSEAL & 1.000 & 0.000 & 45.000 & -1.664 & 79.100 & 0.000 & 1.000 & 0.000 & 1.000 \\
\hline $57.75 \mathrm{MA}$ & 1.000 & 14.000 & 45.000 & -5.123 & 79.100 & 0.100 & 0.900 & 0.000 & 0.000 \\
\hline $98.5 \mathrm{MA}$ & 1.000 & 15.000 & 45.000 & -8.582 & 79.100 & 0.100 & 0.900 & 0.000 & 0.000 \\
\hline BseEocSou & 1.000 & 15.481 & 45.000 & -10.282 & 79.100 & 0.100 & 0.900 & 0.000 & 0.000 \\
\hline TopEocSou & 1.000 & 15.500 & 45.000 & \multirow{13}{*}{\multicolumn{5}{|c|}{ 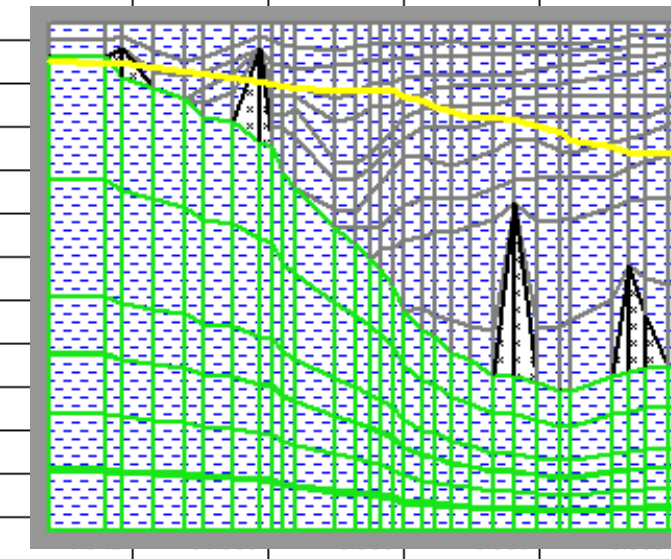 }} & \\
\hline $139.2 \mathrm{MA}$ & 1.000 & 16.000 & 45.000 & & & & & & \\
\hline BseJurSou & 1.000 & 16.438 & 45.000 & & & & & & \\
\hline TopJurSou & 1.000 & 16.500 & 45.000 & & & & & & \\
\hline $180 \mathrm{MA}$ & 1.000 & 17.000 & 45.000 & & & & & & \\
\hline W_2 & 2.000 & 1.000 & 58.900 & & & & & & \\
\hline $0.45 \mathrm{MA}$ & 2.000 & 2.000 & 58.900 & & & & & & \\
\hline $0.75 \mathrm{MA}$ & 2.000 & 3.000 & 58.900 & & & & & & \\
\hline $1.3 \mathrm{MA}$ & 2.000 & 4.000 & 58.900 & & & & & & \\
\hline $1.7 \mathrm{MA}$ & 2.000 & 5.000 & 58.900 & & & & & & \\
\hline $2.8 \mathrm{MA}$ & 2.000 & 6.000 & 58.900 & & & & & & \\
\hline 3.7MA & 2.000 & 7.000 & 58.900 & & & & & & \\
\hline 4MA & 2.000 & 8.000 & 58.900 & & & & & & \\
\hline $5.6 \mathrm{MA}$ & 2.000 & 9.000 & 58.900 & -1.042 & 81.000 & 0.500 & 0.500 & 0.000 & 0.000 \\
\hline 8.3MA & 2.000 & 10.000 & 58.900 & -1.042 & 81.000 & 0.500 & 0.500 & 0.000 & 0.000 \\
\hline 13.4MA & 2.000 & 11.000 & 58.900 & -1.042 & 81.000 & 0.500 & 0.500 & 0.000 & 0.000 \\
\hline $16 \mathrm{MA}$ & 2.000 & 12.000 & 58.900 & -1.042 & 81.000 & 0.500 & 0.500 & 0.000 & 0.000 \\
\hline $17 \mathrm{MA}$ & 2.000 & 13.000 & 58.900 & -1.042 & 81.000 & 0.000 & 0.000 & 1.000 & 0.000 \\
\hline TRANSSEAL & 2.000 & 0.000 & 58.900 & -1.198 & 81.000 & 0.000 & 1.000 & 0.000 & 1.000 \\
\hline
\end{tabular}

Figure 102. SFLAT file of blcases/Examples.BL/GoM_Shelf. $2 d$ case obtained with menu command Geology: Edit/Plot Spreadsheet shows how a transgressing seal is defined by inserting horizons with horizon numbers of 0 (here called TRANSSEAL). The plot is made by depressing the Lithology Pattern button at the top of the spreadsheet. The transgressing seal is shown by the yellow line.

When the section is processed under GeoHistory, pore fluid pressures below the transgressing seal are assumed to be a specified fraction of lithostatic at the depth of the seal in each well (see Figure 109). These pressures affect the present-day porosity and thus also affect decompaction, as we will discuss in the next section. 
A seal can be fixed to particular strata, or can migrate across strata to maintain approximately the same depth below the basin surface. This is discussed at length and illustrated with examples from Eugene Island Block 330 in Revil and Cathles (2001). Figure 103 shows migrating seal elements and fluid pressures at a couple of times in the blcases/Examples.BL/GoM_Shelf.2d case. Whether the seal is fixed, migrating, or there is no seal is determined by toggles at the top of the GeoHistory form (see Figure 88).

$0 \mathrm{Ma}$
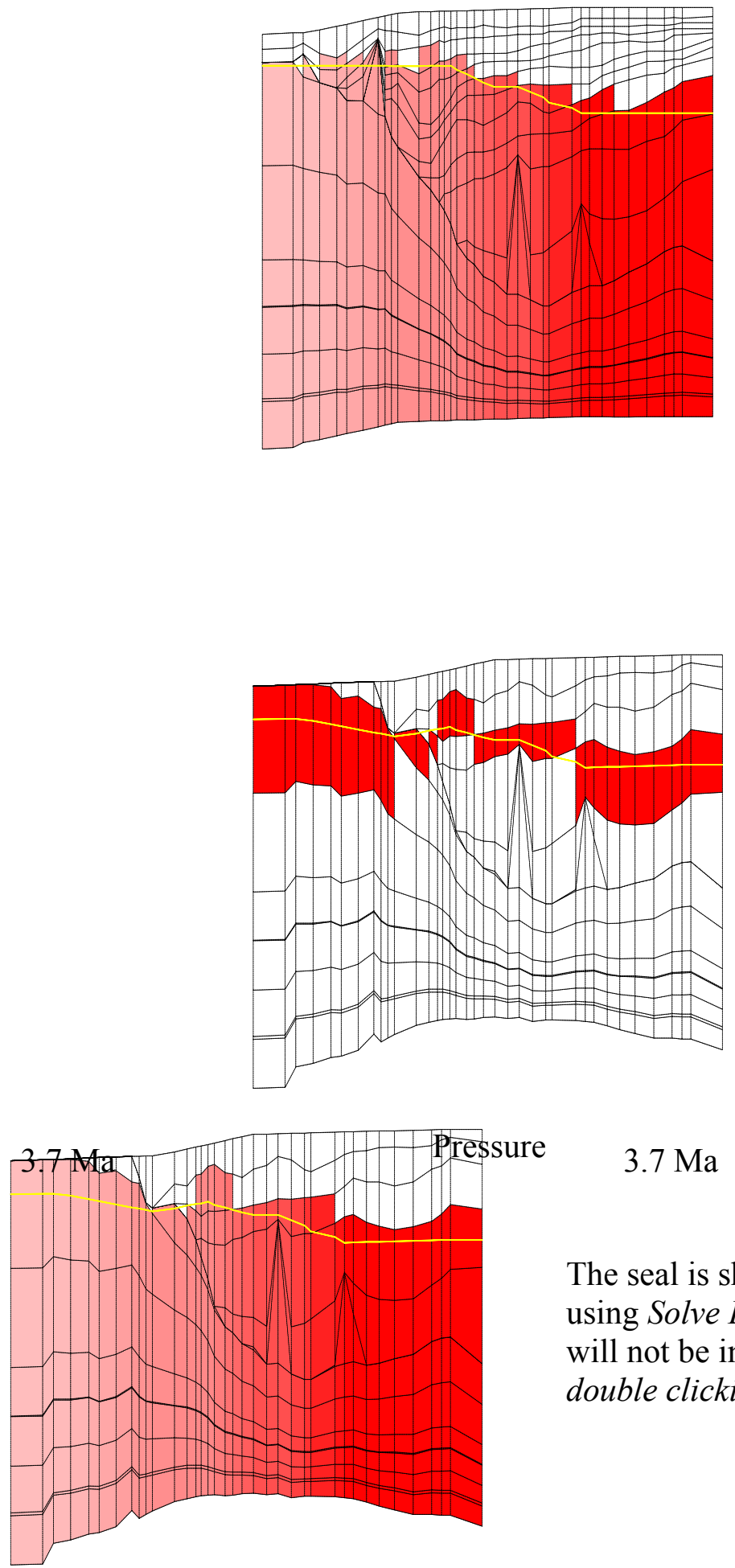

$0 \mathrm{Ma}$

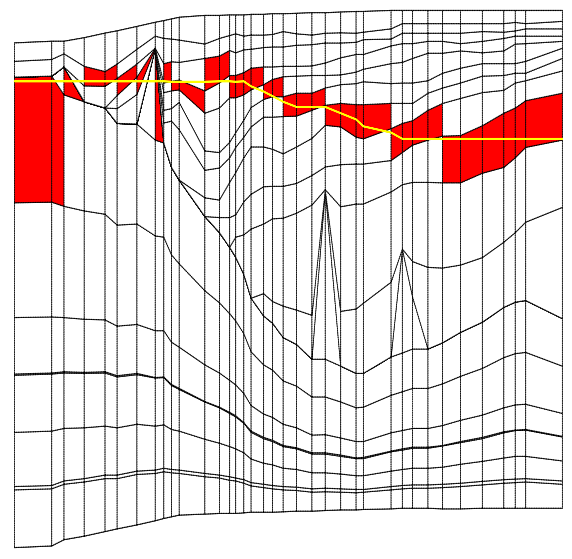

Figure 103. Migrating seal elements and pressure under them at the present day and 3.7 Ma before present for the blcases/Examples.BL/GoM_Shelf.2d case. Seal was defined in the SFLAT file as shown in Figure 102 and processed (to infer the geological history of the section) as a migrating seal (see Figure 88). The images above are obtained from GeoHistory: Plot and Edit: Check Grid and Graphic Edit. Note that the graphic image is placed in the clipboard only when the File button on the workspace toolbar is depressed. A*.wmf file is also placed in folder when this is done.

The seal is shown again when the finite element grid is created using Solve Equations: Create FE Grid. However, the seal will not be in the most ascetic form. It can be adjusted by double clicking a seal element to turn off (or on) the seal flag 
in that element. Figure 104 shown how this procedure can be used to improve the form of a seal.
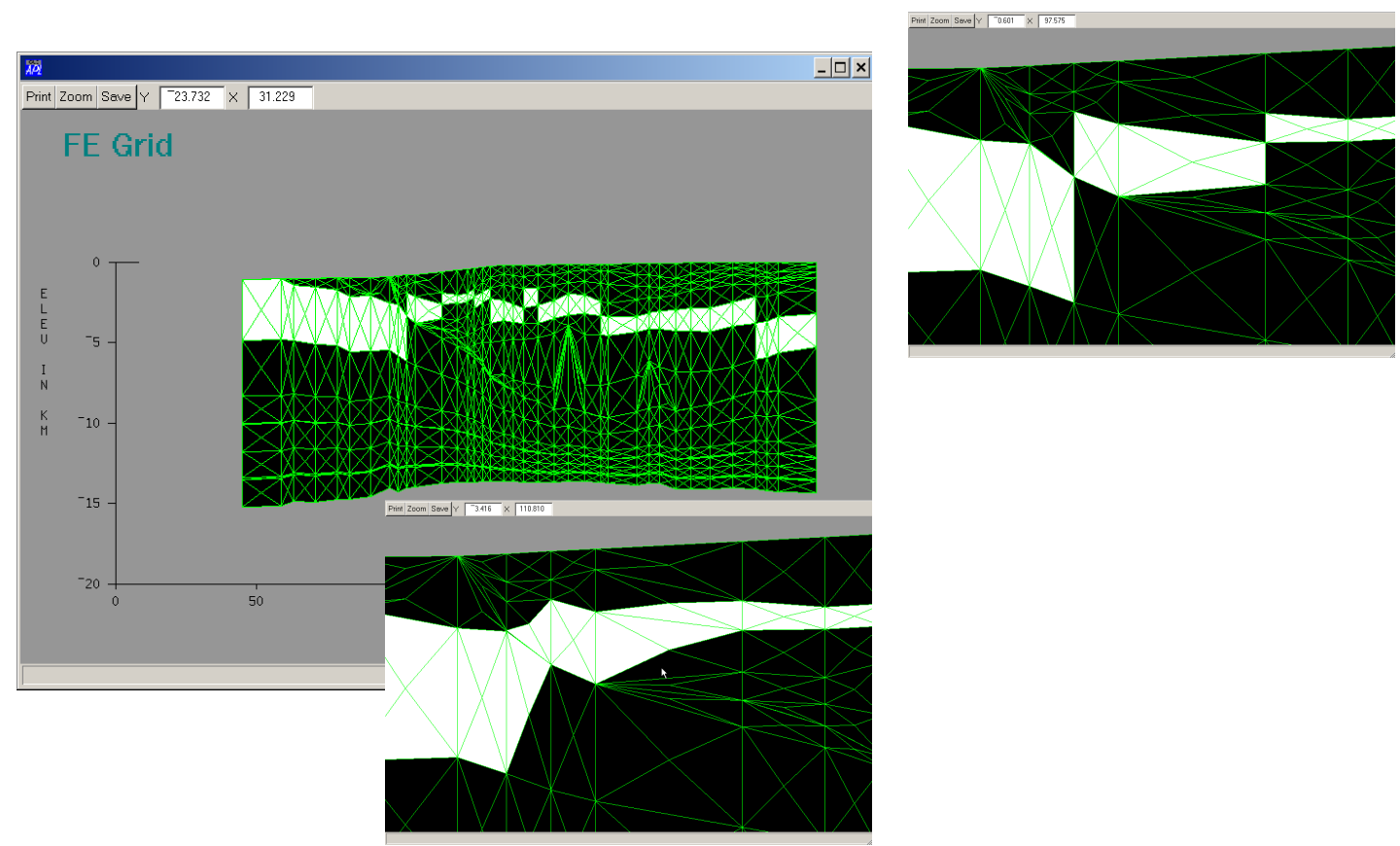

Figure 104. Editing the finite element grid using Solve Equations: View FE Grid allows the definition of a seal to be improved. Double clicking a seal element removes the seal flag; double clicking a non-seal element turns on the seal flag. This procedure can be used to improve the form of the seal as illustrated.

The brine flow calculated by the 2D finite element methods depends on the permeability the sediments, faults, salt and seal. Salt and seal permeabilities are assigned by the Salt, Seals and Hydrofracture form shown in Figure 105.

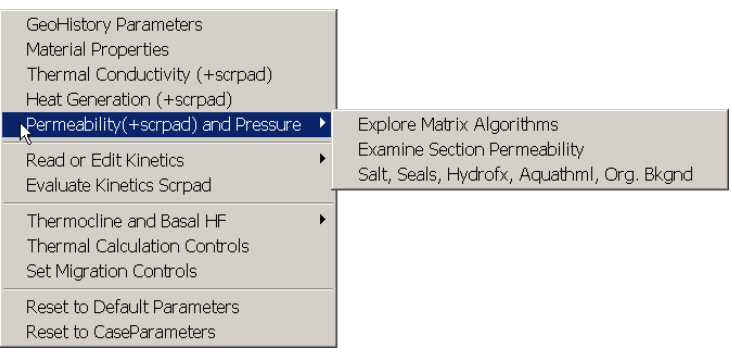




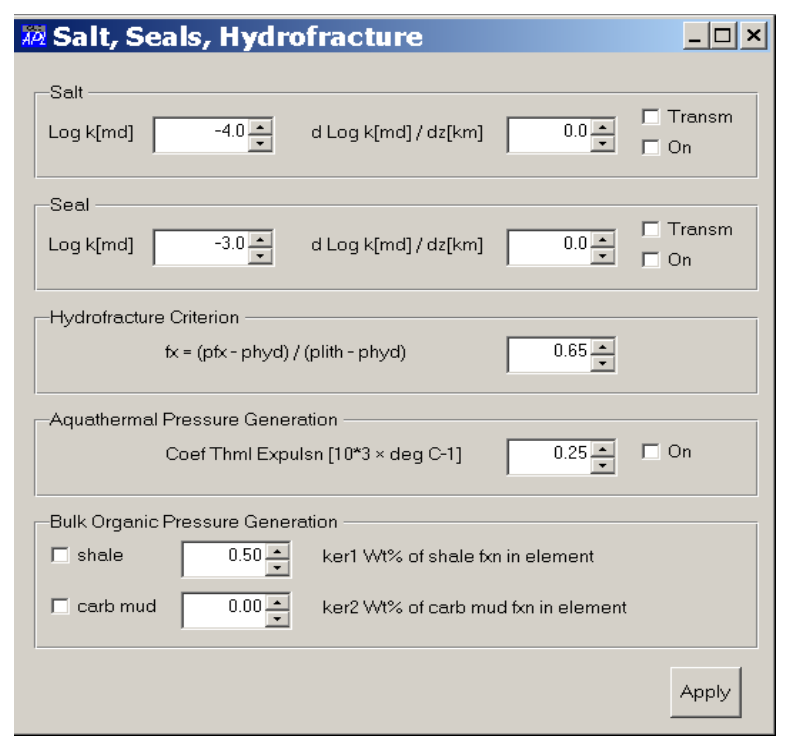

Figure 105. Parameters controlling how salt, seals, hydrofracturing, and aquathermal and the maturation of bulk organic matter will affect the calculated brine flow. Form summoned by Parameters: Permeability (+scrpad) and Pressure: Salt, Seals, Hydrofx, Aquathml, Org. Bkgnd as shown.

The blcases/Examples.BL/GoM_Shelf.2d is calculated for no special changes in salt or seal (no boxes checked in Figure 105) by selecting the temperature and pressure equations for solution (using the Solve Solutions: Equation Controls: Choose Equations menu), and then computing the case using Solve Equations: Run Simulation: All Selected $2 D$ Equations. The case is a large one ( 37 wells, 21 horizons, and 12 timesteps) and will take about 1.5 hours to run. The results are shown in Figure 106.

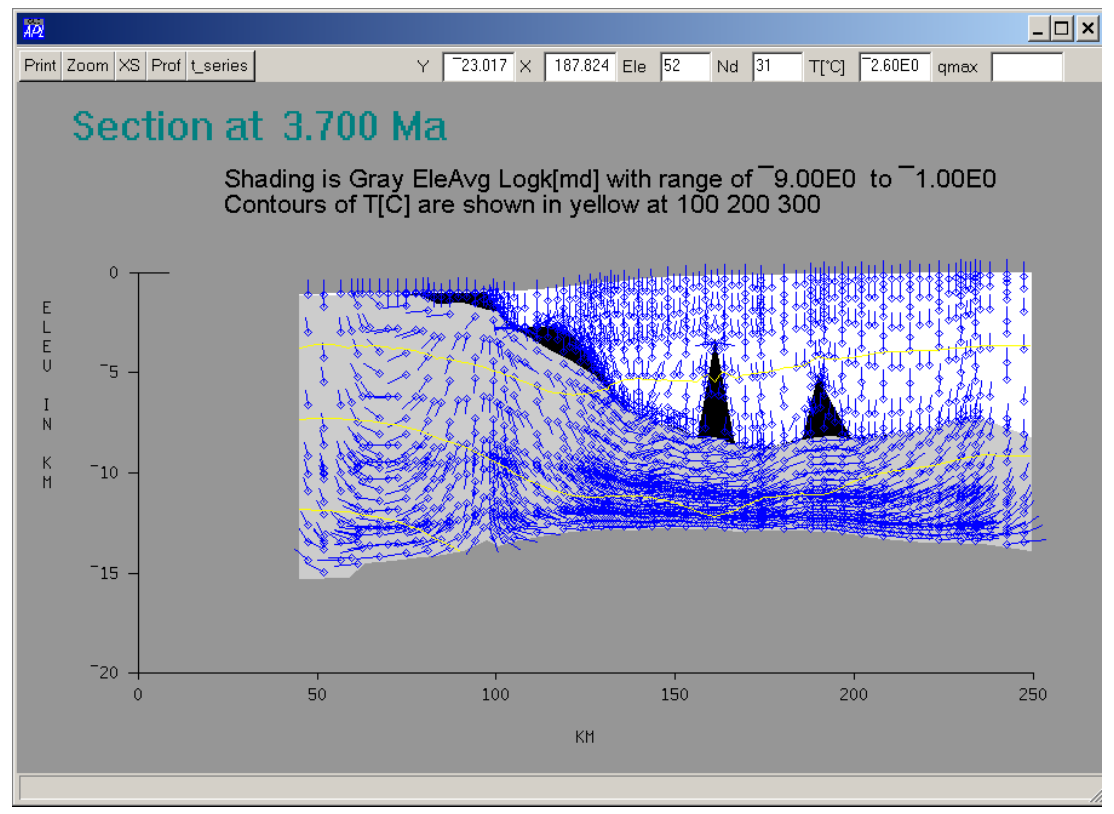




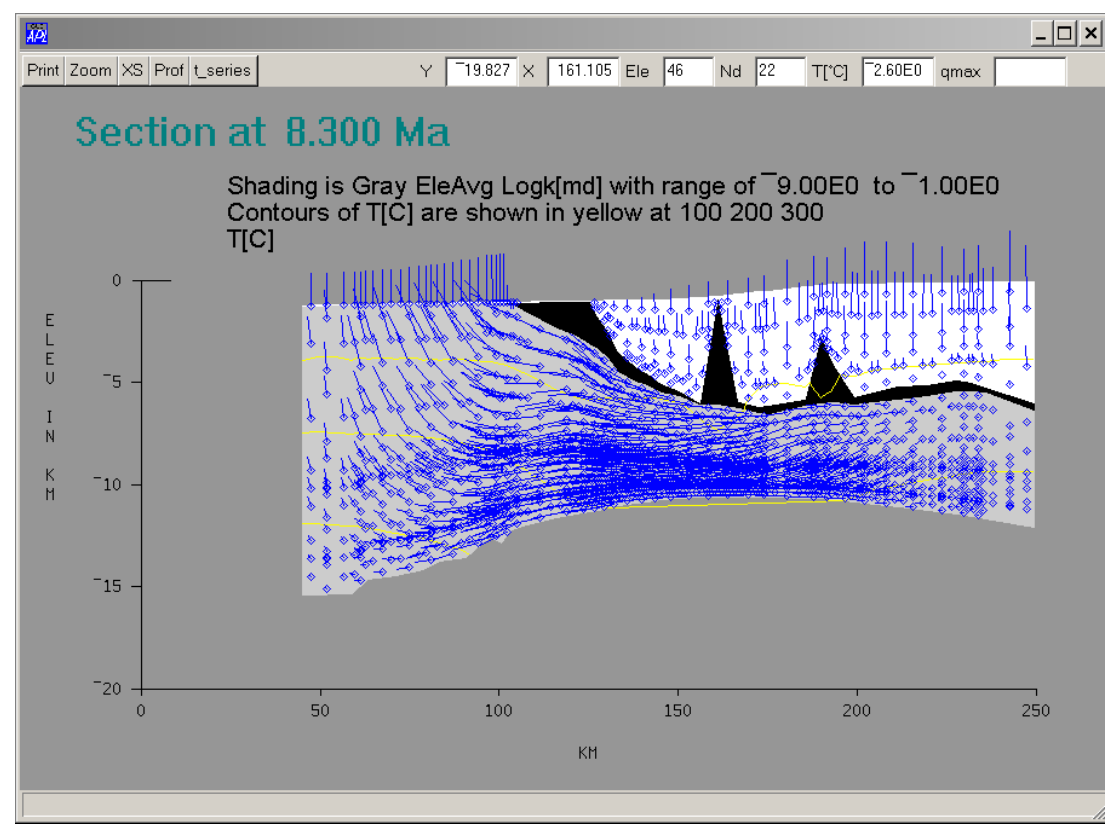

Figure 106. Flow calculated at two different times for the blcases/Examples.BL/GoM_Shelf.2d case with the salt and seal controls set as shown in Figure 105. Because special assignment of salt permeability is not turned on, salt has its lithologic permeability of $10^{-9}$ millidarcies. Brine flow moves around salt bodies. Because the seal permeability is not turned on, the 2D brine flow calculations do not treat seal elements specially, and these elements have their normal lithologically-specified permeability. Figure 107 shows the flow at $8.3 \mathrm{Ma}$ if the salt permeability is set at $10^{-4}$ md rather than $10^{-9} \mathrm{md}$.

The influences of the controls in Figure 105 are illustrated below. First Figure 107 shows that the flow will no longer be diverted $\sim 100 \mathrm{~km}$ to vent seaward to the Sigsbee knolls if the seal permeability is $10^{-4} \mathrm{md}$ rather than $10^{-9} \mathrm{md}$. This change was achieved by turning the salt permeability control on in the form shown in Figure 105 with the salt permeability set at $10^{-4}$ and the change with elevation set at 0 (as shown). The flow and temperature pattern shown in was then computed by solving the $6^{\text {th }}$ step over again, as described in the caption. 


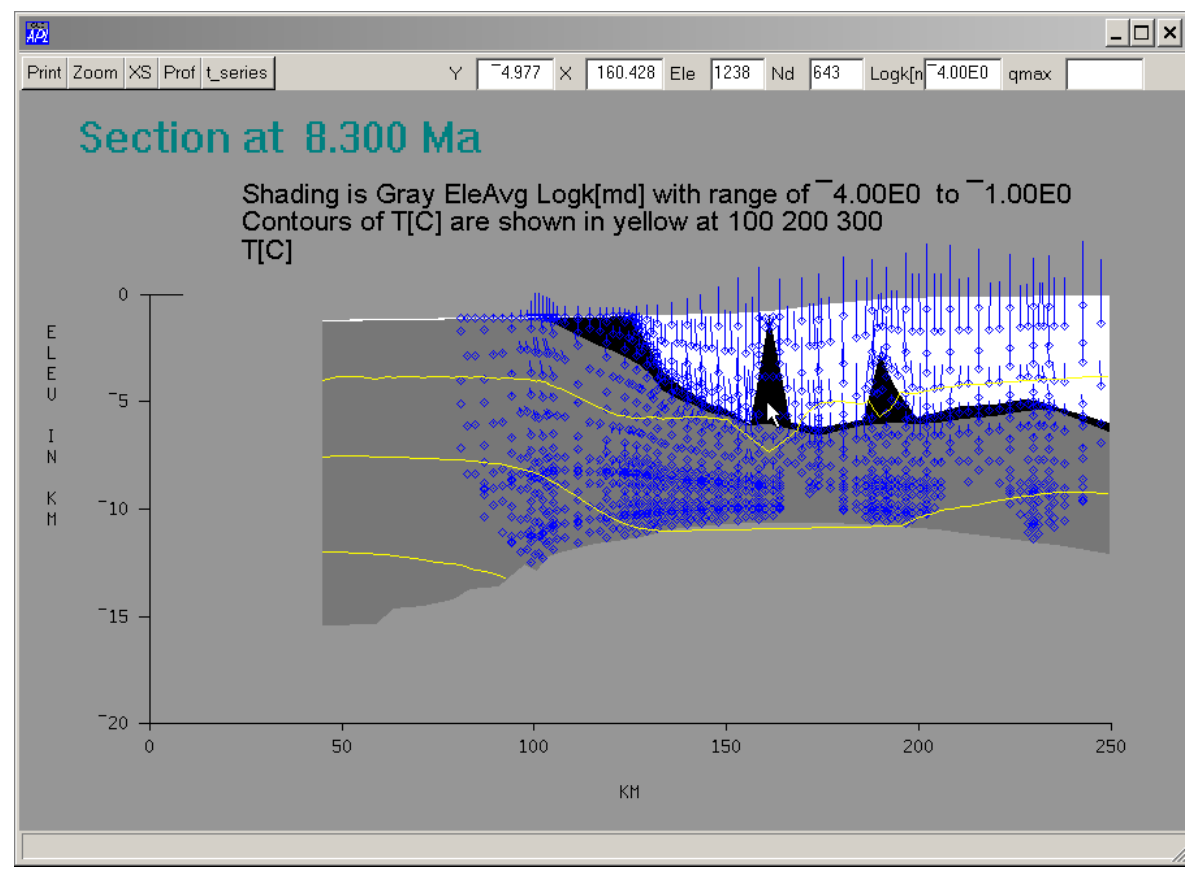

Figure 107. Flow at $8.3 \mathrm{Ma}$ in the blcases/Examples.BL/GoM_Shelf. $2 d$ case shown in the lower figure in Figure 106, but with the salt permeability set to $10^{-4}$ md rather that $10^{-}$ 9 . With this higher salt permeability the flow passes through the salt rather than moving 100 's of kilometers to avoid this transit. Calculation of this flow pattern was achieved using the restart button 1 -step Solve on the toolbar that appears when a case is examined graphically using Solve Equations: View Calculated Solution.

Figure 108 shows the flow pattern if the seal permeability is set to $10^{-4}$ and the elevation dependence of seal permeability set to $-0.5 \mathrm{log}$ units per kilometer in Figure 105. The seal then shows clearly on the permeability section, and the flow is controlled by a combination of salt and seal impedance. The purpose of these tools is to allow possible controls on the flow pattern to be efficiently investigated. The pattern of flow is what is important. The pattern is controlled by permeability contrasts. For reasonable compactive and aquathermal expulsion the calculated fluid pressures may be unrealistically high. In nature hydrofracture will limit the fluid pressures that can be attained, and we could adjust the model pressures the permeability of units in our models so that the pressures do not exceed this fracture limit. We do not need to do this because all we are interested in is the flow pattern. Therefore we calculate a flow pattern and set the pressure to reasonable values afterwards, rather than adjusting permeability. This is an important and perfectly valid methodological simplification. 


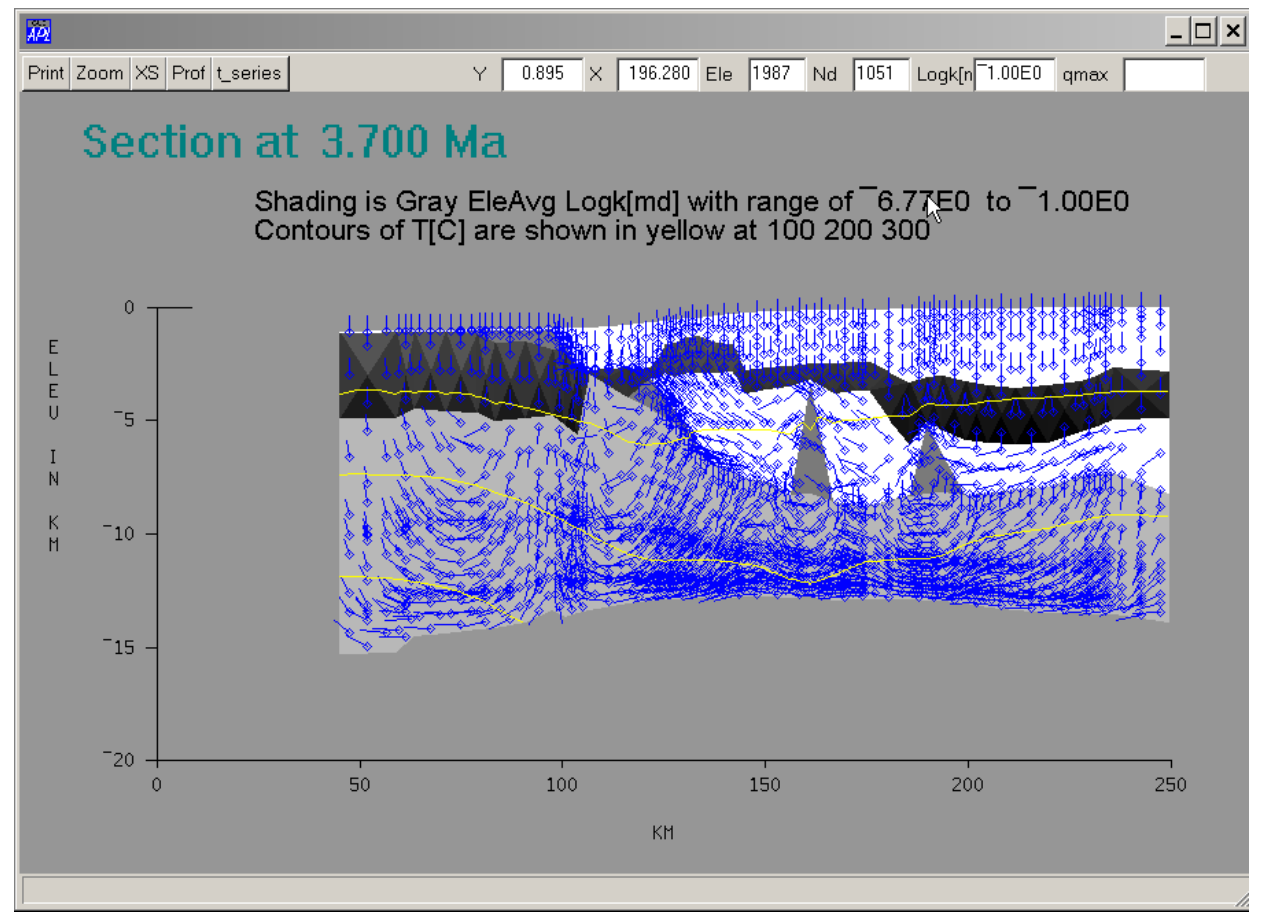

Figure 108. Same case as the upper figure in Figure 106 but the seal has been turned on with a base permeability of $10^{-4} \mathrm{md}$ and a decrease with elevation of $-0.5 \log$ units per kilometer below sea level.

\section{Parameters}

A basin modeler must specify the material properties of the sediments in the basin (compressibility, density, thermal conductivity, internal heat generation, permeability), kinetic parameters characterizing the maturation of organic material in the sediments,

\begin{tabular}{|l}
\hline Parameters GeoHistory Heatflow ar \\
GeoHistory Parameters \\
Material Properties \\
Thermal Conductivity (+scrpad) \\
Heat Generation (+scrpad) \\
Permeability (+scrpad) \\
\hline Read or Edit Kinetics \\
Evaluate Kinetics Scrpad \\
\hline Thermal BC (+scrpads) \\
Thermal Calculation Controls \\
Set Migration Controls \\
\hline Reset to Default Parameters \\
Reset to CaseParameters \\
\hline \hline
\end{tabular}
thermal and flow boundary conditions, and modeling and migration parameters (minimum timestep, etc.). Parameters controlling compaction have already been used, without discussion, in the previous section. Basin $\boldsymbol{L} \boldsymbol{A B}$ provides access to all the parameters it uses, most from the Parameters menu of the workspace toolbar shown to the left. Basin LAB parameters are related to geologic variables:

- Material properties are calculated by algorithms that take lithology and physical parameters (such as temperature, pressure, fault slip rate, etc.) as input.

- Because one objective is to infer property behavior from basin observations, different algorithms specifying the same parameter (especially permeability) can be selected.

- The BasinL $\boldsymbol{A B}$ architecture is designed so that new parameter functions can be added easily. 
This section discussed important aspects of the parameter list shown above. The equations solved by BasinLAB are derived and discussed in Appendix C (p. 340).

\section{A. Geohistory Parameters and Porosity}

Parameters (other than sediment properties) that control GeoHistory calculation are specified when the Parameters: GeoHistory Parameters menu command (see above) is selected. This brings up the form shown in Figure 109. The other parameter forms are called in a similar fashion. These forms are mainly discussed in the figures presented below. Text comments are added only when discussion is extensive.

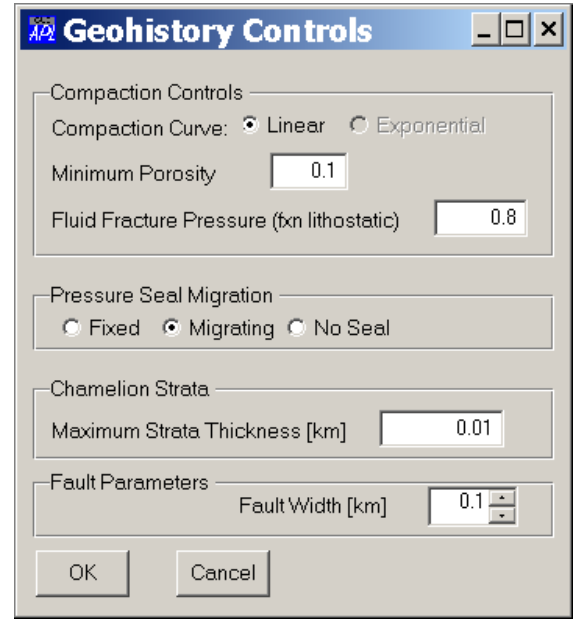

and "disappears".
Figure 109. GeoHistory parameters form produced by menu command Parameters: GeoHistory Parameters.

- Compaction is linear to some minimum porosity where compaction ceases.

- The pressure under a seal is assumed to be a specified fraction of lithostatic.

- The seal may be attached to the sediment matrix (fixed) or can migrate through the sediment matrix (migrating). Migrating seals are assumed to maintain the same depth below the basin surface in past times as they have today.

- There is a maximum strata thickness below which the strata takes on the properties of the underlying strata

- Faults have a set width.

Porosity is a linear function of effective stress to a minimum porosity, and sediment density is a linear function of porosity:

$$
\begin{aligned}
& \phi=\phi_{o} \frac{\sigma^{\prime}-p_{1}}{\sigma_{c}} \quad, \text { for } \phi \leq \phi_{\min } \\
& \phi=\phi_{\min } \quad, \text { for } \phi \geq \phi_{\min } \\
& \rho_{s}=\rho_{G}(1-\phi)+\rho_{w} \phi .
\end{aligned}
$$

Here $\sigma$ ' is the reduced lithologic stress (lithologic stress minus hydrostatic pore pressure). It is calculated by integrating buoyant sediment density from the top surface of the basin to the depth of interest. $\mathrm{p}_{1}$ is the excess pore pressure (pore pressure minus hydrostatic pressure), $\rho_{\mathrm{w}}$ is the density of pore water, $\rho_{\mathrm{s}}$ the density of the basin sediment, and $\rho_{\mathrm{G}}$ and $\phi$ are the grain density and porosity of the sediment computed as linear combinations of the end-member lithology values given in Material Property table. Porosity is not allowed to increase in our models. It might also be noted that the rigidities are about 50 times smaller than the rigidities used in reservoir engineering and hydrologic simulations of fluid flow. Over geologic times, mineral grains dissolve and/or deform where pressed together. This accounts for the greater compressibility. The compressibility is calibrated to Gulf of Mexico sediments in the Eugene Island Block 330 area (Revil and Cathles 2001). 


\section{B. Material Properties}

\begin{tabular}{|c|c|c|c|c|c|c|}
\hline \multicolumn{7}{|c|}{ Aal Material Properties } \\
\hline \multicolumn{7}{|l|}{ Apply } \\
\hline & $\log k x$ & $\log k z$ & $\mathrm{~K}[\mathrm{TCU}]$ & Density [g... & Porosity & Compr[ba... \\
\hline Sand & 1 & 1 & 10.56 & 2.76 & 0.39 & 0.000573 \\
\hline Shale & -3 & -3 & 3.6 & 2.76 & 0.35 & 0.0006136 \\
\hline Carbonate sa... & 1 & 1 & 6.96 & 2.72 & 0.47 & 0.001228 \\
\hline Carbonate $\mathrm{m} \ldots$ & -3 & -3 & 6.96 & 2.72 & 0.43 & 0.001271 \\
\hline Salt & -9 & -9 & 13 & 2.16 & 0 & 0.00000001 \\
\hline
\end{tabular}

Figure 110.

Material

properties form

produced by

menu command

Parameters:

Material

Properties

Sediment material properties are determined from the properties of the lithologic endmembers. The properties of these end-members are defined in the Material Properties table shown in Figure 110.

\section{Matrix Permeability}

Matrix permeability is determined by one of three possible algorithms, depending on user choice:

1) An anisotropic combination of coarse and fine lithology permeabilities,

2) An anisotropic linear combination of strata-parallel and perpendicular log permeabilities

3) An isotropic empirical function of porosity and the fraction coarse sediments.

The first model (Lithology: Aniso fine/coarse Harm Combo) takes linear and harmonic averages of the first permeability entry $\left(\log _{10} \mathrm{k}_{\mathrm{x}}\right.$, which we indicate $\left.\mathrm{k}_{1 \mathrm{i}}\right)$ in the Material Property table over the lithologic fractions, $\mathrm{F}_{\mathrm{i}}$, of these end-members in each sediment element, and combines them according to the fraction coarse sediments:

$$
\begin{array}{ll}
k_{\text {parll }}=F_{i} k_{1 i} & k_{\text {perp }}=1 / F_{i} / k_{1 i} \\
k_{\text {parallel }}=1 / F_{x} / k_{\text {perp }}+\left(1-F_{x}\right) / k_{\text {parll }} & k_{\text {perpendicular }}=F_{x} k_{\text {parl }}+\left(1-F_{x}\right) k_{\text {perp }}
\end{array}
$$

Summing over repeated indices (i) is assumed in the above formulae. $F_{x}$ is the fraction cross-cutting permeability type. For example, sands may be almost entirely strataparallel, but occasionally a sand flame may crosscut stratigraphy. Joints may provide a cross-cutting permeability. Similarly impermeable units may cut across the strata and impair the strata-parallel permeability. $F_{x}$ is adds a little parallel permeability to in the strata-perpendicular direction, and a little perpendicular permeability in the strata-parallel direction. Unless the permeability of the fine and coarse sediments is the same, or the 
sediment mix is all fine or all coarse, $\mathrm{k}_{\text {parallel }}$ and $\mathrm{k}_{\text {perpendicular }}$ will differ and the sediment will be anisotropic. $\mathrm{F}_{\mathrm{x}}$ reduces this anisotropy.

The second model (Lithology: Log-linear mix) is a log-linear combination of lithologic units with volume fraction $F_{i}$ of the first and second permeability columns in the Material Property table shown in Figure 110:

$$
\begin{aligned}
& k_{\text {parallel }}=F_{i} \log k_{1 i} \\
& k_{\text {perpendicular }}=F_{i} \log k_{2 i}
\end{aligned}
$$

In the third model (Porosity: $k$ (porosity, fxcoarse) Permeability, $\mathrm{k}$ in millidarcies, is computed from the fraction coarse-grained sediments (e.g., the sum of the fraction sand and the fraction carbonate sand), $\mathrm{f}_{\text {coarse, }}$ and porosity, $\phi$, of an element:

$$
\begin{aligned}
& \log k=\left(5 f_{\text {coarse }}-3\right)+28.5 f_{\text {coarse }}(\phi-0.2) \\
& f_{\text {coarse }} \leq 0.7 \\
& \log k \leq 5
\end{aligned}
$$

This relationship was deduced from Pennzoil core plug measurements (Coehlo 1997). Depending on the lithologic fraction of coarse material (sand or carbonate sand), the permeabilities range from 1 microdarcy to 100 darcies.

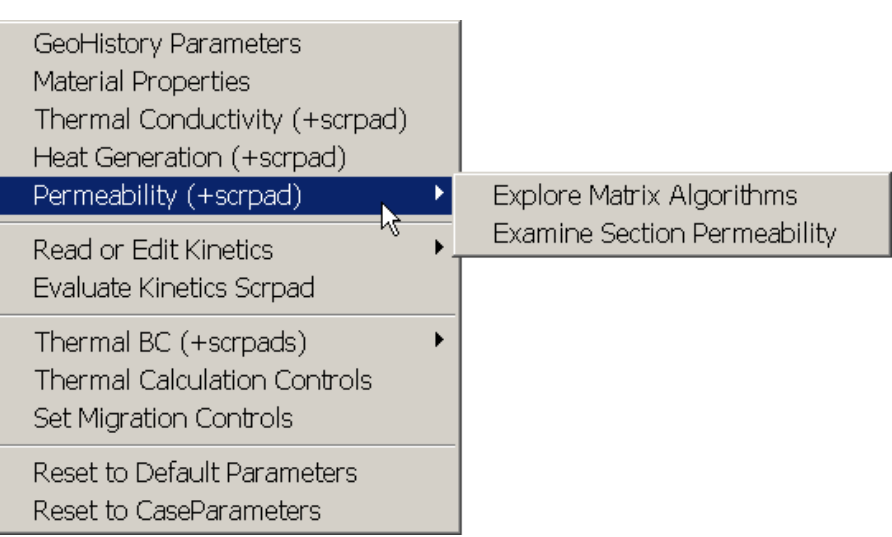

The Parameters: Permeability (+scrpad): Explore Matrix Algorithms and Examine Section Permeability can be used to examine the various matrix permeability algorithms and how they apply to the current section. This is illustrated in Figure 111 and Figure 112. 


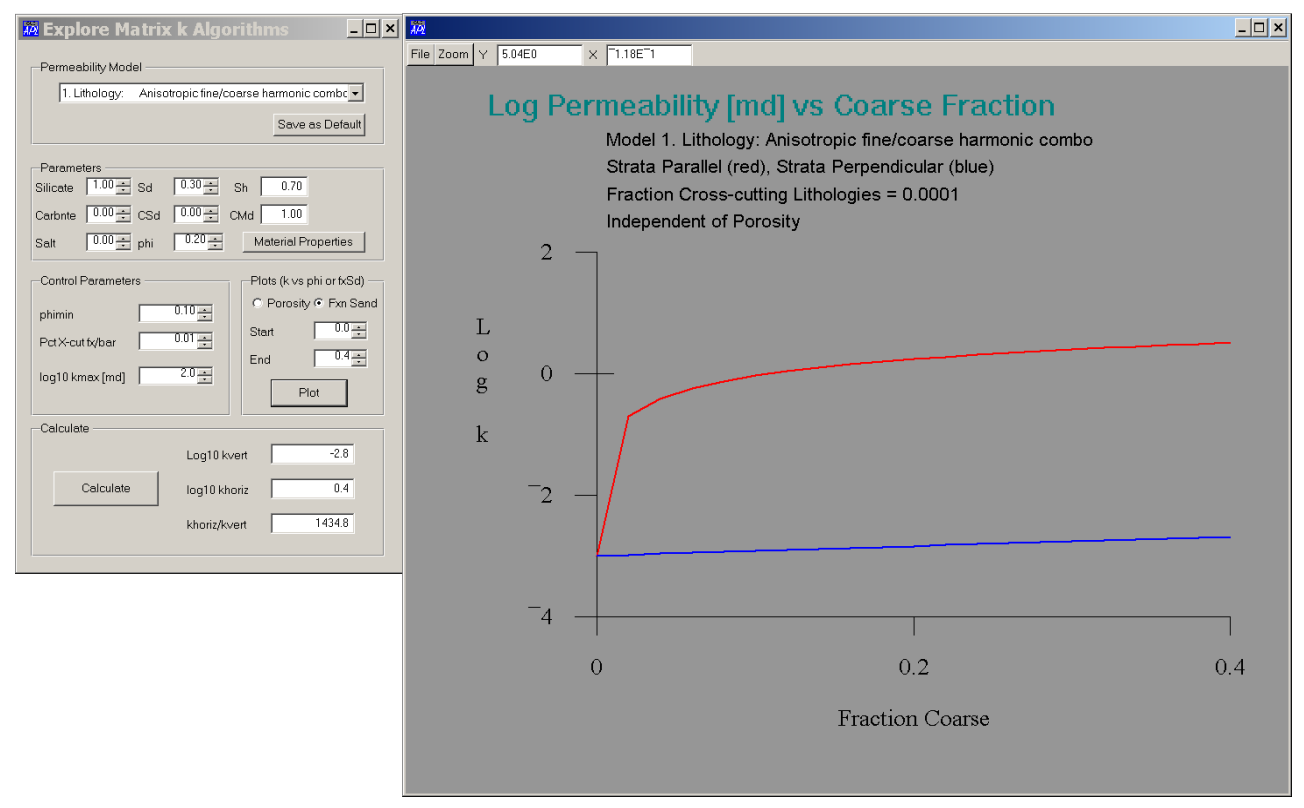

Figure 111. The properties of the matrix permeability algorithms can be explored efficiently with the Parameters: Permeability (+scrpad): Explore Matrix Algorithms form. Plots for the porosity and the harmonic combo models are shown. The Calculate button calculates the permeability at the porosity, lithology, and hydrocarbon saturation specified.

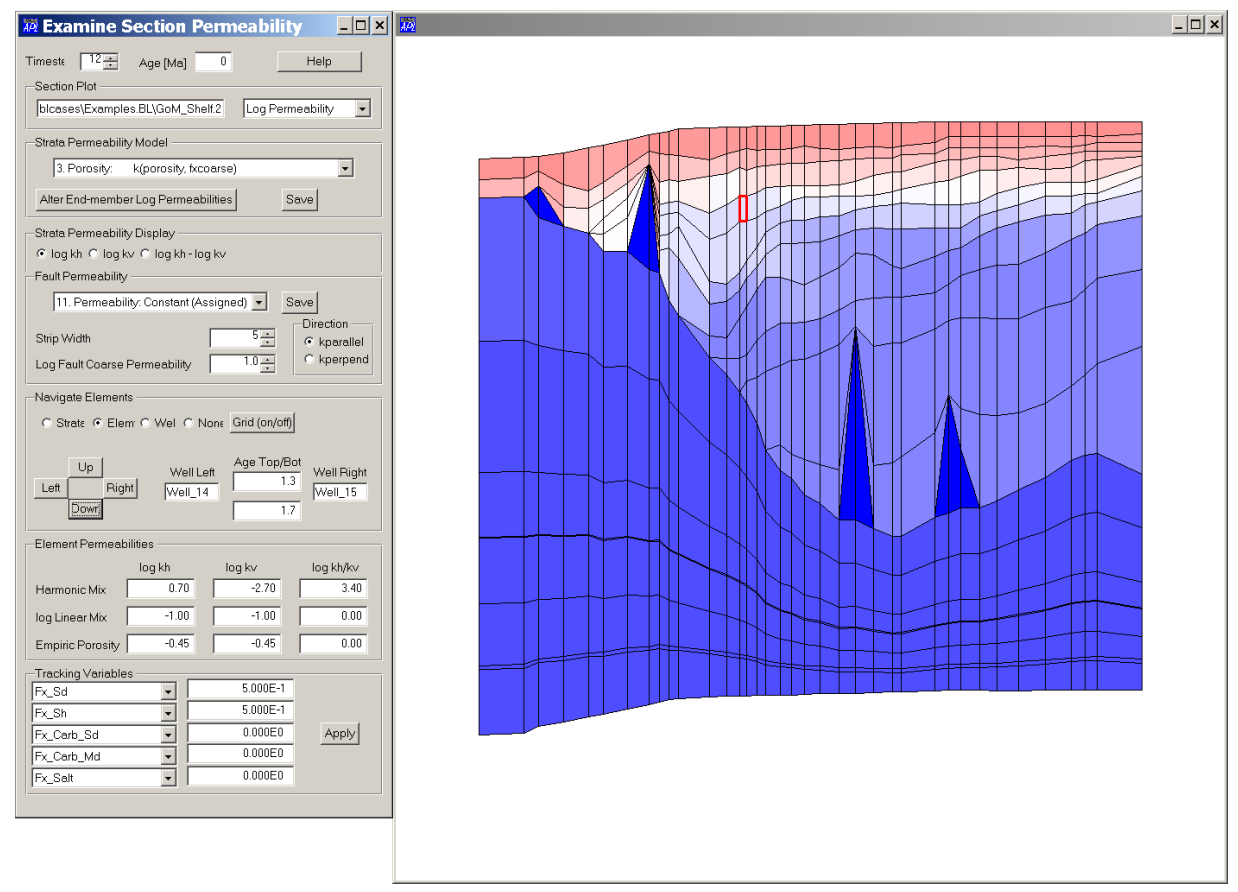

Figure 112. The permeabilities calculated by the various permeability algorithms for the current section can be examined using the Parameters: Permeability $(+$ scrpad): Examine Section Permeability menu command. The Navigate Elements section moves the red element indicator, and the edit boxes of that group show the strata-parallel and 
perpendicular permeabilities ( $\mathrm{kh}$ and $\mathrm{kv}$ ) and the anisotropy. The Strata Permeability combo box allows the different matrix algorithms to be selected. The image immediately changes when this is done. The Material Properties table can be edited using the button below this combo box. The edited properties are then used in subsequent displays and calculations.

\section{Salt and Seal Permeability}

\section{Salt and seal permeability is controlled by the Parameters: Permeability (+scrpad) and Pressure: Salt, Seals, Hydrofx, Aquathml, Org. Bkgnd menu as described above in Figure 105-Figure 108 and the associated text discussion.}

\section{Thermal Conductivity}

The thermal conductivity model used in Basin $\boldsymbol{L} \boldsymbol{A B}$ is a fabric model that mixes harmonic and arithmetic averages of temperature-dependent, thermal conductivities for endmember lithologies following methods described in (Luo, Wood et al. 1994). We adopt Platte River values for the $25^{\circ} \mathrm{C}$ grain $(0 \%$ porosity) conductivities of the lithologic mixtures shown in the Material Property table (Figure 110). The Platte River thermal conductivities of the end members are mostly those expected from a fabric theory combination of the thermal conductivities of the individual mineral grains. The thermal conductivity of shale is about half that expected from a combination of the mineral grains and pore fluids, however. This major discrepancy, which alone doubles the temperature gradient in shale dominated deltaic basins like the Gulf of Mexico Basin, is due to anisotropy. The thermal conductivity perpendicular to bedding in a shale is 2.5 times lower than the thermal conductivity parallel to bedding. The has been very convincingly established by (Blackwell and Steele 1989) and confirmed by others. For a complete review and discussion see (Cathles and Losh 2002).

Thermal conductivity can be investigated using the Thermal Conductivity Scratchpad form shown in Figure 113. It calculates thermal conductivities from the end-member lithology parameters in the Material Properties table. The lithologic mix and values of porosity, temperature and oil and gas saturation can be specified. Thermal conductivities

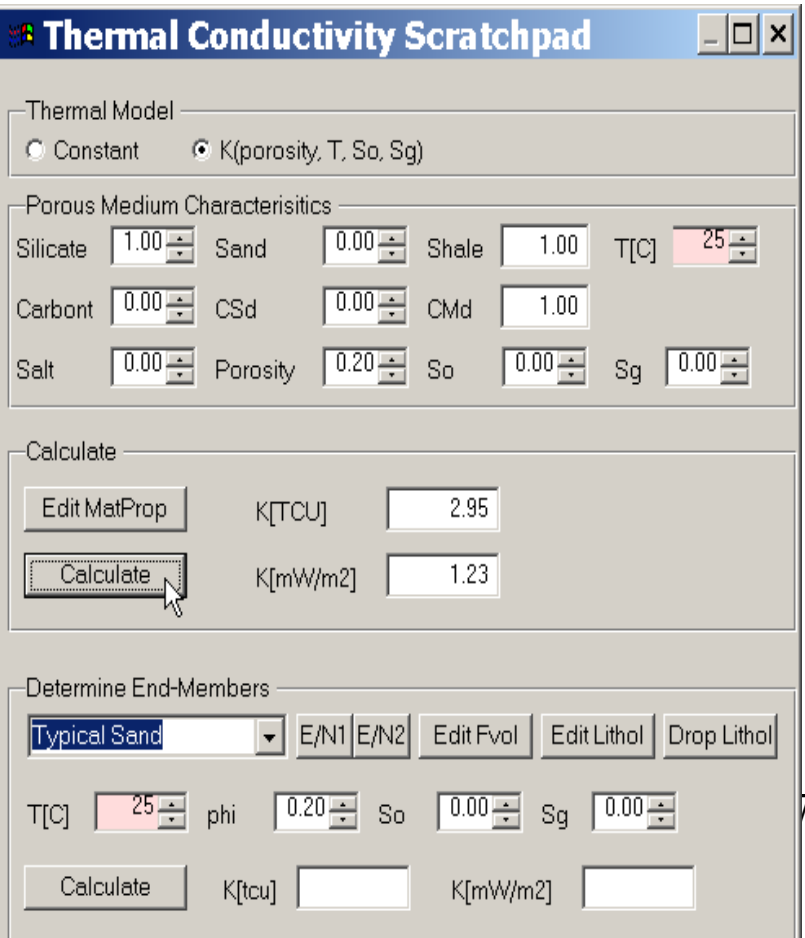
are reported in both cgs and mks units.

Figure 113. Thermal conductivity form produced by menu command Parameters: Thermal Conductivity $(+$ scrpad $)$.

- Lithology, porosity, oil and gas saturation, and temperature are specified in the Porous Medium Characteristics group spinners

- Calculate button calculates thermal conductivities in cgs and mks units $(\mathrm{K}$ of pure shale at $25^{\circ} \mathrm{C}$ and 0.2 porosity is 2.95 tcu or $1.23 \mathrm{~mW} / \mathrm{m}^{2}$ as shown). 
- Edit MatProp button allows Material Property table to be modified.

- Constant radio calculates for a linear mix of lithologic K.

- $K$ (porosity, $T, S o, S g$ ) calculates using the Luo et. al. fabric model and the Material Property end members.

- The bottom group constructs sediments from their mineral grain composition using the Luo et. al. fabric model.

The use of the lower group in the Thermal Conductivity Scratchpad of Figure 113 is described in Figure 114. This group allows construction of rock type end-members from the thermal conductivity of the mineral grains from which they are composed. The $25^{\circ} \mathrm{C}$ thermal conductivities of a broad list of minerals are stored in Basin $\boldsymbol{L} \boldsymbol{A B}$. The user selects first the mineral of which the sediment is composed, and then assigns the volume fraction (excepting porosity) of these minerals, the porosity of the sediment, temperature, and the fraction of the porosity filled oil and gas. Depressing the Calculate button then calculates the thermal conductivity of the sediment.

The designed sediment can be preserved for later reference. This is done by selecting the first sediment in the combo list. The first sediment is a "new" sediment. If this "new" sediment is selected and the Edit Lithol (or the Edit Fvol) button depressed, it will be seen no minerals have yet been designated. Select a set of minerals. When this form is closed the "new" mineral will have been added to the bottom of the sediment list in the combo box. Select this bottom "new" entry and depress the Edit Fvol button. You will see that all the minerals have been assigned equal volume fractions. This can be changed as desired. The name of the "new" sediment may be changed by selecting it, depressing the $E / N 1$ button, typing in the new name, and depressing the $E / N 2$ button. Old, no longer wanted sediment types can be deleted by selecting them and depressing the Drop Lithol button.

Figure 114 shows parts of the mineral composition (mineral basis species) and mineral volume forms. 


\begin{tabular}{|c|c|c|c|c|}
\hline \multicolumn{3}{|c|}{ AP Sedinent Minerals } & \multicolumn{2}{|c|}{$-\square \times$} \\
\hline Mineral & Select & $\mathrm{K}[\mathrm{tcu}]$ & $\mathrm{K}[\mathrm{W} / \mathrm{m}-\mathrm{K}]$ & $\Delta$ \\
\hline Chlorite & $\sqrt{\nabla}$ & 12.3 & 5.13 & \\
\hline Diopside & $\Gamma$ & 11.79 & 4.91 & \\
\hline Dolomite & $\Gamma$ & 13.16 & 5.48 & \\
\hline Epidote & Г & 6.7 & 2.79 & \\
\hline Fluorite & $\Gamma$ & 22.72 & 9.47 & \\
\hline Forsterite & Г & 12.3 & 5.13 & \\
\hline Gibbsite & $\Gamma$ & 6.21 & 2.59 & \\
\hline Gypsum & $\Gamma$ & 3 & 1.25 & \\
\hline Halite & Г & 14.6 & 6.08 & \\
\hline Hornblend & $\Gamma$ & 6.71 & 2.8 & \\
\hline Hematite & $\Gamma$ & 26.95 & 11.23 & \\
\hline Illite-Mica & $\sqrt{\nabla}$ & 5.5 & 2.29 & \\
\hline Ilemenite & $\Gamma$ & 5.7 & 2.38 & \\
\hline Kaolinite-Sericite & $\sqrt{\nabla}$ & 6.6 & 2.75 & \\
\hline K-Feldspar & $\sqrt{\nabla}$ & 5.7 & 2.38 & \\
\hline Magnetite & $\Gamma$ & 12.18 & 5.08 & \\
\hline Magnesite & $\Gamma$ & 13.94 & 5.81 & \\
\hline Microcline & $\Gamma$ & 5.95 & 2.48 & \\
\hline Mixed layer clay & $\Gamma$ & 4.44 & 1.85 & \\
\hline Muscovite & $\Gamma$ & 5.54 & 2.31 & \\
\hline Olivine & $\Gamma$ & 11.6 & 4.83 & \\
\hline Oligioclase & $\Gamma$ & 4.71 & 1.96 & \\
\hline Orthoclase & $\Gamma$ & 5.53 & 2.3 & \\
\hline Plagioclase & $\sqrt{\nabla}$ & 5 & 2.08 &. \\
\hline
\end{tabular}

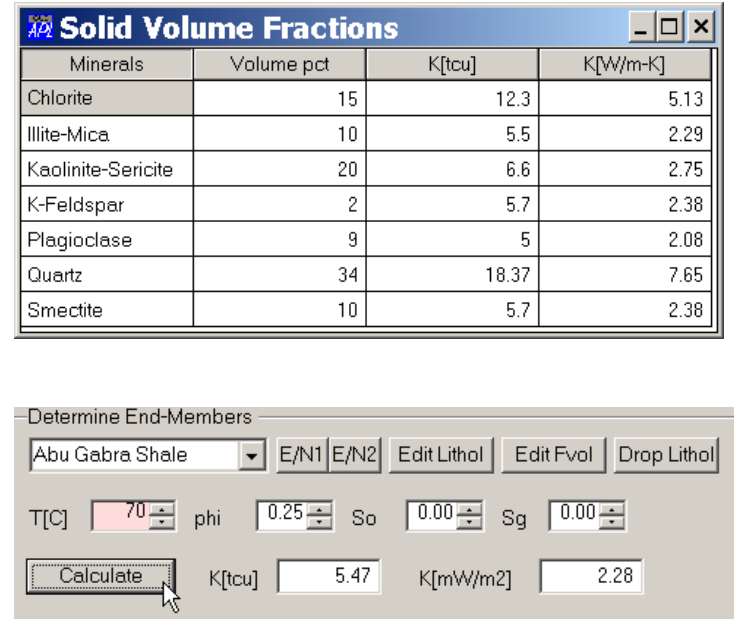

Figure 114. A sediment can be designed by assigning its mineralogy, the solid volume fraction of those minerals, the porosity, and the oil and gas saturation fractions. This is done as described in the text using the lower group of the Thermal Conductivity Scratchpad form shown in Figure 113. Here we show a portion of the mineral selection and volume fractions assigned for the Abu Gabra shale. Depressing the Calculate button for the parameter selections shown gives the result shown in the third panel.

\section{Heat Generation}

Heat generated from the decay of radioactive minerals contributes significantly to basin heat flow. The Parameters: Heat Generation (+scrpad) allows a user to assess this contribution. The heat generation used in the modeling is controlled by the "AT" parameter on the form; the heat generation rate for each lithologic end-member is measured in $\mathrm{nW} / \mathrm{kg}$. The form shown in Figure 115 allows a user to determine reasonable values for of this term from the chemistry of the lithologic end members in an area or from the gamma log response. All parameters are stored in the Case 
Parameters file and are thus recorded. However, when it comes to the calculations only the AT[nW/kg] are needed and used.

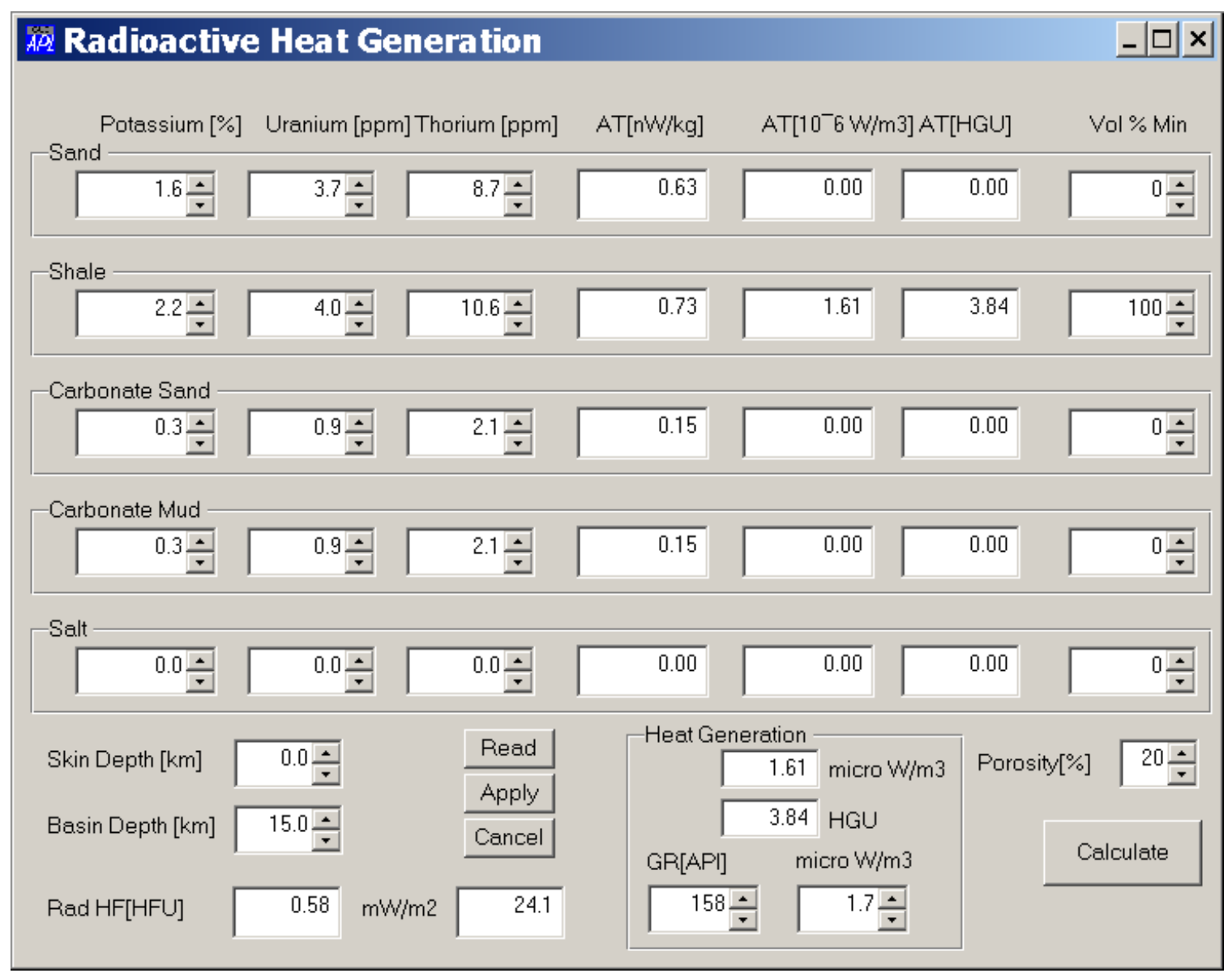

Figure 115. Radioactive Heat Generation form produced by menu command Parameters: Heat Generation (+scrpad). Heat generation is specified by specifying the $\mathrm{Wt} \%$ potassium and the ppm uranium and thorium in a basin's sediments. Depressing the Calculate button then calculates the heat generation in cgs heat generation (HGU) and mks units. If a basin depth is indicated, the radiogenic heatflow in HFU and $\mathrm{mW} / \mathrm{m} 2$ is also calculated. If the skin depth (the depth below the basin surface) is zero radiogenicity is assumed not to decay with depth. Otherwise the radiogenicity is decreased with depth such that it is $1 / \mathrm{e}$ at the skin depth specified. The heat generation expected from the gamma tool response in API units can be estimated by dialing in an average gamma tool reading. Alternatively the gamma tool response expected for a particular heat generation rate can be determined by spinning the heat generation spinner in the Heat Generation group. The relations used are from (Bucker and Rybach 1996).

\section{Fault Permeability}

Fault permeability can presently be specified by one of 4 different algorithms. 
1. Fault Gauge

2. Adjacent Lithology

3. Total Fault Throw

4. Slip Rate

The algorithms depend on the 4 fault parameters defined in Figure 116.

1. Fc fraction coarse in red interval equals the fraction coarse in the interval slipped past, D

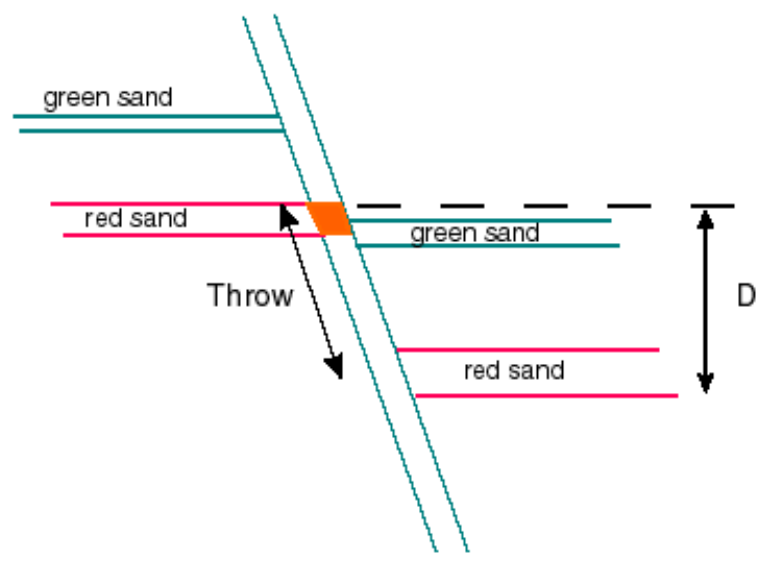

2. Fcc the fraction coarse adjacent to left hand red sand is $1 / 1+0.5=0.666$. E.g., the adjacent coarse fraction is $100 \%$ from the left and $50 \%$ from the right.

3. The throw, Te, of an element is the offset of the midline of the strata.

4. The slip rate, Se, of a strata is the incrimental throw over a timestep divided by the timestep.
Figure 116. Parameters for fault permeability are defined in the figure.

- The fault gauge fraction coarse of a fault element is defined as the fraction coarse of the elements slipped past the element

- The adjacent fraction coarse averages the coarse fraction at each side of the element.

- The throw of an element is the displacement of its midpoint on each side of the fault.

- The slip rate is the increase in throw over a timestep divided by the timestep.

Fault elements should be thought of as elements with horizontal top and bottom attached to the nodes on left side of the fault.

The Fault Gauge and Adjacent Lithology models define the fault-parallel and faultperpendicular permeabilities: 


$$
\begin{aligned}
& k_{\text {parallel fault }}=F_{c}^{e} k_{\text {coarse fault }}+\left(1-F_{c}^{e}\right) k_{\text {fine fault }} \\
& k_{\text {perpendicular fault }}=1 /\left(F_{c}^{e} / k_{\text {coarse fault }}+\left(1-F_{c}^{e}\right) / k_{\text {fine fault }}\right)
\end{aligned}
$$

$F_{c}^{e}$ is the fraction coarse in the fault element calculated either as the fraction coarse in interval $\mathrm{D}$ in Figure 116 (fault gauge model), or as the adjacent fraction coarse $\left(\mathrm{F}_{\mathrm{cc}}\right.$ in Figure 116).

The Total Throw permeability is calculated:

$$
\log k_{\text {parallel fault }}^{\text {throw }}=\log k_{\text {perpendicular fault }}^{\text {throw }}=\log k_{\text {fine fault }}+3 T_{e},
$$

where $\mathrm{T}_{\mathrm{e}}$ is measured in kilometers.

The Slip Rate permeability model is defined:

$$
\log k_{\text {parallel f fault }}^{\text {sliprate }}=\log k_{\text {perpendicular fault }}^{\text {slipate }}=\log k_{\text {fine fault }}+3 S_{e},
$$

where Se is the slip rate in $\mathrm{km} / \mathrm{Ma}$. In the above equations

$$
\begin{aligned}
& \log k_{\text {fine fault }}=-3 \text {, and } \\
& k_{\text {coarse fault }} \text { is defined by the user (see Figure 117). }
\end{aligned}
$$

The fault permeability models and the parameters upon which they depend can be examined using the Parameters: Permeability: Examine Section Permeability form. Figure 117 illustrates the use of this form for examining fault permeability. Fault permeability is displayed as a strip down the fault in which a horizontal base and top joined to the left side of fault elements extend across the fault and are connected to from a parallelepiped. 


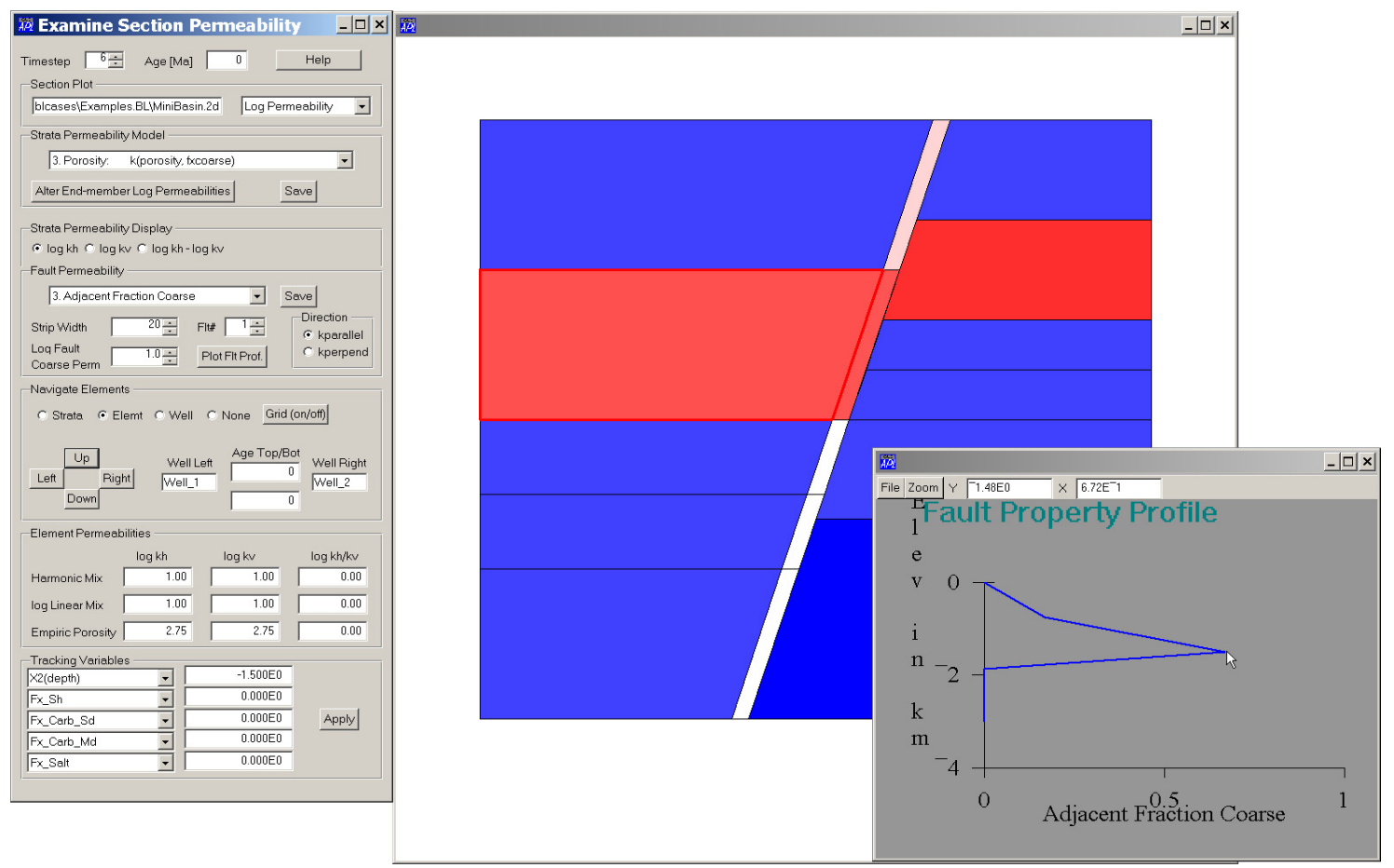

Figure 117. Fault permeability of the fault in the blcases/Examples.BL/MiniBasin.2d case illustrated in the left panel of Figure 89. Fault permeability is examined using the Parameters: Permeability (+scrpad): Examine Section Permeability form. The Adjacent Fraction Coarse fault property has been selected. Its profile down the fault is shown in the insert (obtained by depressing the Plot Flt Prof button. The adjacent fraction coarse is 0 for the first three elements, and zero at the top. It is maximum at the second element down from the top. The base of this element lies at $-1.5 \mathrm{~km}$ (x2 Tracking Variable in the form). The fraction coarse there is 0.67 ( $\mathrm{x}$ value on the toolbar). Figure 118 shows the Adjacent Fraction Coarse and Sliprate fault permeabilities for this case.

10. Permeability: Slip Rate 

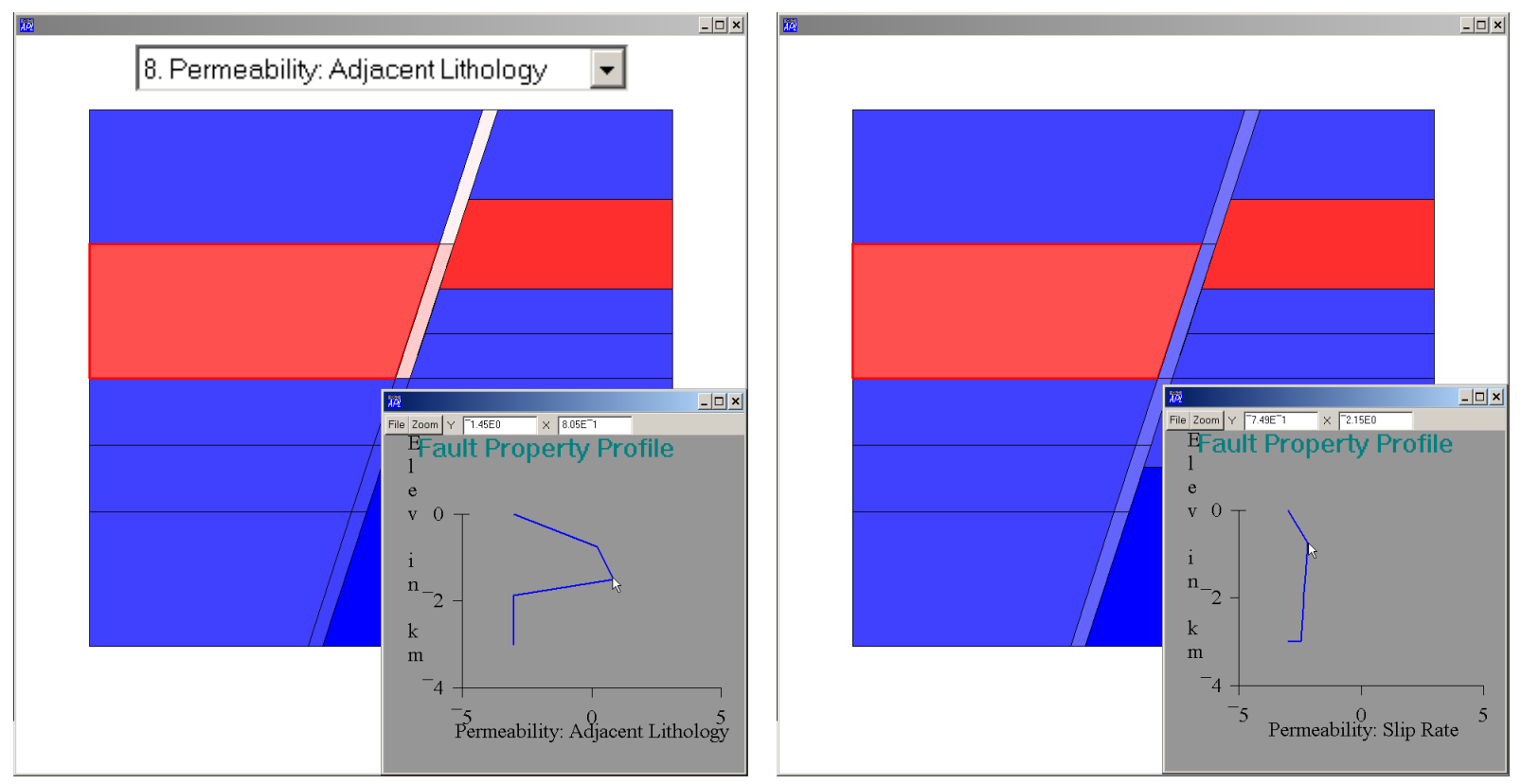

Figure 118. Adjacent Fraction Coarse and Sliprate permeability of the fault in the blcases/Examples.BL/MiniBasin.2d case shown in Figure 113. The Sliprate permeability is low because the fault slip rate is small $(<0.26 \mathrm{~km} / \mathrm{Ma})$. We have pasted the fault model selected on the cross section images of this figure. Profile plots show how permeability varies with depth along the fault and are created using the Plot Flt Prof button on the Examine Section Permeability form in, for example, Figure 117.

\section{Kinetic Maturation Models}

Maturation models are input from askii text files. A set produced by - $\supset$ blcases

†. Ageo_Tex_Nigeri.BL

$\oplus \bullet$ Champlain.BL

PCustom Kinetics

$\exists$ Documentation s

+... Examples.BL

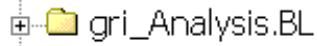

Yongchun Tang at CalTech with funding from

the same DoE grant that supported conversion and development of this program is distributed here in a folder called Custom Kinetics. $A$ convenient place to store this folder is in the blcases folder as shown on the left. The kinetic askii text file for the Green River shale

maturation kinetic model is given in Figure 119. 


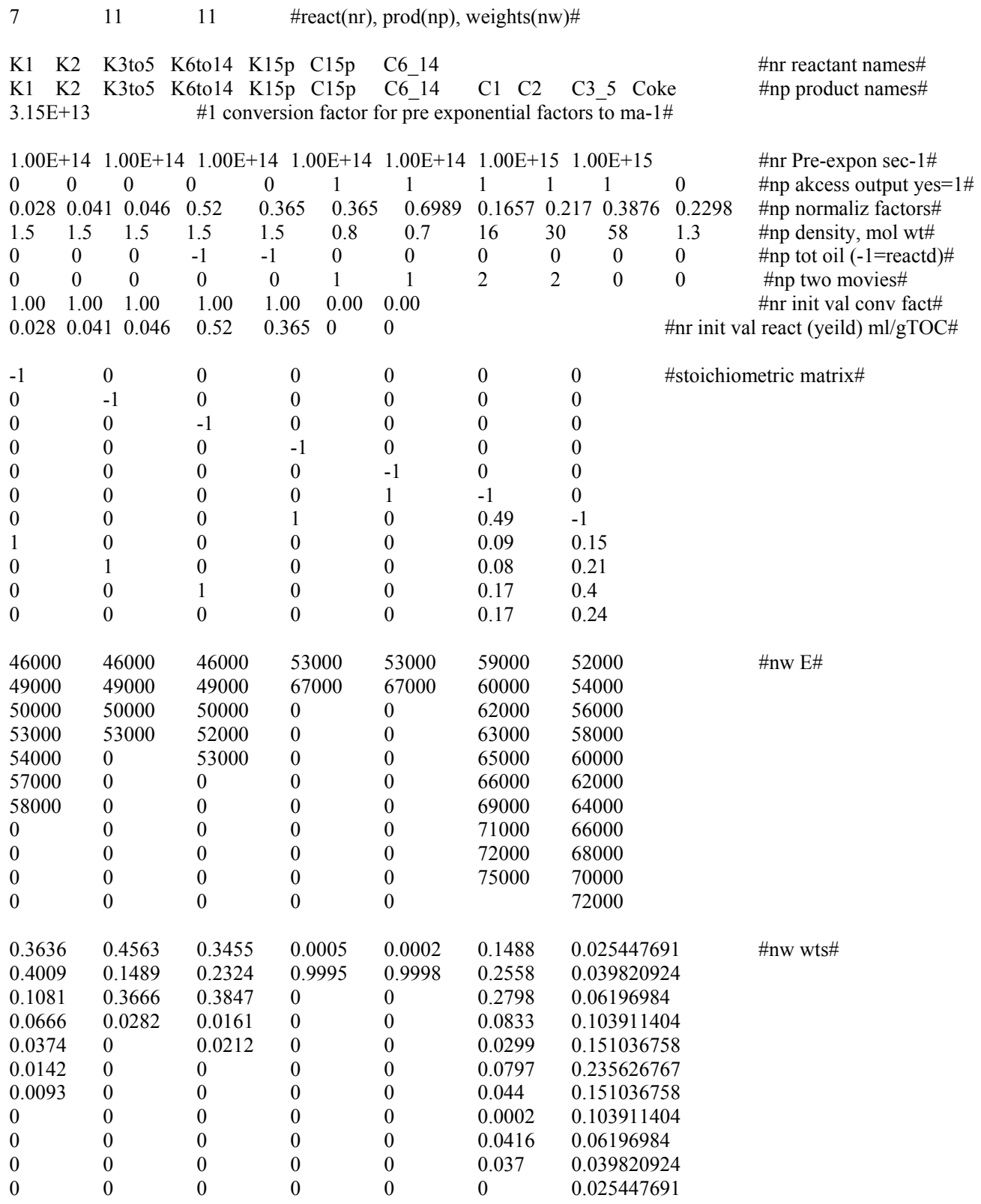

Figure 119. Green River shale Kinetic maturation file. The file is documented with comments enclosed in pound (\#) signs. The first line gives the number of reactants, products, and the (maximum) number of activation energy weights. The second and third lines give the names of the reactants and products. The carbon number range of the products is given in the name connected by an underscore. This convention communicates the range to BasinLAB. BasinLAB uses units of $\mathrm{Ma}$ in its maturation calculations. Hence the $4^{\text {th }}$ line gives the conversion from $1 /$ seconds to Ma. The $5^{\text {th }}$ line gives the pre-exponential for all 7 reactions in $1 /$ seconds. The $6^{\text {th }}$ line can be ignored; it communicates variables to be written to output files in workstation calculations. The $7^{\text {th }}$ line gives the maximum possible mass of the reactant or product. It is the initial value of the kerogen (mass fraction of the total) and the total products that could be produced if all decomposition reactions were turned off. These values are computed in BasinLAB and can be copied into the kinetics file from there. The $8^{\text {th }}$ line gives the density of the solid 
and the average molecular weights of the gaseous products. Basin $\boldsymbol{L A B}$ uses it to distinguish solid from liquid products (liquid petroleum is assumed to have a density $<1$ or $>2$ ). The $9^{\text {th }}$ and $10^{\text {th }}$ lines are specific to the workstation and of no concern here. The $12^{\text {th }}$ line gives conversion factors; the $13^{\text {th }}$ initial reaction masses. The remaining lines give the stoichiometric decomposition matrix and the activation energies and their weights. 
The maturation model in Basin $\boldsymbol{L A B}$ is standard, but very flexible in its ability to handle almost any kind of maturation model. The initial mass of reactive components, $r$, is distributed into bins with assigned activation energies according to a set of weights, $\mathrm{W}_{\mathrm{r}}$. For each reactive component (whether kerogen or hydrocarbon derived from kerogen) there are $\mathrm{w}$ weights which sum to 1 . The number of weights for reactive components can be different. Each reactive component has a pre-exponential factor $A_{r}$. $A_{r}$ is the same for all the activation energy bins of a reactive component (e.g., $A_{w r}=A_{r}$ ).

Initial reactive mass fractions, are distributed according to their activation energy bin weights:

$$
X_{w r}^{0}=\bar{W}_{r} X_{r}^{0} .
$$

The summing convention does not apply in this equation. The vector $W_{r}$ is simply multiplied by the initial mass fraction of reactive component $r$ to obtain the initial mass fractions of $r$ in each activation energy bin of $r$.

The reactive components are then matured assuming Arhennius kinetics:

$$
\frac{\partial X_{w r}}{\partial t}=A_{w r} X_{w r} \exp \left(E_{w r}^{*} / R T\right),
$$

where $\mathrm{R}$ is the gas constant, $\mathrm{T}$ is temperature in degrees Kelvin, and $E_{w r}^{*}$ is the activation energy of each bin, w, of each reactive component. This equation is integrated over time using a second order Runge Kutta (e.g., (Hildebrand 1962)) version of the equation shown below:

$$
\Delta X_{w r}^{t}=\Delta t A_{w r} X_{w r}^{t-1} \exp \left(E_{w r}^{*} / R T\right) .
$$

This equation is a component equation that applies to each individual value of the $\mathrm{w}$ and $\mathrm{r}$ subscripts (summing is not implied by repeated indices). It gives $\Delta X_{w r}^{t}$ from $X_{w r}^{t-1}$. We assure that the mass fraction in each bin is $\geq 0$ :

$$
\Delta X_{w r}^{t}=\max \left|\Delta X_{w r}^{t},-X_{w r}^{t-1}\right| .
$$

The non-reactive components are then computed:

$$
\begin{aligned}
& \Delta X_{r}^{t}=\sum_{w} X_{w r}^{t} \\
& X_{w p}^{t}=X_{w p}^{t-1}+W_{w p} S_{w p}^{\prime} \Delta X_{r}^{t}+\Delta X_{w r}^{t},
\end{aligned}
$$

where $\mathrm{S}^{\prime}{ }_{\text {wp }}$ is the stoichiometric matrix with the depletion of the reactive components removed (they are included in $\Delta X_{w r}^{t}$ term). 
The path to kinetic models previously used in a case are recorded in the Case Parameters file (saved in File: Save Case Parameters), and these kinetic models are read into the workspace when the case is opened. The kinetic models in the workspace can be determined by holding the mouse over the Parameters: Read or Edit Kinetics: HC Kinetics (kerl): Select as indicated in Figure 120.

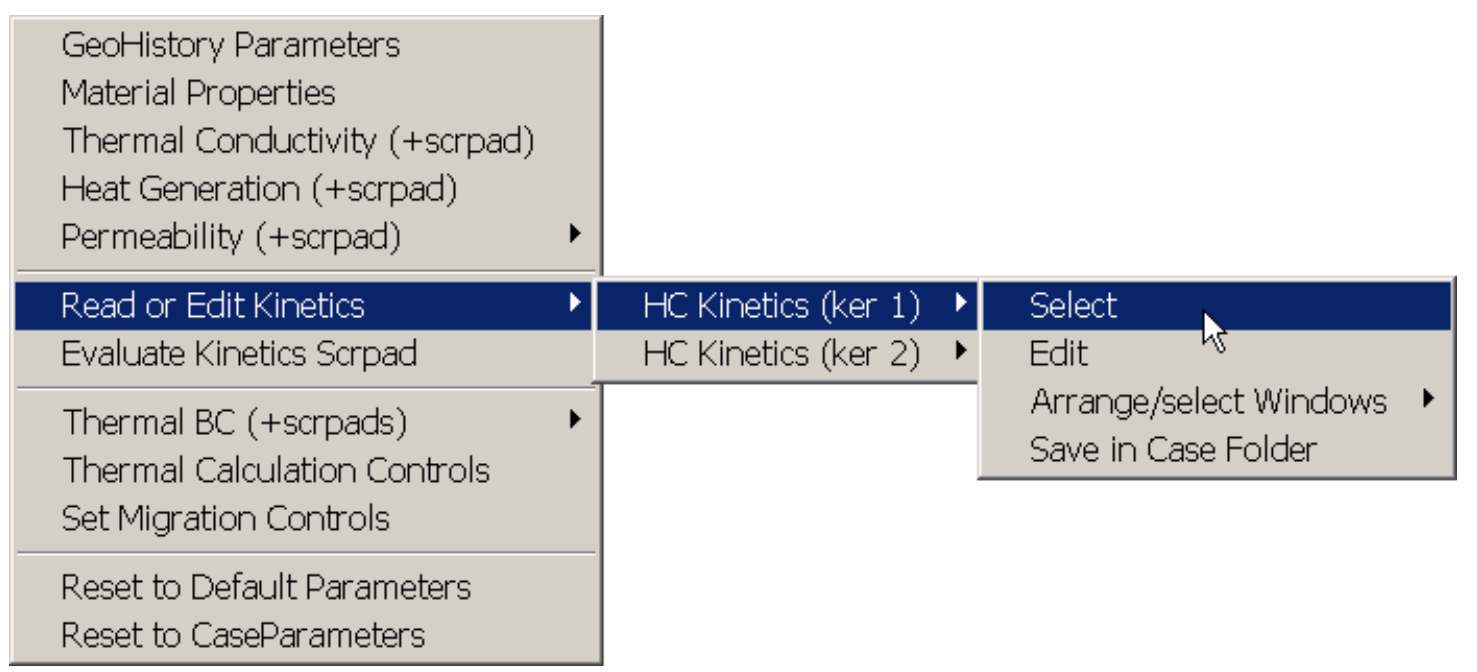

Read file specifyinq hydrocarbon maturation stoichiometry and kinetics. Current kinetic model is: Burnham and SweeneyCBS II

Figure 120. Holding the mouse over the Select or Edit kinetic menus shows the kinetics currently in the workspace. Choices can be changed by selecting a different kinetic file using the navigator in Select. The selected kinetics files can be changed using the Edit menu (see Figure 121). The changes apply to the session only. The kinetic files must be edited in Excel (and saved as tab-delimited text files, and then the " inserted by Excel removed using the replace function in Notepad) to make any changes permanent. 


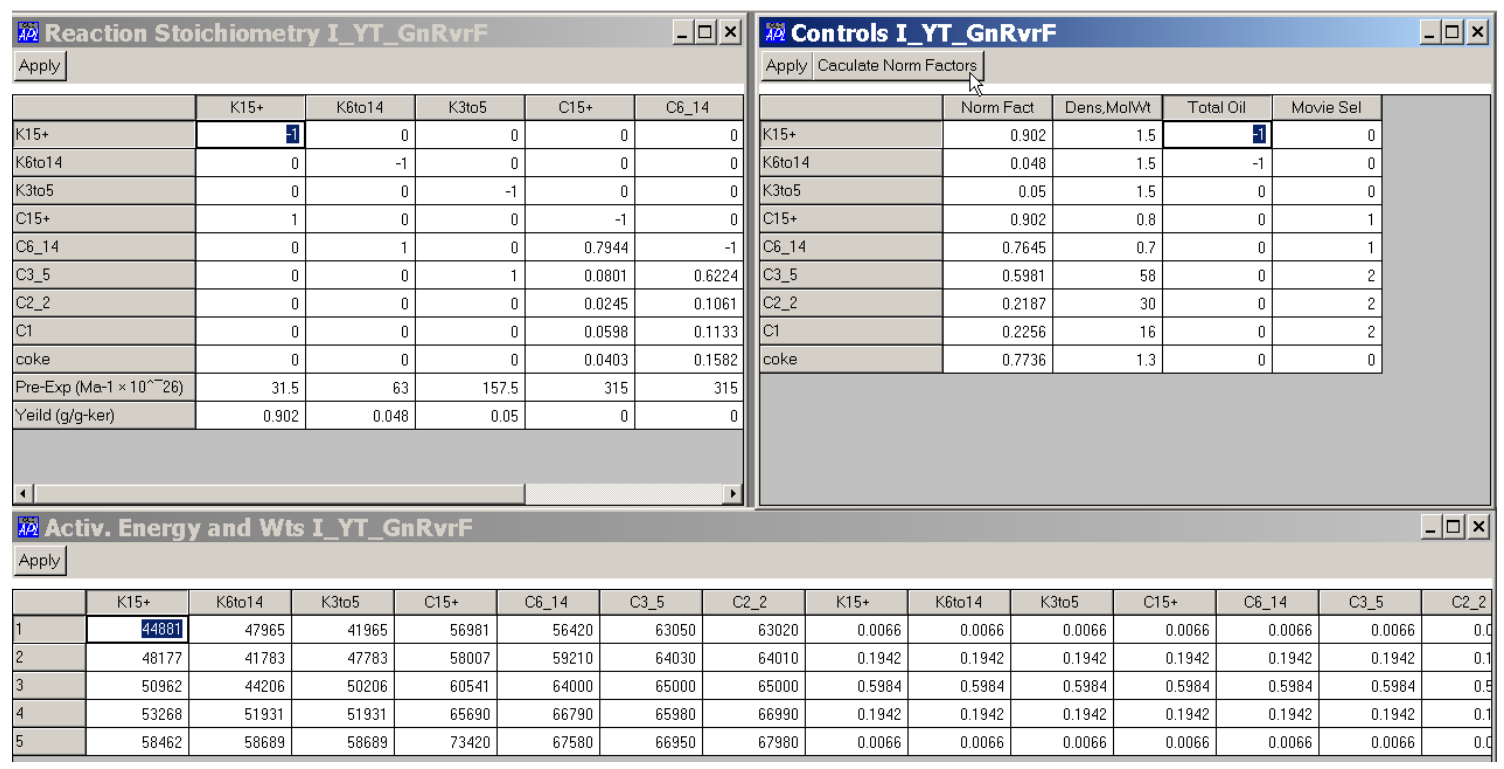

Figure 121. Edit version of the Green River kinetics file shown in Figure 119. Any of the data can be modified and applied to the current case. The Calculate Norm Factors button calculates the maximum possible amounts of all species as discussed in the text.

The Parameters: Evaluate Kinetics menu command (see Figure 122) allows kinetic models to be compared for specified uniform heating rates. This form operates in a fashion similar to the ones that compare kinetic models in pseudo $1 \mathrm{D}$ and 2D thermal simulations (see discussion of Figure 27 and Figure 39). A comparison of two Type II kinetic models for a uniform heating rate of $2^{\circ} \mathrm{C} / \mathrm{Ma}$ is shown in Figure 122.

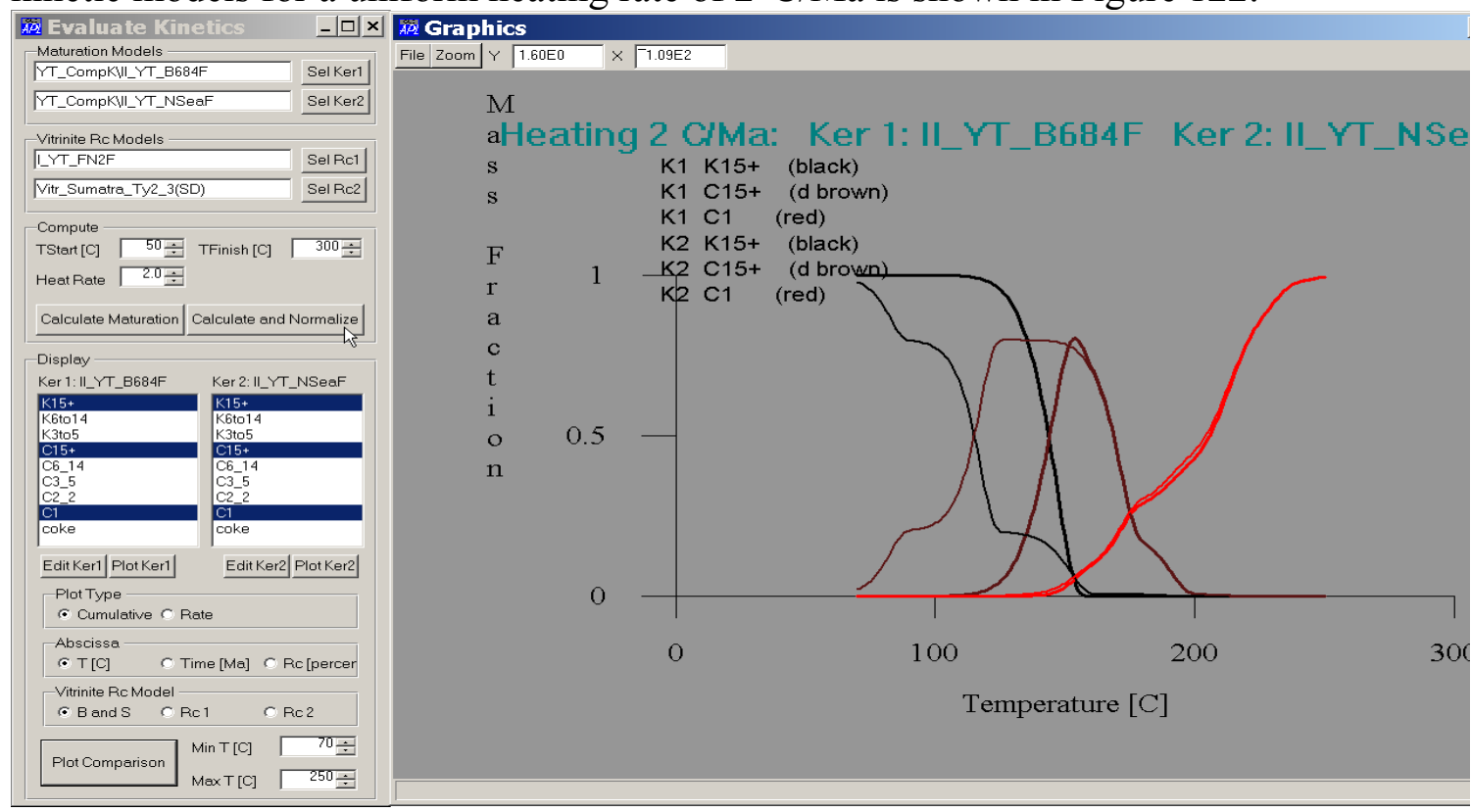

Figure 122. Comparison of two Type II kinetic models using the Parameters: Evaluate Kinetics menu command. The products are normalized to their maximum possible buttons because the maturation was computed using the Calculate and Normalize button (under mouse). The two models differ mainly in the timing of $\mathrm{K} 15+$ conversion to $\mathrm{C} 15+$. 
The production curves for these kerogens at the depth of the Jurassic Source in our Gulf of Mexico section is shown in Figure 39. A pseudo 2D example is shown in Figure 28 and Figure 29.

The maturation calculations use a time-refined version of the time-temperature time series delivered from the pseudo 2D or 2D finite element models (or the uniform heating rate). This refinement is specified in the form summoned by the Parameter: Thermal Calculation Controls menu (see Figure 116). This control (Figure 123) allows the user to set the maximum timestep allowed in maturation calculations.

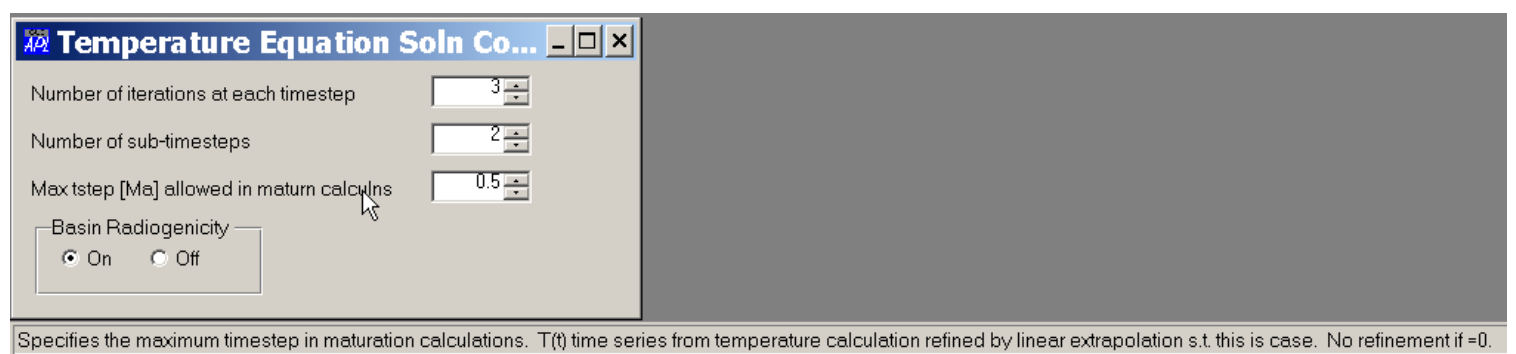

Figure 123. The maximum timestep allowed in maturation calculations is set in the Parameters: Thermal Calculation Controls form. The time series is linearly interpolated and sub-divided to meet this criterion.

\section{E. Temperature Boundary Conditions}

\section{Surface Temperature and the Ocean Thermocline}

The temperature in a basin is strongly influenced by the temperature of its surface. In

\begin{tabular}{l|l|} 
GeoHistory Parameters \\
Material Properties \\
Thermal Conductivity (+scrpad) \\
Heat Generation (+scrpad) \\
Permeability (+scrpad) \\
\hline \begin{tabular}{l|l} 
Read or Edit Kinetics \\
Evaluate Kinetics Scrpad
\end{tabular} \\
\hline Thermocline and Basal HF \\
\hline Thermal Calculation Controls \\
Set Migration Controls \\
\hline Reset to Default Parameters \\
Reset to CaseParameters
\end{tabular}

Basin $L A B$, surface temperature is a function of water depth. The Parameters: Thermal BC (+scrpads) menu allows design of the thermocline that establishes the functional dependence between temperature and water depth. Figure 124 shows how a basin's surface temperature is controlled by a user-defined thermocline. The thermocline may be linear or

exponential. The surface temperature is a single "land" temperature if the surface elevation is positive (above sea level). 


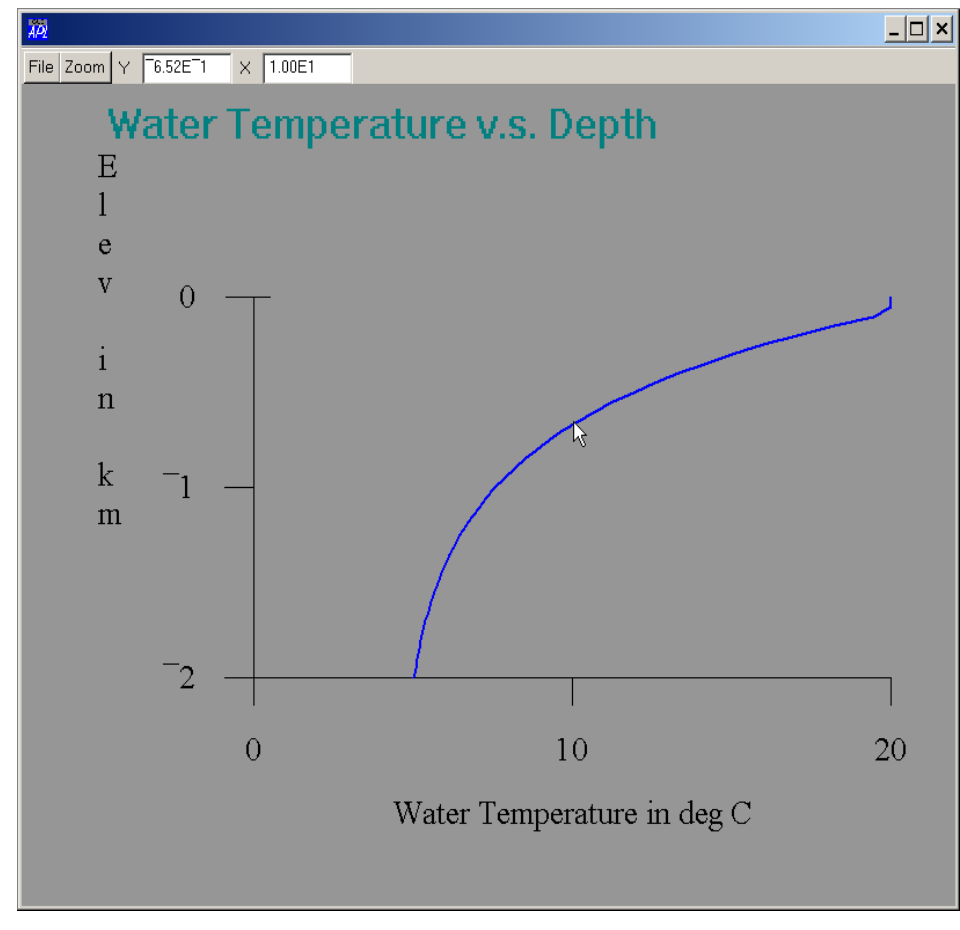

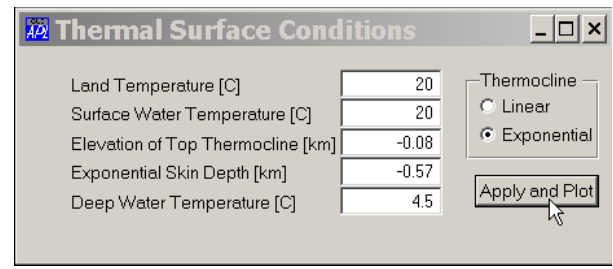

Figure 124. The Parameters:

Thermocline and Basal HF: Define Thermocline form allows design of an ocean thermocline that is either linear or exponential. In this case an exponential thermocline starts at $80 \mathrm{~m}$ water depth and exponentially approaches a deep water temperature of $4.5^{\circ} \mathrm{C}$ :

$$
T=T_{\text {surface water }}+\left(T_{\text {sw }}-T_{\text {deep } w}\right)\left(1-e^{z / \delta}\right)
$$

where $\delta$ is the skin depth in $\mathrm{km}$. The thermocline translates water depth to basin surface temperature in all $\boldsymbol{B a s i n} \boldsymbol{L A B}$ calculations

\section{Table Input of Basal Heat Flow and Water Depth}

The heatflow.txt and waterdepth.txt files allow water depth and heat flow at a few times in a basin's history to be input and interpolated to determine the heatflow and water depths over the full basin history. In each case the data is input at selected age horizons of the first and last pseudowells and any number of pseudowells in between. The data is then linearly interpolated both spatially (along the horizons) and temporally (between strata). The interpolated values are placed in the SFLAT file.

Figure 125 illustrates the format and input of these table files. 
heatflow.txt:

\begin{tabular}{lllllllll}
7 & 8 & \multicolumn{7}{c}{ \#nbr hf pseudowells, nbr hf strata\# } \\
1 & 5 & 11 & 15 & 19 & 25 & 26 & & \#hf wells\# \\
1 & 3 & 8 & 16 & 19 & 23 & 25 & 27 & \#hf strata list\# \\
0.59 & 0.52 & 0.49 & 0.46 & 0.5 & 0.51 & 0.51 & & \#HF[hfu] \\
0.59 & 0.52 & 0.5 & 0.47 & 0.51 & 0.53 & 0.53 & \\
0.61 & 0.53 & 0.53 & 0.54 & 0.69 & 0.76 & 0.76 & \\
0.68 & 0.78 & 0.85 & 0.87 & 0.88 & 0.88 & 0.88 & \\
1.07 & 1.05 & 1.04 & 1.03 & 1.03 & 1.02 & 1.02 & \\
1.46 & 1.47 & 1.47 & 1.46 & 1.45 & 1.47 & 1.47 & \\
3.37 & 3.51 & 3.32 & 3.38 & 3.47 & 3.47 & 3.47 & \\
3.96 & 4.43 & 4.24 & 4.4 & 4.44 & 4.44 & 4.44 & &
\end{tabular}
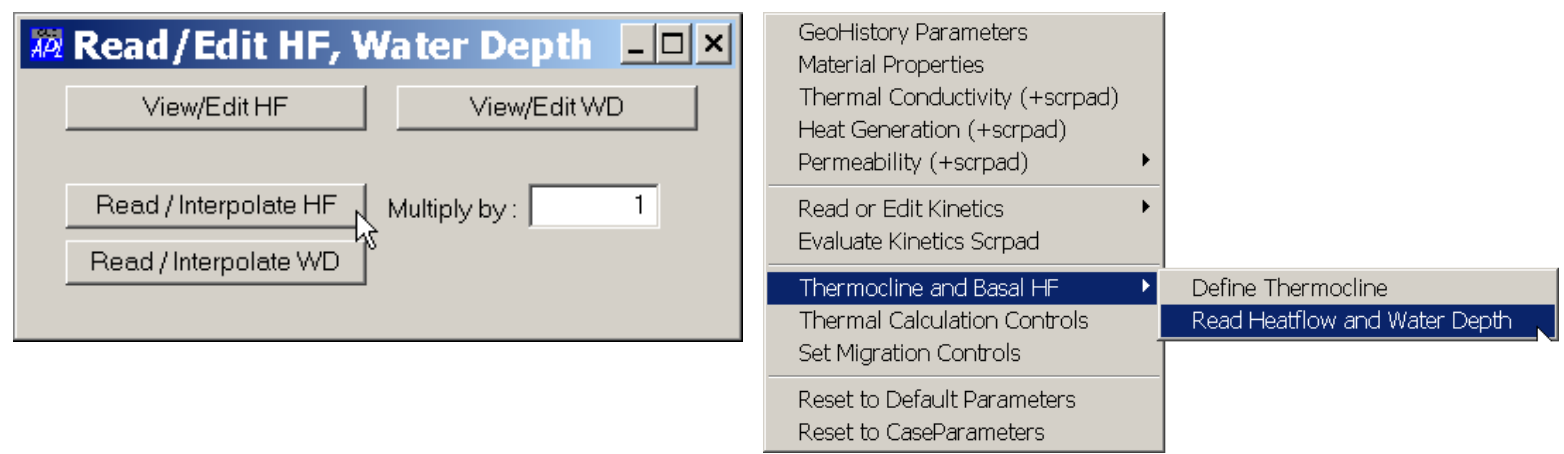

Figure 125. The heatflow.txt and waterdepth.txt files have the same format. An example of a heatflow.txt file is shown above. The first line of the file gives the number of pseudowells and strata at which heatflow is defined. The next two lines gives the number of the pseudowells (from the left) and strata (from the top) at which heatflow is defined. The next remaining lines are a table of heatflow data nwell columns and nstrata rows. The heatflow.txt and waterdepth.txt files are read by the Parameters: Thermocline and Basal HF: Read Heatflow and Water Depth menu command as illustrated. The resulting form allows the files to be read, and the data consequently placed into the SFLAT file to be read and edited as illustrated in Figure 94.

\section{F. Controls for 1D Thermal and Migration Calculations}

Figure 126 sets $1 \mathrm{D}$ thermal calculation and 2D migration controls. The former apply to maturation and temperature calculations that are calculated for a single well. Figure 127 sets parameters that control petroleum migration.

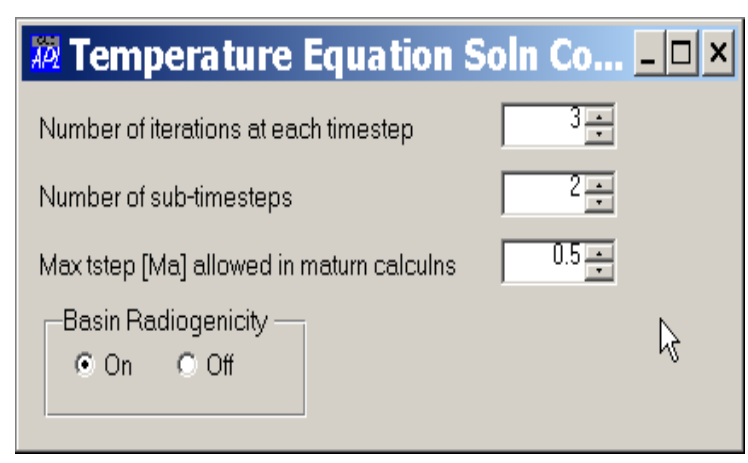

Figure 126. This form from Parameters: Thermal Calculation Controls mainly sets controls for 1D thermal calculations in a single well, but also allows radioactive heat generation to be turned off, and controls the refinement of the T-t timeseries in maturation calculations by setting the maximum timestep allowed in them. The number of iteration in a timestep refers to the iteration loop, which converges thermal conductivity with temperature. 
Sub-timesteps are the number of intervals each timestep is subdivided into for calculation purposes.

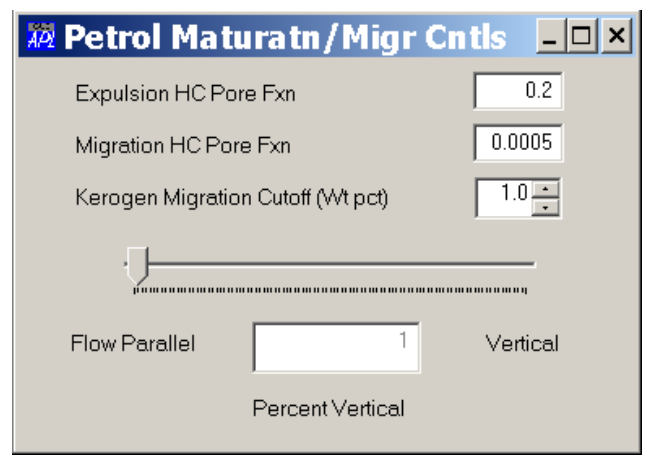

Figure 127. This form from Parameters: Set Migration Controls sets the pore saturation required for hydrocarbons to migrate from source stratum, and the hydrocarbon saturation required to migrate through stratum above the source stratum. It also sets the kerogen cutoff grade. Kerogen maturation is not computed below this amount of kerogen.

\section{G. Saving Control Parameters}

All of the parameters that control how a case is processed are saved in the system CaseParameters file. Parameters are written to this file, using File: Save

\begin{tabular}{|l|}
\hline GeoHistory Parameters \\
Material Properties \\
Thermal Conductivity (+scrpad) \\
Heat Generation (+scrpad) \\
Permeability (+scrpad) \\
\hline Read or Edit Kinetics \\
Evaluate Kinetics Scrpad \\
\hline Thermocline and Basal HF \\
Thermal Calculation Controls \\
Set Migration Controls \\
\hline Reset to Default Parameters \\
\hline Reset to CaseParameters \\
\hline \hline
\end{tabular}

CaseParameters. The parameters that have been written to the CaseParameters file can be retrieved using the Parameters: Reset to CaseParameters menu. Or the parameters can be set to a standard default set using the Parameters: Reset to CaseParameters menu.

One thing to especially note is that the CaseParameters file saves the path to the kinetic maturation models used in a case, not the data itself. If the Custom Kinetics folder is moved or the kinetic files re-labeled, the case will not be able to find the kinetic data. The CaseParameter file is automatically read when a case is opened. 


\section{Verifying the Basin Heatflow History}

Heatflow and Maturation

Evaluate Heatflow

Evaluate Maturation
This section is accessed from the Heatflow and Maturation menu. It consists of only two items whose functionality has already been described. For Evaluate Heatflow see Figure 22, and for Evaluate Maturation see Figure 27.

- Section V.C (p. 182) describes how maturity models can be used to verify the time-temperature history of a basin

- Section V.D (p. 187) describes how hydrocarbon maturation is calculated in a single well, for a selected element (V.F p. 195), and for an entire 2D section (V.H p. 202).

- The kinetic maturation models have been described in Section VII.D (p. 274).

Here we focus on the input files and databases that have not been previously described, and fill in some computational details.

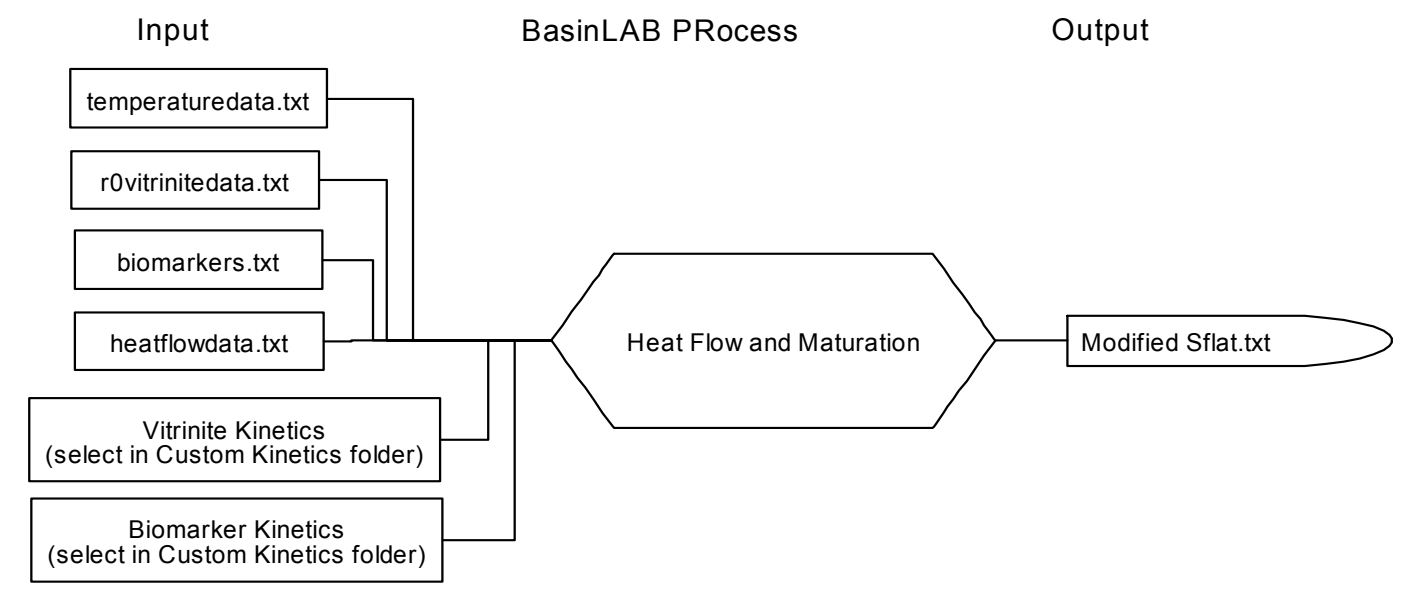

Chart 3. Input files for the Heat Flow and Maturation section of BasinLAB. Box conventions are the same as described in Chart 1. Charts 4 and 2 are on pages 293 and 232.

\section{A. Time-temperature History Confirmation}

Thermal conductivity, the heat flow history, and the history of surface temperatures combine to determine the model time-temperature history of the basin. All these parameters are uncertain to some degree, and therefore it is useful and usual to confirm their combined validity by calculating the conversion of various maturity indicators and comparing these calculations to measured data in the basin, and also by comparing computed temperatures to measured temperatures.

\section{Temperature and Maturity Data Input}

Maturity data is input to $\boldsymbol{B a s i n} \boldsymbol{L} \boldsymbol{A B}$ by ascii text files. Temperature, vitrinite reflectance, and biomarker data are input through the 
- temperaturedata.txt,

- rOvitrinitedata.txt, and the

- Biomarkers.txt

files. These files are all of very similar format as detailed in Figure 128. If present in a case folder, these files are read automatically when the Evaluate Heatflow menu command is issued, and are displayed automatically on the Evaluate/Adjust Heat Flow form as shown in Figure 22.

\begin{tabular}{|c|c|c|c|c|c|}
\hline $\begin{array}{l}1 \\
3\end{array}$ & 2 & 1 & 0 & 0 & $\begin{array}{l}\text { \#m or } \mathrm{ft}, \mathrm{C} \text { or } \mathrm{F}, \mathrm{Tmul} \text {,Tadd,zmul,zadd\# } \\
\text { \#number of wells with data\# }\end{array}$ \\
\hline 1 & 7 & \$Humble_21\$ & & & \\
\hline 3931.92 & 190 & & & & \\
\hline 4084.32 & 195 & & & & \\
\hline 4389.12 & 229 & & & & \\
\hline 4815.84 & 245 & & & & \\
\hline 5151.12 & 290 & & & & \\
\hline 5425.44 & 320 & & & & \\
\hline 5791.2 & 330 & & & & \\
\hline 5 & 7 & \$Atlantic\$ & & & \\
\hline 365.76 & 90 & & & & \\
\hline 1310.64 & 118 & & & & \\
\hline 3017.52 & 175 & & & & \\
\hline 4023.36 & 192 & & & & \\
\hline 4754.88 & 233 & & & & \\
\hline 5303.52 & 284 & & & & \\
\hline 5486.4 & 305 & & & & \\
\hline
\end{tabular}

Figure 128. First two wells of the temperaturedata.txt file in the gri_Analysis.BL/McBride_lst ${ }^{\text {st }}$ third.2d case. The first line indicates whether the depth data are in meters of feet, the temperatures in $\mathrm{C}$ or $\mathrm{F}$, and allows the temperature and depth data to be modified by multiplying by or adding a constant. The next line gives the total number of wells with data. The following lines give the pseudowell number, the number of data points in that well and the name of the well (enclosed in \$ signs, not \# signs). The total vertical depth below seal level and the temperatures are then listed.

\begin{tabular}{|llllll|}
\hline 1 & 0 & 0 & 0 & 0 & $\# \mathrm{~m}(=1)$ or $\mathrm{ft}(=2)$, r0mul, r0add, zmul, zadd \# \\
1 & & & & \\
1 & 2 & \$Humble\$ & & \\
4267.2 & 0.58 & & & \\
5486.4 & 0.75 & & & \\
\hline
\end{tabular}

Figure 129. The r0vitrinitedata.txt file for the gri_Analysis.BL/McBride_ ${ }^{\text {st }}$ _third.2d case. Same comments as in Figure 128 apply. 


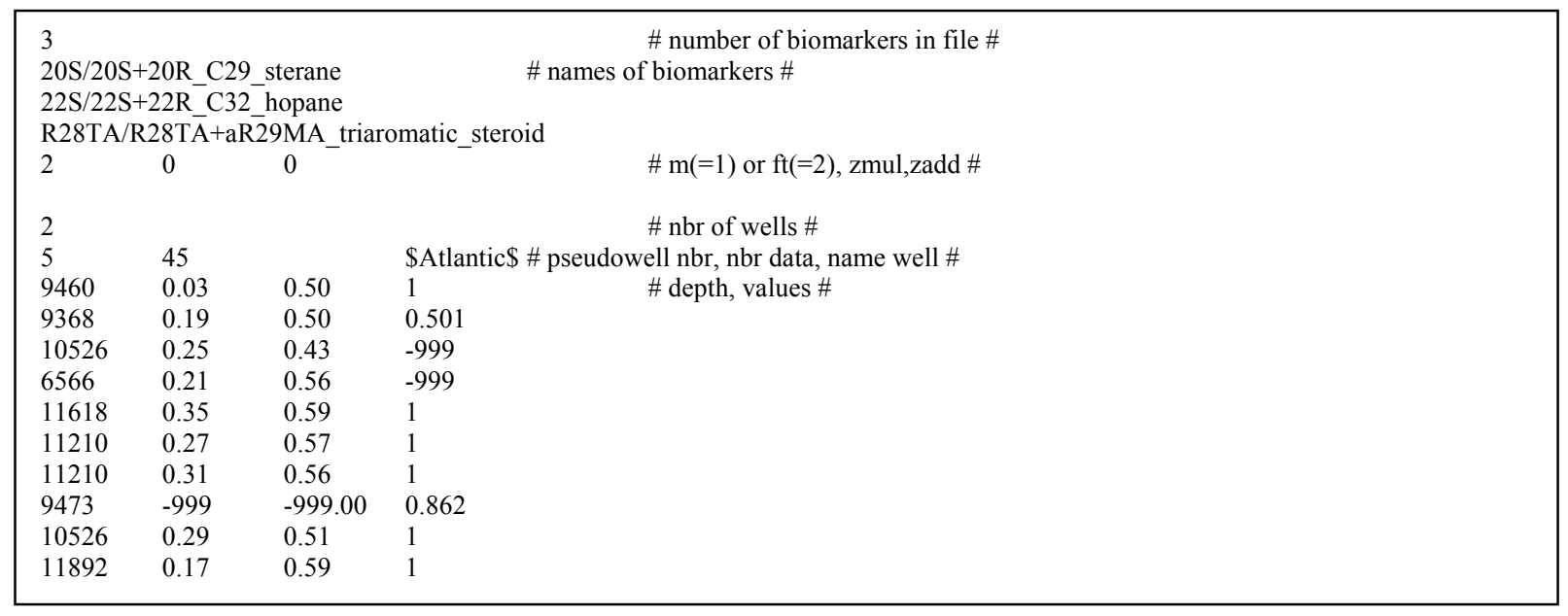

Figure 130. Header and part of the first two wells in the Biomarker.txt file of the gri_Analysis.BL/McBride_1 $1^{\text {st }}$ third.2d case. The first line gives the number of biomarker types, the next lines their names. The names must be exactly the same as in Table 13. The remaining lines are similar to those described in Figure 128, except there are three columns of biomarker ratio data. Notice that if there is no data of a particular biomarker type at a particular depth, the data is input as -999 . The bio

\section{Vitrinite and Biomarker Kinetic Data}

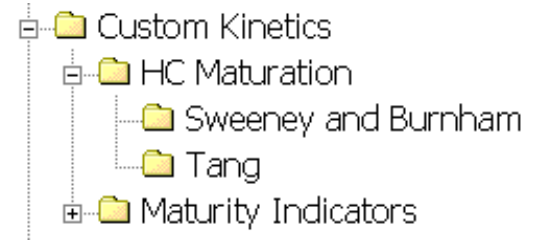

The vitrinite R0 kinetic models are in the blcases/Custom Kinetics/Maturity Indicators folder as illustrated to the left. Table 12 shows the kinetic data available.

Table 12. Vitrinite R0 Kinetic Models in Custom Kinetics/Maturity Indicators

\begin{tabular}{ll}
\hline \multicolumn{1}{c}{ Filename } & \multicolumn{1}{c}{ Brief Description } \\
\hline R0_S\&B1990_EasyR0.txt & Sweeney and Burnham 1990 Easy R0 model \\
RO_I_YT_BrownShale.txt & Tang kinetic data for Type I Brown Shale \\
RO_I_YT_RedWater.txt & Tang kinetic data for Type I Redwater Shale \\
RO_II_YT_BarnettShale.txt & Tang kinetic date for Type II Barnett Shale \\
RO_III_YT_TwinArrow.txt & Tang kinetic data for Type III Twin Arrow (Cameo) Coal \\
RO_III_YT_FushanCoal.txt & Tang kinetic data for Type III Fushan Coal \\
RO_III_YT_Dongfang.txt & Tang kinetic data for Type III Dongfang Coal \\
\hline
\end{tabular}

The vitrinite kinetic data is all of the same form. An example vitrinite kinetic file is given in Figure 131. 


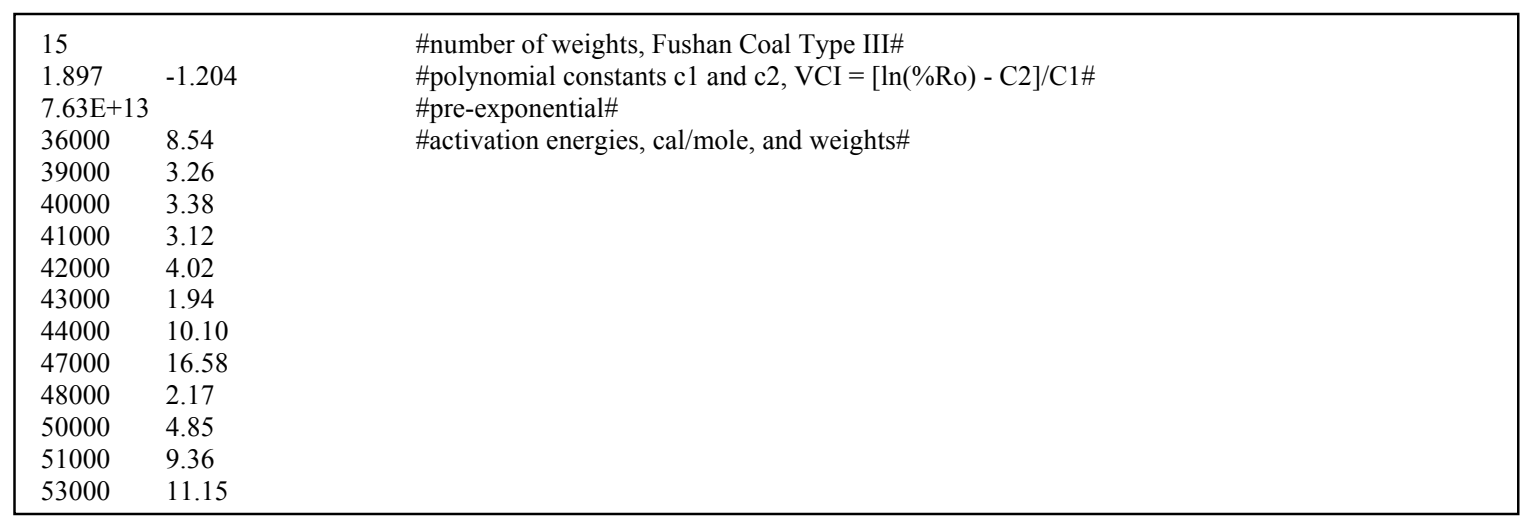

Figure 131. Example of vitrinite kinetic data. The pre-exponential constant is always measured in $\sec ^{-1}$.

All the vitrinite maturation models are computed in the following fashion. First $\mathrm{X}_{\mathrm{i}}$ is initialized:

$$
X_{i}=W_{i}
$$

where Wi are weights associated with each activation energy, and $\sum_{i} W_{i} \leq 1$. The following kinetic equation is then integrated over time to obtain $X_{i}(t)$ :

$$
\frac{\partial X}{\partial t}=X_{i} A \exp \left(-E_{i} / R T\right),
$$

where $A$ is the pre-exponential factor, $R$ the gas constant and $T$ temperature in degrees Kelvin. At any time t,

$$
\begin{aligned}
& X(t)=\sum_{i} X_{i}(t) \\
& V C I(t)=1-X(t) \\
& R_{o}[\%]=\exp \left(C_{1}(V C I)-C_{2}\right)
\end{aligned}
$$

where $C_{1}$ and $C_{2}$ are conversion constants given in the kinetic model, $V C I$ is the conversion index (running from 0 to 1 ), and $R_{o}$ is the computed percent vitrinite reflectance. We integrate the $\frac{\partial X}{\partial t}$ equation using finite difference methods.

\section{Biomarker Kinetic Models}

Parameters for the 7-biomarker kinetic models provided with $\boldsymbol{B} \boldsymbol{a} \boldsymbol{s i n} \boldsymbol{L} \boldsymbol{A B}$ are defined in Table 13. The parameters define how the biomarker conversion index of each biomarker evolves with time and temperature. The conversion index is identical to that presented immediately above for vitrinite $\mathrm{R}_{0}$. These models are use in $\boldsymbol{B} \boldsymbol{a s i n} \boldsymbol{L} \boldsymbol{A B}$ as follows: 
1. The biomarker conversion index (BCI) is computed for the time-temperature history of all nodes in a well

2. At the same time the vitrinite $R_{0}$ for the Sweeney and Burnham Easy Ro and the two selected custom kinetic models are computed.

3. The BCI is regressed against the calculated vitrinite $\mathrm{R}_{0}$ maturities over all times and nodes in the well (see Figure 25):

$$
R_{e}=\frac{a_{1}}{B C I}+a_{2}+a_{3} B C I+a_{4} B C I^{2} .
$$

4. The $\mathrm{BCI}$ ratios are computed from chromatographic data using the limit data in the table:

a. First compute the compound ratio. For example, $\mathrm{I}=22 \mathrm{~S} / 22 \mathrm{~S}+22 \mathrm{R}$.

b. Then compute the normalized ratio or BCI: $\mathrm{BCI}=(\mathrm{I}-\mathrm{lb}) /(\mathrm{ub}-\mathrm{lb})$, where $\mathrm{ub}$ is the upper limit (in this case 0.58), and $\mathrm{lb}$ the lower limit (in this case 0 ).

5. The BCI values of oils collected at various depths in the well are converted to Re using the regression in (3) above and plotted as Re vs. true vertical depth.

6. The range of validity (the limit range) of the Re conversion is indicated on the vitrinite Ro curve that was the basis of the conversion regression by thickening the vitrinite curve over that interval (see Figure 24). 
Table 13. The biomarker conversion models provided by BasinLAB. The limits are the range of equivalent Ro values over which the conversion index is valid. A is the preexponential in $\sec ^{-1}$.

\begin{tabular}{|c|c|c|c|c|c|}
\hline & \multicolumn{3}{|c|}{$\mathrm{E}[\mathrm{cal} / \mathrm{mole}]$ Weights } & \multirow{2}{*}{$\begin{array}{r}\mathrm{E}[\mathrm{cal} / \mathrm{mole}] \\
45000\end{array}$} & \multirow{2}{*}{$\begin{array}{r}\text { Weights } \\
0.0247\end{array}$} \\
\hline 22S/22S+22R_C32_hopane & 33000 & 0.0107 & ienanthrene_Alkylation & & \\
\hline$A=6.92 E 8$ & 34000 & 0.0077 & $A=1 E 14$ & 46000 & 0.0628 \\
\hline \multirow[t]{5}{*}{ Limits $=0$ to 0.58} & 35000 & 0.0911 & Limits $=0$ to 2.8 & 47000 & 0.0252 \\
\hline & 37000 & 0.6574 & & 48000 & 0.1224 \\
\hline & 38000 & 0.2125 & & 49000 & 0.0058 \\
\hline & 39000 & 0.0206 & & 50000 & 0.0998 \\
\hline & & & & 51000 & 0.021 \\
\hline 22S/22S+22R_C32_hopane & 37000 & 0.3067 & & 52000 & 0.1839 \\
\hline$A=3 E 8$ & 39000 & 0.1386 & & 54000 & 0.3176 \\
\hline Limits $=0$ to 0.55 & 40000 & 0.5547 & & 55000 & 0.1368 \\
\hline R28TA/R28TA+aR29MA_ & 43000 & 0.08 & Diamondoid_MAI & 48000 & 0.008 \\
\hline triaromatic_steroid & 44000 & 0.0102 & $A=1 E 15$ & 51000 & 0.0097 \\
\hline $\mathrm{A}=1.0 \overline{2} \mathrm{E} 14$ & 46000 & 0.9098 & Limits $=0$ to 100 & 52000 & 0.0773 \\
\hline Limits $=0$ to 1 & & & & 53000 & 0.0165 \\
\hline Methyl_Phenanthrene & 43000 & 0.003 & & 54000 & 0.1232 \\
\hline$A=1 E 14$ & 45000 & 0.0125 & & 55000 & 0.0041 \\
\hline \multirow[t]{22}{*}{ Limits $=0$ to 4.5} & 46000 & 0.0255 & & 56000 & 0.161 \\
\hline & 47000 & 0.005 & & 57000 & 0.0074 \\
\hline & 48000 & 0.0344 & & 58000 & 0.156 \\
\hline & 50000 & 0.0291 & & 59000 & 0.0231 \\
\hline & 51000 & 0.0014 & & 60000 & 0.1181 \\
\hline & 52000 & 0.0535 & & 61000 & 0.0809 \\
\hline & 54000 & 0.0792 & & 62000 & 0.026 \\
\hline & 56000 & 0.0988 & & 63000 & 0.1603 \\
\hline & 57000 & 0.0003 & & 64000 & 0.0284 \\
\hline & 58000 & 0.1148 & & & \\
\hline & 59000 & 0.0154 & Diamondoid_MDI & 53000 & 0.0823 \\
\hline & 60000 & 0.1083 & $A=1 E 15$ & 55000 & 0.0356 \\
\hline & 61000 & 0.0627 & Limits $=0.5$ to 100 & 56000 & 0.0664 \\
\hline & 62000 & 0.0542 & & 57000 & 0.0241 \\
\hline & 63000 & 0.1661 & & 58000 & 0.0994 \\
\hline & 64000 & 0.0085 & & 59000 & 0.008 \\
\hline & 65000 & 0.1273 & & 60000 & 0.13 \\
\hline & & & & 61000 & 0.0204 \\
\hline & & & & 62000 & 0.1125 \\
\hline & & & & 63000 & 0.1045 \\
\hline & & & & 65000 & 0.2485 \\
\hline & & & & 66000 & 0.0598 \\
\hline
\end{tabular}


$77000 \quad 0.0085$ 


\section{B. Hydrocarbon Maturation}

The kinetic maturation models available in BasinLAB in the Custom Kinetics: HC Maturation folder are described in Section VI.D and are listed in Table 14. The Burnham and Sweeney Type I and II maturation model was modified by us to include hydrocarbon cracking (Sweeney and Burnham folder). A set of original kinetic data determined in the laboratory by Y. Tang under funding for this project is contained in the Tang folder.

Table 14. Petroleum maturation kinetic models provided in the Custom Kinetics $\mid H C$ Maturation

\author{
Tang Maturation File \\ I_YT_FN2F.txt \\ I YT GnRvrF.txt \\ II_YT_B684F.txt \\ II YT DevonSh(RW).txt \\ II_YT_NseaF.txt \\ II_III_YT_CapeRange.txt \\ III_YT_CameoCoal.txt
}

\author{
Brief Description \\ Type I China coal \\ Type I Green River Shale, USA \\ Type II China \\ Type II, Devon Shale, Redwater sample, W. Canada \\ Type II, North Sea, UK \\ Type II/III, Australia \\ Type III, USA
}

\section{Sweeney and Burnham Maturation \\ File}

B\&S_Type_I_full.txt

Braun and Burnham (1990), Burnham and Braun (1990)

CB\&S_II.txt

ditto above, hydrocarbon cracking added

Evaluation of hydrocarbon maturation in the Heatflow and Maturation: Evaluate Maturation menu uses a 1D (well by well) temperature solution subject to the assigned heatflow, water depth, and sedimentation/erosion history. The time-temperature history is computed for the well when the plot is requested. Temperature solutions need not have been previously computed, and are not used even if they have been.

\section{2D Fluid Flow and Temperature Equation Solutions}

The solution of the temperature equation on a 2D finite element grid has been described in Section IV.E. Compaction and convection equations can be solved in addition to the temperature equation, however. The haline convection (salinity) equation has not been implemented at this time and is therefore inactive.

The solution proceeds in two phases. First the pseudo-2D temperature history in the basin is calculated and the maturation reactions in all geogrid nodes for both of the selected kerogen types are computed based on this history and stored in the MATURATION and MATURATION2 APL files. At this stage the porosity history of the basin has already been computed to infer the geohistory (GEOGRID evolution) of the basin. With the pseudo-2D temperature and maturation solutions available, this background data is sufficient to estimate all the fluid expulsion mechanisms over all 
timesteps: porosity change (compactive expulsion), temperature change (aquathermal expulsion), and positive volume change maturation reactions (chemical expulsion both from source beds and from the maturation of low grade sparsely distributed organic matter). The 2D finite element solutions use these source terms in its calculations of brine movements.

The $2 \mathrm{~d}$ finite element solution procedure is:

- Create a finite element grid and store it in the FEGRID file.

- Select the equations to be solved and save them, if desired, in the EQControls file.

- Optionally run a $1 \mathrm{D}$ temperature solution, distribute it over the triangular finite element grid, and store it in the SOLGRID array. This step is necessary to compute the fluid expulsion terms in the pressure equation, if the pressure equation is selected and hydrocarbons are present. The user is warned and provided with the option to compute the pseudo-1D solution.

- Solve the 2D equations selected and store the results in the SOLGRID file.

The flow chart for this part of the program is shown in Chart 4.

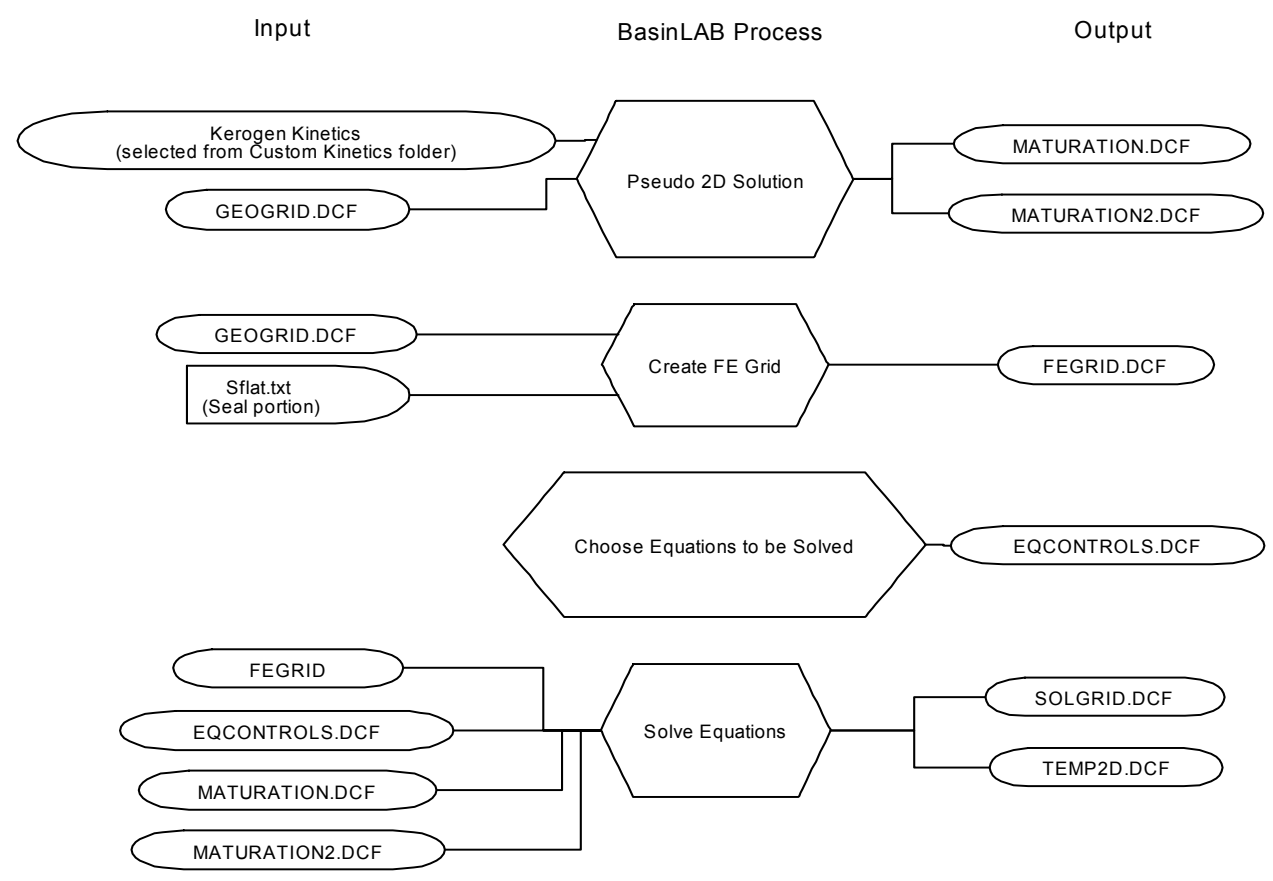

\section{Chart 4. Flow DiAgram FOR TEMPERATURE, MATURATION AND BRINE FLOW SIMULATIONS. CHARTS 5 AND 3 ARE ON PAGES 315 AND 285.}

The flow equations are of course influenced strongly by the permeability models (for the matrix and fault) chosen. We illustrate the solution of flow equations for a simple fault in the next section. The section following that will use this case to examine hydrocarbon migration in faults with variable buoyancy control on the flow. 


\section{A. Flow in a Simple Fault-Antithetic Fault System}

The blcases $\mid$ Examples.BL $\backslash$ Antithetic_Fault. $2 d$ case illustrates how the pressure equation can be solved in conjunction with the temperature equation. We construct the case from a simple Canvas sketch.

\section{Canvas Sketch Input of Antithetic Fault Case}

Figure 132 shows a Canvas sketch of the antithetic fault case. It is a simple cartoon constructed in a few minutes. Its essential elements are: a bounding box, a number of age-stratigraphic horizons including the top surface (age $=0$ horizon) which (must) cut horizontally across the bounding box, and (optional) a number of faults that (must) cut vertically fully across the bounding box. The file is read by Basin $\boldsymbol{L} \boldsymbol{A B}$ using the File: New Section Project option as shown in Figure 133. The case is processed as described in the subsequent series of figures.

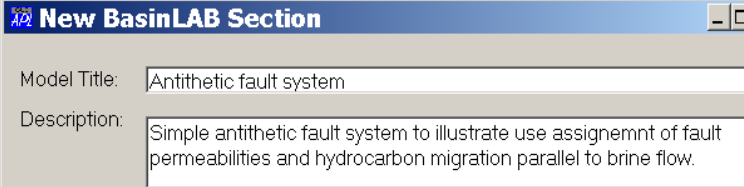

Simple antithetic fault system to illustrate use assignemnt of fault permeabilities and hydrocarbon migration parallel to brine flow.

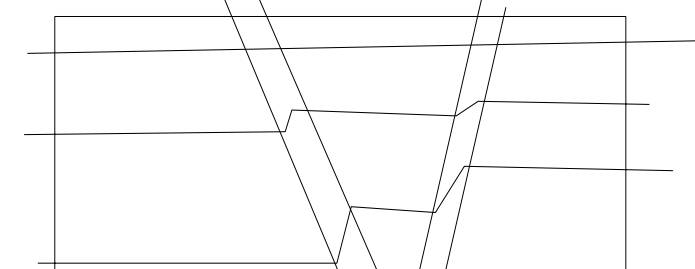

\section{-Define Case Parameter From}

- Default values

C Previous section interpreted

$\checkmark$ CaseParameter file selected

Input Data Source

- Drawing (.dxf from Canvas)

C GoCAD section (.txt)

C SFLAT File (.txt)

File selected

D:BasinModelsłblcasesłExamples. BLLAntithetic Fault.2dKGoCadKAntithetic, dx'

Browse

Model Domain Dimensions $(\mathrm{km})$

Model Domain Dimen
Figure 132. Canvas sketch of a faultantithetic fault system. The model consists of a bounding box, several age-stratigraphic horizons (including the top surface) that cut horizontally across the bounding box, and, optionally, faults that cut vertically across the bounding box. The input Canvas file is placed in the GoCad folder of the Antithetic Fault.2d case. For input to Basin $L A B$ it must be saved as a *.dxf file. The options shown are chosen when it is saved in Canvas.

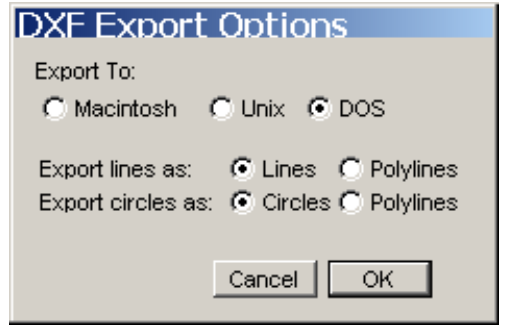

Figure 133. Antithetic.dxf file is read in using File: New Section Project. The title, short description, and dimensions (in $\mathrm{km}$ ) of the section are input and the $O K$ button depressed. This brings up the graphics and form shown in Figure 134. 


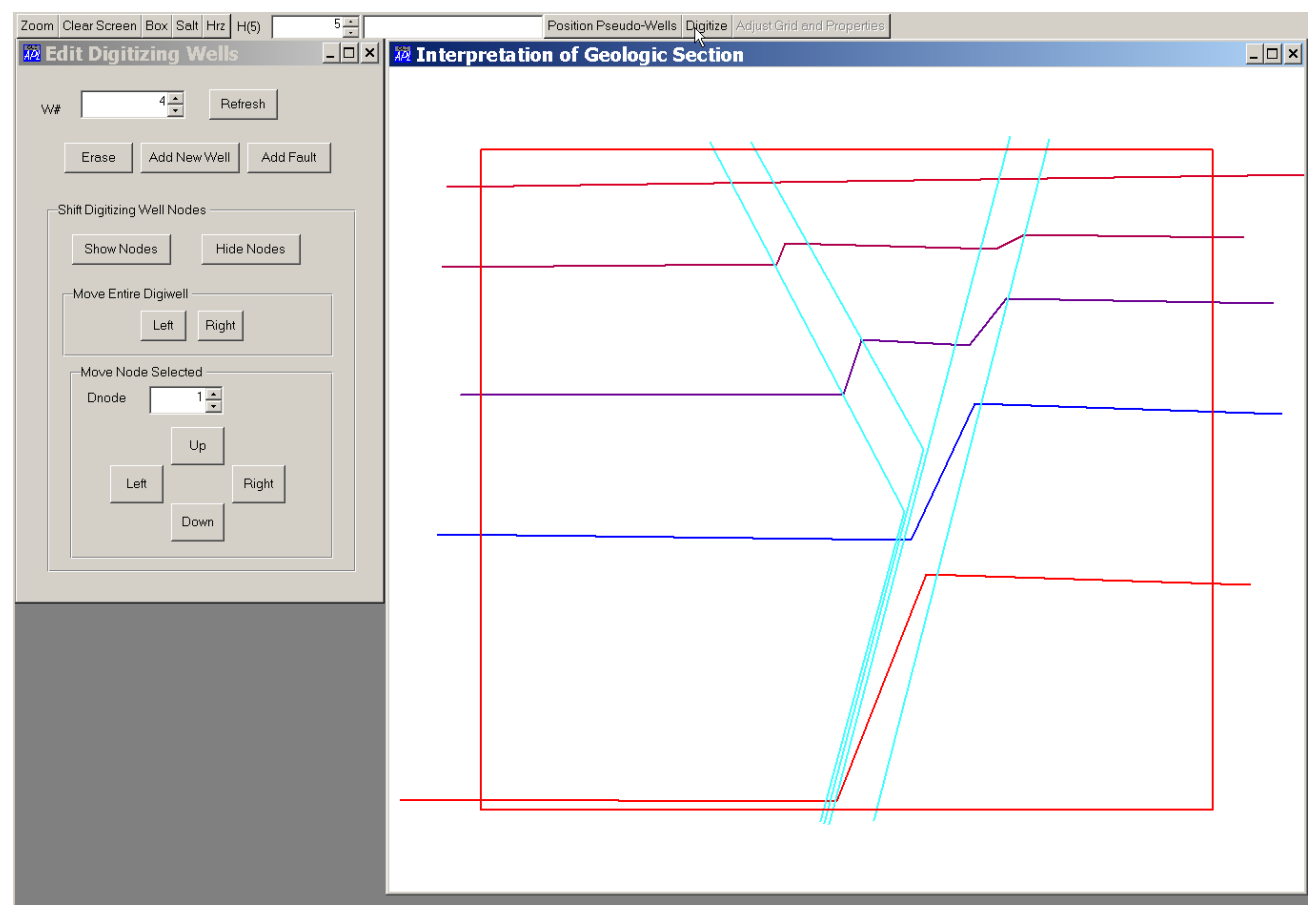

Figure 134. The Canvas sketch as imported to BasinL $\boldsymbol{A B}$ when $\mathrm{OK}$ in Figure 133 is depressed. The form allows pseudowells to be moved and adjusted. Since the faults appear in reasonable positions already, we can immediately depress the Digitize button (at mouse position on toolbar). Depressing this button and then the Adjust Grid and Properties button to its right results in the graphics shown in Figure 135. 


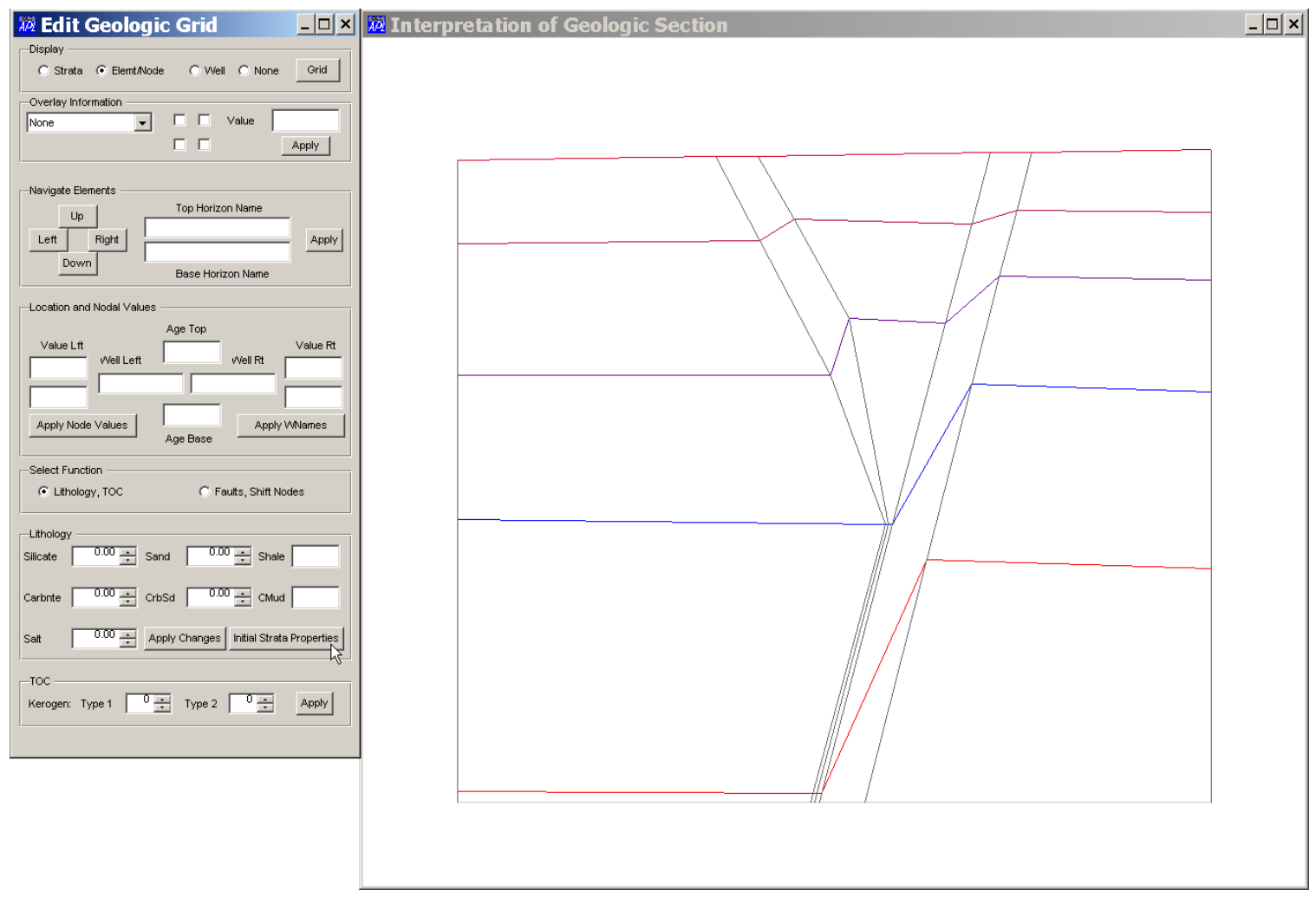

Figure 135. Digitized version of graphic shown in Figure 134. Initial strata properties are most easily input by depressing the Initial Strata Properties button as shown. This brings up the spreadsheet shown in Figure 136.

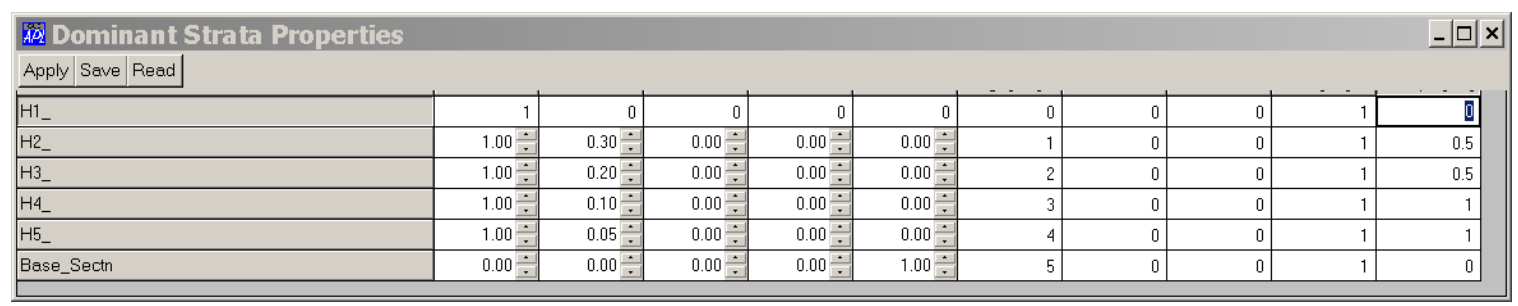

Figure 136. Initial Strata Properties spreadsheet form requested in Figure 135. We have selected an all silicate section except for a salt layer at the base. The sand fraction increases upwards, however. The lithologies are applied by depressing the Apply button. After this is done the lithology can be verified by selecting sand or shale in the Overlay Information combo in Figure 135. 


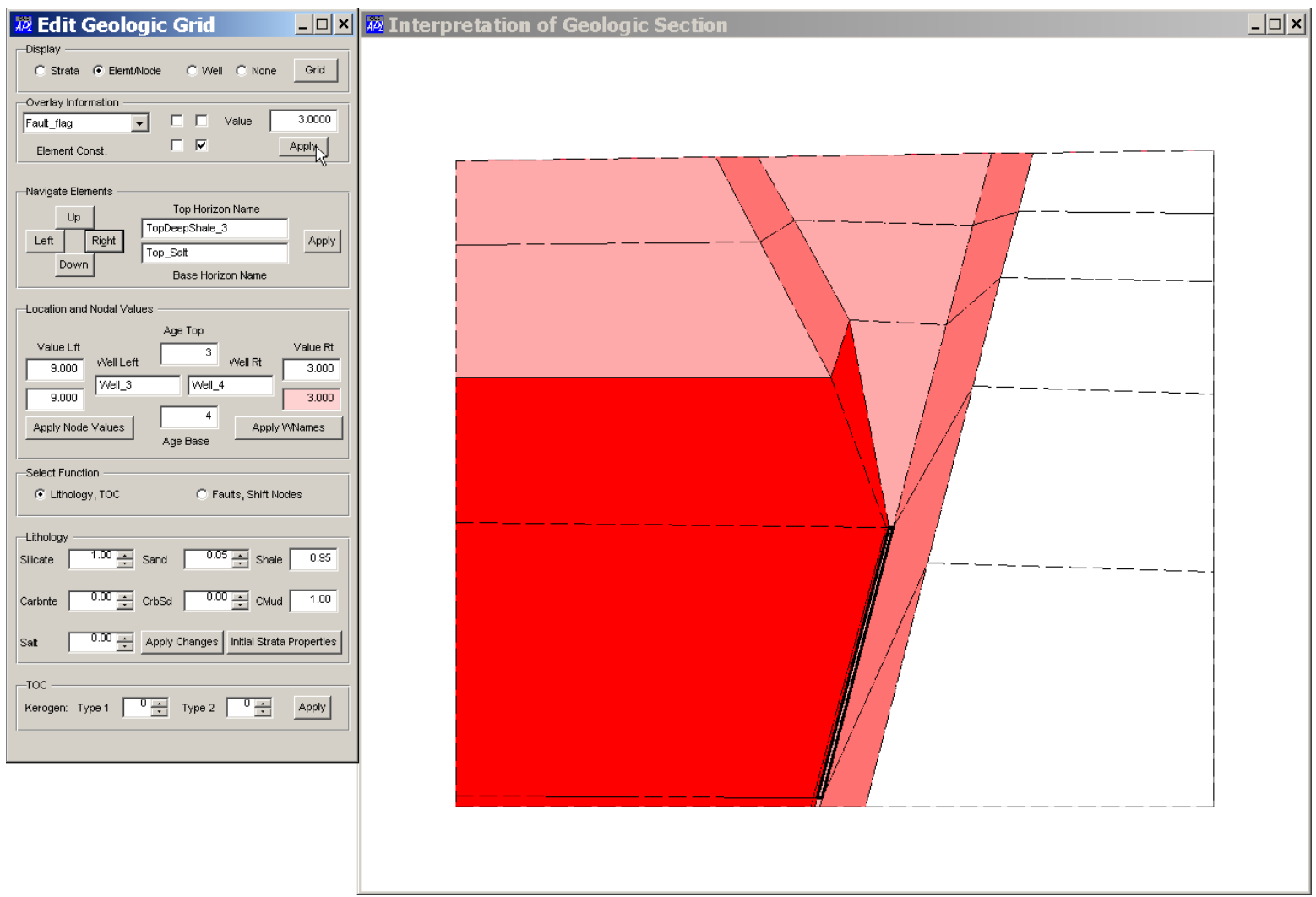

Figure 137. Faults can be assigned by selecting the Fault, Shift Nodes option of the Select Function group. Fault codes are assigned by selecting the Fault flag overlay and labeling the faults with 5 on their right side, 3 on their left, and 9 for nodes that will be attracted to the left side of the system where the antithetic fault merges with the main fault (see V.B). For example the Location and Nodal Values group shows the fault flags at the corners of the black element are 3's (left side main fault) and 9's (nodes to be pasted to this side of the fault). Toggling to the Faults, Shift Nodes option of the Select Function group allows the fault assignments to be checked. 


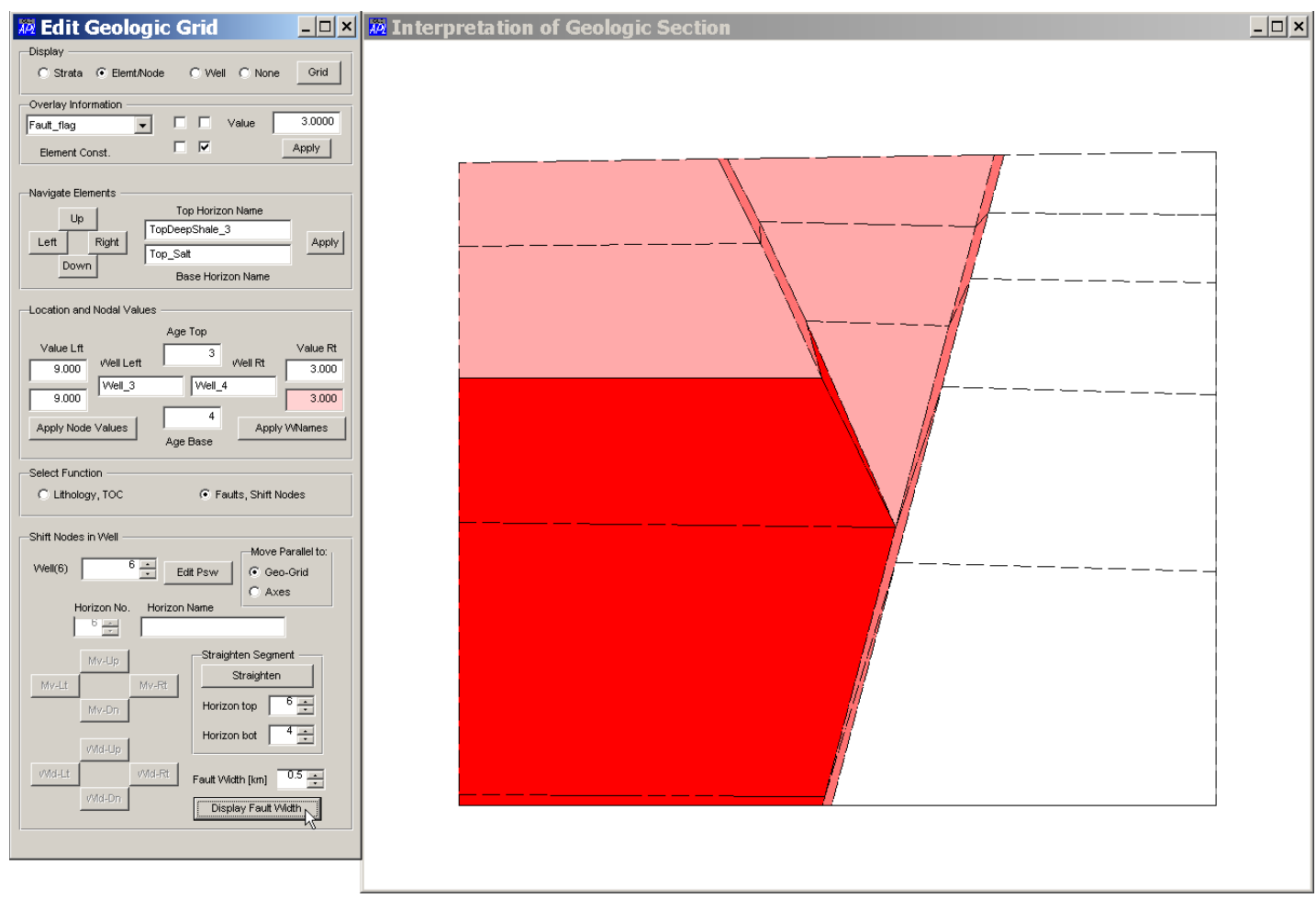

Figure 138. Depressing the Display Fault Width button adjusts the sides of the faults as dictated by the fault flags. The Fault Width $[\mathrm{km}]$ spinner adjusts the width of the fault. The graphics interpretation should be saved using Graphic Input: Save Interpretation or by taking option when form is closed. 


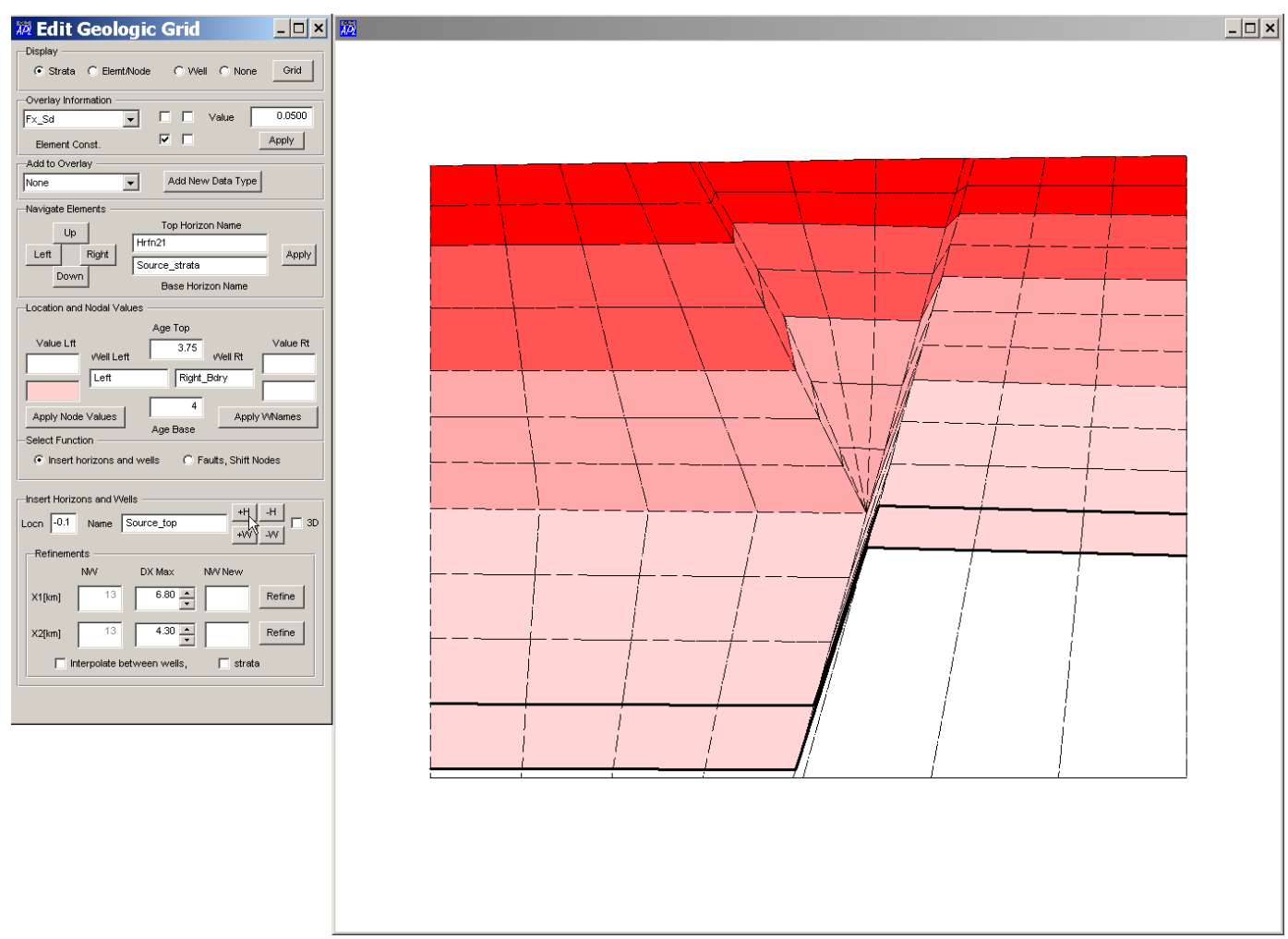

Figure 139. Graphic Input: Place into SFLAT and Save transfers the graphic interpretation to an SFLATorig.txt file in the Antithetic_Fault.2d case folder. The Geology: Graphic Edit menu can now be used to refine the grid, and insert a source strata. This is done first by dialing the DX Max spinners in the Refinements group to the desired refinement. Since this has been done in the image above the results are shown (13 pseudowells by 14 age horizons). Refinement is done separately for X1 (pseudowells) and X2 (age horizons) followed by pressing the Refine button. The source strata is inserted as a horizon by setting the Locn to -0.1 , typing in the name of the inserted strata ("Source_strata") and depressing the H+ button, as shown. The grade of the source strata is initially assumed to be zero. This is changed by selecting Ker1(wtpct) in the Overlay Information combo, navigating to the source strata with the Strata toggle in the Display group selected, and changing the Value in the Overlay Information group to 4 (for $4 \mathrm{wt} \%$ ). A $100 \mathrm{~m}$ thick layer of $4 \mathrm{wt} \%$ TOC has been inserted. Finally a similar approach is used to insert sands into the shale section. The lithologic fractions must sum to 1. The final section is shown in Figure 140.

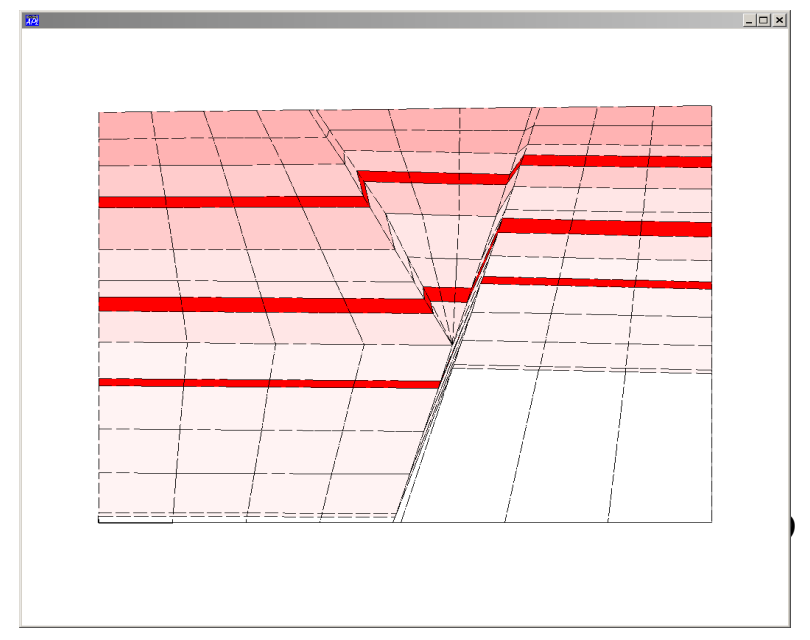

Figure 140. The final antithetic fault section after three sands and a source strata (just above the basal salt) have been inserted into SFLAT file shown in Figure 139. This is the root SFLAT file that now is processed to determine the 
geologic history of the fault system. First, however, the fault flags must be corrected as shown in Figure 141.

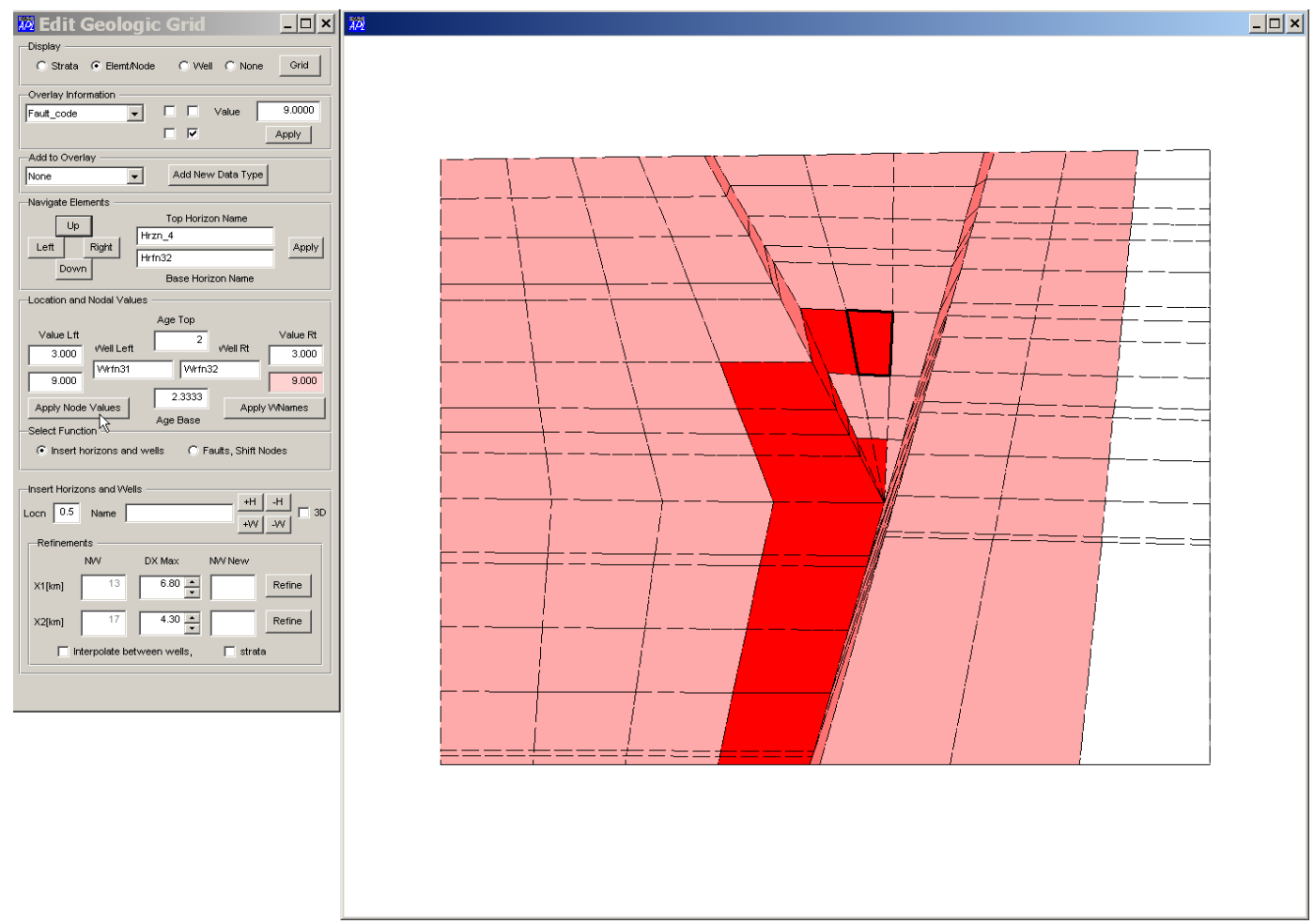

Figure 141. The fault flags have been improperly interpolated. They have been partially corrected in this image, and will be fully corrected when the 9's in the Location and Nodal Values edit boxes (corresponding to the base of the black-outlined element) are changed to 3's, the nodes on the right side of the antithetic fault are changed to 5's, and the extra 9's above where the antithetic fault joins the main fault have been changed to 3's. The properly modified SFLAT file is shown in Figure 142. 


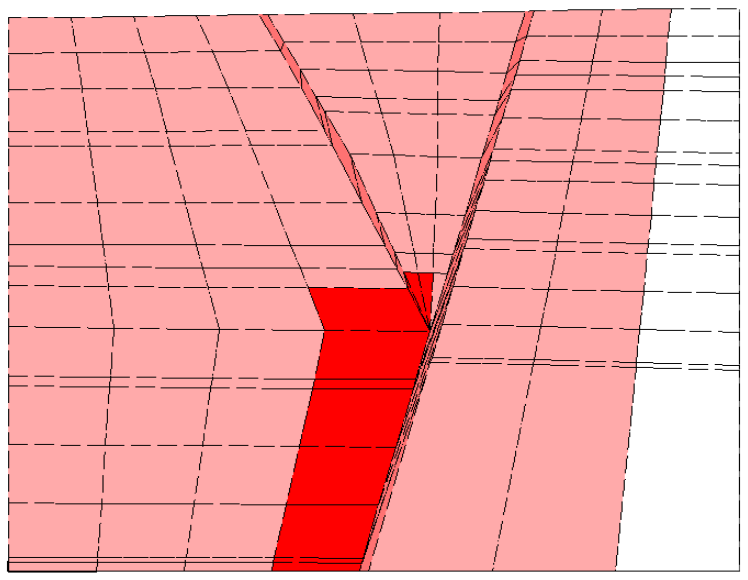

033335333

5330

033335333

5330

033335333

5330

033335333

5330

033335333

5330

033335333

5330

033335333

Figure 142. Proper distribution of the fault code. Element color is controlled by the lower right hand node. Fault flags for all nodes are shown in the insert table. See Section VI.B (p. 234) for definition of fault codes.
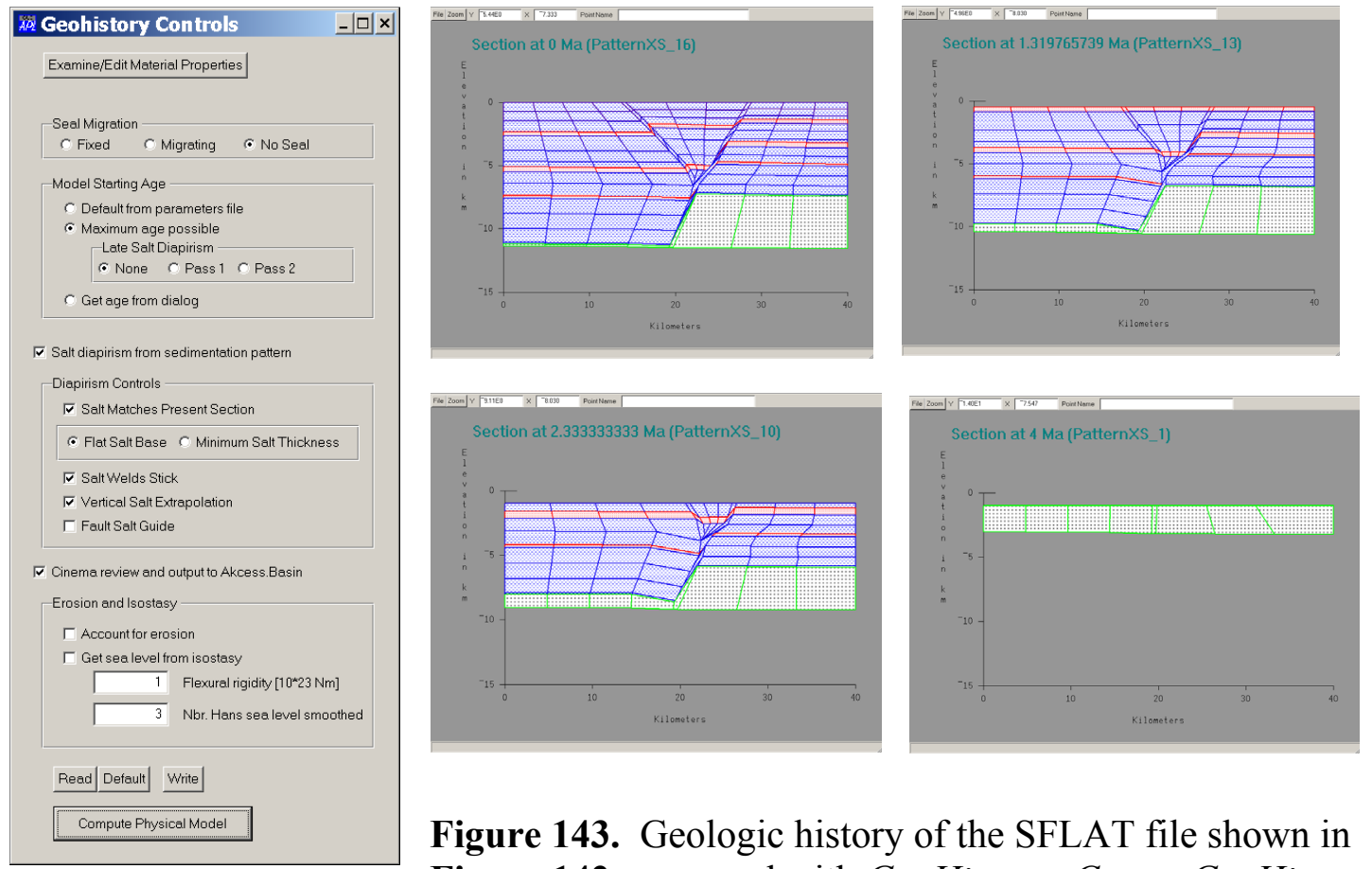

Figure 143. Geologic history of the SFLAT file shown in Figure 142 processed with GeoHistory: Create GeoHistory as shown. GeoHistory plots are produced using GeoHistory: Plot and Edit GeoHistory: Plot. The final step is to assure that the grid is not twisted, as discussed in Figure 144. 


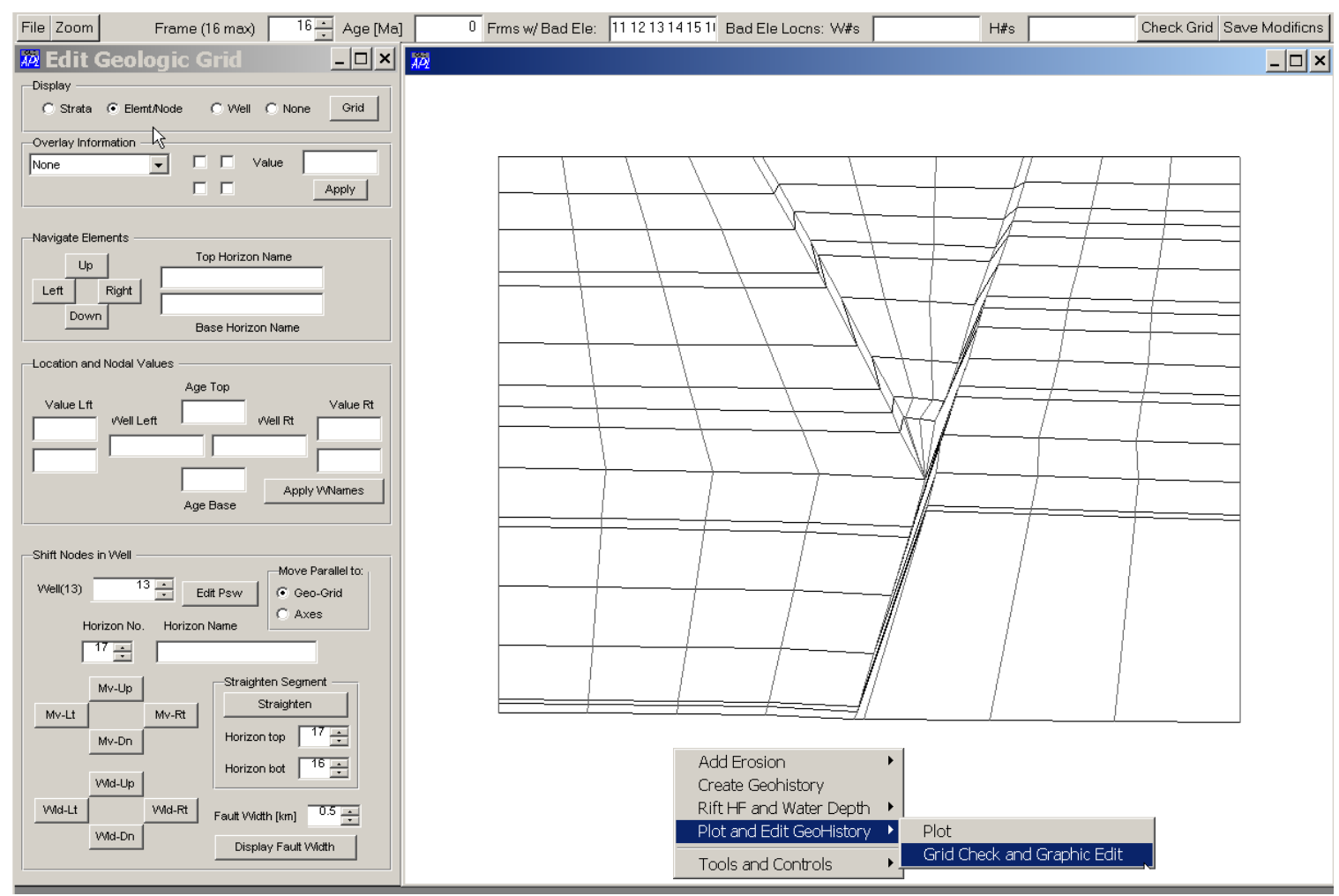

Figure 144. Check the grid using the GeoHistory: Plot and Edit Graphics: Grid Check and Graphic Edit as shown above. The $11-16^{\text {th }}$ frames have bad grid. Depressing the Check Grid button at the top of the toolbar shows where the grid is bad in the displayed frame, and Figure 145 shows how it can be fixed.

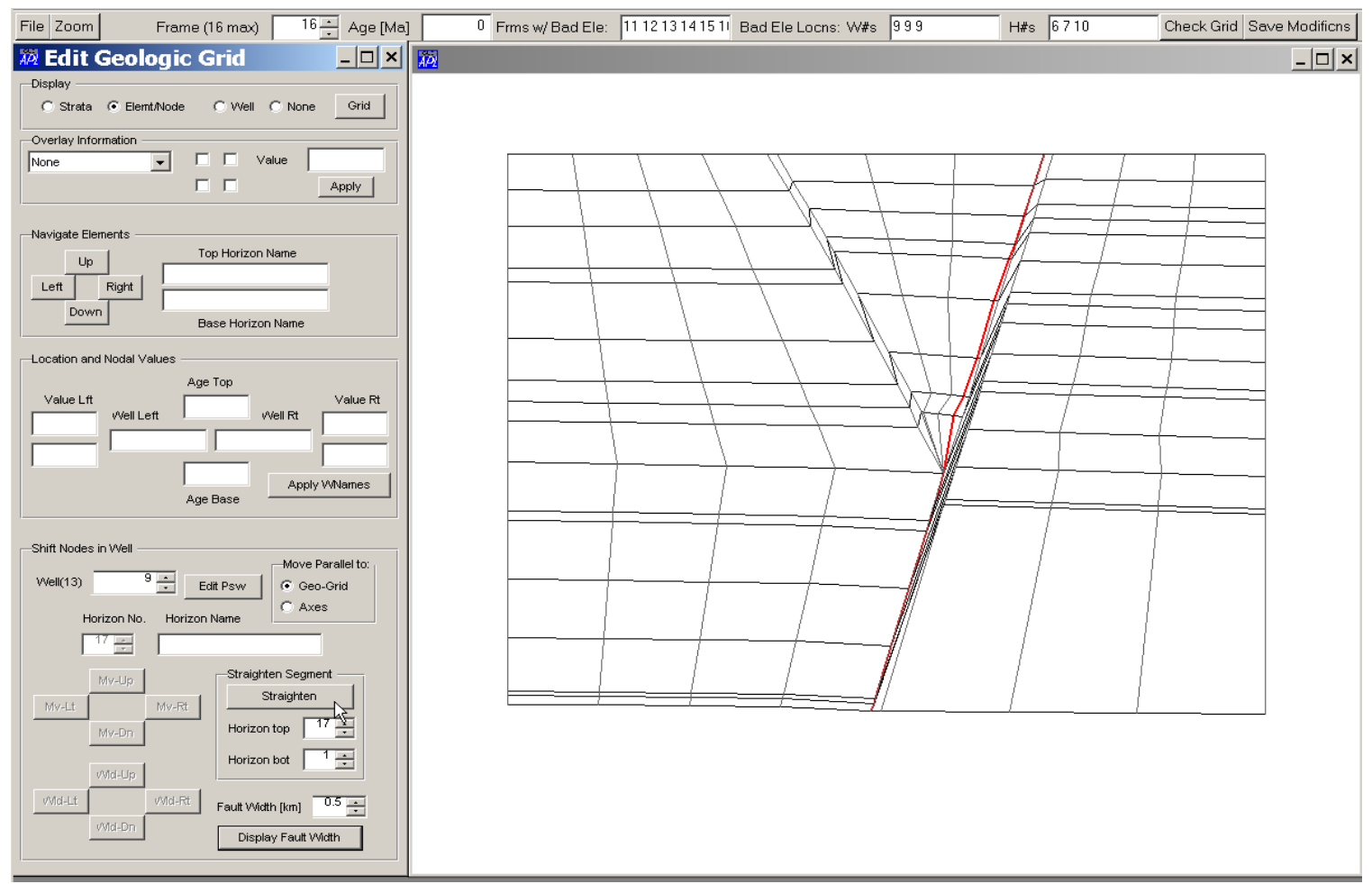


Figure 145. Depressing the Check Grid button on the top toolbar shows that the grid is bad in well 9, horizons (from the base) 6,7, and 10. The problem is that the main fault is not straight and the grid is twisted at irregularities. The easiest fix is to straighten the fault. This is done by selecting the main fault by moving the well marker to the left side of the fault $\left(9^{\text {th }}\right.$ well) using the Well spinner at the top of the Shift Nodes in Well group. The red line in the image shows the pre-straightened position of this pseudowell. Then select the top and bottom of the well in the Straighten Segment group (as shown) and depress the Straighten button under the mouse arrow. In some cases multiple depressions of this button are needed to fully straighten the well. With the left hand side of the well, straightened, depressing the Display Fault Width button adjusts the right side of the fault and produces the image shown above. Depressing the Check Grid button at the top toolbar shows that the grid is fully fixed. The modifications are then saved to the GEOGRID file using the Save Modifications button on the top toolbar. Figure 146 shows how the slight jog in the pseudowells between the main and antithetic faults just above where they join can be corrected.

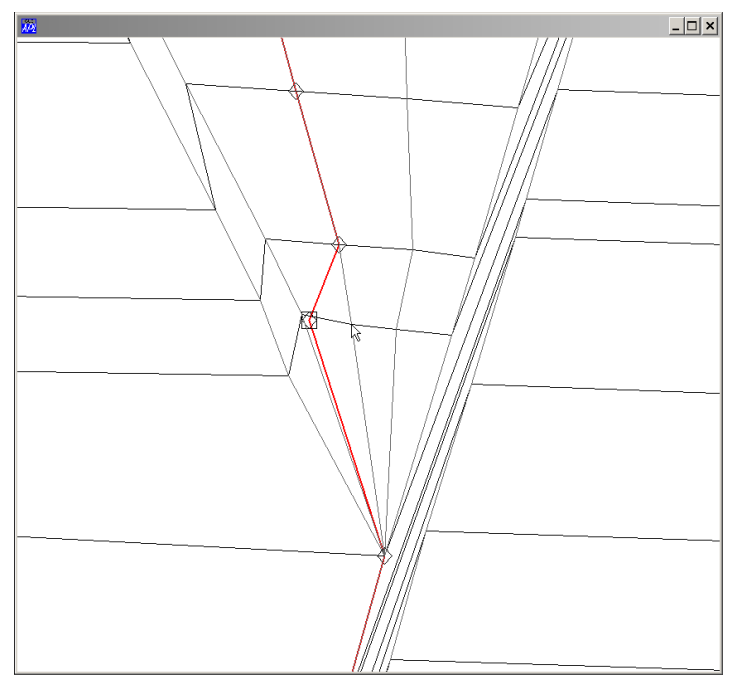

Figure 146. The jog mentioned in Figure 145 can be corrected by navigating the red marker well to the $7^{\text {th }}$ and $8^{\text {th }}$ wells, depressing the Edit Psw button, selecting the node using the Horizon No. spinner, and using the move buttons to shift the node's position. Movement can be parallel to the Geo-Grid or to the vertical and horizontal Axes depending on the toggle settings in the Move Parallel to Group. This figure shows a zoom of the area between the antithetic and main faults. The red line shows the old position of pseudowell 7. The arrow shows the new position of the "boxed" node. Grid can be refreshed using the Grid button at the top of the Figure 145 form. Finer movements are achieved by depressing the control key when moving the nodes. Remember to Save Modification (button on toolbar at top of the workspace form). Unzoom by depressing the shift key while pressing the Zoom button.

\section{Brine Flow in the Antithetic Fault Case}

With the geologic history determined and the grid checked, the equations that determine the temperature evolution and brine flow can be specified and solved. The steps are:

- $\quad$ Create the finite element grid from the GEOGRID at all timesteps using the Solve Equations: Create FE Grid: From GEOgrid menu:

- Once this is done the

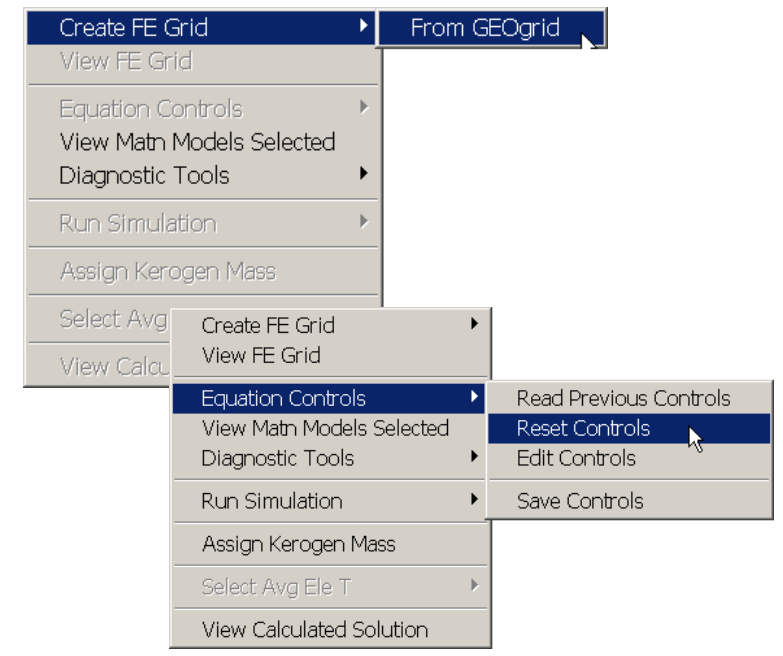


equations to be solved and refinements of the timesteps over which they are solved can be specified:

- Select the equations to be solved from the form summoned by the menu command above. Here we select the temperature and pressure equations, but not the convection equation.

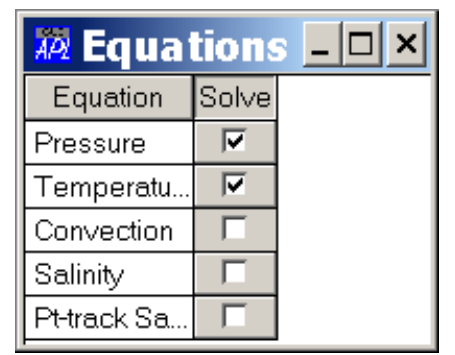

Figure 147 shows the default solution controls that are established when the equations are selected. These controls can be modified as discussed in the figures.

\begin{tabular}{|c|c|c|c|c|c|c|c|c|c|}
\hline \multicolumn{9}{|c|}{ AP Select Equations to be Solved } & $-\square \times$ \\
\hline Age (endst... & $\mathrm{P}$ & $T$ & PSI & Salnty & PATrS... & NDelt & FxDt & 1 stDt & lastDt \\
\hline $4 \mathrm{Ma}$ & $\Gamma$ & $\sqrt{\nabla}$ & $\Gamma$ & $\mathrm{C}$ & $\mathrm{C}$ & $1 \div$ & $1.0 \div$ & 0.000000 & 0.000000 \\
\hline $3.976 \mathrm{Ma}$ & $\sqrt{v}$ & $\sqrt{\nabla}$ & $\Gamma$ & $\mathrm{C}$ & $\mathrm{C}$ & $1 \div$ & $1.0 \div$ & 0.024382 & 0.024382 \\
\hline $3.75 \mathrm{Ma}$ & $\sqrt{v}$ & $\sqrt{\nabla}$ & $\Gamma$ & $\mathrm{C}$ & $\mathrm{C}$ & $1 \div$ & $1.0 \div$ & 0.225618 & 0.225618 \\
\hline $3.5 \mathrm{Ma}$ & $\sqrt{\nabla}$ & $\sqrt{\nabla}$ & $\Gamma$ & $\mathrm{C}$ & $\mathrm{C}$ & $1 \div$ & $1.0 \div$ & 0.250000 & 0.250000 \\
\hline $3.25 \mathrm{Ma}$ & $\sqrt{v}$ & $\sqrt{\nabla}$ & $\Gamma$ & $\mathrm{C}$ & $c$ & $1 \div$ & $1.0 \div$ & 0.250000 & 0.250000 \\
\hline $3.201 \mathrm{Ma}$ & $\sqrt{v}$ & $\sqrt{v}$ & $\Gamma$ & $\mathrm{C}$ & $c$ & $1 \div$ & $1.0 \div$ & 0.048764 & 0.048764 \\
\hline $3 \mathrm{Ma}$ & $\sqrt{v}$ & $\sqrt{v}$ & $\Gamma$ & $\mathrm{C}$ & $\mathrm{C}$ & $1 \div$ & $1.0 \div$ & 0.201236 & 0.201236 \\
\hline $2.667 \mathrm{Ma}$ & $\sqrt{v}$ & $\sqrt{v}$ & $\Gamma$ & $\mathrm{C}$ & $c$ & $1 \div$ & $1.0 \div$ & 0.333333 & 0.333333 \\
\hline $2.51 \mathrm{Ma}$ & $\sqrt{v}$ & $\sqrt{\nabla}$ & $\Gamma$ & $\mathrm{C}$ & $\mathrm{C}$ & $1 \div$ & $1.0 \div$ & 0.156736 & 0.156736 \\
\hline $2.333 \mathrm{Ma}$ & $\sqrt{v}$ & $\sqrt{\nabla}$ & $\Gamma$ & $\mathrm{C}$ & $\mathrm{C}$ & $1 \div$ & $1.0 \div$ & 0.176598 & 0.176598 \\
\hline $2 \mathrm{Ma}$ & $\sqrt{v}$ & $\sqrt{v}$ & $\Gamma$ & $\mathrm{C}$ & $\mathrm{C}$ & $1 \div$ & $1.0 \div$ & 0.333333 & 0.333333 \\
\hline $1.5 \mathrm{Ma}$ & $\sqrt{v}$ & $\sqrt{v}$ & $\Gamma$ & $\mathrm{C}$ & 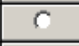 & $1 \div$ & $1.0 \div$ & 0.500000 & 0.500000 \\
\hline $1.32 \mathrm{Ma}$ & $\sqrt{v}$ & $\sqrt{v}$ & $\Gamma$ & $\mathrm{C}$ & $\mathrm{C}$ & $1 \div$ & $1.0 \div$ & 0.180234 & 0.180234 \\
\hline $1 \mathrm{Ma}$ & $\sqrt{v}$ & $\sqrt{\nabla}$ & $\Gamma$ & $C$ & $C$ & $1 \div$ & $1.0 \div$ & 0.319766 & 0.319766 \\
\hline $0.5 \mathrm{Ma}$ & $\sqrt{v}$ & $\sqrt{\nabla}$ & $\Gamma$ & $C$ & $\mathrm{C}$ & $2 \div$ & $1.0 \div$ & 0.250000 & 0.250000 \\
\hline $0 \mathrm{Ma}$ & $\sqrt{v}$ & $\sqrt{v}$ & $\Gamma$ & $C$ & $c$ & $2 \div 1$ & $7.0 \div$ & 0.250000 & 0.250000 \\
\hline
\end{tabular}

Figure 148).
Figure 147. Default solution controls from Solve Equations: Equation Controls: Edit Controls.

- Equations selected are checked.

- Ages apply after the deposition (or erosion) of a stratum.

- Deposition/erosion steps are refined into NDelt refined timesteps.

- FxDt is the ratio of successive refined timesteps.

- First and last refined timesteps are shown in the last two columns.

- Internal controls are revealed by pressing the Age(endstep) button (first column) for any timestep (see

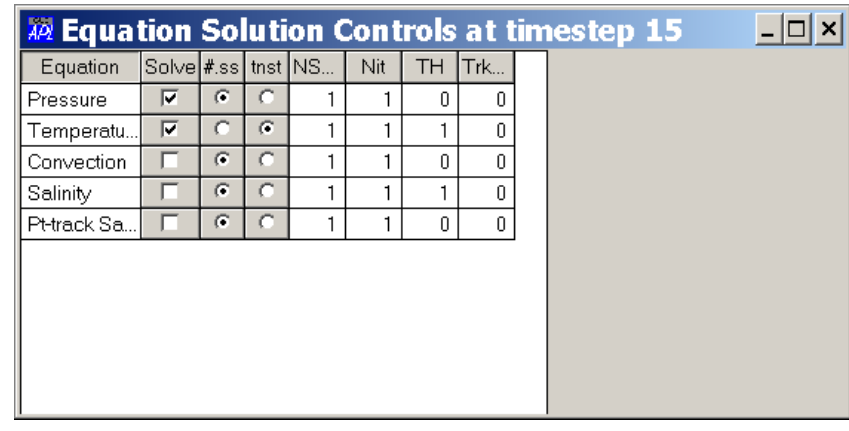

Figure 148. Pressing the Age(endstep) button of any timestep in Figure 147 shows the internal controls for computation of that timestep. The default controls for the timestep at $0.5 \mathrm{Ma}$ is shown. The pressure equation is solved as a steady state equation (the only option currently supported), the temperature

equation as a transient equation. For the temperature equation a maximum of 1 subtimesteps (Nsubt) is taken in the solution of each refined timestep. If the number were -1 the minimum number of sub-timesteps would be one and the maximum would be set such that the temperature change is less than a specified value. These sub-timesteps 
are inside the parameter loop and so are carried out for constant material and fluid properties. One iteration (Nit) is taken across each set of Nsubt sub-timesteps for each equation. The iteration loop includes material property changes and thus iterations converge temperature-dependent parameters such as thermal conductivity. Parameters use time-average (across the iteration loop timestep) state variables (e.g., temperature). Subtimesteps and iterations can be defined individually for each equation but are always 1 for steady state solutions. TH specifies whether the solution is solved implicitly $(=1)$ or explicitly $(=0)$ or in between $(0<\mathrm{TH}<1)$. Trk specifies the node at which the solution is tracked. The value of the variable is written into a text file that is placed in the Metafiles folder of the case, if this variable is not zero. The node number can be determined from Solve Equations: View FE Grid or, if the case has been calculated previously, from View Calculated Solution graphics by positioning the mouse over the node. Note, primary equation solution variables are nodal variables.

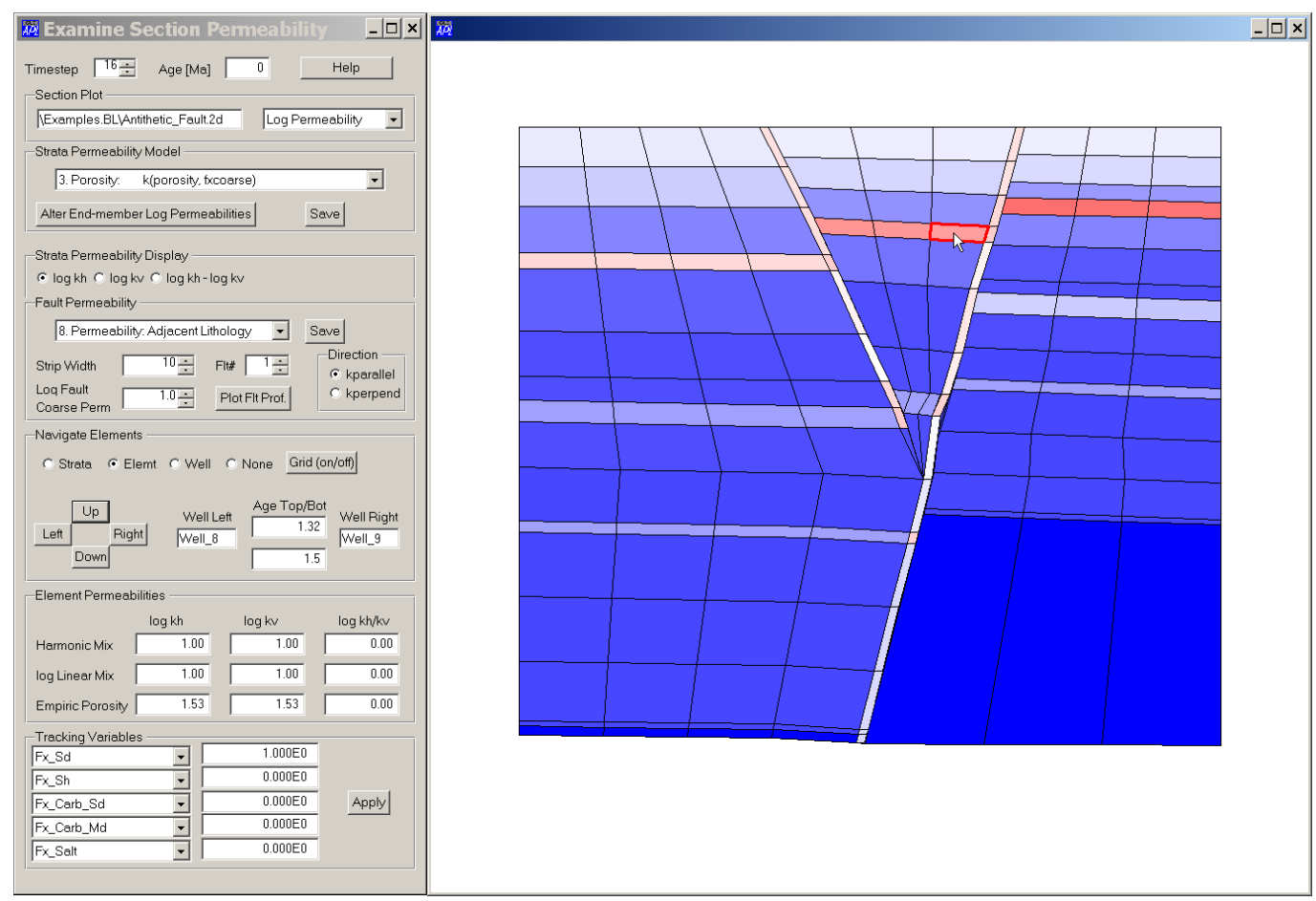

Figure 149. Parameters: Permeability (+Scrpad): Examine Section Permeabilty form. The empirical model in which permeability is a function of porosity and the fraction coarse lithology (Section VI.B.1) has been selected for the matrix permeability, and the adjacent lithology model (Section VI.C) has been selected for fault permeability. The colors in the above figure indicate permeability.

Since flow will be calculated, permeability is an important variable. Permeability models are assigned and their consequences in the case section examined using the Parameters: Permeability (+Scrpad): Examine Section Permeability form. Figure 149 shows the choices made for the Antithetic fault calculations. 
Once the execution controls are set, computing the equations requested is a matter of

\begin{tabular}{|l|l|}
\hline & $\begin{array}{l}\text { Create FE Grid } \\
\text { View FE Grid }\end{array}$ \\
\hline & $\begin{array}{l}\text { Equation Controls } \\
\text { View Matn Models Selected } \\
\text { Diagnostic Tools }\end{array}$ \\
\hline Pseudo 2D Temperature & Run Simulation \\
\hline All Selected 2D Equations & Assign Kerogen Mass \\
\hline & Select Avg Ele T \\
\hline View Calculated Solution \\
\hline
\end{tabular}
simply executing Solution Controls: Run Simulation: All Selected 2D Equations. The temperature solution could be run using the first option (Pseudo 2D Temperature). However, this solution will be run prompted when solving all the equations if the pressure equation is one of the equations whose solution is requested. This is because the volume changes associated with hydrocarbon maturation drives fluid flow, and the volume change term is obtained from the pseudo- $2 \mathrm{~d}$ solution. Execution of the above command thus brings up the following form:

\section{No pseudo 2D solution Exists.}

No volume source term exists for the solution for the pressure equation.

Do you want to create it by solving the pseudo 2D temperature/maturation equation? Respond OK You must re-run 2D FEM solution after pseudo 2D solve is complete.

Or do you want to turn off your request to solve the pressure equation of check the maturation files? Respond CANCEL.

The pressure equation requires a volume source term from hydrocarbon maturation. This is estimated by a $1 \mathrm{D}$ temperature/maturation calculation.

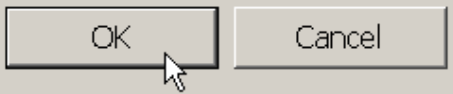

Depressing $O K$ causes first the pseudo 2D solution to be computed. The full 2D solution for all equations must then be requested again using Run Solution Controls: Run Simulation: All Selected 2D Equations. The execution will of this case will take less than 3 minutes on a $997 \mathrm{Mhz}$ PC.

All finite element solution parameters and results are accessed through Solve Equations: View Calculated Solution. The variables that are available for viewing are shown in the form, which appears when this menu command is issued. Those available at this stage of the processing are shown to the right. The variables of first interest are the brine mass flux ( $m$ flux $[\mathrm{g} / \mathrm{cm} 2-a]$ ) and integrated mass flux (Int-

\begin{tabular}{|c|c|c|c|c|c|c|c|c|c|}
\hline Variable & $r$ & $g$ & b & $\operatorname{Tr}$ & $\mathrm{Gx}$ & Gy & $\mathrm{G}$ & V & Con \\
\hline RedLStr & $C$ & $C h$ & $C$ & $\Gamma$ & $\Gamma$ & $\Gamma$ & $\Gamma$ & $\Gamma$ & $\Gamma$ \\
\hline $\mathrm{T}[\mathrm{C}]$ & $C$ & $C \%$ & $C$ & $\sqrt{\nabla}$ & $\Gamma$ & $\Gamma$ & $\Gamma$ & $\sqrt{\square}$ & $\sqrt{v}$ \\
\hline$P$ [bars $]$ & $\Gamma$ & $C 5$ & $C$ & $\Gamma$ & $\Gamma$ & $\Gamma$ & $\Gamma$ & $\Gamma$ & $\Gamma$ \\
\hline $\mathrm{Sal}[\mathrm{g} / \mathrm{g}]$ & $C$ & $C 5$ & $C$ & $\Gamma$ & $\Gamma$ & $\Gamma$ & $\Gamma$ & $\Gamma$ & $\Gamma$ \\
\hline $\mathrm{PSI}$ & $\Gamma$ & $C 5$ & $C$ & $\Gamma$ & $\Gamma$ & $\Gamma$ & $\Gamma$ & $\Gamma$ & $\Gamma$ \\
\hline $\mathrm{K}[\mathrm{tcu}]$ & $C$ & $C \%$ & $C$ & $\Gamma$ & 0 & 0 & 0 & 0 & 0 \\
\hline HSou & $C$ & $C 6$ & $C$ & $\Gamma$ & 0 & 0 & 0 & 0 & 0 \\
\hline $\log k[\mathrm{md}]$ & 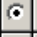 & 6 & $\bullet$ & Г & 0 & 0 & 0 & 0 & 0 \\
\hline $\mathrm{q}^{1}[\mathrm{~g} / \mathrm{cm} 2-\mathrm{a}]$ & $C$ & $\mathrm{C}$ & C & $\Gamma$ & 0 & 0 & 0 & 0 & 0 \\
\hline $\mathrm{q}^{2}[\mathrm{~g} / \mathrm{cm} 2-\mathrm{a}]$ & $C$ & $C 6$ & $C$ & Г & 0 & 0 & 0 & 0 & 0 \\
\hline PHI1 [avg] & $\mathrm{C}$ & $\mathrm{C}$ & $\mathrm{C}$ & $\Gamma$ & 0 & 0 & 0 & 0 & 0 \\
\hline Beta & $C$ & $C 6$ & $C$ & Г & 0 & 0 & 0 & 0 & 0 \\
\hline Aff[Ga-1] & $C$ & $\mathrm{C} / \mathrm{s}$ & $c$ & $\Gamma$ & 0 & 0 & 0 & 0 & 0 \\
\hline$m-f l u x[\mathrm{~g} / \mathrm{cm} 2-\mathrm{a}]$ & $C$ & 56 & $C$ & $\Gamma$ & 0 & 0 & 0 & 0 & 0 \\
\hline Int-mf[kg/cm2] & 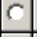 & $\mathrm{C}$ & $c$ & Г & 0 & 0 & 0 & 0 & 0 \\
\hline q dot grad T & $C$ & C 6 & $C$ & $\Gamma$ & 0 & 0 & 0 & 0 & 0 \\
\hline$q$ dot grad $P$ & $\mathrm{C}$ & $C 6$ & $C$ & $\Gamma$ & 0 & 0 & 0 & 0 & 0 \\
\hline Seal Flag & $C$ & $\mathrm{C} / \mathrm{S}$ & $C$ & Г & 0 & 0 & 0 & 0 & 0 \\
\hline
\end{tabular}
$m f[\mathrm{~kg} / \mathrm{cm} 2])$. Figure 150 shows the mass flux vectors superimposed on the log of the integrated mass flux. The importance of the faults in channeling brine flow is apparent. 


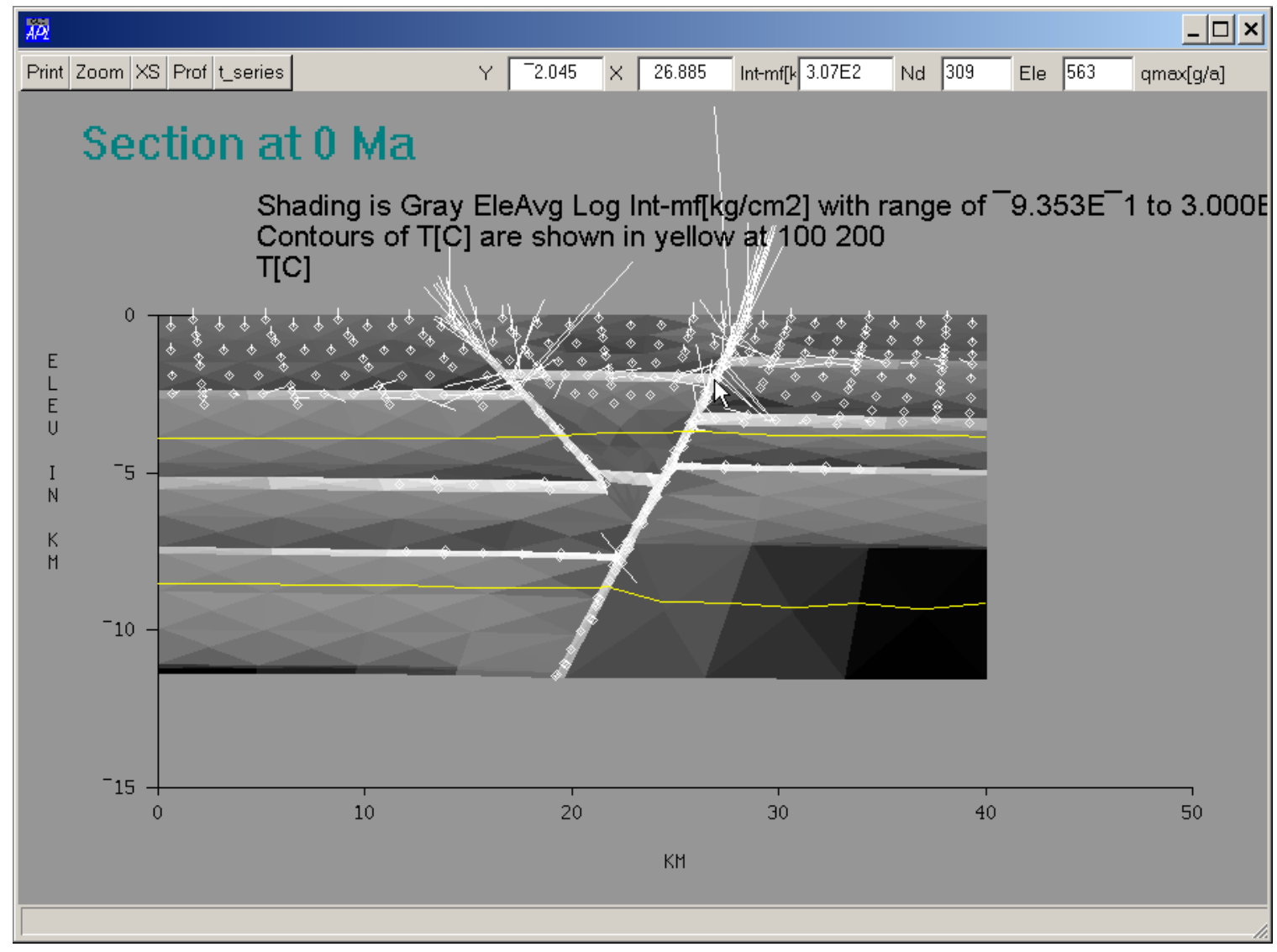

\begin{tabular}{|l|l|l|l|l|l}
\hline $\operatorname{lnt}-\mathrm{m} f[\mathrm{~kg} / \mathrm{cm} 2]$ & $\odot$ & $\odot$ & $\odot$ & $\bar{v}$ \\
\hline
\end{tabular} Calculated Solution: select Int-mf $[\mathrm{kg} / \mathrm{cm} 2-\mathrm{a}]$ and Vector Style - $\log$ Transformation $\mathrm{C}$ off Vector Style $C$ Dir $\odot$ Mag $\triangle$ Log Mag The flag anchor is at the center of each element. Flags are not displayed when the flux is small. The length of the flag can be adjusted in a number of ways (constant length to show direction only, magnitude, and log magnitude). The brine flow is significantly channeled by the main and antithetic faults. The sands low in the section significantly channel brine flow. Most of the brine flow is driven by compaction in the upper $\sim 3 \mathrm{~km}$ of the section. The integrated mass flux is lower in more recently deposited sediments because there has not been as much time for fluids to pass through them. Since Tracking is checked for the integrated mass flux the edit window on the graphic toolbar shows that the integrated mass flux in the main fault at the position of the arrow (element 563 ) is $307 \mathrm{~kg} / \mathrm{cm}^{2}$.

Solution convergence can be studied by running cases with larger NSubt and Nit. The above solution is converged within $\sim 0.5^{\circ} \mathrm{C}$ for the parameters chosen. The impact of different permeability models can be easily explored using the form illustrated in Figure 149. For example one could investigate how the slip-rate model or fault gauge models would impact the 


\section{cumulative flow in the fault, and also the impact of different matrix lithology models by changing these models and re-running the $2 D$ solution.}

\section{B. Brief Comments on Finite Methods}

A complete discussion of the equations and finite element methods of solution is provided in Appendix C. Here we point out a few useful tools.

- The Solve Equations: Diagnostic tools menu allows salt to be temporarily turned

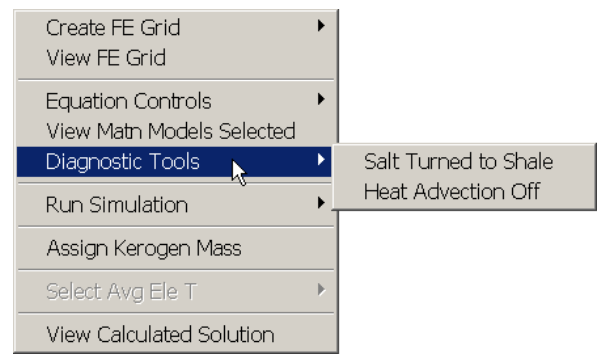

to shale, and thermal advection to be turned off. A common question is how much salt or fluid movement affects the temperature field. These questions can be answered by running a case with salt turned to shale, or with the transport of heat by fluid flow turned off.

- Some cases may encounter problems in convergence at particular timesteps, or a case may blow up at a particular timestep. It is convenient to be able to restart the case at a specified timestep, or compute a single timestep over again. These actions are allowed from the Solve Equations: View Calculated Solution toolbar.

\begin{tabular}{llll|l|}
\hline Frame (18 max) & $18 \div$ Age [Ma $\longdiv { 0 . 0 0 \mathrm { E } 0 }$ & 1-step Solve & Restart \\
\hline
\end{tabular}
Setting the age spinner to a

particular past time and depressing the Restart button will restart the case from that point. Depressing the 1 -step Solve button will re-compute the equation solutions at the next timestep using the present timestep solution as initial conditions. These procedures are possible because BasinLAB stores the full solution in the SOLGRID array at each timestep as the solution progresses. Thus the solution can be restarted or redone at any timestep that has successfully been computed.

- The default solution control parameters shown in Figure 148 can be reset globally (e.g., at all timesteps) using the default assignment menu shown in Figure 151. 

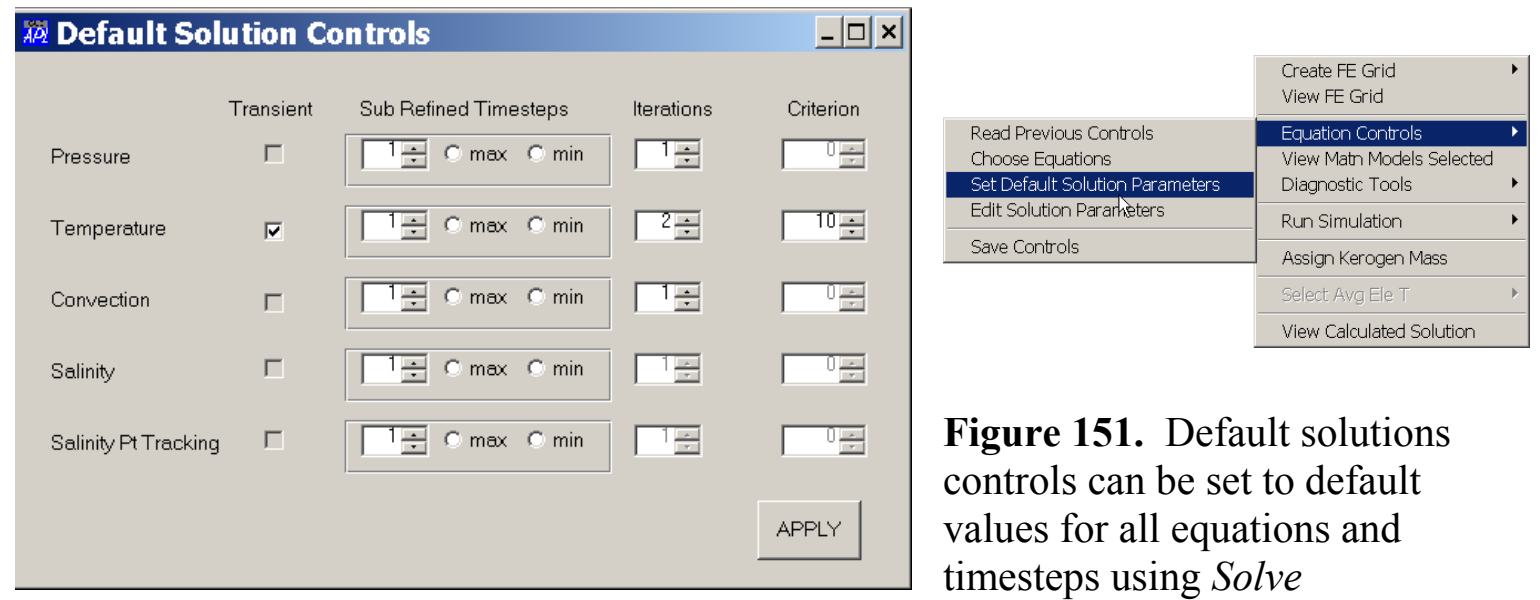

Equations: Equation Controls: Set Default Solution Parameters. In this case we have set the number of iterations for the temperature equation to 2 . The Criterion column specifies, in the case of the temperature equation, the maximum temperature change allowed for a sub-timestep solution. If the temperature change anticipated from the sediment deposition over a sub-timestep exceeds this value, the subtimestep is subdivided until this is not the case. If the min button is selected, the sub-refined timestep will be lor this value, whichever is greatest. If the max button is selected, the sub-refined timestep will be 1 .

- The maximum values of the solution variables (and the maximum change in $\mathrm{T}$ ) are posted on the status bar at the base of the workspace as the solution progresses. This is useful in assessing convergence. Note, however, that the changes are for the last iteration. Thus when the change is small, the iteration number can be reduced by 1 (because the solution was already well resolved with one less iteration). The convergence in the above case was tested in this way. The change with $\mathrm{Nit}=2$ for the temperature equation was less than $\sim 0.5^{\circ} \mathrm{C}$ and thus we could back off to 1 iteration. The maximum temperature for the test case (with the last two deposition events unrefined, e.g. NDelt=1) for $\mathrm{Nit}=2$ was 269.7C and for Nit=1 269.5C. The first case took 2.5 minutes to compute, the second 1 minute 20 seconds. 


\section{Petroleum Migration}

\section{A. Primary Migration}

Capillary forces can be a powerful mechanism for expelling hydrocarbons from source rock. If their matrix is water wet, the fine-grained nature of many source rocks will pull water in and expel any non-wetting phase that has been generated. In the extreme case this water suction will expel petroleum once it reaches the saturation required for mobility (usually considered to be $\sim 20 \mathrm{vol} \%$ of the porosity). We set the expulsion porosity in our models in the Supercritical Migration: Set Migration Controls menu.

There is a possibility, however, that the capillary expulsion of petroleum from thick, finegrained source rocks may be delayed relative to petroleum generation- perhaps for a significant period of time. Basin $\boldsymbol{L} \boldsymbol{A B}$ provides a scratchpad to evaluate this possibility (Figure 152).

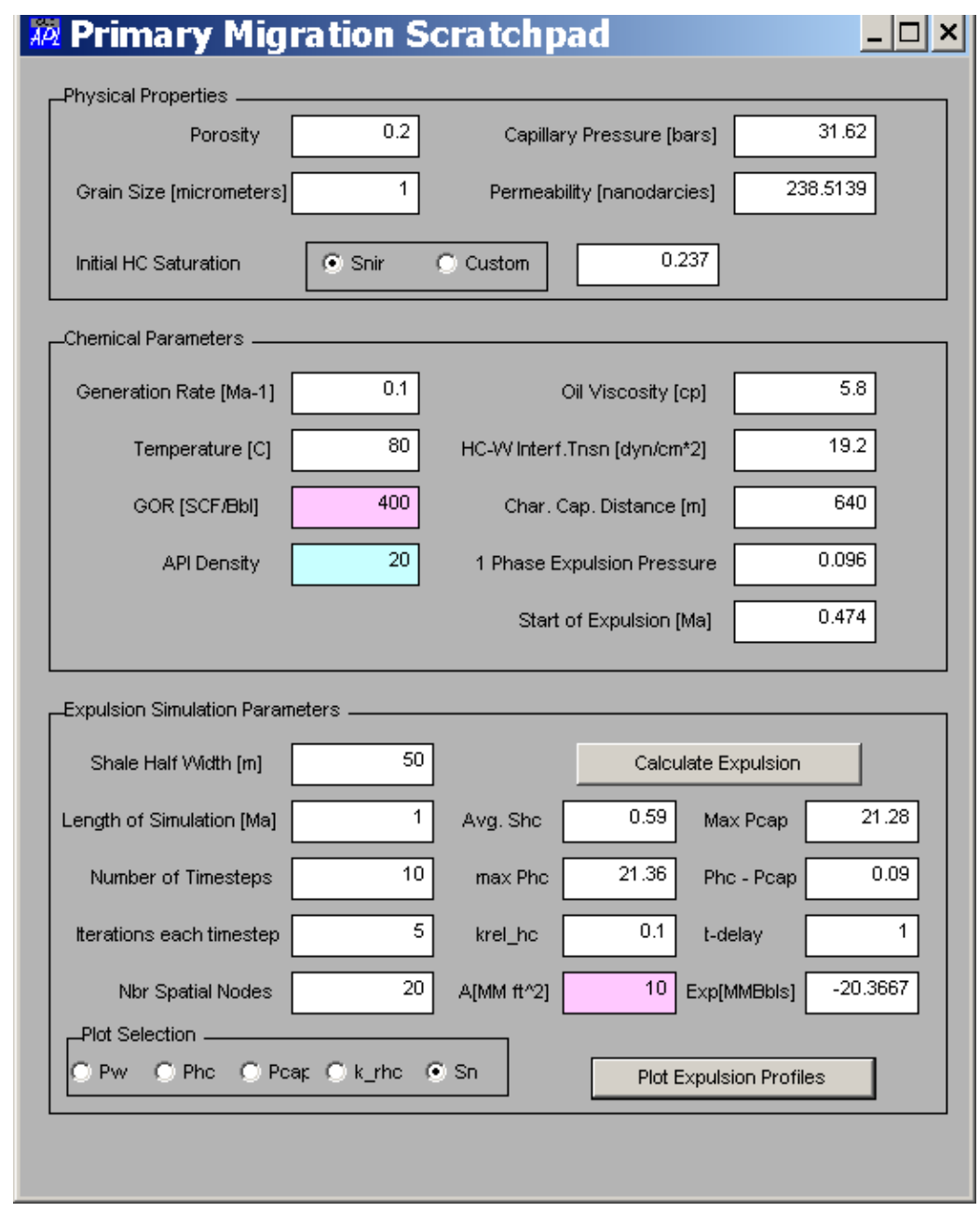

Figure 152. Scratchpad for evaluating the rate of capillary expulsion of petroleum from a source stratum. Access through Primary Migration: Scratchpad. 
The physical properties of the source strata are set in the top group in the form. Input parameters are porosity and grain size. When these parameters are changed, the capillary pressure (difference in pressure between static wetting an non-wetting phases) and the permeability for fluids in the source strata are calculated from the equations listed below.

$$
\begin{aligned}
& k=d^{2} \phi^{3 m} / 24, \\
& p_{c}=2 \sigma / r_{c}, \\
& r_{c}=(2 d / 3) /\left(\phi^{-m}-1\right) .
\end{aligned}
$$

In these equations $\mathrm{k}$ is permeability in units of length squared, $\mathrm{p}_{\mathrm{c}}$ is capillary pressure, $\sigma$ is interfacial tension, $r_{c}$ is the effective pore radius for capillary forces, $d$ is the grain diameter (one of the input parameters), $\phi$ is the porosity (the other input parameter), and $\mathrm{m}$ is the Archie law cementation factor. The equations are discussed in Revil (1998, 2001) and Schowalter (1979).

The second grouping on the form allows the user to specify the petroleum generation rate, the temperature of the source strata, and the petroleum GOR and API gravity. When the mouse leaves these entries, the form calculates the viscosity of the petroleum, the interfacial tension between water and petroleum, a characteristic capillary expulsion distance, the pressure difference required to expel the petroleum at the rate at which it is generated, and the time required for the source strata pores to saturate with petroleum at the generation rate specified. The dead oil (no gas) petroleum viscosity is obtained by regressions against temperature and API gravity, and the gas-saturated viscosity is obtained from a GOR regression. The relationships upon which the regressions are based are shown in Figure 153.
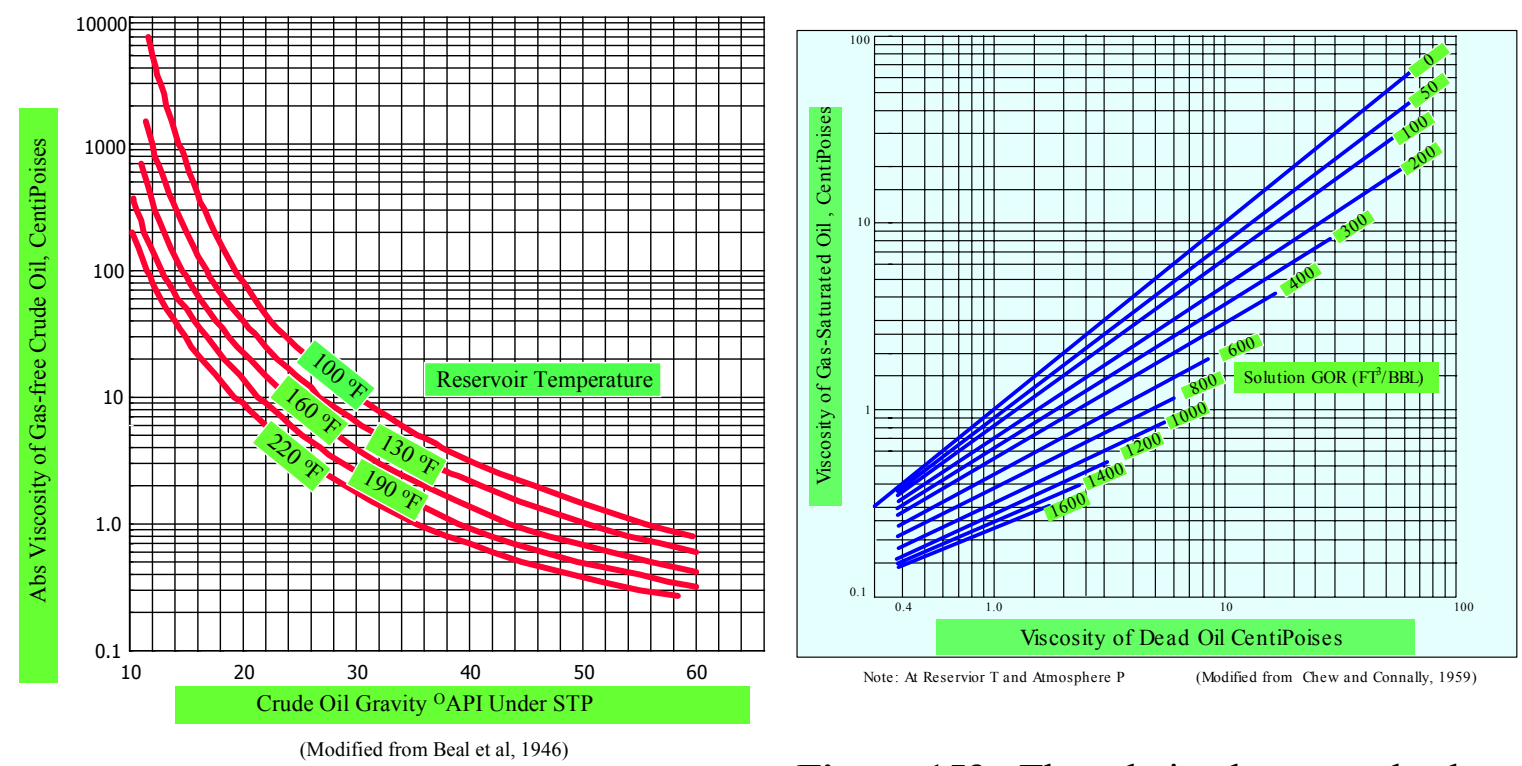

Figure 153. The relation between dead oil (gas free viscosity) temperature and API gravity, and between gas-saturated viscosity and dead oil viscosity and GOR. 
The bottom grouping in the form specifies the dimensions of the source strata and parameters that control the capillary expulsion simulation. The calculations are carried out when the Calculate Expulsion button is depressed. The calculated results can be plotted in a variety of ways (Plot Selection group) if the Plot Expulsion Profiles button is depressed. The calculations are carried out by solving the standard multiphase equations shown below. The fully coupled equations are solved implicitly in 1D using finite difference techniques.

$$
\begin{aligned}
& \nabla \bullet\left(\frac{k k_{w}^{r}}{\mu_{w}} \nabla p_{w}\right)+Q_{w}=\frac{\partial\left(\phi S_{w}\right)}{\partial t}=\phi C_{T} S_{w} \frac{\partial p_{w}}{\partial t}+\phi S_{w}^{\prime} \frac{\partial p_{c}}{\partial t} \\
& \nabla \bullet\left(\frac{k k_{n}^{r}}{\mu_{n}} \nabla p_{n}\right)+Q_{n}=\frac{\partial\left(\phi S_{n}\right)}{\partial t}=\phi C_{T} S_{n} \frac{\partial p_{w}}{\partial t}-\phi S_{w}^{\prime} \frac{\partial p_{c}}{\partial t} \\
& S_{w}^{\prime}=\frac{\partial S_{w}}{\partial p_{c}}=-\frac{\partial S_{n}}{\partial p_{c}}, \quad C_{T}=\frac{1}{\phi} \frac{\partial \phi}{\partial p_{w}}, \quad S_{w}+S_{n}=1
\end{aligned}
$$

In these equations $\mathrm{k}$ is intrinsic permeability, $k_{w}^{r}$ and $k_{n}^{r}$ are the relative permeability to the wetting and non-wetting phase, $\mathrm{Q}_{\mathrm{w}}$ is the source term for water $(=0)$ and $\mathrm{Q}_{\mathrm{n}}$ the source term for the non-wetting phase (the petroleum generation rate), $S_{w}$ and $S_{n}$ are the saturation of the wetting and non-wetting phases (the fraction of the pore space filled with these phases), $\mathrm{C}_{\mathrm{T}}$ is the pore compressibility, $\phi$ is porosity, and $\mathrm{p}_{\mathrm{c}}$ is the capillary pressure.

The relative permeability curve used can be displayed using the Primary Migration: Plot RelPerm Curves menu command. The relative permeability curves used in the examples given below are shown in Figure 154.

\section{Relative Permeablity Curves ( $\mathrm{W}=$ blue, $\mathrm{N}=\mathrm{red})$}

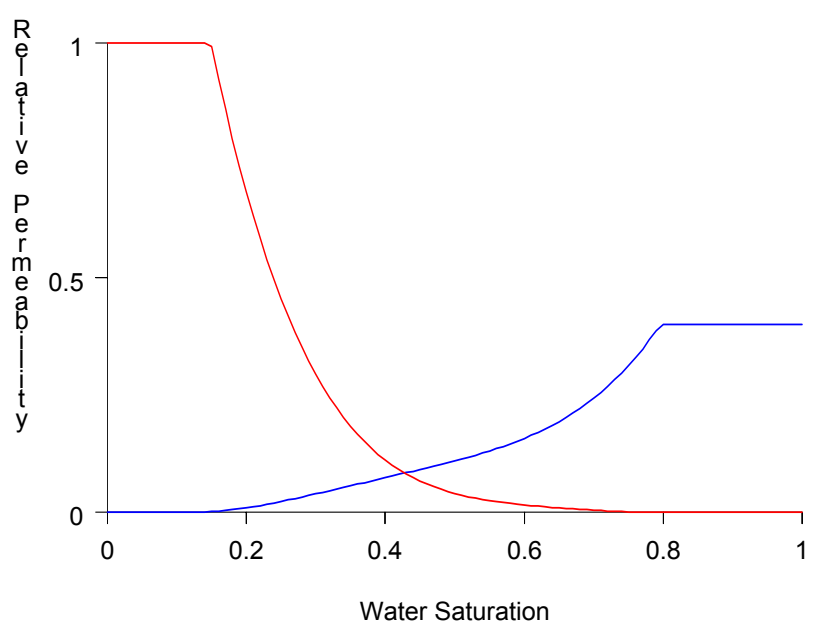

Figure 154. The relative permeability curves used in the hydrocarbon expulsion simulations shown in the following figures. Obtained using the Primary Migration: Plot RelPerm Curves menu. 
Figure 155 shows that at $80^{\circ} \mathrm{C}$ a $100 \mathrm{~m}$ thick source bed with a 0.5 -micron grain size could take $1 \mathrm{Ma}$ to drain about half way to the irreducible petroleum saturation of 0.2 .

\section{Hydrocarbon Saturation Profile}

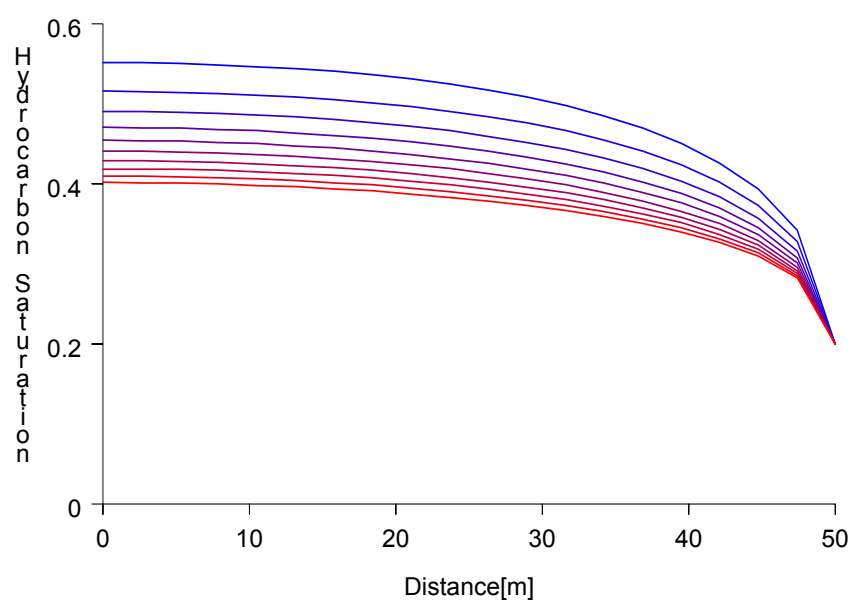

Figure 155. The time required to expel petroleum from a $100 \mathrm{~m}$ thick source bed if the grain size is 0.5 microns. The initial hydrocarbon saturation is set at 0.6 , the generation rate to 0 , and saturations are calculated every 100,000 yrs for $1 \mathrm{Ma}$ (Number of Timesteps $=10)$. After $1 \mathrm{Ma}$ the saturation has dropped from 0.6 to $\sim 0.44$. Useful statistics are given on the form as shown below.

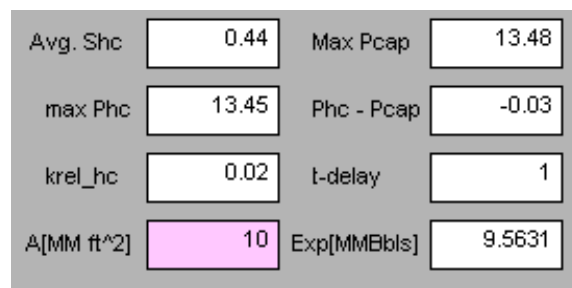

Figure 156 shows that $1 \mathrm{Ma}$ after the irreducible petroleum saturation is reached in the source strata, the petroleum saturation has reached over 0.6 and the expulsion rate is approaching the petroleum Hydrocarbon Saturation Profile generation rate.

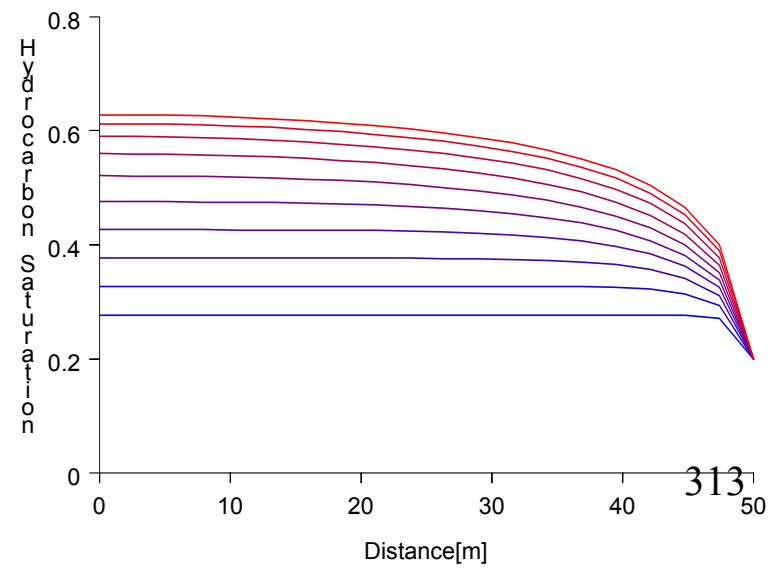

Figure 156. The petroleum saturation is $\sim 0.6$ and the expulsion and generation rates are approximately equal after $1 \mathrm{Ma}$ for the $100 \mathrm{~m}$ thick source strata discussed above. For this calculation the Generation Rate was set to 0.1 
$\mathrm{Ma}^{-1}$. The Length of Simulation is $1 \mathrm{Ma}$. The Number of Timesteps to 10. This petroleum would bleed from the source strata for millions of years after petroleum generation had ceased (see Figure 155).

We should note that the hydrocarbon retention and time delay of its expulsion is very dependent on the source strata thickness. Figure 157 shows that the retention for the conditions of Figure 156 in a $10 \mathrm{~m}$ thick source bed (or a source bed that was fractured every $10 \mathrm{~m}$ ) would be $\sim 0.3$ and would be reached in $\sim 200 \mathrm{ka}$ at a hydrocarbon generation rate of $0.1 \mathrm{Ma}^{-1}$. For a source strata thickness of $1 \mathrm{~m}$ the retention would be only slightly greater than the irreducible petroleum saturation.

\section{Hydrocarbon Saturation Profile}

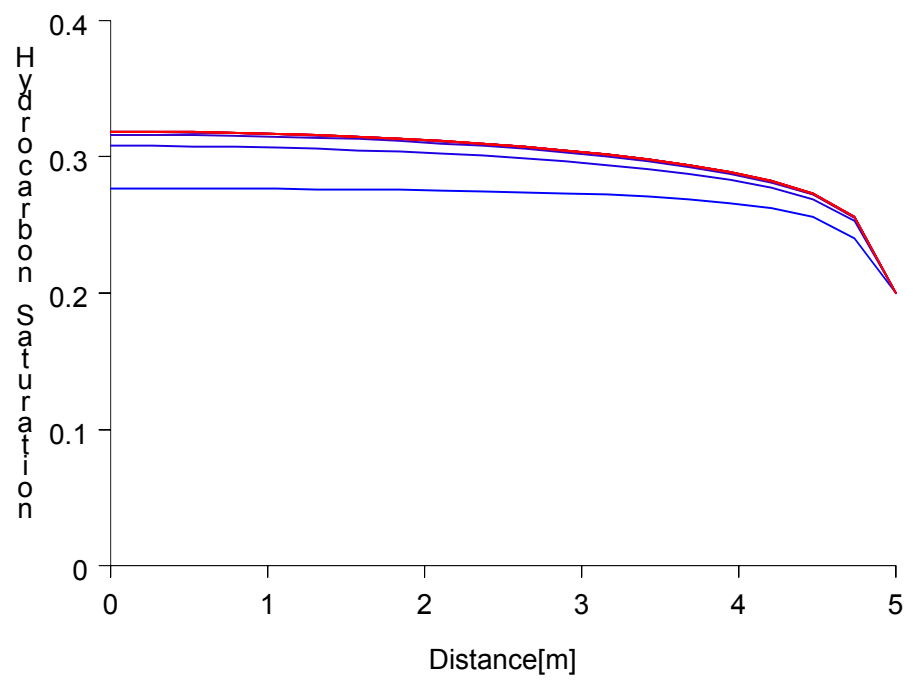

Figure 157. The retention of petroleum under the conditions of Figure 156 but with a $10 \mathrm{~m}$ thick source strata are reached in $\sim 200$ ka rather that in $\sim 1 \mathrm{Ma}$ and the retention is $\sim 0.3$ rather than 0.6 .

\section{B. Secondary Migration}

Migration was vertical in the case discussed in Section $\mathbf{V}$ because we did not solve the flow equation. In the Antithetic fault case we have solved for flow, and thus hydrocarbon migration can be directed vertically, parallel to the brine flow, or any vector rotation in between. The procedure for computing secondary migration is summarized in Chart 5 . It is exactly as described in Sections $\boldsymbol{V} . \boldsymbol{F}$ and $\boldsymbol{V} . \boldsymbol{G}$ and is discussed in a number of bullet steps below. 

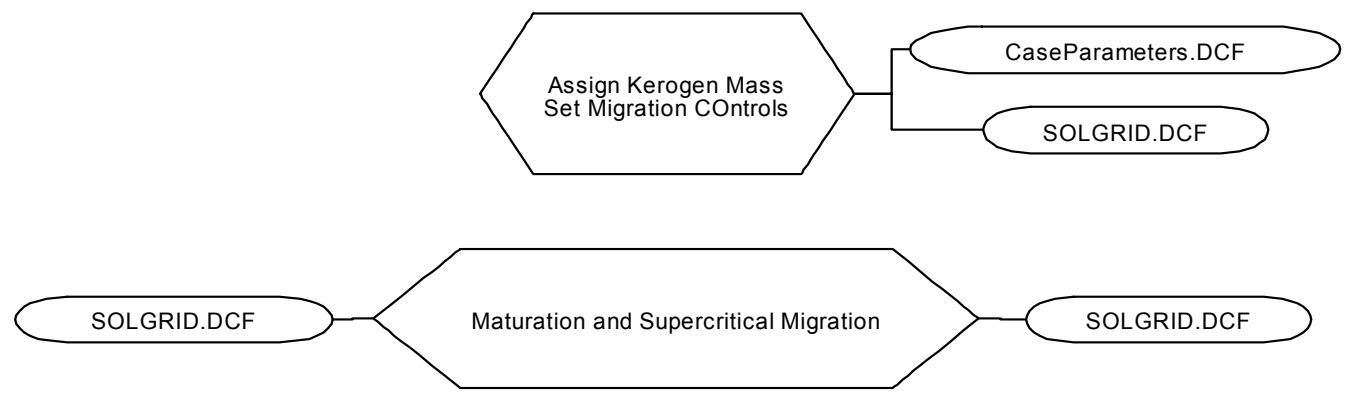

Choose Pressure Convention Pick Petroleum CaseParameters.DCF Set Decomposition Parameters

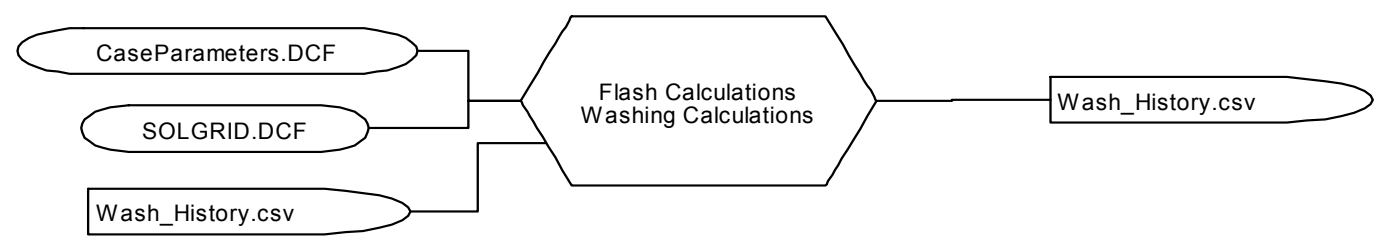

Chart 5. Flow chart for hydrocarbon maturation, migration, petroleum decomposition, flashing and gas washing. Box conventions are the same as shown in Chart 1. Chart 4 is on page 293.

- Select a hydrocarbon maturation models for Type I kerogen using Parameters: Read or Edit Kinetics: HC Kinetics (Ker1): Select. In this case we select a Type II kerogen kinetics II_YT_B684F.

- Assign the kerogen masses to the source strata using Solve Equations: Assign Kerogen Mass.

- Calculate the Solid Phase maturation using Calculate Supercritical Migration: Calculate Solid Phase Migration:

\section{Calculate Solid Phase Maturation}

GeoHistory Parameters

Material Properties

Thermal Conductivity (+scrpad)

Heat Generation (+scrpad)

Permeability (+scrpad)

Read or Edit Kinetics

Evaluate Kinetics Scrpad

HC Kinetics (ker 1)

Thermocline and Basal HF

Thermal Calculation Controls

Set Migration Controls

Reset to Default Parameters

Reset to CaseParameters 
- Set the migration controls using Supercritical Migration: Set Migration Controls: The direction of petroleum migration (parallel to brine flow, vertical, and in between) is set by the Migration Buoyancy Rotation Track Bar shown on the right.

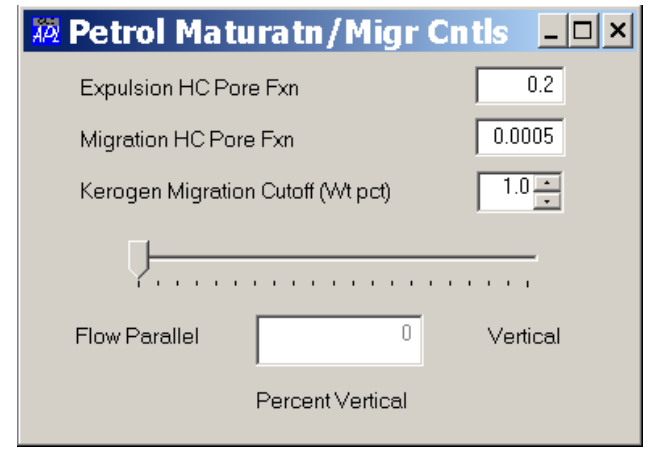

- Calculate the cases using Supercritical Migration: Calculate Mobile Phase Maturation. Note this menu is made active

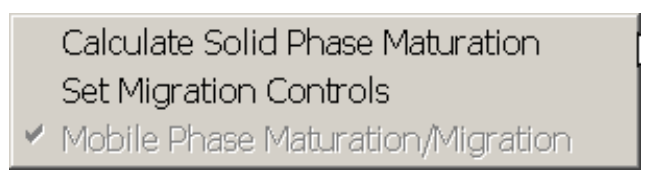
after Calculate Solid Phase Migration and then made inactive with a check mark as shown below after it is executed. An new case (with a different buoyancy rotation) can be computed by recalculating the Solid Phase Maturation, setting the Migration Controls to different values, and recomputing the Mobile Phase Matur/Migr.

Figure 158 shows the instantaneous mass transfer of hydrocarbons between elements at a number of times for a buoyancy rotation of 0 and $1 \%$ at $1.44 \mathrm{Ma}$. The source bed in the left half of the section matures and expels hydrocarbons at about $1.8 \mathrm{Ma}$. The source in the right half of the section matures and expels starting $\sim 0.67 \mathrm{Ma}$. Figure 159 shows the petroleum transfer between elements at the present day. The influence of faults on petroleum migration can be dramatic, but even a little buoyancy rotation greatly diminishes their effect. Figure 160 shows profiles of petroleum flux illustrating the fault control. Figure 161 shows that the petroleum mass conservation in the section is excellent. Figure 162 shows the sedimentation history. 


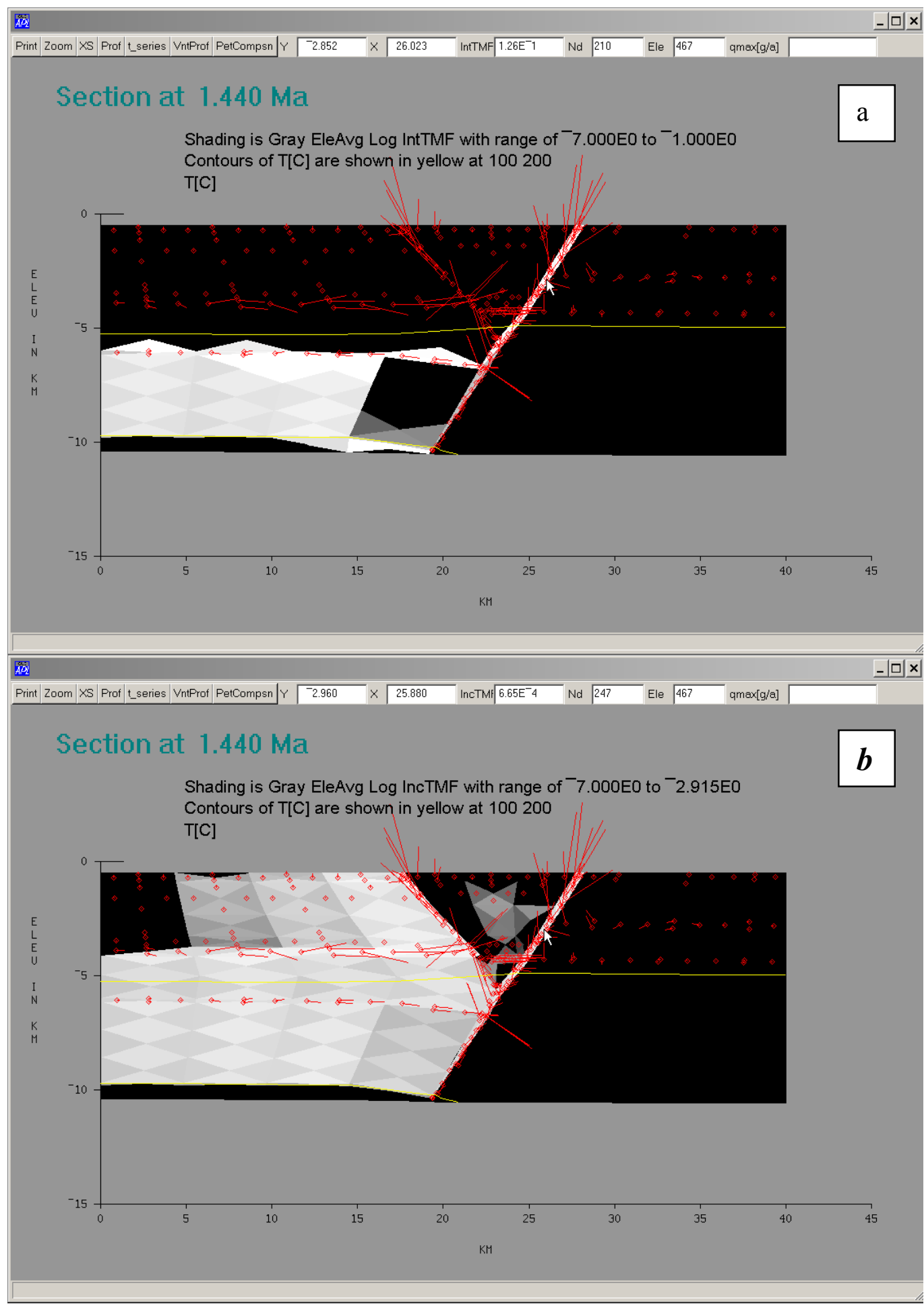

Figure 158. Methane distribution at $1.833 \mathrm{Ma}$ for (a) petroleum migration parallel to brine flow (0\% vertical) and (b) 1\% vertical. Calculated with Supercritical Migration: Mobile Phase Maturation/Migration. The red wind flags show brine flow with the flag length proportional to the - $\log$ Transformation C on $C$ off $-7 \div-1 \div$ brine flux. The log petroleum mass transfer (IncTMF) 
between elements (in billions of tones) over the timestep is shown in grayscale. The grayscale range is from $10^{-7}$ to $10^{-1}$.

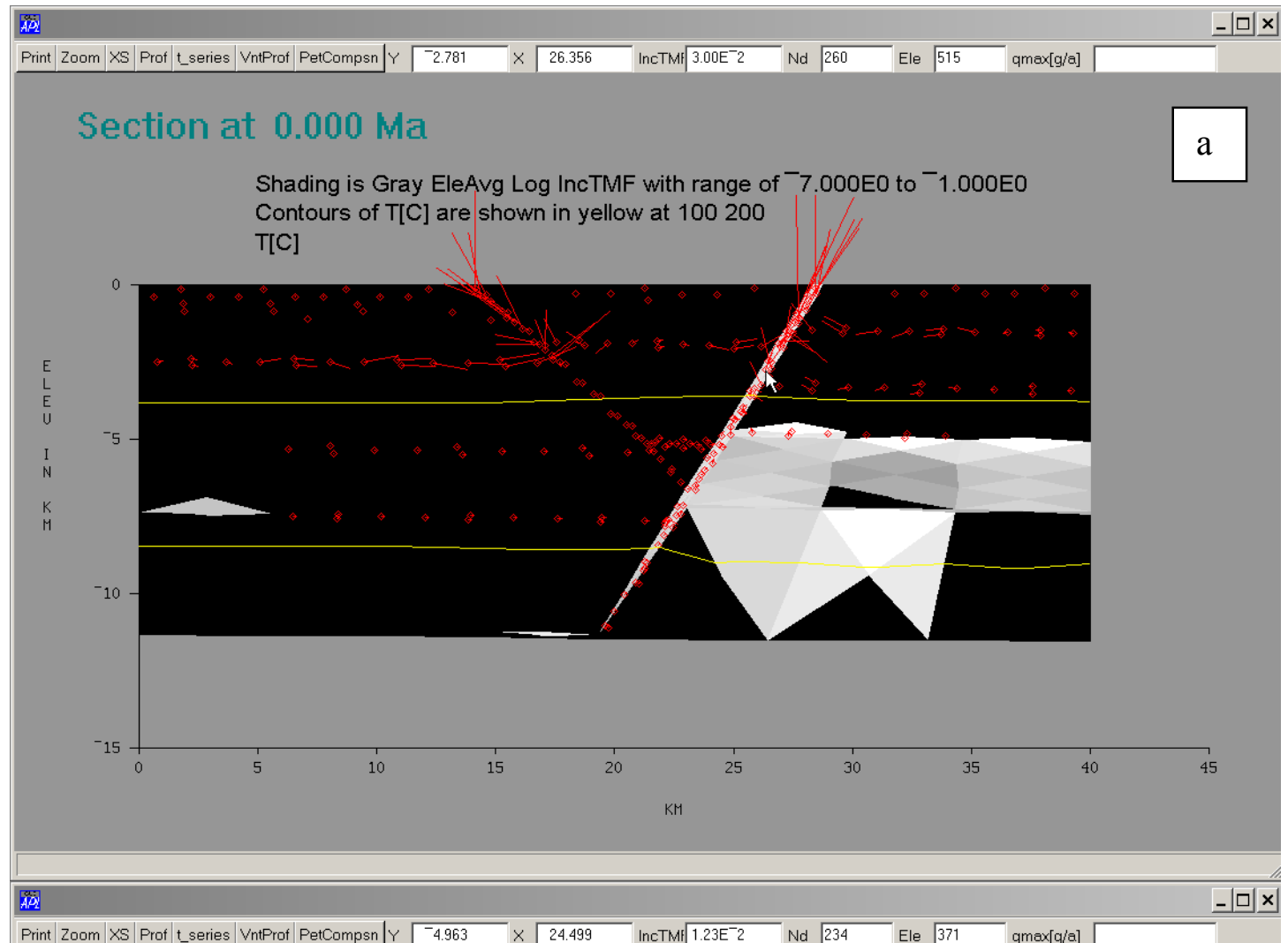

Print Zoom XS Prof t_series VntProf PetCompsn $\mid Y{ }_{-4.963}^{-4} \times \sqrt{24.499}$ IncTMF $1.23 E^{-2}$ Nd 234 Ele 371 qmax[g/a]

\section{Section at $0.000 \mathrm{Ma}$}

Shading is Gray EleAvg Log IncTMF with range of ${ }^{-} 7.000 \mathrm{E} 0$ to ${ }^{-} 1.542 \mathrm{E} 0$

Contours of T[C] are shown in yellow at 100200

$\mathrm{T}[\mathrm{C}]$

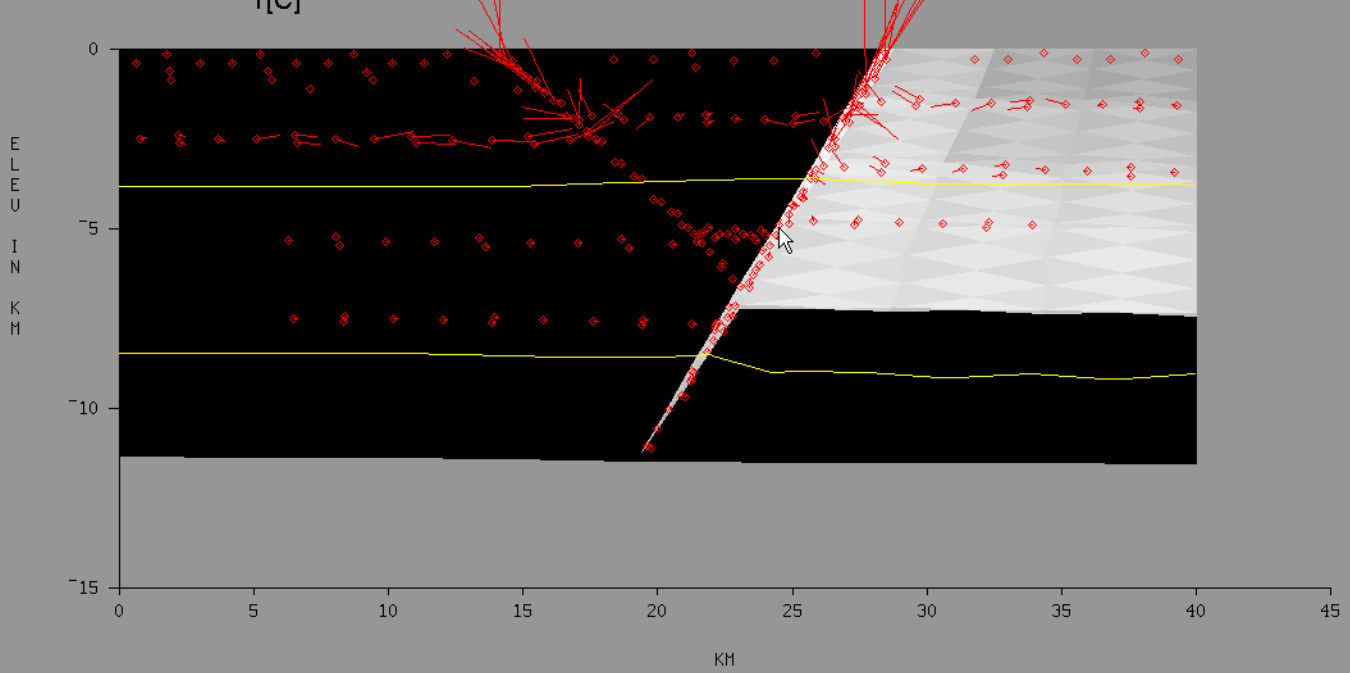

Figure 159. Methane distribution at $0 \mathrm{Ma}$ for (a) petroleum migration parallel to brine flow (0\% vertical), and (b) $1 \%$ vertical. Calculated with Supercritical Migration: Mobile 
Phase Maturation/Migration. The conventions are the same as in Figure 158, except the age of the section has been changed using the toolbar spinner. Petroleum generation begins at about $0.67 \mathrm{Ma}$ in the right half of the section and is near maximum at the present day.

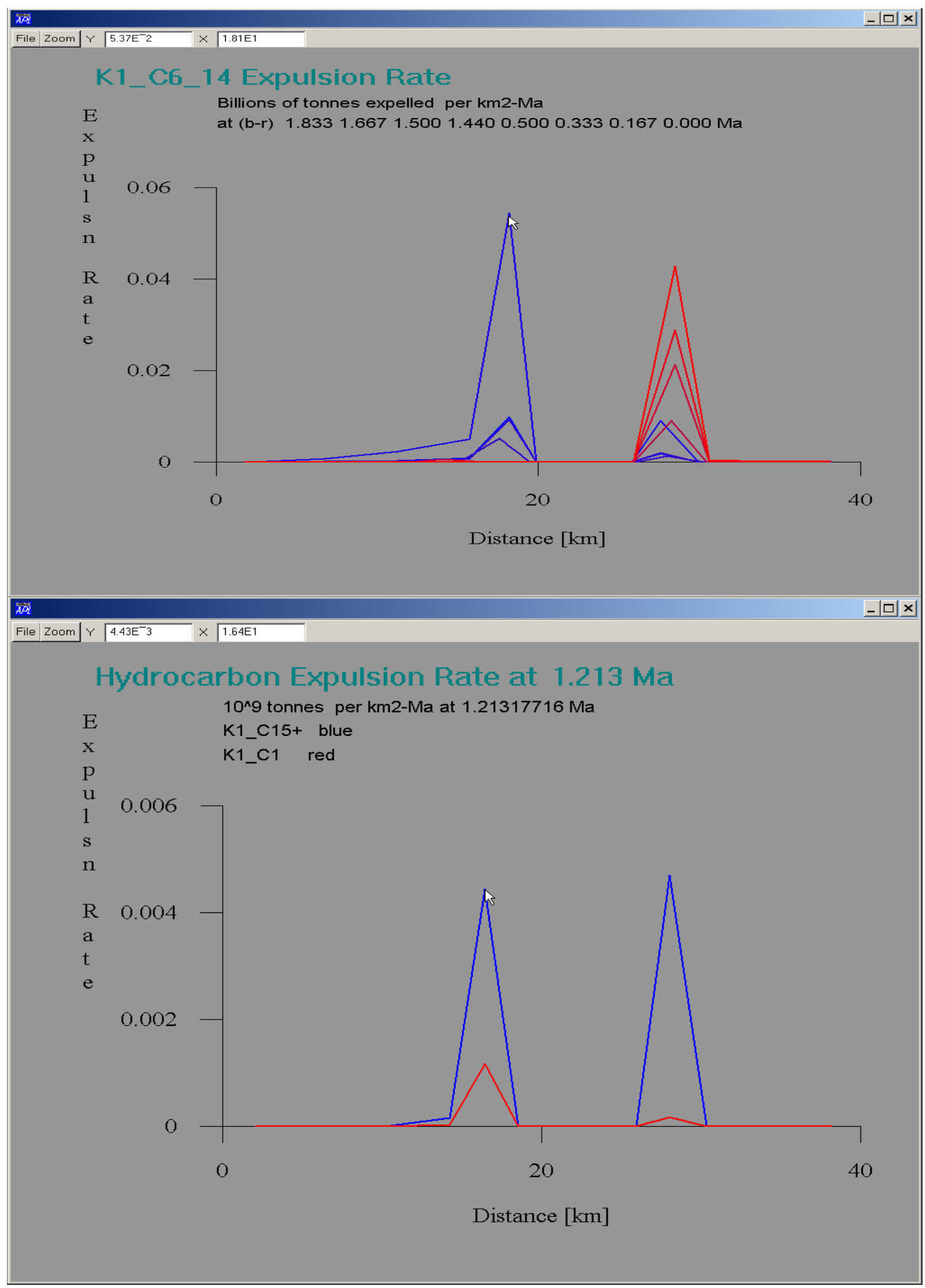


Figure 160. $\mathrm{C}_{6-14}$ venting in $10^{9}(\mathrm{Bt})$ tones $/ \mathrm{km}^{2}$-Ma at past times for the $1 \%$ buoyancy rotation. Plotted using Solve Equations: View Calculated Solution: VntProf button: selecting either a single product and multiple ages or multiple products and a single minimum age: and using the $\mathrm{v} \mathrm{P}$ button (for present venting).

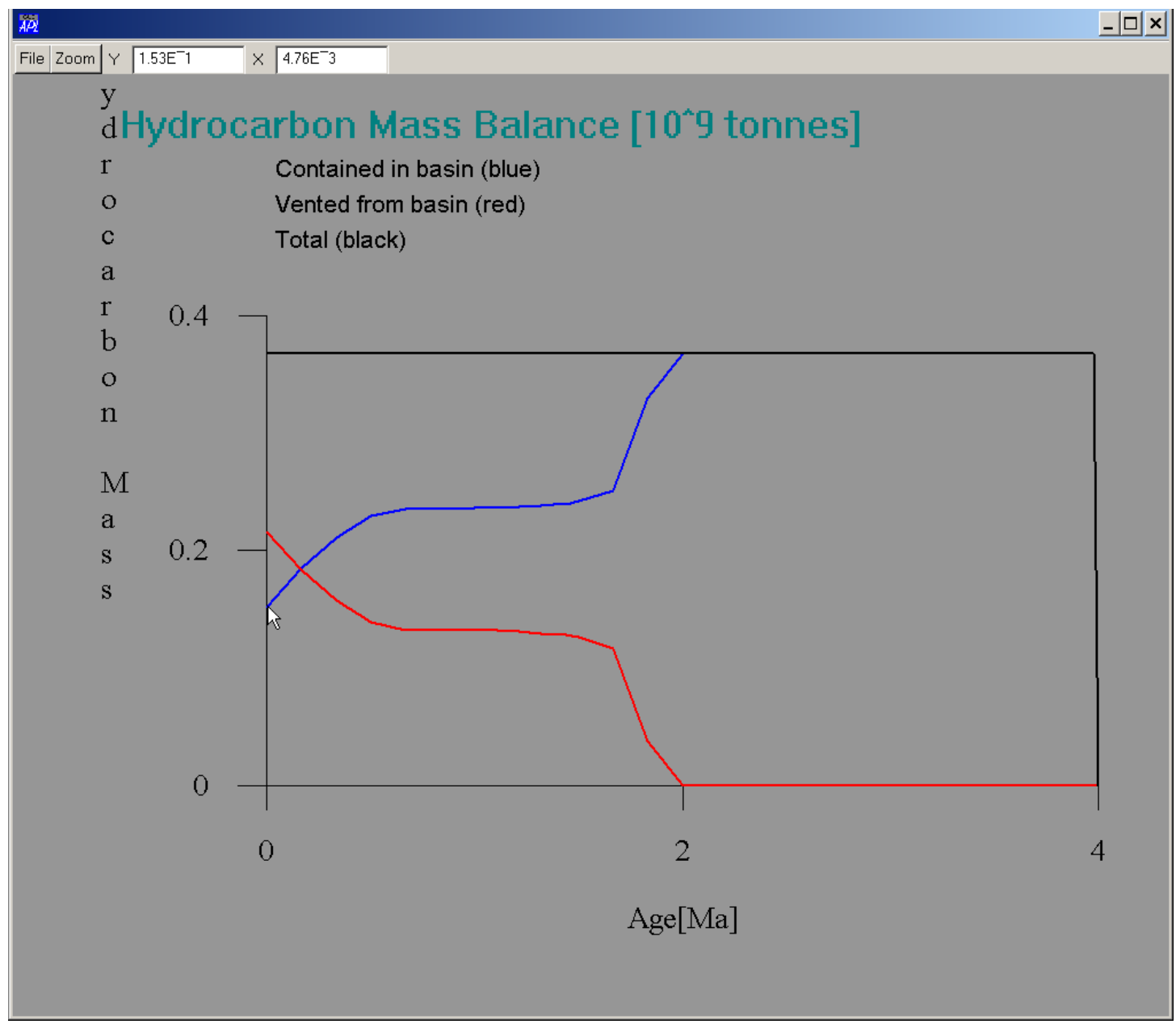

Figure 161. The black curve shows the total petroleum-generating potential of the section (and $+/-0.5 \mathrm{~km}$ to either side), the red curve the petroleum expelled from the basin (to the ocean) and the blue curve the petroleum (generated and potential remaining in the basin). The black curve is the sum of the red and blue. Its flat top shows that petroleum mass has been faithfully conserved in the HCMassBal simulation. Plot is for $1 \%$ buoyancy rotation. The pulses of petroleum generation and

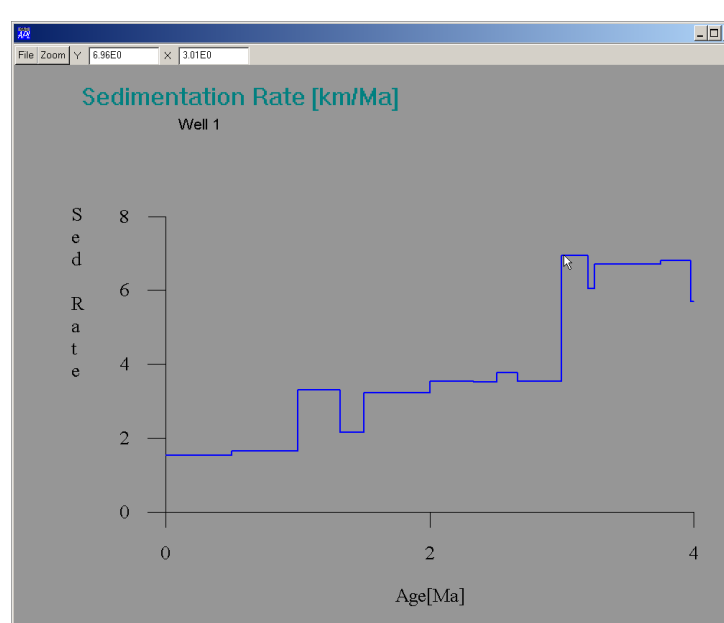
migration on the left and right of the section are evident in the balance curves. Curves are generated by depressing the HCMassBal button when the Solve Equations: View Calculated Solutions has been activated. 
Figure 162. Sedimentation history for the BasinModels|blcases $\mid$ Examples.BL $\backslash$ Antithetic_Fault.2d $\mid$ case discussed above. Obtained by Solve Equations: View Calculated well: depress Sedimentation Rate[t] Solution: Prof button: navigate to desired Prof button. Sedimenation Rate[t]

\section{Comments on Secondary Migration Methods}

The methods of calculating hydrocarbon migration are straightforward. The full history of solid phase maturation is calculated first. No expulsion is allowed and no maturation of the liquid products is allowed. The incremental amount of liquid product generated over each time interval is recorded in the SOLGRID array (and can be examined using the Solve Equations: View Calculated Solution menu). This step is accomplished with the Supercritical Migration: Calculate Solid Phase Maturation menu.

The next step is to calculate the migration and maturation of the mobile products of solid maturation. This is done, subject to the migration controls (source and migration saturation, and buoyancy rotation), by first identifying the petroleum mass in excess of that required to meet the volume saturation requirement for expulsion from the source. This mass is then swept (by buoyancy and flow) into successive elements. The petroleum is moved parallel to the brine flow or vertically depending on the buoyancy rotor setting. The petroleum can move to an adjacent element only when the intervening elements contain a petroleum mass equal to that required to fill the element pore space to the migration saturation.

Transfer from element to element is computed as follows. The dot product of the brine flow vector and the outward normal vectors of the element sides is computed, and the transfer of the excess petroleum mass in the element is made to adjacent elements in proportion of the positive magnitudes of their dot products. If the element is in a fault, the petroleum transfer is vertical regardless of the dot product. Swapping of petroleum across the common side of two elements is eliminated letting the largest flux vector control the transfer unless the vectors are within $10 \%$ of being equal in size whereupon the petroleum is moved vertically upward.

An excess mass threshold of $10^{-5} \mathrm{Bt}$ is used to select overfilled elements. If elements remain after the first sweep, a second sweep is carried out. All over-filled elements are processed in a cycle. The status bar shows how many flowing elements there are at a given cycle, and also shows the number of cycles in the timestep sweep. Typically the number of flowing elements rises as the petroleum is swept out of the source strata. The number then decays as the petroleum is removed to the migration saturation of the elements. All mobile components are kept track of in all elements and all times. These variables are available for plotting (Solve Equations: View Calculated Solution). When more than one kerogen is matured, mixing relations between the two products can easily be calculated from the recorded data. 
The petroleum migration scheme is simple, rigorously conserves mass, accommodates fault-control, and is easily extensible to include other factors influencing migration such as gravity migration along the top of a carrier sand. 


\section{Petroleum Composition and Phase Chemistry}

\section{A. Petroleum Composition}

The ability to examine all variables in sophisticated ways is particularly useful in evaluating the complex chemical changes that may attend petroleum migration. Here we illustrate again (see also Sections IV.I-K) some of the plots that are available. We consider the Antithetic fault case with a $1 \%$ buoyancy rotation. The source expulsion pore saturation is $20 \%$ and the migration pore saturation is $0.05 \%$ as previously discussed.

Figure 161 has already suggested that hydrocarbon generation in the left side of the section is completed by $\sim 1.8 \mathrm{Ma}$. Figure 163 shows how the cumulative and instantaneous hydrocarbon flux through the element just above the source strata adjacent to well 1 changes with time. It can be seen that, indeed, hydrocarbon generation is completed by $\sim 1.5 \mathrm{Ma}$.
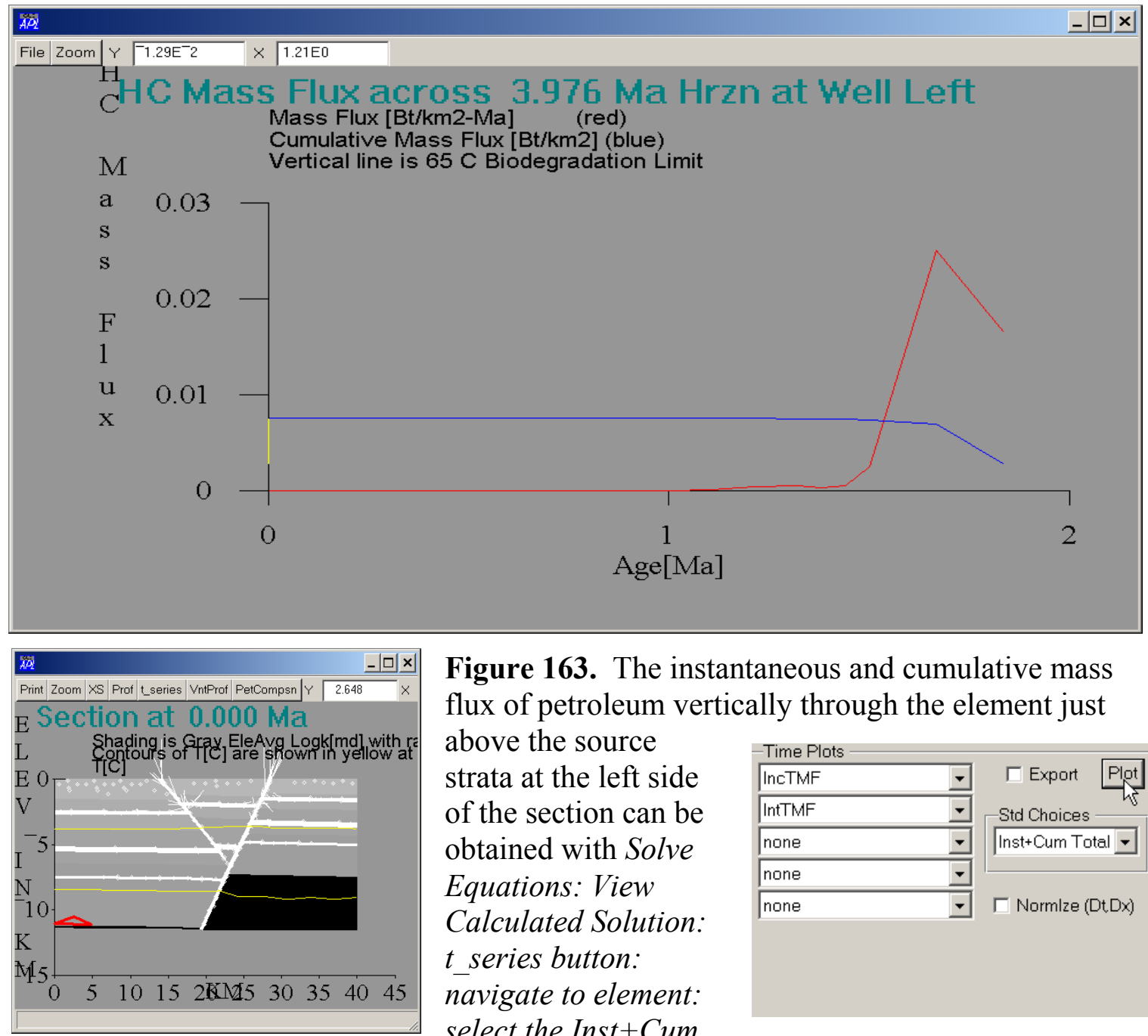

Figure 163. The instantaneous and cumulative mass flux of petroleum vertically through the element just above the source strata at the left side of the section can be obtained with Solve Equations: View Calculated Solution:

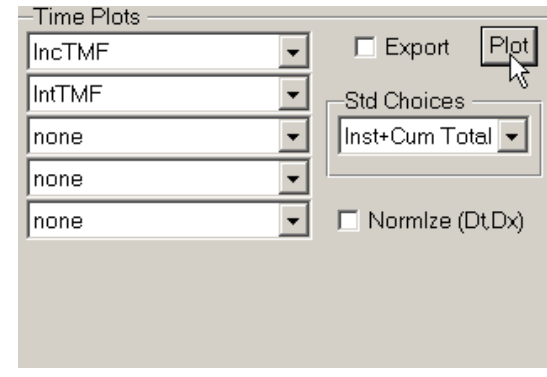
series button: navigate to element: select the Inst+Cum 
Total mass flux from the Std Choices group of the $t$ _series form and depress the plot button as shown.

THE HYDROCARBONS THAT HAVE BEEN GENERATED HAVE NOT FLOODED THE ENTIRE SECTION BECAUSE THEY HAVE BEEN CHANNELED INTO THE FAULTS. FIGURE 164-FIGURE 165 SHOW THE DISTRIBUTION AND COMPOSITION OF HYDROCARBONS IN THE SECTION. FIGURE 166 SHOWS THE HYDROCARBON COMPOSITION IN THE FAULT OVER TIME AND FIGURE 167 THE COMPOSITION LEAVING THE SOURCE.

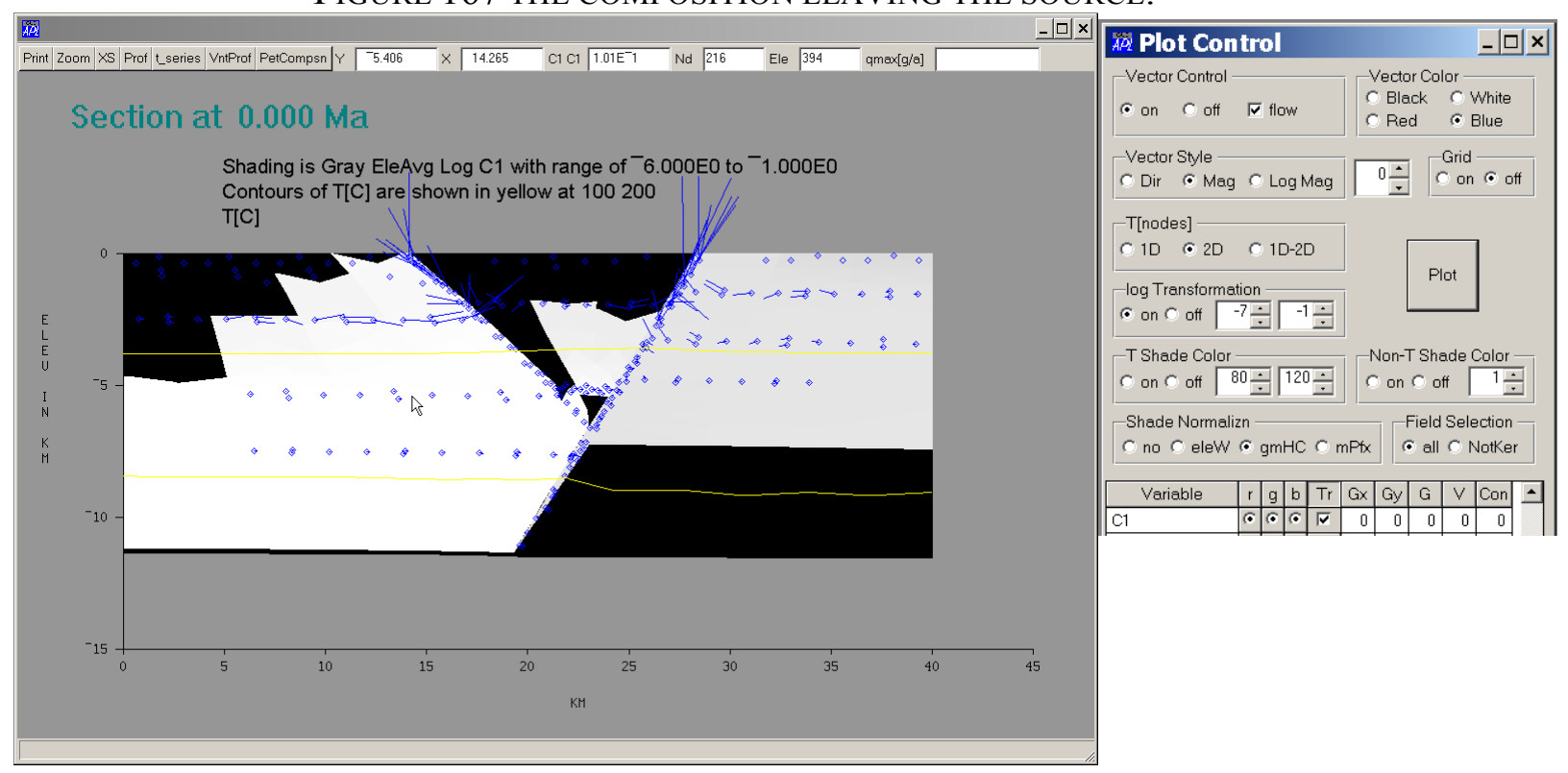

Figure 164. The distribution of $\mathrm{C} 1$ in the section. Plot obtained by Solve Equations: View Calculated Solution: XM Plot Control form with selections made as shown to the right. $\mathrm{C} 1$ has been selected (spreadsheet is scrolled up so it is the top entry). Grams of hydrocarbon ( $g m H C)$ have been selected as the Shade Normalization so that the mass fraction $\mathrm{C}_{1}$ is displayed. The log Transformation is turned on with limits of $10^{-1}$ and $10^{-7}$ so that the full distribution of $\mathrm{C}_{1}$ is evident. A blue Vector Color has been selected so that the brine mass flux vectors show against the gray and black background. Figure 165 shows a profile of hydrocarbon compositions in the middle left portion of the section.
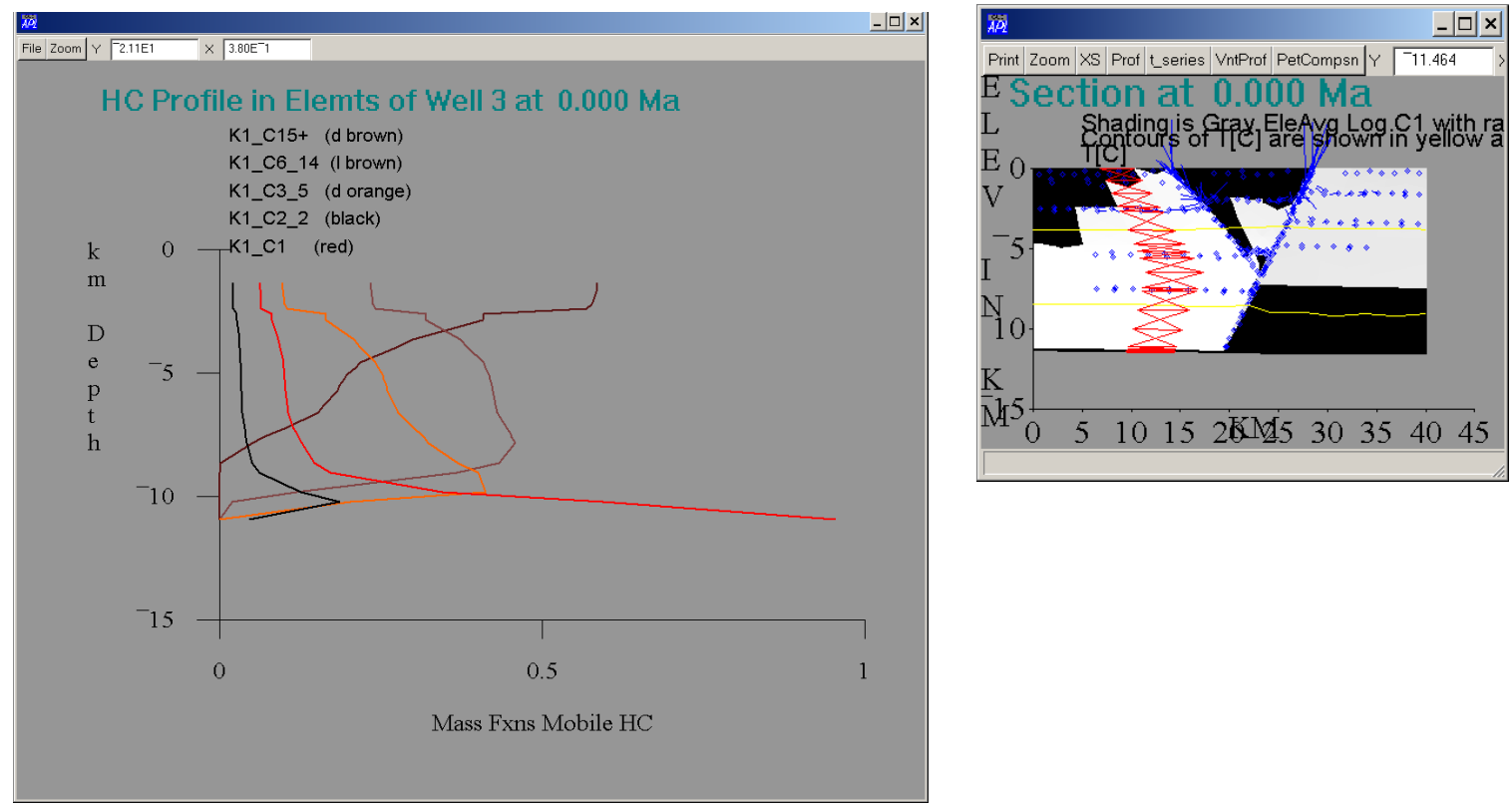


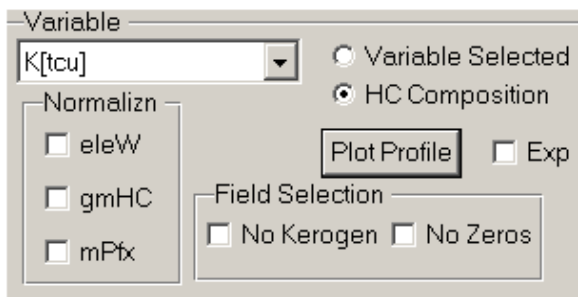

Figure 165. Profile through the section in the position shown obtained using Solve Equations: View Calculated Solution: Prof form: navigating to position shown: selecting HC Composition: depressing Plot Profile. The $k 1$ mass fraction has also been selected. Methane dominates the deep section; oil the shallow.

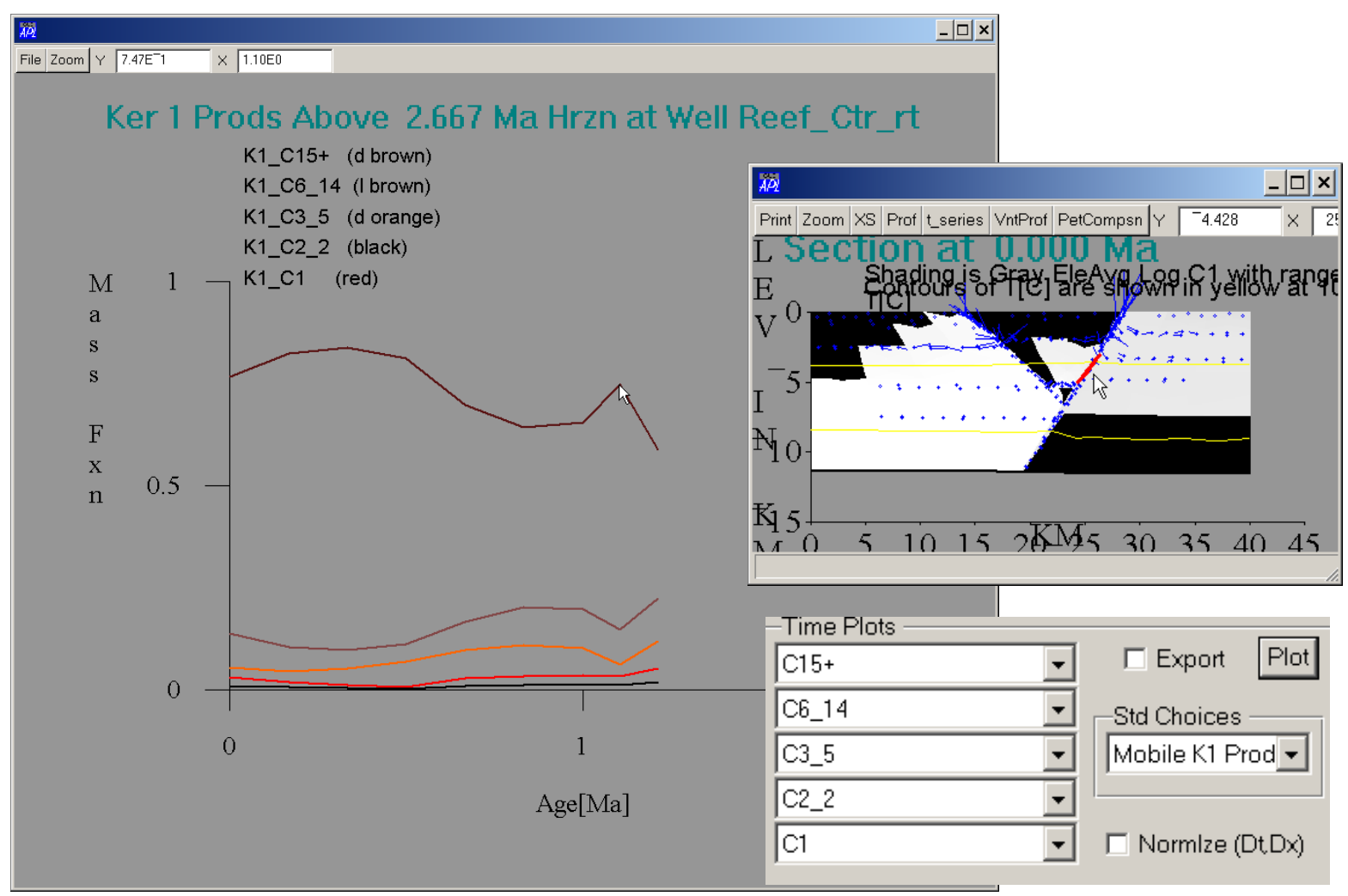

Figure 166. A time series plot of hydrocarbon composition at the location of the main fault shown by the red outline and arrow is obtained with Solve Equations: View Calculated Solution: $t$ series. The Mobile K1 Products standard choice has been selected. The hydrocarbon composition at first becomes more gas-rich, but this tendency is overcome by the later generation of oil in the right half of the section. Figure 167 shows a similar time series plot for hydrocarbons leaving the source strata in the left side of the section. 

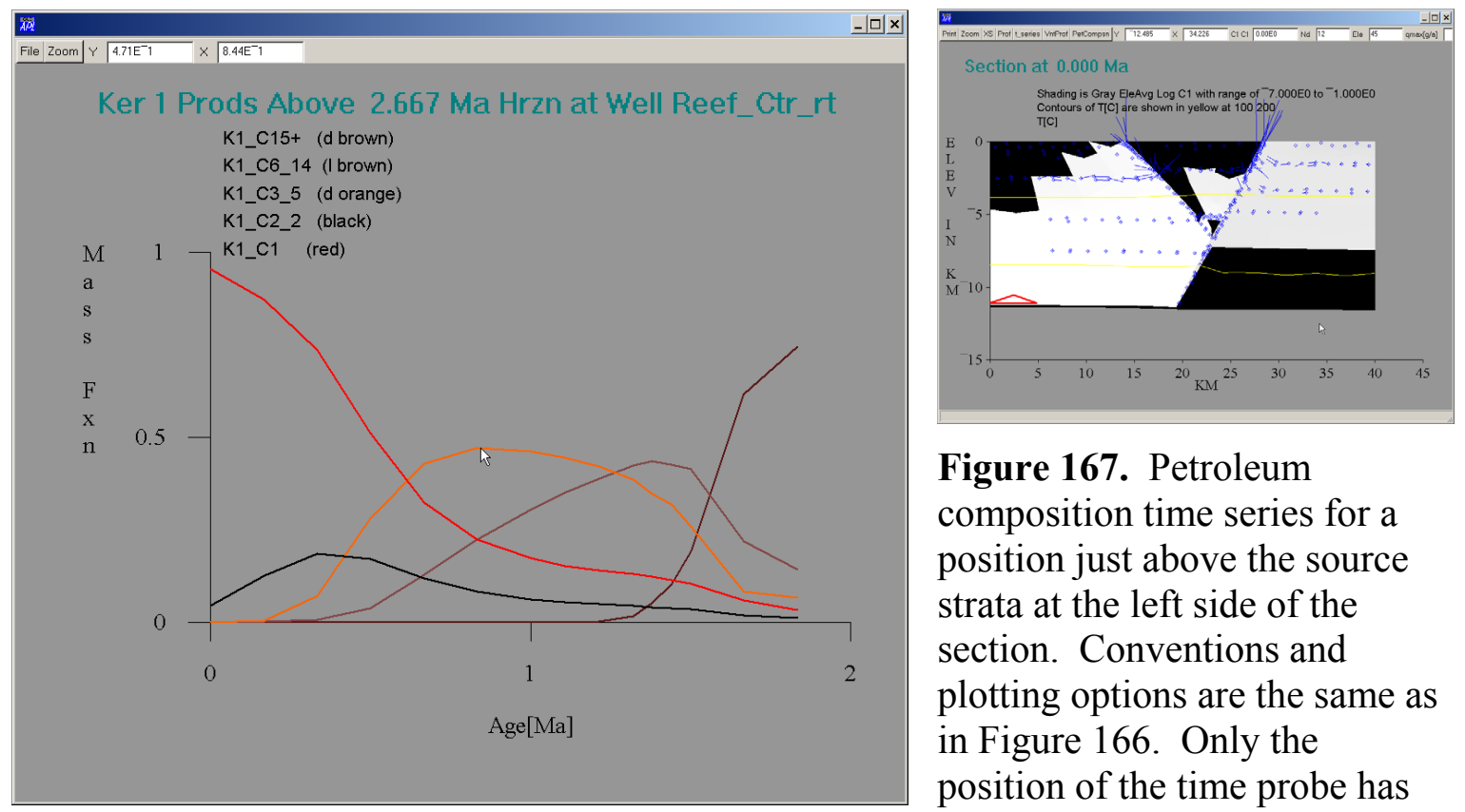

Figure 167. Petroleum composition time series for a position just above the source strata at the left side of the section. Conventions and plotting options are the same as in Figure 166. Only the position of the time probe has

been changed. The $\mathrm{C}_{3-5}$ component reaches a maximum mass fraction of $\sim 0.47$ (as shown bythe arrow).

Finally the time series probe can be used to compute how kerogen would mature in any element in the section. The average time-temperature history in the element is used to compute the maturation of this single element from scratch. This tool can be used to evaluate quickly how much impact another choice in kerogen maturation kinetics might make in a particular section location. An example is shown in Figure 168. 

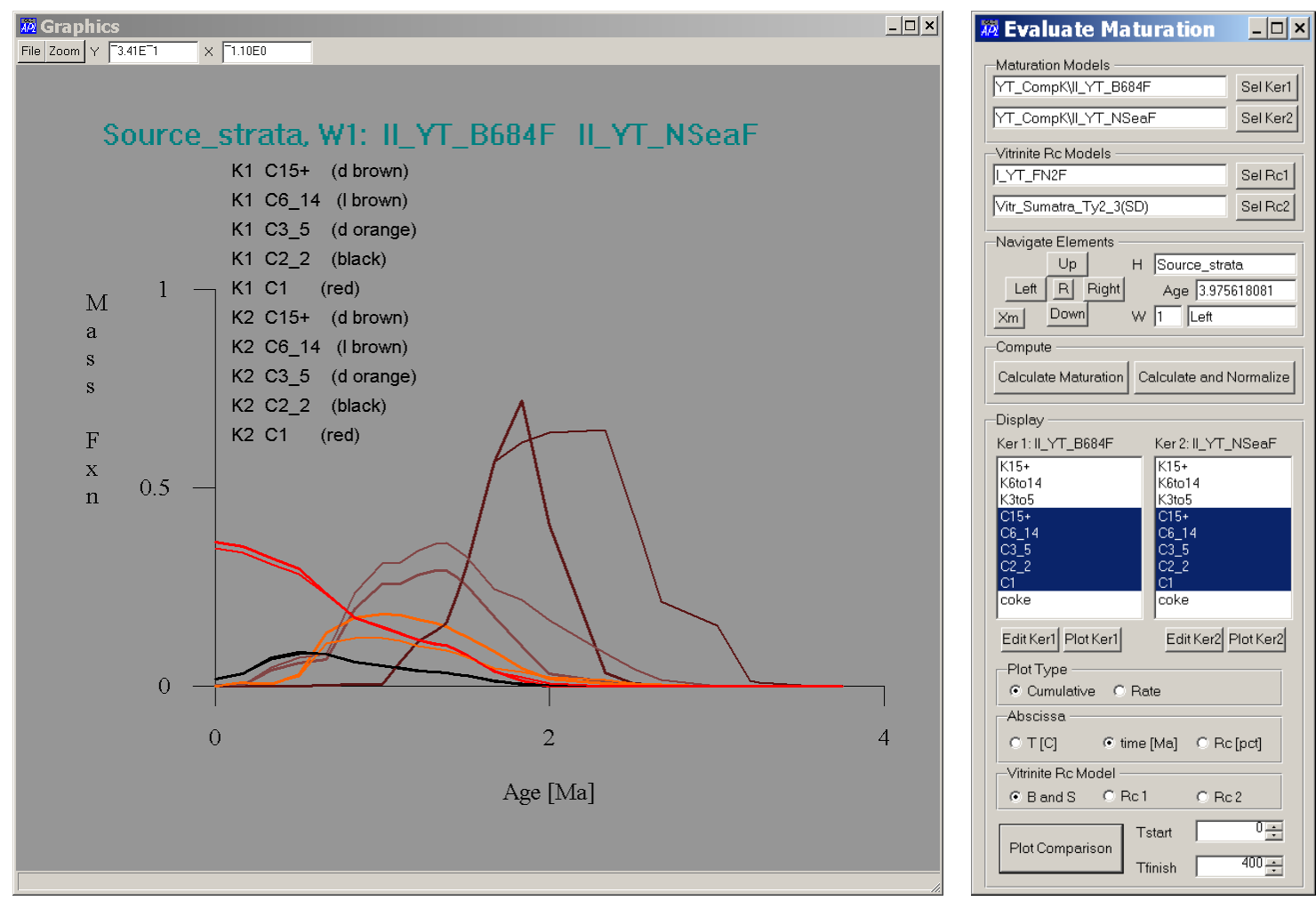

Figure 168. Comparison of maturation of two type II kerogen models for the location shown in Figure 167. The figure is computed using the $t$-series probe described in Figure 167, except that the Maturation Comparison button is pressed. This brings up the Evaluate Maturation form shown to the right. The maturation is calculated by depressing the Calculate Maturation button, and the comparison is selected and plotted as indicated.

\section{B. Petroleum Decomposition and Phase Chemistry}

Petroleum decomposition is carried out just as described in Section IV.J except that the mass fractions of the $\mathrm{C}_{\mathrm{n}}$ cuts are those in the element selected (rather that a set of fractions specified by the user or a set taken at some temperature in the maturation of a kerogen). The procedures are again:

- Select a petroleum decomposition model from Flash: Select Petroleum Model. See Figure 62 for discussion. 
- Select the element whose petroleum you wish to decompose and investigate using Solve Equations: View Calculated Solution: PetroComp button: navigate to element. This procedure will bring up the Petroleum Composition form shown below. Use of this form to select an element for investigation is illustrated in Figure 169.

- If desired (optional step), keeping all forms above open, design the petroleum decomposition using Flash: Design Petroleum Decomposition. This will overlay the Design Hydrocarbon Composition form over the Petroleum Composition form just summoned by the Petcompsn button. This form allows the used to design the decomposition scheme as discussed in Figure 64 and its discussion. This is illustrated in Figure 170.

- Once the petroleum decomposition scheme is determined, all investigation or processing is done through the Petroleum Composition form. Figure 171-Figure 181 illustrate the PetCompsn procedures available.

- The flash calculations depend on pressure as well as temperature. Because calculated pressures may exceed the hydrofracture limit (remember we choose to compute the pattern of flow without requiring that pressures be reasonable), the convention under which pressures will be specified needs to be selected in the Flash Menu. Choices are the excess pressure computed (which can be unrealistic), hydrostatic pressure under any seal, or the maximum of the calculated pressure and the sub-seal pressure with a cap of a specified fraction of lithostatic.

Excess $p 1$ from soln of P-equn Subseal $P=0.8$ lithost at base seal Excess $p<$ hydrofx and max of $p 1$, subseal
Pressure Convention

Select Petroleum Model

View Molecular Components of Selected Model

Design Petroleum Decomposition

Scratchpad: Flash/Wash Decomposed Petroleum 

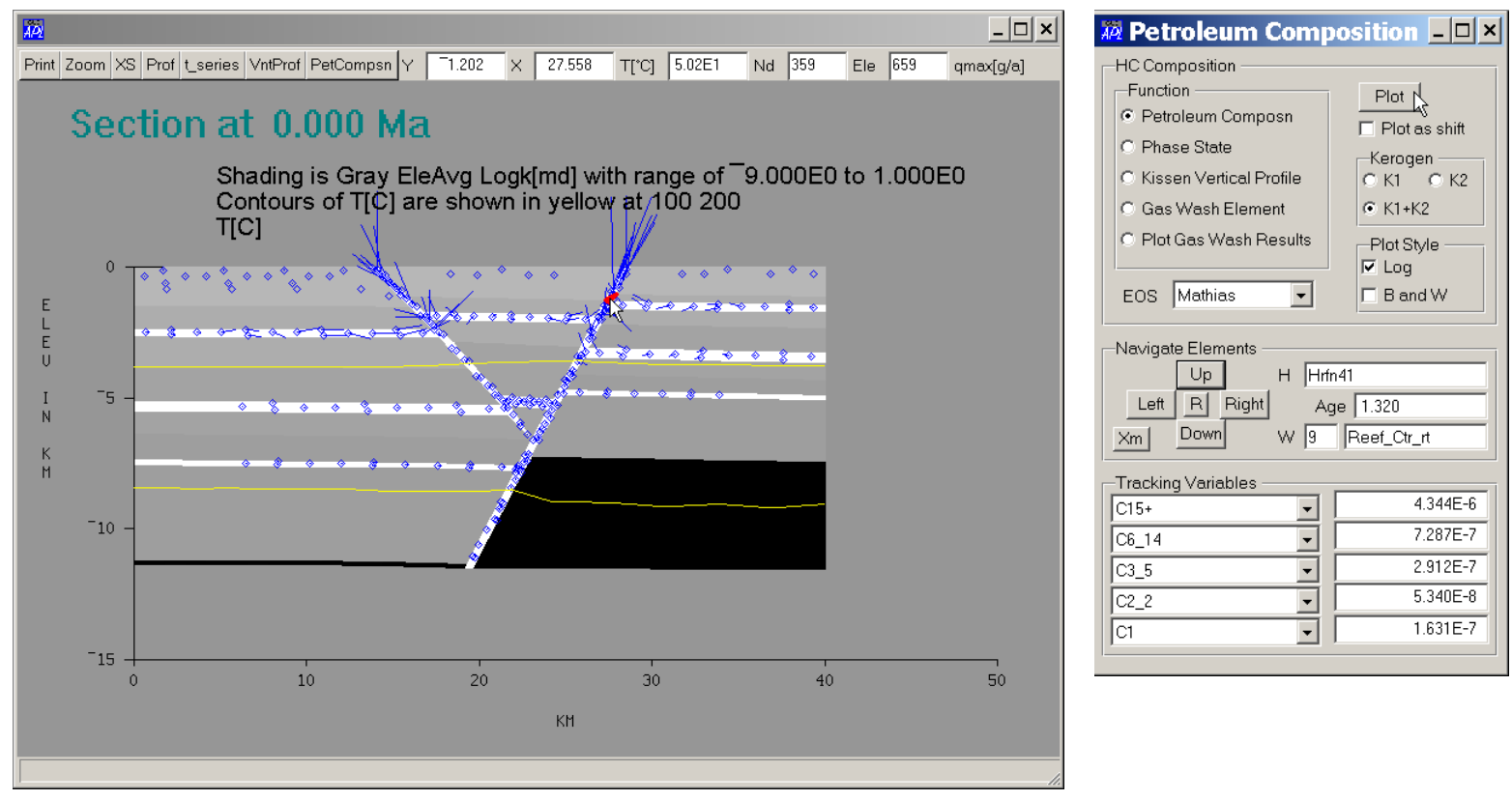

Figure 169. Selection of an element for detailed chemical investigation of the petroleum migrating though it is achieved via the Solve Equations: View Calculated Solution: Petroleum Composition form summoned with the PetCompsn button on the section toolbar. This figure shows that we have used the navigate buttons to select an element in the upper part of the main fault (arrow location). The mouse positioned over the element shows that the element (\# 659) lies $1.2 \mathrm{~km}$ below the surface and has a temperature of about $50^{\circ} \mathrm{C}$. The Tracking Variables are set to the mobile decay products of the first kerogen. Depressing the Plot button (with the Petroleum Composn radio selected), produces the plot in Figure 170. 

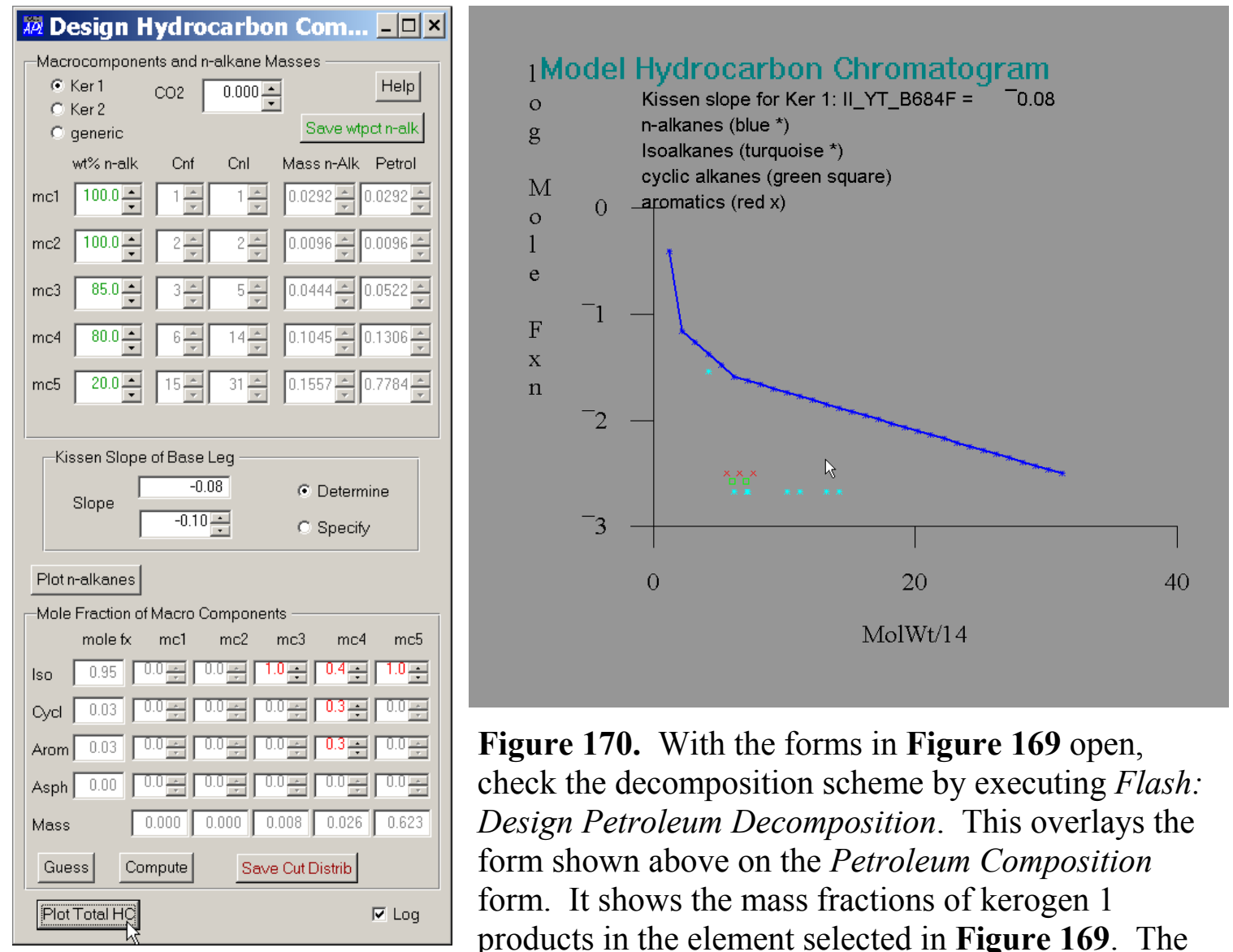

Figure 170. With the forms in Figure 169 open, check the decomposition scheme by executing Flash: Design Petroleum Decomposition. This overlays the form shown above on the Petroleum Composition form. It shows the mass fractions of kerogen 1 products in the element selected in Figure 169. The percent n-alkanes in each carbon number cut (green numbers in first column on the top) can be changed to produce a reasonable kissen slope. The distribution is saved for future use using the Save wtpct n-alk button. The form functions as described in the discussion associated with Figure 64. As discussed there the alkane kissen slope is determined by their mass of the heaviest two carbon number cuts, with lighter cuts attached to the heavier $\mathrm{C}_{\mathrm{n}}$ with slopes adjusted to accommodate their mass. The non-alkanes are then distributed as shown in the lower group. The oil decomposition allows components to be assigned where the numbers are red. The red numbers must sum to 1 in each vertical column. A uniform distribution is provided by depressing the Guess button. This distribution can be changed using the spinners, but be sure the numbers sum to 1 in each vertical column. The total oil composition is plotted by depressing the Plot Total HC button. The result is shown on the right. The impact of changing the red non-alkane distribution numbers can be evaluated by hitting the Plot Total HC button after a change is made. The distribution can be applied to the current case (and saved for subsequent cases unless altered) by depressing the red-lettered Save Cut Distrib button. Once the hydrocarbon decomposition is designed save the Cut and wtpct distributions using the color-lettered buttons and close the Design Hydrocarbon Distribution form. Depressing the Plot button on the Petroleum Composition form (Figure 169) then shows the same plot as above. 

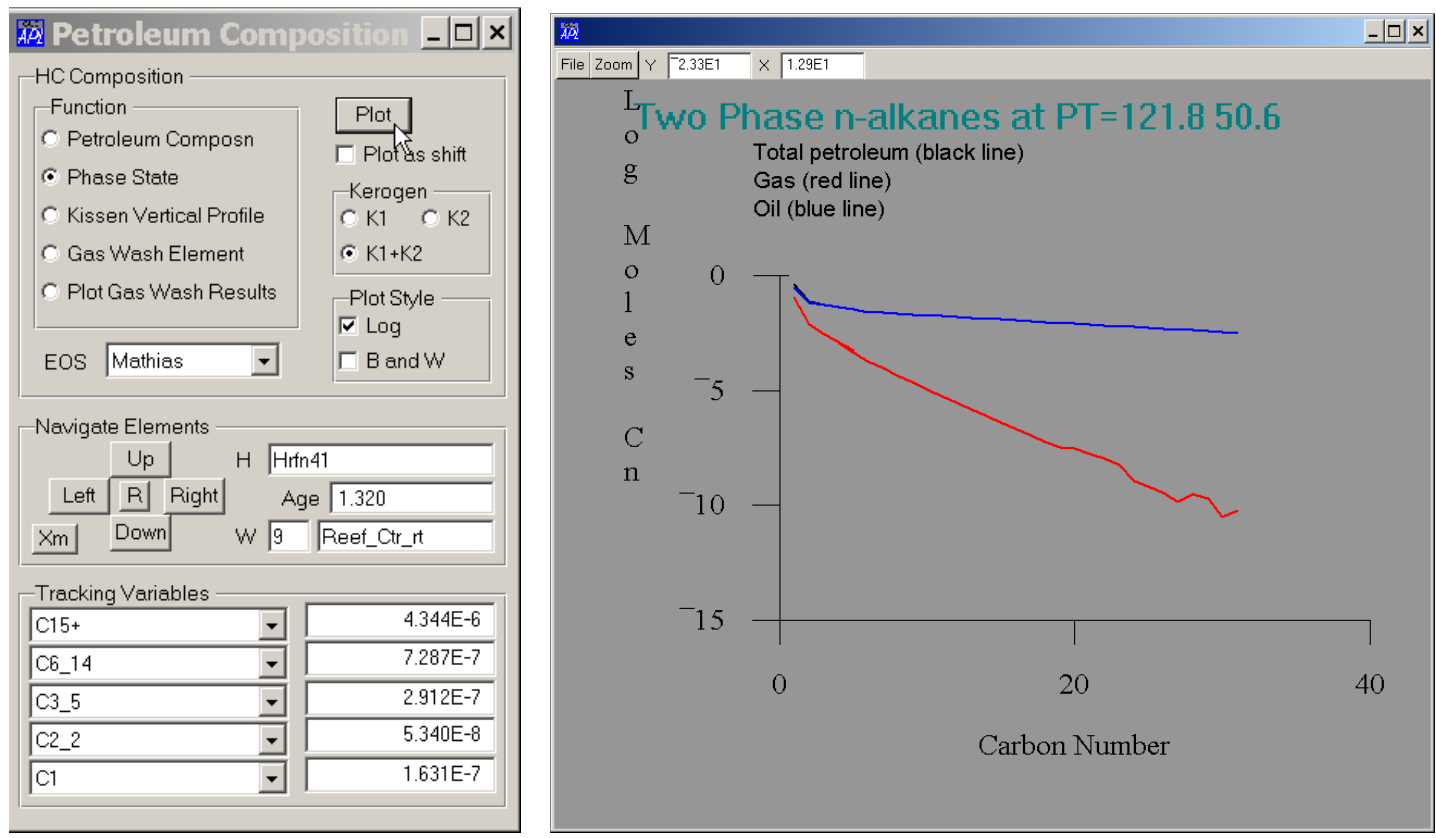

Figure 171. The phase state of the petroleum in the element selected in Figure 169 can be determined by selecting the Phase State toggle and depressing the Plot button. The phase calculation takes $\sim 25$ seconds to compute for the Mathias equation of state. If the petroleum is two phases at the average pressure and temperature in the selected element, the n-alkane compositions of both phases are plotted. If the petroleum is single phase only the liquid n-alkane composition is plotted. Moving down the main fault we find that the phase boundary is at $\sim 1.5 \mathrm{~km}$ depth $\left(57^{\circ} \mathrm{C}\right)$.

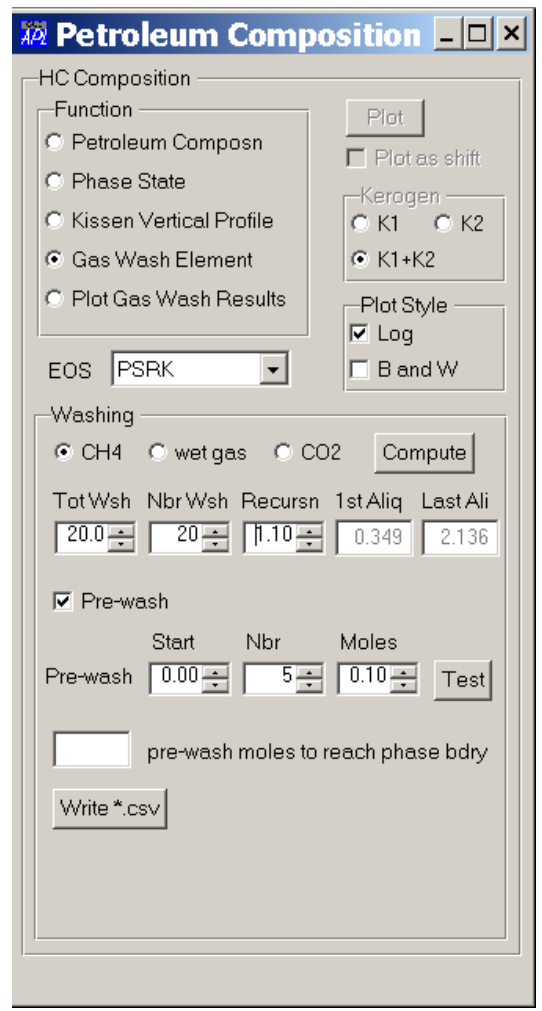

Figure 172. Gas washing can be calculated for the petroleum in element 659 (Figure 169) by selecting the Gas Wash Element radio and specifying the moles and type of washing gas (20 moles $\mathrm{CH} 4)$, and the ratio between washing aliquots, and depressing the Compute button. In this case we know the petroleum is above the phase boundary so we start with 0 moles of methane added to the oil. If the petroleum is below the phase boundary, we find the phase boundary by adding a number of aliquots of washing gas, starting with an aliquot whose size is indicated under the Pre-wash Start label and increasing at the moles indicated under the Moles label for a total of Nbr aliquots. This range of aliquots should be designed to span the phase boundary. The program finds the two wash gas additions that span the phase boundary and subdivides this interval 4 times to hone in on the boundary. This amount of washing gas is then added to the petroleum, and the washing 
sequence started. Then the phase boundary is determined the number of moles of washing gas needed to reach it is posted in the edit box at the bottom of the form. A washing case can be written to the Metafiles folder as a comma separated variable file labeled Wash_History_pic\#_(date and time stamp).csv. This file can be read and processed in Excel and is self-documented as shown in. This file can also be read by BasinL $\boldsymbol{A B}$ at any later time and plotted (see Figure 174). It format is indicated in Figure

Element Washing History
Moles Liquid Petroleum
Well Nbr
Age(Ma)
Strata Name
Well Name
Elevation $(\mathrm{km})$
$\mathrm{P}($ bars $)$
$\mathrm{T}($ deg $\mathrm{C})$
Element $\mathrm{Nbr}$
Petroleum $\mathrm{Nbr}$
Case Path
Cumulative moles washing gas
Moles residual liquid
Mole fraction gas removed
carbon dioxide
methane
ethane
propane

173.

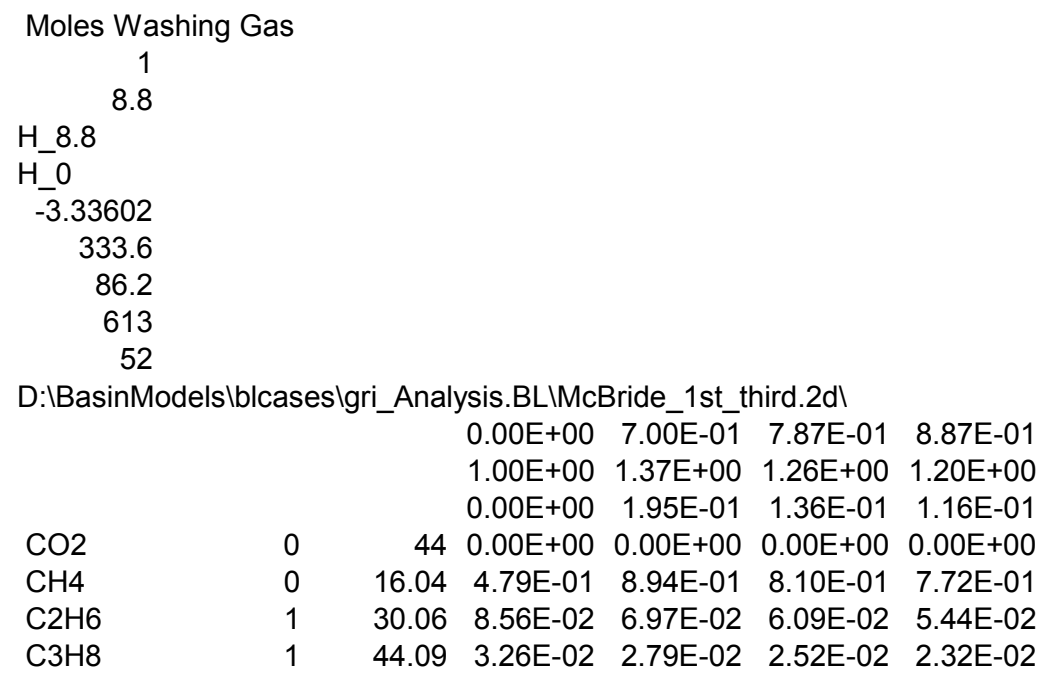

Figure 173. First few lines of the Washing_History.csv file. The first lines give information on the location in the section that was washed. The case path is given. The cumulative moles of washing gas is given, as well as the moles of liquid remaining (not removed by the washing gas) and the mole fraction of liquid removed by each wash. The remainder of the table gives the composition of the liquid fraction as a after each aliquot of washing. 

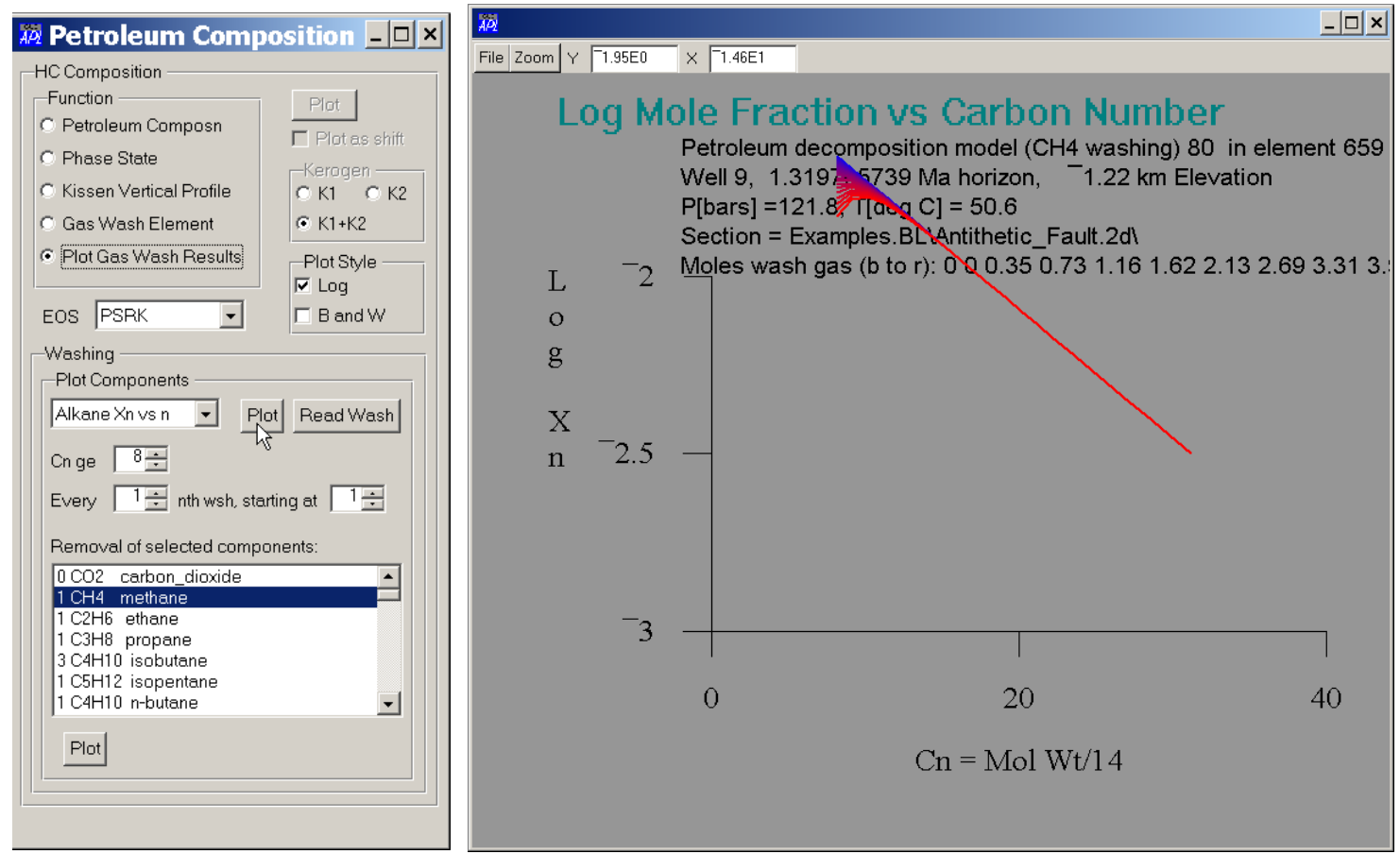

Figure 174. Plotting of a gas washing case is achieved by selecting the Plot Gas Wash Results in the Petroleum Composition form. Previous Wash_History files can be read from the metafiles folder of any case and displayed, or the case just computed can be displayed. A number of plot styles are available as shown in Figure 175-Figure 176. The conventional style shown above shows that the washing break number is $\sim 17$ in this case. Note that the equation of state shown in the image is irrelevant; the equation of state used in the calculation is controlling. For recent discussions of methane washing see Losh et. al., (2002).
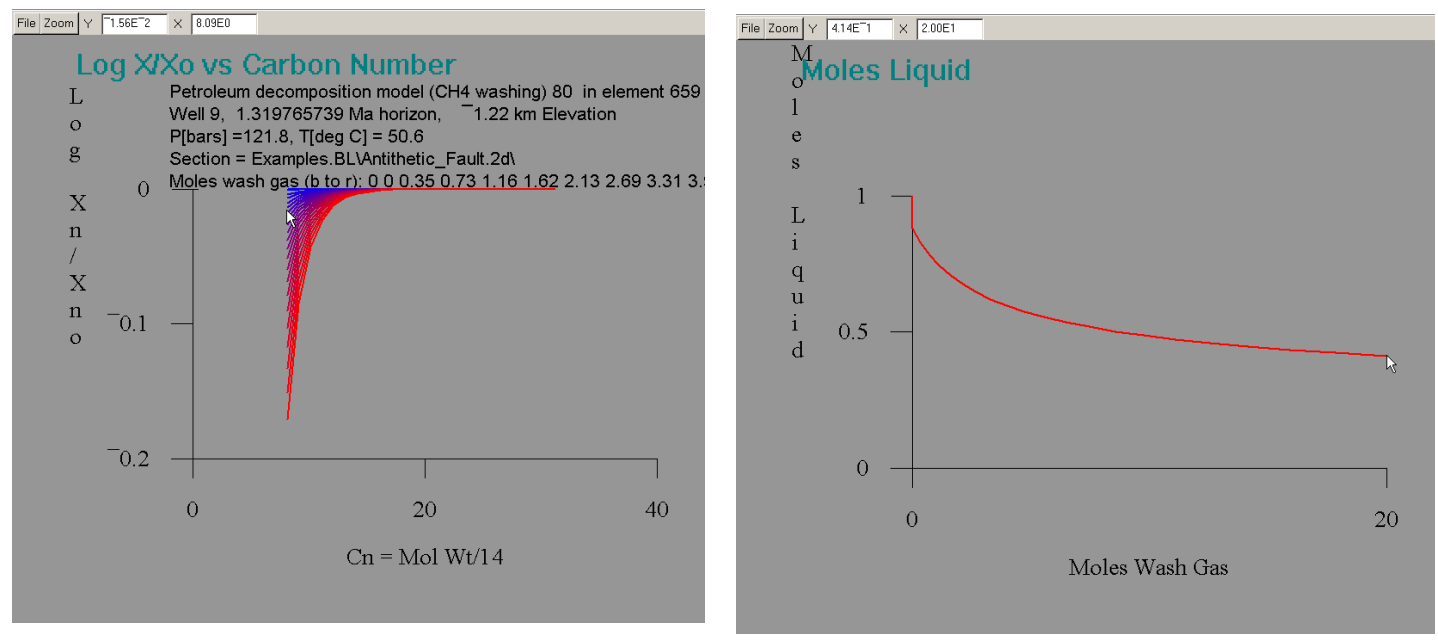

Figure 175. Other plotting options can be selected from the Plot Components combo. For example the petroleum removal can be plotted relative to the original n-alkane distribution AlkaneXn/Xnovs - as shown in the figure on the left. The remaining moles of petroleum can Alkane Xnvs $\mathrm{n}$ Alkane Xnvs $n$ Alkane Xn/ Xno vs n Moles Liq vs Moles $W$ Mtxn gas vs Moles W be plotted using the Moles Liq vs. Moles Wash Gas option. This plot (where we have 
unchecked the log checkbox) shows that about 60 mole percent of the original petroleum has been removed by a combination of phase separation and gas washing.
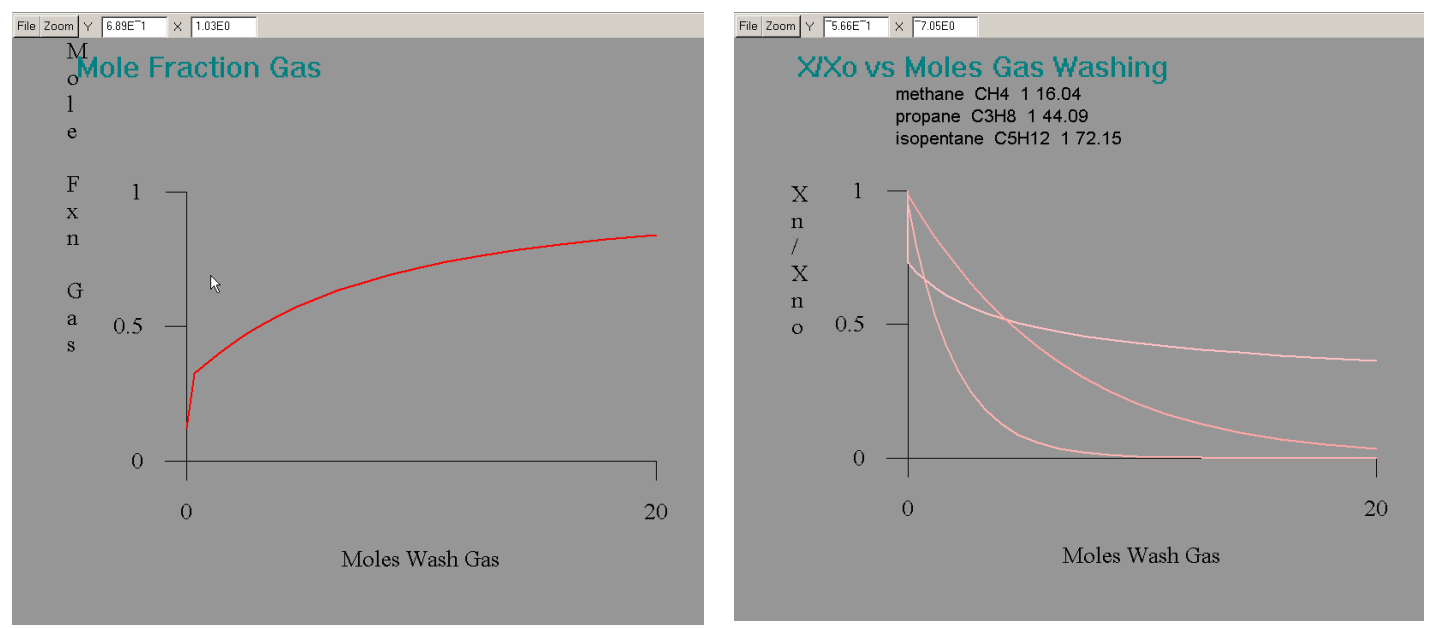

Figure 176. The mole fraction of wash gas in the phase separates declines as washing progresses. This plot is obtained using the Mlfxn gas vs. Moles Wash Gas option shown in the caption of Figure 175. The plot to the left tracks the washing of some of the principle n-alkane components. It is described in Figure 177.
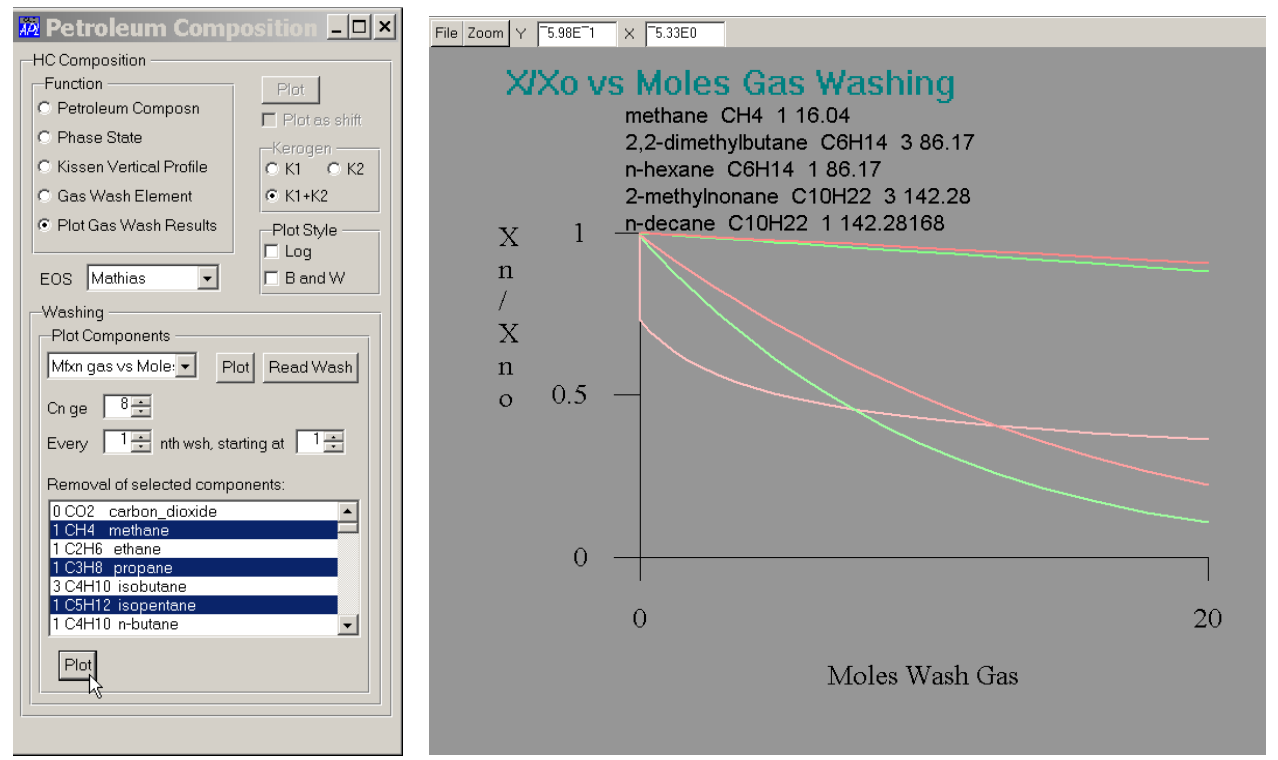
Figure 177. Finally changes in the abundance of any of the components in the oil can be plotted as a function of washing. This is done using the removal of selected components section of the plotting Petroleum Composition form. In Figure 177 we have plotted the selection of components shown selected above. In the figure to the right we have plotted the log depletion of a set of alkane (pink to red with type =1) and branched alkane (green with type $=3$ ) components (log checkbox checked). The caption gives the component name, the chemical formula, the structure type $(1=$ alkane, $2=$ cyclic alkane, $3=$ branched alkane, $4=$ aromatic), and the molecular weight. This kind of plot could allow the identification of components that are diagnostic of washing.

\section{Washing a Standard Petroleum}

The petroleum decompositions in Section XI.A above connect maturation models to detailed descriptions of hydrocarbon component composition and allow phase predictions to be made from maturation/migration models. It is useful, however, to be able to perform flash and washing calculations on an oil whose composition has been carefully defined by laboratory analysis. The Flash: Flash/Wash Decomposed Petroleum allows this. The procedures and forms are very similar to those discussed above. We illustrate them in Figure 178-Figure 181 by washing a well-defined petroleum with $\mathrm{CO}_{2}$. This was the study of a recent Cornell MS thesis by Philip Stalcup (Stalcup 2004).
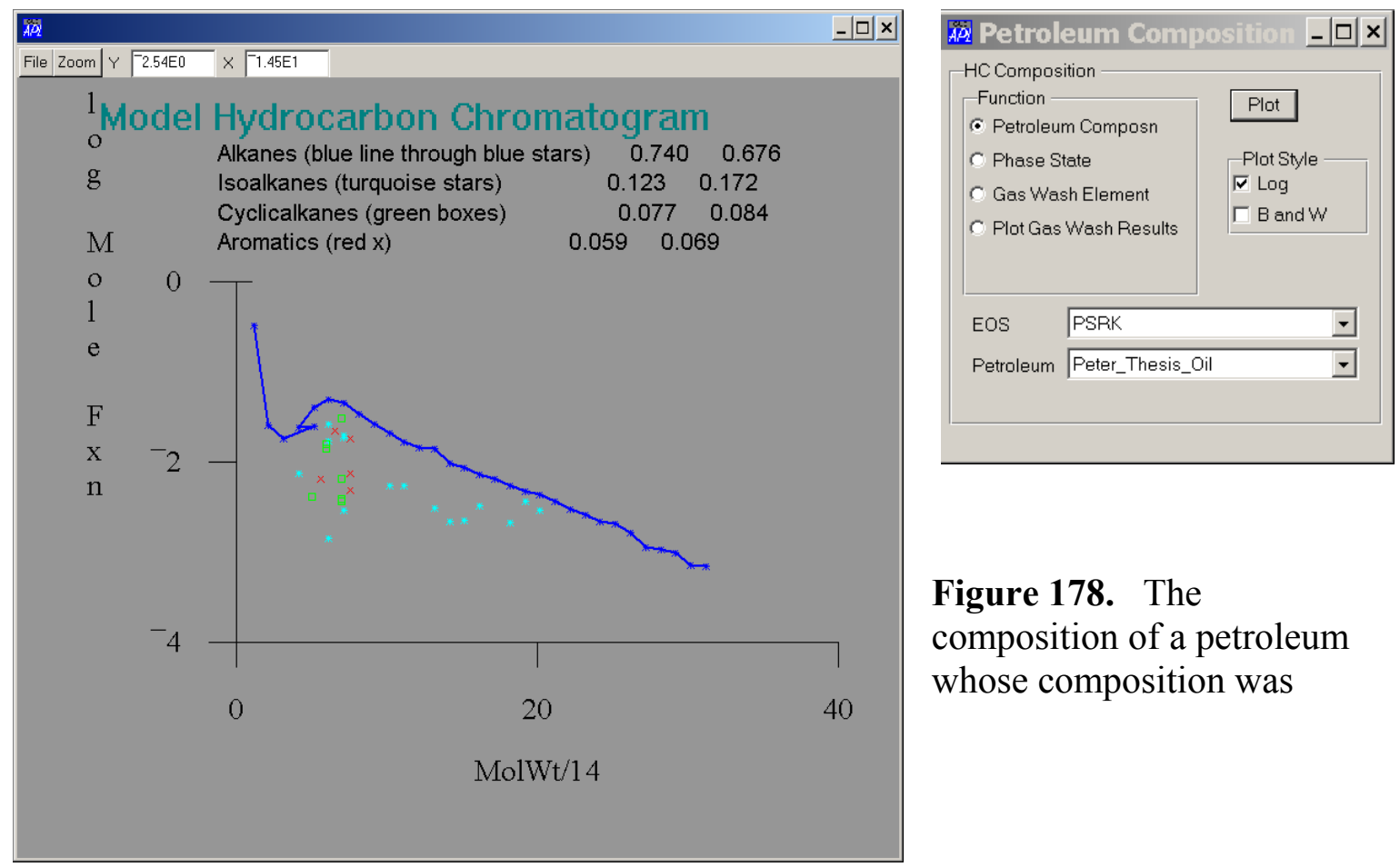

Figure 178. The composition of a petroleum whose composition was 
determined in the laboratory from Peter Meulbroek's $\mathrm{PhD}$ thesis is plotted using Flash: Flash/Wash Decomposed Petroleum: Petroleum Composn toggle: Plot button. The numbers in the caption are the mole and mass fractions of each component type in the petroleum.
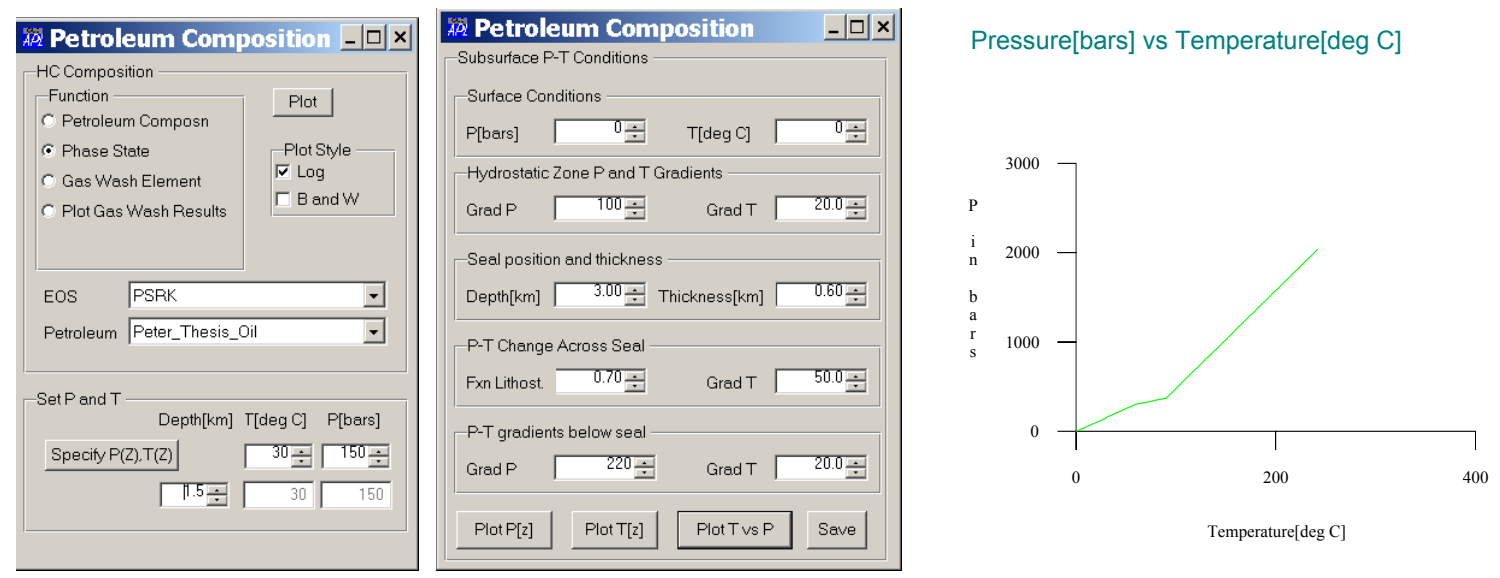

Figure 179. The phase properties of the Petroleum shown in Figure 178 can be determined with the Phase State toggle. The Set $\mathrm{P}$ and $\mathrm{T}$ portion of this form defines pressure and temperature as a function of depth for a sealed basin of the user's design. The Specify $P(z), T(z)$ button defines the pressure and temperature profile in the basin in terms of the $\mathrm{P}$ and $\mathrm{T}$ at its surface, the $\mathrm{P}$ and $\mathrm{T}$ gradients above and below the seal, the temperature gradient in the seal and its rupture pressure, and the thickness of the seal. This supports plots of P vs. depth, T vs. depth, and P vs. T (shown above). The spinner on the form sets $\mathrm{P}$ and $\mathrm{T}$ according to depth using the defined relationship. Here we have selected a depth of $1.5 \mathrm{~km}$ ( $\mathrm{T}=30$ bars, $\mathrm{P}=150$ bars). Alternatively we could have specified the $\mathrm{P}$ and $\mathrm{T}$ directly with the $\mathrm{T}$ and $\mathrm{P}$ spinners.
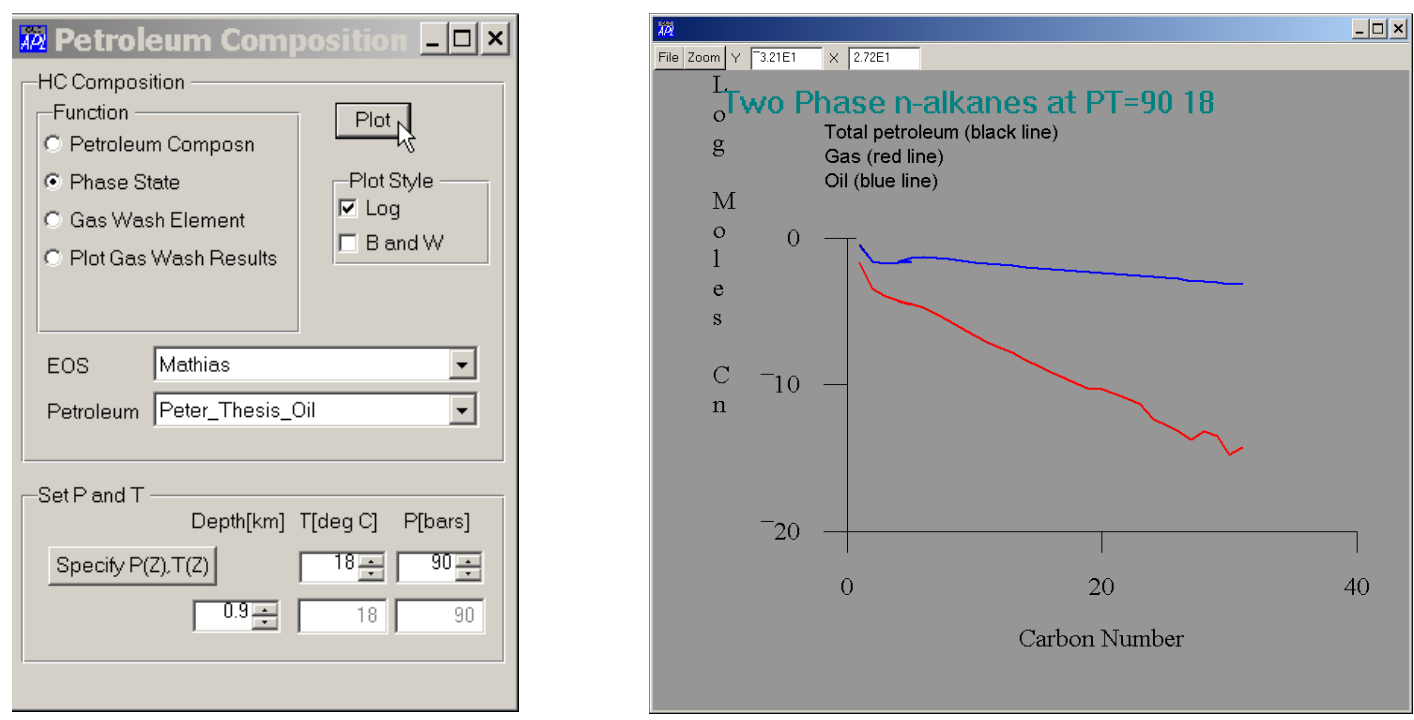
Figure 180. The phase state of the Thesis petroleum is determined for the $\mathrm{P}$ and $\mathrm{T}$ specified by depressing the Plot button in Figure 179. This procedure shows that the petroleum becomes single phase only above $\sim 0.9 \mathrm{~km}$ depth. This is because the petroleum contains less $\mathrm{CH}_{4}$ than it probably should. Addition of $\mathrm{CH}_{4}$ can cause the
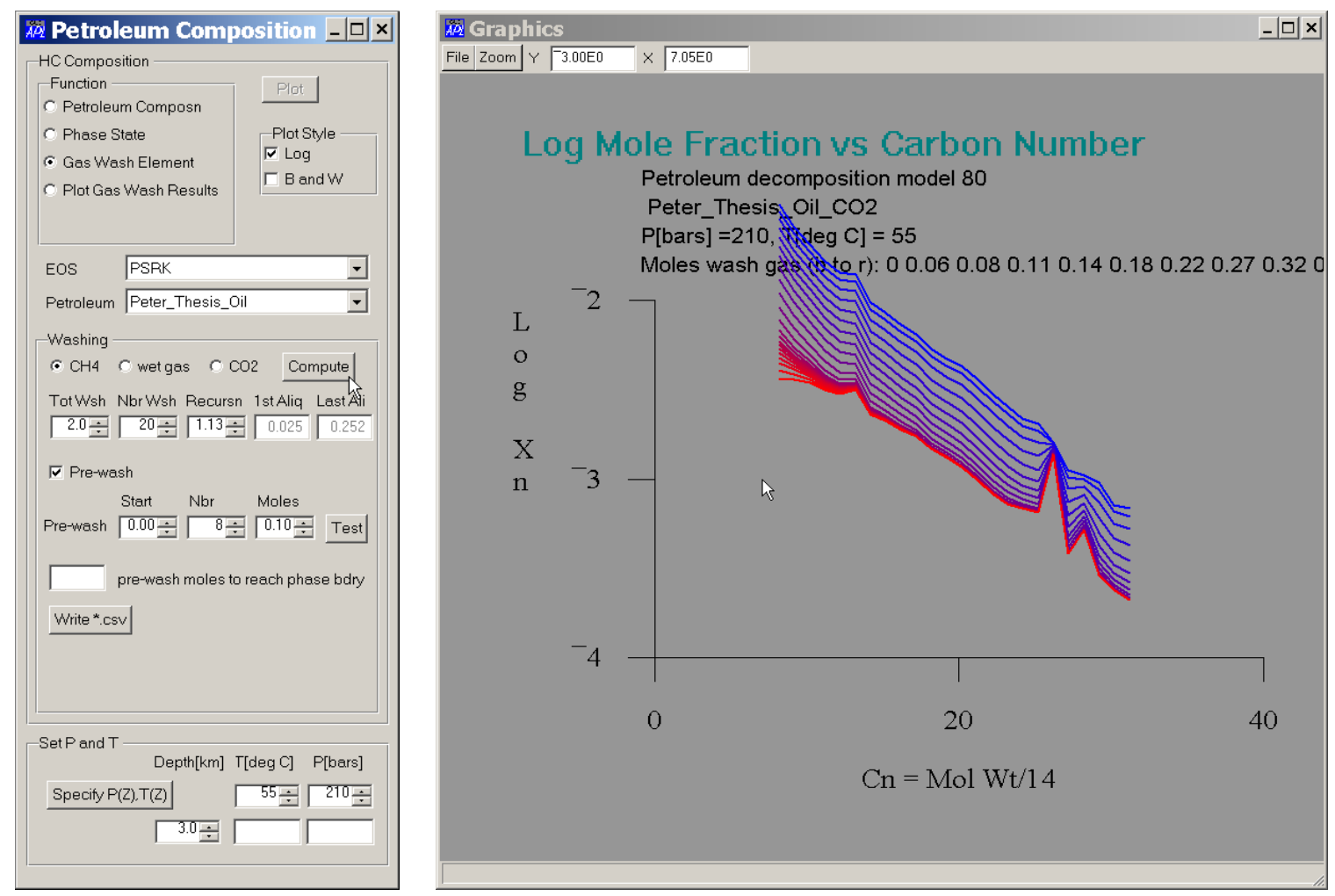

petroleum to phase separate at greater (even much greater) depths.

Figure 181. The petroleum can be washed with $\mathrm{CO} 2$ as well as methane. Here we select a washing depth of $2 \mathrm{~km}$ (55C and 210 bars) and have specified pre-wash additions starting at 0.1 moles $\mathrm{CO}_{2}$ and increasing by 0.1 moles for 8 test aliquots. The pre-wash testing shows that 0.16 moles of $\mathrm{CO}_{2}$ are sufficient to bring the petroleum to the phase boundary. The Figure on the right shows how petroleum components are removed by washing with 2 moles of $\mathrm{CO}_{2} . \mathrm{CO}_{2}$ is a much more potent washing agent than methane; 2 moles does more than 20 moles of $\mathrm{CH}_{4}$. 


\section{Public Web Site and Future Planned Developments}

This manual is a working document. It has been placed on a public web site with executable and source versions of $\operatorname{Basin} \boldsymbol{L} \boldsymbol{A B}$. BasinL $\boldsymbol{A B}$ will be periodically upgraded to remove bugs and advance its capabilities. This documentation will be periodically upgraded as well. Project reports, theses, published papers or references thereto, and links to related material will be placed on the web site. It is our hope that this material and procedure will lead to a greater appreciation of the information content of organic samples and their greater use.

We will use Basin $\boldsymbol{L} \boldsymbol{A} \boldsymbol{B}$ for scientific purposes. In the near term our goals will be to:

- Complete a study of capillary expulsion of oil (Primary Migration)

- Integrate basin seals into computational procedure

- Continue to investigate how patterns of gas washing reflect subsurface sources of gas and gas migration pathways

- Continue to use mixing methods and other tools to constrain the fluxes of organic material leaving active basins such as the Gulf of Mexico Basin.

- Investigate fault control of brine flow and hydrocarbon migration

- Investigate convective flow phenomena in carbonate basins

In the longer term we plan to:

- Integrate inorganic chemical alteration into $\boldsymbol{B a \operatorname { s i n }} \boldsymbol{L} \boldsymbol{A B}$

- Integrate BasinL $\boldsymbol{A B}$ with workstation models capable of 3D flow and alteration simulations

- Include $\mathrm{CO}_{2}$ generation and titration in $\boldsymbol{B a \operatorname { s i n } L \boldsymbol { A B }}$

Upgrades to the web site and Basin $\boldsymbol{L} \boldsymbol{A} \boldsymbol{B}$ will begin in August 2004. We encourage interested parties to collaborate with us in further developments and will appreciate information on bugs and any suggestions for program improvements that would be useful. Communications should be to cathles@geology.cornell.edu with a subject title of BasinLAB. 


\section{Appendices}

\section{A. User Input Files}

Type of File

*.DXF

sflat.txt

erosion.txt

heatflow.txt

waterdepth.txt

paleodepth.txt

heatflowdata.txt

temperaturedata.txt

r0vitrinitedata.txt

biomarkers.txt

Maturation Kinetics

Vitrinite Kinetics

Biomarker Kinetics

Washing History.csv
Function

Input of canvas sketch

Minimal description of geologic section

Description of erosion

Minimal description of the heat flow history

Minimal description of the water depth history

Description of water depth relative to shelf edge

Heatflow data measured along section

Present temperatures in wells along section

Present vitrinite R0 data in section wells

Biomarker data in section wells

Maturation kinetic model description

Vitrinite kinetic model description

Biomarker kinetic model description

Record of washing history readable in Excel
Text Reference

Figure 8, Figure 132

Table 10, Figure 90

Figure 83

Figure 125

Figure 125

Figure 100

Figure 93

Figure 128

Figure 131

Figure 129

Figure 119

Figure 131

Table 13

Figure 167 


\section{B. Variable Names Recognized in Sflat}

Sflat columns with the specific format given below are recognized automatically by BasinLAB. The parameters that will be of most concern to users are indicated in bold type. Several of the parameters are of historical (program development) or future interest only. Nbr indicates the column in the FLAT file that is constructed by Basin $\boldsymbol{L} \boldsymbol{A} \boldsymbol{B}$ from the sflat file input in the course of processing the section. Some of the FLAT columns are filled out by $\boldsymbol{B a s i n} \boldsymbol{L} \boldsymbol{A B}$ during this processing and are of interest only for diagnostic purposes.

\begin{tabular}{l|l|l} 
Nbr & Name & Description \\
\hline 1 & W_Nbr & Well number (from left) \\
\hline 2 & H_Nbr & Horizon number (from top surface down) \\
\hline 3 & X1 & Distance along section from left to right in km \\
\hline 4 & X2(depth) & Elevation relative to present sea level (positive up) in km \\
\hline 5 & X3 & Coordinate perpendicular to section in km \\
\hline 6 & Fx_Sd & Lithologic volume fraction sand (lower left corner element) \\
\hline 7 & Fx_Sh & Lithologic volume fraction shale \\
\hline 8 & Fx_Carb_Sd & Lithologic volume fraction carbonate sand \\
\hline 9 & Fx_Carb_Md & Lithologic volume fraction carbonate mud \\
\hline 10 & Fx_Salt & Lithologic volume fraction salt \\
\hline 11 & Seal_code & Flag in lower left corner indicating element is a seal \\
\hline 12 & I(N_V_H_VH) & Interpolation code (obsolete) \\
\hline 13 & Age(Ma) & Age of horizon \\
\hline 14 & Wat_depth & Water depth of horizon at time it was the basin surface \\
\hline 15 & HF(hfu) & Heat flow into basin when horizon was the basin surface \\
\hline 16 & Sed_rt(m/a) & Sedimentation rate at \\
\hline 17 & diap_tie & Internal variable relating element to diapiric thickness changes \\
\hline 18 & Fault_code & Flag on lower right corner of indicating element is a fault \\
\hline 19 & Sat_oil & Saturation of oil at present day in element (lower left corner) \\
\hline 20 & Sat_gas & Saturation of gas at present day \\
\hline 21 & Mat_param & Data column for maturation parameters \\
\hline 22 & P1 & Excess hydrostatic pressure at present day \\
\hline 23 & avgchordpor & Average porosity in cord joining nodes at present day \\
\hline 24 & T(degC) & Temperature in degrees C at present day \\
\hline 25 & Ro & Vitrinite reflectance at node at present day \\
\hline 26 & CtoD & Carbonate to dolomite ratio of carbonated in element \\
\hline 27 & Ker1(wtpct) & Weight percent first kerogen type in element \\
\hline 28 & Ker2(wtpct) & Weight percent second kerogen type in element \\
\hline & &
\end{tabular}




\section{Sketch Derivation of Equations}

The equations that describe basin processes are conservation equations that can be represented (Fitts, 1962) by a Eulerian formula of the following general form:

(1) $\frac{\partial \rho_{\alpha} \phi_{\alpha} G_{\alpha}}{\partial t}+\nabla \cdot \bar{J}_{G_{\alpha}}-A_{G_{\alpha}}=0$,

where $G$ is any extensive parameter (mass, momentum, energy), $\alpha$ is a material phase (solid, water, gas, or oil), $\rho$ is the density, $\phi$ is the volume fraction of the porous media occupied by $\alpha, G_{\alpha}$ is the extensive parameter per gram of phase $\alpha, \bar{J}_{G_{\alpha}}$ is the flux of a through phase $\alpha$, and $A_{G_{\alpha}}$ is the rate of generation of $G$ per $\mathrm{cm}^{3}$ per second. Following Palciauskas and Domenico (1989), the Eulerian equation can be transformed to equations in a reference frame attached to the porous media by making the following substitutions:

$$
\frac{D}{D t}=\frac{\partial}{\partial t}+\bar{v}_{s} \cdot \nabla
$$

$$
\bar{v}_{f \alpha}=\bar{v}_{\alpha}+\bar{v}_{s},
$$

where $\bar{v}_{f \alpha}$ is the velocity of the fluid relative to the Eulerian coordinate system (fixed reference frame), $\bar{v}_{\alpha}$ is the velocity of a relative to the sediment grain framework, and $\bar{v}_{s}$ is the velocity of the grain framework. Separating the flux of $G_{\alpha}$ into advective and nonadvective parts:

$$
\bar{J}_{G_{\alpha}}=\phi_{\alpha} G_{\alpha} \rho_{\alpha} \bar{v}_{\alpha}+\bar{J}_{G_{\text {non-atva }}},
$$

substituting into (1), and defining $\bar{q}_{\alpha}=\phi_{\alpha} \rho_{\alpha} \bar{v}_{\alpha}$ yields the material coordinate form of the general conservation equation:

$$
\frac{D \rho_{\alpha} \phi_{\alpha} G_{\alpha}}{D t}+\nabla \cdot G_{\alpha} \bar{q}_{\alpha}+\phi_{\alpha} \rho_{\alpha} G_{\alpha} \nabla \cdot \bar{v}_{s}+\nabla \cdot \bar{J}_{G_{\text {non-adiva }}}-A_{G_{\alpha}}=0 .
$$

The fluid and solid phase conservation equations follow immediately by letting $G_{\alpha}=1$ and $\bar{J}_{G_{\text {non-alvo }}}=0$.

$$
\frac{D \rho_{\alpha} \phi_{\alpha}}{D t}+\nabla \cdot \bar{q}_{\alpha}+\phi_{\alpha} \rho_{\alpha} \nabla \cdot \bar{v}_{s}-A_{G_{\alpha}}=0
$$


It should be noted that for the solid phase $\phi_{\mathrm{s}}=1-\phi$, where $\phi$ is the porosity of the porous media. Subtracting $G_{\alpha}$ times (6) from (5) eliminates the $\nabla \cdot \bar{v}_{s}$ term and yields the most useful form of the general conservation equation in material coordinates:

$$
\rho \phi \frac{D G}{D t}+\bar{q} \cdot \nabla G+\bar{J}_{G_{\text {non-adv }}}-\left(A_{G}-G A_{f}\right)=0 .
$$

We have dropped the subscript $\alpha$ because henceforth we will consider only single-phase brine flow. The conservation equations used in basin modeling are all of the form indicated by (5) and (7) and are summarized in Table A-1.

Table A-1: Basin Modeling Equation Summary. The terms in (5) and (7) are defined that yield conservation equations for Fluid Mass, Solid Mass, etc. The final line gives the number of the conservation equation.

\begin{tabular}{llllll}
\hline Component & $\begin{array}{l}\text { Fluid Mass } \\
(5)\end{array}$ & $\begin{array}{l}\text { Solid Mass } \\
(5)\end{array}$ & $\begin{array}{l}\text { Chemical Mass } \\
(7)\end{array}$ & $\begin{array}{l}\text { Momentum } \\
(7)\end{array}$ & $\begin{array}{l}\text { Energy } \\
(7)\end{array}$ \\
\hline$G$ & 1 & 1 & $x_{i}$ & $\mathrm{v}$ & $\mathrm{h}$ \\
$\bar{J}_{G_{\text {non-adv }}}$ & 0 & 0 & $-\overline{\bar{D}}^{E} \cdot \nabla \rho x_{i}$ & $p \overline{\bar{I}}$ & $-\phi \overline{\bar{K}} \cdot \nabla T$ \\
$A_{G}$ & $A_{f}$ & $A_{s}$ & $A_{x_{i}}$ & $\rho \bar{g}-\frac{v}{\overline{\bar{k}}} \cdot \bar{q}$ & $A_{T}$ \\
Equation & $(6)$ & $(6)$ & $(9)$ & $(8)$ & $(10,12)$ \\
\hline
\end{tabular}

As an example of the use of Table A-1, substituting the gravitational sources and viscous sinks of momentum, and the pressure momentum flux into the general conservation equation (7) and assuming that the entire (including $\bar{q} \cdot \nabla G$ ) time derivative term (representing turbulence) is negligible results in Darcy's Law:

$$
\frac{v}{\overline{\bar{k}}} \cdot \bar{q}=-\nabla p+\rho \bar{g}
$$

The conservation of chemical mass in phase a results from substitution of the terms in column 3 of Table A-1 into the general conservation equation (7):

$$
\rho \phi \frac{D x_{i}}{D t}+\bar{q} \cdot \nabla x_{i}-\nabla \cdot D^{E} \cdot \nabla \rho x_{i}-\left(A_{x_{i}}-x_{i} A_{f}\right)=0 .
$$

Note that if $A_{x_{i}}$ derives from a fluid source with solute concentration $x_{s i}$, then the last term becomes $\left(x_{s i}-x_{i}\right) A_{f}$. Thus if the source has the same concentration of $x_{i}$ as the fluid (e.g., if $x_{s i}=x_{i}$ ), the source term is zero, as it logically should be. 
The energy equation follows in a similar fashion and is simplified by assuming that $\mathrm{T}$ in al phases is locally the same, defining a media volumetric heat capacity $\left(\rho_{m} c_{m}\right)$ and thermal conductivity $\left(K_{m}\right)$, and defining a total heat source $A_{T}=A_{T}^{\prime}-h A_{f}$ :

$$
\rho_{m} c_{m} \frac{D T}{D t}+\bar{q} \cdot \nabla h-\nabla \cdot K_{m} \cdot \nabla T-A_{T}=0
$$

It should be noted that a small amount of oil or gas can disproportionately reduce $\mathrm{K}_{\mathrm{m}}$.

The fluid equation of motion (the pressure equation) is a combination of the solid and fluid conservation equations with some time derivative manipulation to produce appropriate thermodynamic coefficients. Taking $\mathrm{A}_{\mathrm{s}}=0$, and remembering that $\phi_{s}=1-\phi$, the solid version of (6) becomes:

$$
\nabla \cdot \bar{v}_{s}=\frac{-1}{\rho_{s}(1-\phi)} \frac{D \rho_{s}(1-\phi)}{D t}
$$

The mass of solid in a material volume element, $\mathrm{V}$, is: $\mathrm{m}_{\mathrm{s}}=\mathrm{V} \rho_{s}(1-\phi)$. Conservation of solid mass, $\mathrm{Dm}_{\mathrm{s}}=0$, then leads to the following identity:

$$
\frac{D V}{V}+\frac{D \rho_{s}}{\rho_{s}}+\frac{D(1-\phi)}{(1-\phi)}=\frac{D V}{V}+\frac{1}{\rho_{s}(1-\phi)} \frac{D \rho_{s}(1-\phi)}{D t}=0
$$

Thus

$$
\nabla \cdot \bar{v}_{s}=\frac{1}{V} \frac{D V}{D t}
$$

The fluid conservation equation obtained by multiplying (6) by $1 / \rho_{f}=1 / \rho$ (note we assume only a liquid water phase in the pore space at this point), and summing over all non-solid phases is:

$$
\frac{\phi}{\rho} \frac{D \rho}{D t}+\frac{D \phi}{D t}+\frac{1}{\rho} \nabla \cdot \bar{q}+\phi \nabla \cdot \bar{v}_{s}-\frac{A_{f}}{\rho}=0 .
$$

Finally we need to specify the material properties. Traditionally, this is done by applying the chain rule on the time derivative terms, which depend on stress, fluid pressure, and temperature. To do this we first transform $\frac{D \phi}{D t}+\phi \nabla \cdot \bar{v}_{s}$ : From (12) it is seen that 
$\frac{D \phi}{D t}=\frac{1-\phi}{V} \frac{D V}{D t}+\frac{1-\phi}{\rho_{s}} \frac{D \rho_{s}}{D t}=0$, and from (13) $\phi \nabla \cdot v_{s}=\frac{\phi}{V} \frac{D V}{D t}$ so that $\frac{D \phi}{D t}+\phi \nabla \cdot \bar{v}_{s}=\frac{1}{V} \frac{D V}{D t}+\frac{1-\phi}{\rho_{s}} \frac{D \rho_{s}}{D t}$. Substituting this in (14) yields:

$$
\frac{\phi}{\rho} \frac{D \rho}{D t}+\frac{1}{V} \frac{D V}{D t}+\frac{1-\phi}{\rho_{s}} \frac{D \rho_{s}}{D t}+\frac{1}{\rho} \nabla \cdot \bar{q}-\frac{A_{f}}{\rho}=0
$$

The time derivative terms are now expanded as functions of $\mathrm{p}, \mathrm{T}$, and $\mathrm{s}$. A full discussion is given in Palciauskas and Domenico (1989), and will not be repeated here. Palciauskas and Domenico argue that for inelastic deformation the time derivatives of fluid and solid density depend only on temperature (water and the solid grains are relatively

incompressible compared to the solid matrix), and the solid matrix (the $\frac{1}{V} \frac{D V}{D t}$ term) depends on effective stress and temperature. With these restrictions, the constituitive relations reduce as shown below, and the matrix deformation depends on effective stress:

$$
\begin{aligned}
& \frac{D \rho}{\rho}=\beta_{f} D p-\alpha_{f} D T \\
& (1-\phi) \frac{D \rho_{s}}{\rho_{s}}=\beta_{s} D \sigma-\phi \beta_{s}^{\prime} D p-(1-\phi) \alpha_{s} D T \\
& \frac{D V}{V}=-\beta D \sigma+\left(\beta-\beta_{s}\right) D p+\alpha_{b} D T
\end{aligned}
$$

If $\beta_{s}, \beta_{s}^{\prime}, \beta_{f}<<\beta$ then,

$$
\frac{D \rho}{D t}=\phi \alpha_{f} \frac{D T}{D t}
$$

$$
\begin{aligned}
& \frac{(1-\phi)}{\rho_{s}} \frac{D \rho_{s}}{D t}=(1-\phi) \alpha_{s} \frac{D T}{D t} \\
& \frac{1}{V} \frac{D V}{D t}=\beta \frac{D p}{D t}-\beta \frac{D \sigma}{D t}+\alpha_{b} \frac{D T}{D t} .
\end{aligned}
$$

If we now define the total thermal expansion coefficient $c_{\Sigma T}=\phi \alpha_{f}-(1-\phi) \alpha_{s}+\alpha_{b}$, and substitute (16) into (15), we obtain the final pressure equation:

$$
\beta \frac{D p}{D t}-\beta \frac{D \sigma}{D t}-c_{\Sigma T} \frac{D T}{D t}+\frac{1}{\rho} \nabla \cdot \bar{q}-\frac{A_{f}}{\rho}=0 .
$$

Substituting: 


$$
\begin{aligned}
& p=\rho_{o o} g x_{2}+p_{1} \\
& \rho=\rho_{o o}+\rho_{1},
\end{aligned}
$$

where $\rho_{\text {oow }}$ is such that:

$$
-\nabla p_{o}+\rho_{\text {oow }} \bar{g}=0
$$

into (8), and defining a reduced lithostatic stress (lithostatic stress minus hydrostatic pore pressure):

$$
\sigma=\sigma^{\prime}+p_{o}
$$

and substituting into (17) results in:

$$
\begin{aligned}
& \bar{q}=-\frac{\bar{k}}{v} \cdot\left(\nabla p_{1}+\rho_{1} g \hat{x}_{2}\right) . \\
& \beta \frac{D p_{1}}{D t}-\beta \frac{D \sigma^{\prime}}{D t}-c_{\sum T} \frac{D T}{D t}+\frac{1}{\rho} \nabla \cdot \bar{q}-\frac{A_{f}}{\rho}=0 .
\end{aligned}
$$

A form of (19) and (20) particularly amenable to solution by linear finite element methods is obtained by solving for the non-convective parts of the flow solution from (20) with $q=-\frac{k}{v} \bullet \nabla p_{1}$ (e.g., $\rho_{1}=0$ ), and solving for the convective part of the solution by substituting $\bar{q}^{\text {conv }}=\nabla \times \bar{\Psi}$ into (8) and taking the curl of the entire equation. Taking $\beta D \sigma^{\prime} / D t=-D \phi / D t$, the pressure, stream function, and flow equations are:

$$
\beta \frac{D p_{1}}{D t}-\nabla \cdot \frac{\bar{k}}{v} \cdot \nabla p_{1}-\left(\frac{A_{f}}{\rho}+c_{\Sigma T} \frac{D T}{D t}-\frac{D \phi}{D t}\right)=0
$$

$$
\nabla \cdot A \cdot \nabla \Psi+\frac{\partial p_{1}}{\partial x_{1}}=0, \text { where } A=\left[\begin{array}{cc}
\frac{v}{k_{22}} & 0 \\
0 & \frac{v}{k_{11}}
\end{array}\right]
$$

$$
\bar{q}=-\frac{\bar{k}}{v} \cdot \nabla p_{1}+\nabla \times \bar{\Psi}
$$

This completes derivation of the equations used in the text. Note (21-23) are equivalent to (19-20), but (19-20) are more difficult to solve accurately with linear finite element methods when temperature increases with depth. The reason is that when temperature 
increases (linearly) with depth the zero order (no flow) pressure solution increases parabolically with depth. Thus pressure cannot be approximated with linear elements.

There are discontinuities in $\frac{\partial p_{1}}{\partial x_{2}}$ where the linear segments join and these produce fictitious sources and sinks of fluid. Successful solution of (19-20) when temperature is not constant requires a quadratic finite element method. Note also that the brine source terms have been expressed as the sum of three parts. The first is from chemical reactions with positive volume change, the second is from to thermal expansion of the fluid and rock, and the last from porosity changes caused by compaction. In Basin $\boldsymbol{A} \boldsymbol{A} \boldsymbol{B}$ these sources are estimated from loading and a pseudo 1D solution of the temperature change and maturation carried out before the 2D solution is computed, as discussed in the text of this manual.

Equations (9), (10) and (21-23) are the equations solved in Basin $\boldsymbol{L} \boldsymbol{A} \boldsymbol{B}$ to define the temperature, fluid flow, and solute evolution of a basin.

\section{References Cited}

Baker, A. J. and D. W. Pepper, 1991, Finite Elements 1-2-3: New York, McGraw Hill, $341 \mathrm{p}$.

Blackwell, D. D. and J. L. Steele, 1989, Thermal conductivity of sedimentary rocks: measurement and significance: in N. D. Naeser and T. H. McCulloh, ed., Thermal history of sedimentary basins: New York, Springer-Verlag, p. 13-36.

Bucker, C. and L. Rybach, 1996, A simple method to determine heat production from gamma-ray logs: Marine and Petroleum Geology, v. 13(4), p. 373-375.

Carslaw, H. S. and J. C. Jaeger, 1959, Conduction of Heat in Solids: Oxford, Clarendon Press, $510 \mathrm{p}$

Cathles, L. M., 2002, Quantifying gas, oil, and brine migration in a 124 x $200 \mathrm{~km}$ area of the offshore Louisiana Gulf of Mexico: Executive Summary: in L. M. Cathles, ed., Seal Control of Hydrocarbon Migration and its Physical and Chemical Consequences: Chicago, Gas Research Institute, GRI-03/0065, CD-ROM.

Cathles, L. M., 2002, Seal Control of Hydrocarbon Migration and its Physical and Chemical Consequences, Chicago, Gas Research Institute, 373 p.

Cathles, L. M., E. L. Colling, et al., 2003, 3D flow modeling in complex fault networks: Illustration of new methods with and exploration application in offshore Nigeria: in S. Duppenbecker and R. Marzi, ed., Multidimensional Basin Modeling: Tulsa, AAPG, CD-ROM.

Cathles, L. M. and S. Losh, 2002, A modeling analysis of hydrocarbon chemistry and gas washing, hydrocarbon fluxes, and reservoir filling: in L. M. Cathles, ed., Seal Control of Hydrocarbon Migration and its Physical and Chemical Consequences: Chicago, Gas Research Institute, GRI-03/0065, CD-ROM.

Cathles, L. M. and J. D. Shosa, 2002, A theoretical analysis of inorganic alteration by the flow of brines through seals: in L. M. Cathles, ed., Seal Control of Hydrocarbon 
Migration and its Physical and Chemical Consequences: Chicago, Gas Research Institute, GRI-03/0065, CD-ROM.

Cathles, L. M., M. WIzevich, et al., 2002, Geology, Geophysics, Geochemistry, and GoCAD database: in L. M. Cathles, ed., Seal Control of Hydrocarbon Migration and its Physical and Chemical Consequences: Chicago, Gas Research Institute, GRI-03/0065, CD-ROM.

Coehlo, D. F., 1997, Three dimensional analysis of the temperature field in Block 330, South Eugene Island, Gulf of Mexico: Geological Sciences, Ithaca, Cornell University, p. 292.

Cornelius, R. R., L. M. Cathles, et al., 2003, An automated method for inferring 3D salt movements from the supra-salt sedimentation pattern: in S. Duppenbecker and R. Marzi, ed., Multidimensional Basin Modeling: Tulsa, AAPG, CD-ROM.

Fitts, D. D., 1962, Nonequilibrium Thermodynamics: New York, McGraw Hill, 173 p.

Gatenby, G., 2001, Phase changes: A major aspect of deep water hydrocarbon migration: Petroleum Systems of Deep-Water Basins: Global and Gulf of Mexico Experiencel, Houston, Texas, GCSSEPM, p. 453-468.

Hildebrand, H. B., 1962, Advanced Calculus for Applications: New Jersey, Prentice Hall, $260 \mathrm{p}$.

Hutchison, I., 1985, The effects of sedimentation and compaction on oceanic heat flow: Geophysical Journal of the Royal Astronomical Society, v. 82, p. 439-459.

Losh, S. and L. M. Cathles, 2002, Gas washing of oil and its implications: in L. M. Cathles, ed., Seal Control of Hydrocarbon Migration and its Physical and Chemical Consequences: Chicago, Gas Research Institute, GRI-03/0065, CDROM.

Losh, S. D., L. M. Cathles, et al., 2002, Gas washing of oil along a regional transect, offshore Louisiana: Organic Geochemistry, v. 33, p. 655-663.

Luo, M., J. R. Wood, et al., 1994, Prediction of thermal conductivity in reservoir rocks using fabric theory: Journal of Applied Geophysics, v. 32, p. 321-334.

McBride, B., 1998, The evolution of allochthonous salt along a megaregional profile across the northern Gulf of Mexico Basin: Amer. Assoc. Petrol. Geol. Bull., v. 82, p. 1037-1054.

McKenna, T. E. and J. M. Sharp, 1998, Radiogenic heat production in sedimentary rocks of the Gulf of Mexico basin: AAPG Bulletin, v. 82(3), p. 484-496.

McKenzie, D., 1978, Some remarks on the development of sedimentary basins: Earth and Planetary Science Letters, v. 40, p. 25-32.

Meulbroek, P., 1998, Phase fractionation at South Eugene Island Block 330: Organic Geochemistry, v. 29, p. 223-229.

Palciauskas, V. V. and P. A. Domenico, 1989, Fluid pressure in deforming porous rocks: Water Resources Research, v. 25(2), p. 203-213.

Pollack, H. N. and D. S. Chapman, 1977, On the regional variations of heat flow, geotherms, and lithosphere thickness: Tectonophysics, v. 38, p. 279-296.

Revil, A. and L. M. Cathles, 1998, Permeability of shaley sands: Water Resources Research, v. 35(3), p. 651-662.

Revil, A. and L. M. Cathles, 1999, Permeability of shaly sands: Water Resources Research, v. 35(3), p. 651-662. 
Revil, A. and L. M. Cathles, 2001, The porosity-depth pattern defined by 40 wells in Eugene Island South Addition, Block 330 Area, and its relation to pore pressure, fluid leakage, and seal migration: Petroleum Systems of Deep-Water Basins: Global and Gulf of Mexico Experience, Houston, Texas, GCSSEPM, p. 687-712.

Royden, L., J. G. Sclater, et al., 1980, Continental margin subsidence and heat flow: important parameters in formation of petroleum hydrocarbons: AAPG Bulletin, v. 64(2), p. 173-187.

Sass, J. H., C. A. Brott, et al., 1981, Heat flow from the crust of the United States: in Y. S. Touloukian, W. R. Judd and R. F. Roy, ed., Physical properties of rocks and minerals: New York, McGraw Hill, v. II-2, p. 503-548.

Schowalter, T. T., 1979, Mechanics of secondary hydrocarbon migration and entrapment: American Association of Petroleum Geologists Bulletin, v. 63, p. 723-760.

Stalcup, P. R., 2004, Compositional Changes in Petroleum Caused by Carbon Dioxide: Earth and Atmospheric Sciences, Ithaca, Cornell, p. 47.

Watts, A. B., 1981, The U.S. Atlantic continental margin: subsidence history, crustal structure and thermal evolution, Atlantic City, AAPG Short Course. 


\section{Acknowledgements}

This work was supported by the Department of Energy (FETL) under contract DE-AC2699BC15217 to the California Institute of Technology, Cornell University, and GeoGroup Inc. The work completed under this contract built on work funded by the Gas Research Institute (Grant 5097-260-3787) and benefited from funding from the oil industry to the Global Basins Research Network. We are grateful to all these funding sources, especially the DOE for support of the work reported here. 


\section{Conclusion}

The Advanced Chemistry Basin Model Project was awarded in 1999 to a consortium of the California Institute of Technology, Cornell University, and GeoGroup, Inc, to prepare a public domain, user-friendly basin modeling software under PC or low-end workstation environment that predicts hydrocarbon generation, expulsion, migration and chemistry. The main features of the software are that it:

- Affords users the most flexible way to choose or enter kinetic parameters for different maturity indicators;

- Affords users the most flexible way to choose or enter compositional kinetic parameters to predict hydrocarbon composition (e.g., gas/oil ratio (GOR), wax content, API gravity, etc.) at different kerogen maturities,

- Calculates the chemistry, fluxes and physical properties of all hydrocarbon phases (gas, liquid and solid) along the primary and secondary migration pathways of the basin and predict the location and intensity of phase fractionation, mixing, gas washing, etc., and predict the location and intensity of de-asphaltene processes.

Included within the model distribution is a database of chemical properties for fluid phase calculations, a database of maturity indicator kinetics, and a database of oil maturation parameters. The model can be downloaded from

http://ruby.wag.caltech.edu/ 